NIST NCSTAR 1-5A

Federal Building and Fire Safety Investigation of the World Trade Center Disaster

\title{
Visual Evidence, Damage Estimates, And Timeline Analysis
}

(Chapter 9 - Appendix C)

William M. Pitts

Kathryn M. Butler

Valentine Junker 



\section{Chapter 9 \\ FiRE BEHAVIOR IN WORLD TRADE CENTER 2}

\section{$9.1 \quad$ INTRODUCTION}

This chapter describes the fire behaviors observed in World Trade Center (WTC) 2 during the period following the impact of United Airlines Flight 175 at 9:02:59 a.m. until the tower collapsed at 9:58:59 a.m. The fires had very different behaviors than those observed for WTC 1. In general, there was much less fire spread, and the total area of the outer façade where fires were observed was much smaller. For this reason, the discussion will focus on general characteristics and major changes in fire distribution instead of the details of the fire spread as in Chapter 8 describing the fires in WTC 1.

Façade maps describing window conditions, fire behavior, and smoke observations similar to those developed for WTC 1 have been prepared based on data sheets derived from the visual database. The time-dependent results are shown in Appendix G to Appendix $\mathrm{J}$ for the four sides of the tower.

Other major differences between the two towers are observations of streamers and falling people. Unlike for WTC 1, very limited numbers of streamers and falling people were observed for WTC 2 .

Observations of these events will be described in the text, but, unlike for WTC 1, separate appendices summarizing these observations have not been provided.

\section{$9.29: 03$ A.M. TO 9:15 A.M.}

Figure 9-1 shows an image of the east face of WTC 2 recorded at 9:03:42 a.m., which was $43 \mathrm{~s}$ after the aircraft impact. The numerous white dots are pieces of paper that were expelled from the building due to the aircraft impact and subsequent fireballs. Extensive fires are visible on the 81st, 82nd, and 83rd floors. On the two lower floors, intense fires, which fill the windows with flame, appear to be nearly continuous from around windows 81-321 and 82-322 to the north edges of the floors. On the 83rd floor, the fires are intense, with considerable flaming outside of windows between windows 83-335 and 83-346. There is also a region with a lower intensity fire nearer the center of the face on the 81 st floor, and there is an indication of a small fire near window 80-313 on the 80th floor. Despite the numerous fires present, the amount of smoke being released is relatively small, and it is possible to see most of the east face above the fires.

The intensity levels of the photograph in Figure 9-1 were adjusted to enhance the visibility of an object that is identified by the arrows added to the photograph. A blowup of the area of the photograph including the arrows is provided in Figure 9-2. The object appears as a narrow line that has been draped inside windows on the 82nd floor extending from at least window 82-321 to window 82-339. This hanging object will be observed numerous times in images to be shown below. While it is difficult to identify the object with certainty, the evidence will be strong that it is the end of a part of the 83rd floor concrete floor slab that has been dislodged from the spandrel located above the 82 nd floor and has settled downward several feet to appear below the spandrel. 


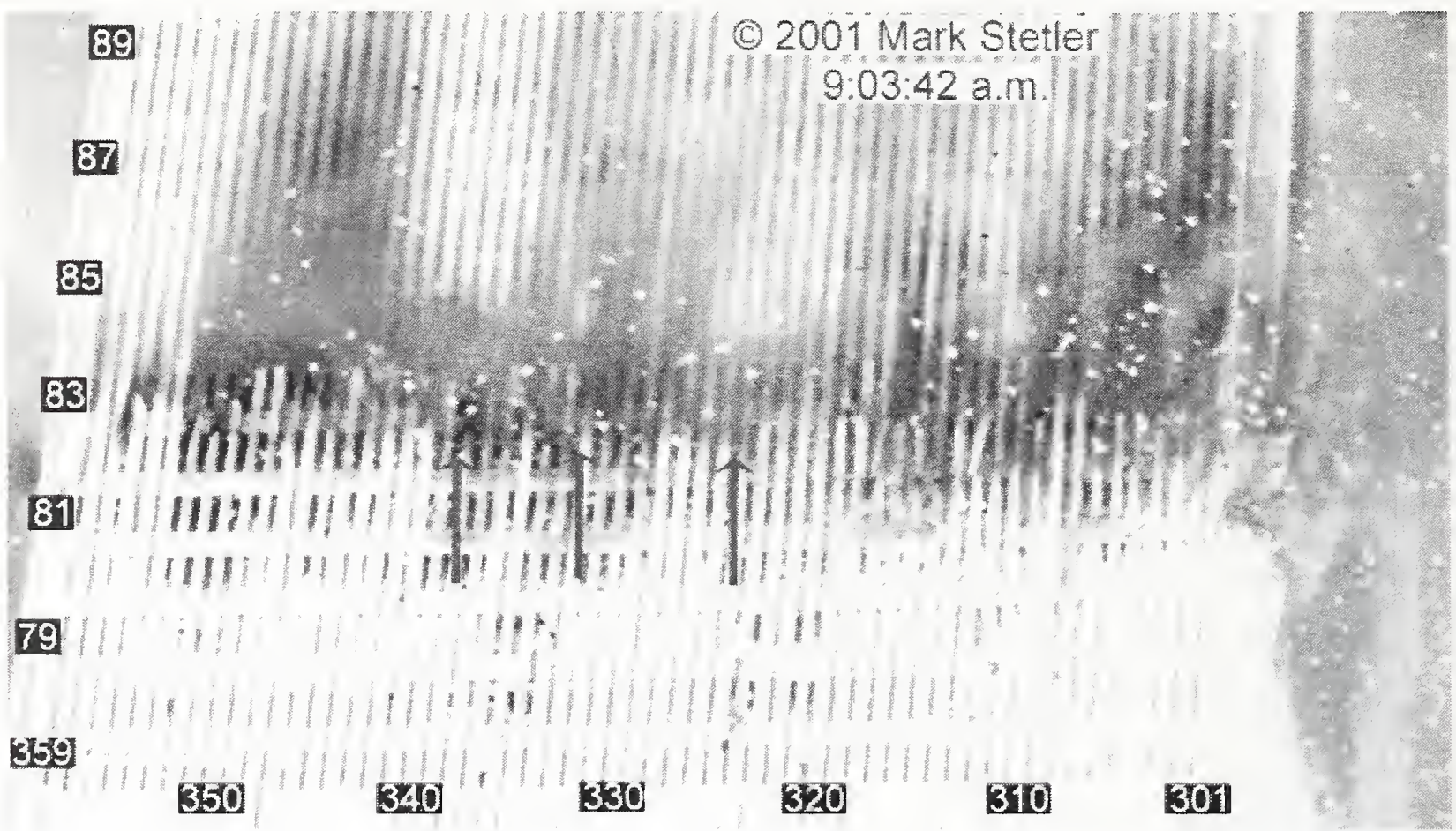

Figure 9-1. A view of the east face of WTC 2 recorded at 9:03:42 a.m. is shown. The original photograph has been rotated, cropped, and enhanced by adjusting intensity levels. Column and floor numbers have been added. The arrows highlight a hanging object observed through open windows on the 82nd floor.

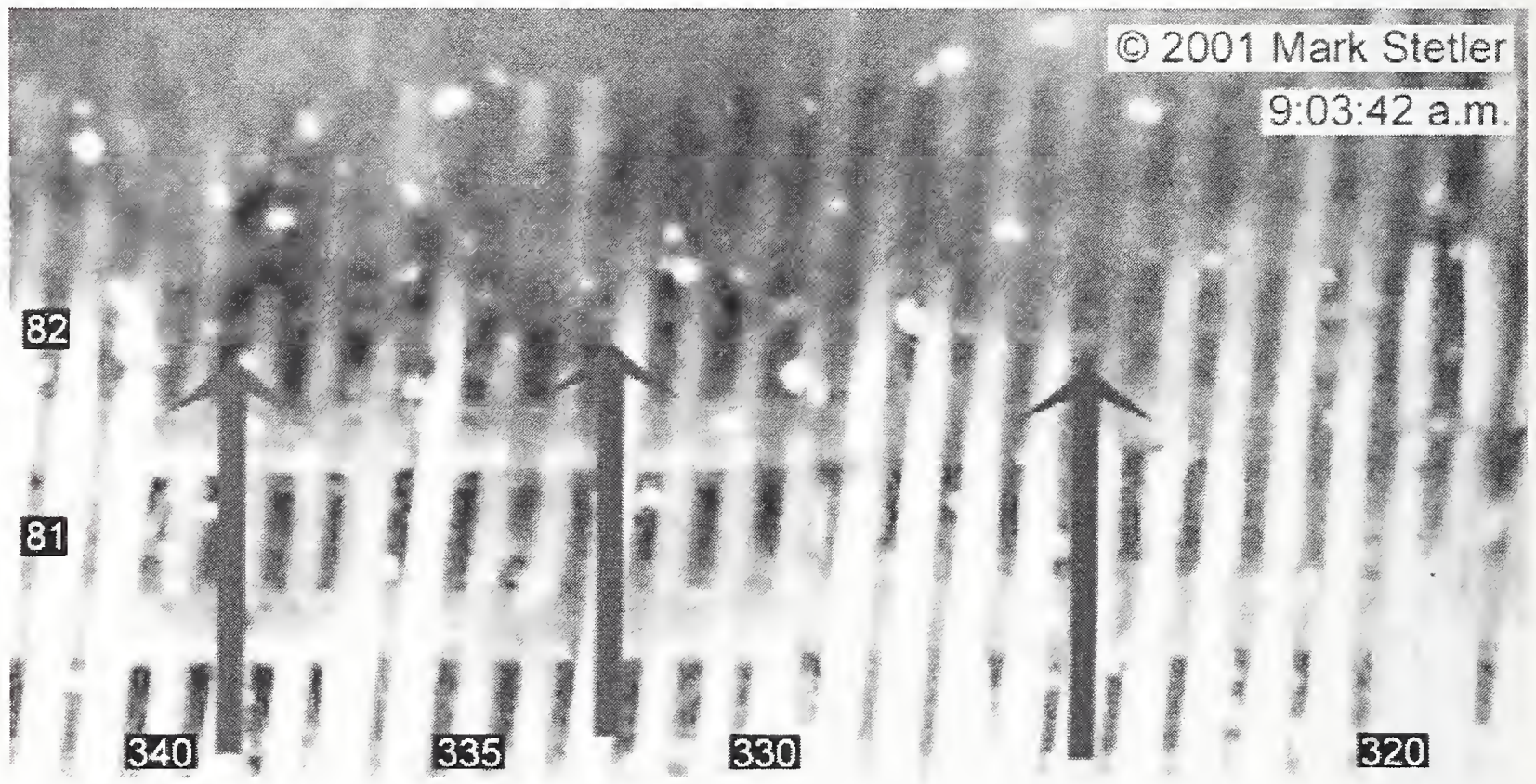

Figure 9-2. A section of the image shown in Figure 9-1 is blown up. The arrows point to the location of an object that is visible through open windows on the $82 \mathrm{nd}$ floor on the east side of WTC 2. 
Closer inspection of Figure 9-1 and Figure 9-2 shows that small flames are visible above the object at the tops of windows 82-335 to 82-337. While not as clear as in these windows, similar fires appear to be present elsewhere along the top of the line. Heavy flames are coming from windows on the 83rd floor immediately above this area. This suggests that the flames observed at the tops of windows on the $82 \mathrm{nd}$ floor are burning at the base of a fuel source located on top of the hanging object that is fueling the much larger flames visible on the 83rd floor. It is likely that this fuel is on top of the concrete floor slab that was originally attached to the spandrel above the 82 nd floor.

The visual evidence supports a conclusion that a substantial length of the concrete floor slab for the 83rd floor along the east facade of the WTC 2 has been dislodged and has come to rest below the spandrel. The edge of the object appears to be continuous, suggesting that even though the floor slab has dropped down, it is essentially intact and is still acting as a single system.

The fact that the hanging object is visible so shortly after the aircraft impact on WTC 2 suggests that it was dislodged at some point during the impact and subsequent fireball. The discussion in Section 7.4.1 indicates that the 83 rd floor was struck by a portion of the aircraft's starboard wing. Other floors struck by much larger sections of the aircraft. however, appeared to have intact floor slabs.

In Chapter 7 it was also noted that a substantial flash fire lasting several seconds occurred on the 82nd floor due to the release of aviation fuel. The sudden appearance of this fire is believed to have generated an overpressure, that may have been on the order of several psi on the floor (see the discussion in Section 6.5). This overpressure was likely responsible for the removal of large sections of the curtain wall on the north and east faces, and it drove additional atomized fuel from the building, resulting in the formation of fireballs. It is possible that the separation of the 83 rd floor slab from the spandrel was due the generation of sufficient overpressure on the 82 nd floor to push the 83 rd floor slab upward off of its seats, allowing it to then settle down below the spandrel.

A view of the north face of WTC 2 recorded at 9:04:13 a.m. is shown in Figure 9-3. The large amount of paper expelled from the tower due to the aircraft impact and subsequent fires is apparent. Two areas of intense fire are seen. One of these is located near the east edge of the face on the 81 st floor, and the second is on the 79th floor, extending from roughly windows 79-231 to 79-238. In Chapter 7 visual evidence was shown indicating that large piles of debris, presumably a mixture of aircraft and building debris, were present at these two locations. Evidently, these debris piles were ignited during the aircraft impact and subsequent fireballs and continued to burn intensely. Even though there is a large amount of dust evident, there does not appear to be a substantial fire in the region between the two burning areas.

A view of the south face of WTC 2 recorded at 9:04:36 a.m. is shown in Figure 9-4. It was taken $97 \mathrm{~s}$ after the aircraft impact on this face. A number of sheets of paper are also visible on this side of the building. The most prominent feature is the damage caused by the aircraft impact. Two areas of bright flame are evident in the image on the 78th and 79th floors. On the 78th floor, the fire is located just to the right of the cavity generated by the aircraft impact, while on the 79th floor the fire appears to be burning within the cavity. On the 82 nd floor there appears to be an orange glow from windows $82-420$ to $82-425$, suggesting that a fire is present in this area as well. 


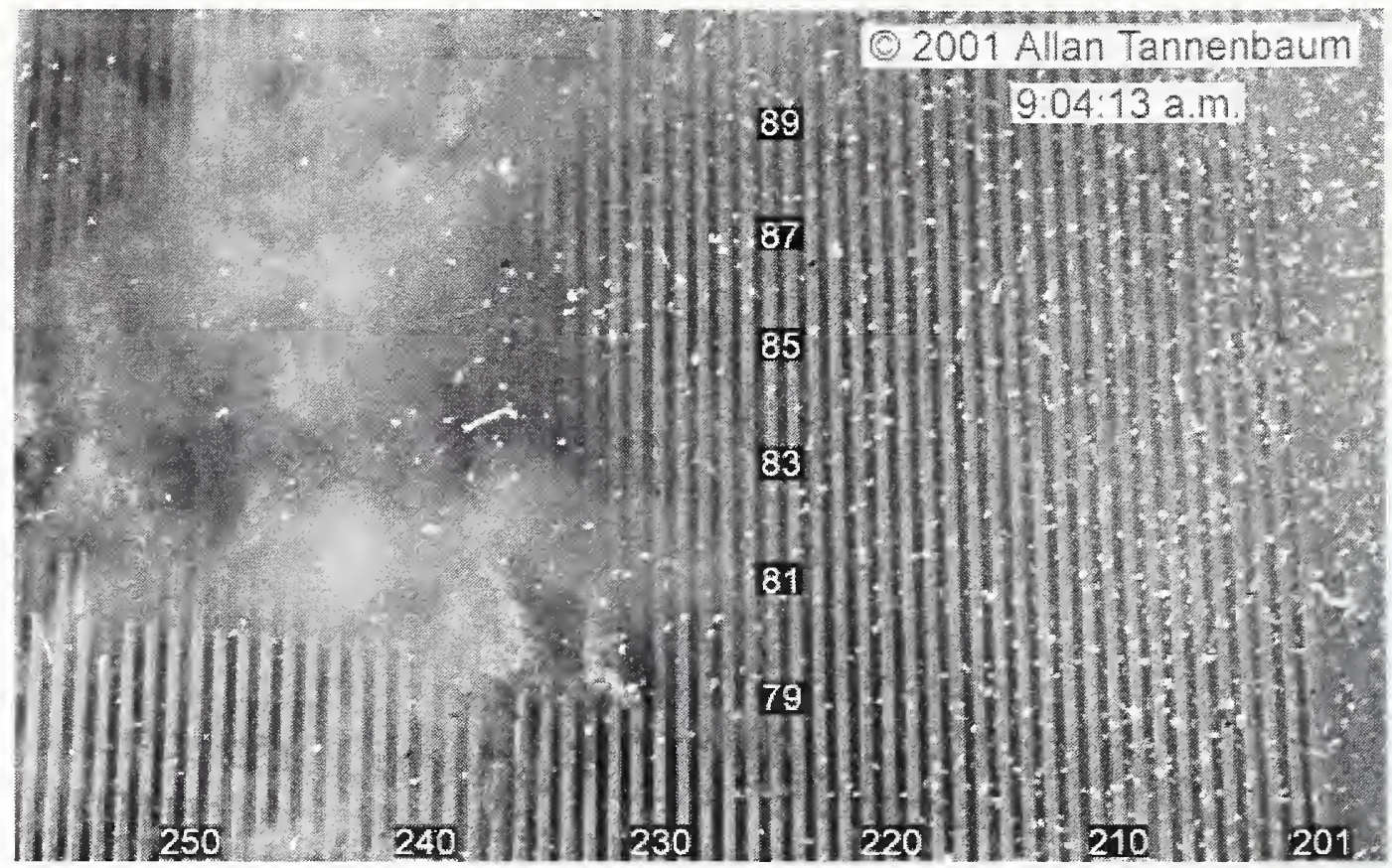

Figure 9-3. This cropped photograph of the north face of WTC 2 was recorded at 9:04:13 a.m. The image intensity levels have been adjusted. Column and floor numbers have been added.

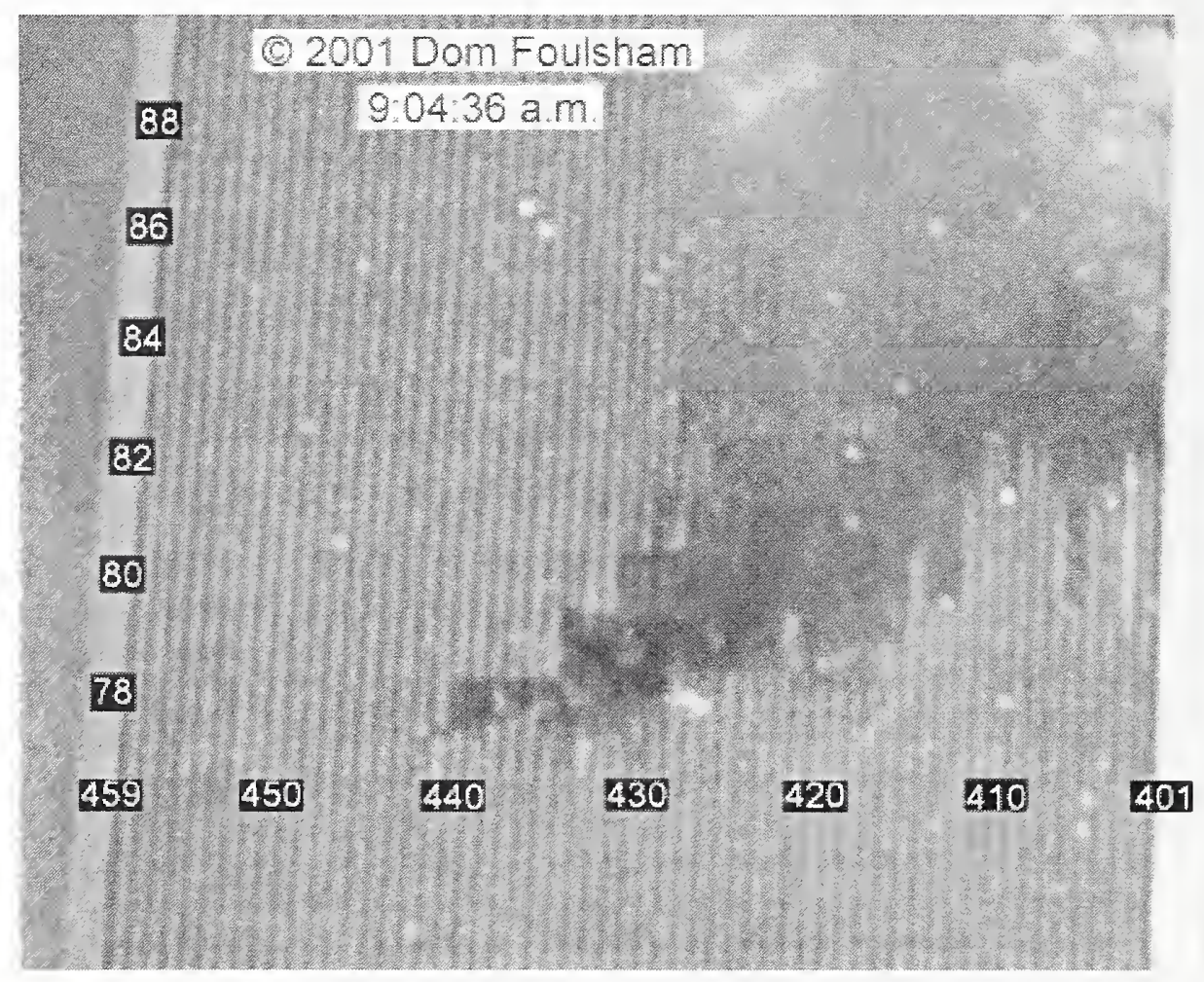

Figure 9-4. This cropped photograph shows the south face of WTC 2 at 9:04:36 a.m. Column and floor numbers have been added. 
There is relatively little smoke coming from windows on the south face at this time. For later comparison purposes, note that the entire lengths of the 81 st and 82 nd floors can be seen and that very little smoke is evident coming from windows on these floors. The only significant plume of smoke is coming from the 83 rd floor near the east edge of the face.

The database does not contain clear images of the west face of WTC 2 in the early period following the aircraft impact. Figure 9-5 shows an image of the face taken from a video recording made from a news helicopter at 9:09:34 a.m. At this time there was no visible smoke or fire on the face. This is consistent with the conclusion in Chapter 7 that the aircraft impact and subsequent fireballs caused no visible damage to the west face of WTC 2.

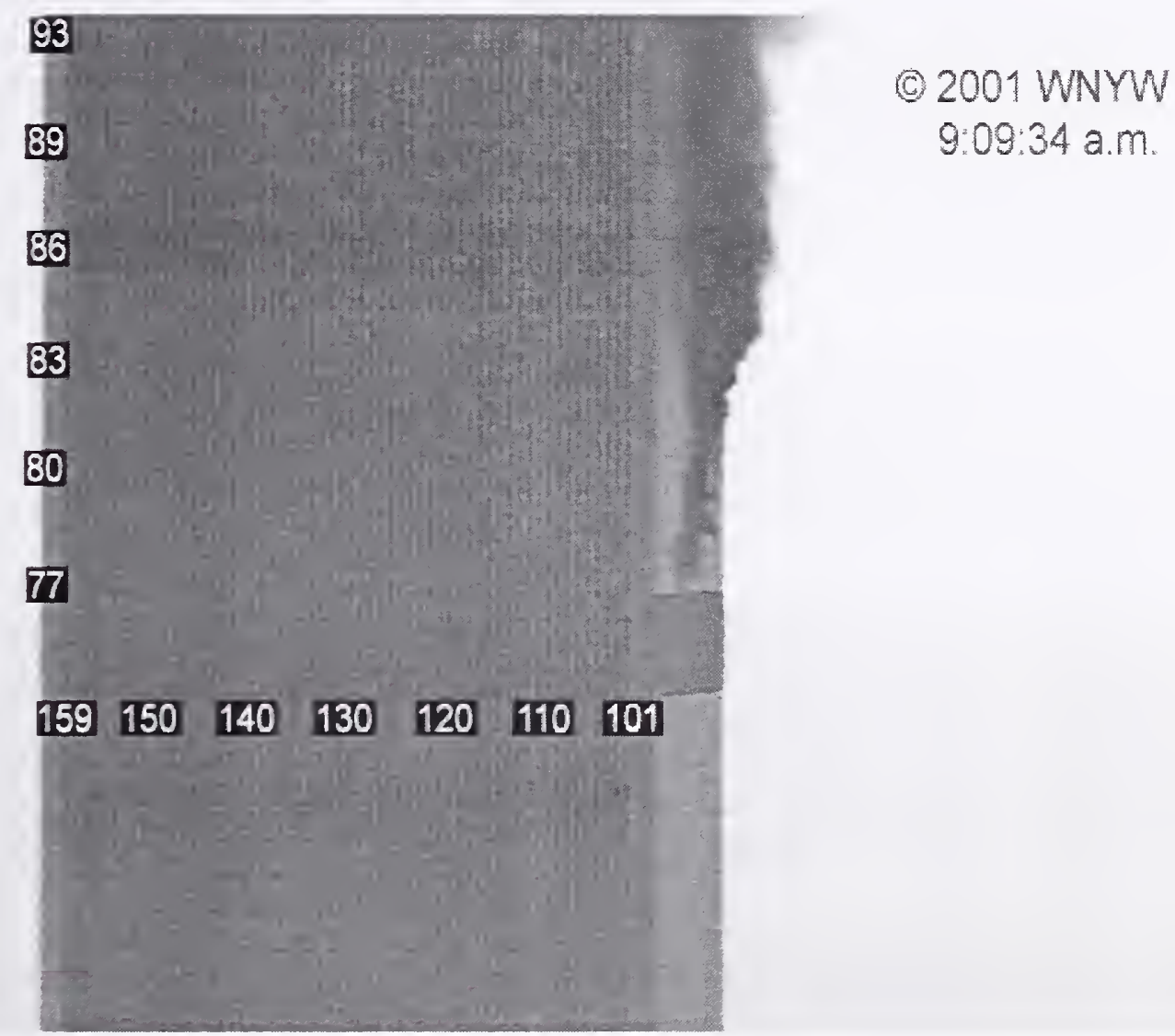

Figure 9-5. This image of the west face of WTC 2 was captured from a video shot from a news helicopter at 9:09:34 a.m. Column and floor numbers have been added.

The west face of WTC 2 remained clear of smoke until 9:10:29 a.m. At this time, a plume of smoke appeared on the 86th floor from near window 86-118. This location is well above the floors where fires had been observed, and there is no indication of an active fire on this floor on the other three faces. A possible explanation for the appearance of smoke at this time is that people in the tower broke out a window and released smoke that had built up on this floor. This explanation suggests that smoke was traveling upward through the tower. 


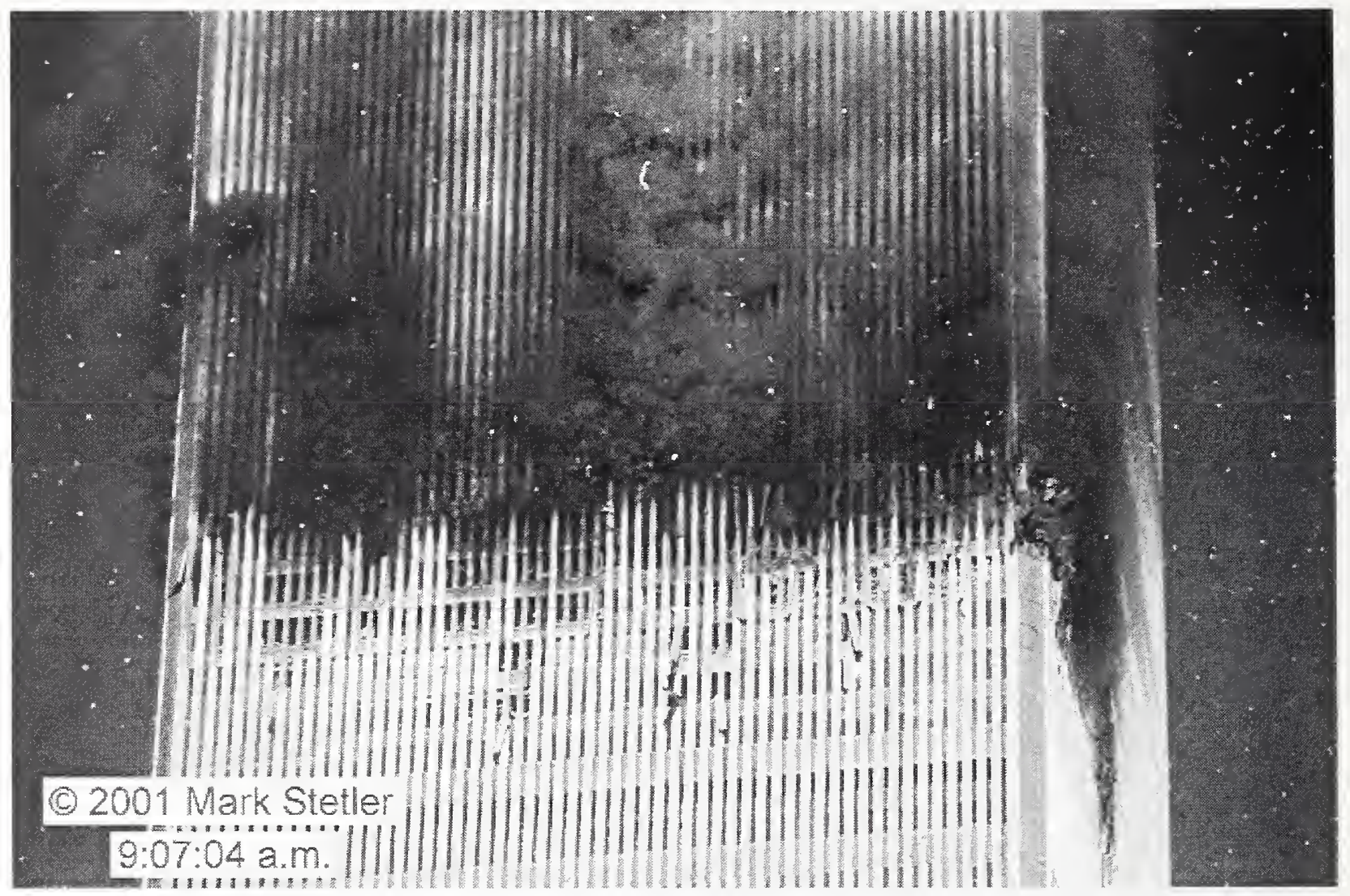

Figure 9-6. This photograph showing the east face of WTC 2 was recorded at 9:07:04 a.m.

A close-up view of the east face of WTC 2 recorded at 9:07:04 a.m. is shown in Figure 9-6. During the 3 min 20 s since Figure 9-1 was taken, the fire distribution on the face has changed. The large fires that were burning earlier on the $81 \mathrm{st}, 82 \mathrm{nd}$, and $83 \mathrm{rd}$ floors appear to have died down somewhat. A new area of intense fire has appeared on the 82 nd floor just to the south of where the large fire was visible on the 83 rd at the earlier time.

A substantial increase in the amount of smoke coming from open windows is evident when the two photographs of the east face are compared. The smoke appears to be coming from two separate locations on the 82 nd floor. Videos of the east face show that heavy smoke appeared first from the area on the south side of the face, increasing rapidly around 9:04:30 a.m. The smoke flow coming from windows just to the north of the center of the face increased markedly about $50 \mathrm{~s}$ later.

The image in Figure 9-6 provides a great deal more resolution than is evident from the way it has been sized. Figure 9-7 shows blowups taken from the photograph of the northern and southern halves of the east face for the floors where fires were present. The north side is at the top of the figure. In this image the damage to the aluminum façade is evident. The debris that has been piled along the east wall in the northeast corners on the 80 th and 81 st floor is visible. There is also a large amount of debris protruding from the opening created by removing the aluminum panel from the northeast corner of the 81 st floor. 

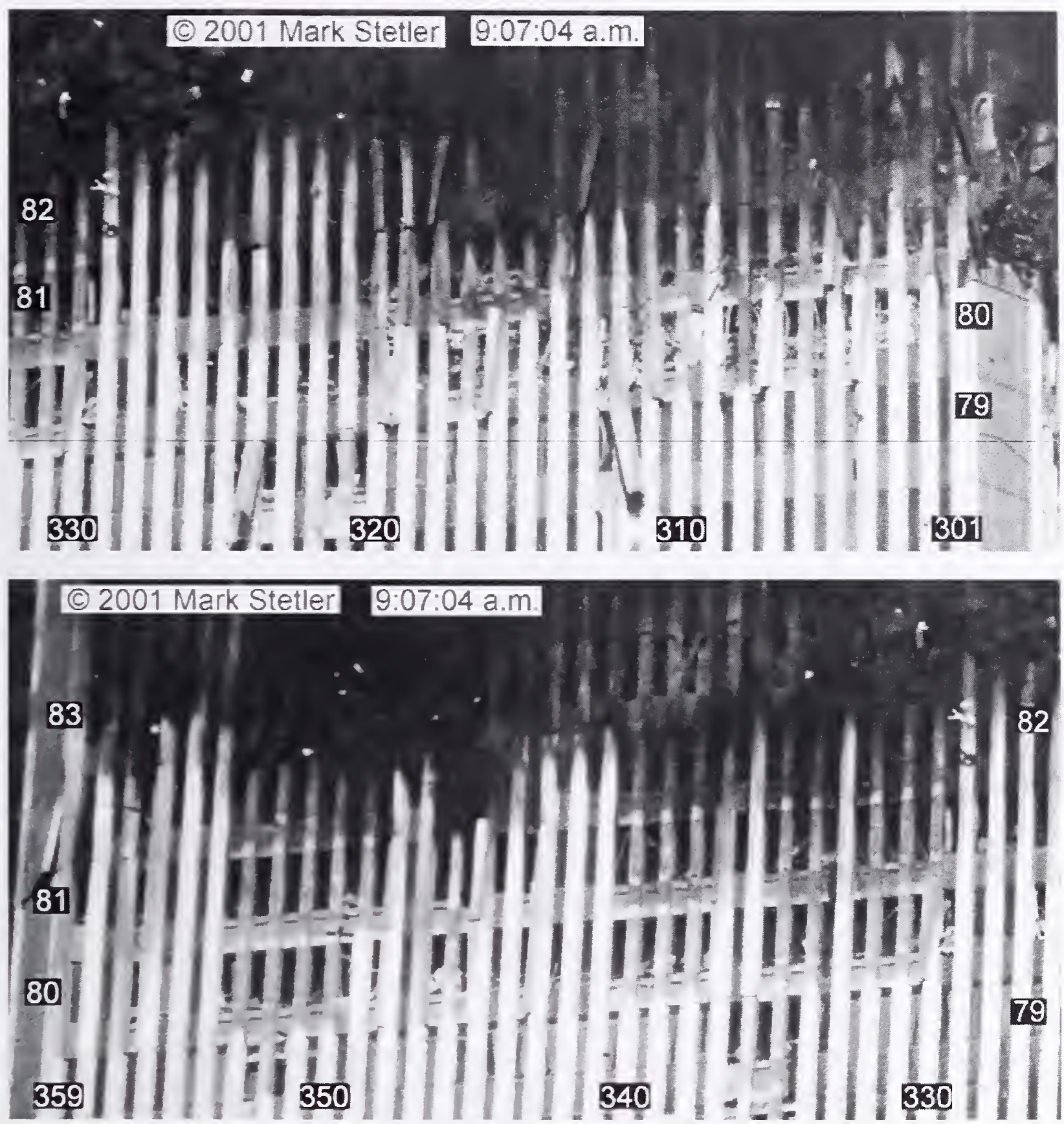

Figure 9-7. These two images show blowups of the north and south sides of the east face of WTC 2 taken from the photograph included in Figure 9-6, which was shot at 9:07:04 a.m. Column and floor numbers have been added.

Heavy fires are burning in the northeast corners of the 81 st and 82 nd floors. On the 81 st floor flames are coming from windows 81-301 to 81-304 as well as from the northeast corner where the aluminum panel has been removed. Lower intensity flames are evident in many windows further to the south, extending at least as far as the last visible window, 81-332. It is likely that the fire is continuous across this entire length. On the 82 nd floor flames are coming from windows 82-301 to 82-309. Flames further to the south do not appear to be as widespread as on the 81 st floor, but there is much more smoke coming from open windows on this floor. The smoke flow is particularly heavy from windows 82-323 to 82-332. The 
smoke flows fill the upper portions of the windows, indicating that a smoke layer exists inside that descends well below the tops of the windows. A flame can be seen coming from window 82-331.

Small isolated fires are also visible on the 80th floor at windows 80-313,80-321, and 80-330. Even though there is a large amount of debris on this floor, the fires are still relatively small. No fire or smoke is evident coming from windows on the 79 th floor, and many windows still have intact glass.

A blowup of the southern side of the fire floors is included at the bottom of Figure 9-7. Flames are visible on the 83 rd floor over a length from near window 83-331 to window 83-338. The intensity of these flames has died down considerably from that observed at the earlier time in Figure 9-1. It can be seen that a considerable amount of the aluminum facing on this floor has been removed.

Unlike in the earlier photograph, intense flames are now visible in windows 82-341 to 82-349. Flames fill these windows and in many cases extend from the opcnings. Heavy black smoke is coming from the flame area as well as from windows immediately below on the 81st floor, particularly window 81-345 and window 81-346. Smoke is also coming from numerous windows to the north of the visible fire on the 82 nd floor. This smoke has obscured the windows where the hanging object was identified in Figure 9-1 and Figure 9-2. Note that the aluminum panel on the southcast corner of this floor is pushed out, but is still attached.

On the 81 st floor the last window to the south where a low-intensity fire is observed is window $81-333$. It is interesting that despite the fact that there is a great deal of damage to the aluminum façade on this floor, there are several windows for which the glass is still in place. The small spot fire present in window 80-330 can be seen in the overlapping lower image as well. Several window panes are also still in place on this floor. No fire or smoke is visible on this half of the 79 th floor.

Figure 9-8 shows the north face of WTC 2 at 9:07:23 a.m. This is $3 \mathrm{~min}$ and $10 \mathrm{~s}$ later than the image of the north face included in Figure 9-3. The view of the fires in the current image is more complete due to the angle and distance from which it was shot. The two large fires that were observed on the 79th and 81 st floors at the earlier time continue to burn vigorously. The fire on the 79th floor still consists of two large tongues of flame at the same locations however, flames are visible further to the west than at the earlier time, now reaching at least as far as window 79-228. In the earlier photograph, windows 79-228 and 79-229 appeared to have intact glass. The intense fire on the east edge of the 81 st floor is in roughly the same location as earlier. In Figure 9-3 it was not possible to determine whether the 82 nd floor was burning. It is clear in the later image that there is a fire on the 82 nd floor, with flames coming from windows $82-252$ to $82-258$. It was difficult to see this area immediately following the aircraft impact because of the amount of debris released and the large flames present on the 81 st floor. A review of images indicates that the $82 \mathrm{nd}$ floor was burning in this corner as early as 9:04:25 a.m.

In Section 7.4.2 it was noted that there was a large rectangular area on the east side of the north face where large fires did not grow. For this reason, the area was referred to as the "cold spot". The area ran between columns 238 and 250 and included the 80 th, 81 st, and 82 nd floors. Later in this chapter an infrared image will be reproduced that shows that this area was indeed at a much lowcr temperature compared to the surrounding locations where large fires were present. The rectangular area stands out distinctly in Figure 9-8 because it lies between two intense fires, and most of the aluminum cladding in the area has been removed. Recall from the discussion in Chapter 7 that the largest fireball that formed on the north face of the tower following the aircraft impact came from the general area of the cold spot. 


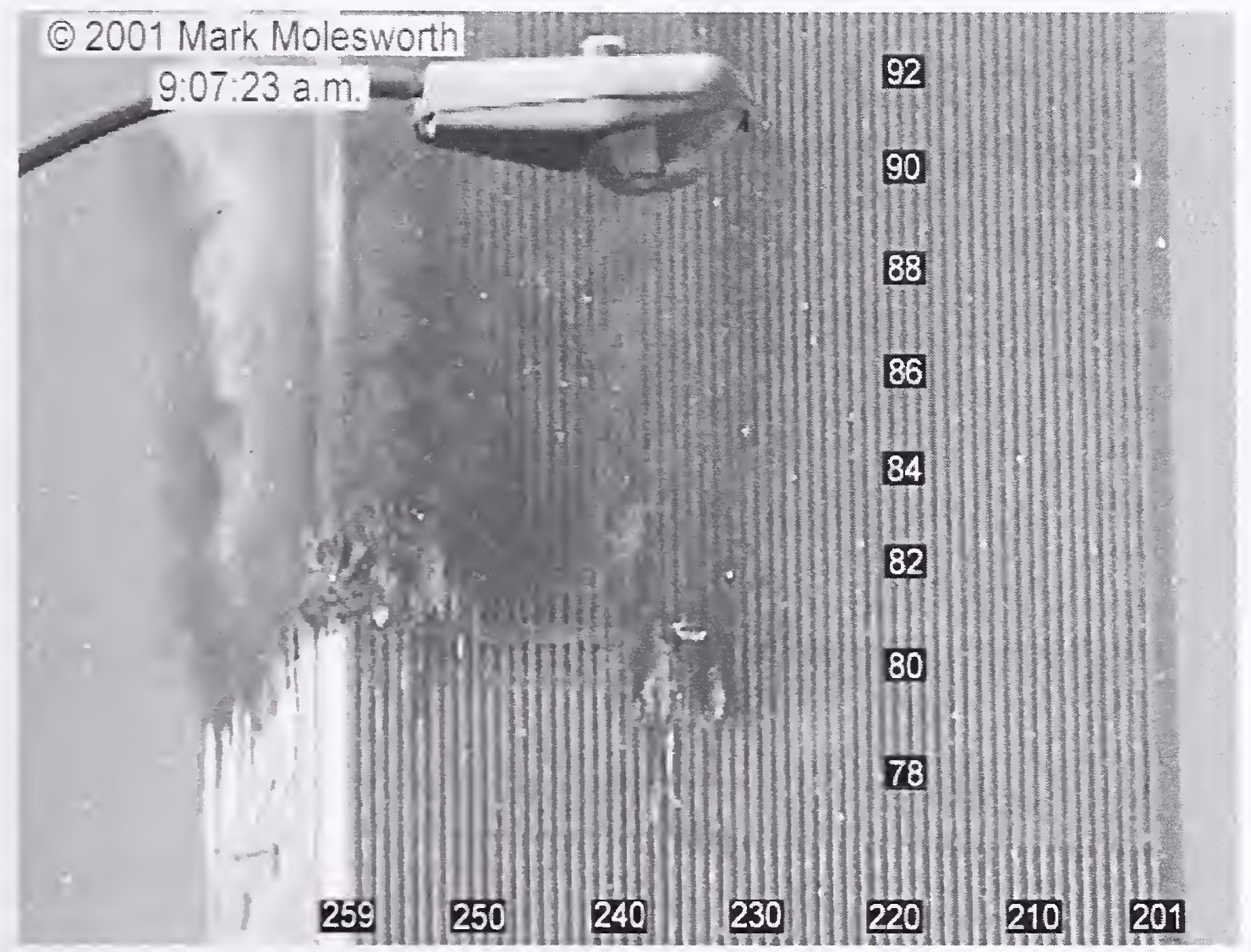

Figure 9-8. This image captured from a video shows the north face of WTC 2 at 9:07:23 a.m. Column and floor numbers have been added.

Figure 9-8 provides a good view of much of the north face with sufficient resolution to resolve details on the face. For instance, the open windows on the 78th floor near window 78-225 can be discerned. There is no indication of fire or smoke at locations removed from the area that was originally damaged during the aircraft impact and subsequent fireballs.

An image of the fires on north and east faces shot from the northeast at 9:06:53 a.m. is shown in Figure 9-9. It provides a good view of the debris that was protruding from the northeast corner of the 81 st floor, as well as an indication of the actual extent and intensity of the fires burning in the northeast comers of the 81 st and 82 nd floors. The cold spot on the north face is easily identified from this angle.

An image of the south face of WTC 2 taken from a video recorded at 9:06:31 a.m. is shown in Figure 9-10. This is slightly less than two minutes later than the image shown in Figure 9-4. Even though the latter image is clearer and shows more detail, it can still be seen that the appearance of the face has changed somewhat during this short period. A new fire has appeared on the 81 st floor near window $81-430$ that was not apparent earlier. The fire that was burning within the cavity created by the aircraft impact on the 79th floor seems to have moved toward the west and can now be seen on top of a pile of debris through open window 81-436, as well as at multiple locations on the debris within the impact cavity. In the later image there is no indication of a fire burning on the $82 \mathrm{nd}$ floor above the aircraft impact cavity. 


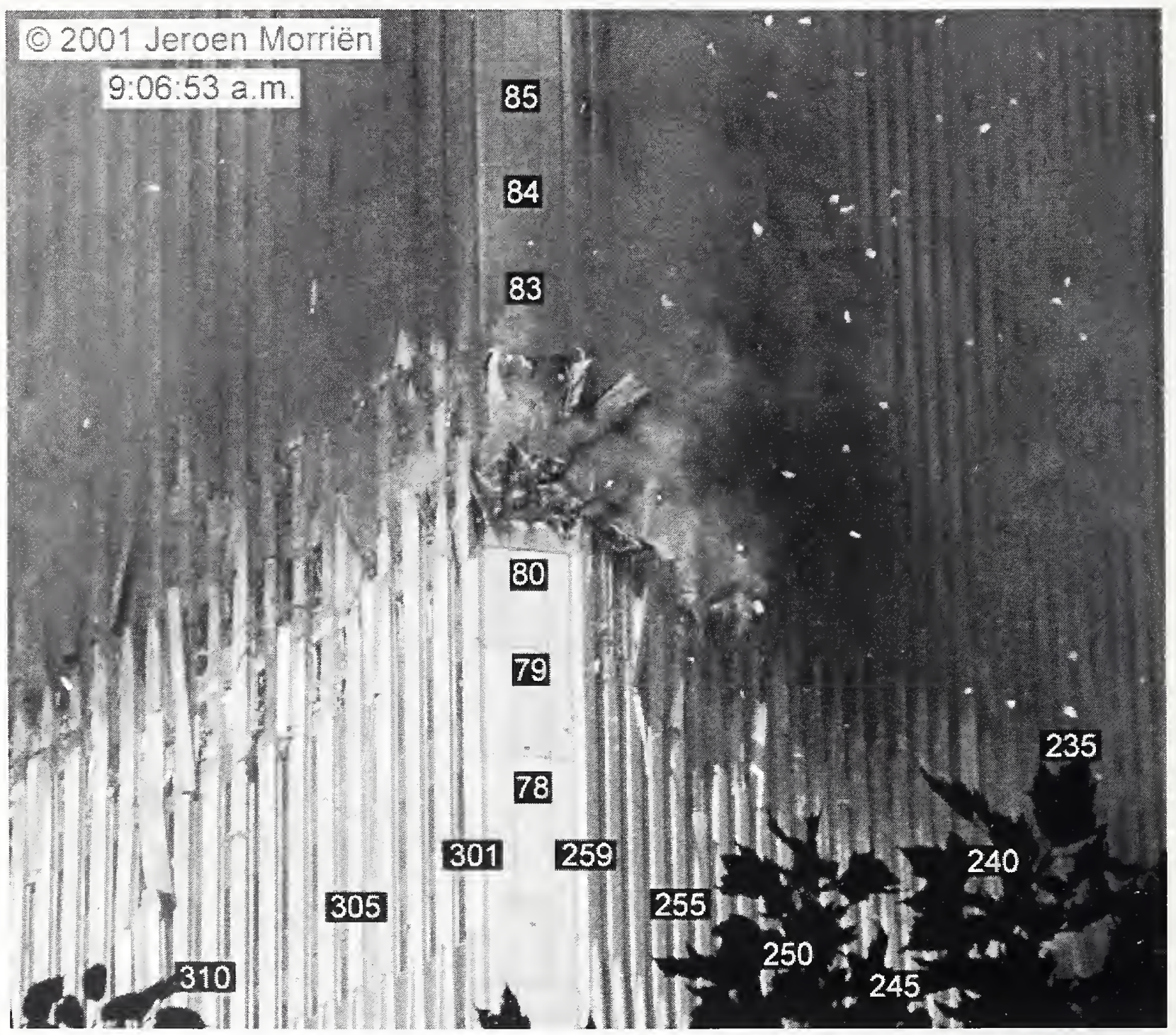

Figure 9-9. This cropped photograph showing the northeast corner of WTC 2 was taken at 9:06:53 a.m. The image has been enhanced by adjusting the intensity levels. Column and floor numbers have been added.

In Figure 9-10, smoke is coming from open windows to the east of the aircraft impact cavity on the 81 st floor, and there is an indication of flames in the area. In the earlier photograph, this area was clear of smoke, and some details on this floor as well as the one above could be identified. Apparently, a fire has grown quickly on the 81 st floor in this area. Interestingly, a photograph of the east face taken around this time (see Figure 9-7) shows very little smoke and no visible flame near the south edge of the face. This suggests that the fire growing at this location was ignited near the aircraft impact location and is moving toward the east.

Another view of the south face at roughly the same time is shown in Figure 9-11. It was shot from further east than Figure 9-10. From this angle fires are visible at multiple spots inside the aircraft impact cavity, including locations corresponding to the 78th, 79th, and 80th floors. The smoke coming from the windows to the east of the aircraft impact cavity on the 81 st floor is also visible. Apparently, the fires 


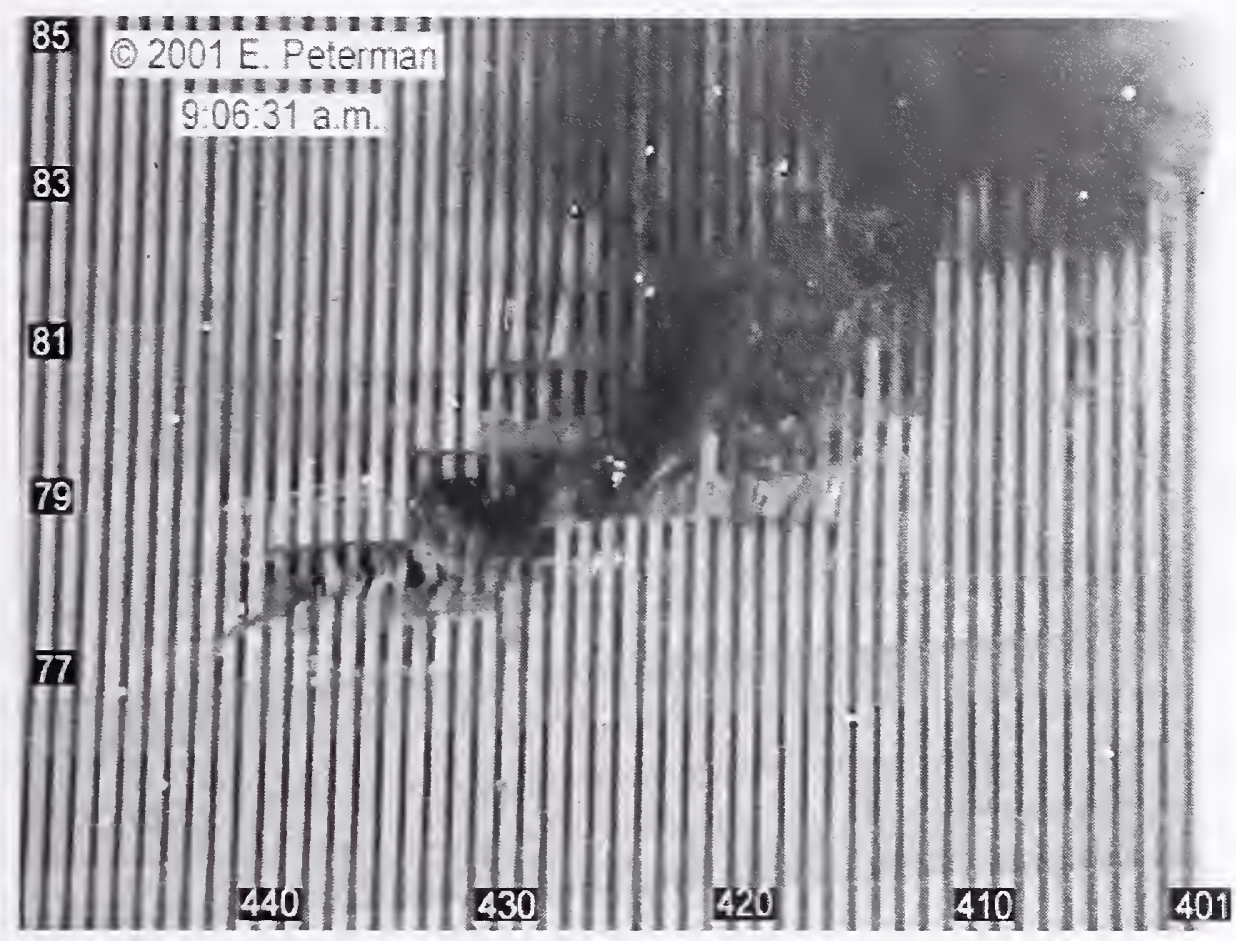

Figure 9-10. This image showing the south face of WTC 2 is a frame captured from a video recorded at 9:06:31 a.m. It has been enhanced by adjusting the intensity levels, and column and floor numbers have been added.

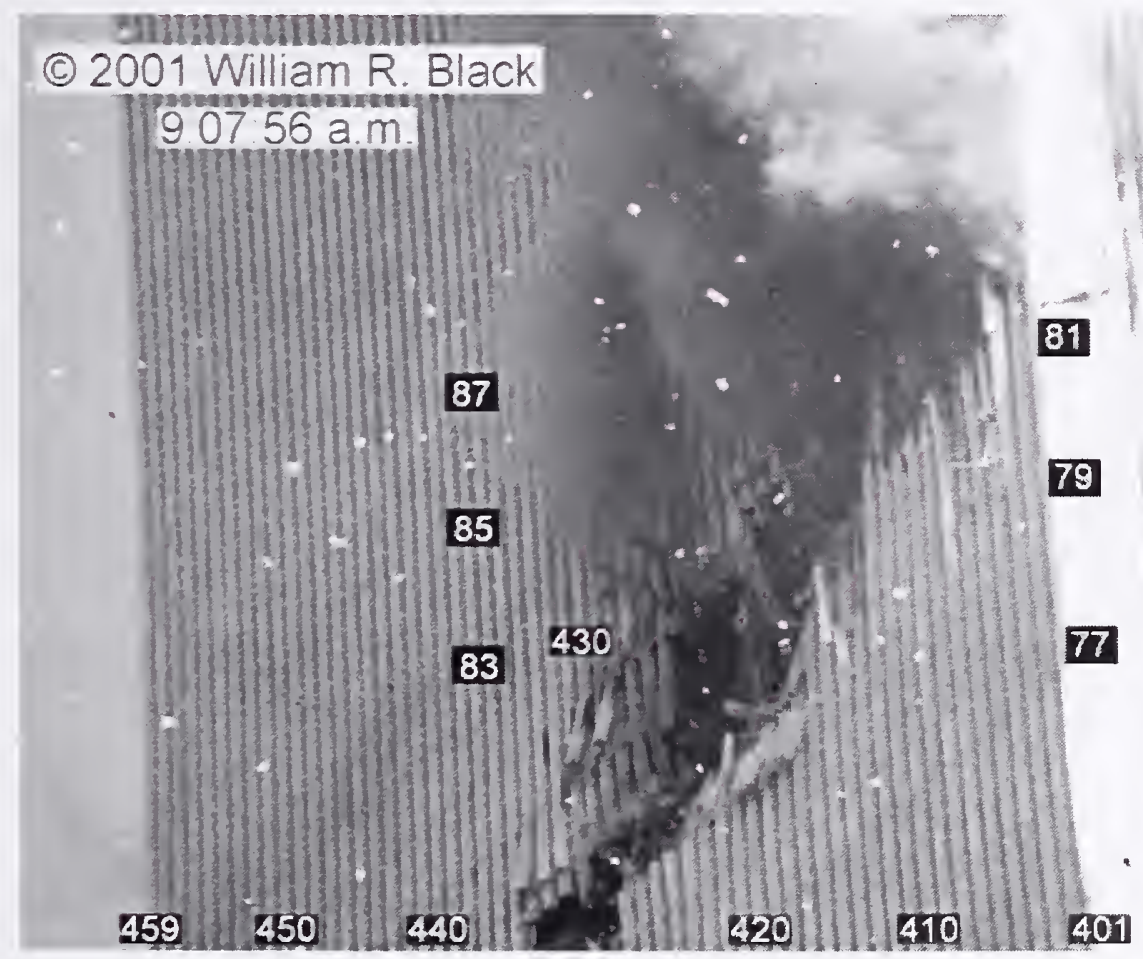

Figure 9-11. This photograph of WTC 2 was taken at 9:07:56 a.m. from the southeast and shows the south face and a portion of the east face. The intensity levels have been adjusted, and column and floor numbers have been added. 
growing on this floor are also venting through the aircraft impact cavity since a large amount of smoke is flowing from the opening just to the west. This photograph confirms that little smoke and no flames are visible coming from windows on the 81 st floor of the east face that are adjacent to this area.

The fire distribution and growth seen on the south face of WTC 2 suggests that small fires were ignited at multiple locations surrounding the aircraft impact cavity. The ignitions most likely resulted from the fireball that formed on the south face following the aircraft impact. These fires had very different behaviors than the large intense fires established on the large piles of debris in the northeast corner during the same period.

At 9:10:01 a.m. a videographer recorded a closer view of the north face of WTC 2 than those presented thus far. Figure 9-12 shows a frame captured from this video. The image shows that the fires burning on the 79th, 80th, and 81st floors have not changed markedly since 9:07:23 a.m. (see Figure 9-8). The cold spot is also distinct. The increased resolution of Figure 9-12 allows a detail to be resolved that was not easily identified in the earlier images. There appear to be two objects hanging across a length of open windows on the 80th floor. The arrows added to the image indicate these objects. One of the objects appears as a line running from at least window 80-255 to window 80-243. The second object also appears as a curved line. It is visible above the first object starting near the top of window 80-247 and extending west to at least window 80-241.

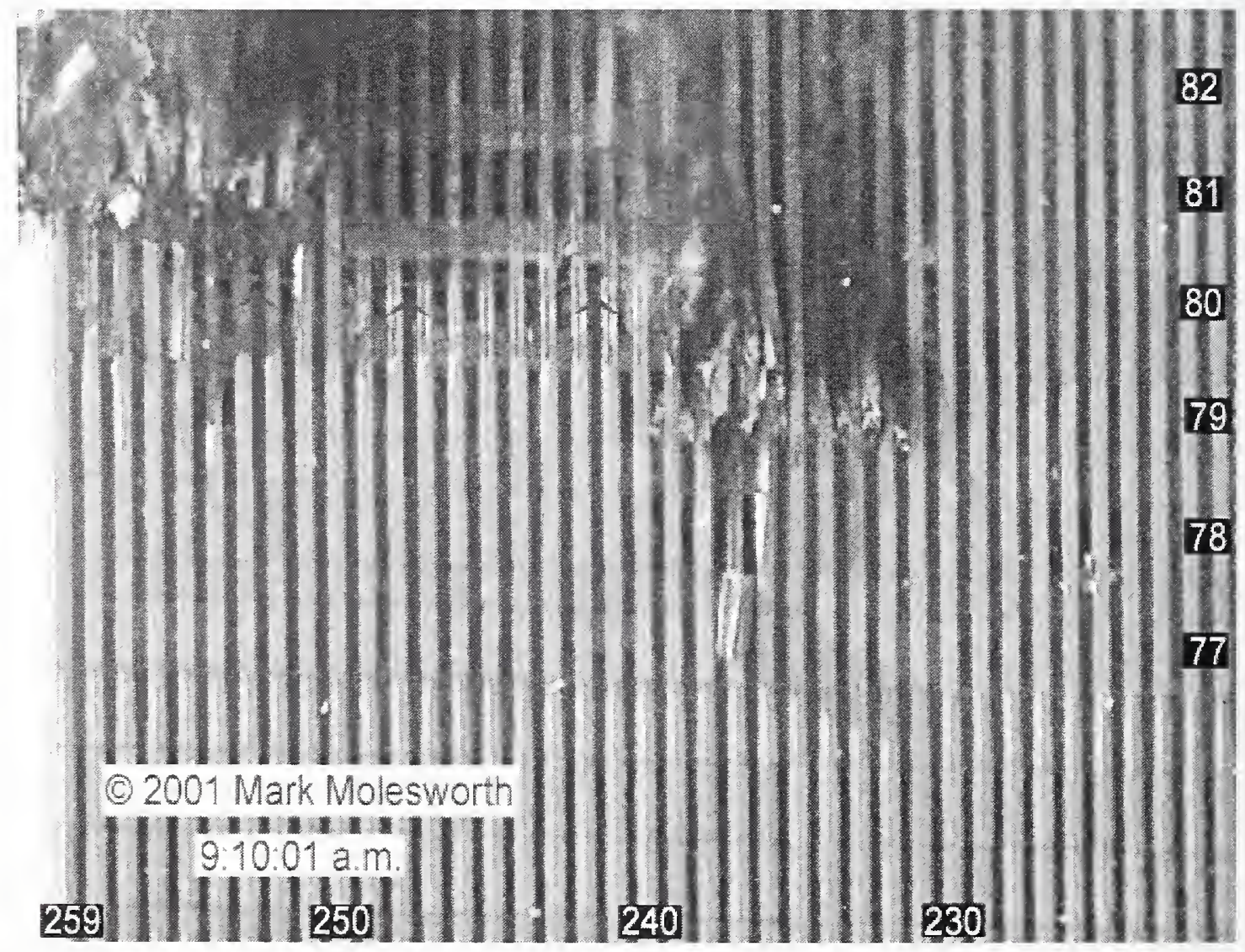

Figure 9-12. An image of the north face of WTC 2 captured from a video recording at 9:10:01 a.m. is shown. The intensity levels of the image have been adjusted, and column and floor numbers have been added. The arrows highlight hanging objects observed through open windows on the 80th floor. 
Closer inspection of Figure 9-12 shows that flames are visible above the first object in windows 80-249, $80-252$, and $80-253$. These fires have similar locations and appearances to the numerous fires that were observed at the tops of windows in WTC 1 (see Chapter 8) and were attributed to burning thermal insulation located above the windows. In this case this explanation seems unlikely since the aluminum façade has been removed from the columns adjacent to these windows. Using Figure 9-12 as a guide, Figure 9-8 was reviewed more carefully to look for indications of these objects and fires. It is possible to barely discern the first of the objects, and the fires above the objects are clearly visible at the earlier time.

The appearance of the objects and the presence of fires immediately above them are similar to the long hanging object seen across a number of windows on the 82 nd floor of the east face (see Figure 9-1 and Figure 9-2 and related discussion). Based on the same arguments, it is possible that the hanging objects in Figure 9-12 are portions of one or more floor slabs that have detached from spandrels on the north face and have settled down below the spandrel at the base of the 81 st floor. If the objects are portions of a floor slab, their appearance indicates that they are continuing to function as intact systems.

There is insufficient detail available in Figure 9-12 to provide a confident explanation for the presence of two objects. If both are indeed hanging floor slabs, one could postulate that the floor slab on the 81st floor has pulled away from the façade and. in the process of settling downward, has split into two sections along a line parallel to the face to form the two separate objects or perhaps that two separate floors (e.g., 81 st and 82 nd floors) have settled to this location from above.

Since images of the north face at the earliest times following the aircraft impact lacked sufficient resolution to observe the hanging objects, it is not possible to conclude that they were formed during the aircraft impact and subsequent fireballs. However, this seems to be the most likely scenario. It may be significant that they are present at the same locations where the largest fireball formed on the north face immediately following the aircraft impact (see Chapter 7). This is consistent with the hypothesis, as surmised for the hanging floor slab on the east face, that the partial collapse of the floor slab was due to overpressure created by the rapid burning of aviation fuel on the floor(s) below.

More details concerning the rectangular cold spot running from columns 238 to 250 on the 80 th, 81 st, and 82nd floors are visible in Figure 9-12. There is a pile of debris in the lower right-hand corner, and white smoke is coming from the 80th floor at window 80-238. Small flames are also visible in windows 80-249 and 81-249. Even so, there continues to be a remarkable lack of smoke and fire in the area.

A close-up view of the northern portion of the east face of WTC 2 at 9:11:14 a.m. is shown in Figure 9-13. The debris piles on the 80 th and 81 st and in the opening on the northeast corner of the 81 st floor are particularly clear. The fires on the 81 st and 82 nd floors continue to burn intensely. The only fire visible on the 80 th floor is in window 80-317. Most of the glass is intact for windows on the 79th floor.

Another photograph of the east face of WTC 2 taken at 9:12:11 a.m. is shown in Figure 9-14. Figure 9-15 includes blowups of the north and south sides of the fire floors from this photograph. Comparison of Figure 9-14 with the similar photograph in Figure 9-6, which was taken just over 5 min earlier, shows that the heavy smoke flows from near the center and closer to the southern edge of the face have continued. In Figure 9-14, it appears as if a portion of the smoke flow near the center may be coming from the 83 rd floor. It can also be seen that the fire burning on the southern half of the 81 st floor has grown substantially. 


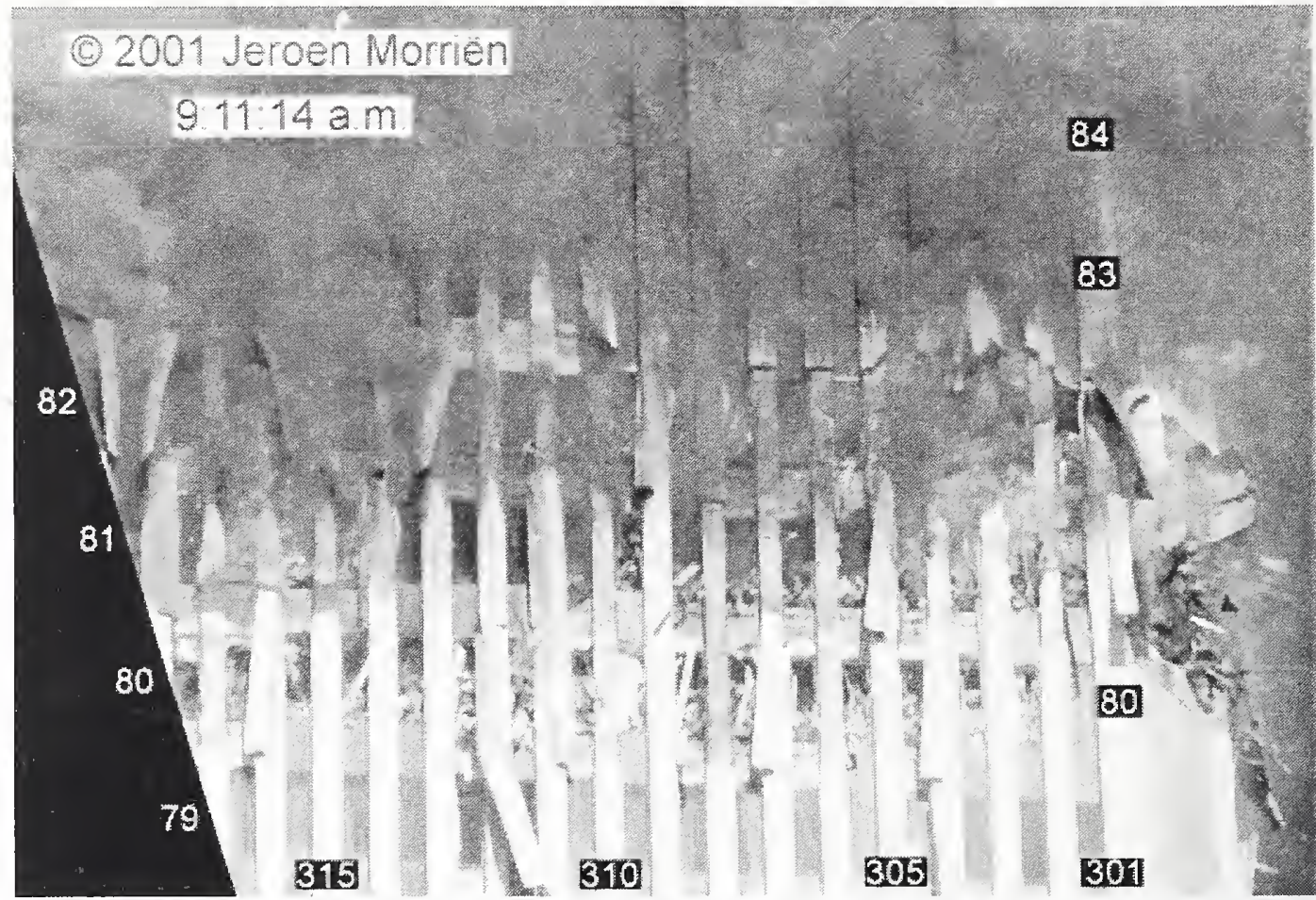

Figure 9-13. This photograph shows the northern portion of the east face of WTC 2 at 9:11:14 a.m. The image has been cropped and rotated, and the intensity levels have been adjusted. Column and floor number have been added.

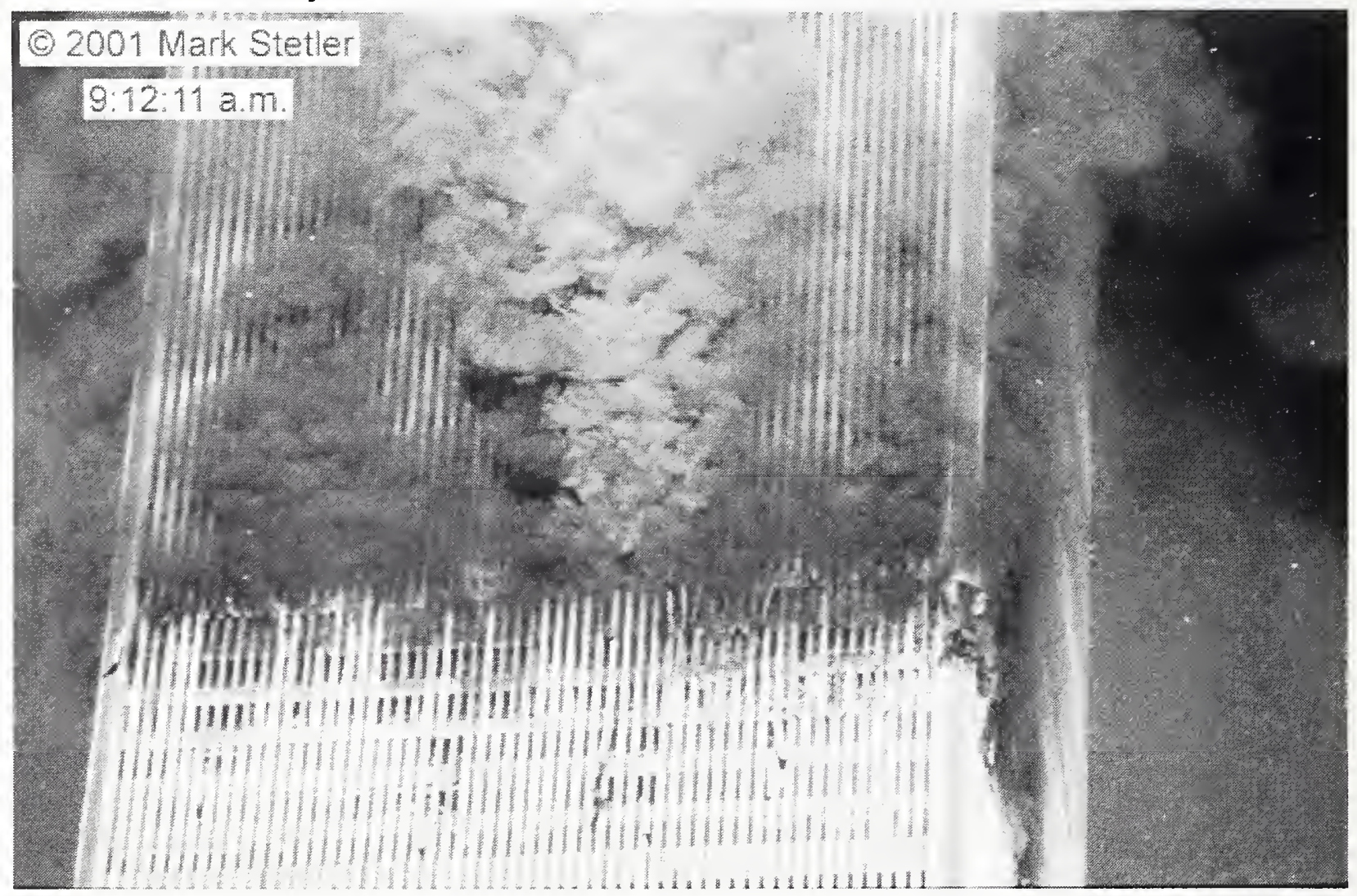

Figure 9-14. This photograph showing the east face of WTC 2 was recorded at 9:12:11 a.m. 

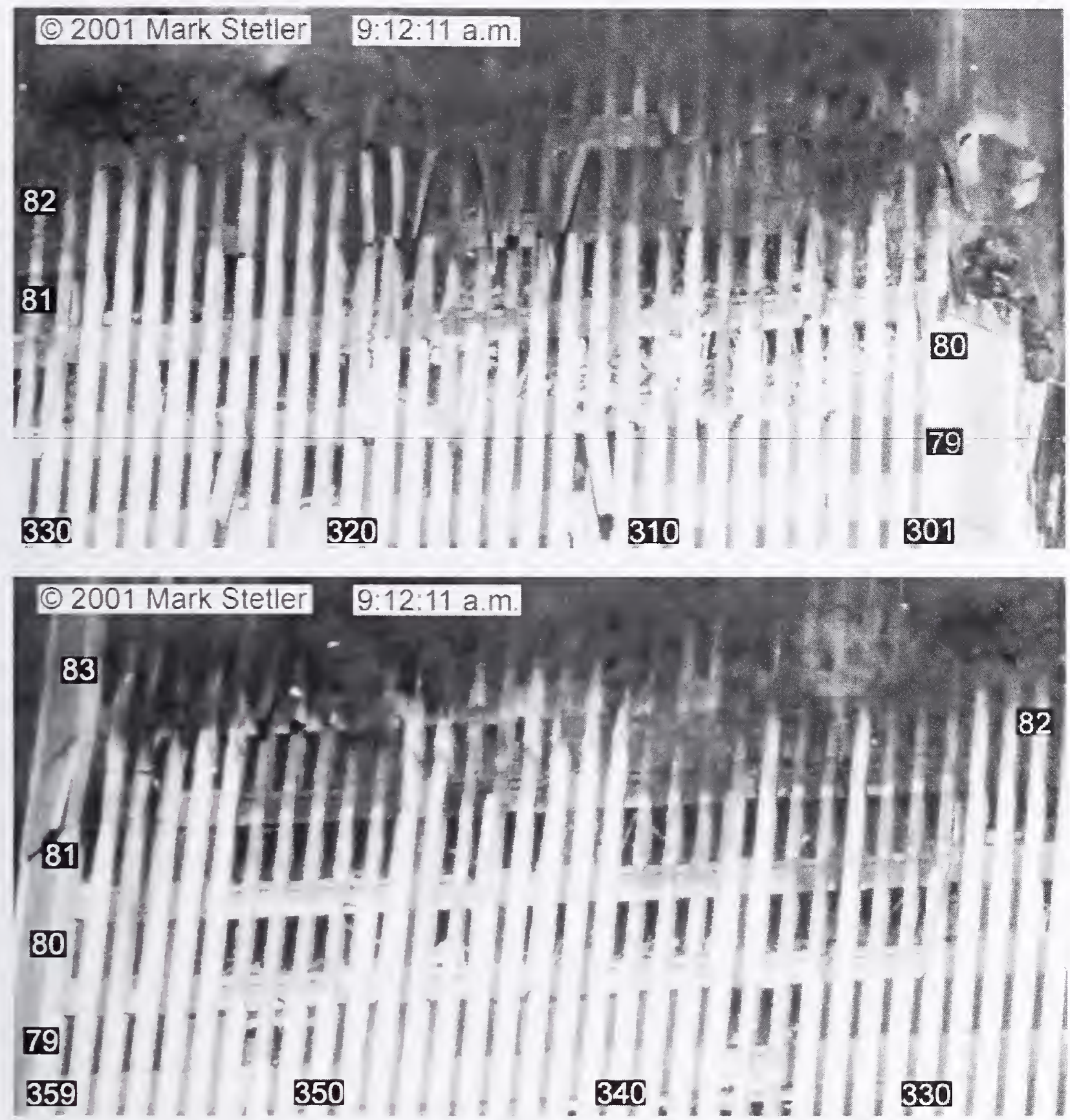

Figure 9-15. These two images show blowups of the north and south sides of the east face of WTC 2 taken from the photograph included in Figure 9-14, which was shot at 9:12:11 a.m. Column and floor numbers have been added.

The fire spread during the period is more easily characterized by comparing the blowups of the southern part of the face shown in Figure 9-7 and Figure 9-15. At the earlier time, flames were visible on the 82 nd floor in windows 82-341 to 82-349. By 9:12:11 a.m., the flames had spread to cover a length of the floor, running from window 82-332 to window 82-350. At the earlier time, smoke was coming from some of the windows where fire has now appeared. This is likely an indication that growing fires were near these windows at 9:07:04 a.m. As the fires to the north have grown larger, the earlier fires in windows 82-341 to 82-349 have decreased in intensity, as reflected by their external flame lengths. This 
decrease in fire size is confirmed by the presence of carets, indicating that sometime earlier flames had extended from the adjacent windows, on the aluminum column covers that are still in place on columns $82-341,82-342,82-346$, and 82-347. Flames are now coming from many of the windows located in the new fire area to the north.

In contrast to the rapid fire spread and growth seen on southern side of the east face on the 82 nd floor, the small fire that was visible on the 80th floor at 9:07:04 a.m. in window 80-330 has grown and spread slowly. Small flames are now visible through windows 80-332 to 80-335. A new region of small flames has appeared in windows 80-353 and 80-356. Conversely, the small fires evident on the 81 st floor near windows 81-331 and 81-333 appear to have died down between the times when the two photographs were taken.

Due to the heavy smoke and fire on the 82 nd floor, it continues to be impossible to observe the hanging object observed through the windows on this floor in Figure 9-1 and Figure 9-2.

The close up views of the north side of the east face shown at the tops of Figure 9-7 and Figure 9-15 look very similar. There appears to have been a slight decrease in the intensity of the fires burning in the northeast corners of the 81 st and 82 nd floors. Two small spot fires burning on the 80 th floor also seem to have grown smaller.

Figure 9-16 shows a close-up view of the north face of WTC 2 taken at 9:14:02 a.m. This is just over 4 min later than the image shown in Figure 9-12. This photograph provides somewhat higher resolution than the earlier image. The arrows point to objects that appear to be hanging across open windows on the 80 th and 81 st floors. The area of the image containing the arrows is blown up in Figure 9-17.

Even though the later image has higher resolution and shows more detail, a comparison of Figure 9-12 and Figure 9-16 indicates that the locations of the hanging objects identified in Figure 9-12 have not shifted appreciably during the 4 min period between the photographs. There are additional hanging objects resolved in the later photograph (seen most clearly in Figure 9-17) that are not easily identified in Figure 9-12. In addition to the two hanging objects on the 80th floor that were previously described, there is another similar object visible on the 81st floor near the tops of windows 81-239 to 81-242. This hanging object appears to consist of two closely spaced lines. There is yet another object in windows $80-251$ to $80-254$ that is below the much longer object that is visible hanging across window $80-242$ to window 80-256.

In Figure 9-16 and Figure 9-17, small fires are visible above the longest hanging object seen through windows on the 80th floor at the tops of window 80-248 and windows 80-252 to 80-254, again suggesting that fuel located on top of the hanging objects is burning. The large fires nearest the eastern edge on the 81 st and 82 nd floors and in the opening on the northeast corner continue to burn intensely in the same windows as earlier. In Figure 9-16, it appears as if a fire is burning on the 83rd floor in window 83-257, but it is possible that these flames are an extension from the large fire on the 82 nd floor. There is evidence in the photograph for strong heating of the façade at the base of the 83rd floor in this area. The lover portions of the aluminum covers on columns 83-256 to 83-259 are missing, and the bottom edges of the remaining aluminum have an inverted "v" shape. These observations indicate that these aluminum column covers have been melted by the intense heat coming from below. 


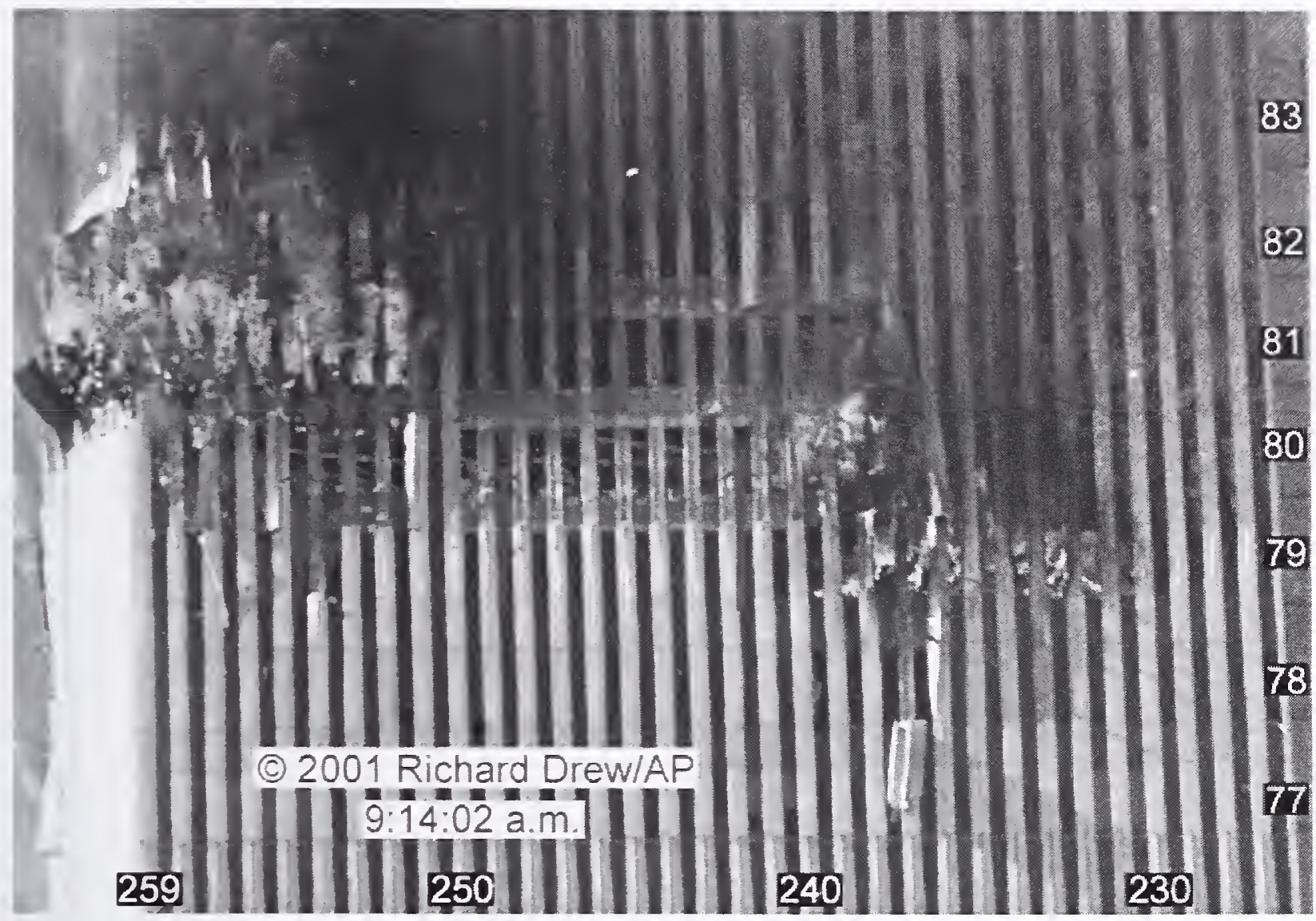

Figure 9-16. This close-up image of the north face of WTC 2 was taken at 9:14:02 a.m. The original photograph has been cropped and rotated, and the intensity levels have been adjusted. Column and floor numbers have been added. The arrows highlight hanging objects observed through open windows on the 80th and 81 st floors. The brown area to the right is the eastern edge of WTC 7.

Most of the cold spot continues to be smoke and fire free. In the lower right-hand corner of the area a small fire is now visible on the 80 th floor in windows $80-238$ to $80-240$, burning on a debris pile. The image in Figure 9-16 has sufficient resolution to determine the condition of windows. All of the windows on the 83rd floor immediately above the cold spot have glass in place while windows 79-241 to 79-249 on the 79 th floor below the spot were also closed at this time.

The debris pile on the 79th floor continues to burn vigorously. Comparison of Figure 9-12 and Figure 9-16 shows that flame lengths are decreasing slowly. This fire is visible in the same windows as earlier and does not appear to be spreading in either direction along the north side of the building.

A view of the south face of WTC 2 at 9:11:17 a.m. is shown in Figure 9-18. It was taken approximately 4 min after the images shown in Figure 9-10 and Figure 9-11. During this time a large fire has appeared on the 81 st floor to the east of the aircraft impact cavity. Flames are now visible on this floor in windows $81-401$ to $81-409$, and heavy smoke is coming from the area. Given the apparent intensity of this fire, it is noteworthy that there continues to be no indication of a fire, i.e., smoke and/or flame, from adjacent windows on the east face at this time. Figure 9-15, which was taken nearly a minute later, shows that this is the case. 


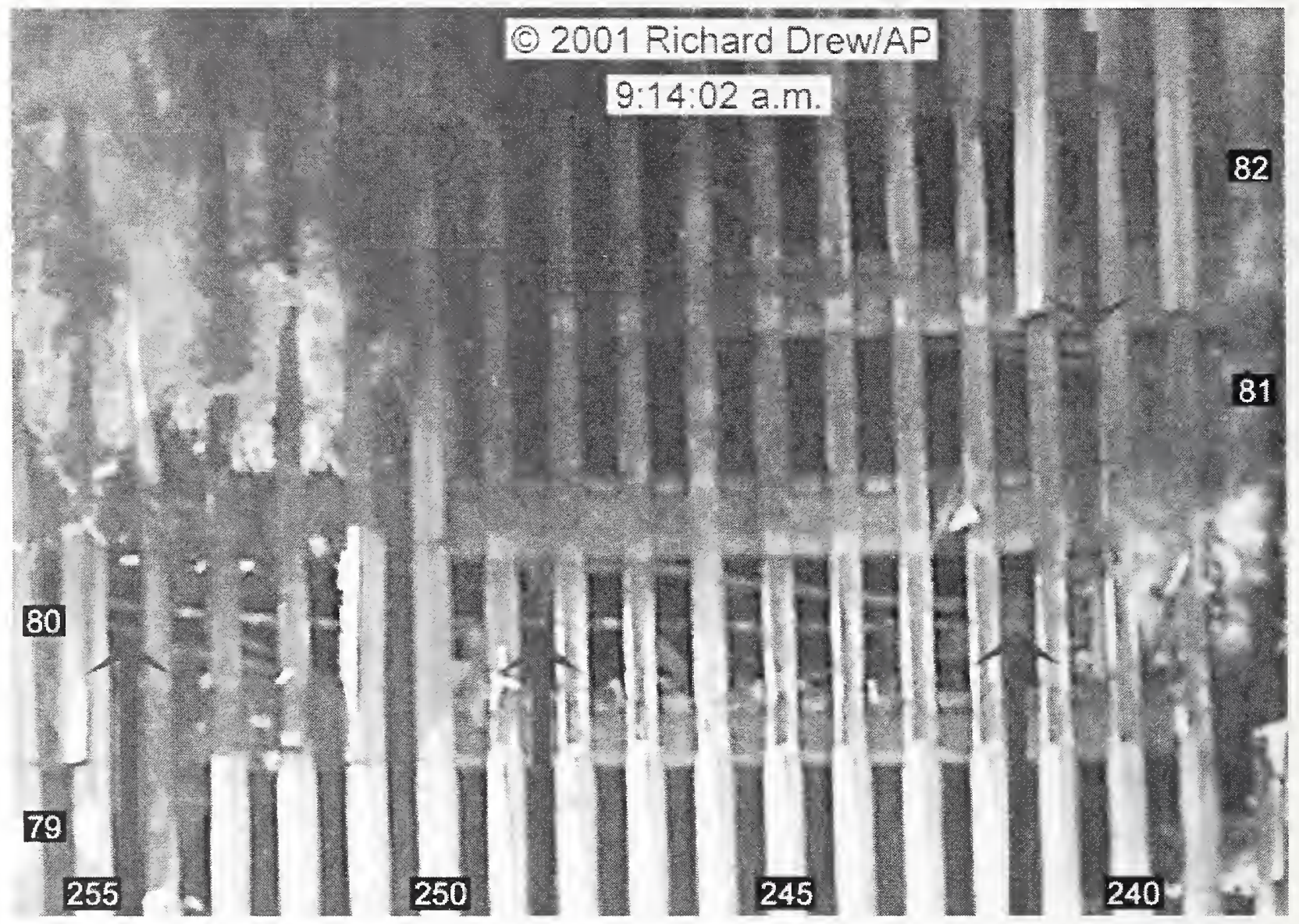

Figure 9-17. A section of the image shown in Figure 9-16 is blown up. The arrows point to the locations of hanging objects that are visible through open windows on the 80th and 81 st floors on the north side of WTC 2.

Comparison of Figure 9-10 and Figure 9-18 indicates that the fires ignited at other locations within and immediately adjacent to the aircraft impact cavity have continued to grow and spread slowly. At the earlier time, the glass in window 81-431 appeared to be intact, while in Figure 9-18 flames are coming from this window and are visible in window $81-432$ as well. The appearance of the fire burning on the debris pile located at the western edge of the aircraft impact cavity on the 79th floor is similar to that seen earlier, while the fire on the 78th floor seems to be slowly spreading across debris piled within the cavity.

A video shot at a long distance around 9:14:45 a.m. showed that the fire burning on the south face near the east edge of the 81 st floor had died down considerably. At this time it was possible to see a fire burning immediately above on the 82 nd floor. This observation suggests that fires grew on the east side of both the 81 st and 82 nd floors around the same time.

Major observations concerning the fire behavior in WTC 2 during the initial twelve minutes following the aircraft impact are summarized here. The datasheets for the fires have been integrated by identifying the most intense fire observed in a given window during the period. The results are shown graphically in Figure 9-19 for the four faces of WTC 2. The same cautions concerning datasheet accuracy apply as discussed in the preceding chapter with regard to WTC 1. Nevertheless, Figure 9-19 should prove 
helpful for following the summary. A similar map, showing the observed fires shortly after the aircraft impact, was included in Figure 7-39 in Section 7.5.

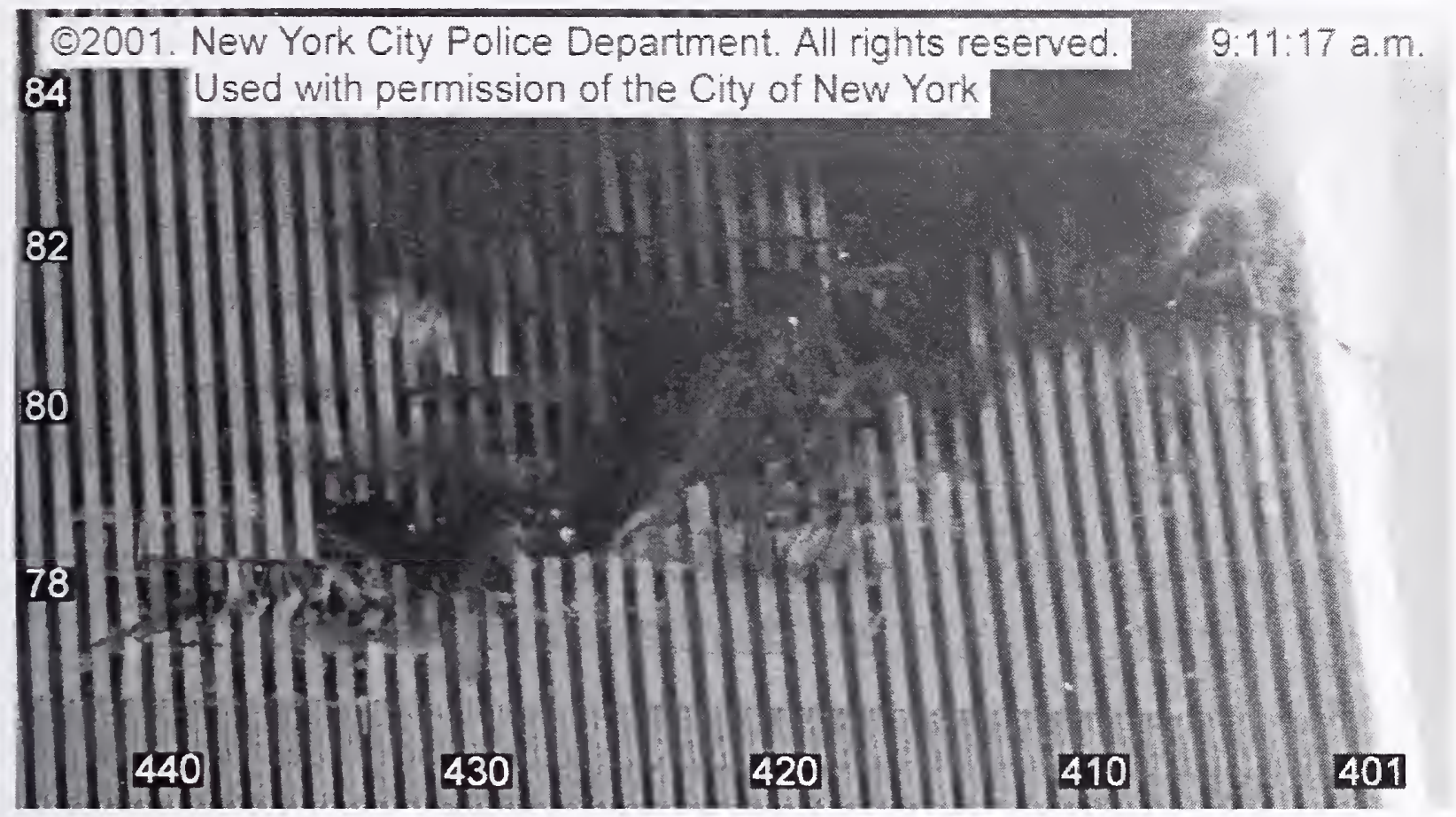

Figure 9-18. This cropped photograph shows the south face of WTC 2 at 9:11:17 a.m. The image has been rotated and enhanced by adjusting the intensity levels. Column and floor numbers have been added.

On the north face large fires were initially ignited on the eastern edges of the 81 st and 82 nd floors and at a location more toward the center on the 79th floor. On the 79th and 81 st floors these fire were burning on large piles of debris located just inside the façade. These fires continued to burn intensely during the entire period. There was little indication of fire spread or additional growth. Little smoke and flame were observed in an area of the façade, covering the 80th, 81 st, and 82 nd floors and extending from column 238 to column 250, lying between the fires on the 81 st and 82 nd floors and the fire on the 79th floor.

This area is referred to as the cold spot.

Immediately following the aircraft impact, extensive fires were visible on the east face over the northern halves of the 81 st and 82 nd floors and on the southern portion of the $83 \mathrm{rd}$ floor. An intense fire was also burning within the debris-filled opening created in the northeast corners of the 81 st and 82 nd floors by the aircraft impact. Smaller isolated fires were present on the 80th floor. The fire on the southern side of the 83 rd floor died down rapidly, while a large fire appeared and rapidly grew on this side of the 82nd floor. The fires on the northern ends of the 81 st and 82 nd floors and in the northeast corner continued to burn vigorously during the entire period. Only small fires were visible on lower floors. 

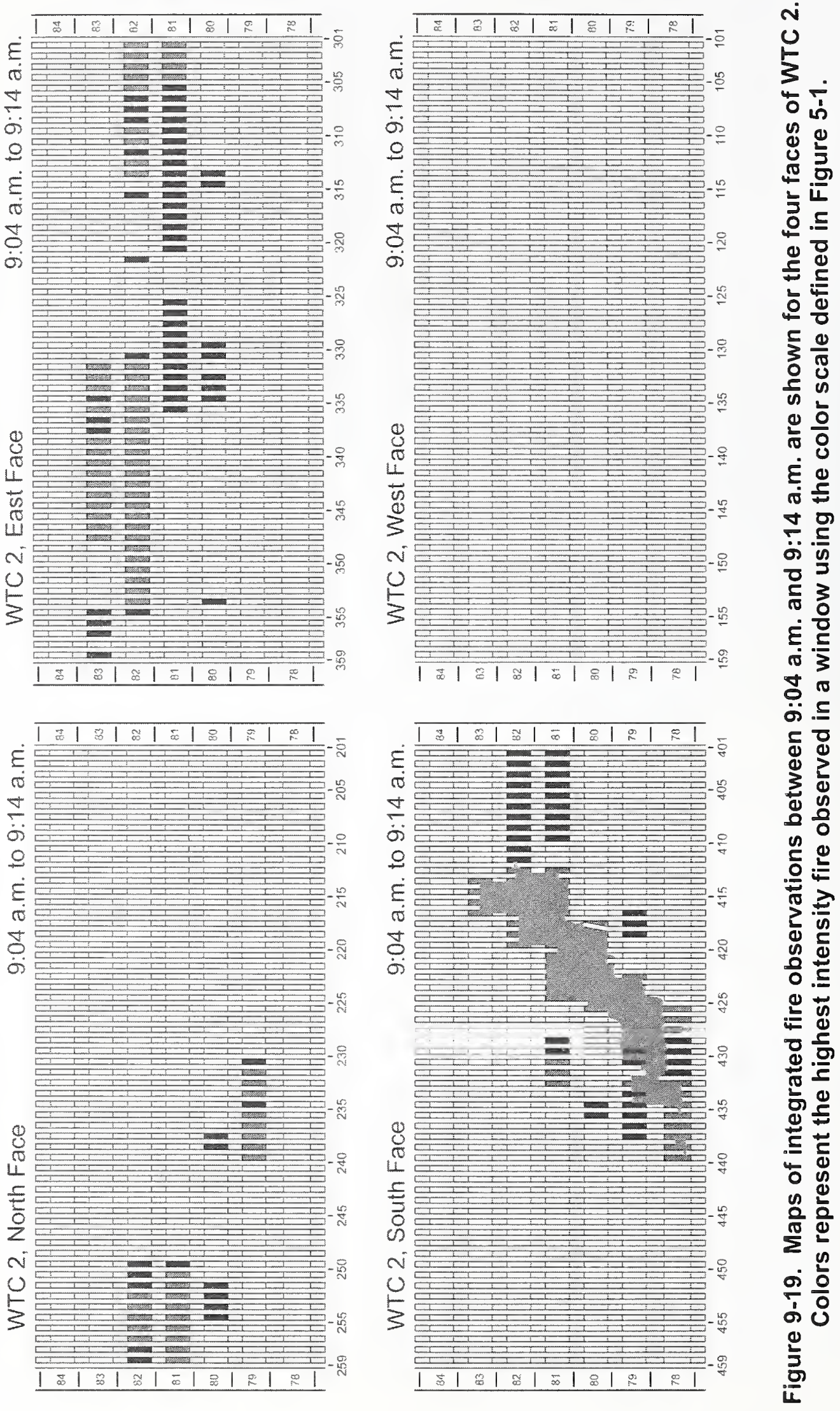
Several small fires were visible on the south face shortly following the aircraft impact. These fires were located within the aircraft impact cavity and immediate adjacent areas. During the period considered here, these fires spread and grew relatively slowly. The only visible area of extensive fire spread was on the eastern side of the 81st floor, where a large fire grew between 9:07 a.m. and 9:11 a.m., and immediately above on the $82 \mathrm{nd}$ floor, where a fire was observed just prior to 9:15 a.m., after the fire on the 81 st floor had died down.

No flames or fire were evident on the west face of WTC 2 for over seven minutes following the aircraft impact. After this time, a flow of smoke appeared well above the fire floors. This observation has been attributed to windows being broken open by people.

With the exception of the fire burning on a debris pile near the center of the north face and a small fire on the south face. there were no indications of extensive fires on the 79th floor during the period. This is somewhat unexpected since the analysis in Chapter 7 suggested that much of the aviation fuel from the aircraft"s port tank was dispersed on this floor, and widespread ignitions might have been expected.

In addition to characterizing the fire behavior, details in the images have revealed what may be partial floor slab collapses on the north and east sides of the tower due to the aircraft impact and subsequent fireballs. On the east face it appears that a substantial length of the 83rd floor slab sank below the spandrel at the base of this floor. On the north face hanging objects were visible at several locations through window's on the 80 th and 81 st floors. It may be worthy of note that the locations of the hanging objects are in or near the cold spot, which is an area on the north face wherc much of the local curtain wall was likely removed due to the overpressure generated by the ignition of aviation fuel inside the tower.

\section{$9.3 \quad 9: 15$ A.M. TO 9:29 A.M.}

In the last section it was mentioned that smoke appeared from the 86th floor on the west face of WTC 2 at 9:10:29 a.m. An image of the west face captured from a news helicopter vidco at 9:16:54 a.m. is shown in Figure 9-20. Smoke is visible near the bottom of the photograph and coming from near the 107th floor. A view of the tower recorded just prior to that shown in Figure 9-20 in the same vidco indicates that the smoke in the lower part of the image is coming from a number of open windows on the 86th floor, which are just below the view of Figure 9-20. The windows emitting smoke spanned a length from roughly window $86-417$ to window $86-423$. The smoke flowing from the 107 th floor was coming from windows ccntered near window 107-427.

The observations from the vidco indicate that several additional windows located on the 86th floor had broken out since 9:10:29 a.m. It also appears that heavy smoke had traveled to the top of the tower, and people on the 107 th floor broke out windows.

A photograph of the east face of WTC 2 at 9:18:38 a.m. is shown in Figure 9-21. It was taken almost 61/2 min after the photograph reproduced in Figure 9-14 and Figure 9-15. Comparison with the earlier images indicates that the fire burning on the southern side of the 82 nd floor has subsided during the intervening time. At the earlier time, intense flames completely filled windows 82-333 to 82-342 and hid the hanging object that had been visible in this area shortly after the aircraft impact. As indicated by the arrows added to Figure 9-21, thc object was once again visible at 9:18:38 a.m. A review of photographs and videos recorded during the intervening period indicated that the view of this area had gradually 
improved between the times when the two photographs were taken. This resulted from a continuation of the trend, described in the last section, in which the most intensely burning area on the floor moved to the north at the same time that fires to the south continued to die down.

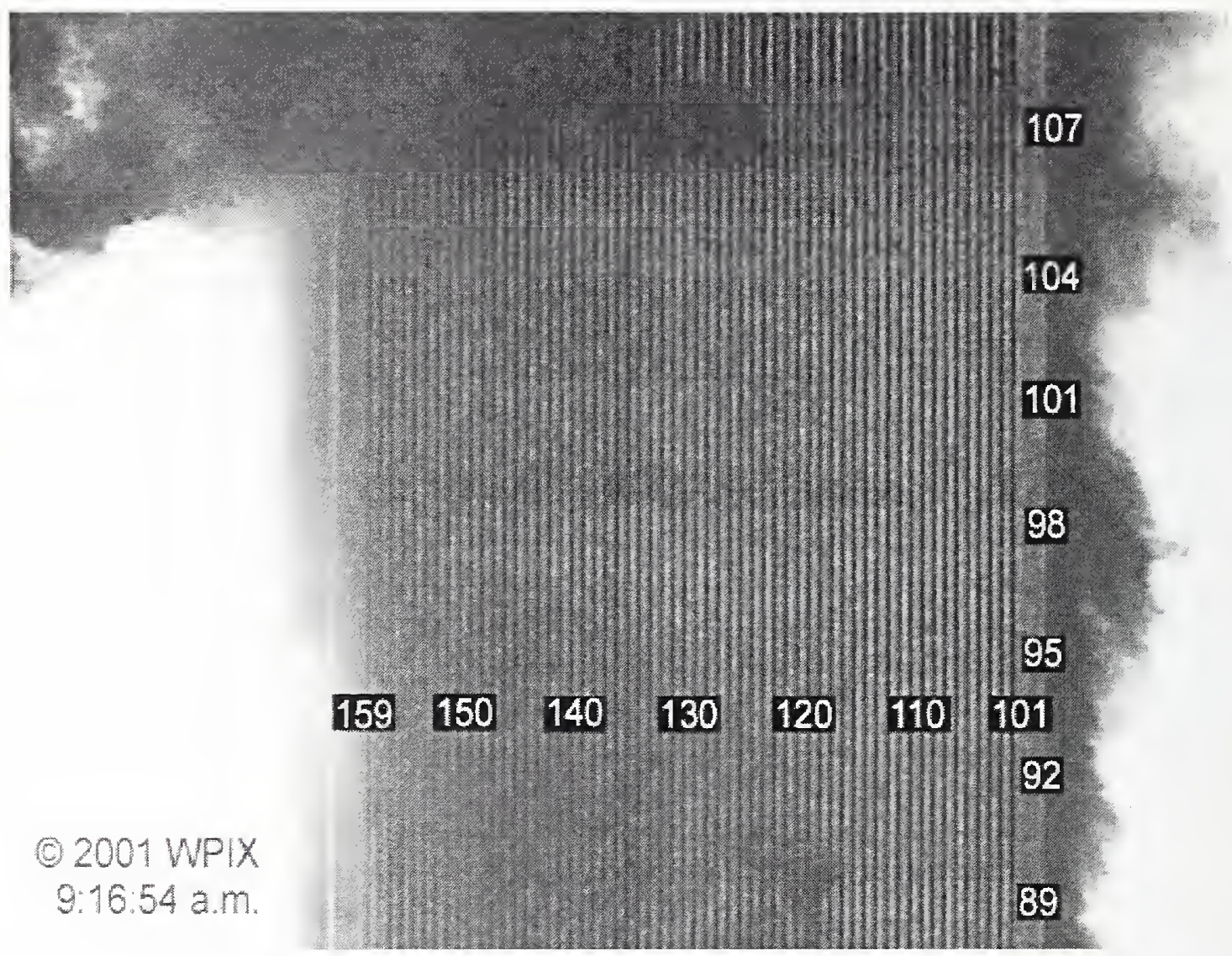

Figure 9-20. This image of the west face of WTC 2 was captured from a video shot from a news helicopter at 9:16:54 a.m. It has been enhanced by adjusting the intensity levels, and bright areas to the right and left of the tower are saturated. Column and floor numbers have been added.

The hanging object on the 81 st floor is now visible through windows $81-331$ to $81-335$ and windows 81-338 to 81-342. Windows further to the north remain obscured. Comparison of the location of the object with the image reproduced in Figure 9-1 and Figure 9-2, which was recorded at 9:03:42 a.m., shows that the object is at roughly the same height in the windows and has a similar shape.

The fires on the northern edges of the 81 st and 82 nd floors and in the opening on the northeast corner continue to burn intensely at a level comparable to that seen at 9:12:11 a.m. in Figure 9-14. There is still very little indication of a fire near the southern edge of the 81 st floor. A small fire remains on the 80th floor near window 80-335. Heavy flows of smoke continue from the southern side of the 83rd floor and from near the center of the 82 nd floor.

In the photograph taken at 9:18:38 a.m. there is no indication of significant smoke flow on the east face from windows lower than the 82 nd floor. This was generally true around this time, but was not always the case. Starting around 9:12:45 a.m. a series of short-lived, apparently coordinated smoke releases were observed in which smoke was pushed out of multiple open windows on the 79th, 80th, and 81st floors. 
At times these puffs moved across the face in sequence, looking much like an old fashioned steam-driven pipe organ. The available imagery provides only limited clues as to the cause(s) of these smoke puffs.

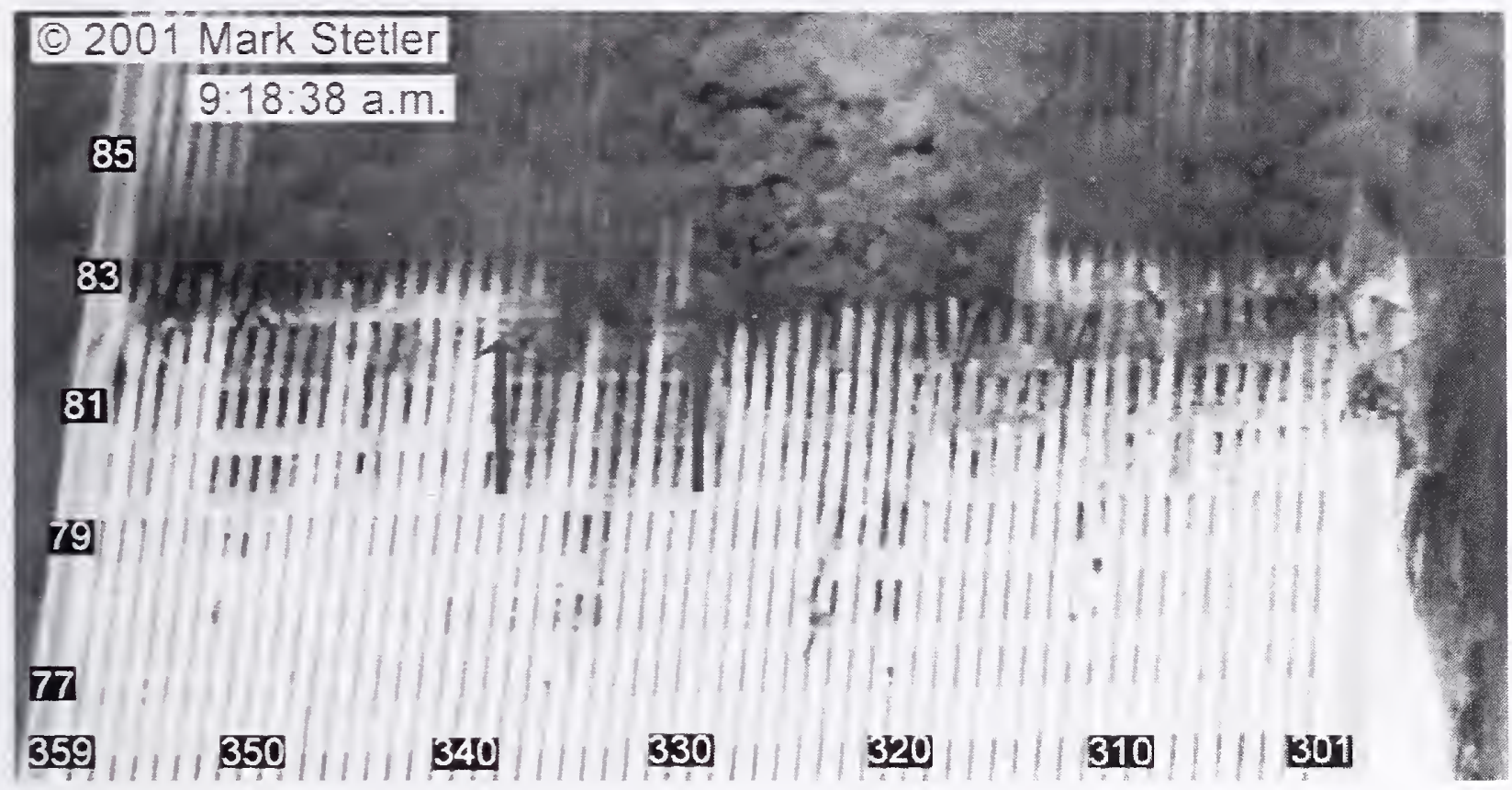

Figure 9-21. View of the east face of WTC 2 recorded at 9:18:38 a.m. The original photograph has been cropped and enhanced by adjusting intensity levels. Column and floor numbers have been added. The arrows highlight a hanging object observed through open windows on the 82 nd floor.

Figure 9-22 and Figure 9-23 include infrared images of WTC 2 that were shot around 9:15:15 a.m. and 9:17:20 a.m., respectively, from northeast of the WTC site. Time uncertainties are estimated as $15 \mathrm{~s}$. Figure 9-22 also includes a frame taken from a standard video that was shot from the same vantage point at 9:17:37 a.m. The size and orientation of the video frame has been adjusted to match the infrared photograph, allowing relative locations on the infrared photograph to be identified. The image in Figure 9-23 was created by connecting the output of the infrared camera to the input of a standard video camcra. The infrared camera was set to output a false-color scale for temperature, unlike the grey scale used for the image in Figure 9-22. Recall from the discussion in Section 8.1 that the temperature scales for the infrared images are not quantitative and that they provide only a relative indication of the amount of heating at a given location. Much of the heated area is saturated in the infrared imagcs.

The most striking feature in the infrared image shown in Figure 9-22 is the dark region between the two bright areas on the north face. The low level of infrared emission coming from the dark region indicates that the temperature of this portion of the facade is close to the outside ambient temperature. By comparing the infrared image with normal images of the north face, such as that shown in Figure 9-16, it can be secn that the unheated region is located in the same area of the face that has been identified as the cold spot. It is clear that not only is the cold spot mostly free of smoke and fire, but that it is very nearly at room temperature, despite the presence of nearby fires on either side. The area is indeed a "cold spot" relative to its immediate surroundings. 

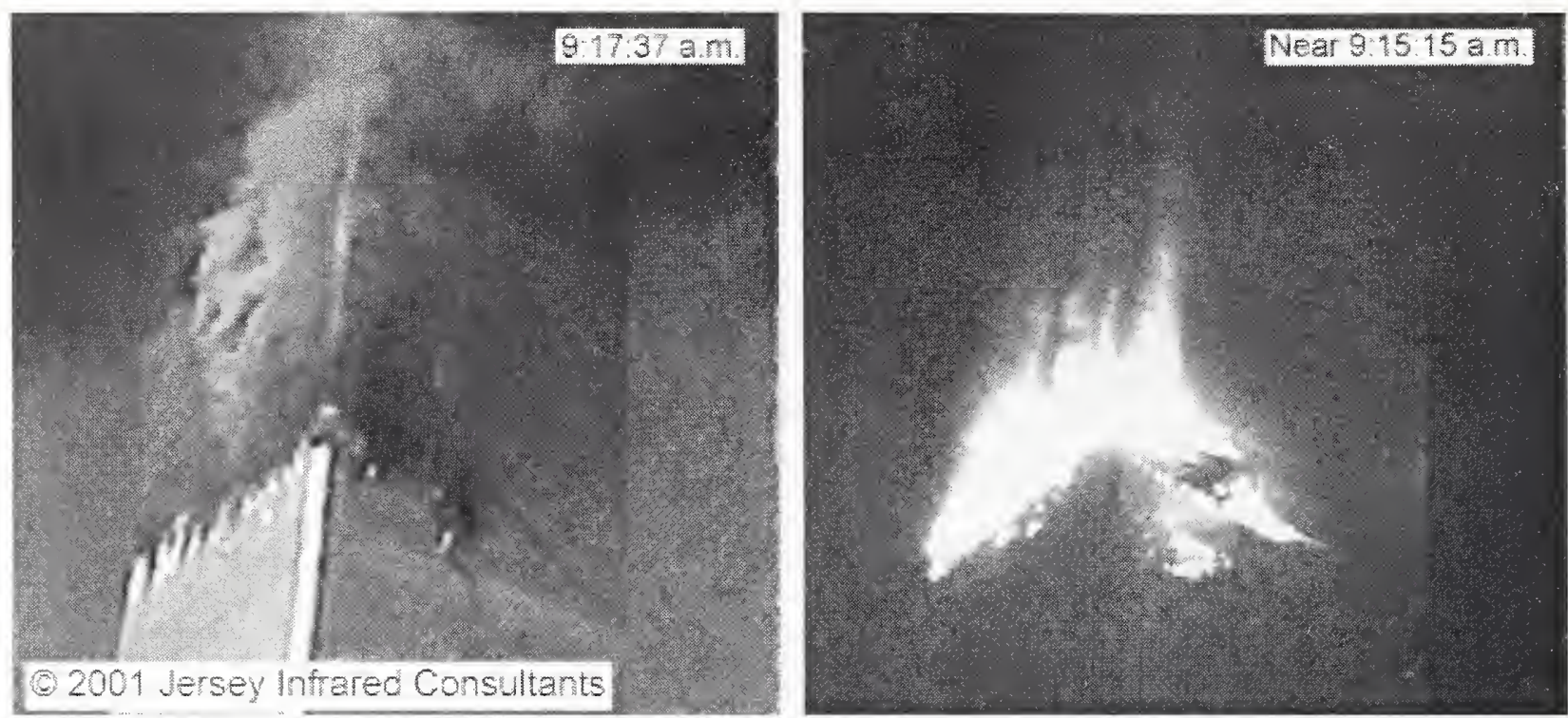

Figure 9-22. This figure compares an infrared photograph (on the right) and an image of WTC 2 captured from a video that was shot from the same vantage point and scaled and oriented to match the infrared shot. The north and east faces of the tower are visible. The video frame was shot at 9:17:37 a.m., and the infrared image was taken around 9:15:15 a.m. (an uncertainty of $15 \mathrm{~s}$ has been assigned).

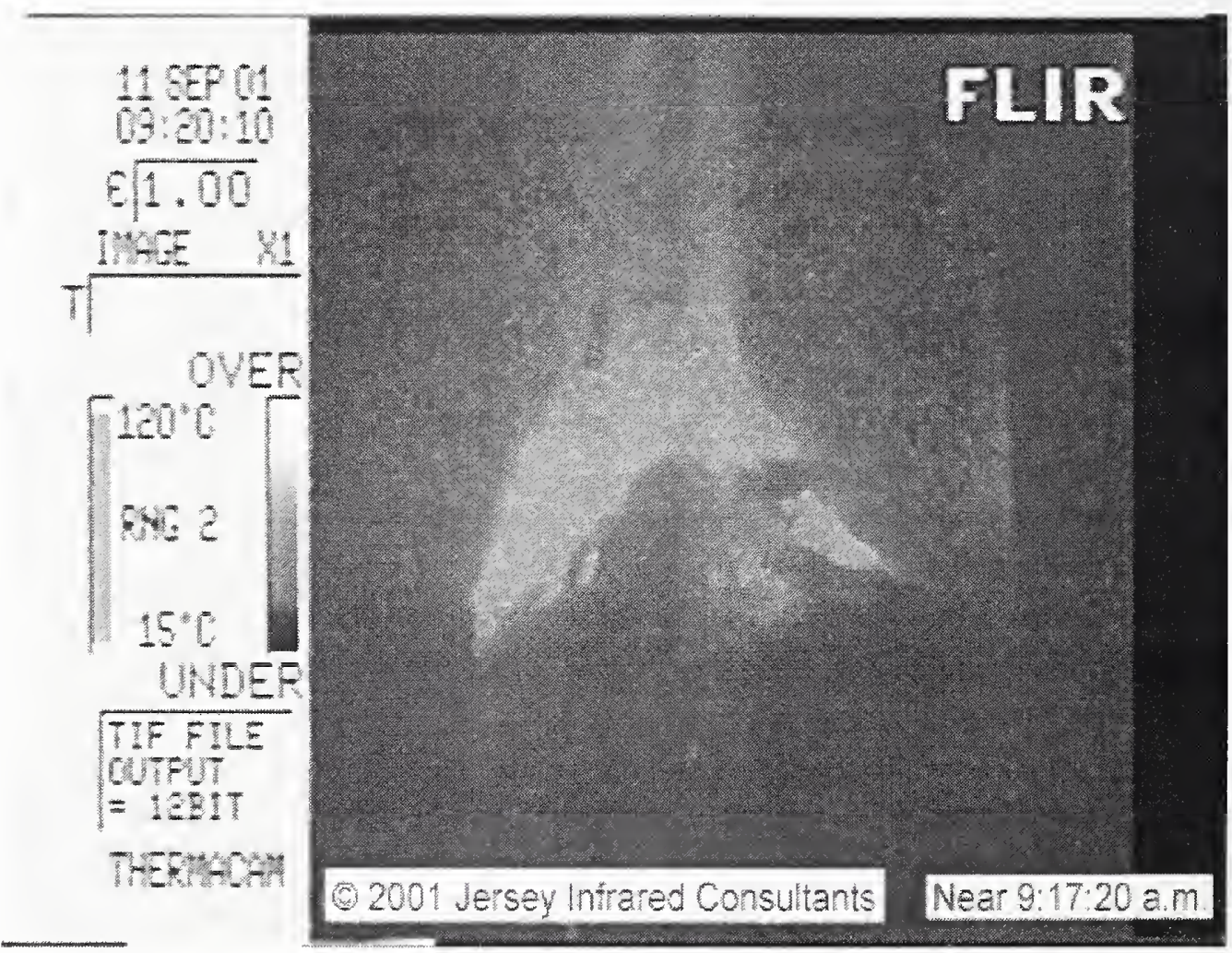

Figure 9-23. This image shows a frame from the false-color output of an infrared camera that was connected to a standard video camera. The time was around 9:17:20 a.m. (an uncertainty of $15 \mathrm{~s}$ is assigned). Blue represents the coolest temperatures and red the warmest temperatures. 
The cold spot does not appear to have a completely rectangular shape in Figure 9-22. There is a slightly heated area at the upper left corner that is most likely due to the flames on the 82 nd floor that are visible in window 82-249 in Figure 9-16. Similarly, there is small heated region in the lower right corner on the 80 th floor that is most likely associated with the small fire burning on the debris pile in window 80-241.

By using the heated areas within the cold spot as markers, it is possible to correlate other parts of the infrared image with Figure 9-16. The bright area below and to the west of the cold spot is the fire visible over many window's on the 79th floor. The narrow lighter line that extends westward from the brightest region indicates that heat is being transported across the floor, even though imagery indicates that these windows were still in place in the area. Similar heating is also apparent on this floor below the cold spot. A narrower bright region above the 79 th floor reflects the fire burning on the pile of debris on the 80th floor that can be seen in Figure 9-16.

Individual open windows to the left of the cold spot on the 80th floor appear as points of light, indicating that some hot gases are likely flowing through the openings. The image is saturated immediately above these openings due to the intense fires burning on the 81 st and 82 nd floors. Interestingly, there also appears to be significant heating above the cold spot on the 83rd floor. In Figure 9-16 the windows on this part of the 83 rd floor appear to have glass in place, suggesting that heat is being transported to this location from elsewhere on the floor. Finally, there is a bright region on the north face that is located well below those discussed thus far. Comparison with the video frame in Figure 9-22 indicates this heat source is located at the mechanical floors, i.e., the 75 th and 76 th floors. Apparently, this area was either heated earlier by the smoke and dust observed coming from these floors (see the discussion in Chapter 7), or heated gases are still being released from the mechanical floors.

The heated area on the mechanical equipment room floors is at a location where there is an exhaust spill louver for the ventilation system on the 76th floor between columns 242 and 258 (see the ventilation diagram in Figure A-6 in Appendix A). The heated area appears to cover roughly half of the length of the spill louver on the west side. Most of the heating seems to be on the 76th floor, but therc is also evidence for heating at the 75 th floor level. The amount of air allowed to pass through the spill louvers was varied depending on conditions. It is possible that a portion of the louvers were closed on September 11, 2001, which could explain the heat distribution. The presence of heating on the mechanical equipment room floors suggests that the exhaust ventilation system may possibly have continued to operate after the aircraft impacted WTC 2.

In Figure 9-22 and Figure 9-23, heated plumes can be seen rising near the east edge of the north face and at several locations on the east face. Comparison with the video frame shows that the hcated plumes are associated with the heavy smoke flows coming from the tower.

On the east face there is a strongly heated area where the lower side forms a nearly straight line across the face. Heating is evident over a number of floors above this line. The location of the base of the heated area indicates that it corresponds to the 81 st floor. In Figure 9-21, there is visual evidence that there was a substantial fire burning on the east face of the 81 st floor at this time, but the fires did not appear to be distributed across the entire floor. This suggests that interior fires were present on this floor, but were not visible from the outside, or that heat was being transferred to other parts of the floor from the fire areas, or that heat absorbed by the walls on this floor at earlier times had not yet dissipated. 
The small bright areas below the heated base at the 81 st floor indicate that localized high temperature regions are present on the 79 th and 80 th floors. On the 80th floor, these areas are likely associated with the low intensity fires that have already been described. One of these areas is visible in windows 80-332 to 80-335 in Figure 9-21. This location correlates well with the largest of the four heated areas that are apparent on this floor in Figure 9-22 just to the left of the center of the face. There is also a small heated region just to the north of the center of the face on the 79th floor. The fire responsible for this heating is not visible in Figure 9-21.

Even though the exact times when Figure 9-22 and Figure 9-23 were taken are not available, it is known that the image in Figure 9-23 was taken 2 min and 4 s after Figure 9-22 was shot. The false-color image in Figure 9-23 is more saturated, but it is clear that the heat distributions on the north and east faces are similar to those described for Figure 9-22. This indicates that the thermal distributions on the north and east face of WTC 2 changed relatively slowly around this time.

A photograph showing the fire areas on the north face of WTC 2 at 9:19:19 a.m. is reproduced in Figure 9-24. This image was taken just over five minutes later than that shown in Figure 9-16. Even though the later image is not as resolved, it is clear that the fire distribution and intensities have remained essentially unchanged during the intervening five minutes. The most prominent hanging objects, which are barely visible in Figure 9-24, also seem to be at the same locations. The similarity of Figure 9-16 and Figure 9-24 indicates that comparisons of the infrared images with Figure 9-16 are justified since they wcre most likely recorded during the period between the two photographs.

Figure 9-25 shows a view of the south face of WTC 2 recorded at 9:16:33 a.m. Comparison with Figure 9-18, which was recorded almost five minutes earlier, indicates that both the fire distributions and fire intensities have changed during this time. The most obvious change is the growth of a substantial fire on the 79th floor at windows 79-414 to 79-417. Consistent with earlier observations for fires on this face, it appeared at a location immediately adjacent to the aircraft impact cavity.

The fires on the east side of the face on the 81 st and 82 nd floors have also changed substantially. On the 81 st floor, the fire intensity over most of the area has decreased noticeably, and the amount of smoke coming from the open windows has also decreased. The exception is window 81-401, where flames, which were not prominent at the earlier time, now fill the window. In the earlier photograph most of the windows on the 82 nd floor were hidden by smoke coming from the 81 st floor, and no flames were visible. It was noted in the last section that a distant video taken just prior to 9:15 a.m. showed that the fire on the 81 st floor had died down and that a fire had become visible just above on the 82 nd floor. At 9:16:33 a.m. these flames are visible in window 82-401 to window 82-408, and flames are extended from the windows in the center part of this length.

Two fires remain visible to the west of the aircraft impact cavity on the 79th and 81st floors. In both cases their intensity appears to have decreased during the five minute period. Meanwhile, the fire on the east side on the 79th floor next to the aircraft impact cavity seems to have grown larger. 


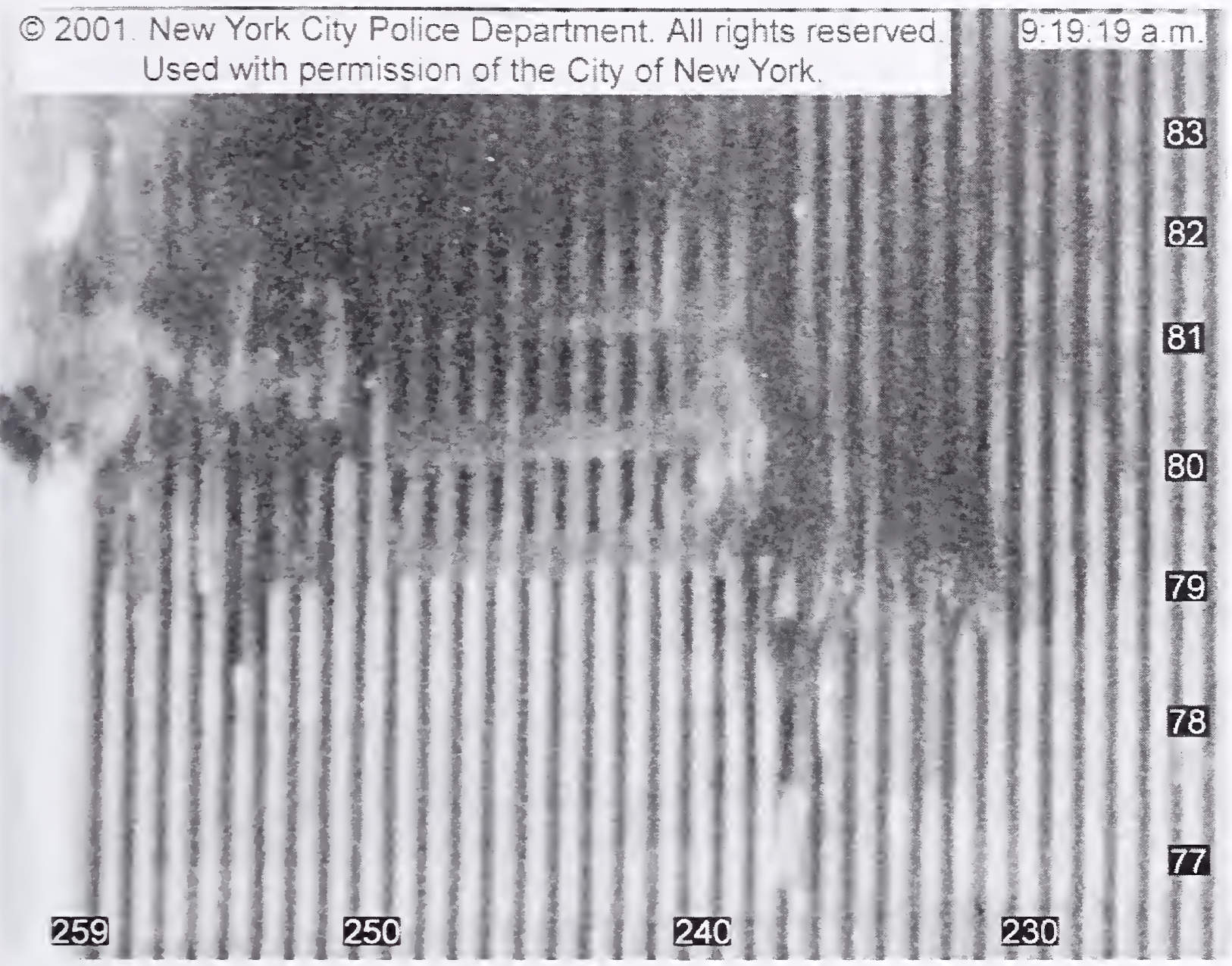

Figure 9-24. This cropped photograph shows the north face of WTC 2 at 9:19:19 a.m. The image has been enhanced by adjusting the intensity levels, and column and floor numbers have been added.

A later view of the south face taken at 9:23:08 a.m. is shown in Figure 9-26. During the nearly seven minutes since Figure 9-25 was shot, the fire that had appeared on the east side of the aircraft impact cavity on the 79th floor has grown and spread toward the east. The most intense flames are now observed in windows 79-413 to 79-415, and heavy smoke is coming from windows 79-411 and 79-412. At the earlier time flames were coming out of windows $79-415$ to $79-417$, and heavy smoke was coming from windows 79-412 to 79-414. A heavy smoke flow is also coming from window 79-406 at the later time.

The fires on the east sides of the 81 st and 82 nd floors have similar appearances in the two photographs. The same is true for the lower intensity fire burning on the 78th floor just to the east of aircraft impact cavity. The small fire to the west of the aircraft impact cavity on this floor is still, just barely, visible. The fire higher up on the 81st floor has grown brighter and now extends over windows 81-429 to 81-433. Comparison with Figure 9-10 and Figure 9-18, which were shot at 9:06:31 a.m. and 9:11:17 a.m., respectively, indicates that this fire has not spread significantly, but that its intensity seems to fluctuate with time. 


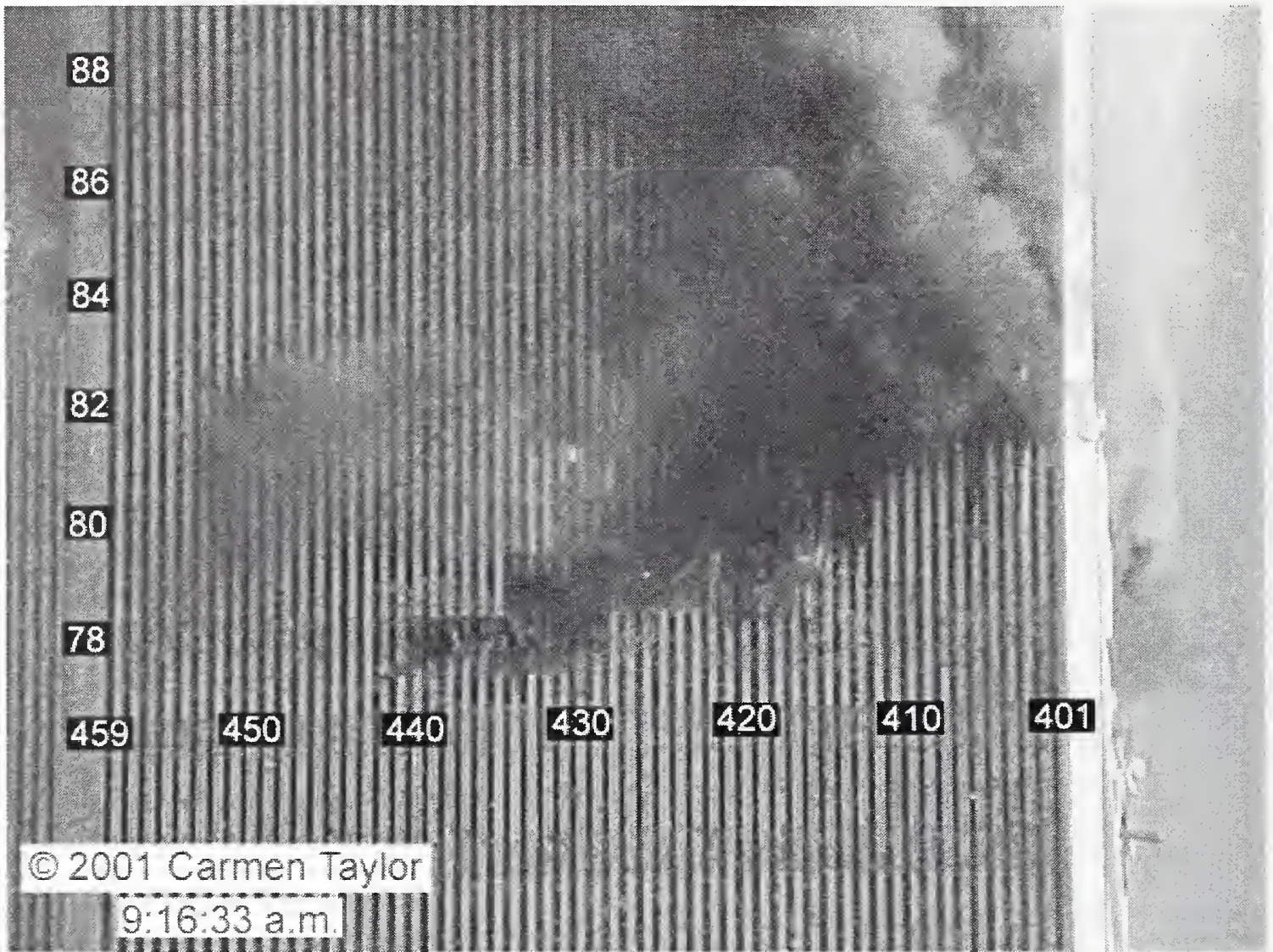

Figure 9-25. This photograph shows the south face of WTC 2 at 9:16:33 a.m. The south face of WTC 1 is visible to the left of the western edge of WTC 2. Column and floor numbers have been added.

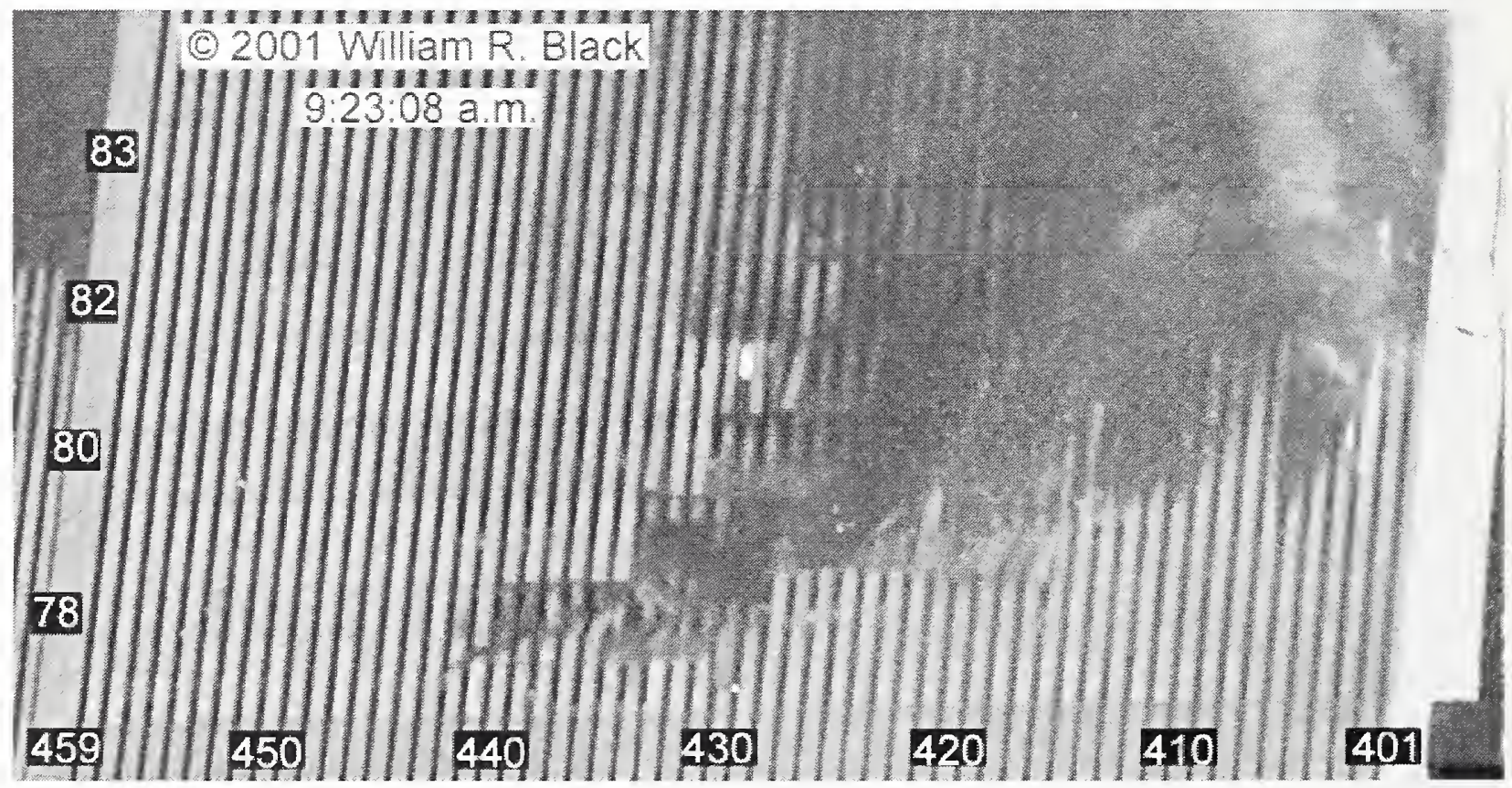

Figure 9-26. This cropped photograph shows the south face of WTC 2 at 9:23:08 a.m. The image has been rotated and enhanced by adjusting the intensity levels. Column and floor numbers have been added. 
A photograph of the east face of WTC 2 at 9:25:47 a.m. is shown in Figure 9-27. This image was taken $7 \mathrm{~min}$ and $9 \mathrm{~s}$ later than the similar photograph reproduced in Figure 9-21. Arrows added to the figure indicate the hanging object observed earlier through windows on the $82 \mathrm{nd}$ floor. Its appearance has changed substantially from earlier times (compare with Figure 9-1, Figure 9-2, and Figure 9-21). In the vicinity of window $82-336$, it is now visible lower down in the open windows. The hanging object no longer has the appearance of a thin line, but now looks like a curved sheet since the top of the object has been revealed. On either side of the dip the object remains high in the windows and still has the appearance of a line.

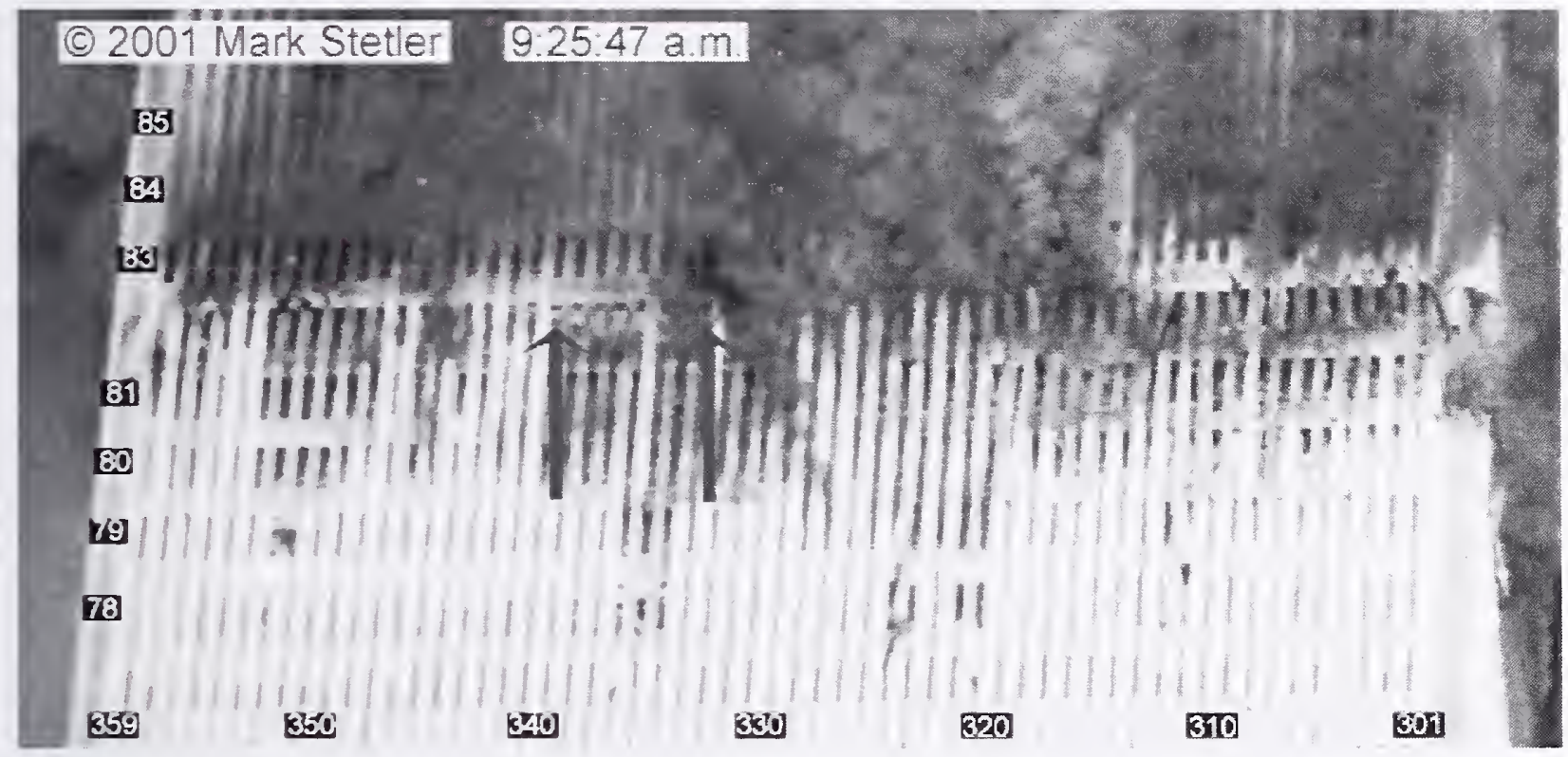

Figure 9-27. View of the east face of WTC 2 recorded at 9:25:47 a.m. The original photograph has been cropped and enhanced by adjusting intensity levels. Column and floor numbers have been added. The arrows highlight a hanging object (floor slab) observed through open windows on the 82 nd floor.

Another view of this object, shot from a different angle, is shown in Figure 9-28. This photograph was taken from a location on a nearby street to the southeast of the tower. As a result, the photograph is looking up at the hanging object from below. The arrows indicate the object. An enlarged view of the area with the object is shown in Figure 9-29. From this angle the object also has a sheet-like appearance, but, unlike in Figure 9-27, the underside of the object is in view. This is most easily seen in window 82-332. The sheet-like structure of the object is that which would be expected if the hanging object were a portion of the $83 \mathrm{rd}$ floor slab that, after having been dislodged from the east perimeter wall, had settled down further below the open windows on the 82 nd floor. This closer view shows that the object was visible as far south as window 82-342 at this time. At earlier times the object was visible further to the north than in Figure 9-27, but these windows remain hidden by dark smoke.

By comparing Figure 9-6, Figure 9-14, and Figure 9-21 with Figure 9-27, it is apparent that the fire that grew on the south side of the east face on the 82 nd floor shortly after the aircraft impact has continued spreading to the north. Intense flames are now coming from windows 82-321 to 82-329, while the fires have died down in windows further to the south. Heavy smoke is coming from windows as far north as window 82-316. 


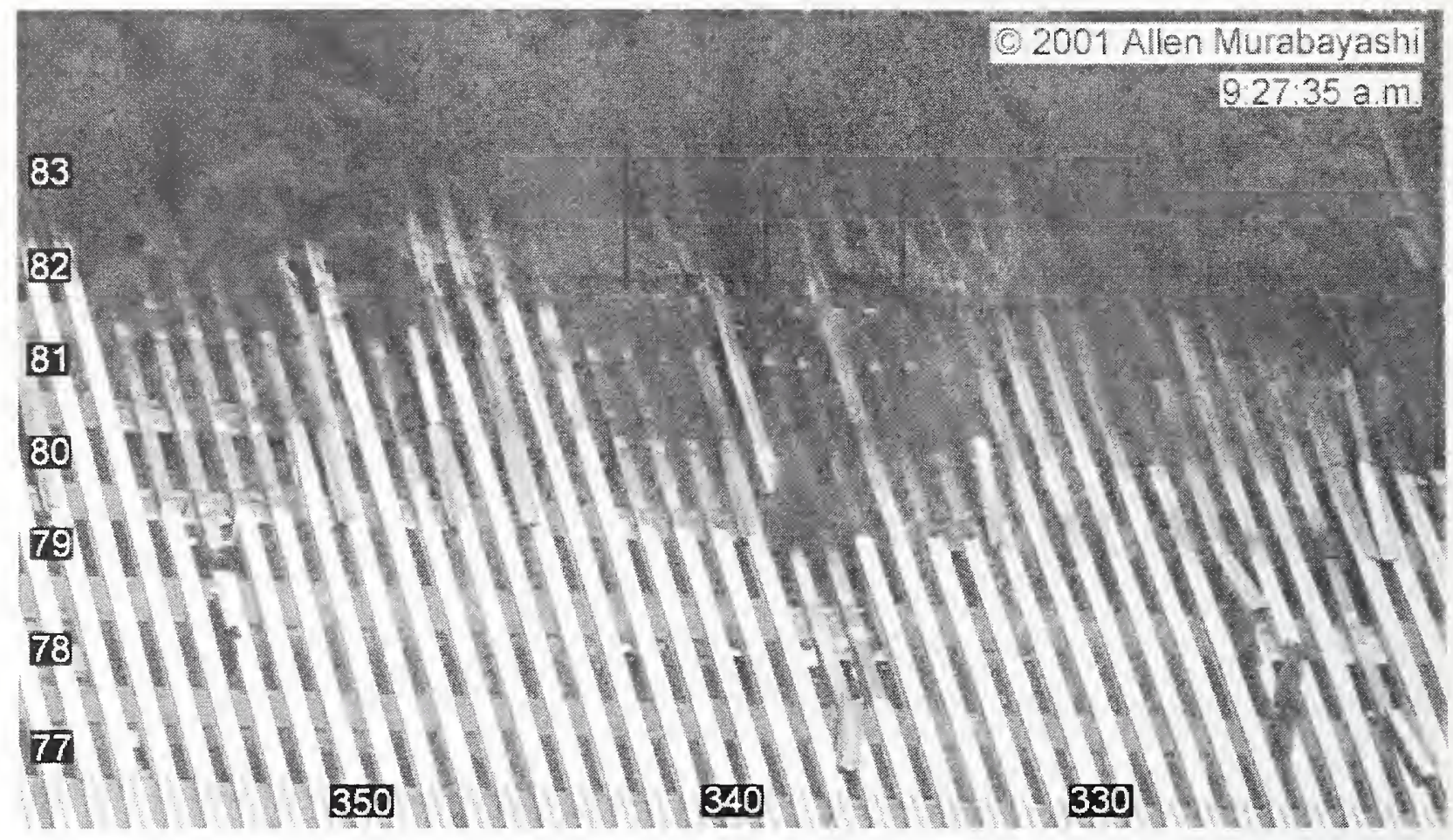

Figure 9-28. This cropped photograph shows the east face of WTC 2 at 9:27:35 a.m. The original image has been rotated, and column and floor numbers have been added. The arrows highlight a hanging object observed through open windows on the 82 nd floor.

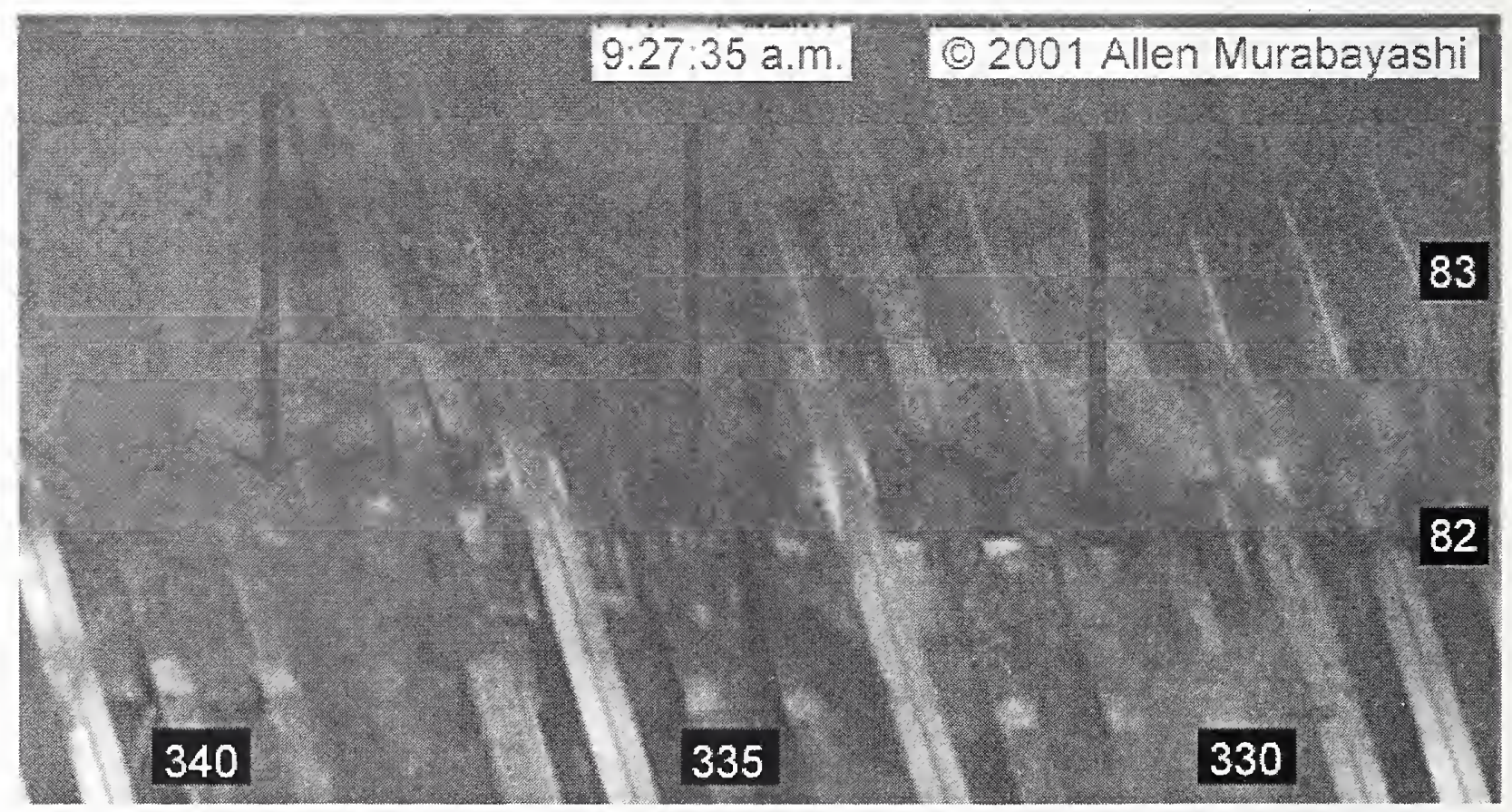

Figure 9-29. This image is an enlargement of a portion of Figure 9-28 showing the east face of WTC 2, where a hanging object is visible through open windows on the $82 \mathrm{nd}$ floor. Column numbers have been added. 
The fire distributions also changed at other locations on the east face between the times when Figure 9-21 and Figure 9-27 were taken. At the earlier time there was a large fire visible on the 82 nd floor in windows 82-305 to 82-312 on the north side. By 9:25:47 a.m. this fire appears to have died down, and flames are no longer evident in this area. A flaming region is now present immediately above on the 83rd floor. Flames are visible on this floor in windows 83-302 to 83-312. In the earlier photograph heavy smoke was coming from these windows. but flames were not as evident. The fire burning in the northeast corner of the 81 st floor appears to have decreased somewhat in intensity during the period. The heaviest flames are now coming primarily from the opening on the northeast corner of this floor. Even in this area. the flame lengths have decreased somewhat. It is now possible to barely discern the corner column 82-300, which is still in place, through the opening on the 82 nd floor.

The fire that was visible on the 80th just to the south of the center of the east face in Figure 9-21 is no longer evident in Figure 9-27, but there does appear to be an internal fire visible on the 79th floor in windows 79-227 and 79-228.

In Figure 9-27, the heaviest smoke is coming from windows on the 82nd and 83rd floors, and the lower floors are visible. A smoke plume is flowing from one of the windows, 79-227, on the 79th floor where there appears to be some flame visible, and light smoke can be seen exiting from a few other windows on the lower floors. Nevertheless, the general impression is that of little smoke flowing from floors lower than the $82 \mathrm{nd}$. As mentioned above, this conclusion is somewhat misleading since brief periods of smoke release from lower floors had been observed starting before 9:13 a.m. Review of videos and photographs showed that these smoke releases increased in both frequency and the amount of smoke released as time progressed.

After 9:20 a.m. some of these smoke bursts released sufficient smoke that portions of the lower floors were partially obscured for several seconds. Some of the more intense smoke releases were observed around 9:21:37 a.m., 9:23:25 a.m., 9:24:02 a.m., 9:26:17 a.m., 9:26:28 a.m. and 9:28:55 a.m. Figure 9-30 shows a photograph of the east face that was taken at 9:26:20 a.m. during the release of smoke that began around 9:26:17 a.m. Note that Figure 9-30 was shot only $33 \mathrm{~s}$ later than Figure 9-27. Blowups of the north and south sections of the east face from Figure 9-30 are included in Figure 9-31.

Comparison of Figure 9-27 with Figure 9-30 and Figure 9-31 provides an indication of how quickly the appearance of the east face could change during the smoke release events. In Figure 9-30 heavy smoke almost completely obscures the hanging object seen clearly less than a minute earlier through the 82nd floor windows. This smoke had been pushed out of open windows on the 79 th through 82 nd floors. In the blowup of the south side of the face in Figure 9-31, the hanging object is just visible at the tops of windows 82-329 through 82-333, as indicated by the arrow placed on the lower image in Figure 9-31. A small fire is visible at the top of window $82-229$ above the object.

In this photograph (see lower image in Figure 9-31) the heaviest smoke flow on the 79th floor is from windows 79-333 to 79-336, but lighter smoke is also flowing from windows 79-320 to 79-325, 79-327, and 79-328. The glass is still in place for the intermediate windows where no smoke is observed, as well as on either side of windows 79-320 and 79-336. A long-distance video of the smoke release indicates that the relative density of smoke flowing from these windows varied rapidly with time and location and that heavy smoke was pushed from the other open windows during a portion of this release. 


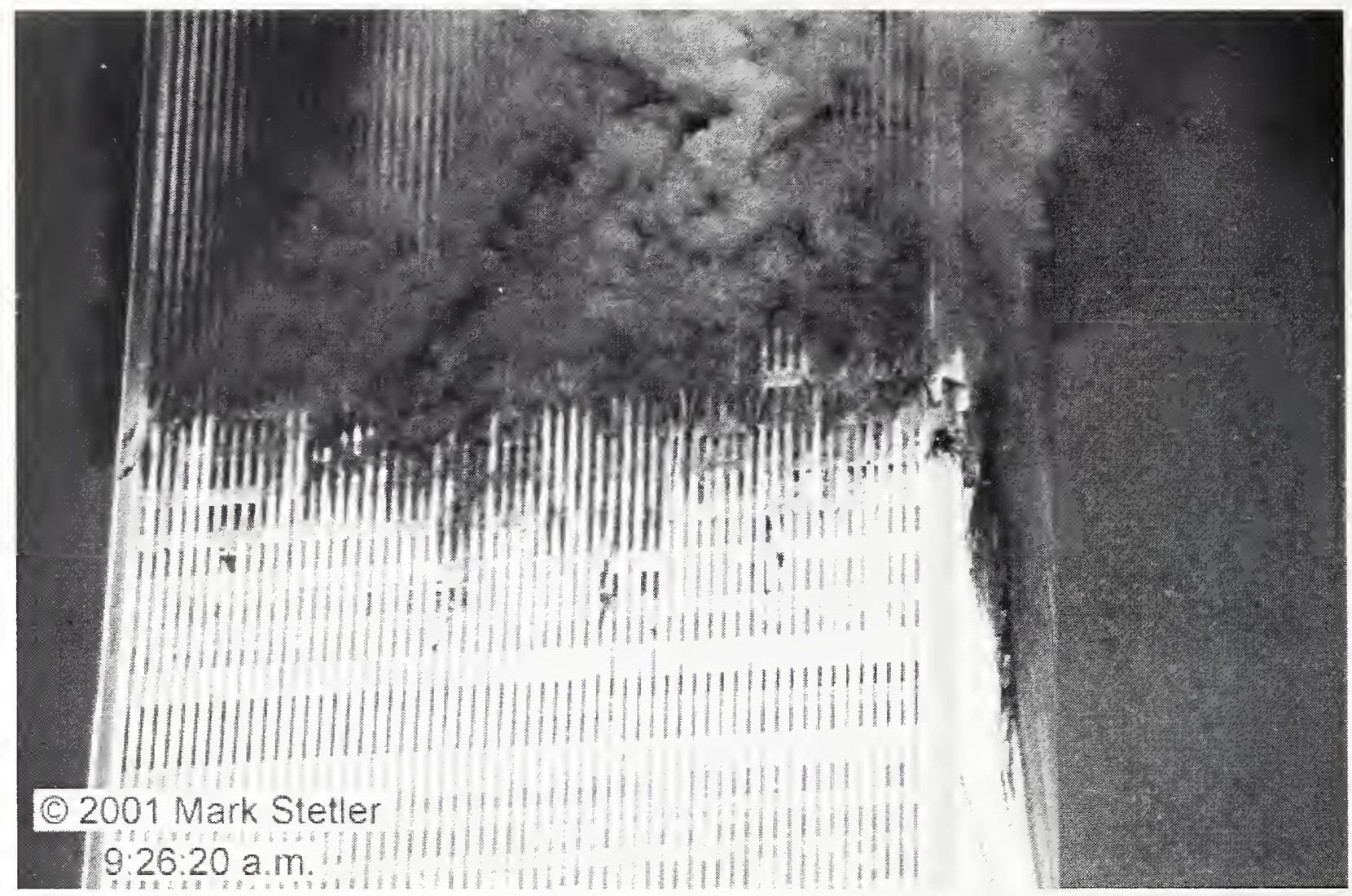

Figure 9-30. This photograph showing the east face of WTC 2 was taken at 9:26:20 a.m.

A person was observed falling from a window on the 79th floor, window 79-351, at 9:24:06 a.m. The appearance of smoke on the 79th floor suggests that local conditions on this floor were beginning to deteriorate around this time. This may explain why this person fell.

In Figure 9-31 smoke is also coming from open windows at the southern end of the east face on the 80th, 81 st, and 82nd floors. Unlike on the 79th floor, flames as well as smoke are visible on the 80th floor in windows 80-333 to 80-340. Fire was present in this vicinity as early as 9:07:04 a.m. (see Figure 9-7). It has apparently grown slowly during the intervening $20 \mathrm{~min}$. A small fire is also visible in window 81-335 on the 81 st floor.

The heavy flames coming from windows 82-321 to 82-329 on the 82nd floor 33 s earlier in Figure 9-27 have retreated back into the windows. In Figure 9-31 extended flames are only visible in windows 82-326 and 82-327. The intense fire on the northern edge of the 83rd floor in Figure 9-27 has also died down. In particular, the flames that filled windows 83-310 to 82-312 earlier appear to have completely dissipated in Figure 9-31. The appearance of the fire burning on the northeast corner of the 81 st floor has also changed. At the earlier time large flames were visible coming from the opening on the northeast corner. Thirty-three seconds later, the fire covered a much broader extent of the debris in the corner, while the flames in the northeast corner opening appeared to have died down. A long-distance video of this corner around this time shows that the intensity of the fire on the 81 st floor was highly variable. 

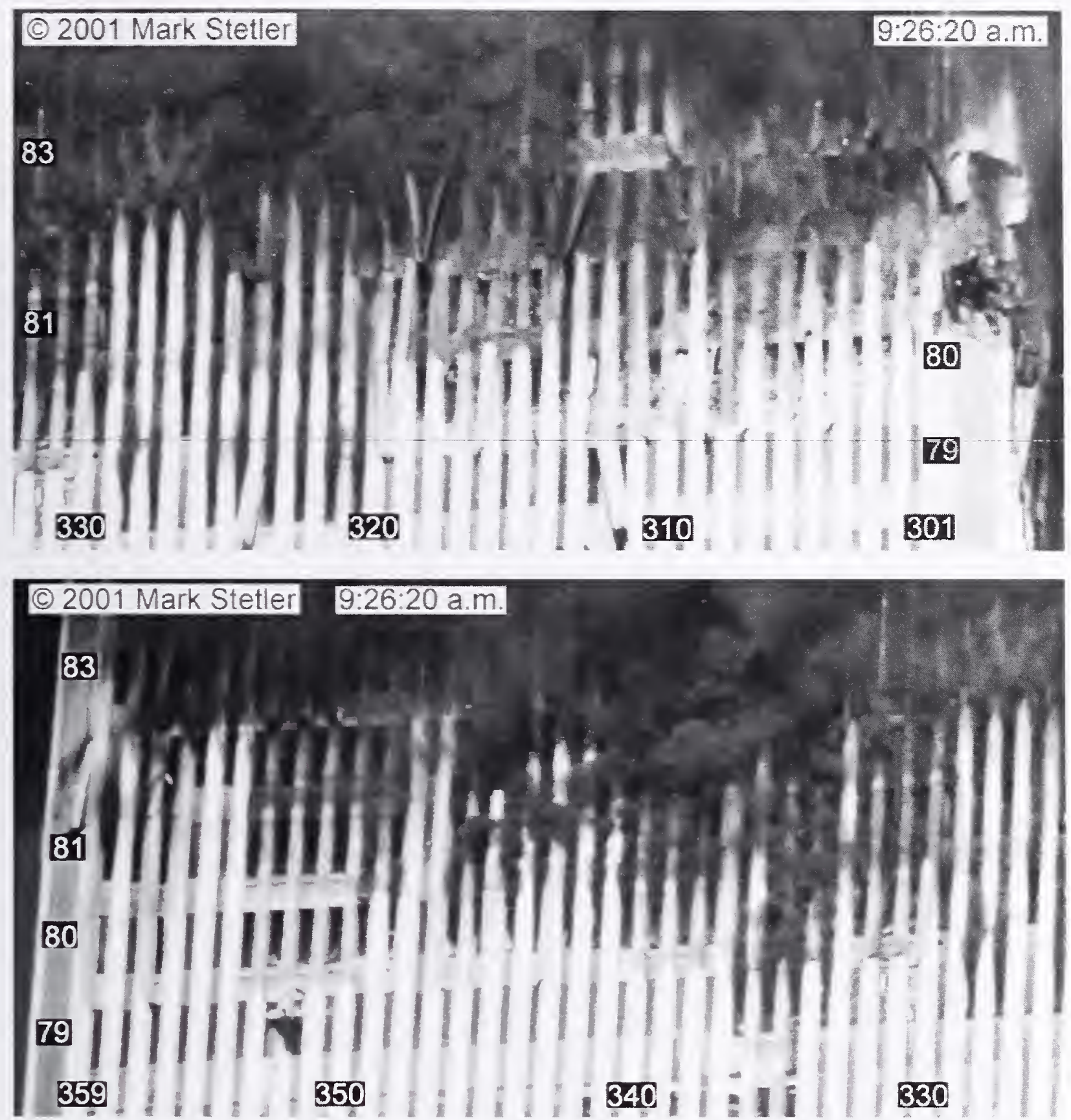

Figure 9-31. These two images show blowups of the north and south sides of the east face of WTC 2 taken from the photograph included in Figure 9-30, which was shot at

9:26:20 a.m. Column and floor numbers have been added. The arrow highlights a hanging object visible through open windows on the 82 nd floor.

It has proven difficult to identify causes from the visual record for the short smoke releases and rapid changes in fire distribution observed on the east face around this time. In general, these behaviors are not typical of building fires, and such occurrences were not as apparent in the fires that occurred in WTC 1. They could be associated with such causes as local wind changes around the tower or possible localized movement of debris or structural elements within the tower. 
A view of the north face recorded at 9:27:04 a.m. is shown Figure 9-32. It was shot nearly 8 min after the image shown in Figure 9-24. The fire distribution on this face has changed very little during this period. The intense fires ignited immediately following the aircraft impact on the east edges of the $81 \mathrm{st}$ and 82 nd floors continue to burn, even though the fire intensity on the 82 nd floor has continued to decrease. The fire in the northeast corner opening on the 81 st floor also decreased in size. The column in the corner of the 82nd floor, column 82-300, which was hidden by flames in Figure 9-24, is now fully visible.

The fire burning on the 79th floor to the west of the cold spot remains in the same location and is burning with a comparable intensity to the earlier time. Flames are visible in both of the lower corners of the cold spot on the 80th floor in Figure 9-32. Due to the lower image quality of Figure 9-24, it is difficult to determine whether these flames have grown more intense during the $8 \mathrm{~min}$ period.

\section{(C) 2001 CBS Broadcasting Inc. $9.27,04 \mathrm{a} . \mathrm{m}$}
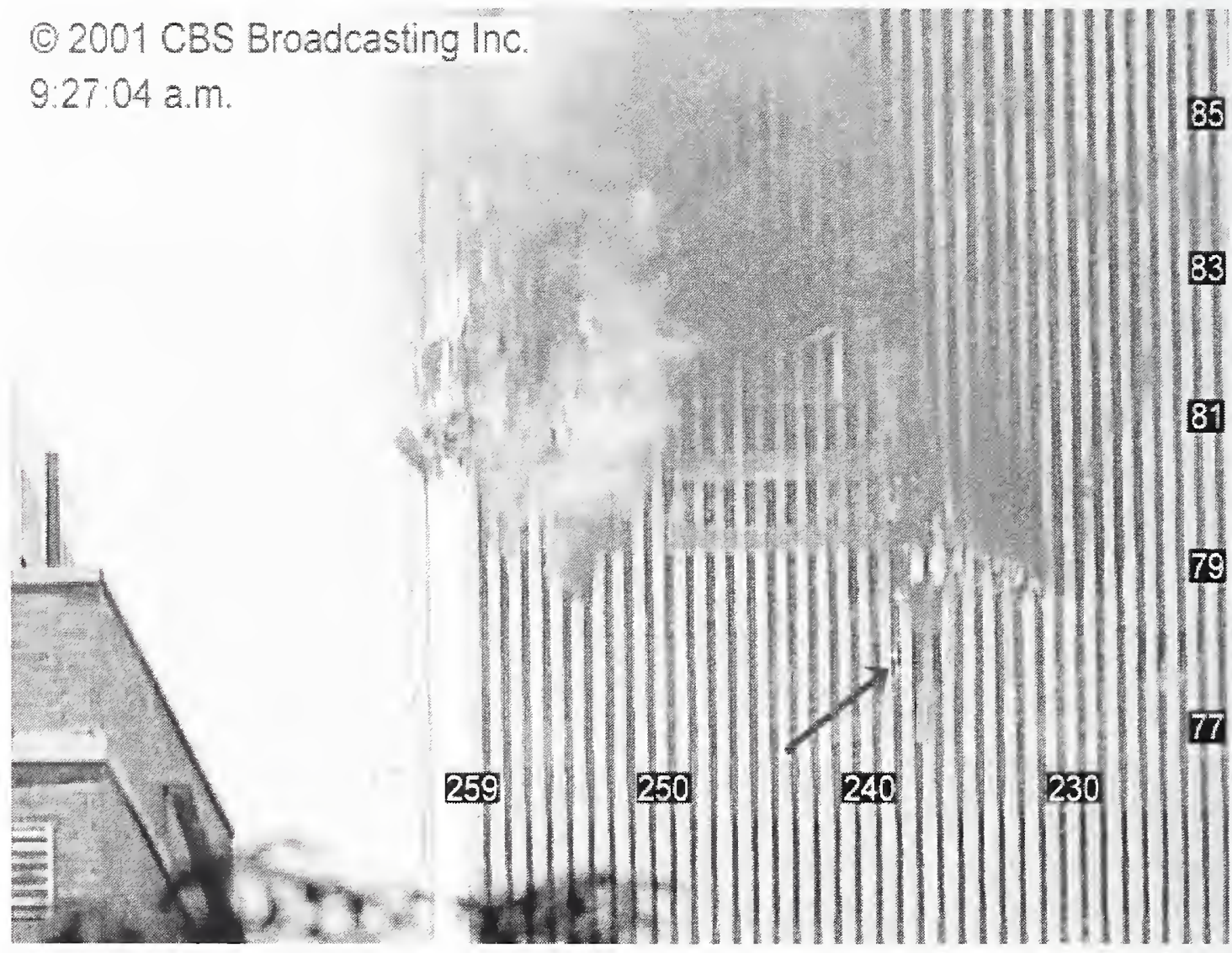

Figure 9-32. This captured video frame showing the north face of WTC 2 was recorded at 9:27:04 a.m. The intensity levels have been adjusted, and column and floor numbers have been added. The arrow indicates a stream of liquid-like material that was observed on the 78th floor falling in window 78-238.

There are some distinct differences between the two images. In the earlier photograph it is difficult to identify whether there is fire on the eastern edge of the 83rd floor due to the height of the flames on lower floors. At 9:27:04 a.m. fire is visible in windows 83-257 and 83-258. No other flames are visible on the north side of the $83 \mathrm{rd}$ floor. In the later image heavy smoke is coming from windows 79-253 and 79-254 on the 79th floor, while the area is clear in Figure 9-24. Review of videos shows that the smoke flows from these windows, as well as windows 79-257 and 79-256, were intermittent around this time and 
tended to be correlated with each other. This behavior was similar to that already described for the east face.

Closer inspection of Figure 9-32 shows what appears to be a shiny silver string (indicated by a red arrow) passing from the top to the bottom of one of the windows, 78-238, on the 78th floor. In the video from which this image was taken, the material actually appeared to be a liquid pouring intermittently from the top of the window and splashing at the window base. The liquid looked as if it was glowing. It was only observed during the six seconds that this video recorded this area of the tower. In Section 9.5, a flow of similar material from windows 80-255 and 80-256 on the 80th floor of the north face will be described. It will be hypothesized that the material was most likely aluminum from the aircraft that had melted and was pouring down through an opening from the floor above. This is also a plausible explanation for the current observation. If correct, it suggests that the pile of debris located on the 79th floor above this window may have contained aluminum from the aircraft. Recall that a pile of debris in this area of the 79th floor was present immediately following the aircraft impact. An intense fire had been burning on this debris since the impact that would have provided the heat necessary to melt the aluminum.

During this period, it was noted that smoke was flowing from several open windows on higher floors of the north face, including windows 93-201 to 93-203 on the 93rd floor, windows 103-120 to 103-122 on the 103rd floor, and windows 105-131 and 105-132 on the 105th floor. These windows were possibly broken open by people in these locations.

A view of the south face of WTC 2 taken at 9:28:17 a.m. is shown in Figure 9-33. It was shot just over 5 min later than Figure 9-26. The locations of the fires on the face did not change a great deal during the time between the images. The fire on the 81 st floor to the west of the aircraft impact cavity appears to have moved west a couple of windows, and the fire on the floor below is somewhat larger. Bascd on the amount of smoke coming from open windows and the appearance of flames, the intensity of the fire to the east of the impact cavity on the 79th floor has decreased somewhat. The visible fires on this side of the cavity on the 78th and 82nd floors have similar appearances to those at the earlier time.

A video of the south face shot around 9:28:50 a.m. showed a brief flow of a large amount of smoke and dust from the aircraft impact cavity on the 78th and 79th floors. This event coincided with one of the releases of heavy smoke on the east face listed above. It provides an indication that the mechanism(s) responsible for the rapid smoke variations on the east face could simultaneous affect the smoke flows on the other faces of the tower.

Most of the images showing the west face of WTC 2 during the period were taken from long range. The smoke plumes described earlier on the 86th and 107th floors of the tower are the dominant features visible in the images.

Major observations concerning WTC 2 between 9:15 a.m. and 9:29 a.m. are summarized here. Visual representations of integrated fire intensities over the period for the four faces are shown in Figure 9-34. Changes in fire distributions during the period can be tracked by comparing the results with Figure 9-19, which shows similar maps for the first time period. It should be kept in mind that the integrated fire maps for this period incorporate some very rapid changes in fire distribution. 


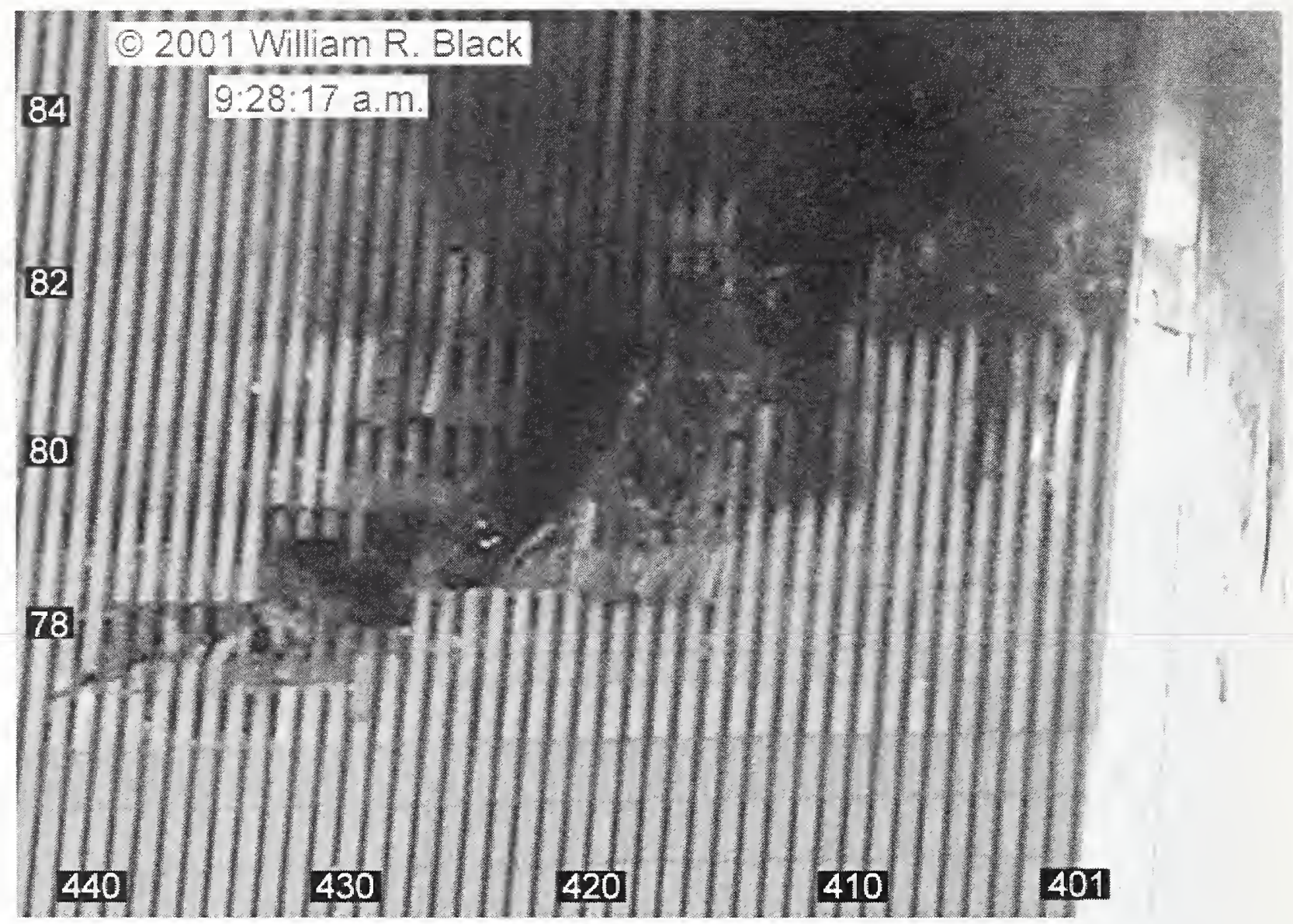

Figure 9-33. This cropped photograph showing the south face of WTC 2 was shot at 9:28:17 a.m. The image has been rotated, and the intensity levels have been adjusted. Column and floor numbers have been added.

On the north face, the primary observation was the continued burning of fires on the 81 st and 82nd floors on the east side of the cold spot and on the 79th floor to the west side. These fires were ignited during the aircraft impact and subsequent fireballs and appeared to be burning on piles of debris that resulted. While still substantial, these fires appeared to decrease in intensity during the period. Infrared images recorded near the start of the period confirmed that temperatures in the cold spot on the north face were near ambient. While some fires were observed at various corners of the rectangular cold spot, in general, the area remained smoke and fire free. Near the end of the period, flames were obscrved in windows near the cast side of the 83 rd floor. The appearance and positions of the numerous hanging objects visible through open windows on the north face, some of which may be floor slabs that have dropped down from the floor above, did not change noticeably during the period. 

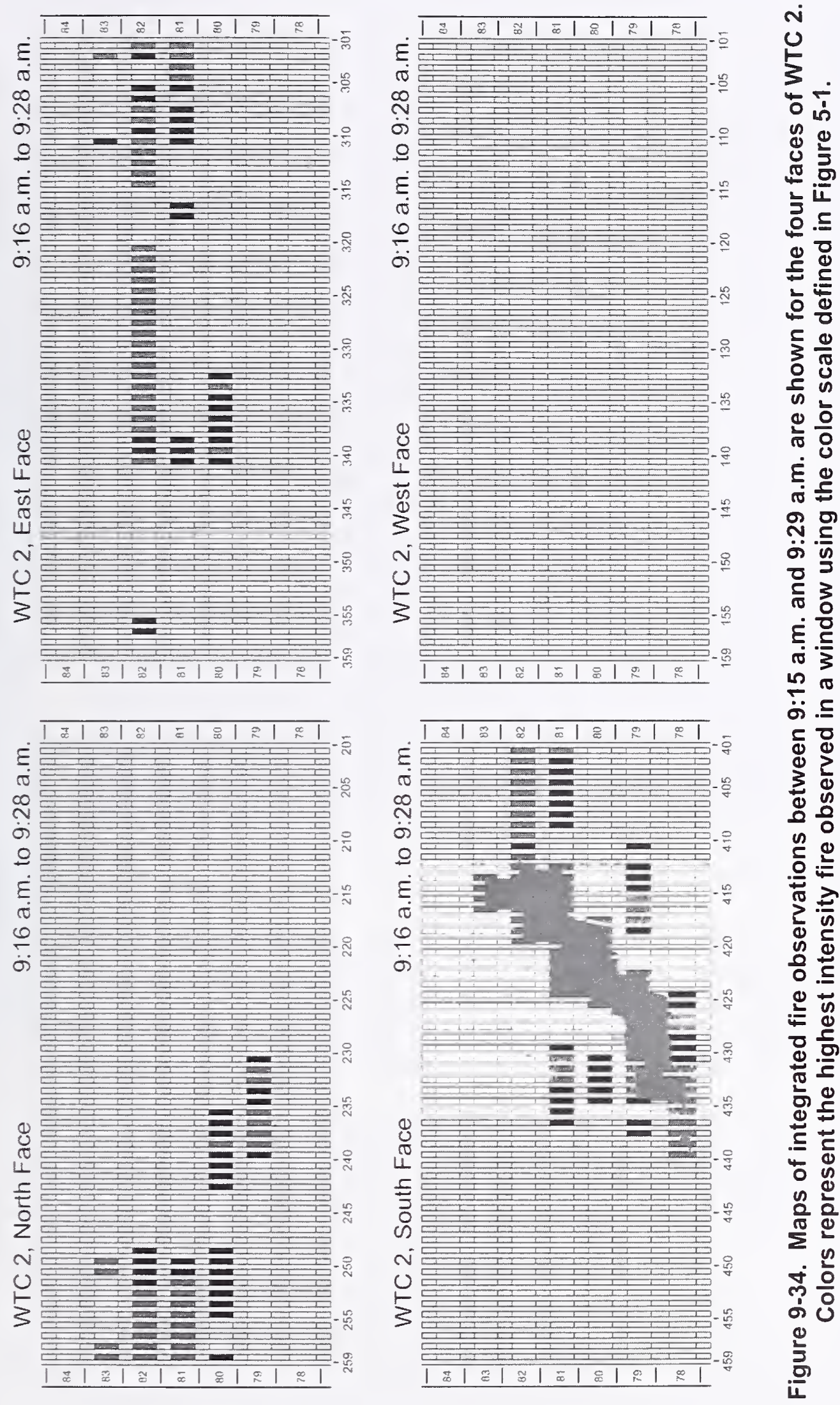
On the east face a large fire that had started on the south side of the 82 nd floor spread slowly to the north, going through a cycle of initial growth to an intense burning phase, followed by decline. Fires that were ignited on piles of debris located in the northcast corners of the 81 st and $82 \mathrm{nd}$ floors continued to burn over the period, but their intensities decreased as did the intensity of a fire burning in the opening in the northeast corner on these two floors. During the period, a substantial fire was observed briefly near the east edge of the 83 rd floor. Infrared images recorded during the early part of the period indicated that there was strong heating on the 81 st floor and only regions of localized heating on the 79th and 80th floors. Over the course of the period, increasingly frequent and intense intermittent bursts of smoke were observed coming from windows on several floors of the face. Rapid variations in fire distribution were also observed.

The hanging object observed through a number of open windows on the east face at the 82nd floor dropped down lower in some of the windows during the period. This allowed oblique shots to show that it appeared to be a thin, sheet-like object, providing additional support for the hypothesis that it was a portion of the 83rd floor slab hanging down from above.

Most of the fires on the south face remained localized near the aircraft impact cavity during the period. This included fires to the west of the cavity on the 79th, 80th, and 81st floors and to the east on the 78th and 79th floors. Fires already present to the east of the impact cavity on the 81 st and 82 nd floors did not seem to spread to the east face. During the period, the fire on the 81 st floor decreased in intensity, while the fire on the 82 nd floor continued to burn vigorously. Near the start of the period, a substantial fire grew on the 79 th floor to the immediate east of the aircraft impact cavity and then spread slowly to the east.

There was no indication of smoke or fire on the aircraft impact floors on the west side of the tower. Smoke flows coming from the 85 th, 86 th, and 107 th floors may possibly be attributed to windows being opened by people.

\section{$9.4 \quad$ 9:29 A.M. TO 9:45 A.M.}

Multiple videos show that at 9:29:15 a.m. an intense flame suddenly erupted from window 83-237 on the 83 rd floor of the north face of WTC 2. Figure 9-35 shows an image of this face taken $27 \mathrm{~s}$ after the flames first appeared. The new area of flame is just above and to the right of the cold spot and is coming from windows 83-236 to 83-238. There are also flames visible to the left of the cold spot near window 83-250, as well as in windows 83-255 to 83-258. The intervening windows are obscured by smoke.

At first glance, it appears the new area of fire on the 83rd floor in Figure 9-35 is well separated from other flames on the floor. However, a dull red glow is barely visible in windows 83-240 to 83-245, suggesting there is a low intensity fire burning behind closed windows immediately above the cold spot. It seems this low intensity fire had spread further west, where it eventually broke out windows and then grew rapidly.

Comparison of Figure 9-35 with Figure 9-32, which was taken 2 min and 38 s earlier, shows how quickly the fire on the northern side of the 83 rd floor grew and spread. At the earlier time there was no indication of a fire on the floor to the west of the small fires visible through windows 83-257 and 83-258. The appearance of fire at this location on the $83 \mathrm{rd}$ floor is the first visual evidence of significant fire spread on the north face of the tower. It took place almost 27 min after the aircraft struck the tower. 


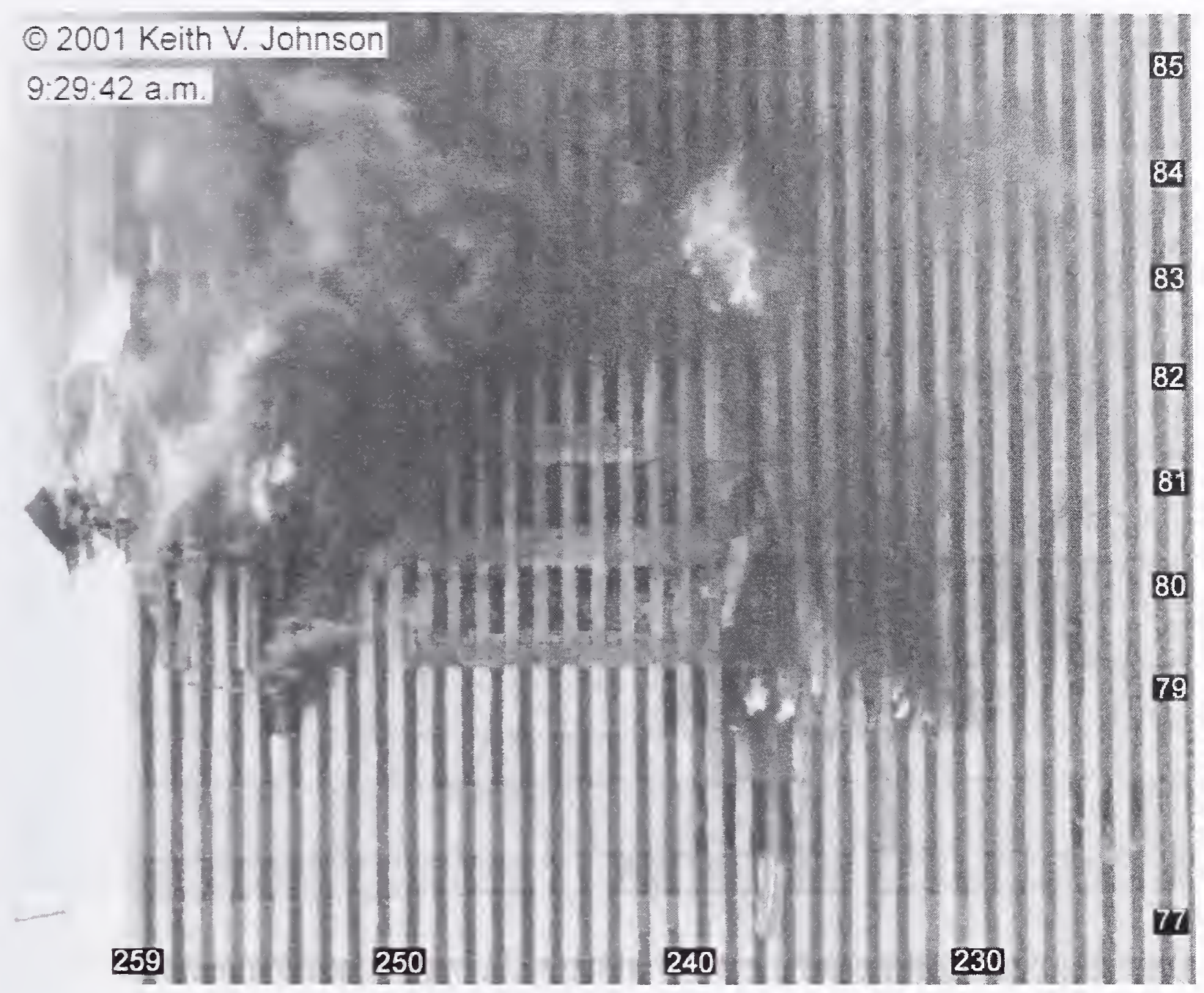

Figure 9-35. This cropped photograph shows the north face of WTC 2 at 9:29:42 a.m. The intensity levels have been adjusted, and column and floor numbers have been added

Elsewhere on the face, the appearance of the fires at 9:29:42 a.m. is much the same as at 9:27:04 a.m. The fires continued to burn on the debris piles at the eastern edges of the 81st and 82nd floors and closer to the center of the face on the 79th floor. The fires at the lower comers of the cold spot on the 80th floor are visible in both images. The hanging objects visible earlier in Figure 9-12 and Figure 9-16 are not immediately apparent in Figure 9-35. This is somewhat unexpected given the quality of the image.

Another view of the north face taken at 9:32:24 a.m. is shown in Figure 9-36. Comparison with Figure 9-35 reveals that the intense area of fire on the 83rd floor to the west of the cold spot has spread during the $2 \mathrm{~min}$ and $42 \mathrm{~s}$ period between the times the images were taken. Long flames are now coming from a length of windows running from window 83-234 to window 83-239. Flames are also visible on this floor starting at window 93-249 and extending to the eastern edge. Flames are not visible on the floor in windows immediately above the cold spot. 


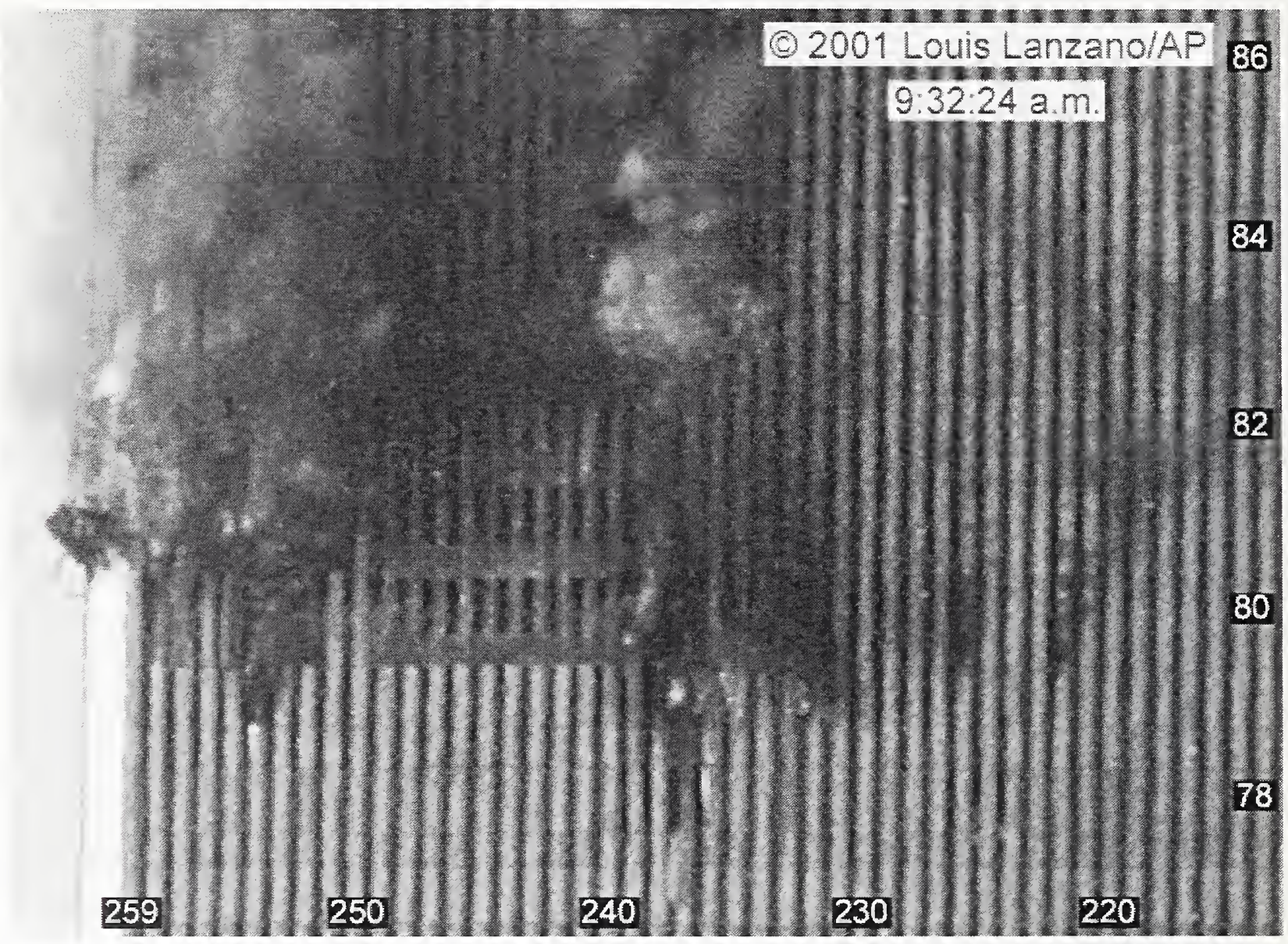

Figure 9-36. This cropped photograph shows the north face of WTC 2 at 9:32:24 a.m. The image has been rotated and enhanced by adjusting the intensities. Column and floor numbers have been added.

Another difference between Figure 9-35 and Figure 9-36 is apparent on the 79th floor. At the later time a dull red glow is visible in windows 79-224 to 79-228, and smoke is coming from window 79-226 and window 79-222. This is evidence that the fire that has been burning on the 79th floor since the aircraft impact has begun to spread across the face toward the west.

Elsewhere on the face, the fires present in Figure 9-36 have not changed markedly for many minutes.

At 9:33:52 a.m. the fire burning on the 83rd floor of the north face seemed to jump several windows to the west when window 83-226 broke open, and a large flame exited. The resulting flare can be seèn in Figure 9-37, which was recorded $0.9 \mathrm{~s}$ after the window opened. This large flame only lasted a few seconds, but after it died down heavy smoke and a shorter flame continued to come from the window. 


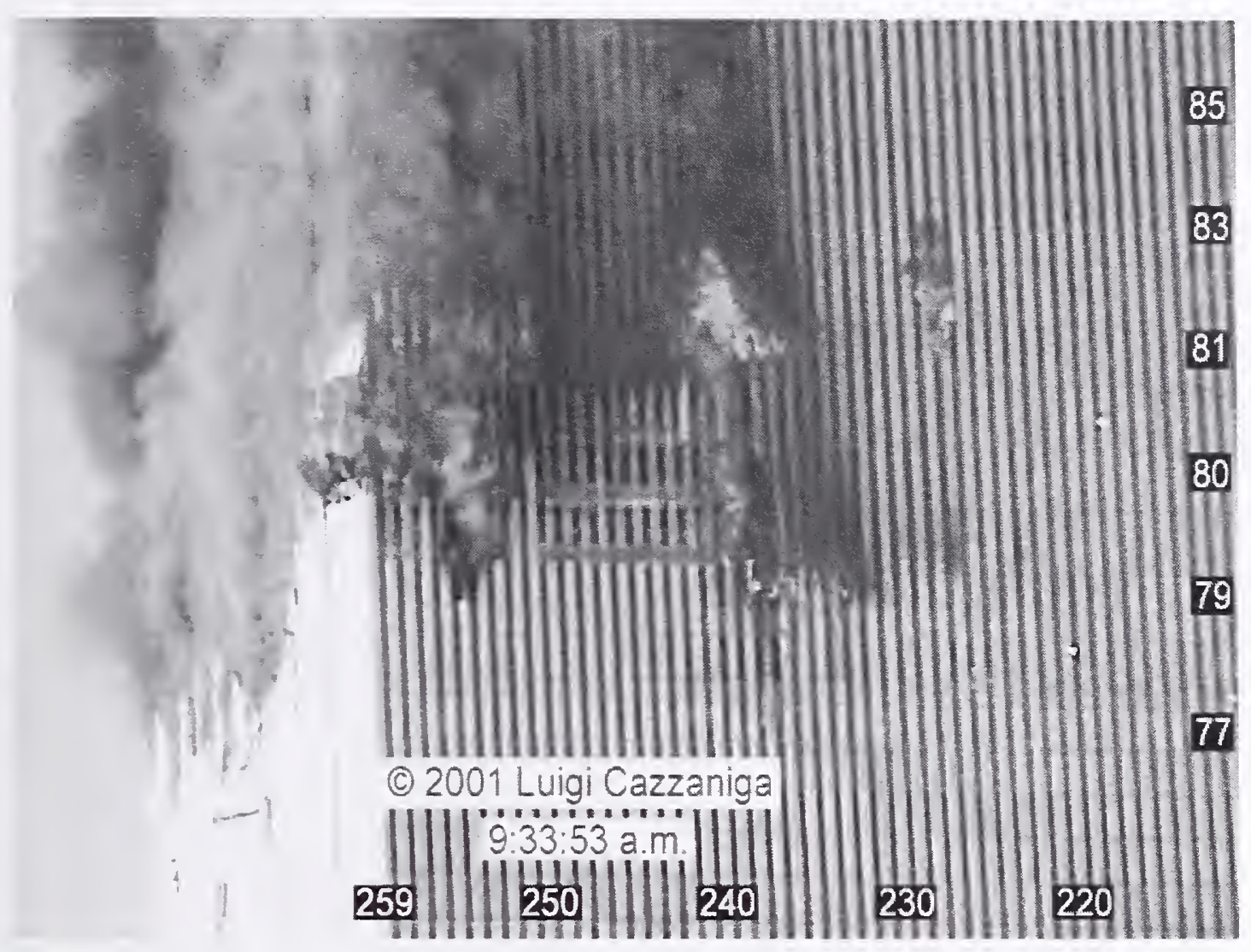

Figure 9-37. This frame taken from a video shows the north face of WTC 2 at 9:33:53 a.m. The intensity levels of the image have been adjusted, and column and floor numbers have been added.

In Figure 9-37 small flames are visible in windows 83-240 to 83-244, which are located above the cold spot on the 83 rd floor. This confirms that fires were present on this part of the floor. As an aside, since flames are not observed on the floor below, it also suggests that the 83rd floor slab is in place above the cold spot. The fires observed elsewhere on the face have similar distributions and intensities to those in Figure 9-32, Figure 9-35, and Figure 9-36.

Two views of the northeast corner of WTC 2 are shown in Figure 9-38. The two images were captured from a video recording at 9:31:21 a.m. and 9:31:28 a.m. They were chosen to illustrate the appearance of the smoke puffs that had been observed periodically on the east face of WTC 2 for nearly twenty minutes. In the earlier image shown at the top of Figure 9-38, the visible portion of the east face is relatively clear of smoke. The only flame visible in windows on the east face at this time is the relatively small fire on the 81 st floor in window 81-302, even though a fire continues to burn in the opening at the northeast corner of the 81 st floor. Otherwise, the fires that were previously observed on this part of the east face seem to have died down. A video shot from a longer distance shows that shortly after the upper frame was recorded a smoke release occurred from multiple windows over multiple floors on the east face. This particular release was not as intense, i.e., less smoke was released, as others that were observed around this time. 

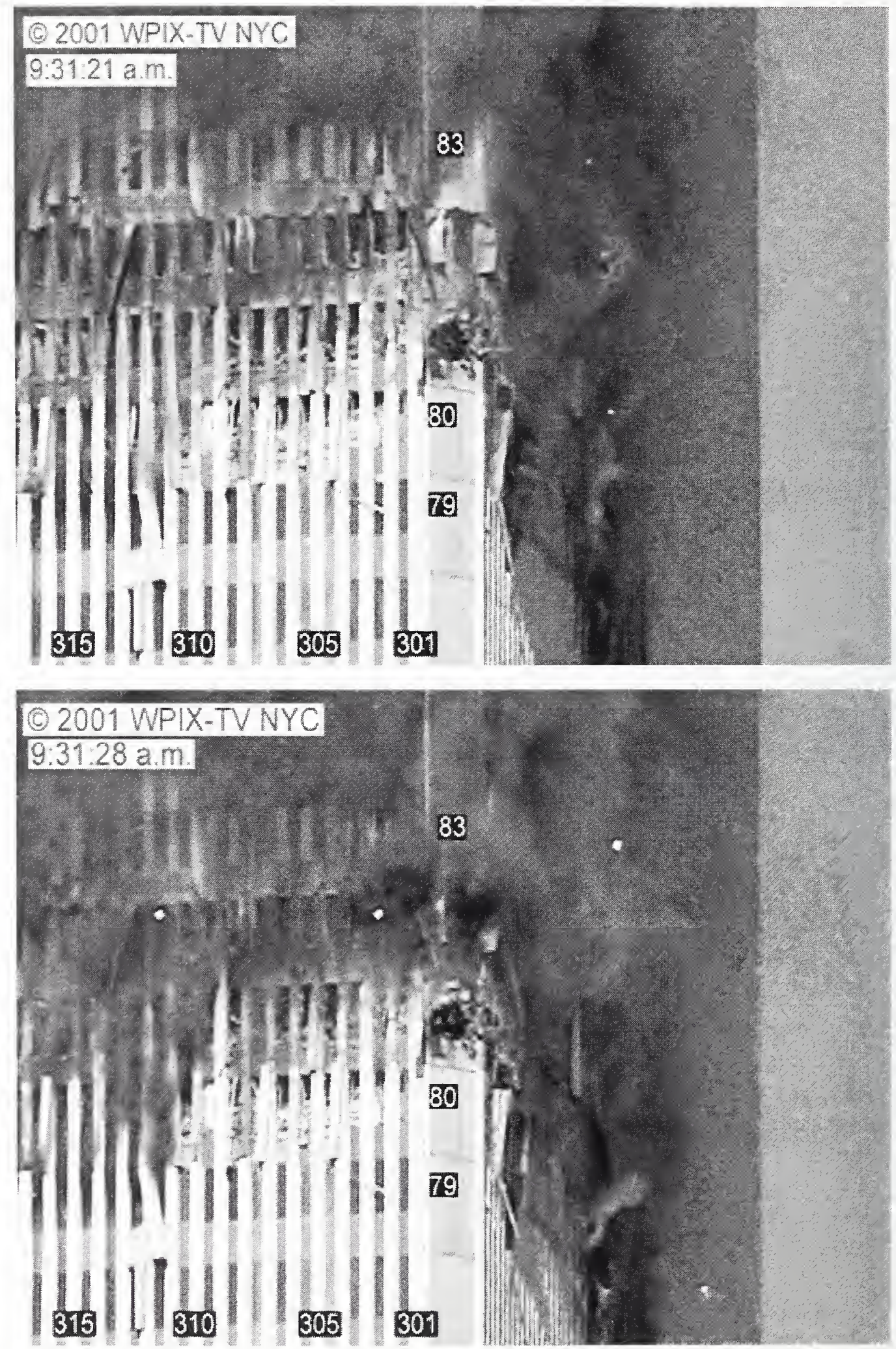

Figure 9-38. Two frames captured from a video at 9:31:21 a.m. and 9:31:28 a.m. show the northeast corner of WTC 2. The intensity levels of the images have been adjusted, and column and floor numbers have been added. 
The effects of the smoke release are evident in the lower image shown in Figure 9-38. Smoke has been pushed out of open windows on the 79th floor, i.e., windows 79-311 to 79-316, and can bc scen rising above these windows. Comparison of the frames shows that the appearance of the smokc and fire in the vicinity of the burning corner on the 81 st floor has also changed. Not only has smoke been pushed out of windows 81-301 and 81-302 and the opening on the northeast corner, but the fire intensity has also increased in the immediate area. Flames have been pushed out of windows 81-301 and 81-302. In the video it is clear that smoke was also pushed from windows on the 80th floor on the north side of the tower. In the lower image, this smoke appears as a white stream that has been pushed away from the building. In a video shot from a longer distance it was observed that smoke also appeared from windows on the 80th floor located on the south side of the east face. These observations suggest that the smoke puffs could be associated with pressure variations that were extensive enough to affect multiple floors and faces of the tower simultaneously. It should be noted that while the pressure variations that create the puffs may be the result of structural changes within the tower, the resulting pressure increases are likely to be much too small to have any impact on the structure.

Close-up videos and photographs showing the entire east face of WTC 2 are limited during the initial part of this time period. In order to gain insights into what was happening on this face between 9:29 a.m. and 9:34 a.m., long-range videos showing the face were reviewed. The videos indicated that the unusual short-lived smoke releases described previously, including the moderate example captured in Figure 9-38, occurred randomly throughout the period. Many of these releases displayed the pipe organ appearance discussed earlier, but a few pushed large amounts of smoke from windows that briefly obscured the lower floors. Such large smoke releases occurred around 9:30:20 a.m., 9:31:45 a.m., 9:31:55 a.m.. 9:33:25 a.m., and 9:34:15 a.m.

The smoke release around 9:30:20 a.m. appeared to be particularly intense. It was observed in videos shot from the northeast and southeast. The video shot from the northeast includes a view of the north face. At the time of the smoke release on the east face, fire and smoke were also pushed from windows centered near column 238 on the 79th and 80th floors of the north face. A long distance video shot from north of the tower revealed that a puff of dust was also pushed from open windows on the 78th floor at windows 78-224 to 78-226. The southeast view included the south face. On this face smoke was pushed from open windows to the west of the aircraft impact cavity on the 77 th, 79 th, and 81 st floors. These observations provide additional evidence that the repeated puffs of smoke obscrved on the east face of WTC 2 were associated with pressure pulses that were sufficient to modify smoke flows over multiple floors and faces of the tower.

An oblique view of the east face taken from a video shot at 9:32:08 a.m. is shown in Figure 9-39. It shows the tower during one of the smoke releases. Heavy smoke is coming from the tops of numerous open windows on the 79th floor. Lighter smoke is also coming from windows on the west sides of the 79th, 80th, and 81 st floors. The bright flame visible coming from near the 81st-floor window 81-302 was pushed out of the tower at the same time as the smoke.

A relatively close view of the east face shot from southeast of WTC 2 at 9:34:40 a.m. is shown in Figure 9-40. This photograph was taken during a period when the flow of smoke coming from windows on the northern side of the 79th floor was fairly heavy, but when little smoke was coming from other windows below the 82nd floor. In Figure 9-27, taken at 9:25:47 a.m., windows 79-314 to 79-320 on the 79th floor had glass in place. These windows are open in Figure 9-40, suggesting that a fire must be burning nearby. The southern half of the face is visible to the tops of $83 \mathrm{rd}$ floor windows. Flames are 
visible on the 82 nd floor from window $82-322$ to window $82-328$. The fire on this floor has continued its spread toward the north since 9:27:35 a.m. (see Figure 9-28 and Figure 9-30 and related discussion). The fire burning in and near the northeast corner of the 81 st floor is also visible in the photograph.

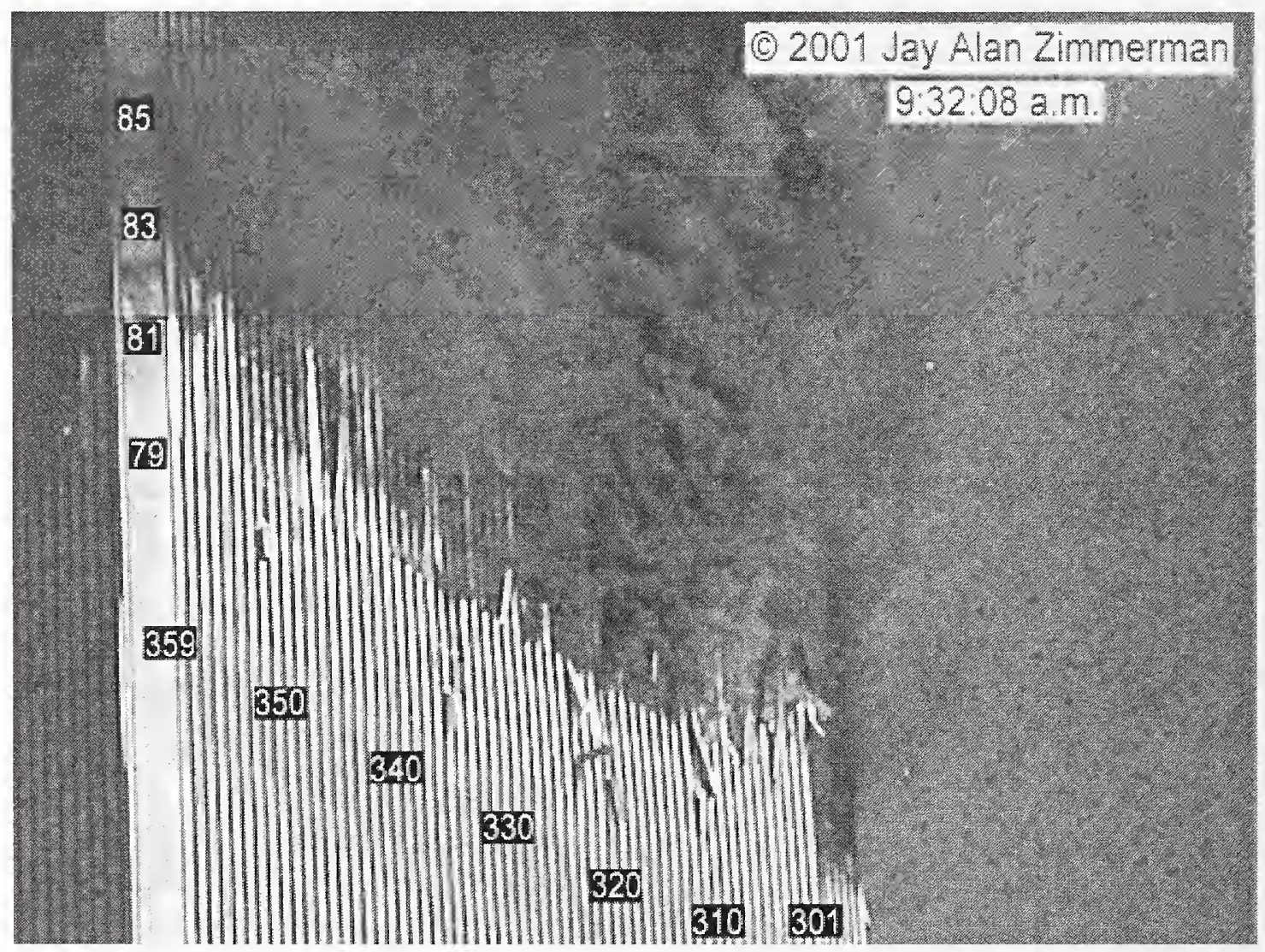

Figure 9-39. This view of the east face of WTC 2 is a frame from a video taken at 9:32:08 a.m. Column and floor numbers have been added. The northeast corner of WTC 1 is visible in the background.

The arrows in Figure 9-40 indicate the hanging object that was described earlier. The appearance of the object is much the same as in Figure 9-28, which was shot from a similar angle at 9:27:35 a.m. This indicates that the portion of the object that is visible has not moved a great deal in the intervening period.

A possible indication of the deteriorating conditions on the east side of the tower around this time is provided by the actions of a person. At 9:30:14 a.m. a person began to climb down the outside of the tower from a window, 79-351, on the 79th floor. This person reached the top of the mechanical floors, i.e., the 76th floor, before falling at 9:31:07 a.m. Recall that another person had fallen from window 79-351 at 9:24:06 a.m.

A view of the south face of WTC 2 at 9:30:19 a.m. is shown in Figure 9-41. It was taken just over 2 min later than the image of the face in Figure 9-33. One difference between the images is immediately apparent. Smoke is coming from windows to the west of the aircraft impact cavity on the 79th, 80th, and 82 nd floors that was not present in the earlier photograph. The flames visible on the 81 st floor are also much brighter. This picture was taken during one the smoke releases identified on the east face, and the presence of smoke and increased flame is likely associated with the same event. 


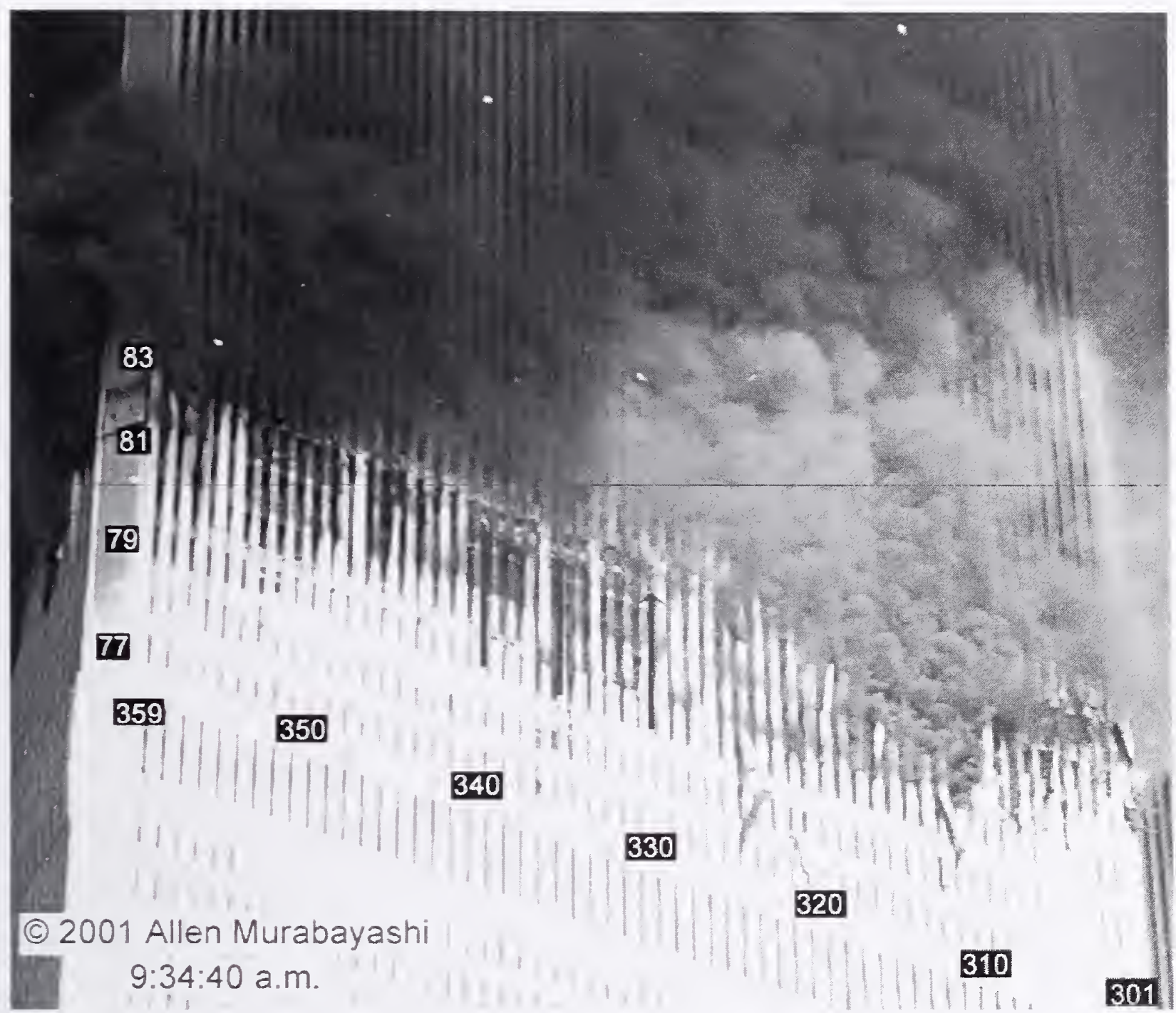

Figure 9-40. This cropped photograph, shot from the southeast at 9:34:40 a.m., shows the east face of WTC 2 . The intensity levels have been adjusted, and column and floor numbers have been added. The arrows highlight a hanging object observed through open windows on the 82 nd floor.

In Figure 9-41, flames are visible on the 83rd floor in window 83-424. This is the first visual evidence of a fire burning on the 83 rd floor on the south face. The fire on the 82 nd floor between the aircraft impact cavity and the east edge has decreased in intensity, and the smoke has cleared up since Figure 9-33 was taken. Part of the 83 rd floor is now visible. It appears as if the spandrel covers at the base of this floor are missing below windows 83-401 to 83-407. There does not appear to be an active fire on this part of the 83 rd floor. The small fires burning elsewhere around the aircraft impact cavity have similar appearances to those observed at the earlier time.

An oblique view of the west face of WTC 2 at 9:34:19 a.m. is shown in Figure 9-42. Smoke is visible at several locations on the west face. The densest smoke is flowing from windows $86-117$ to $86-122$ on the 86th floor. Lighter smoke is flowing from near window 88-142 and window 84-149 on the 88th and 84th floors, respectively. Smoke is also visible at two locations near the top of the tower; on the 107th floor, 
where it was observed earlier, and at the top next to the southwest corner. The latter smoke flow is much lighter and is barely discernable in the photograph. In other images (not shown) it is more distinct. This smoke appears to be flowing from windows 108-101 and 108-102, which were located on the lower level of the mechanical equipment room that spanned the 108th and 109th floors. The nearest louvers to these windows are for a fresh air intake for the 107th floor observation area located opposite windows 108-101 to 108-109 (see Figure A-1 in Appendix A). This suggests that the smoke coming from the upper mechanical equipment room is back flowing through the fresh air intake for the 107th floor. Each of the locations with smoke flow on the west face is above the floors where fires have been observed, which is consistent with the possibility that people broke the glass out and released local build-ups of smoke.

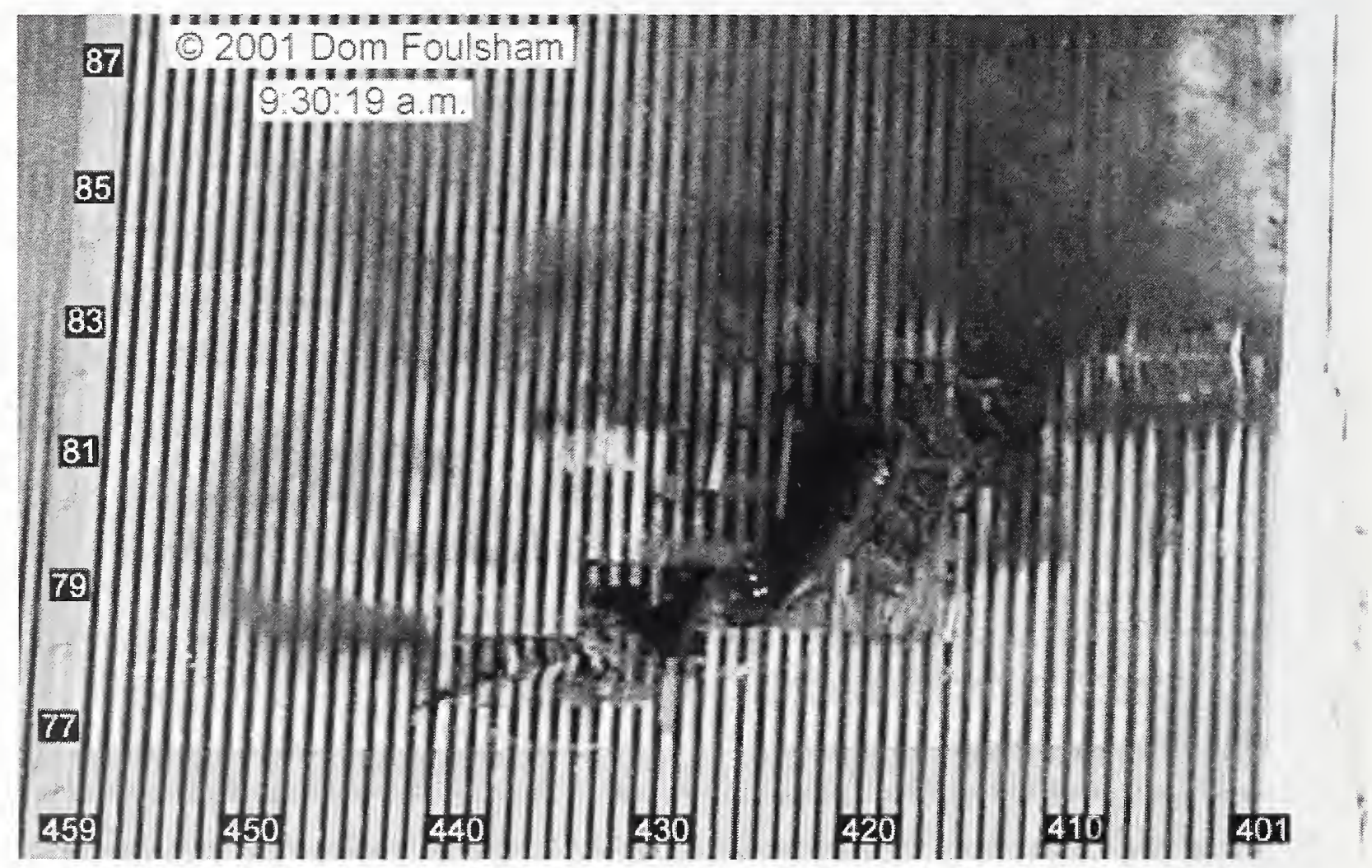

Figure 9-41. This cropped photograph shows the south face of WTC 2 at 9:30:19 a.m. The image has been rotated, and column and floor numbers have been added.

Videos shot from the northeast and southeast show a sudden increase in the amount of smoke flowing from the east face around 9:35:45 a.m. This turned out to be the most intense release of smoke from the lower floors observed up to this time. Unlike earlier releases, intense bursts of flame were also visible, even in the long-distance videos. Two particularly intense flame bursts were observed on the 80th floor near window 80-339 at 8:36:09 a.m. and 9:36:38 a.m. The heavy smoke and flames were present for just over a minute. At around 9:36:50 a.m. the smoke flow abruptly decreased, and the fires abated.

Figure 9-43 shows a photograph that was shot during this period of heavy smoke flow at 9:36:14 a.m. The northern and southern sides of the floors with smoke and fire are shown as blow-ups in Figure 9-44. The enormous increase in smoke is evident by comparing Figure 9-43 with Figure 9-40, which was shot only $1 \mathrm{~min} 34 \mathrm{~s}$ earlier. In the earlier photograph relatively light smoke was coming from open windows on the 79th floor roughly located between windows 79-311 and 79-317. In the later photograph smoke 
flow from these windows has apparently completely halted, but now heavy smoke and fire are visible over a range of windows from window 79-318 to window 79-336. In Figure 9-44 it is apparent that smoke and, in many cases. fire are coming from every window that is open over this range. Windows $79-326,79-329,79-330$, and 79-332 still have glass in place.

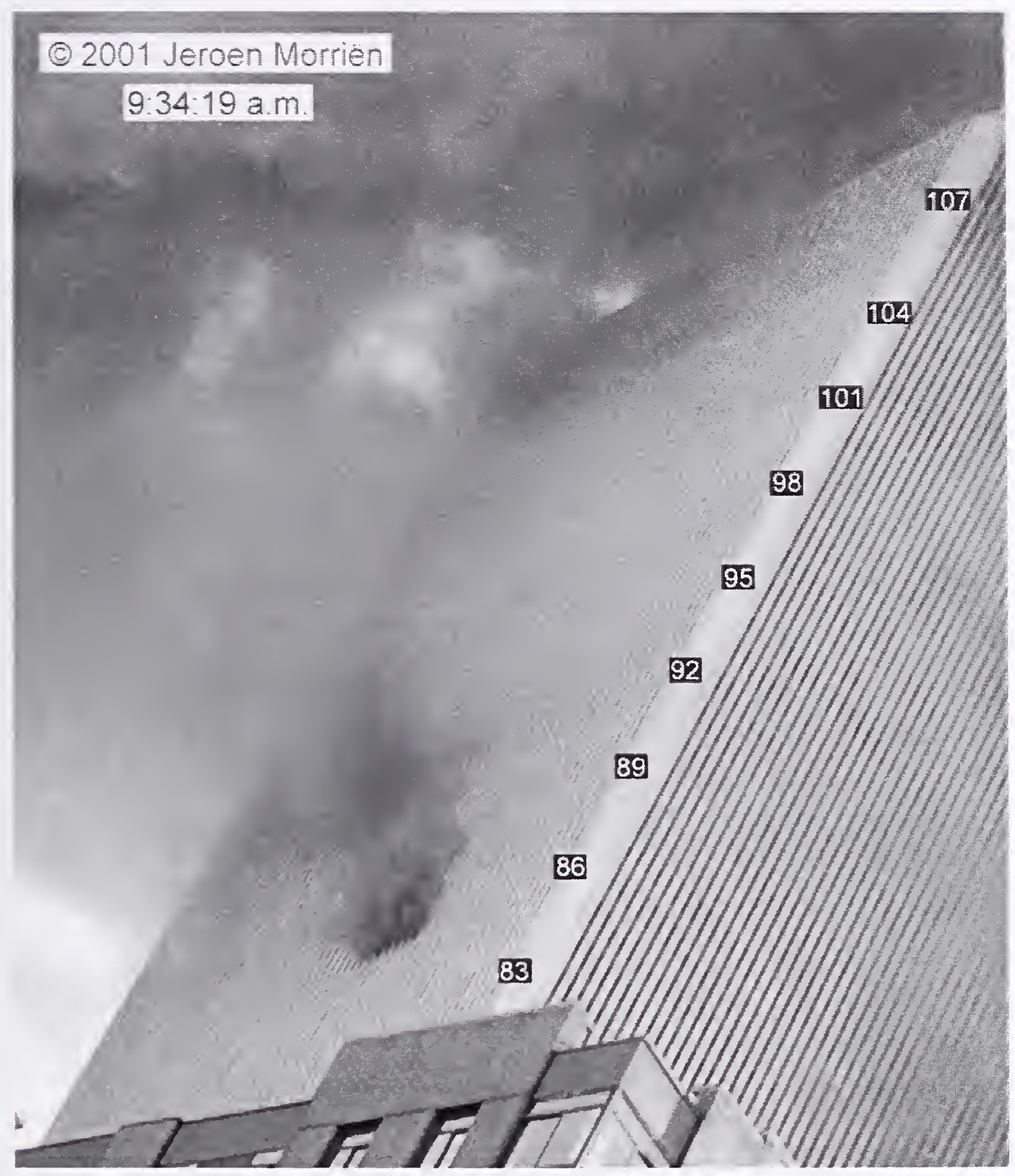

Figure 9-42. This cropped photograph shot from the southwest shows an oblique view of the south and west faces of WTC 2 at 9:34:19 a.m. The intensity levels have been adjusted, and floor numbers have been added. 


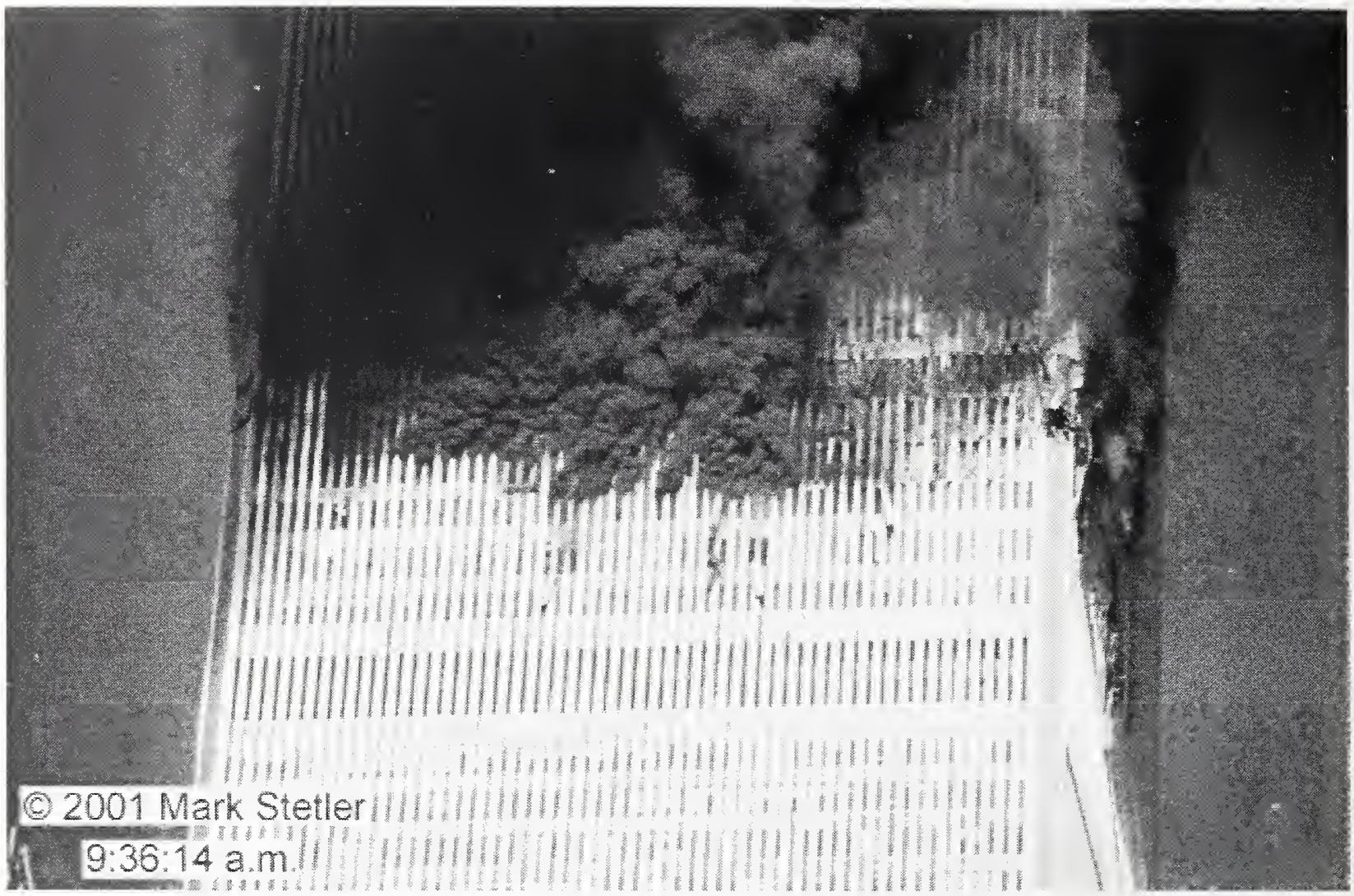

Figure 9-43. This photograph showing the east face of WTC 2 was taken at 9:36:14 a.m.

Heavy smoke and fire are also visible on the 80th floor from window 80-229 to window 80-249. Flames are particularly prominent in windows $80-336$ to $80-343$. These fires might extend over an even wider range, but many windows are hidden by smoke rising from the 79th floor. Flames are barely visible higher up on the face, which suggests that the fire on the 82nd floor continues to burn near column 330 .

The intense fire in the northeast comer opening of the 81 st floor is still present. An unusual flame is visible within this fire. In the upper photograph in Figure 9-44 a very bright white flame, as opposed to the typical yellow or orange surrounding flames, which is generating a plume of white smoke, stands out. The intensity of this flame is considerably brighter than normal flames. It was easily identified in numerous photographs and videos shot from long distances at which the surrounding "normal" flames were not visible. The brightness of the flame, along with the white smoke, suggests that some type of metal is burning. Metal combustion is known to generate much higher flame temperatures than hydrocarbon combustion, and, as a result, to burn much brighter. It is difficult to identify what type of metal is burning. Aluminum will burn, but in normal fires it usually melts instead because the metal surface is protected by an oxide layer that must be breeched before ignition can take place. Aluminum oxide melts at high temperatures that are not typically reached in normal fires. There were limited quantities of other metals on the aircraft that might also burn. Whatever the metal, the ignition of a metal fire is an indication of the significant heating of the debris that took place in the northeast corner of the 81 st floor due to the prolonged intense burning in this area following the aircraft impact. 

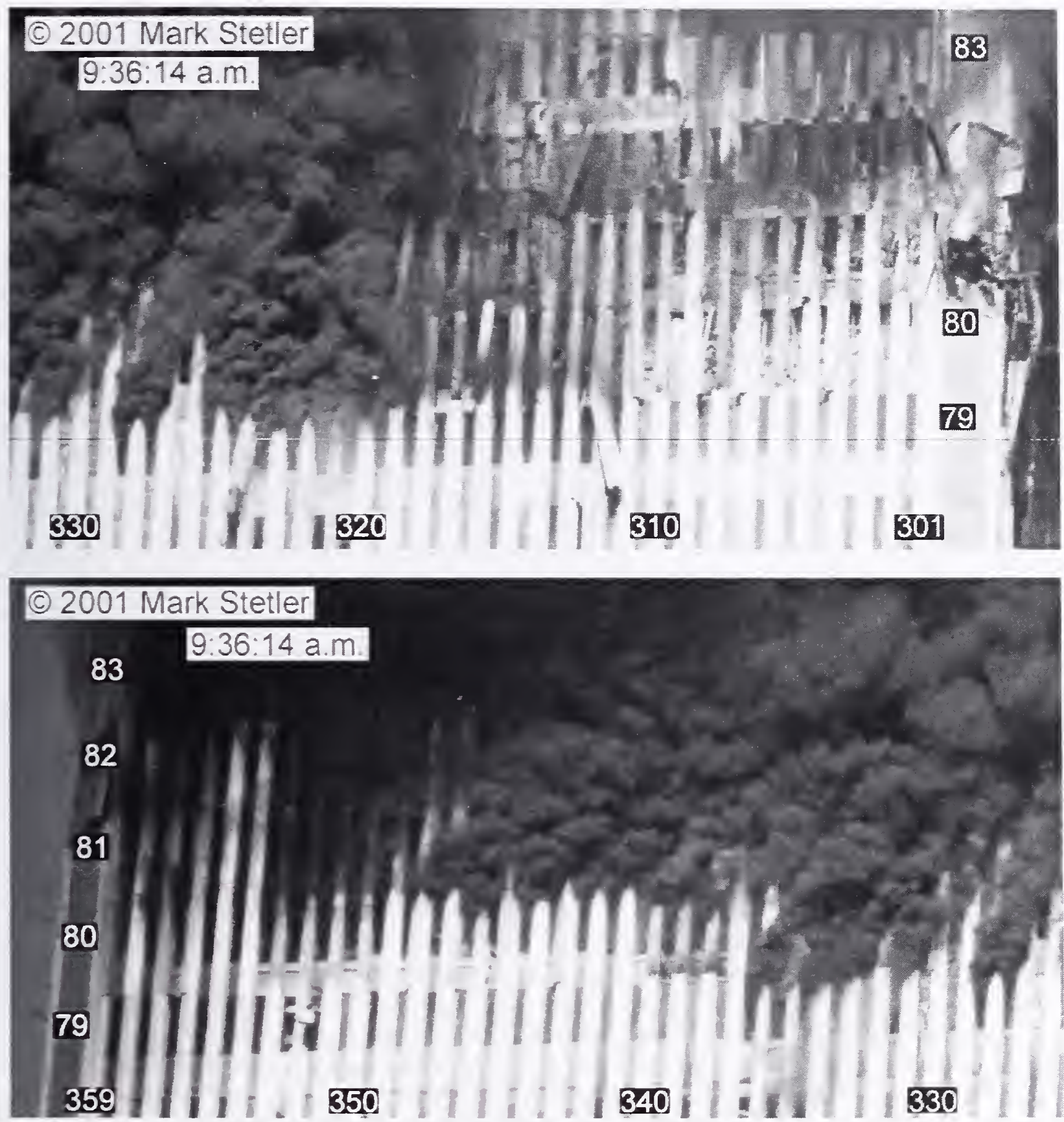

Figure 9-44. These two images show blow-ups of the north and south sides of the east face of WTC 2 taken from the photograph included in Figure 9-43, which was shot at 9:36:14 a.m. Column and floor numbers have been added

Given the large amounts of smoke coming from the central regions of the 81 st and 82 nd floors on the east face, there is surprisingly little smoke at the north and south ends of these floors. With the exception of a light smoke flow from windows 78-334 and 78-335 on the 78th floor, no smoke is observed coming from below the 79th floor. Videos show that short bursts of smoke did come from other open windows on the 77 th and 78 th floors during the heavy smoke release. 
A video provides additional insights into this short period of heavy fire and smoke. A news camera crew was on the plaza immediately below WTC 2 and filmed a close-up view of the south side of the east face, between columns 345 and 358, from 9:36:07 a.m. to 9:37:28 a.m. This video shows heavy smoke

flowing from the windows on the 80th floor and occasional flames extending out of window 80-350. It is remarkable that in the long-distance videos these smoke flows look relatively light compared to the heavier flows towards the center of the face. This is an additional indication of how intense the primary smoke flows actually were. The video also shows a lighter smoke flow coming from window 79-351 on the 79th floor. The nearby fire on the 80th floor and the smoke coming from window 79-351 forced a third person at this location to climb outside onto the face of the tower. This person tried to climb down the outside of the tower and ultimately fell at 9:38:18 a.m.

The video shot from the WTC plaza captured an intriguing event at 9:37:04 a.m. A jet of air, dust, and a large piece of debris was ejected from a window, 77-355, on the 77th floor at an extremely high velocity. Longer distance videos show that puffs of smoke and or dust appeared simultaneously on the east face from several open windows near the center of the 78th floor and from open windows on the north side of the 79th floor. Interestingly, the smoke flow from windows on the west sides of the 79th and 80th floors, which had decreased markedly by this time, did not increase. Within $14 \mathrm{~s}$ of this release, a large fire either grew or became visible near the center of the east face on the 82 nd floor.

A long-distance video shot from the south showed that fire and smoke were pushed from multiple locations on the south face at the same time the strong jet occurred on the 77 th floor of the east face. A bright flash of fire occurred from the fire burning to the east of the aircraft impact cavity on the 79th floor, and smoke poured from this area as well as from window 79-406. Smoke also came from lines of windows on the 81 st and 82 nd floor between the cavity and east edge. Puffs of smoke were pushed out of open windows to the west of the aircraft impact cavity on the 78th, 79th, 80th, and 81 st floors. This video was shot from an angle that provided an oblique view of the west face, and it showed that the smoke flow from open windows on the 86th floor increased at the same time. A video shot from the upper floors of a building to the southeast of the tower provided similar observations. Videos shot from the north around this time showed that puffs of smoke appeared on the north face from the open windows on the 79th floor near the east edge of the face and from the open windows on the 78th floor at windows 78-224 and 78-226, but otherwise the event was not as prominent from this direction.

A sequence of three images shot over $14 \mathrm{~s}$ showing sections of the east face of WTC 2 starting at 9:37:24 a.m., i.e., shortly after the end of the large smoke release and the pressure pulse, is shown in Figure 9-45 to Figure 9-47. Comparison of these three photographs with the photograph reproduced in Figure 9-43 and Figure 9-44 reveals how much the smoke and fire intensity on the east face decreased around 9:36:50 a.m., which was less than a minute earlier.

Figure 9-45 shows the northeast corner of the tower. The bright flame in the 81st floor corner opening, which has been attributed to metal combustion, stands out. A check of the original image shows that the pixels at this location are essentially saturated, providing an indication of how much brighter this flame was than the surrounding flames, which had intensities that were far from saturating the pixels. From this angle, flames are visible extending from the 82nd floor at windows 82-204 to 82-207. These windows appeared to be flame-free at 9:36:14 a.m. 


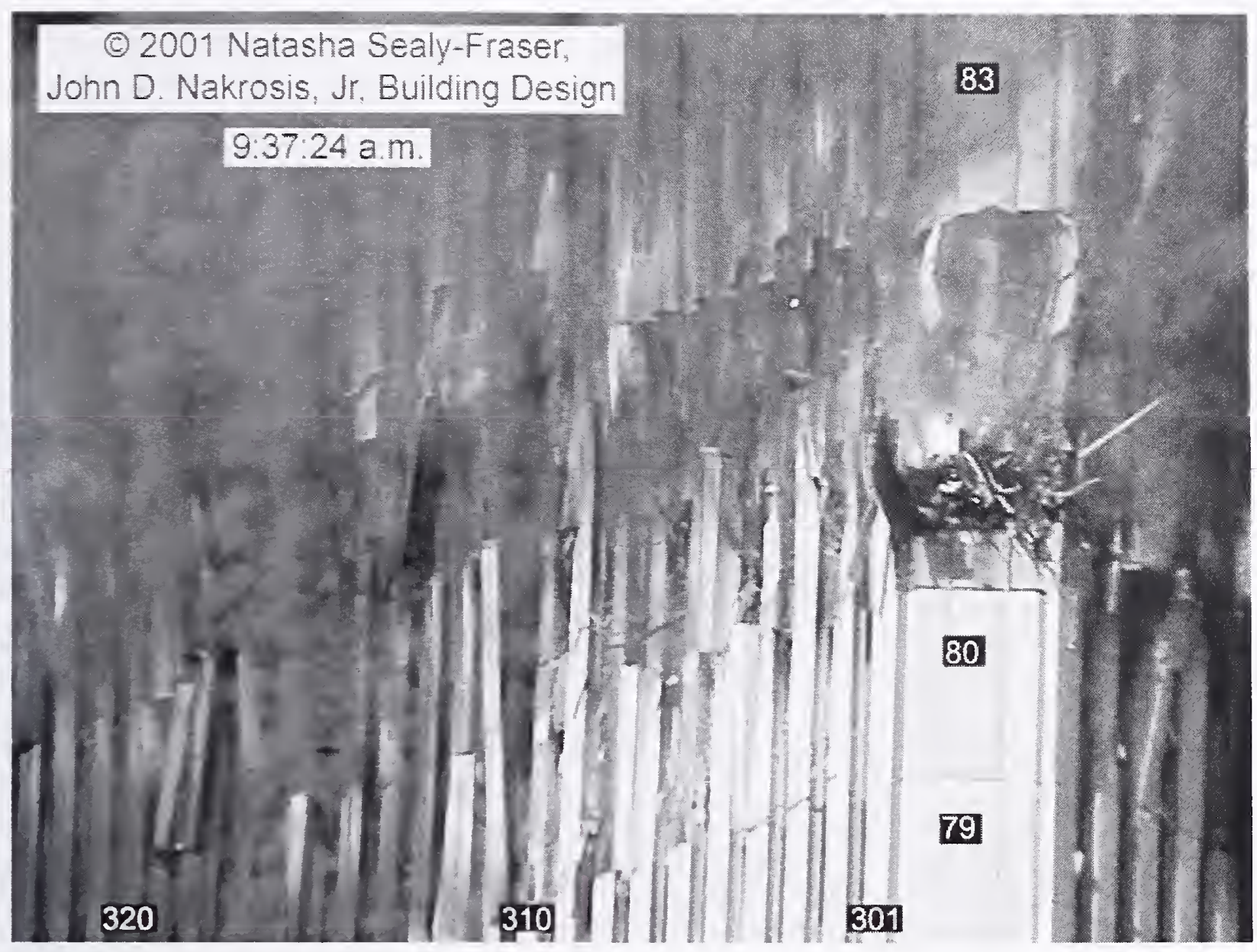

Figure 9-45. This photograph was taken from the northeast at 9:37:24 a.m. and shows portions of the north and east faces of WTC 2. The intensity levels have been adjusted, and column and floor numbers have been added.

The largest changes in appearance occurred near the center of the east face. In Figure 9-46 the only heavy smoke and flames visible on lower floors is coming from windows 79-310 to 79-317 on the 79th floor. At 9:36:14 a.m. these windows appeared to be free of smoke and fire. On the other hand, the only evidence of the intense fires raging on the 79th floor seconds earlier in windows 79-318 to 79-325 are the carets visible at the tops of the adjacent column covers. Heavy smoke hid much of the upper floor façade in Figure 9-44. The only flames visible on the 82nd floor appeared to be just to the south of column 330 . At the later time, large flames are coming from windows 82-320 to 82-333. Recall that intense flames became visible in this region around 9:37:18 a.m.

Closer inspection of Figure 9-46 reveals that the columns on the 80th and 81 st floors on either side of column 330 appear to be bowed inward. This bowing could be identified in images taken as early as 9:21:29 a.m. Several additional photographs to be described below show a similar bowing. This indicates that columns on these floors were distorted at least 37 minutcs prior to the collapse of the tower. 


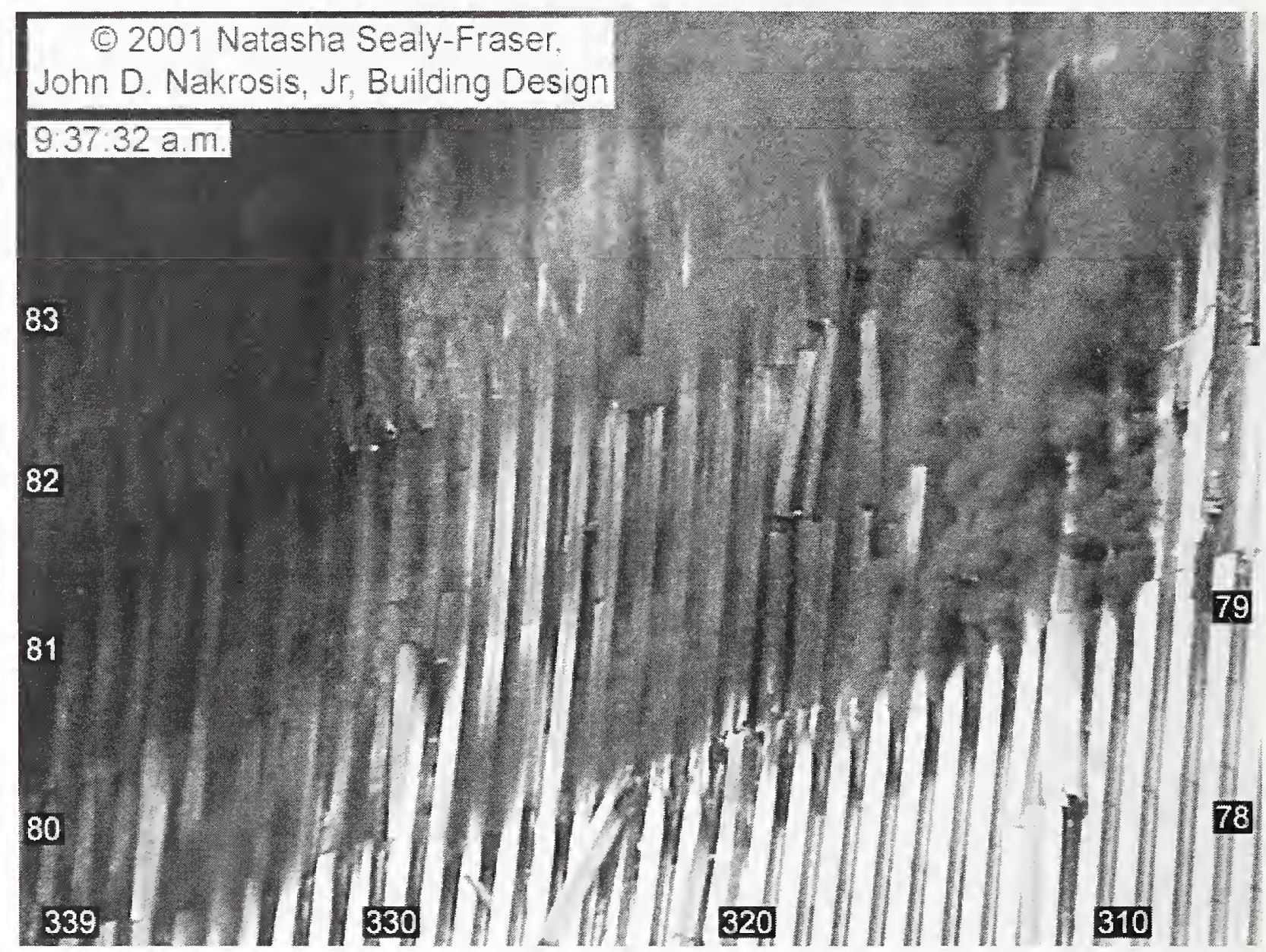

Figure 9-46. This photograph was taken from the northeast at 9:37:32 a.m. and shows the center portion of the east face of WTC 2. The intensity levels have been adjusted, and column and floor numbers have been added.

Figure 9-47 show the southern portion of the east face. There is very little smoke and no fire coming from windows in this area. This contrasts sharply with Figure 9-44, in which intense flames and large amounts of smoke were visible coming from numerous windows on the 79th and 80th floors.

Views of the northern and southern sides of the east face enlarged from the same photograph taken at 9:38:22 a.m. from the east are shown in Figure 9-48. The intensities have been adjusted to overexpose much of the images in order to highlight the object marked with arrows. This is the same hanging object described earlier that has been tentatively identified as the 83rd floor slab. Its appearance has now changed from those seen in Figure 9-1 and Figure 9-2 (9:03:42 a.m.), Figure 9-21 (9:18:38 a.m.), Figure 9-28 (9:27:35 a.m.), Figure 9-31 (9:26:20 a.m.), and Figure 9-40 (9:34:40 a.m.). At these earlier times, the object was visible hanging near the tops of open windows 82-321 to 82-342, even though it did shift downward locally between 9:18:38 a.m. and 9:26:17 a.m. In the bottom image of Figure 9-48, it can still be seen at the top of window $82-342$, but now it drops very rapidly and seems to disappear below the base of window 82-337. The object is no longer visible in windows at least as far to the right as window 82-330. Windows immediately to the north of window 82-330 are filled with fire that would hide the object even if it was above the window base. 


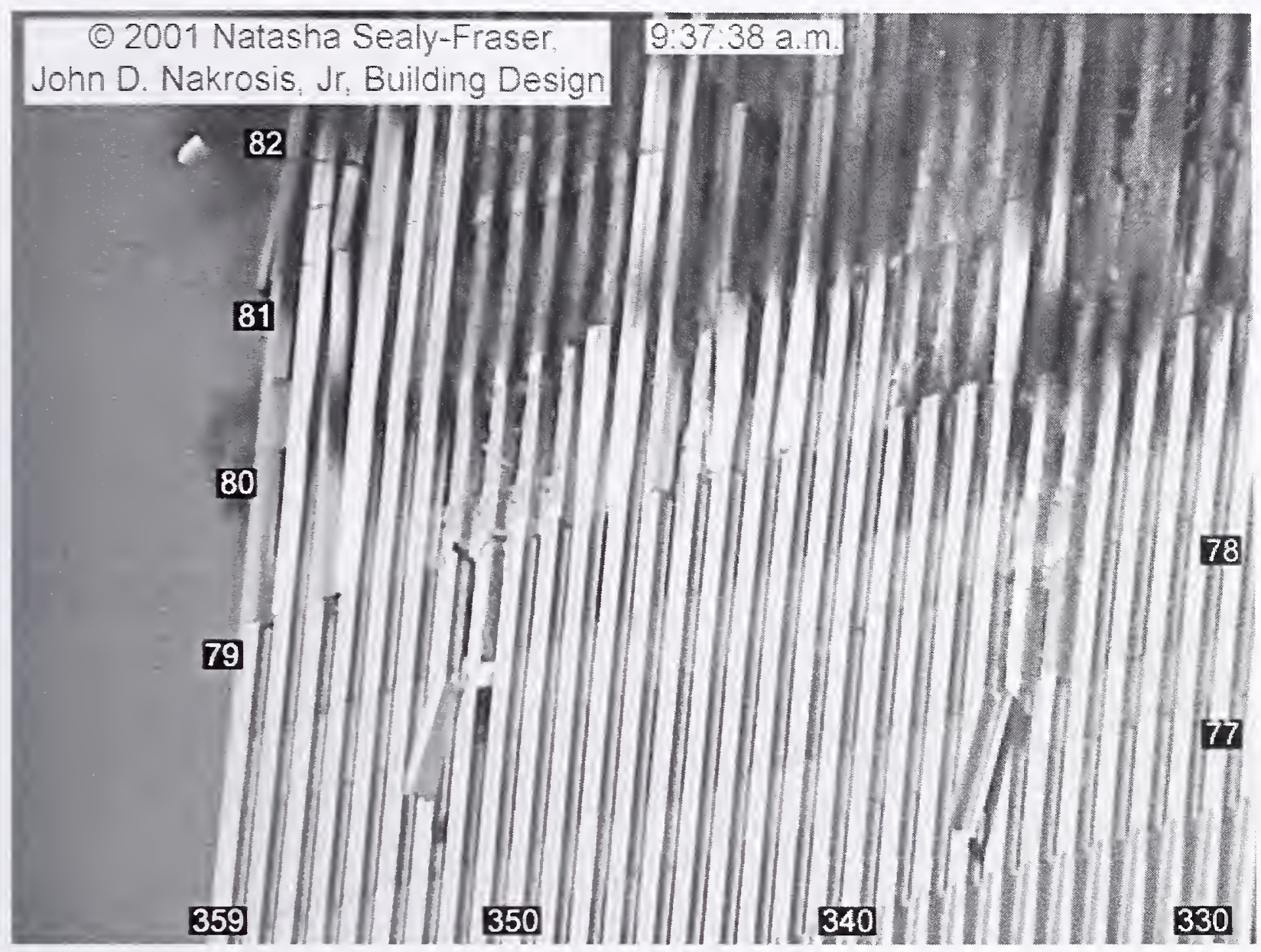

Figure 9-47. This photograph was taken from the northeast at 9:37:32 a.m. and shows the western portion of WTC 2 . The intensity levels have been adjusted, and column and floor numbers have been added.

In the top portion of Figure 9-48, the visible fire area near the center of the 82 nd floor ends at window 82-318. Beyond this point, it is possible to see inside the windows again, and the hanging object (marked with arrows) is visible starting near the base of window 82-317 and rising toward the top of window 82-311. This location is 10 windows further north than where the object was seen earlier. These observations suggest that the object, which may have been the 83 rd floor slab, that had been hanging near the tops of windows on the 82 nd floor since shortly after the aircraft impact sank much lower sometime between 9:34:40 a.m. and 9:38:22 a.m. At some unknown time an additional length of the object became visible on the north side. The fire that had reappeared at the northern edge of the 82 nd floor in Figure 9-45 continued to burn at 9:38:22 a.m.

In the top part of Figure 9-48, an extensive fire can be seen on the 79th floor between windows 79-310 and 79-320. There had been indications of a fire in this area prior to the sudden release of smoke and fire (see Figure 9-40). Isolated fires are also visible on the 81st floor at window 81-331 and on the 80th floor at windows 80-338 and 80-339, with flames extended from window 80-339. These latter fires are the only visible remnants of the large fires visible on these floors in Figure 9-43 and Figure 9-44. 

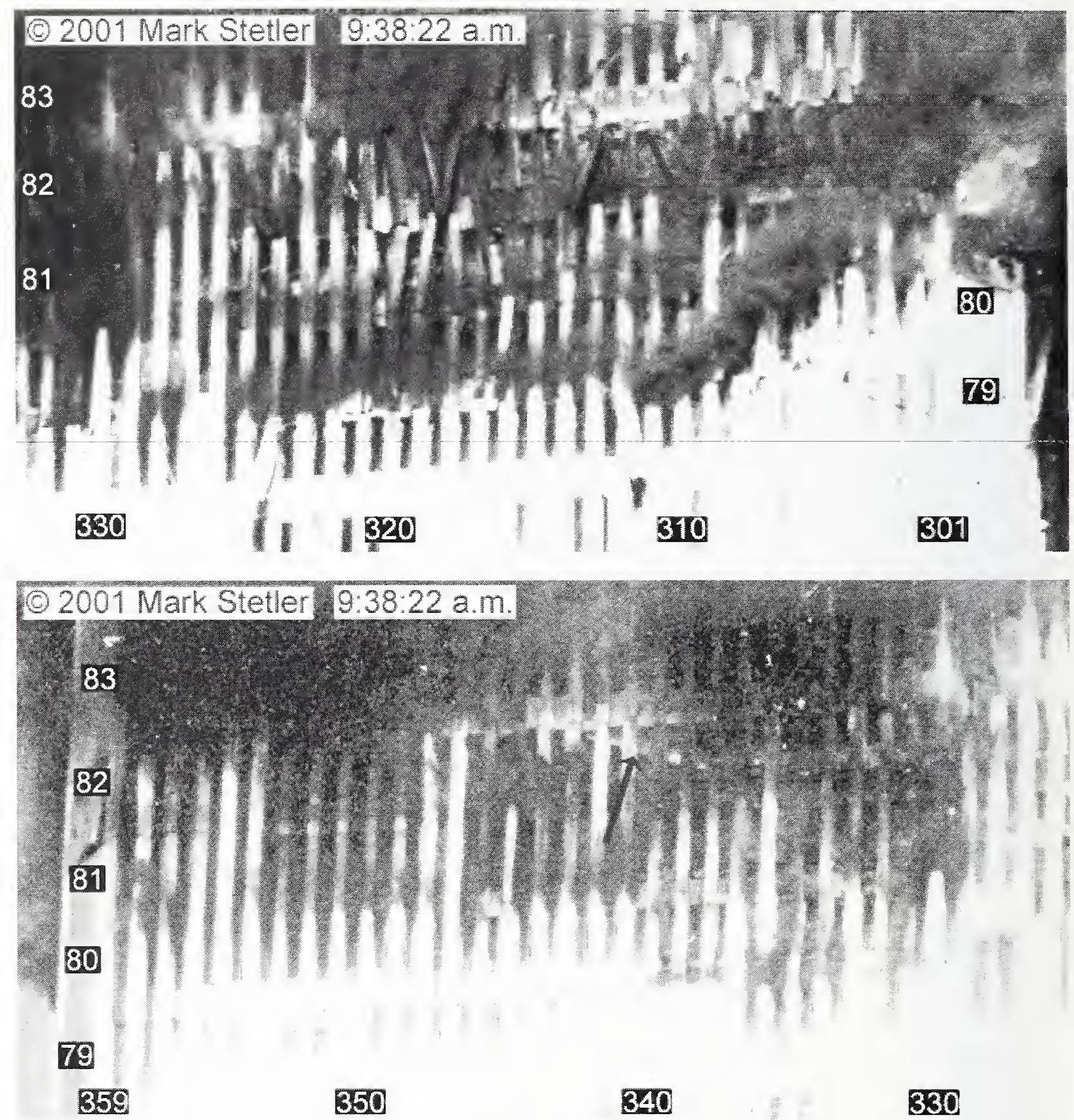

Figure 9-48. These two images show blow-ups of the north and south sides of the east face of WTC 2 taken from a photograph that was shot at 9:38:22 a.m. The intensities have been adjusted to purposefully overexpose parts of the images. Column and floor numbers have been added

Three unusual events were observed on the east face of WTC 2 between 9:34:40 a.m. and 9:38:22 a.m.-the increase in fire and smoke coming from the 79th and 80th floors between 9:35:45 a.m. and 9:36:50 a.m., the short-lived (order of seconds) smoke release at 9:37:04 a.m., and the sinking of the object visible through windows on the 82 nd floor at an unknown time. These observations might be an indication that structural changes were taking place within the tower during this time. Unfortunately, the visual evidence is insufficient to determine exactly what happened and why. 
The sudden increase and decrease in smoke and fire over such a large area is not a typical behavior for building fires. One possible explanation is that a pressure increase (perhaps generated by the falling of the object visible in the $82 \mathrm{nd}$ floor windows or a portion of the core) pushed the fire and smoke out of the windows from interior locations. The length of the event (roughly $1 \mathrm{~min} 5 \mathrm{~s}$ ) and the absence of similar changes on the other faces of the tower argue against this hypothesis.

As described in Chapter 6 and Chapter 7, similar burning behaviors were observed following the aircraft impacts on both towers. Initial periods of intense burning lasting several tens of seconds were followed by abrupt decreases in flame size. These fires were attributed to burning aviation fuel. In Chapter 7 it was noted that very little of the aviation fuel released from the port fuel tank of United Airlines Flight 175 burned during the fireball generated by the aircraft impact. The fuel from this tank should have been dispersed primarily on the 79th floor. The fate of this unburned fuel is unknown, but it is considered likely that portions of it remained somewhere on the floor, collected in one or more liquid pools. It is possible that such a pool of fuel was ignited somewhere on the 79th floor around 9:35:45 a.m. and burned until it was depleted $1 \mathrm{~min} 5 \mathrm{~s}$ later. In this scenario, the flames and dark smoke observed on the east face would be generated following transport of rich combustion gases and unburned soot, created by underventilated burning at the site of the aviation fuel, to the east face where they would then burn further upon coming in contact with the fresh air outside the windows. In the absence of additional evidence. this scenario is considered the likely explanation for the roughly 1 min release of smoke and fire.

It is conceivable that the pressure pulse generated within WTC 2 at 9:37:04 a.m. could have resulted from the sudden collapse of the hanging object. presumed to be a portion of the 83rd floor slab, observed through open 82 nd floor windows. While the rapid collapse of a large section of a floor slab would likely generate a substantial pressure pulse, evidence supporting such a conclusion is circumstantial, at best. The actual collapse of the object was not observed, and there is no evidence that it occurred rapidly or even at this time. The appearance of large fires on the $82 \mathrm{nd}$ floor shortly after the pressure pulse is an indication that something happened on this floor around this time. On the other hand, most of the direct effects of the pressure pulse were observed on lower floors, particularly floors 77 through 79 . The available pathways for transporting the pressure pulse are unknown, but it might have been expected that its largest effects would have been apparent near the floor where it was generated. It must be concluded that, while it is possible that the pressure pulse resulted from a partial collapse of the hanging object seen on the east side of the tower, the evidence is far from conclusive.

Twenty-one seconds after fire erupted from window 83-226 on the 83rd floor of the north face at 9:33:52 a.m. (see Figure 9-37), window 83-227 opened, and smoke and fire were released. By 9:34:14 a.m. windows 83-228 and 83-229 were also open, and flames were coming from all four windows. A photograph of the area taken at 9:35:39 a.m. is shown in Figure 9-49. The flames in windows 83-226 to 83-229 are visible. Flames are not visible in windows 83-230 to 83-232, but a continuous region of fire extends from window 83-233 to window 83-245. Comparison with Figure 9-36 and Figure 9-37, taken at 9:32:24 a.m. and 9:33:53 a.m., respectively, shows that this fire had died down somewhat from the earlier times and had apparently moved one window to the west. The absence of visible fire immediately adjacent to the new fire location, along with the rapid appearance of flames at multiple windows, observations that indicate flashover took place around this time, suggests that the fire seen in windows 83-226 to 83-229 was burning within an enclosed room. It is not possible to check whether a room was present at this location since floor plans are unavailable for this floor. 


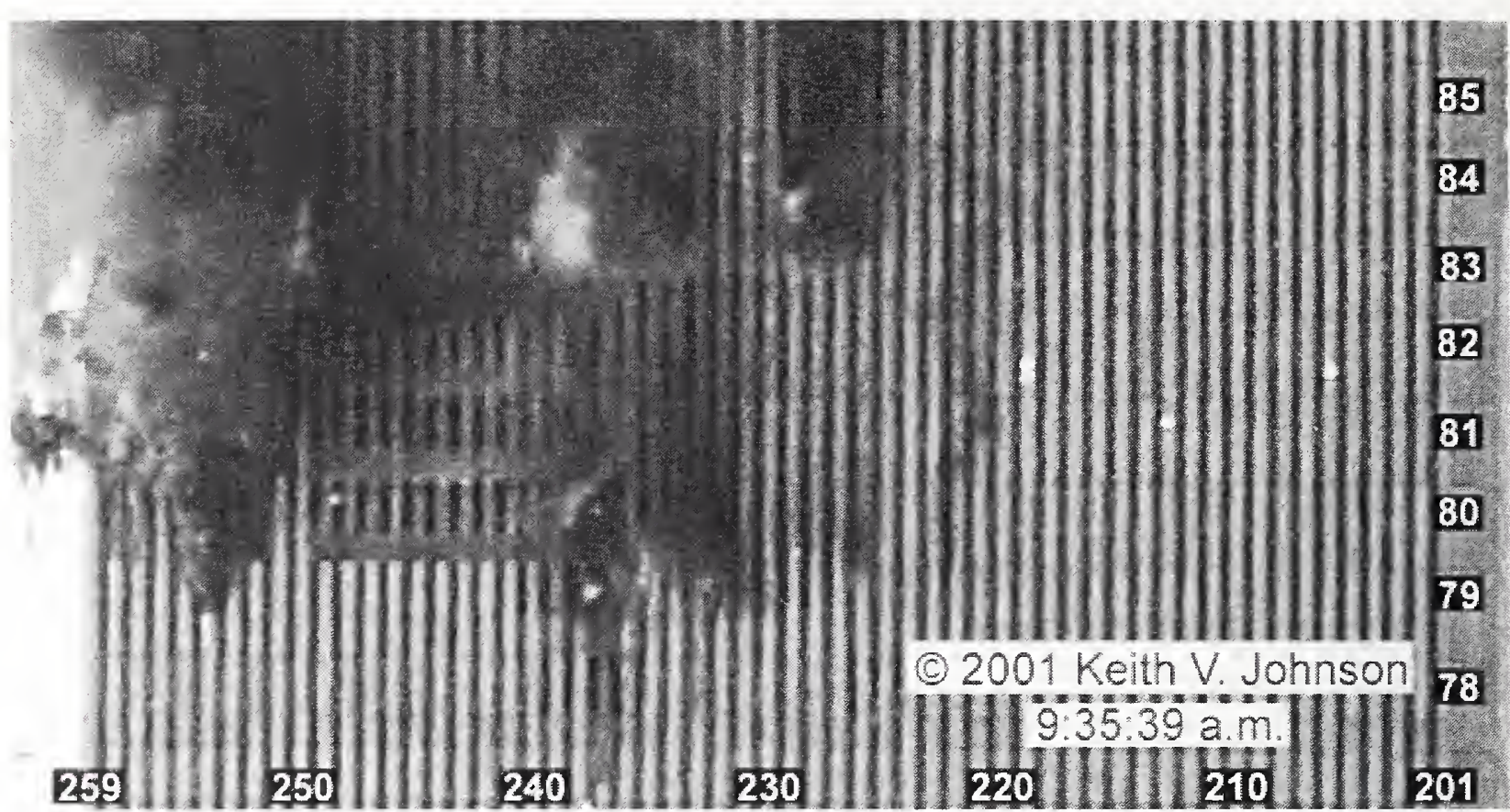

Figure 9-49. This cropped photograph shows the north face of WTC 2 at 9:35:39 a.m. The intensity levels have been adjusted, and column and floor numbers have been added.

The fire distributions elscwhere on the north face in Figure 9-49 are similar to those in Figure 9-36 and Figure 9-37. Fires were still burning at the east edge of the tower on the 81st, 82nd, and 83rd floors. The debris piles on the 78th floor and in the lower right-hand corner of the cold spot continued to burn, and fire was visible as far west as window 79-225 on the 79th floor. A plume of smoke is visible coming from window 79-222. There are no visible indicators of fire to the west of this window on any of the floors.

A somewhat closer view of the north face taken at 9:40:01 a.m. is shown in Figure 9-50. Even though the image contains some digital noise, it is sufficiently resolved that open and closed windows can be identified. By this time, the fire visible in windows 83-226 to 83-229 on the 83rd floor no longer had extended flames. The carets on the surrounding aluminum column covers reflect their earlier presence. The three windows immediately to the east, windows 83-230 to 83-232, appear to still have glass in place. Flames continue to extend from windows on this floor above the right-hand side of the cold spot, but flames in windows 83-234 to 83-236 have died down sufficiently that they are no longer visible. Carets around these windows indicate that extended flames were present earlier. The fire on the eastern edge of the 81 st floor continues to burn vigorously, hiding the locations immediately above on the $82 \mathrm{nd}$ and 83 rd floors. The fires burning on piles of debris on the 79 th and 80 th floors have comparable intensities to those seen in recent images.

A high-resolution photograph of the area around the cold spot on the north face is reproduced in Figure 9-51. It was shot at 9:42:31 a.m. Arrows have been added to the image to indicate hanging objects that are visible through open windows on the 80th and 81 st floors. The area containing the arrows is blown up further in Figure 9-52. It is instructive to compare this image with Figure 9-17, which shows a blowup of roughly the same area at 9:14:03 a.m., and Figure 9-12, which was recorded at 9:10:01 a.m. 


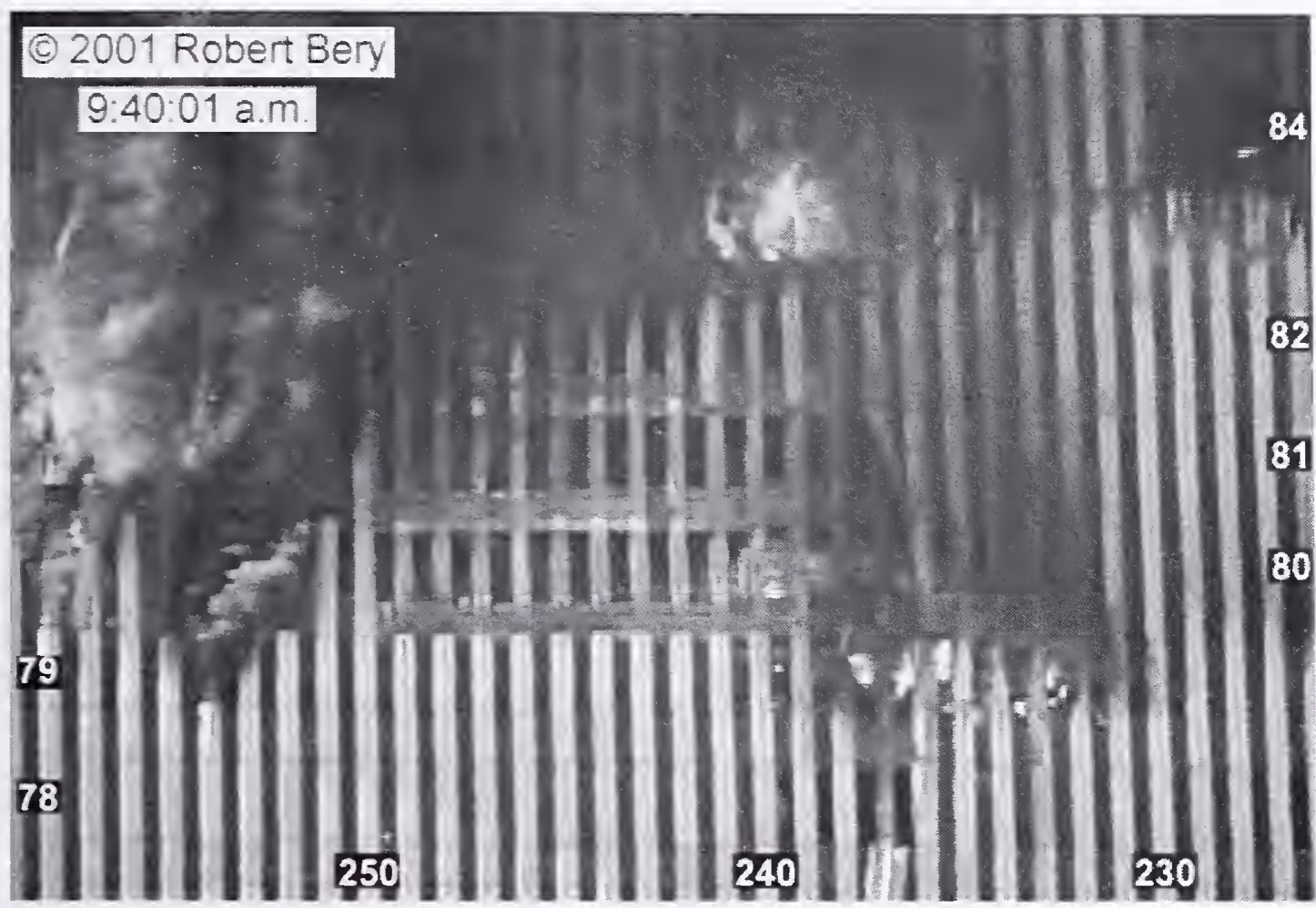

Figure 9-50. This frame captured from a video shows the north face of WTC 2 at 9:40:01 a.m. The intensity levels have been adjusted, and column and floor numbers have been added.

Hanging objects are visible in the two blow-ups shown in Figure 9-17 and Figure 9-52, but it is apparent that their locations and appearances have changed between the times when the two photographs were taken. At the earlier time. two objects were observed through open windows in the cold spot on the 80th floor. The first was located near the center of the windows and extended across the entire area, reaching as far east as window 80-255. The second was visible curving downward in window 80-247 and extending to near the center of window 80-242. In Figure 9-52 the lower object has apparently disappeared completely from view, and the object that was hanging higher up in the windows has dropped down. It now first appears in window 80-248 and curves rapidly downward, seeming to disappear below the spandrel at the base of window 80-243.

In the earlier high-resolution photographs, hanging objects were visible to the east of the cold spot on the 80 th floor to at least window 80-255. Much of this area is now hidden by smoke, but no objects are discernable in windows 80-250 and 80-251.

In the earlier blow-up there appeared to be two objects hanging in the cold spot on the 81 st floor just below the tops of windows 81-239 to 81-242. In Figure 9-52 only a single object is visible through windows in this area of the 81 st floor. It now first appears in window 81-245 and curves downward to the base of window 81-240. This object has also apparently shifted position. 


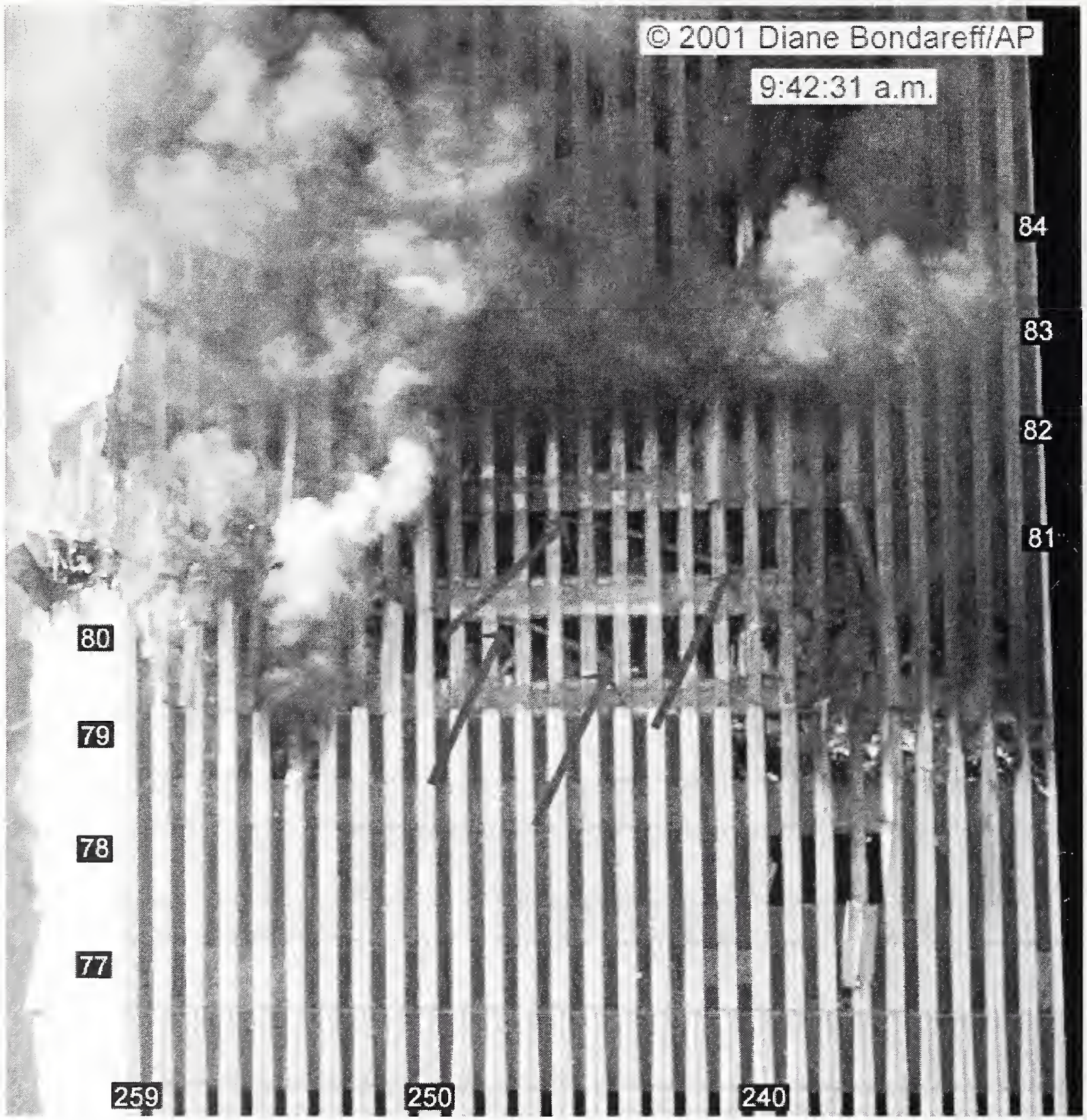

Figure 9-51. This cropped photograph shows a portion of the north face of WTC 2 at 9:42:31 a.m. The image has been rotated and enhanced by adjusting the intensity levels, and column and floor numbers have been added. The arrows highlight hanging objects visible through open windows on the 80 th and 81 st floors.

Recognizing that the appearances of the hanging objects changed to such a large extent between 9:14 a.m. and 9:42 a.m., lower-resolution images, in which the objects were not well resolved, taken between these times were reviewed. In many of these images there are faint indications of the hanging objects, and it proved possible to narrow down the times when the changes occurred. In Figure 9-32 the objects are barely visible on the 80th and 81st floors at 9:27:04 a.m. Their locations are consistent with those seen in Figure $9-17$ on both floors. This is no longer true in Figure 9-35. On the 80th floor the faint hanging object appears to have the same shape as in Figure 9-52, while on the 81st floor the hanging object seems 
to be close to the top of the windows. These observations indicate that changes in the location of the hanging objects took place between 9:27:04 a.m. and 9:29:42 a.m. on the 80th floor and sometime between 9:29:42 a.m. and 9:42:31 a.m. on the 81st floor.

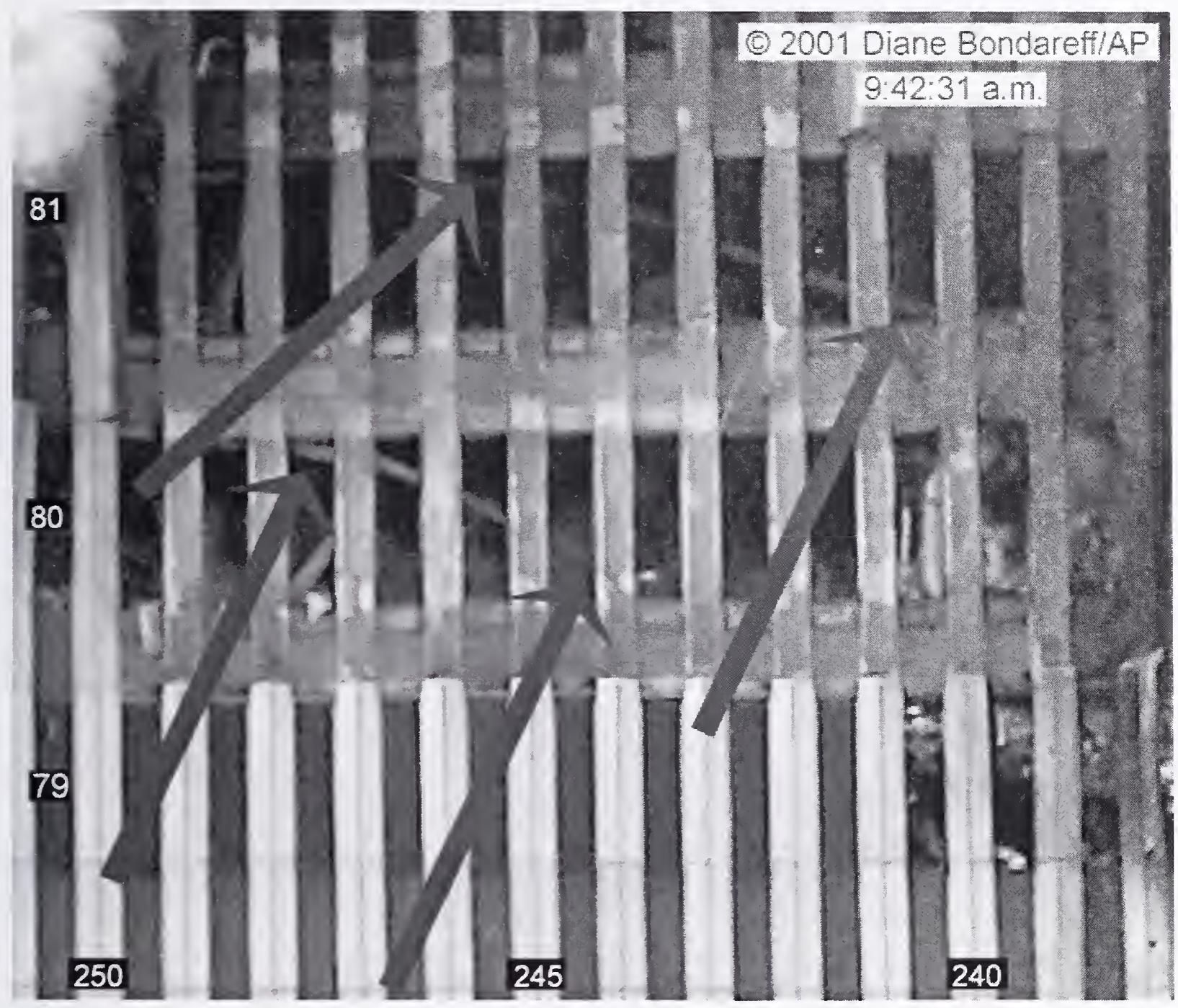

Figure 9-52. A section of the image shown in Figure 9-51 is blown up. The arrows point to the locations of hanging objects that are visible through open windows on the 80th and $81 \mathrm{st}$ floors on the north side of WTC 2. Column and floor numbers have been added.

Figure 9-51 provides good views of the fires that continued to burn on the east edge of the north face on the 81 st and $82 \mathrm{nd}$ floors and on the piles of debris on the right side of the cold spot on the 79th and 80th floors. Flames are also visible on the 83rd floor in windows 83-238 to 83-244. All of these fires have similar appearances to those seen in recent earlier photographs.

A video shows that a stream of smoke appeared from window 83-224 on the 83rd floor of the north face at 9:42:26 a.m., and a small object (window glass?) fell from the location at the same time. Twenty-seven seconds later, the smoke plume burst into flames. At 9:45:02 a.m. there was another burst of smoke and a falling object from window 83-222, followed by flames $5 \mathrm{~s}$ later. This flame spread to window 83-221 at 9:45:25 a.m. A photograph face taken $7 \mathrm{~s}$ later is shown in Figure 9-53. Two long tongues of flames are 
coming from windows 83-221 and 83-224. Flames are no longer visible in windows 83-226 to 83-230, even though carets are visible on adjacent column covers. Windows 83-225, 83-231, and 83-232 appear to have glass in place. The sudden and isolated development of a fire on the 83 rd floor in windows 83-221 to 83-224 suggests again that flashover within a room has occurred. The fire on this floor near the left edge of the cold spot has died down with internal flames only visible in windows 83-238 and 83-239.

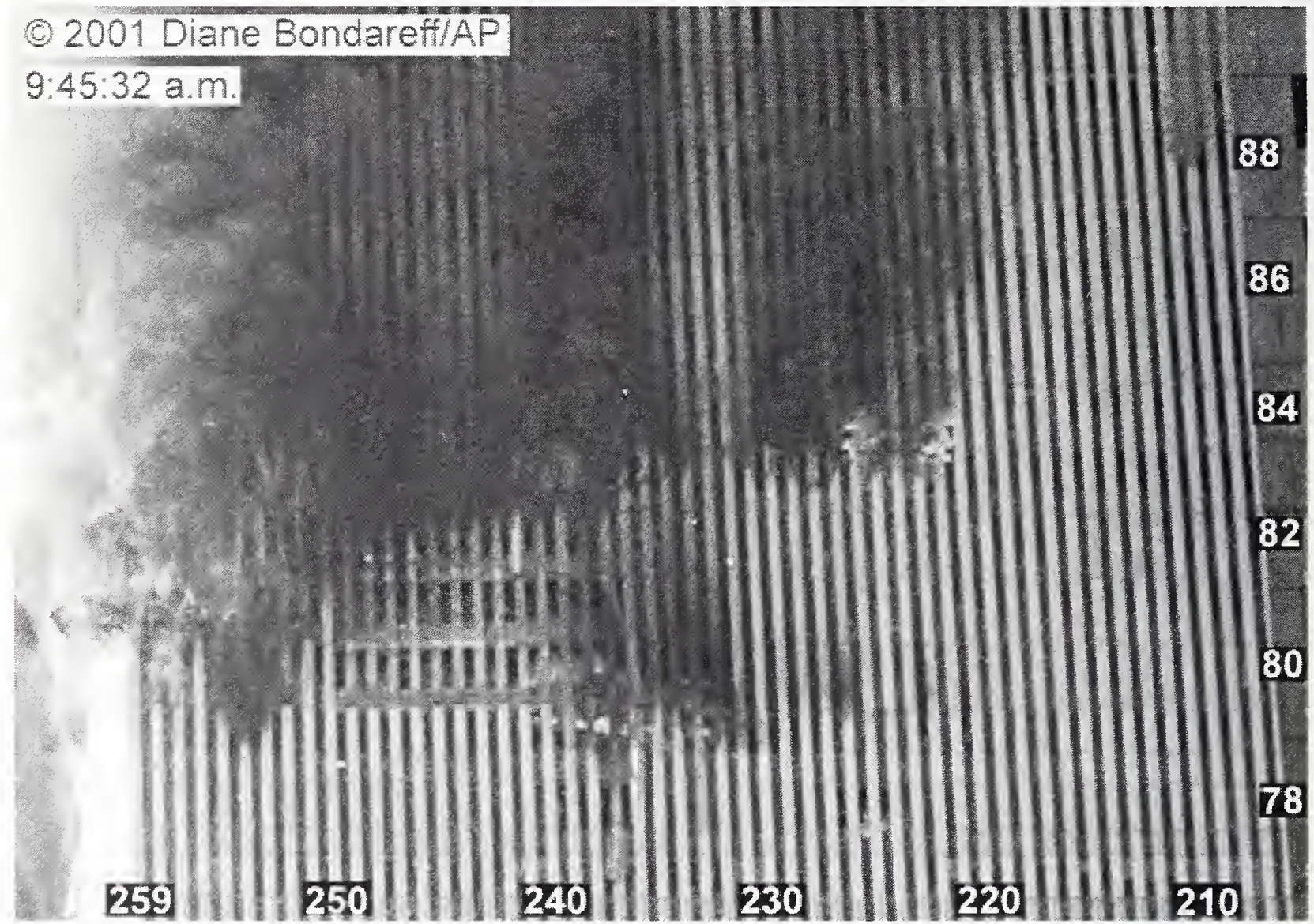

Figure 9-53. This image of the north face of WTC 2 was taken at 9:45:32 a.m. The photograph has been cropped and rotated, and the intensity levels have been adjusted. Column and floor numbers have been added. The brown area to the right is the eastern edge of WTC 7 .

The fire burning on the eastern edge of the north face on the 81 st floor continues to burn vigorously, but flames are not visible in the 82 nd floor windows at this time. A small fire is visible in window 83-259 on the 83 rd floor. The fires on the 79th and 80th floors near the center of the north face continue to burn on the piles of debris. Fires are not evident on the 79th floor to the west of window 79-230, even though smoke marks and open windows at 79-220 and 79-222 are evidence of the earlier burning observed in this area.

In Figure 9-53 an isolated smoke plume is visible on the 88th floor coming from window 88-208. This is another location where it is possible that people have broken out a window. A photograph shot just before 9:45 a.m. showed that windows 88-201 to 88-203 were also open. 
Clear close-up images of the fires on the south face of WTC 2 are limited between 9:30 a.m. and 9:38 a.m. Figure 9-54 shows a frame taken from a video of the upper portion of the south face at 9:36:23 a.m. It has been included because at this time the smoke on the face cleared sufficiently to observe that heavy smoke was coming from multiple windows, extending from roughly window 105-430 to window 105-438, on the 105th floor. This is another location where people may have broken open windows. This was the only location above the fire floors where smoke flow from open windows was evident on this face. In particular, no smoke was observed flowing from the upper mechanical equipment room floors.

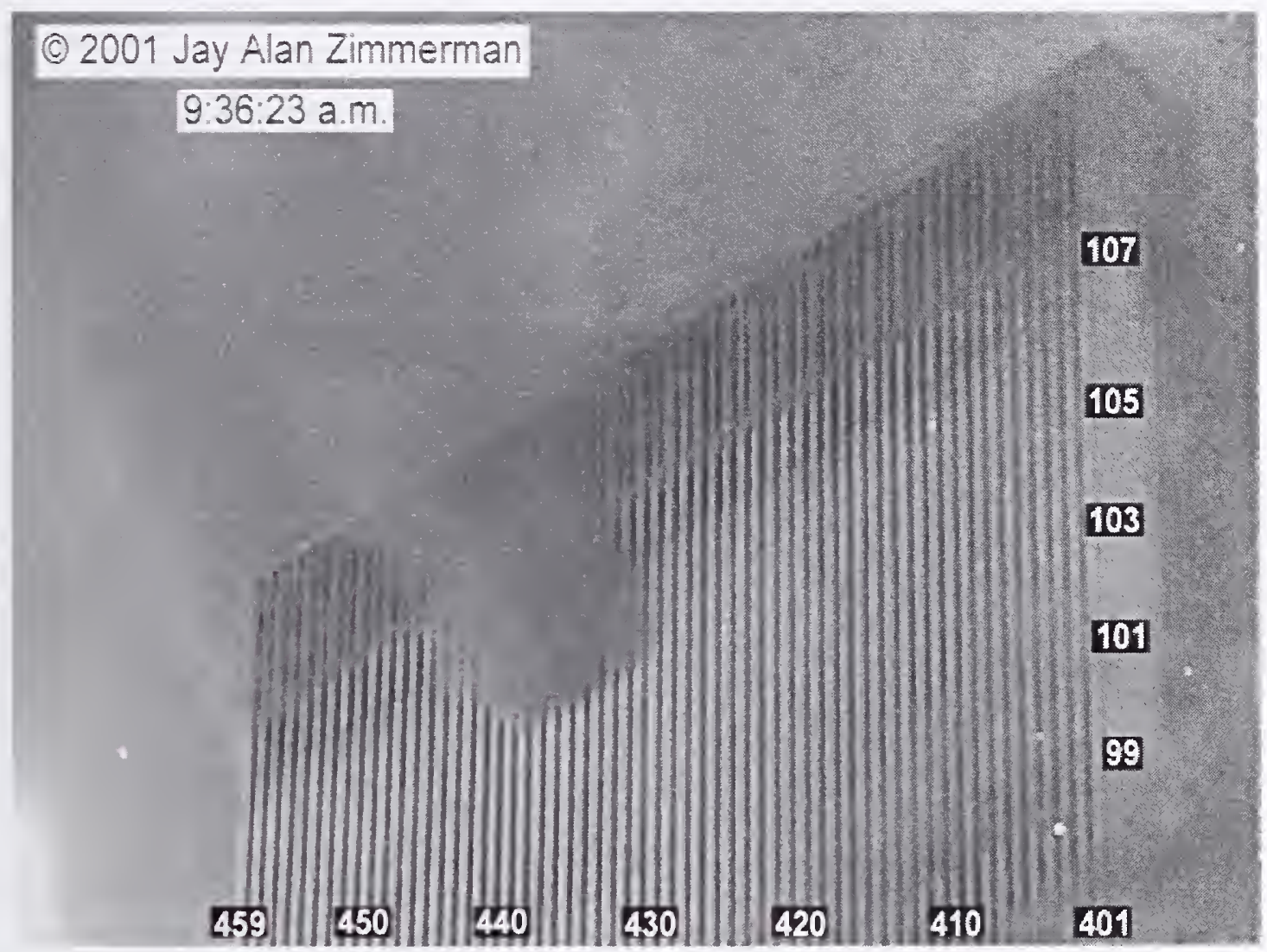

Figure 9-54. This image is taken from a video shot from the southeast and shows the upper portion of the south face of WTC 2 at 9:36:23 a.m. The intensity levels have been adjusted, and column and floor numbers have been added.

A video shows that heavy smoke began flowing from windows at the eastern edge of the south face on the 80 th floor at 9:38:12 a.m. This smoke is visible coming from windows 80-401 to 80-408 in Figure 9-55, which shows a frame captured from a video recorded at 9:38:50 a.m. In the video, fire, which is visible inside windows 80-407 and 80-408, occasionally extended from these windows. The smoke coming from the 80 th floor tended to hide the windows on the floors immediately above, but there were brief glimpses of a fire burning on the 83 rd floor.

Comparison of Figure 9-55 with Figure 9-41, which was taken roughly $81 \frac{1}{2}$ min earlier, shows that changes in fire locations and intensities have taken place on the south face. In comparing the images it should be kept in mind that Figure 9-55 was shot from further east and, as a result, low intensity fires are likely to be hidden by columns. With this caveat in mind, it is clear that the large fire visible in windows 81-431 to 81-435 to the left of the aircraft impact cavity in Figure 9-41 has died down. In Figure 9-55 
only a small spot fire is visible near the bottom of window 81-435. On the 79th floor, flames are no longer evident near window 79-426, but the fire has migrated to the east from this area, and burning is now visible in windows $79-408$ to $79-410$.

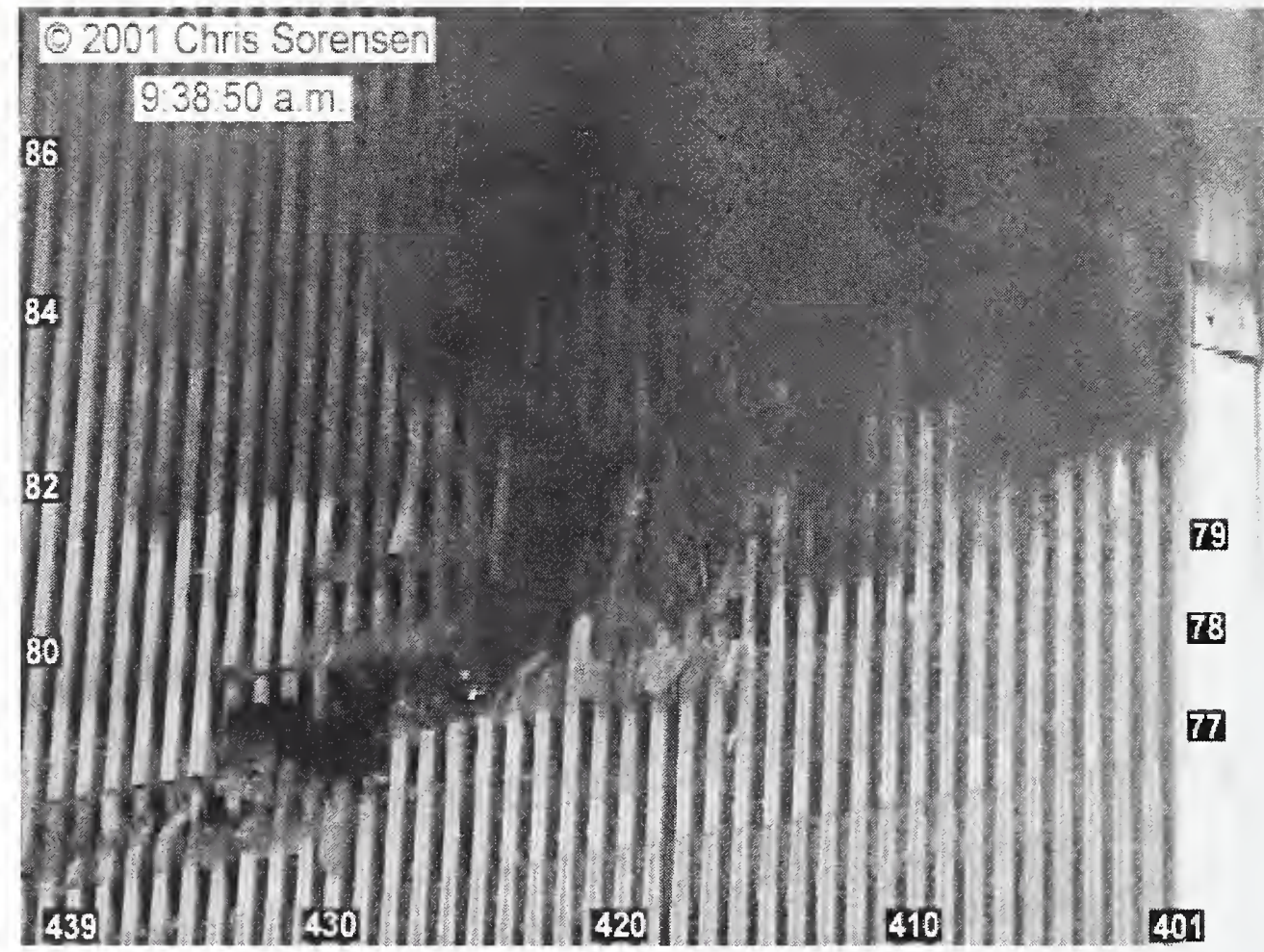

Figure 9-55. This image of the south face of WTC 2 was captured from a video recorded at 9:38:50 a.m. The intensity levels have been adjusted, and column and floor numbers have been added.

Another view of the south face taken at 9:43:34 a.m. is shown in Figure 9-56. More widespread flames are visible than in Figure 9-55 because it was shot from a location more directly to the south and further away. Low intensity fires are visible on the 80th and 81 st floors to the west of the aircraft impact cavity. The fire on the 81 st floor is a remnant of the intense fire seen in this area at 9:30:19 a.m. (see

Figure 9-41), while the fire on the 80th floor has been burning at a low level since at least 9:23 a.m. (see Figure 9-26). A small fire, which has been burning on the 78th floor just to the east of the aircraft impact cavity since shortly after the impact, is also evident.

The fire on the east side of the 79th floor in windows 79-408 to 79-410 in Figure 9-55 was also present at 9:43:34 a.m., and there is now an indication of flame at window 79-406. The heavy smoke and fire present on the 80th floor at 9:38:50 a.m. seem to have dissipated, and it is possible to see the base of the 83rd floor on the eastern edge. Videos suggest that this change occurred around 9:40 a.m. There are now no flames visible east of the aircraft impact cavity on the 80th through 82 nd floors as far east as column 404 , which is the last column visible in the image. 


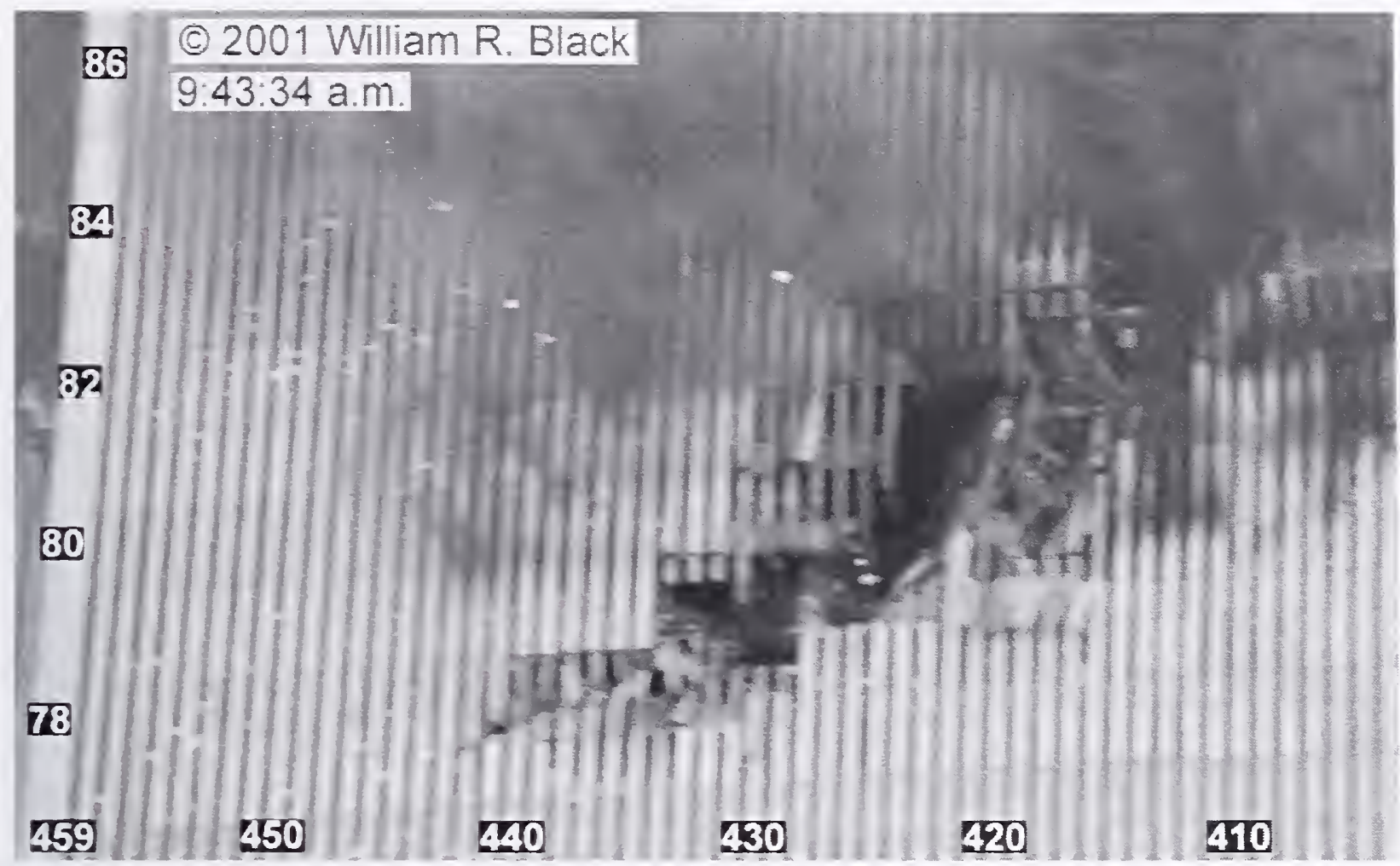

Figure 9-56. This cropped, slightly out of focus photograph of the south face of WTC 2 was shot at 9:43:34 a.m. The intensity levels have been adjusted, and column and floor numbers have been added. The flames visible to the left of the western edge of WTC 2 are on the south side of WTC 1.

A clear view of the west face of WTC 2 at 9:42:33 a.m. is shown in Figure 9-57. Smoke is visible coming from several windows on the 86 th, 88 th, $91 \mathrm{st}, 105$ th, and 107 th floors, as well as from the mechanical equipment room on the 108 th and 109 th floors. These locations include windows $86-117$ to 86-122, 88-142. 91-125 and 91-126, 105-148, 107-123 to 107-129, and the two right-most windows on the 108 th floor. Smoke was observed coming from these same locations at 9:34:19 a.m. (see Figure 9-42), with the exception of the windows on the 91 st and 105 th floors. These two locations had light smoke flows, and it is difficult to determine when the windows were opened.

In Figure 9-42 smoke was also coming from a window, 84-149, on the 84 th floor. Smoke is not visible at this location in Figure 9-57, but the image has sufficient resolution to confirm that this window was open. Windows 84-141 and 84-127 also appear to be open on this floor. It is interesting that no smoke seems to be flowing from the open windows that are located closest to the fire floors. No other open windows are apparent on lower floors (note that the image from which Figure 9-57 was cropped extended as far down as the mechanical equipment room on the 76 th floor).

On several floors electric lights are visible through windows on the west face in Figure 9-57. These include the 104th floor. The fact that lights remained on for floors well above the aircraft impact floors means that the aircraft did not cut all of the power lines running to the top of the tower as it passed through the core. 


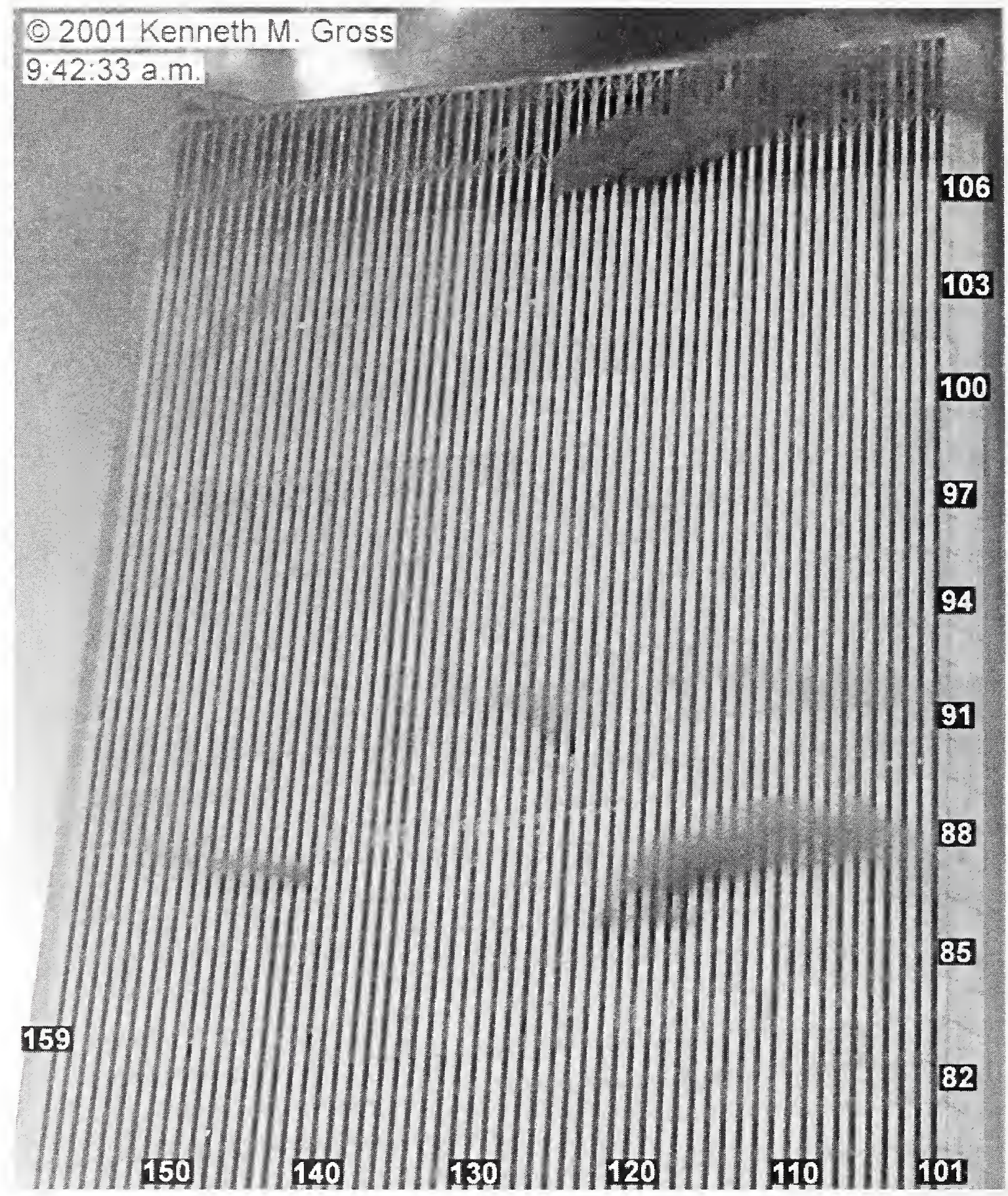

Figure 9-57. This cropped photograph shows the west face of WTC 2 at 9:42:33 a.m. It has been rotated and enhanced by adjusting the intensity levels. Column and floor numbers have been added.

The relatively quiescent appearance of the east face at 9:38:22 a.m. is shown in Figure 9-48. Videos reveal that between 9:38 a.m. and 9:45 a.m. the fires on the east face of WTC 2 went through two additional periods, similar to that described above, in which heavy smoke and intense fires occurred for relatively short periods on the 79th and 80th floors. The first took place from roughly 9:39:53 a.m. to 9:40:41 a.m. Figure 9-58 shows a photograph of the east face taken at 9:40:29 a.m. during this time. 
Fires are visible in Figure 9-58 on the 79th and 80th floors at roughly the same locations as in the photograph shown in Figure 9-43 and Figure 9-44, which was taken at 9:36:14 a.m. The fires are particularly heary on the 80 th floor near windows $80-331$ to $80-339$. The duration (roughly $50 \mathrm{~s}$ ) of the heary smoke flows and fires is comparable to that of the earlier event observed between 9:35:45 a.m. and 9:36:50 a.m. This suggests that the cause for the short-lived burning is the same. Therefore it is also tentatively attributed to the burning of a pool of aviation fuel somewhere inside the tower.

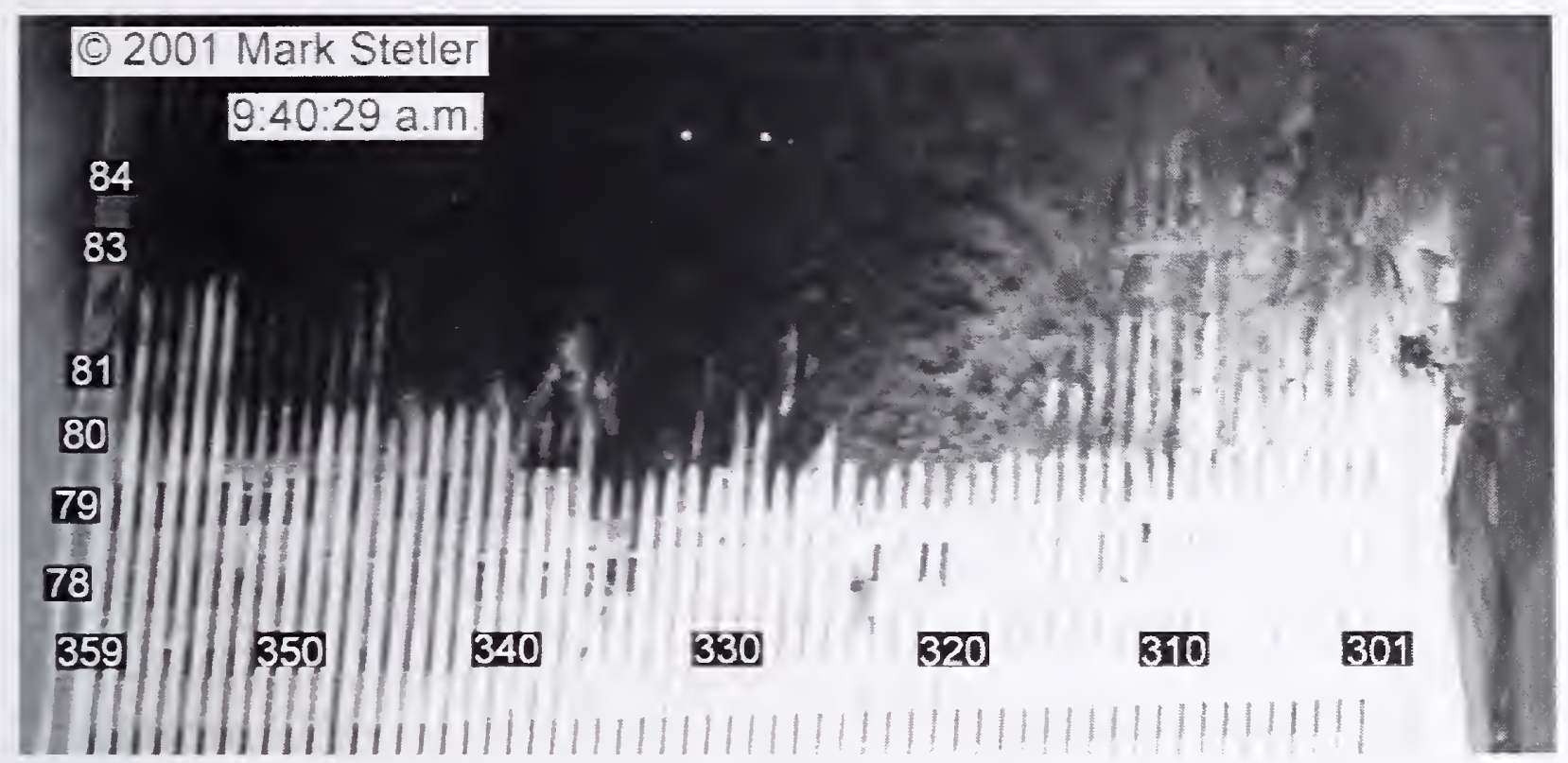

Figure 9-58. This cropped photograph showing the east face of WTC 2 was taken at 9:40:29 a.m. Column and floor numbers have been added.

A similar increase in smoke and fire was seen at roughly the same locations between 9:41:17 a.m. and 9:42:27 a.m. The duration and appearance were similar to the two earlier events. It is also tentatively attributed to the burning of an isolated pool of aviation fuel.

During the periods when the heavy smoke and fires were not present on the east face, its appearance was similar to that seen in Figure 9-48. Occasionally. coordinated puffs of smoke similar to those described earlier were observed coming from windows on the face. Figure 9-59 shows the east face at 9:44:50 a.m. in a photograph shot from the northeast. The amount of smoke coming from lower floors is light, and most of the face is visible. From this angle very little fire is evident, indicating that flames have receded back into the building or died down at many locations on the face. In particular, the heavy flames present near the center of the face in Figure 9-46 and Figure 9-48 seem to have died down by this time. There is evidence for a fire burning on the 82 nd floor near the northern edge of the face. Fire had been observed here earlier. The fire in the northeast corner of the $81 \mathrm{st}$ floor continued to burn vigorously at 9:44:50 a.m. The bright flame attributed to metal combustion is still evident, but has markedly decreased in intensity.

In Figure 9-59 the columns near the center of the east face on the 80th and 81st floors appear to be bowing inward. A similar feature was identified earlier in Figure 9-46. This photograph provides additional visual evidence that the bowing was actually taking place and for its extent. 


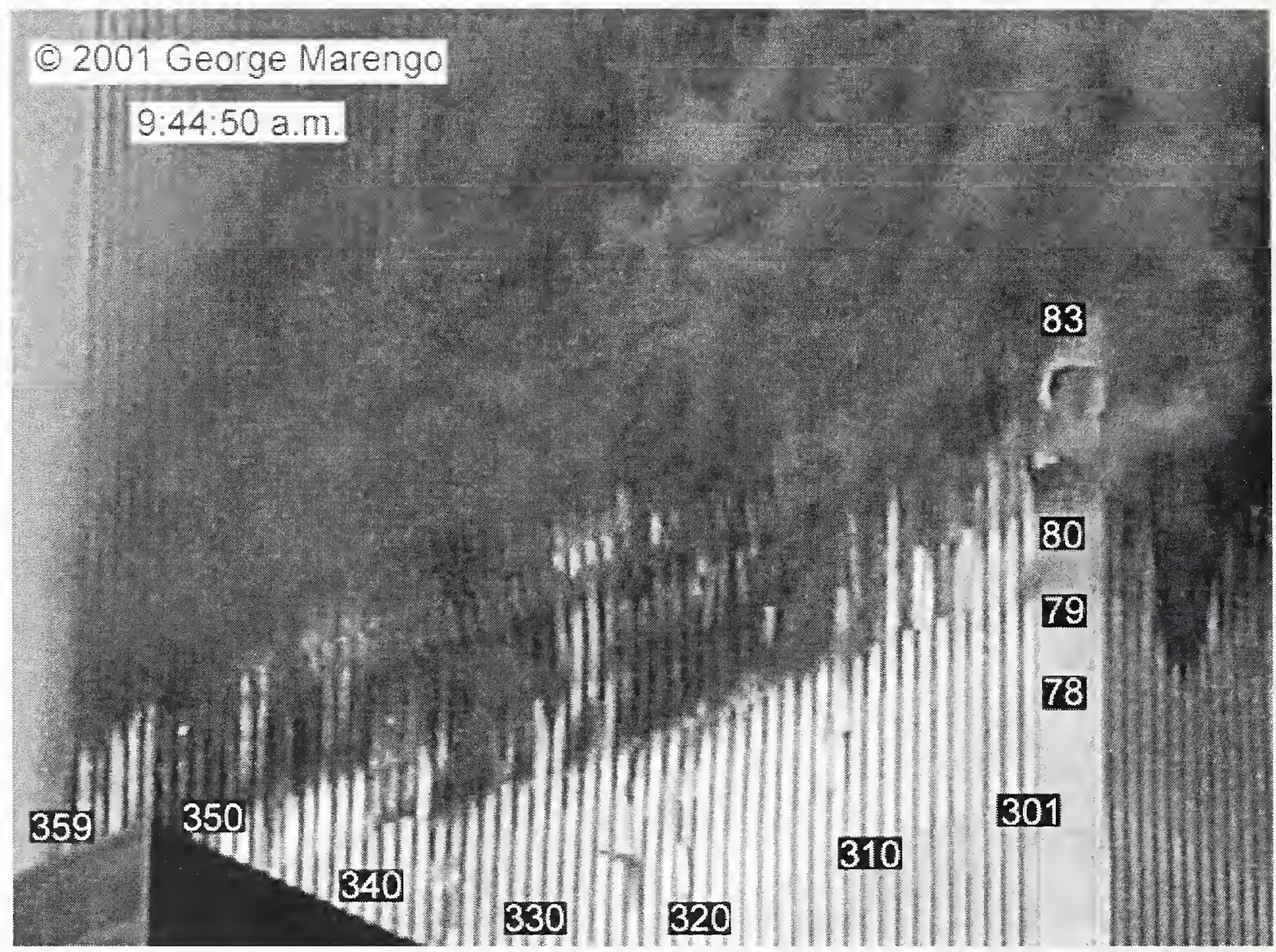

Figure 9-59. This cropped photograph was shot from the northeast and shows the east face of WTC 2 at 9:44:50 a.m. The original intensity levels have been adjusted, and column and floor numbers have been added.

The dominant features during the current period were the three short-lived bursts of smoke and fire observed on the east face of WTC 2, the observation of pressure pulses that affected multiple floors and faces, and changes in the positions of hanging objects, which may indicate that sinking or settling of floor slabs was taking place. Additionally, images recorded during this period suggested that multiple columns on the east face were bowing inward. This feature was observed as early as 9:21:29 a.m.

The short-lived (roughly $1 \mathrm{~min}$ ) bursts of smoke and fire were hypothesized to be due to the ignition and burning of pools of aviation fuel that formed on the 79th and 80th floors during the aircraft impact. The visual evidence for the occurrence of short pressure pulses with magnitudes large enough to redirect smoke flows on multiple faces and floors was strong, but the visual evidence did not provide sufficient information to identify their source(s). The possibility that the collapse of the 83rd floor slab was a potential source of a pressure pulse was discussed.

The fire behaviors and changes in distribution are more easily followed by comparing Figure 9-60, which shows integrated fire intensity maps for the four faces of WTC 2 during the period, with the corresponding maps for the period from 9:15 a.m. to 9:29 a.m. shown in Figure 9-34. 

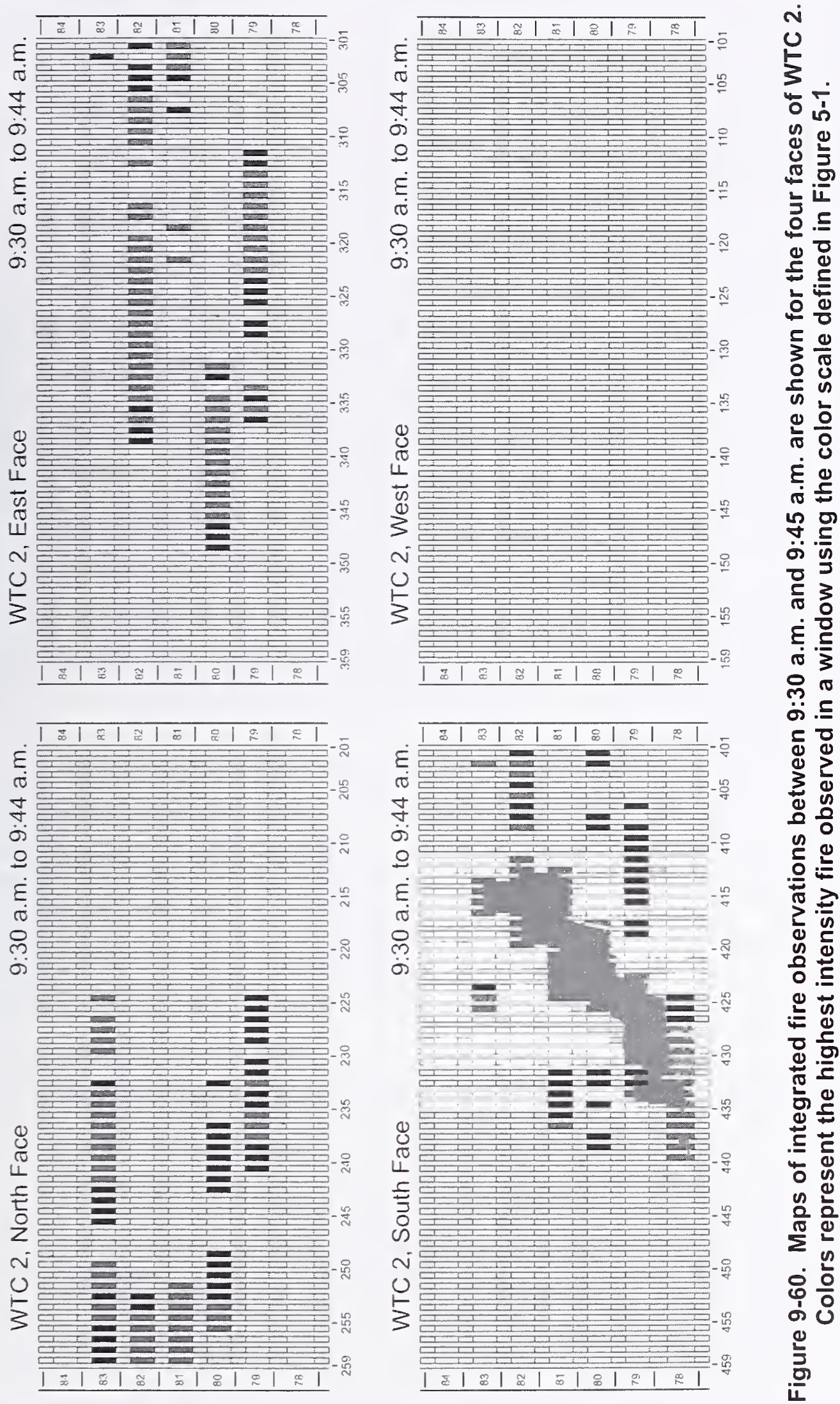
Fires on the north face that were ignited on piles of debris on the 79th, 81st, and 82nd floors shortly after the aircraft impact, along with a fire that grew somewhat later on a debris pile on the 80th floor continued to burn throughout the period. At 9:29:15 a.m. a fire grew and spread rapidly on the 83 rd floor just above the west side of the cold spot. During the period this fire spread to the west in two separate steps, reaching window $83-221$ by 9:45:25 a.m. There was also evidence for westward fire spread on the 79th floor. Even though some small fires continued to burn at the periphery of the cold spot, the area continued to be mostly free of fire during the period. The fire behavior on the east face during this period was complex. In addition to the short periods of intense smoke release and burning on the 79th and 80th floors discussed above, a large fire with external flames burned for a short period on the 82 nd floor near the center of the face. A fire on the northern edge of the 82 nd floor, that had apparently died down earlier, reappeared. The 81 st floor fire burning near and in the northeast corner continued to burn throughout the period. An unusually bright flame was observed in the corner that was attributed to metal combustion.

On the south face several fires on various floors around the aircraft impact cavity died down. A small fire was observed on the $83 \mathrm{rd}$ floor above the aircraft impact area. A new region of fire, as indicated by the presence of smoke and flame in windows 80-401 to 80-408, burned briefly on the eastern side of the 80th floor. A fire that had first appeared on the 79th floor to the east of the impact cavity around 9:15 a.m. continued to spread toward the east.

There were no indications of fire reaching the west face during the period. Smoke flow from additional open windows above the fire floors was observed and, as for earlier times, possibly caused by people breaking out the glass.

\section{$9.5 \quad 9: 45$ A.M. TO 9:59 A.M.}

Figure 9-61 shows a photograph of the north face taken at 9:46:32 a.m. It was shot exactly 1 min after the image shown in Figure 9-53. While the fire distributions look similar in the two photographs, there has been a significant change. At 9:46:32 a.m. a substantial fire is visible near the center of the cold spot on the $82 \mathrm{nd}$ floor. Prior to this time, there had been no indication of a fire at this location. The photograph in Figure 9-61 was shot with high resolution and magnification. It is possible to enlarge it substantially. Two blow-ups of areas in the image are shown in Figure 9-62 and Figure 9-63. In the blow-ups the intensities have been adjusted to highlight darker regions.

Figure 9-62 shows an enlargement of the area of the north face encompassing the cold spot. Here it can be seen that the fire that has grown on the 82 nd floor consists of the bright flame visible through window 82-245 and duller flames that appear to be further back from windows 82-242 to 82-244 and 82-246. There is no indication of where this fire spread from. A hanging object, marked with arrows, is visible through the open windows on the floor immediately below the fire. This object was not immediately apparent in Figure 9-61, but became visible when the image intensities were further adjusted to highlight dark areas. This object was observed at the same location at 9:42:31 a.m. in the image shown in Figure 9-51 and Figure 9-52. Note that there is a section of the object visible near the tops of windows 81-248 to 81-250 that was not clear in Figure 9-52. 


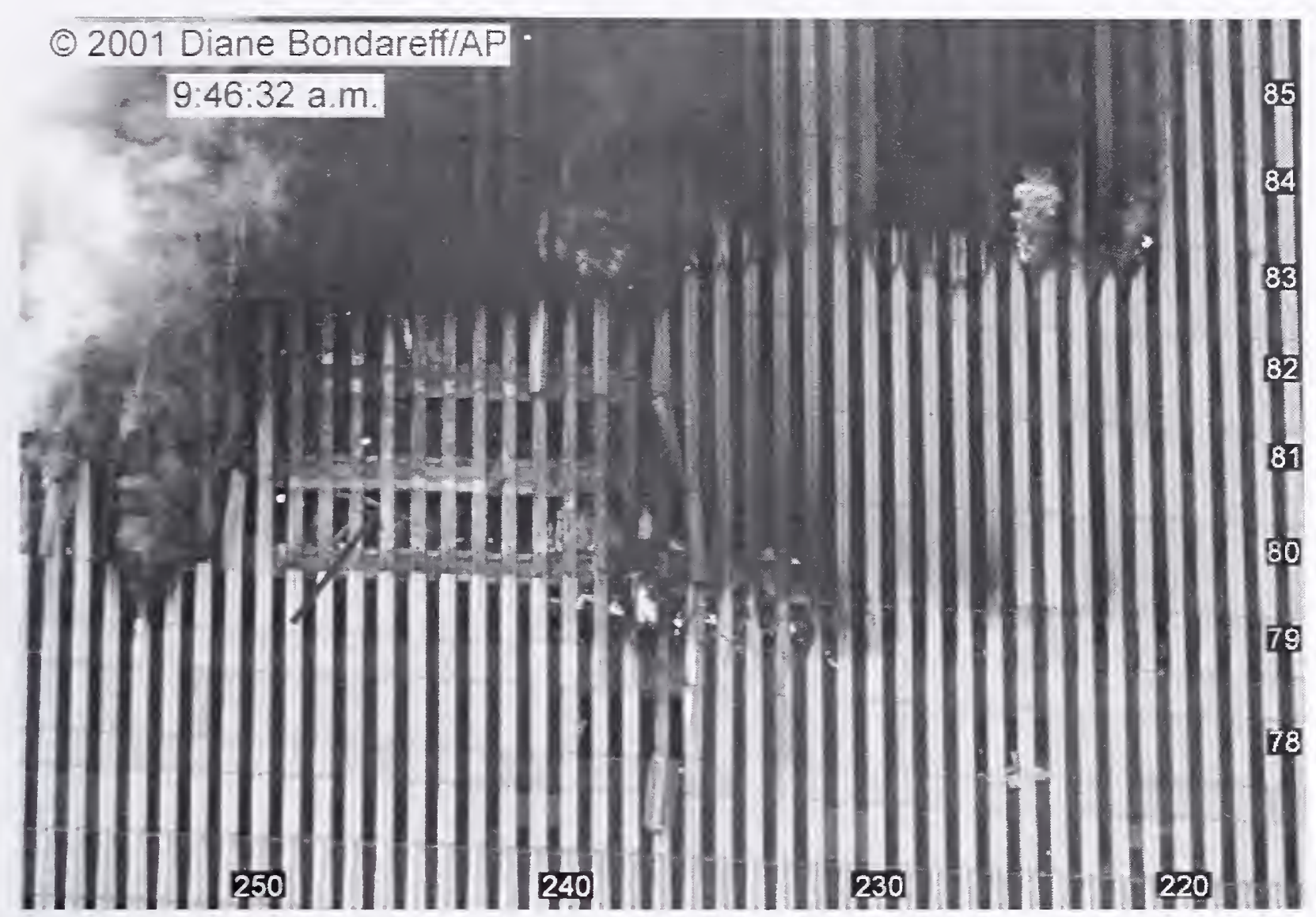

Figure 9-61. This cropped photograph shows the north face of WTC 1 at 9:46:32 a.m.

The intensity levels have been adjusted, and column and floor numbers have been added. The arrow indicates a hanging object visible through windows on the 80th floor.

Closer inspection of Figure 9-62 shows that small dull-colored flames are visible on the 81st floor through windows 81-243 and 81-244 at the level of and above the hanging object. This suggests that a fire is burning on top of the object. It is possible that this fire is burning at the base of a debris pile that is also providing fuel for the fire visible immediately above on the $82 \mathrm{nd}$ floor. If true, this means that the hanging object is most likely a portion of the 82 nd floor that has broken away from the spandrel at the base of the $82 \mathrm{nd}$ floor and settled to its current location from above.

The hanging object visible in Figure 9-62 on the 80th floor is also in the same position as observed at 9:42:31 a.m. In this closer view it has a very similar appearance to the hanging object on the 81st floor. Therefore, it appears to be a portion of the 81 st floor slab that has separated from the spandrel and dropped down from above.

The fire that had been burning at the lower left-hand corner of the cold spot since before 9:15 a.m. is still burning vigorously in Figure 9-62. This long burning time suggests that the fuel is a large debris pile since typical office fuels should have burned out by this time. It may be that the 80 th floor slab is missing in this area and that the fires visible on the 79th and 80th floors (see Figure 9-61) are actually burning on the same pile of debris. There is weak evidence to support such a conclusion. The arrows in Figure 9-63 have been added to highlight what may be a hanging object in windows 79-229 to 79-233 on the 79th 


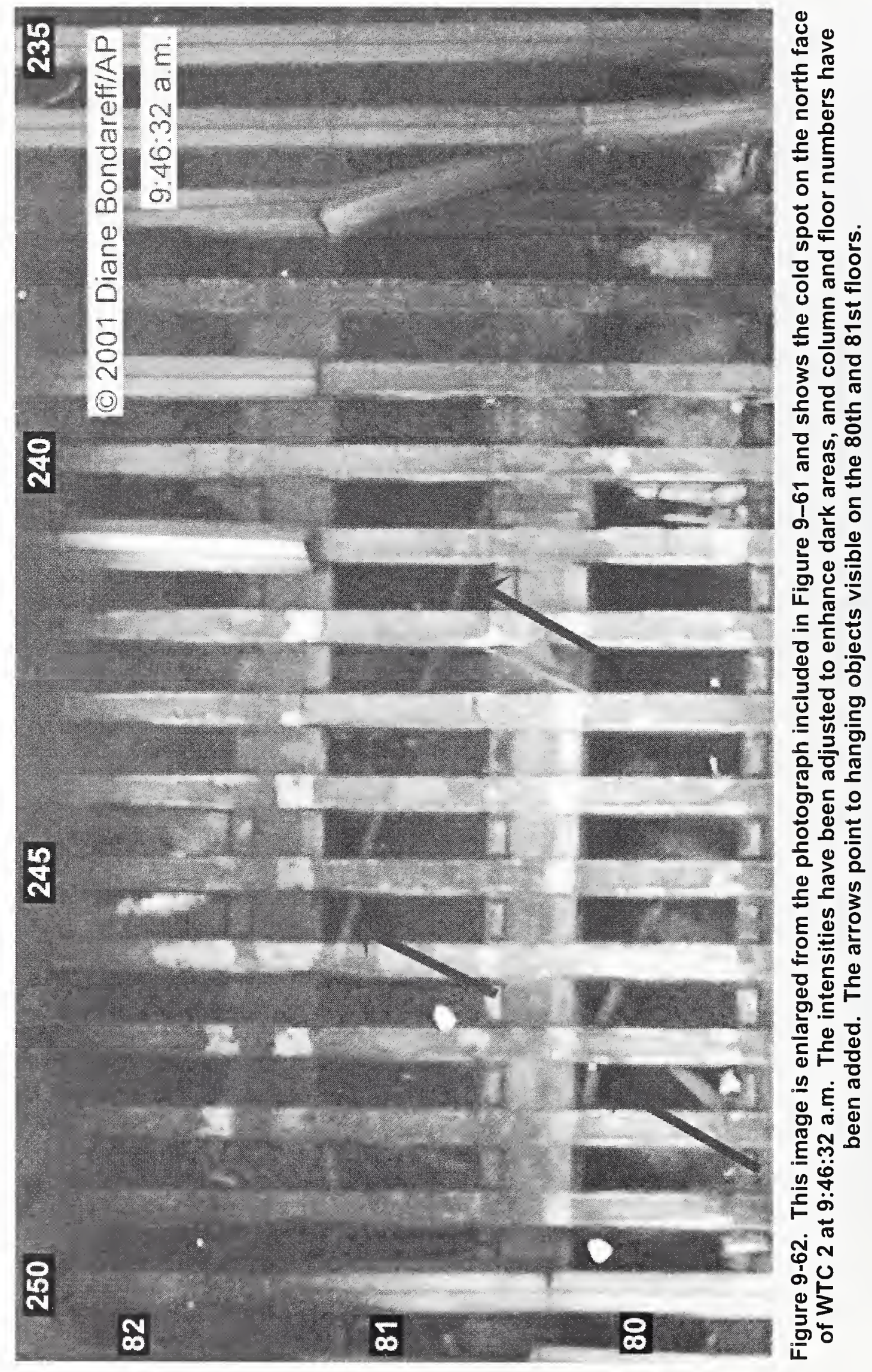



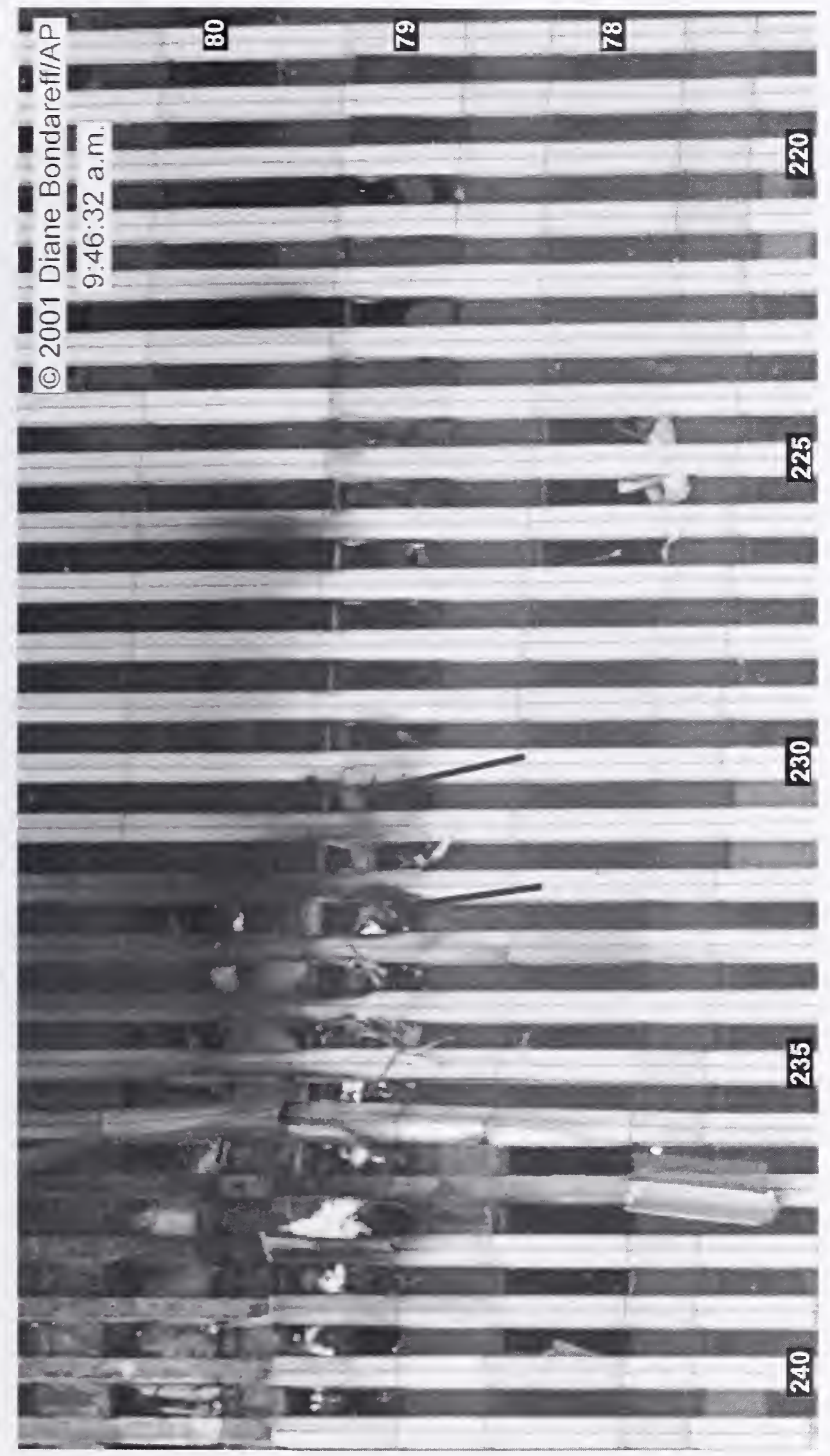

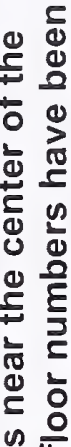

di

는 응

舫

उำ 든

的至

응 숭

흐을

एक

1 in

0 赵

는

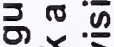

政究>

등 넝

음

d

옹

든 돋

잉

등으 동

융

ㅎํㅇㅇㅛ

융

등

등

我方

ํํㅇㅇํㄴ

츤

동

\&

o.․․

究

도웡음

․ㅗ..

ฮั

正

ह

nu

단

म่

ดู่

क

는

은 등 
floor. The appearance of the object in these windows is somewhat different than thosc observed elsewhere on the north and east faces of WTC 2. This introduces some uncertainty into its identification as one of the hanging objects.

Figure 9-63 provides a detail that has not been apparent previously. There is a distinct outward bulge in the stecl pcrimeter wall along the spandrel at the base of the 80th floor. It appears to extend from column 235 to column 239. This bulge is roughly in the center of the pile of debris deposited at this location during the aircraft impact. It is likely that it was created when debris that passed through the tower (see the discussion in Chapter 7) struck the wall.

The contrast available in Figure 9-62 and Figure 9-63 is sufficient to identify whether or not glass has been broken out of windows. In Figure 9-62 the only window that is closed is window 81-235. In Figure 9-63 several windows are visible on the 79th floor in which glass has been partially removed, presumably due to heating from the nearby fire. The westernmost of these is window 79-220. A small spot fire is visible at the base of this window. On the 80th floor the westernmost open window is window 80-232. A small fire is also present in this window. The glass in the window to the immediate left, window 80-233, appears to be intact. Several open windows are visible on the 78th floor. These have been previously identified as having been broken during the aircraft impact and subsequent fireballs.

A close-up image of the burning debris pile on the 79th floor is shown in Figure 9-64. This image is a video frame that was captured at 9:48:01 a.m. The bulge in the spandrel at the base of the 80th floor is pronounced. Despite the degree of detail available in the image, it is not possible to determine whether the fires visible on the 79th and 80th floors are burning on a single large pile of debris, i.e., the intervening floor slab is missing, or there are two separate fires on the two floors.

An image of the north face of WTC 2 taken at 9:50:55 a.m. is shown in Figure 9-65. Comparison with Figure 9-61 shows that some changes occurred in the approximately $4 \frac{1}{2} \mathrm{~min}$ between the two photographs. The intense fire burning on the 83 rd floor moved west one window. A video shows that fire appeared in window 83-225 at 9:49:11 a.m.

The fire that was visible in the cold spot in the earlier photograph on the 82 nd floor has spread over more windows. It is now visible in windows $82-247$ to $82-244$. The small flames observed earlier one floor down in window 81-243 are still visible, but a more substantial fire has grown just to the west, and flames are now visible in windows 81-239 and 81-240. The fire on the west side of the cold spot on the 80th floor has died down somewhat. 


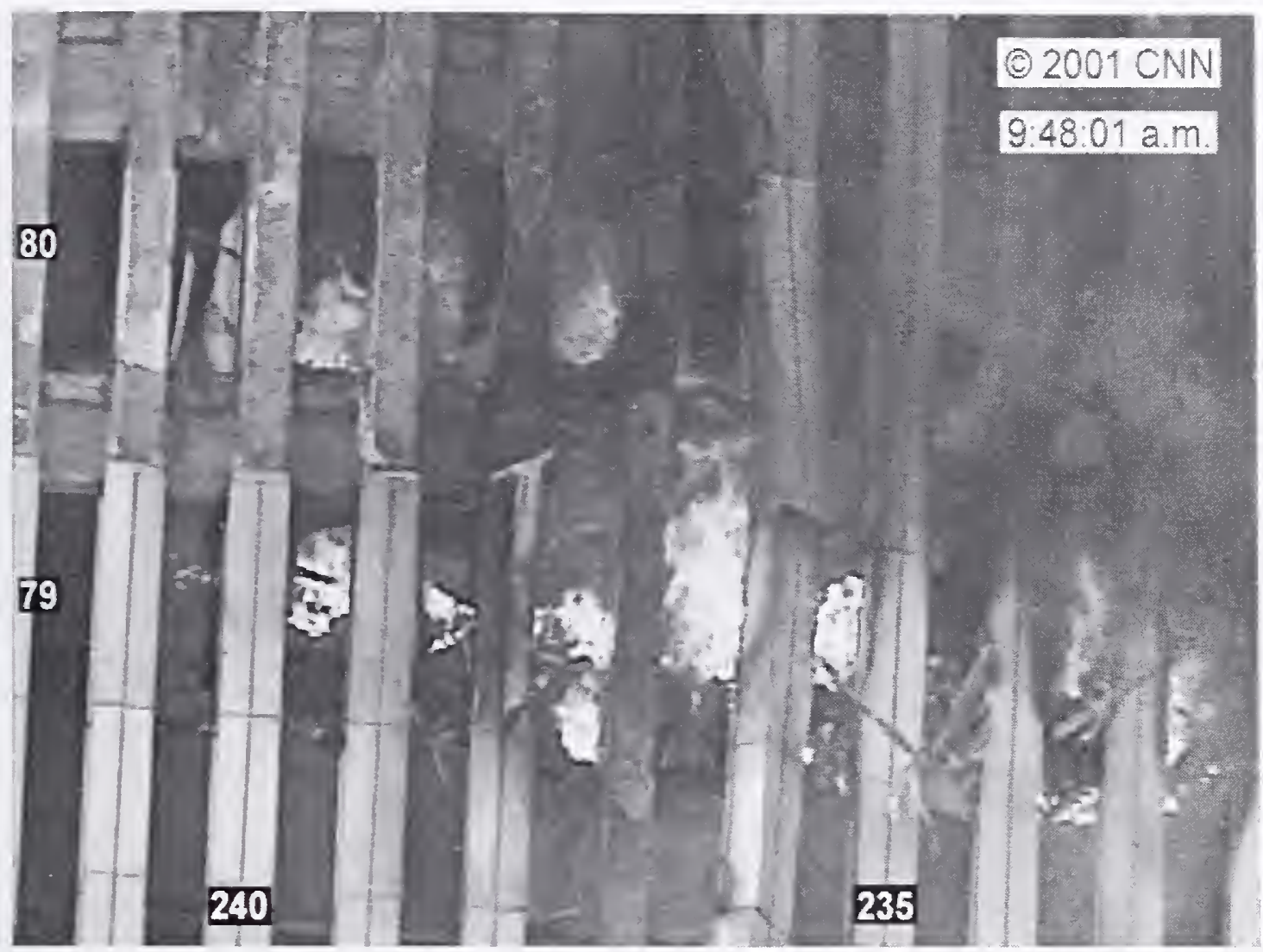

\section{Figure 9-64. This close-up image of the north face of WTC 2 was captured from a video recorded at 9:48:01 a.m. The intensity levels have been adjusted, and column and floor numbers have been added.}

The fire on the eastern edge of the 81st floor seen in Figure 9-65 continues to burn on the dcbris pile in this area. The appearance of the fire that had earlier spread west on the 79th floor has not changed markedly. Earlier it was mentioned that windows on the 89 th floor possibly had been broken open by people at windows 89-201 to 89-203 and window 89-208. Light smoke flows are visible coming from these windows in Figure 9-65.

The hanging object on the 80th floor is just visible in this photograph as indicated by the arrow.

Starting around 9:44:58 a.m. there was another period during which heavy smoke flows came from numerous open windows on the 79th and 80th floors of the east face. This release lasted until approximately 9:46:05 a.m. Figure 9-66 shows an image recorded during this smoke release at 9:45:40 a.m. Comparison with Figure 9-43 and Figure 9-58 indicates that the amount of smoke flow and visible flame are much less than observed during the three similar earlier events. Nonetheless, the smoke flow obscured much of the face, and videos show that intermittent flames appeared outside of some windows. Near the end of this smoke release episode, there were two large pressure pulses at 9:45:57 a.m. and 9:46:01 a.m. that pushed large amounts of smoke out of windows on the 79th and 80th floors. 


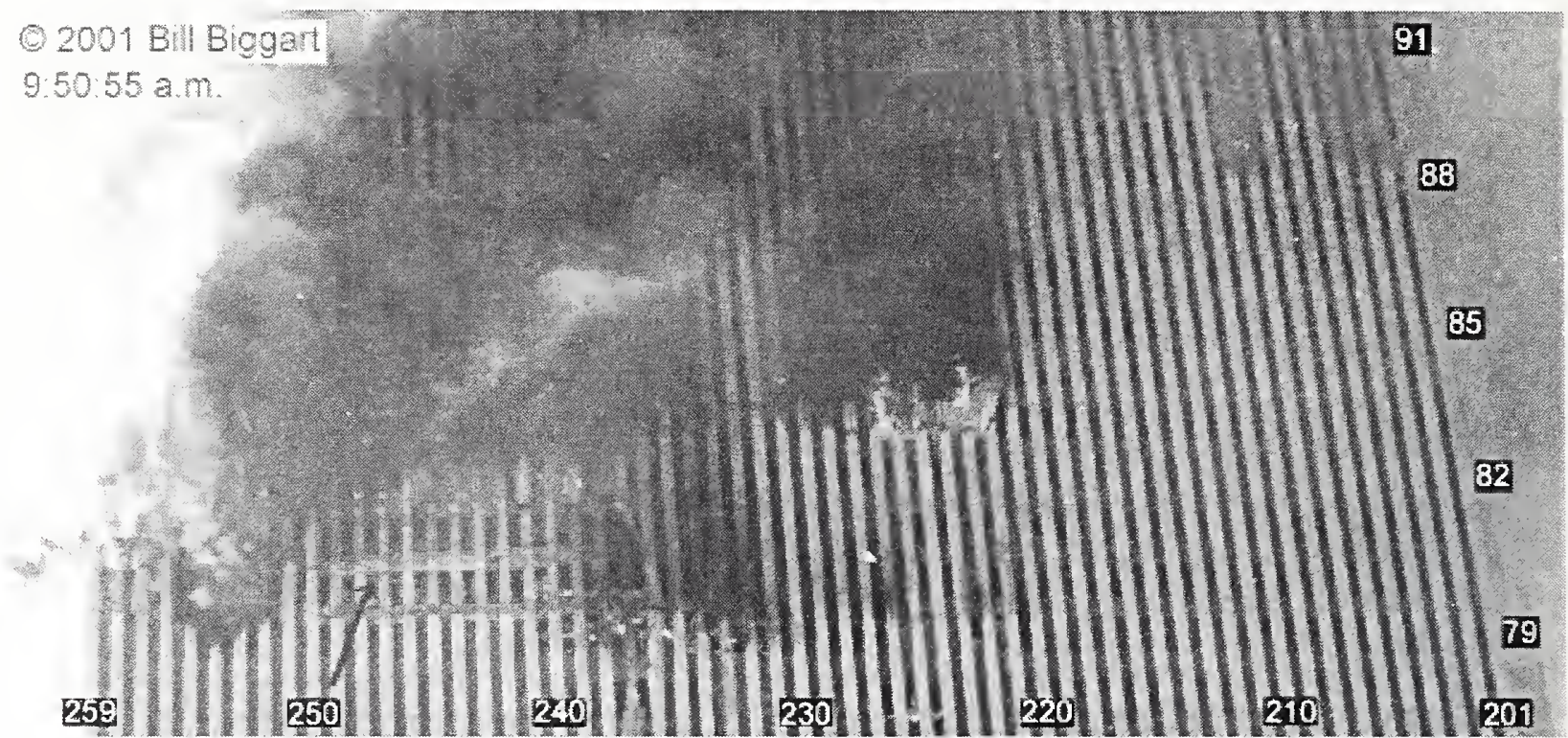

Figure 9-65. This cropped photograph shows the north face of WTC 2 at 9:50:55 a.m. The original has been rotated and enhanced by adjusting the intensity levels. Column and floor numbers have been added. The arrow points to a ganging object visible through open windows on the 80th floor.

In Fig. 9-66 flames are visible on the 79th floor between windows 79-313 and 79-319 and are coming from window 82-306 to window 82-308 on the 82nd floor. At 9:38:22 a.m. (see Fig. 9-48) fires were observed at the same locations. The earlier photograph was shot in between the periods of heavy smoke flow and external flaming. This shows that these fires are well established and are not associated with the periods of heavy smoke flows and external burning. The fire in the 81 st floor northeast corner continued to burn at 9:45:40 a.m.

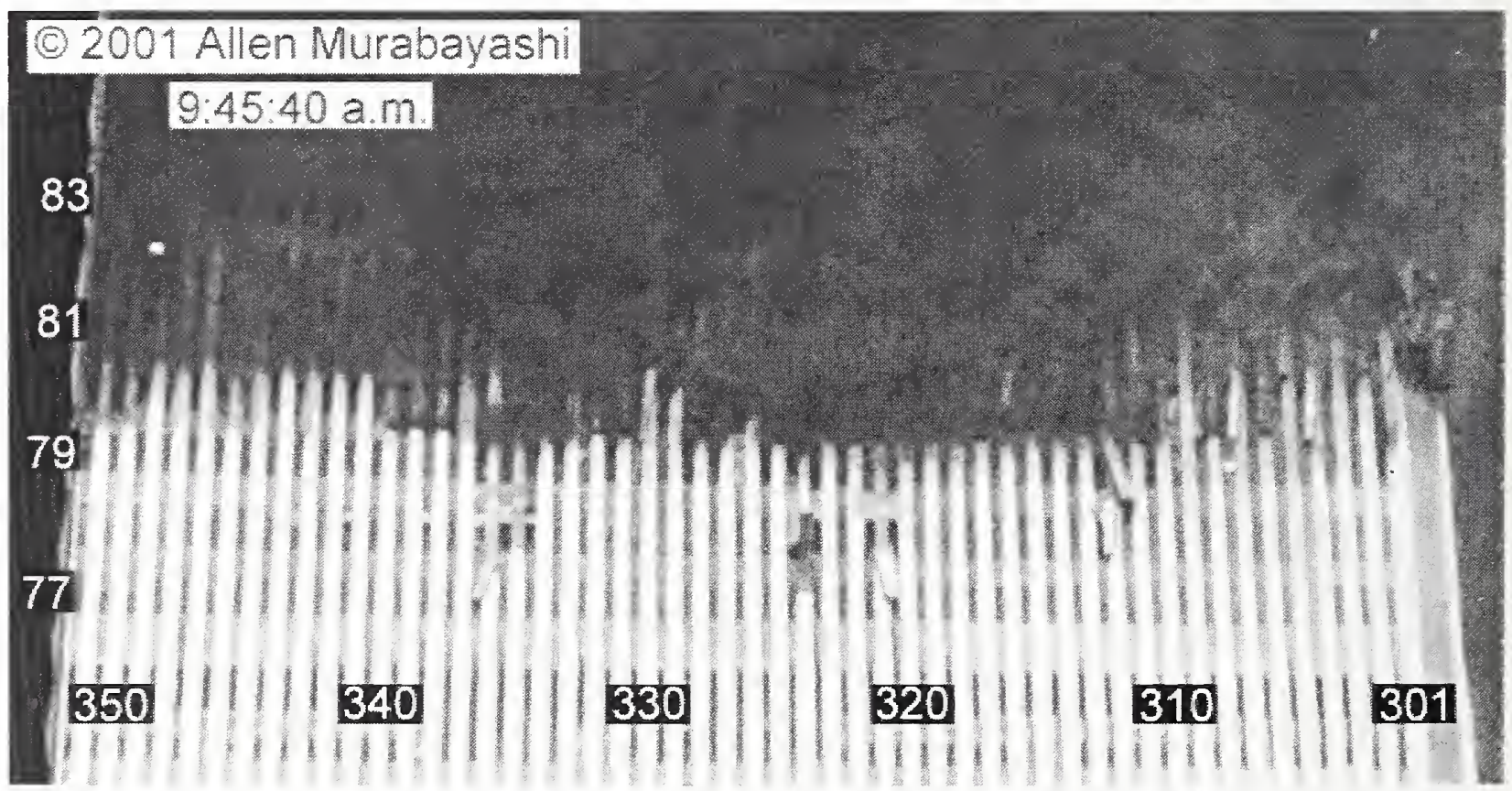

Figure 9-66. This cropped photograph shows the east face of WTC 2 at 9:45:40 a.m. The image has been rotated, and the intensity range has been adjusted. Column and floor numbers have been added. 
Figure 9-67 shows a higher resolution photograph of the east face taken 11/2 min after Figure 9-66 at $9: 47: 10$ a.m. The smoke has cleared up, and it is now possible to see many more windows at the south side of the face on the 80th and 81 st floors, as well as higher up on the face. A substantial fire, visible in windows $79-313$ to $79-320$, continues to burn on the 79th floor. Flames are also present in windows $82-303$ to $82-307$. The fire on the 81 st floor is isolated to the first couple of windows along the northern edge of the east face and the pile of debris in the northeast comer. At this time flames were not visible elsewhere on the east face.

The arrows in Figure 9-67 highlight the hanging object visible through open windows on the 82nd floor. Comparison with the blow-ups in Figure 9-48, which were shot at 9:38:22 a.m., shows that the hanging object has not shifted position appreciably since this time. A shorter length of the object on the north side of the face is visible in the later photograph due to smoke obscuration.

There was another period of smoke release and a limited amount of external flaming on the 79th and 80th floors starting around 9:50:57 a.m. It appeared to be of comparable intensity to the event that started around 9:44:58 a.m. and lasted until approximately 9:51:50 a.m., before once again subsiding. A photograph (not reproduced here) shot from immediately below the east face of WTC 2 at 9:51:30 a.m. showed that heavy smoke and fire were coming from a number of windows on the 79th floor centered near window 79-333.

In addition to the sustained periods (roughly one minute) of smoke and fire, pressure pulses, during which smoke was rapidly pushed out of open windows for short periods, were also observed moving across the east face at 9:49:16 a.m. and 9:52:13 a.m.

Close-up views of the south and west faces of WTC 2 are limited during the first part of this time period. Videos shot from news helicopters at long distances show that between 9:45 a.m. and 9:52 a.m. there were no large fires visible on either face. On the south face there was a heavy smoke plume on the east side of the face with its base on the 80th floor. At 9:47:50 a.m. a brief flash of fire was observed in window 80-439. These flames appeared to die down rapidly. There were also intermittent releases of smoke from a line of open windows on the 81 st floor to the west of the aircraft impact cavity.

Figure 9-68 shows a view of the south face rccorded at 9:52:00 a.m. It is taken from a video shot from a location to the southeast of the towver. Several small fires are visible on the 78th (window 78-418), 79th (window 79-408), 80th (windows 80-438 and 80-439), and 81 st (window 81-436) floors. Heavy smoke is flowing from windows near the eastern edge of the 80 th floor. There is a stream of smoke coming from the 81 st floor in the same area. At this time the smoke flow from other areas of the face was relatively light. Markings on column covers near the tops of windows indicate that smoke had earlier come from windows on the $79 \mathrm{th}, 80 \mathrm{th}$, and 81 st floors just to the west of the aircraft impact cavity. The smoke band on the 81 st floor, which extends from window 81-431 to 81-439, is the darkest. The presence of this smoke band is consistent with the recent smoke releases observed from this floor. 


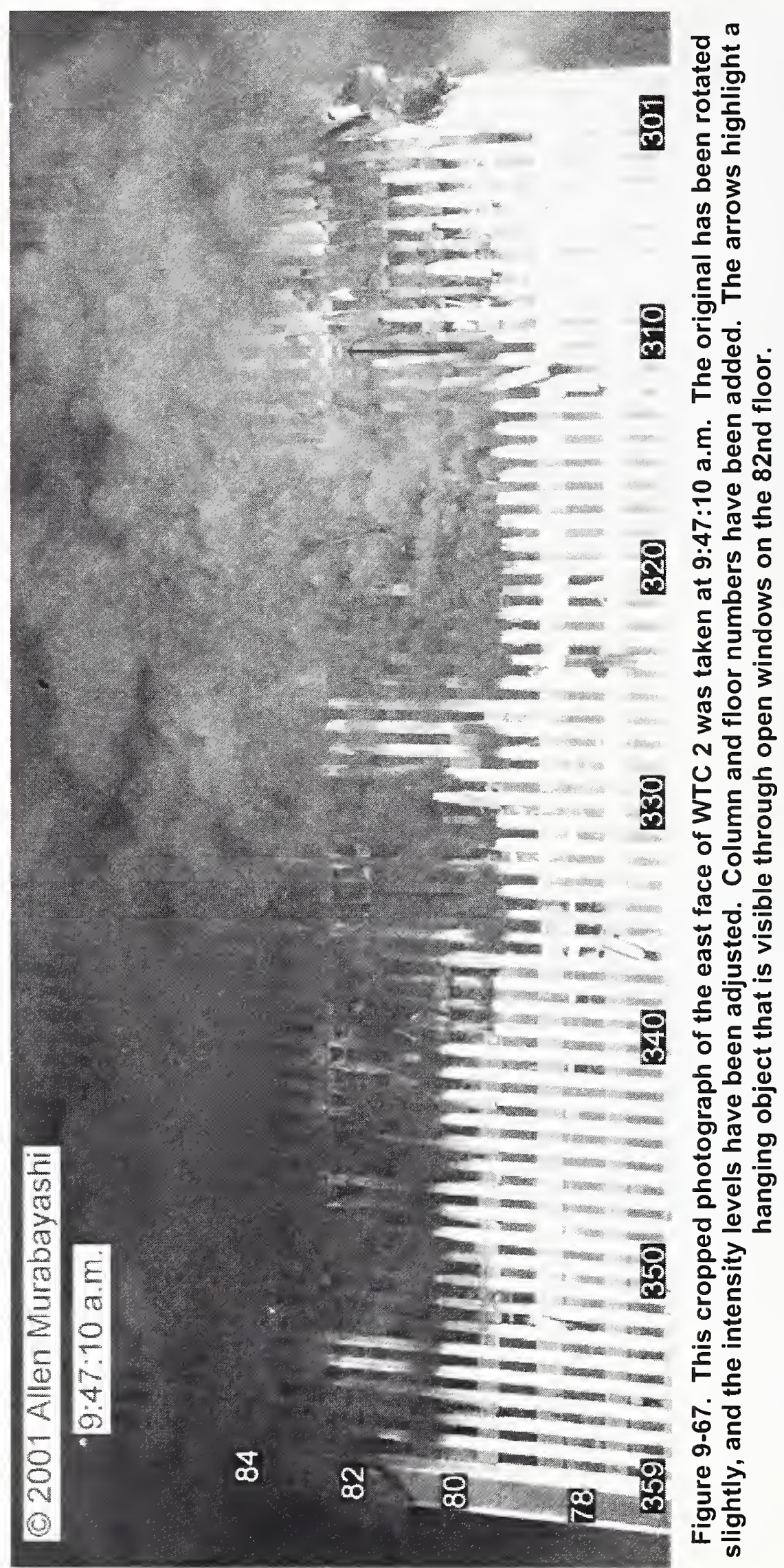




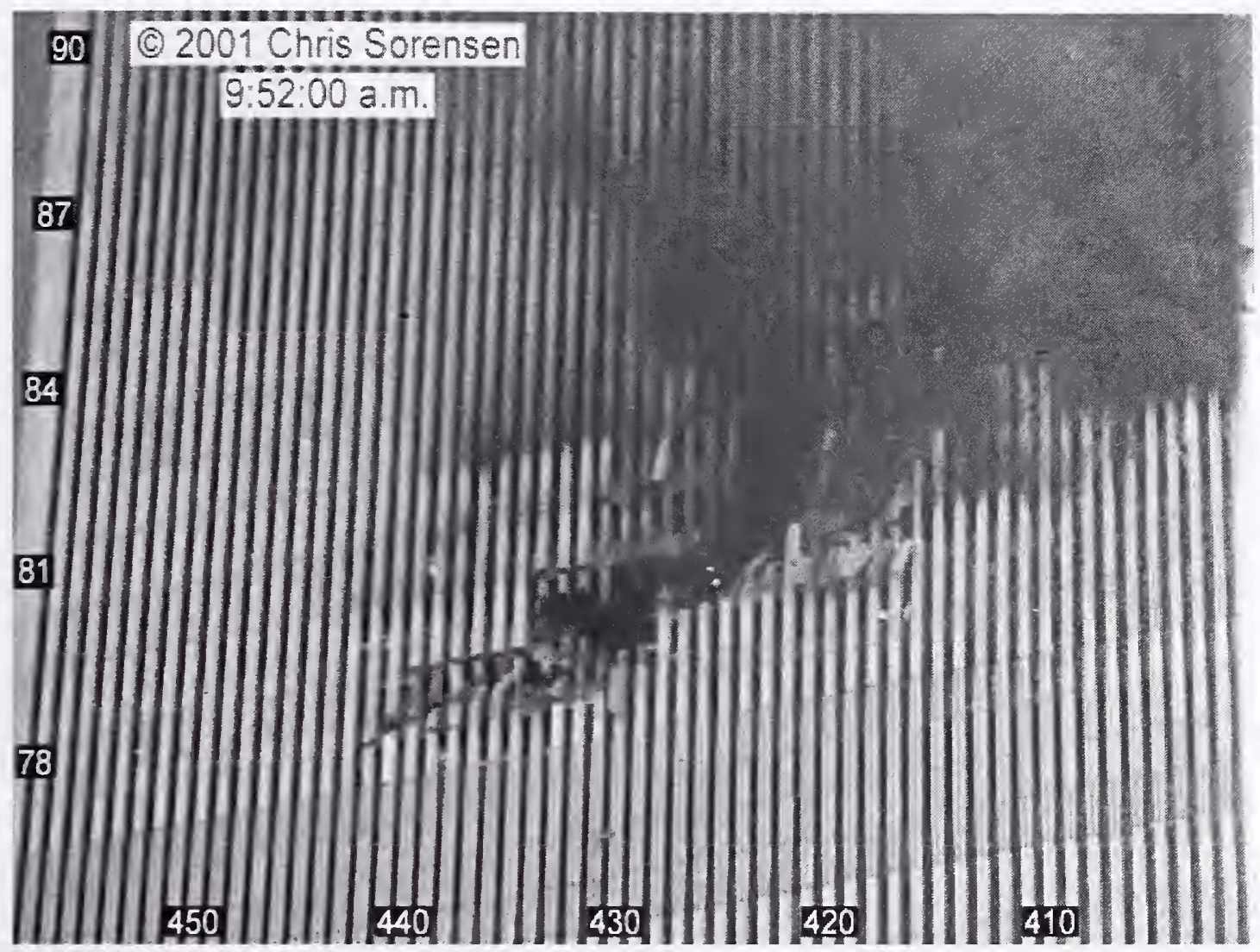

Figure 9-68. This view of the south face of WTC 2 was taken from a video recorded at 9:52:00 a.m. The intensity levels have been adjusted, and column and floor numbers have been added.

Figure 9-69 shows a long-distance view of the west face of WTC 2 at 9:45:56 a.m. Smoke streams are coming from open windows on several floors, many at the same locations observed in earlier images. The discussion associated with Figure 9-57 refers to open windows on the 84th, 86th, 88th, 91st, 105th, and 107th floors. The three lower smoke trails visible in Figure 9-69 are coming from the windows previously identified on the 86th, 88th, and 91 st floor. Closer to the top of the face, three separate smoke trails are visible on the 95th floor. Slightly closer views of the area show that the northern most stream is coming from window 105-148, which is the location visible in Figure 9-57. The other two streams are from near windows 105-116 and 105-140. These two smoke flows were observed to be intermittent in videos of the west face. They were detected briefly as early as 9:43:45 a.m. The heavy smoke flow from the 107th floor observed earlier is prominent in Figure 9-69. The light smoke coming from the southern edge of the mechanical equipment room on the 108 th floor is also visible. 


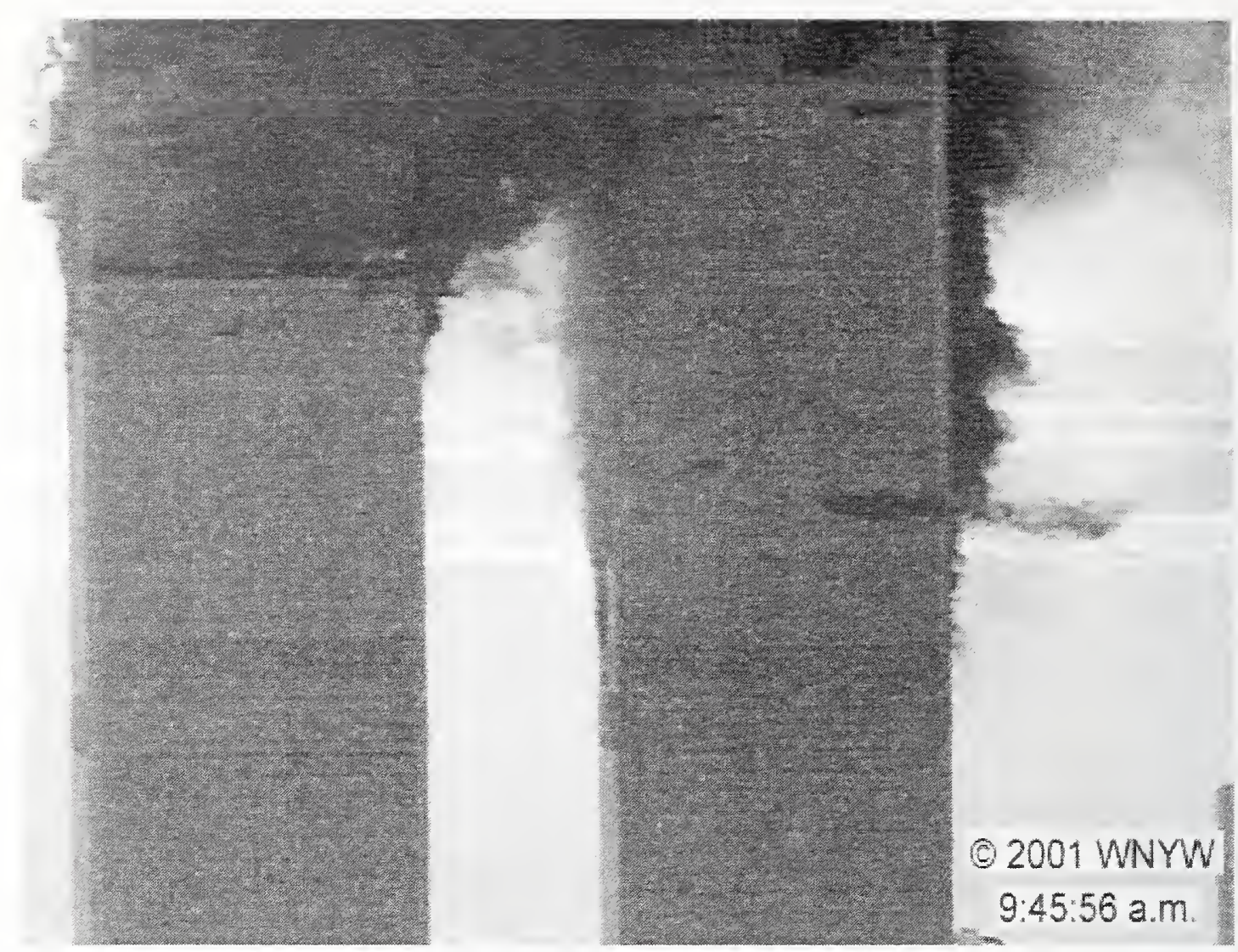

Figure 9-69. This west view of WTC 1 and WTC 2 is a frame captured from a news helicopter video at 9:45:56 a.m. The intensity range has been adjusted.

Figure 9-69 provides an indication of the complexity of the wind patterns present on the west face of WTC 2 on September 11, 2001. The smoke trails on the lower floors are flowing in opposite directions. Around this time, the heavy smoke coming from windows on the 86th floor was observed streaming across the south face of the tower. A similar flow pattern is evident in Figure 9-57. Videos show that at other times the smoke from these windows would reverse direction and actually flow toward the north. Figure 9-42 shows an example of this flow at 9:34:19 a.m. As discussed in Section 4.1, these complex wind patterns developed because WTC 2 was on the leeward side of WTC 1, which shielded it from the direct impact of the ambient wind and created complex flow patterns that interacted with WTC 2.

At 9:51:51 a.m. puffs of smoke and/or dust were expelled from open windows on the 79th, 80th, and 81st floors on the north face of WTC 2 near the east edge. These included windows 79-253 and 79-254, 80-257 and 80-258, and 81-254. Almost simultaneously, smoke was pushed from windows on the 79th and 80 th floors of the east face. Just over a second later, a bright spot appeared at the top of one window, $80-255$, on the 80th floor, and a glowing liquid began to pour from this location. As it fell, the glowing liquid struck the base of window 80-255 and splashed before falling further. Figure 9-70 shows a frame captured from a video at 9:51:54 a.m. in which the falling liquid and the splashing can be seen. This liquid flow lasted approximately $4 \mathrm{~s}$ before subsiding. After the flow stopped, the glowing spot was still visible at the top of window 80-255. 


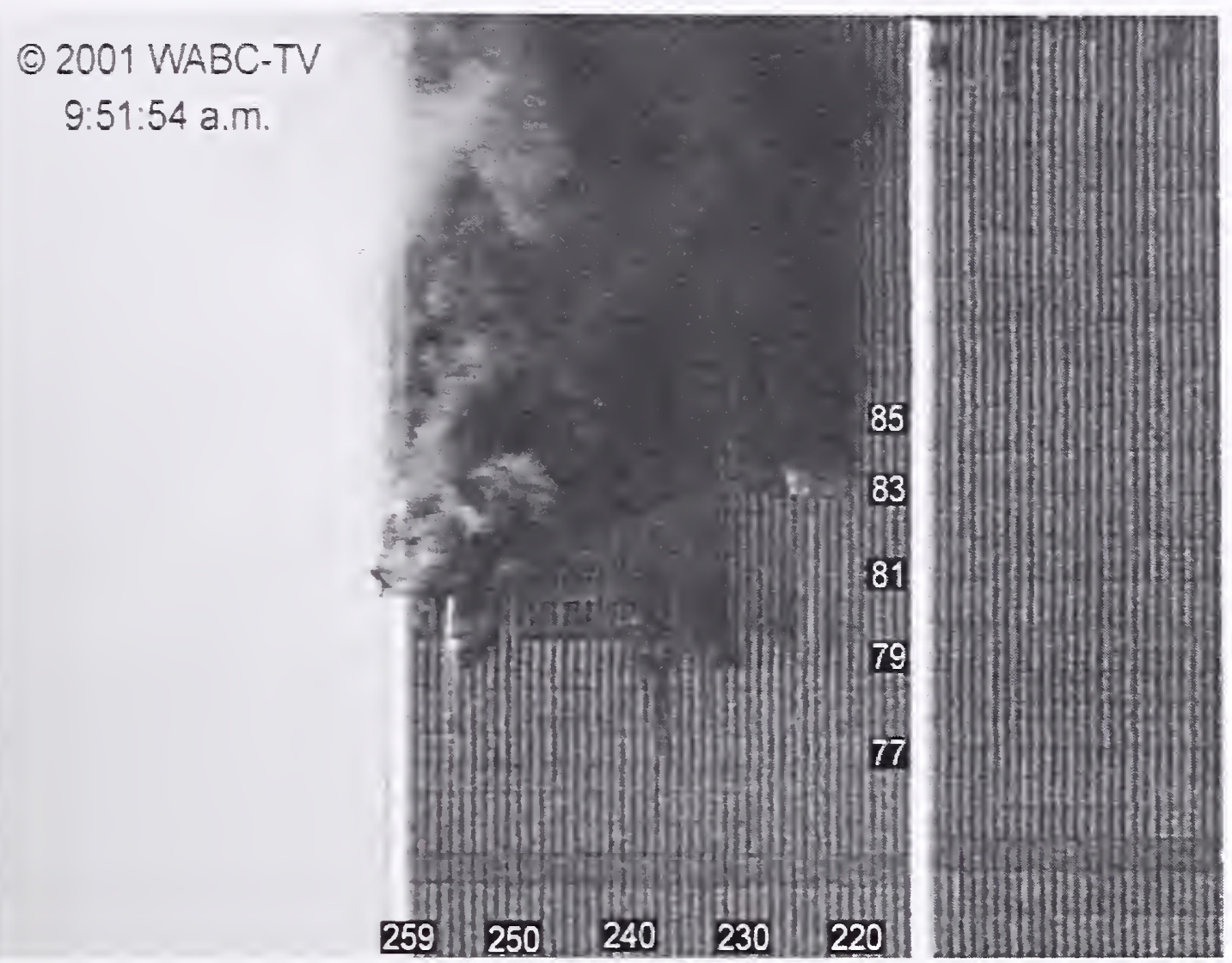

Figure 9-70. This image of the north faces of WTC 1 and WTC 2 was captured from a video recorded at 9:51:54 a.m. The intensity levels have been adjusted, and column and floor numbers have been added to WTC 2.

It has been reported in the FEMA report (McAllister 2002) as well as in the media that what appeared to be molten metal was observed pouring from the north face near the northeast corner prior to the collapse of WTC 2. This is the area where the sustained fires on the 81 st and 82 nd had been burning since the aircraft impact. The likely explanation for the observation of pouring liquid is that the material had originally pooled on the floor slab above, i.e., the 81 st floor. and that it was allowed to pour out of the building when the floor slab in the immediate vicinity either pulled away from the spandrel or sank down to the point where the window was exposed. The puff of smoke and/or dust just prior to the first appearance of the molten material suggests that the ultimate event responsible for the release of the material occurred suddenly, in the process creating a pressure pulse that forced smoke and/or dust out of open windows over three floors.

The composition of the flowing material can only be the subject of speculation, but its behavior suggests it could have been molten aluminum. Visual evidence already discussed shows that significant wreckage from the aircraft passed through the building and came to rest in the northeast corner of the tower on the 81 st floor, i.e.. at the location where the molten material apparently originated. Much of the structure of the Boeing 767 is formed from two aluminum alloys that have been identified as 2024 and 7075 (NIST NCSTAR 1-3). The melting points for these alloys vary as the material melts. The Aluminum Association handbook (The Aluminum Association 2003) lists the melting point ranges for the alloys as roughly $500^{\circ} \mathrm{C}$ to $638^{\circ} \mathrm{C}$ and $475^{\circ} \mathrm{C}$ to $635^{\circ} \mathrm{C}$ for alloys 2024 and 7075 , respectively. These 
temperatures are well below those characteristic of fully developed fires (ca. $1000{ }^{\circ} \mathrm{C}$ ), and any aluminum present is likely to have been at least partially melted by the intense fires that had been in the area for nearly $48 \mathrm{~min}$.

There was another release of smoke and/or dust from near the east edge of the north face at 9:52:38 a.m. Immediately afterward, the spot near the top of window 80-255 brightened considerably. At 9:52:47 a.m. a series of three much larger pressure pulses, which took place over $35 \mathrm{~s}$, pushed smoke and/or dust from several locations on multiple floors of the north face. These locations included those described above, the two areas on the north side of the 83rd floor where fires were burning, the vicinity of the pile of debris near the center of the 79th floor, an area of newly observed fire on the 79th floor near windows 79-209 to $79-213$, and the opening on the northeast corner of the 81 st floor. The fire burning near the center of the 79th floor flared up noticeably. Immediately following one of the pressure pulses (at 9:52:48 a.m.) intense flames suddenly reappeared in windows 81-301 and 81-302 on the east face just to the south of the northeast corner of the 81 st floor. At roughly the same time, part of the debris lodged at the northeast corner of the 81 st floor fell out of the opening. Relatively small amounts of molten material poured from window 80-255 near the start and end of the series of pressure pulses. Videos show that smoke was also expelled from windows near the north edges of the 79 th and 80 th floors of the east face during these pressure pulses.

A high-resolution photograph of the north face shot at 9:52:51 a.m., i.e., just $4 \mathrm{~s}$ after the start of the first of the three pressure pulses, is shown in Figure 9-71. The white cloud in the photograph is the rising dust and/or smoke that was pushed from windows on multiple floors during the pressure pulse. Blow-ups of the area around window 80-255, which is the location on the 80th floor where the molten material poured from, and the cold spot are shown in Figure 9-72 and Figure 9-73, respectively.

First, consider Figure 9-72. Videos show that a small amount of molten material poured from window $80-255$ within a second or two after this photograph was taken. In the photograph the bright material is visible inside this window at the top. The area seems to be surrounded by a large number of glowing drops, which are likely associated with the liquid that will pour out shortly. Dull, deep-red glowing areas are just visible at the tops of windows $80-252$ and $80-254$. These might be an indication that the 81 st floor slab has sunken below the spandrel at the base of this floor. 


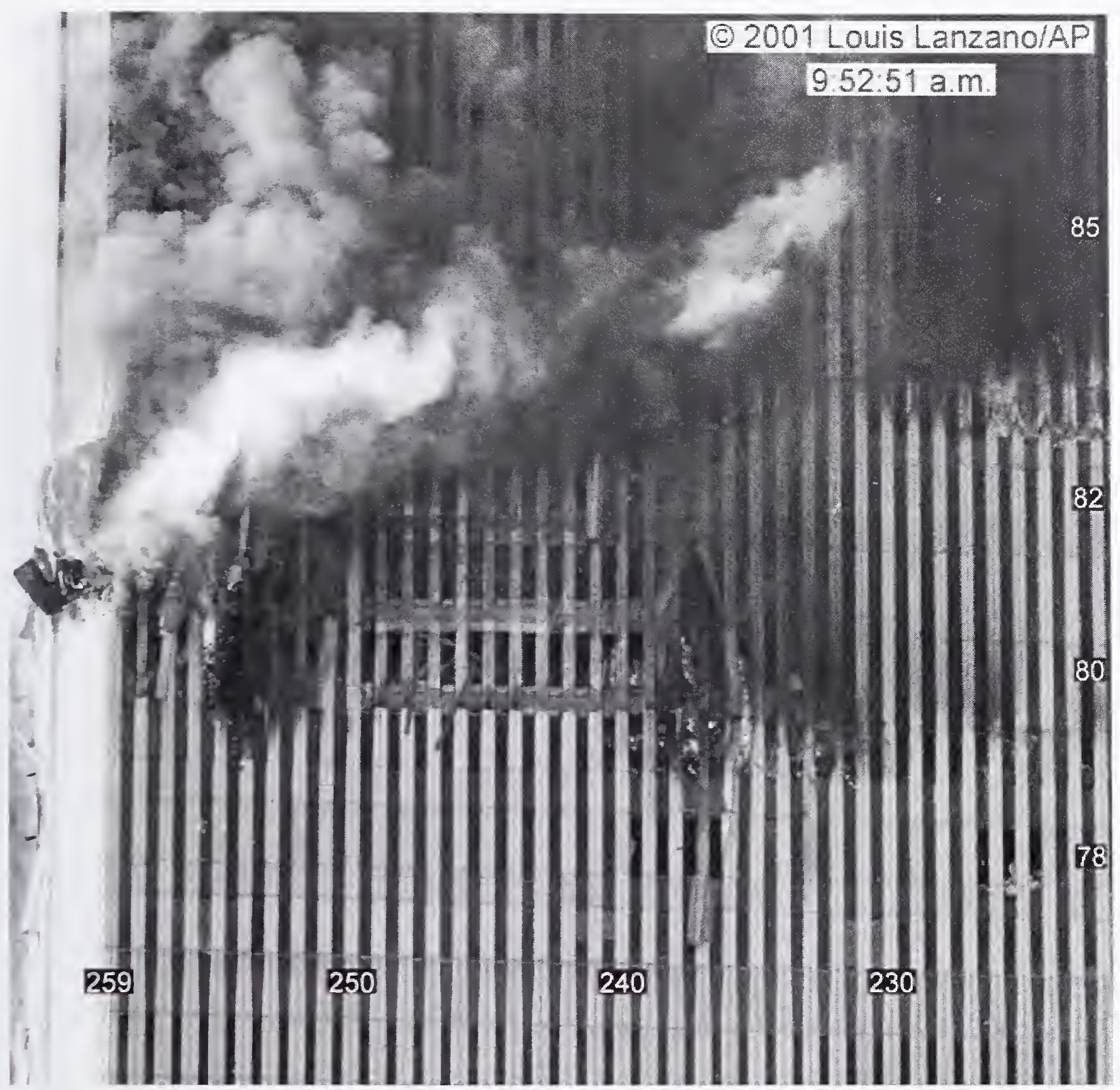

Figure 9-71. This cropped photograph shows the north face of WTC 2 at 9:52:51 a.m. The image has been enhanced by adjusting the intensity levels, and column and floor numbers have been added. The arrows highlight a hanging object visible through open windows on the 80th floor. 


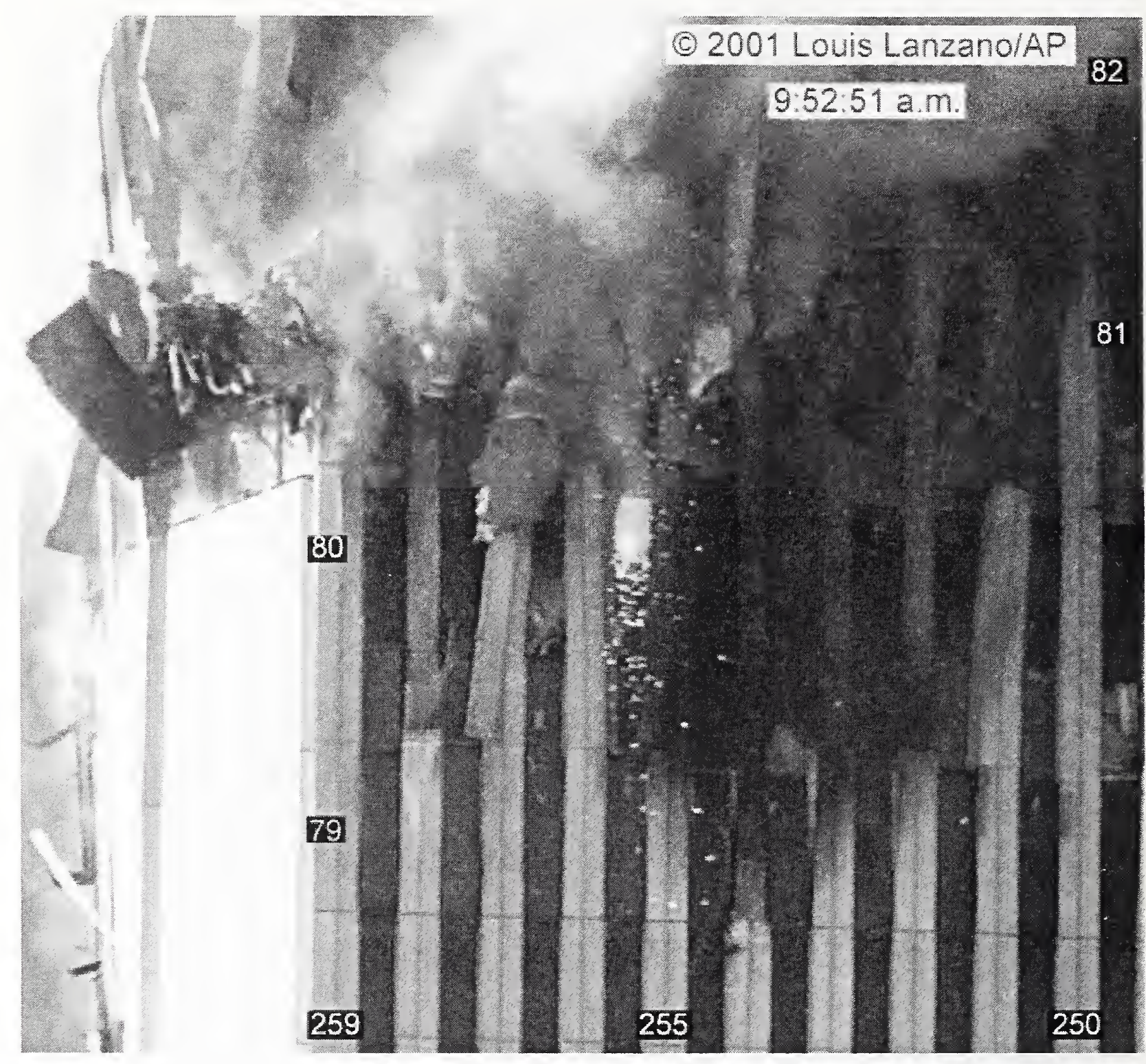

Figure 9-72. This image is a blow-up of a portion of Figure 9-71 showing the north face of WTC 2 around window 80-255 at 9:52:51 a.m. Column and floor numbers have been added.

This image provides a good view of the fire that continues to burn in the northeast corner on the 81 st floor. It can be seen that column 81-254 on this floor is severely distorted. This damage may be à result of the landing gear that was identified as passing through the area during the aircraft impact (see the discussion in Section 7.4.3). Flames are also visible on the 79th floor through open windows 79-252 to 79-254. The orange color visible through the glass that remains in place in window 79-251 and windows $79-255$ to $79-258$ shows that fire is present behind these closed windows as well. This is one of a limited number of cases in which flames were seen through windows with glass still in place. In Figure 9-61, which was shot at 9:46:32 a.m., small flames could be seen in window 79-254. At this time the glass for window 79-252 was still intact. 
It is instructive to compare the image of the cold spot in Figure 9-73 with the close up shown in Figure 9-62, which was taken nearly $6 \mathrm{~min}$ and $20 \mathrm{~s}$ earlier. The first thing to note is that the hanging object on the 80th floor did not change position between 9:46:32 a.m. and 9:52:51 a.m. The fire burning on the west side of the cold spot on this floor has died down somewhat.

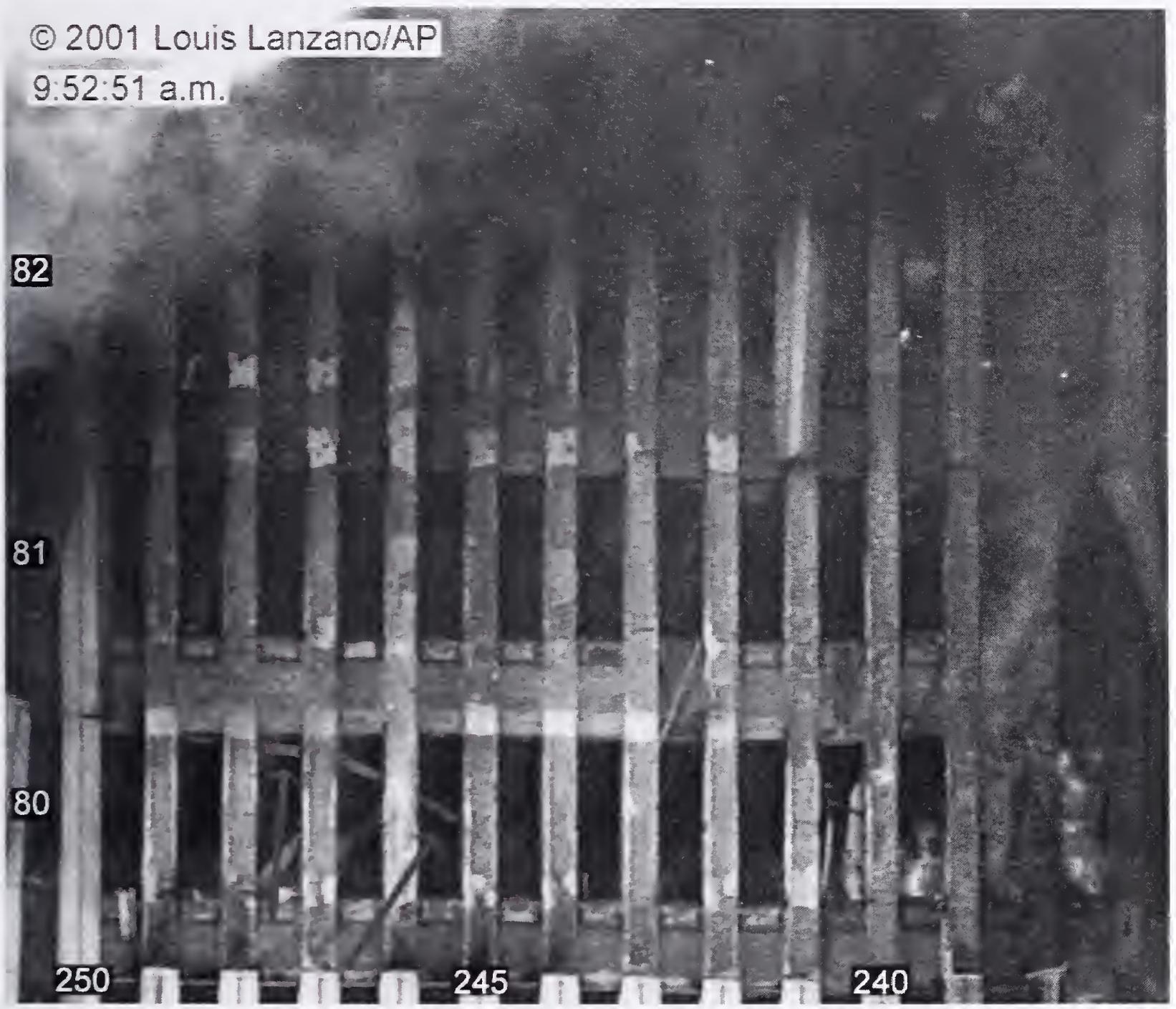

Figure 9-73. This image is a blowup of a portion of Figure 9-71 showing the cold spot on the north face of WTC 2 at 9:52:51 a.m. Column and floor numbers have been added. The arrows highlight a hanging object visible through open windows on the 80th floor.

The hanging object visible on the 81 st floor at the earlier time is not apparent in Figure 9-73. This could simply be due to lighting, since the later photograph is somewhat darker, even though the intensities have been adjusted. In Figure 9-62 small flames were visible through window 81-243. In the later image these flames are not evident, but there is a curved region of dull red flames running from the top of window 81-242 to the middle of window 81-239. It is unclear why these flames are raised, i.e., what is holding them up in the window. One possibility is that the fuel for the fire is on top of a section of the 82 nd floor slab (not visible) that became dislodged and settled below the spandrel at the base of the floor. 
The bright flames visible on the 82nd floor at 9:46:32 a.m. have died down by 9:52:51 a.m., but lower intensity flames are still visible from window 82-237 to window 82-242. The dying fire on the 83rd floor is still visible in windows $83-238$ and 83-239.

As seen in Figure 9-71, the fire located on the 83rd floor in windows 83-221 to 83-225 continued to burn vigorously at 9:52:51 a.m. Recall that flames first appeared in this area at 9:42:53 a.m. Other images available around this time show no apparent additional spread of this fire towards the west. Carets are visible on many of the aluminum column covers between columns 83-222 and 83-236. Many of these carets are near the base of the windows, providing an indication for the intensity of the flames that were present at these locations earlier.

There appears to be a small fire burning on the 84th floor in window 84-223. This is an interesting observation because it is likely that this fire was ignited by flames coming from below, possibly through a window opened by the heat coming from the $83 \mathrm{rd}$ floor. If so, this would represent the clearest example of upward flame spread by an external pathway observed on September 11,2001. On the 79th floor the fire burning on the debris pile is still visible in windows 79-232 to 79-239. The fire on this floor that had earlier spread to the west has died down and is no longer visible.

Videos were reviewed to determine when the fire on the 79th floor first became visible in the area of windows 79-209 to 79-213. The review showed that a flame briefly flickered in window 79-212 at 9:50:58 a.m. A burst of light smoke was released from this window at the same time. At 9:51:13 a.m. a heavier release of smoke came from window 79-213, and a brief flicker of flame was seen here at the same time. A similar flicker of flame and smoke release occurred in window 79-209 at 9:52:45 a.m. The flames in the area appeared to grow rapidly around 9:52:51 a.m.

A photograph of the full width of the north face of WTC 2 at 9:53:33 a.m. is shown in Figure 9-74. Flames are visible on the 79th floor in windows 79-209, 79-211, and 79-212, and smoke is coming from window 79-213. It appears as if window 79-210 still has glass in place. The fires visible elsewhere on the face are very similar to those present in Figure 9-71, which was taken $42 \mathrm{~s}$ earlier. Of particular relevance is the bright emission from the top of window 80-255, which was the source of the molten flow on the 80th floor and the small fire visible on the 84th floor in window 84-223.

Smoke is visible coming from multiple windows near the western edge of the face on the 88th and 93rd floors. These windows have previously been identified as possibly having been broken open by people. Clearly, smoke is continuing to pass up through the core of the tower to these floors.

Around 9:53:47 a.m., bright flames suddenly appeared near window 82-234 on the 82nd floor just to the west of the cold spot. This fire grew rapidly. A video of the area taken at 9:54:02 a.m. shows extended flames coming from windows 82-234 to 82-237.

At 9:53:41 a.m. and 9:53:46 a.m. two pressure pulses forced additional smoke from windows on the north face. Both of these occurrences were accompanied by short flows of molten material from the same window, 80-255, on the 80th floor observed earlier. The largest flow occurred during the second release. Figure 9-75 shows a view of the northeast corner of WTC 2 taken from a video at 9:53:51 a.m. The bright molten stream flowing from the top of window 80-255 is prominent. Unlike earlier images (e.g., see Figure 9-71 and Figure 9-74, both of which were taken within a minute of 9:53:51 a.m.) showing this area of the tower, the intense fire burning on the 81 st floor at the eastern edge of the north face has 
substantially died down, and it is now possible to see clearly the $82 \mathrm{nd}$ and 83 rd floors above for the first time. Videos suggest that the fire intensity at this location decreased around the time of the first pressure pulse at 9:53:41 a.m.

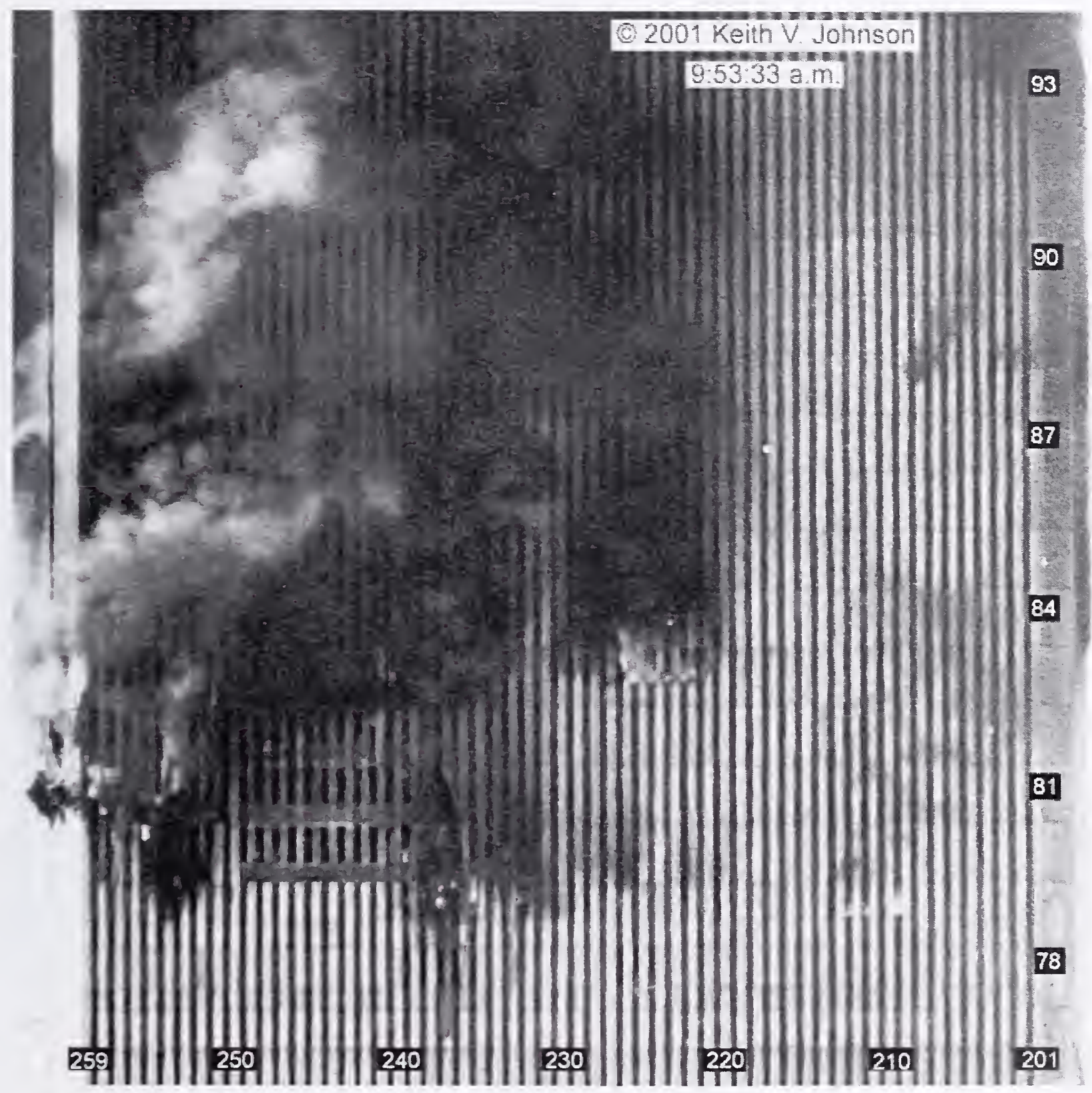

Figure 9-74. This cropped photograph shows the north face of WTC 2 at 9:53:33 a.m. The intensity range has been adjusted, and column and floor numbers have been added.

The arrows in Figure 9-75 highlight a hanging object that is visible through the open windows on the 82 nd floor. In the windows nearest the east edge of the face, the object appears to have a flat surface that is being viewed from below and is drooping downward, away from the windows. The object also seems to be separated into two sections. Further to the west, the hanging object looks as if the edge of a flat object has dropped down below the top of the window from above. Its appearance is similar to the hanging objects seen elsewhere on the north and east faces of WTC 2. 


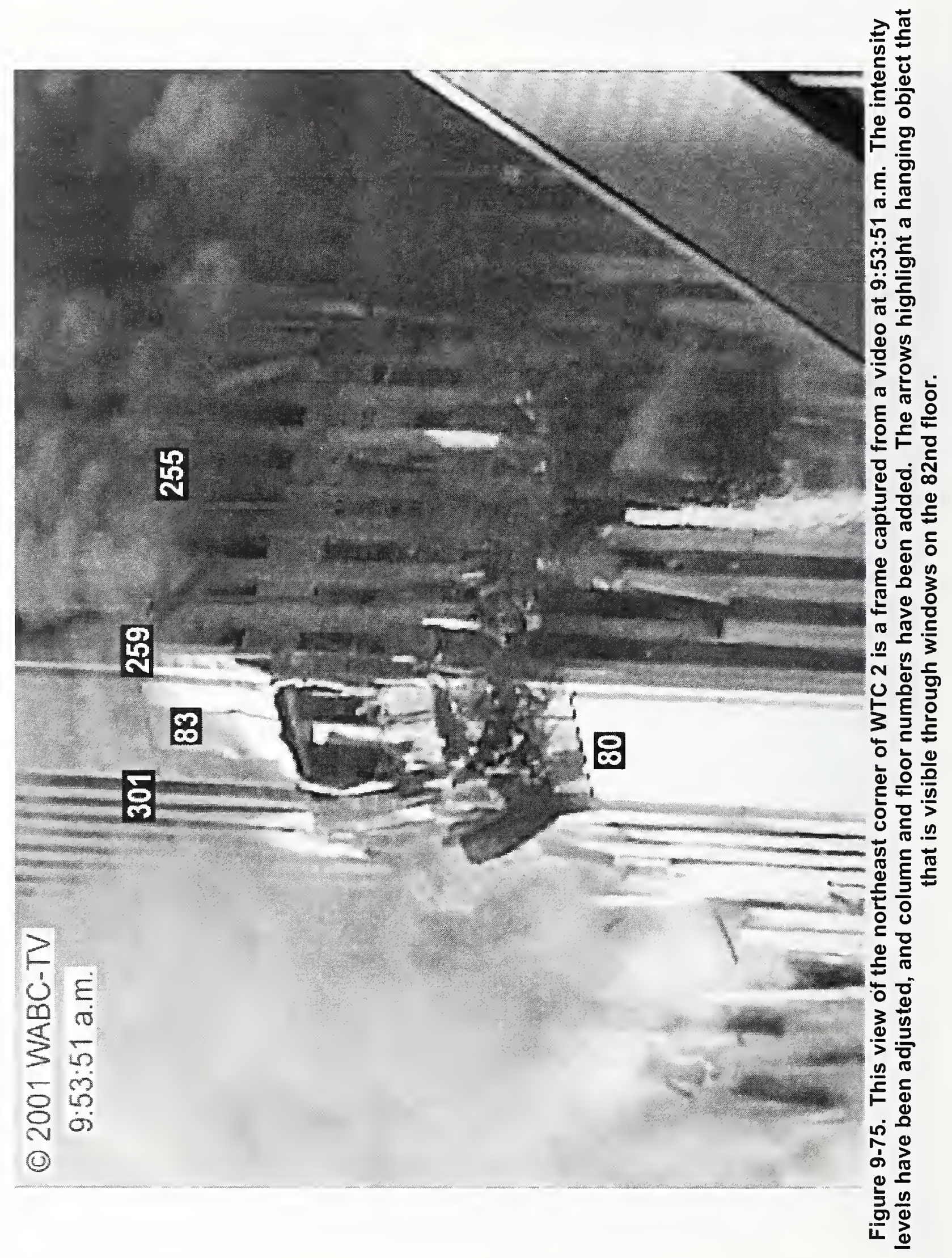


An image of the north face taken at 9:55:18 a.m. is shown in Figure 9-76. This image was shot $1 \mathrm{~min}$ $45 \mathrm{~s}$ after the one shown in Figure 9-74. The fire distributions are very similar in the two photographs, with the exception of the fire on the 82nd floor to the right of the cold spot in Figure 9-76. As discussed above, this fire was first observed at 9:53:47 a.m. burning in windows 82-234 to 82-337. At 9:55:18 a.m. flames were visible in windows 82-231 to 82-236, and flames were coming out of window 82-233. The bright spot at the top of window $80-255$ is visible on the 80 th floor, but there is no obvious molten liquid flow at this time. Unlike in the earlier photograph, fire is now visible in the adjacent window, 80-256.

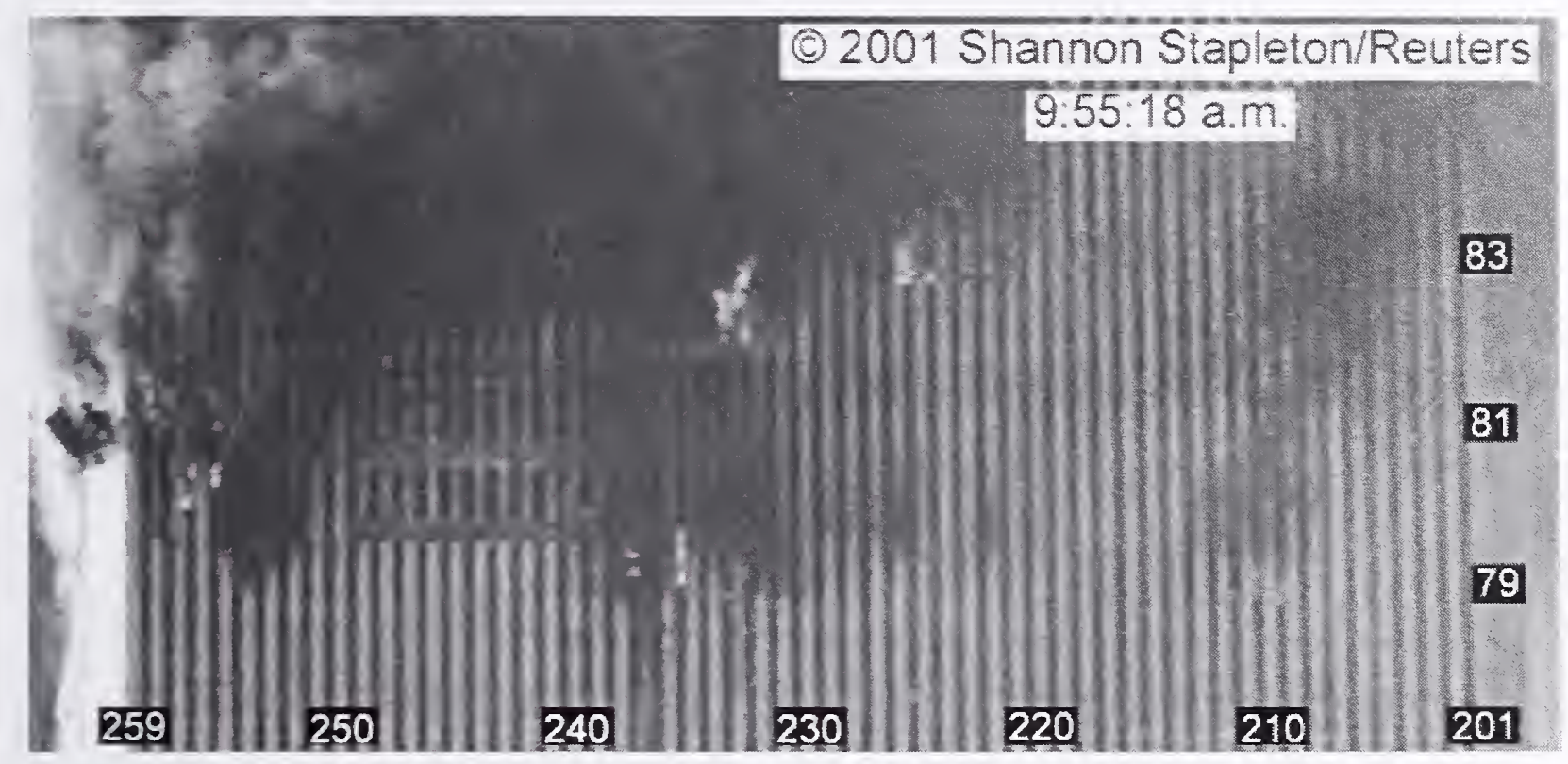

Figure 9-76. This cropped photograph shows the north face of WTC 2 at 9:55:18 a.m. The image has been rotated, and the intensity levels have been adjusted. Column and floor numbers have been added.

During the next several minutes, following the two pressure pulses around 9:53:45 a.m., numerous pressure pulses were noted that pushed smoke from open windows on the north and east faces of WTC 2.

A particularly strong pressure pulse started at 9:56:11 a.m. It lasted nearly $10 \mathrm{~s}$. This particular pulse was strong enough to push flames from the tower at many locations. Following a pressure pulse at 9:56:31 a.m., the flames present on the 81 st floor in the northeast corner opening and nearby flames on the north face abruptly died down. The large flame in window 81-301 on the east face also declined within $2 \mathrm{~s}$. All of these flames reappeared after a few seconds.

At 9:57:21 a.m., shortly after another pressure pulse, the bright light reappeared at the top of the window, $80-255$, on the 80th floor from which the flow of molten material had been observed earlier. Almost immediately, it appeared to jump one window to the east, i.e., to window 80-256. Five seconds later a light flow of molten metal began pouring out of window 80-256. The flow of material from this window would now be nearly continuous until the tower collapsed. At 9:57:32 a.m. there was a fairly intense pressure pulse within the tower. The flow rate of the molten metal increased dramatically at this time. 
Figure 9-77 is a frame from a video that was shot at 9:57:45 a.m. It shows the falling molten metal as it was approaching the ground. The large amount of falling material is evident. Most of the molten metal seems to have broken up into small "droplets," but there are a couple of large pieces falling as well. Apparently, the material flowing out of window 80-256 also included pieces of debris that had not fully melted. There was another very heavy flow of molten metal at 9:58:35 a.m.

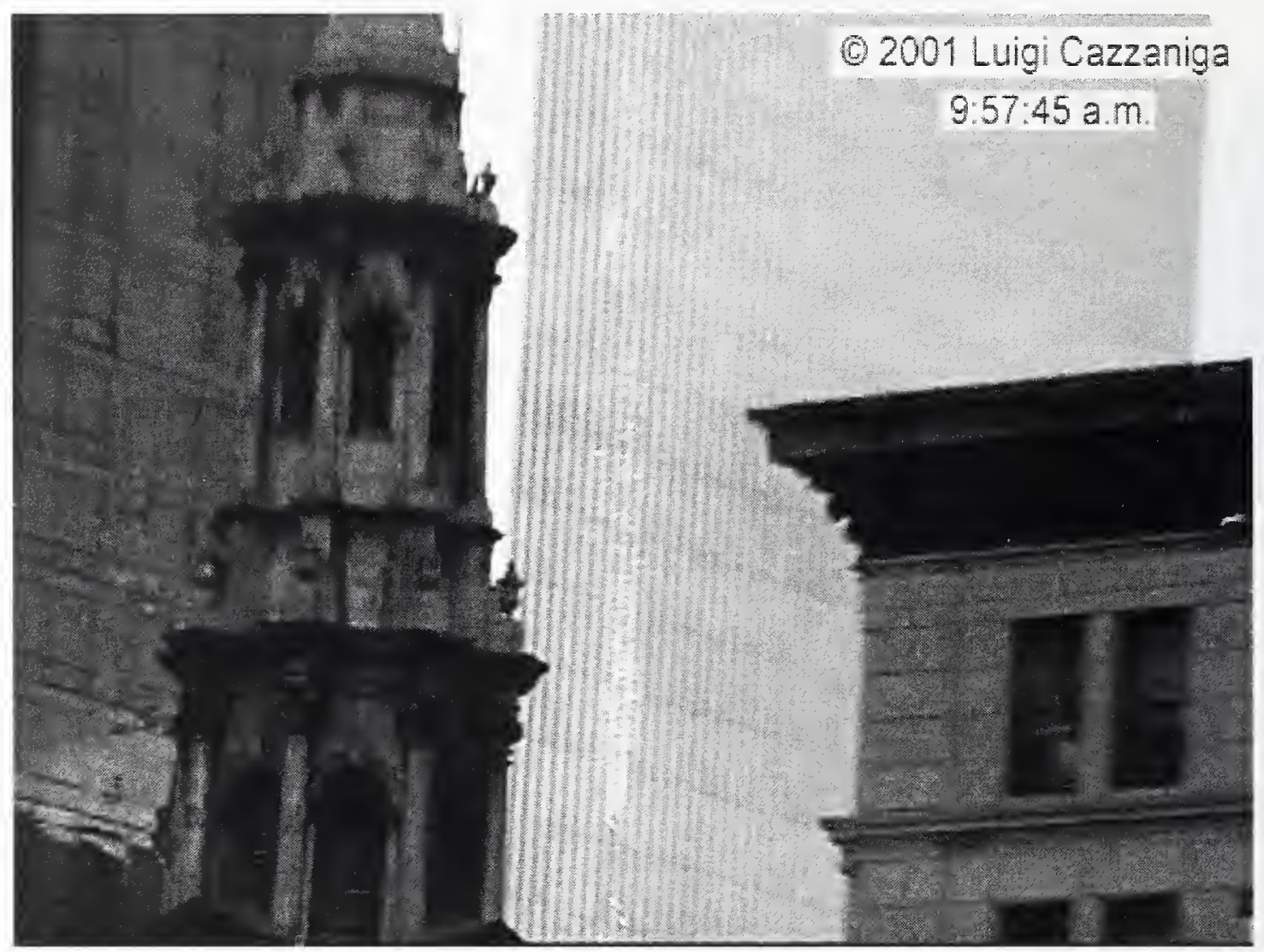

Figure 9-77. This frame showing the lower portion the north face of WTC 2 was captured from a video shot from the northeast at 9:57:45 a.m. The intensity levels have been adjusted.

Figure 9-78 shows a photograph of the north face of WTC 2 taken at 9:58:37 a.m. The bright source of the recent molten metal flows stands out at the top of window 80-256 on the 80th floor. Some metal is visible flowing from the window at this time. An area, including this window and a portion of the cold spot, is blown up in Figure 9-79.

It is useful to compare Figure 9-78 with Figure 9-71, which was taken 5 min and 46 s earlier. Starting on the 83 rd floor, it can be seen that the fire in windows 83-231 to 83-235 has died down considerably over the period between the photographs. In both cases the most intense flames are in window 83-225.

Photographs and videos taken just prior to the collapse provide no indication of westward fire spread on this floor beyond window 83-220. The fire in this area grew rapidly just after 9:45 a.m. Apparently, it did not spread further after this time. The carets visible at the top of the adjacent columns show how intense the burning was in the area. The incipient fire on the floor above in window 84-223 is barely visible. 


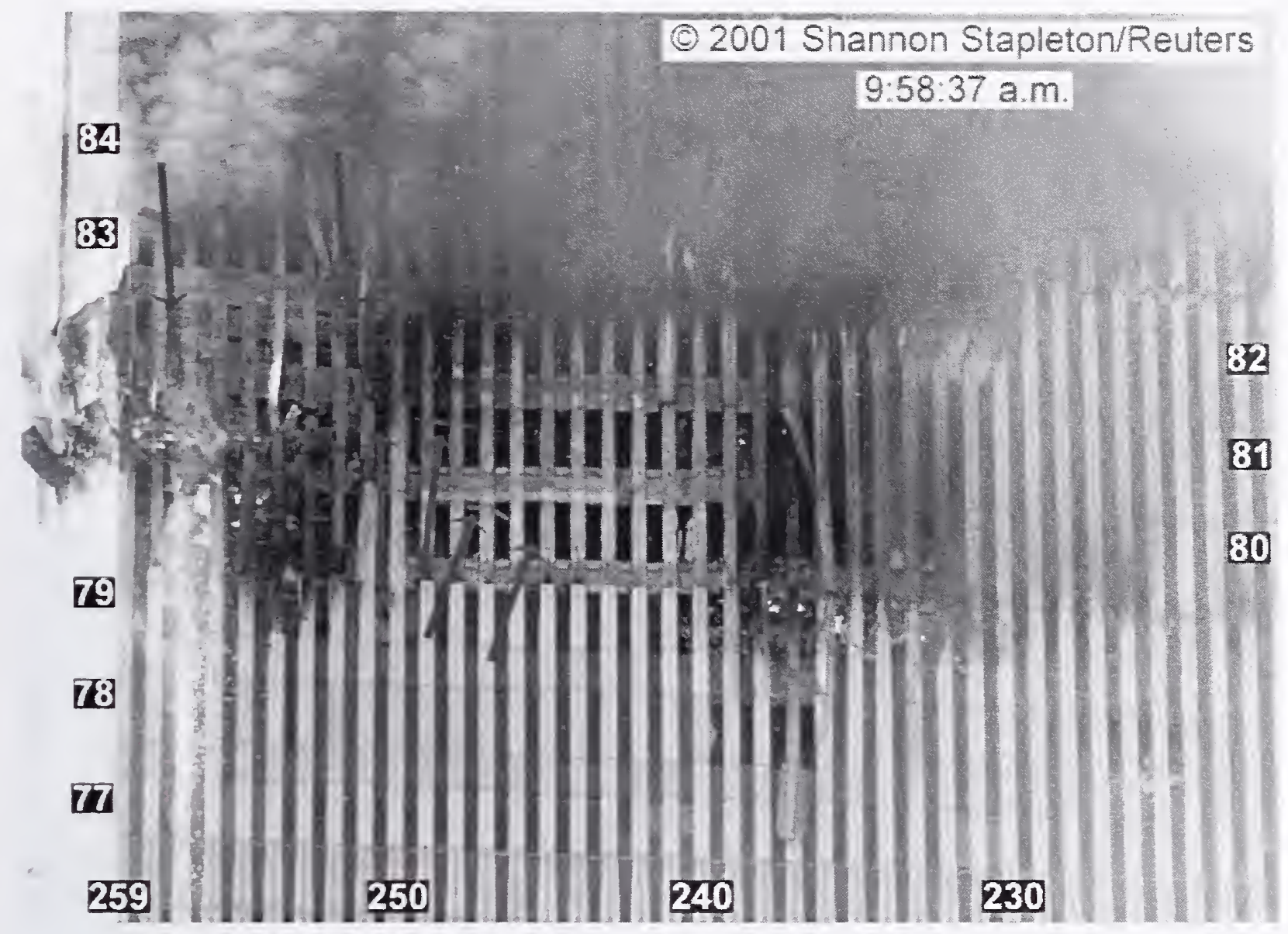

Figure 9-78. This cropped photograph shows the north face of WTC 2 at 9:58:37 a.m. The intensity levels have been adjusted, and column and floor numbers have been added. Arrows highlight hanging objects visible through open windows on the 79th, 80 th, 81st, and 82nd floors.

The fire on the $82 \mathrm{nd}$ floor to the west of the cold spot, which grew rapidly just before 9:54 a.m., has continued to spread and grow. In Figure 9-71 there was no indication of this fire. At 9:58:37 a.m. flames are coming from windows $82-230$ to $82-232$. There are carets on the aluminum column covers around these windows, as well as on the covers next to windows $82-233$ to $82-236$. This fire seems to not only be spreading rapidly, but also to be going through a rapid cycle of growth and decline. Windows to the west of window 82-230 appear to have intact glass. No flames are visible in or to the east of the cold spot on this floor. At the earlier time the area was hidden by smoke.

During the period between when Figure 9-71 and Figure 9-78 were taken, the fires on the eastern edge and the northeast corner of the 81 st floor have died down considerably. Prior to 9:53 a.m. intense fires had burned in these areas continuously following the aircraft impact. After this time it was observed that these fires suddenly died down and then reappeared several times. These cycles seemed to be associated with pressure pulses that pushed smoke and/or dust out of open windows on multiple floors of the north and east faces. This may be an indication that these fires died down due to changes in their local environment, e.g., a partial collapse of a floor, as opposed to simply burning out the available fuel. A low-level fire continued to burn at the western edge of the cold spot on the 81 st floor. There had been no 
apparent westward spread on this floor since window glass was still in place for windows to the east of window 81-236.

The fires burning on the 79th and 80th floors between columns 230 and 241 have also died down. As a result, the large amount of debris piled up in windows 79-234 to 79-240 is more apparent than in earlier images. It is still not possible to identify whether the fires on these two floors were burning on a single pile of debris, i.e., the intervening floor had collapsed, or as two independent fires.

The only indication in Figure 9-78 of a fire burning on the 79th floor to the immediate west of window 79-229 is a small flame visible through a partially broken window 79-224. The fires that had been burning in this area earlier have nearly died out. Windows 79-221 and 79-223 to 79-225 still have glass. Half of the window pane is in place in window 79-222. These observations suggest that the fire that burned in this area was not too intense. Videos show that the fire that grew further west in windows 79-109 to 79-113 (see Figure 9-76) continued to burn at a comparable intensity until the tower collapsed. In Figure 9-78 it can be seen that window glass remains in place on the 80th floor in window 80-231 and windows to the west. Videos show occasional smoke coming from window 80-204 starting after 9:55 a.m. There is no indication of fire at this location. This window may have been broken earlier by a person.

The only fires visible in the cold spot are small spot fires at the western side of the 81 st floor and in the lower east side of the 80 th floor.

The blow-up of a section of Figure 9-78 shown in Figure 9-79 provides a closer view of the area around the 80th-floor window 80-256 and a portion of the cold spot. Since smoke is no longer obscuring the east side of the 81 st floor, it is possible to see additional details concerning the damage in this area. The distortion of column 254 on the 81 st floor, which was described earlier, is apparent. The column just to the west, 253 , has been cut through near the top of the window. The spandrel at the base of the 82 nd floor between column 251 and column 252 appears to be torn. It is not possible to say with certainty, but it is likely that this steel damage resulted from the large object observed exiting near this location during the aircraft impact (see the discussion in Section 7.4.3) and that it is not due to subsequent events.

A number of arrows have been added to Figure 9-79 to highlight hanging objects visible through open windows. These objects are possibly sections of floors slabs that became dislodged and settled down from above. The appearance of the object visible through windows 82-250 to 82-258 on the 82nd floor supports such a conclusion. This object was previously identified in a somewhat lower resolution image shown in Figure 9-75. Its appearance does not seem to have changed since 9:53:51 a.m. In windows 82-250 to 82-252 the object is hanging just above the tops of the windows. In these windows it looks like the hanging objects observed elsewhere on the north and east faces. However, further east it is apparent that a portion of the object located away from the edge has collapsed, and what is visible is the bottom of the object that is sloping away from the tops of the windows. It can be seen that the object has split into two sections in the east-west direction. This object is most likely a section of the $83 \mathrm{rd}$ floor that has locally collapsed and, in the process, broken along the line between two prefabricated sections. Drawings of the floor system indicate the sections were laid in this direction.

On the 81st floor a hanging object is apparent in Figure 9-79 at the tops of windows 81-247 to 81-250. A faint trace of the object in the two windows to the west indicates the object is sloping sharply downward in windows 81-246 and 81-245. This object was not visible in the close-up of the area shown in 
Figure 9-73, which was taken at 9:52:51 a.m. This was true even when the intensity levcls for the photograph in Figure 9-73 were adjusted to emphasize the darkest levels. Interestingly, a hanging object is visible in this same vicinity in Figure 9-62, which was taken at 9:46:32 a.m., but it was located further west and had a different shape. A detailed comparison shows that the objects in the two photographs are at roughly the same height in window 81-250, but while the object curved upwards in windows 81-249 and 81-248 and disappeared above the spandrel in window 81-247 in Figure 9-62, in the latcr image it slopes downward in windows 81-249 to 81-247. At the earlier time the object reappeared in window 81-245 and then sloped gradually downward toward the west. In Figure 9-79 the object nearly disappears in window 81-246 and seems to be curving strongly downward to the west.

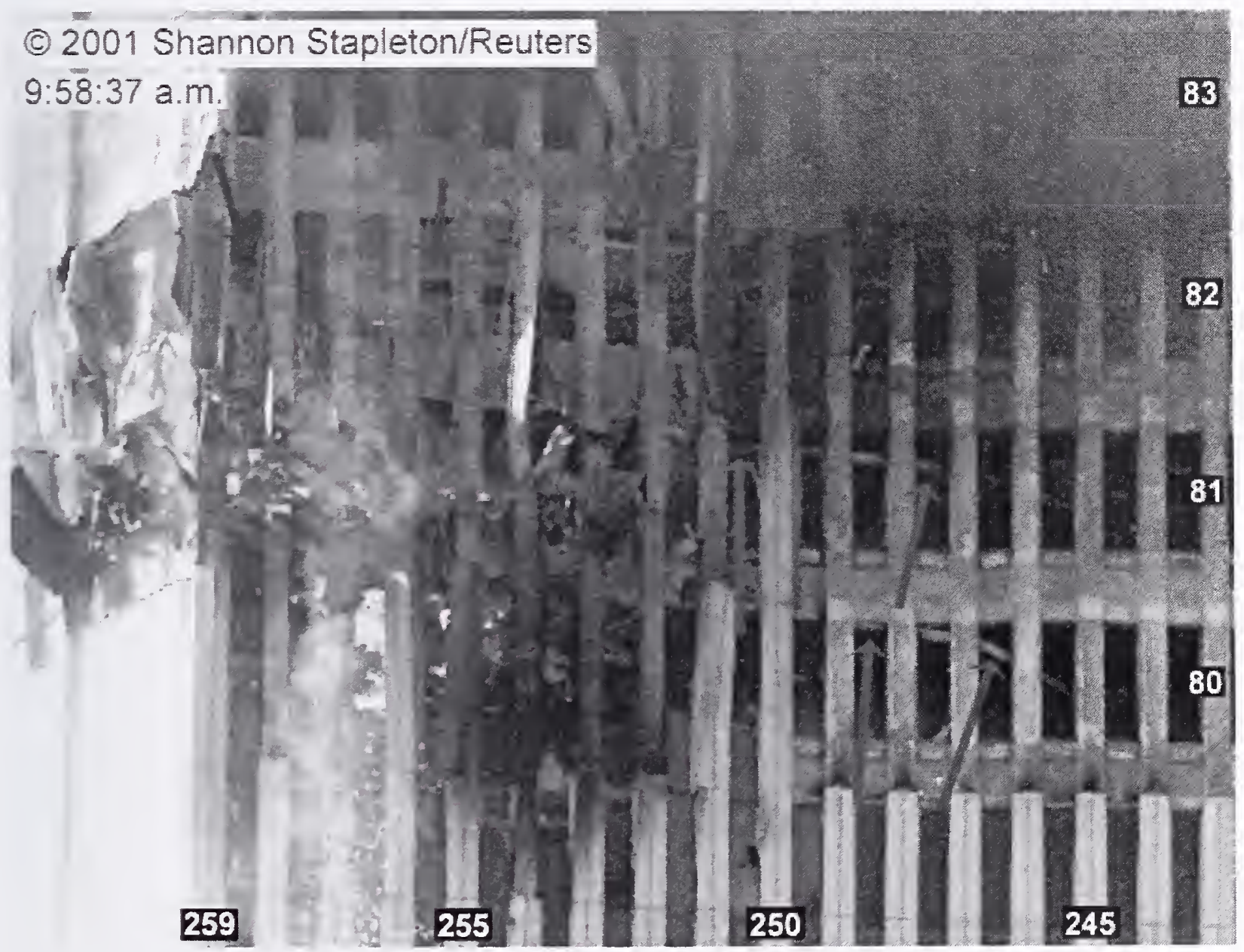

Figure 9-79. This photograph is a blow-up of a portion of the photograph shown in Figure 9-78. It shows the north face of WTC 2 and includes window 80-256 and part of the cold spot. The intensity levels of the original have been adjusted, and column and floor numbers have been added. Arrows highlight hanging objects visible through open windows on the 79th, 80th, 81st, and 82 nd floors.

It is difficult to understand why the object is not visible in Figure 9-73 at 9:52:51 a.m. Either it is hidden by some unanticipated optical effect or the object moved between 9:46:32 a.m. and 9:52:51 a.m. If the former is the case, it means that the object visible at 9:46:32 was obscured sometime between 9:46:32 a.m. and 9:58:37 a.m. On the other hand, if the latter is the case, it suggests that the object visible at 9:46:32 a.m. sank down out of sight between 9:46:32 a.m. and 9:52:51 a.m. and that a second object 
appeared in the windows from above sometime between 9:52:51 a.m. and 9:58:37 a.m. It has not proven possible to make a clear choice between these two alternatives.

In contrast to the 81 st floor, the appearance of the hanging object visible through windows $80-245$ to 80-248 on the 80th floor in Figure 9-79 is very similar to that seen in Figure 9-52 (taken at 9:42:31 a.m.), Figure 9-62, and Figure 9-73. Recall that this object appeared at this location sometime between 9:27:04 a.m. and 9:29:42 a.m. It has remained stable for around $30 \mathrm{~min}$.

There appears to be a short hanging object visible in 79th floor windows 79-252 to 79-254. In most of the earlier high resolution images this area was hidden. Comparisons with Figure 9-12 (9:10:01 a.m.) and Figure 9-17 (9:14:03 a.m.) are not conclusive, but suggest that this object has been present since shortly after the aircraft impact.

At 9:58:59 a.m. WTC 2 began to collapse. Videos show that the only place fire was pushed out of windows on the north face during the collapse was from the burning area near windows 79-209 to 79-213 on the 79th floor. Here there was a bright burst of flame similar to those observed when WTC 1 collapsed. Smoke, but no flames, was pushed out of the open window 80-204 on the west side of the 80th floor. The active fire burning near the center of the $82 \mathrm{nd}$ floor appeared to be snuffed out during the collapse.

Review of videos shows that there were two additional periods of heavy smoke flow from windows located on the east faces of the 79th and 80th floors between 9:52 a.m. and the time of collapse of WTC 2 . The first of these releases lasted from roughly 9:52:25 a.m. to 9:53:01 a.m. and the second from 9:55:55 a.m. to 9:56:50 a.m. Both of these primarily involved smoke, but external flames were observed on the 79th floor near window 79-133 during the first release. Videos of the second release were shot from longer distances, and any flames present may not have been visible from this distance.

A partial view of the east face of WTC 2 recorded at 9:52:54 a.m. is shown in Figure 9-80. This image was taken just after the conclusion of the first smoke release described in the last paragraph. The light smoke flow on the face is evident. Even though this picture is fairly close up, the only flames visible on the 81 st and 82 nd floors are in windows 81-301,81-302, and 82-303. The photograph was shot from a location near the towers, and, as a result, parallax effects limit views of fires inside the tower. Even so, it is clear that the fires are considerably less intense than seen in Figure 9-67, which was shot at 9:47:10 a.m.

In Figure 9-67 heavy smoke was flowing from windows 79-314 to 79-320 on the 79th floor, and flames were extended from a number of these windows. In Figure 9-80 relatively light smoke is coming from windows 79-319 to 79-321, and there is no indication of external flaming. Apparently, the fires present in this area roughly $51 / 2$ min earlier have died down considerably. Carets at the tops of the covers for columns 79-312 to 79-324 indicate the relative local intensities of the fire that burned in this area earlier. In the photograph taken at 9:47:10 a.m. (Figure 9-67) the most intense burning at the northern edge of the 82 nd floor was in windows 82-306 and 82-307. At the later time flames are only visible in window 82-303. On the other hand, the flames on the northern edge of the 81 st floor are clearly more intense at the later time. Overall, the number and intensities of the fires on the east face appear to be at their lowest levels since the aircraft impact 50 min earlier. 


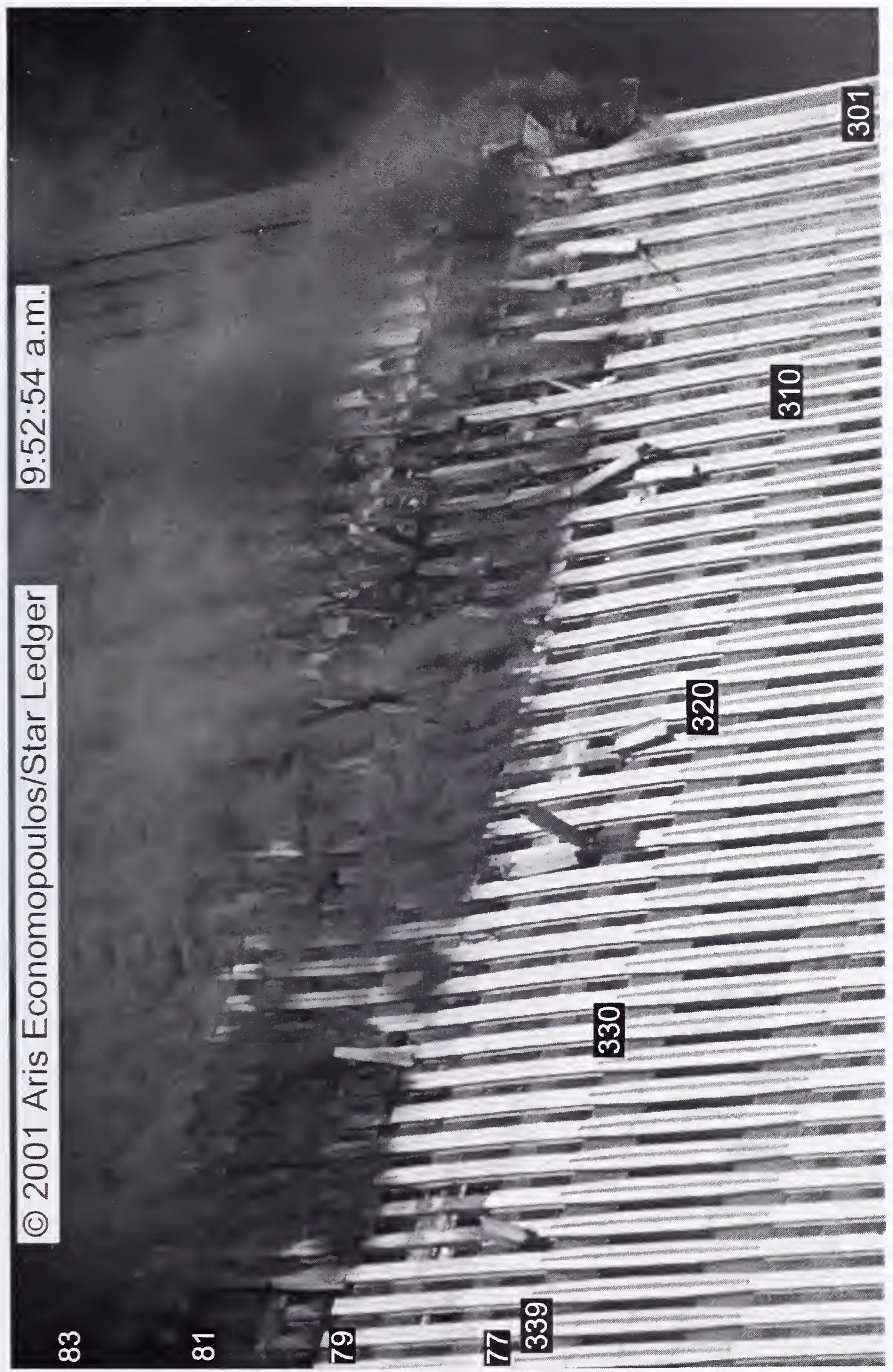


Figure 9-80 was shot from the southeast. Due to the angle involved, it is possible to see the column bowing on the 80 th and 81 st floors that was previously discussed with regard to Figure 9-46 and Figure 9-59, images shot from the northeast. The largest deflections appear to be in the vicinity of column 330 .

Figure 9-81 is a photograph of the east face that was taken at 9:55:04 a.m. This image was shot from a longer distance and from a higher position than Figure 9-80. In this image it is apparent that flames are present inside windows on the 79th floor over a length running from window 79-311 to window 79-319. This is roughly the same location as seen in Figure 9-67 at 9:47:10 a.m. Consistent with Figure 9-80, there is little smoke and no flame coming from the windows where fire is visible in Figure 9-81.

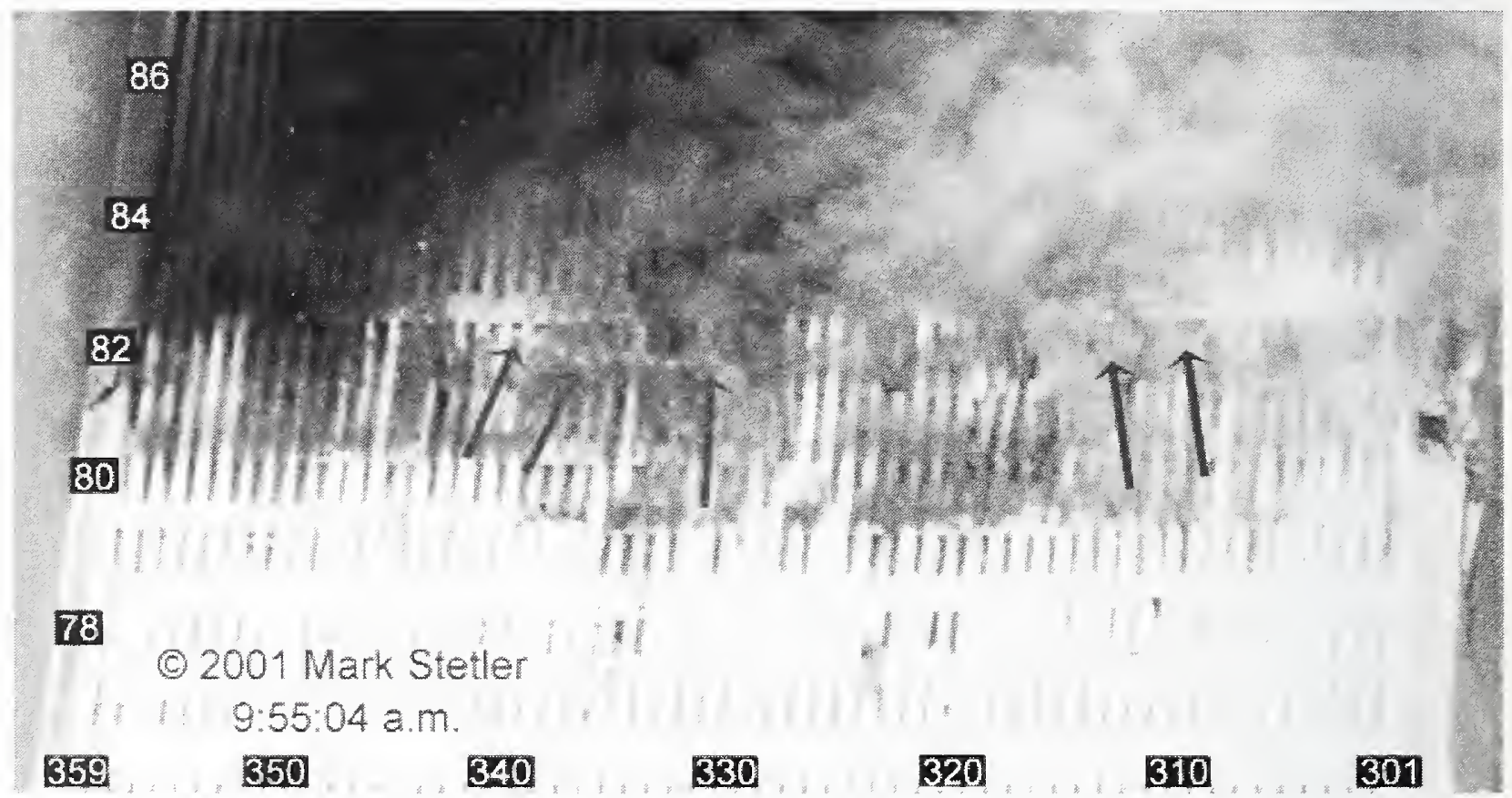

Figure 9-81. This cropped photograph of the east face of WTC 2 was shot at 9:55:04 a.m. The image has been rotated, and the intensities have been adjusted. Column and floor numbers have been added. Arrows indicate a hanging object visible through open windows on the 82 nd floor.

There is also an area of flame visible on the 80th floor in Figure 9-81 extending from window 80-316 to window 80-326. Windows to the north of this length are hidden by smoke, while windows 80-327 and 80-328 on the other end still have glass in place. It is difficult to identify when this fire developed. These windows are visible in the east face view shown in Figure 9-48, which was taken at 9:38:22 a.m. At this time there was no indication of a significant fire in this area of the 80th floor, even though the fire on the 79th floor was already well developed. In similar views of the face shown in Figure 9-58 (9:40:29 a.m.), Figure 9-66 (9:45:40 a.m.), and Figure 9-67 (9:47:10 a.m.), smoke from the 79th floor hides these windows. Since the flames appear to be well inside and there is no smoke coming from the windows, it is not surprising that this fire can not be seen in Figure 9-80.

The only other area where a significant fire is visible on the east face at 9:55:04 a.m. is in windows near the north edge of the 81 st floor and in the opening on the northeast corner. Since these flames extend from the opening, they are also visible in Figure 9-80. 
The arrows in Figure 9-81 highlight the hanging object visible through open windows on the 82nd floor. This object has been observed in a number of images, including Figure 9-67 at 9:47:10 a.m. On the southern end around window 82-340 the apparent position of the object has not changed during the almost seven minutes between the times when these photographs were taken. This is not the case at the northern end. At the earlier time the object was visible near the tops of windows 82-310 and 82-311. At 9:55:04 a.m. the object is visible at the top of window 82-309 and can be seen sloping downward across several open windows toward the left, approaching the base of window 82-315. This is anothcr example of hanging objects, which may be dislocated floor slabs, changing position during the event.

A photograph (not reproduced here) taken at 9:55:31 a.m. from the same location as Figure 9-81 is very similar to Figure 9-81. In particular, the fires on the 79th and 80th floors are visible. The hanging object can be seen and does not appear to have changed position. There are large flames coming from the $81 \mathrm{st}$ floor at the opening on the northeast corner. This photograph is the last image of the east face in the data base shot from a location that is advantageous for seeing fires inside windows on WTC 2. Images of the face after this time are shot from less direct angles or from locations close to the towers.

An image of the east face of WTC 2 shot from near the corner of Church and Fulton Streets at 9:58:02 a.m. is shown in Figure 9-82. From this angle the only flames visible on the east face are in windows 81-301 and 81-302 on the 81 st floor. Unlike at 9:55:04 a.m. (see Figure 9-81), there are no large flames visible in the opening on the northeast corner of the 81 st floor. There is relativcly little smoke coming from open windows on the face.

The bowing of the columns as they pass through the 81 st and 82 nd floors is easily identified in Figure 9-82. The extent of the bowing seems to be largest around column 330 and appears to decrease in both horizontal directions moving away from the center. On the south side of the face the bowing is not apparent for columns to the left of column 347. Columns on the north side are partially obscurcd by smoke. making definite conclusions difficult, but it appears as if column 309 remains straight.

The bright spot on the 80 th floor of the north face in window $80-256$ is the source of the molten metal that was pouring from the tower around this time.

Pieces of debris were observed falling from WTC 2 at various times after the aircraft impact. At around 9:58:34 a.m. a large number of pieces fell over a period of $3 \mathrm{~s}$ from the vicinity of the 80th and 81st floors along a line running between the north edge of the cast face and column 318 . The largest number of pieces seemed to come from an area centered near column 312. The falling debris coincided with a heavy flow of molten metal from window 80-256 on the 80th floor of the north face.

An even larger number of pieces of debris started falling from the samc area around 9:58:49 a.m. This shower of debris lasted until the tower collapsed $10 \mathrm{~s}$ later. Figure 9-83 shows a photograph taken at 9:58:56 a.m., i.e., 3 s prior to the start of the collapse of WTC 2, from roughly the same location as Figure 9-82. A number of pieces of falling debris are visible on the north side of the east face.

The bowing of the columns near the center of the face stands out in Figure 9-83. Quantitative comparisons are difficult, but the bowing appears greater than in Figure 9-82. The fire visible at the northeast corner has a similar appearance to that seen earlier. 


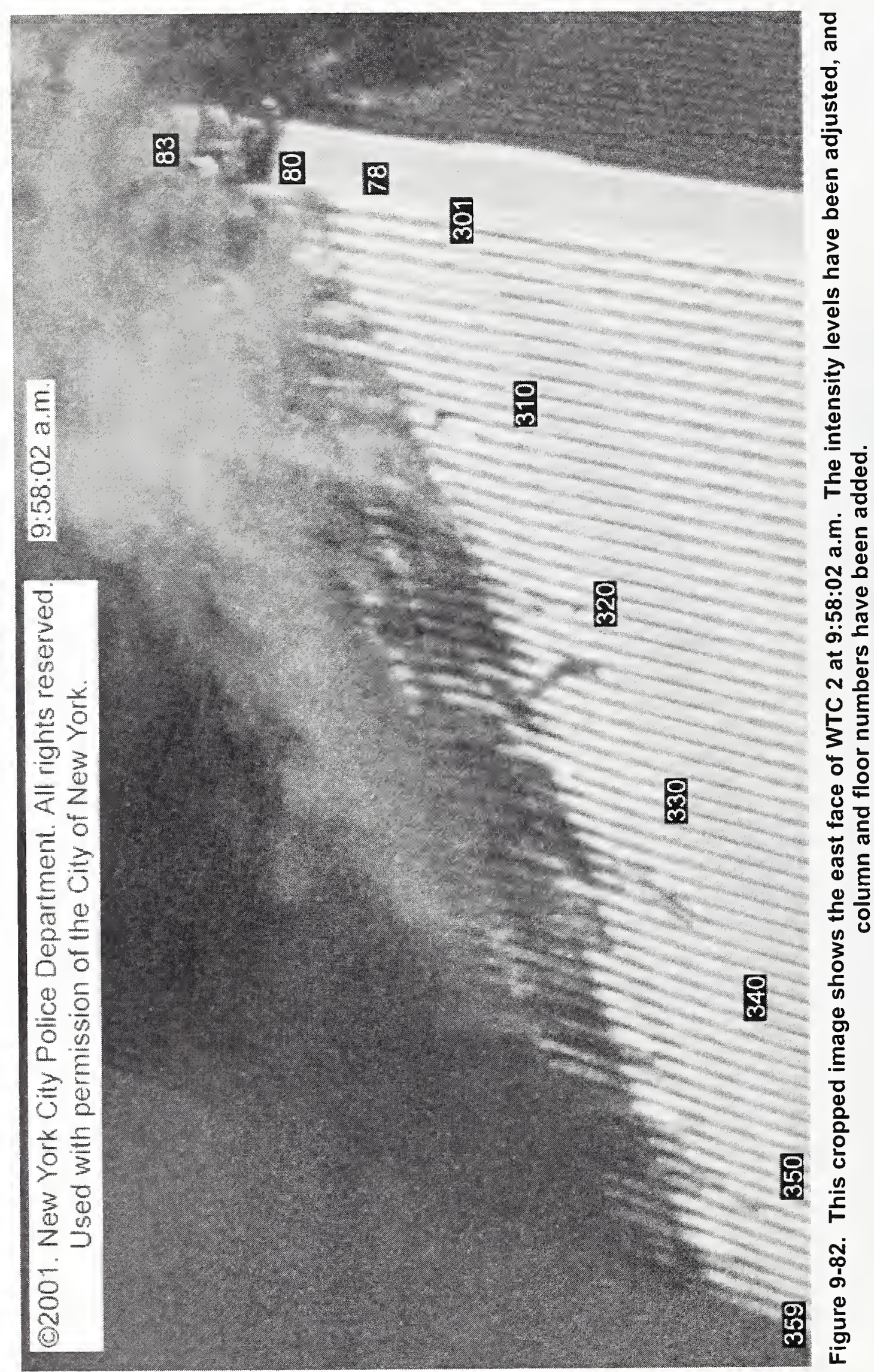




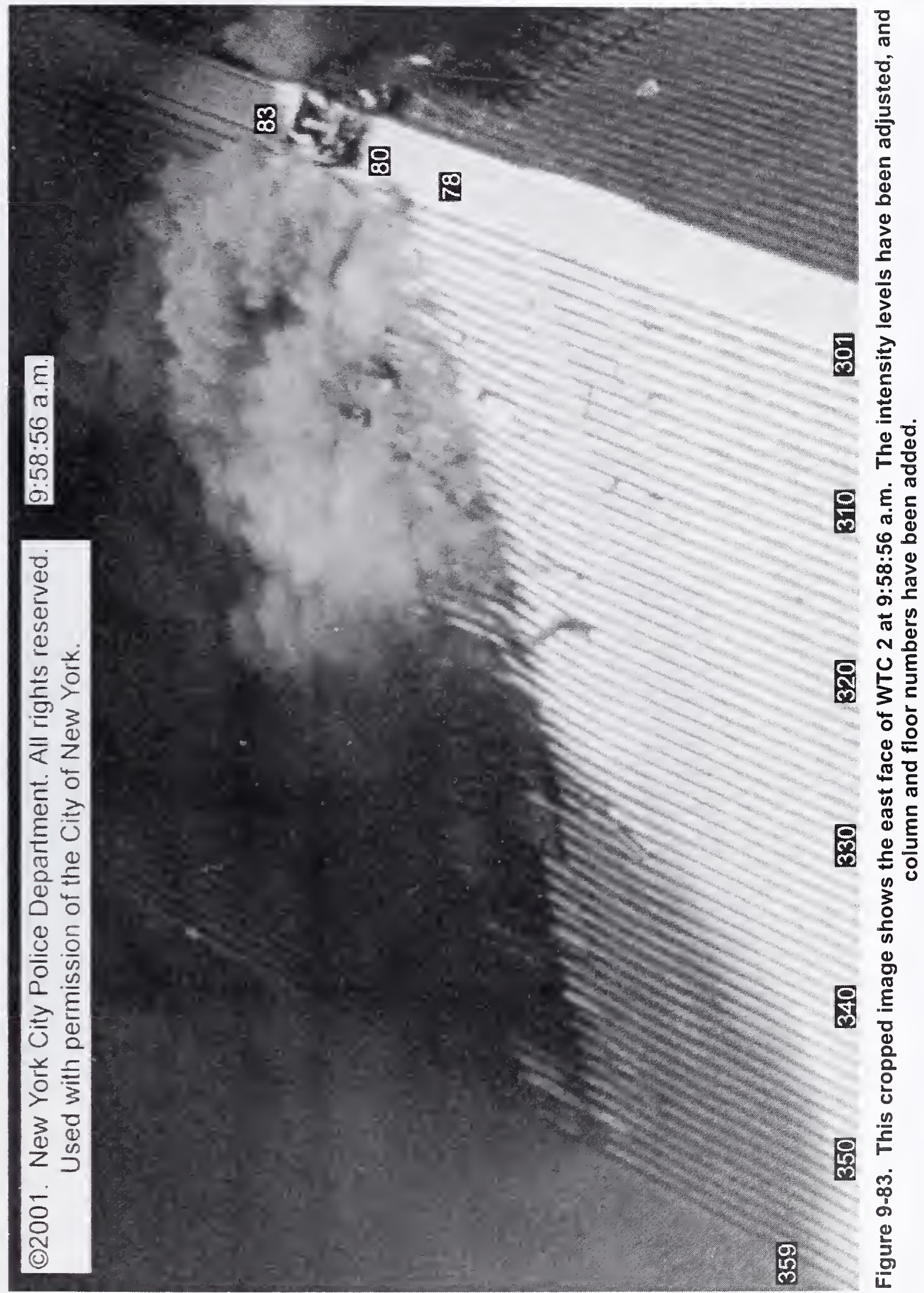


When WTC 2 collapsed, very little flame was expelled from east face windows. The fire on the northeast corner brightened briefly, and a small amount of flame was observed coming from near window 80-126 on the 80th floor. Based on observations during the collapse of WTC 1 and on other faces of WTC 2, regions of intense fire would be expected to generate short-lived jets of flames from nearby windows during the start of the collapse. The absence of such jets indicates that areas of intense burning were not present on the east side of the tower at the time of collapse. This conclusion is consistent with visual observations of fires prior to the collapse.

Figure 9-68 shows a view of the south face of WTC 2 shot from the southeast at 9:52:00 a.m. Another view of this face shot from the southwest at 9:52:27 a.m. is shown in Figure 9-84. A couple of spot fires are visible in Figure 9-68, and no fires are visible with the resolution and viewpoint of Figure 9-84. A long-distance video shot by a news helicoptcr shows the south face of WTC 2 around this time. At 9:51:55 a.m. a flame could be detected on the 80th floor to the west of the aircraft impact cavity. At around 9:52:35 a.m. the video zoomed in somewhat closer (still a distant view), and fire could be identified on the 80th floor roughly located between windows 80-436 and 80-446. Recall that a shortlived burst of flame had been observed in window 80-439 at 9:47:50 a.m. Around 9:52 a.m. the fire in the area appeared to have a relatively low intensity and to be intermittent. A small fire was also visible on the 79th floor near window 79-444.

At 9:52:52 a.m. there was a substantial release of smoke from the 80th floor near window 80-446. It was accompanied by a smaller puff of smoke from near window 80-440. Another release of smoke from the area occurred at 9:54:01 a.m. This release was further west, perhaps as far as window 80-450.

Figure 9-85 shows a low resolution image of the south faces of WTC 1 and WTC 2 taken at 9:54:24 a.m. in which the fire on the 80th floor of WTC 2 appears as a bright orange line to the left of the aircraft impact cavity. A light stream of smoke coming from near window $80-450$ is also visible to the west of the fire. The presence of this smoke indicates that this rapidly growing fire is also likely spreading to the west, since this window has been opened. The fire on the 80 th floor is the only fire visible on the face in this long-distance shot.

The video shot from the news helicopter shows that over the next few minutes the line of fire on the 80th floor had a steady appearance similar to that in Figure 9-85. Smoke flow from the fire area was generally light, even though there were occasionally streams of smoke released from various windows along the observed fire length. This situation changed at 9:56:44 a.m., when a heavy release of smoke lasting over $20 \mathrm{~s}$ took place from windows along the entire length of the fire. At this time the smoke appeared to be coming from as far west as window 80-454. Within $5 \mathrm{~s}$ of the start of the heavy smoke flow, large flames erupted from several windows centered near window 80-442. There was also a short flame flare from near window 80-450.

By 9:57:49 a.m. the smoke and flames on the 80th floor of the south face had subsided somewhat. The view of the south and west faces of WTC 2 at 9:57:49 a.m. shown in Figure 9-86 was taken from a NYPD helicopter looking down on the tower from the southwest. The line of smoke and flame to the west of the aircraft impact cavity on the south face is visible extending nearly to the west edge. Heavy smoke also appears to be coming from the 81st floor. There is a bright flame region visible near the 79th floor inside the aircraft impact cavity. Flames were observed in this general vicinity in several groundbased images including those in Figure 9-26 (9:23:08 a.m.) and Figure 9-41 (9:30:19 a.m.), even though such a large fire was not evident. 


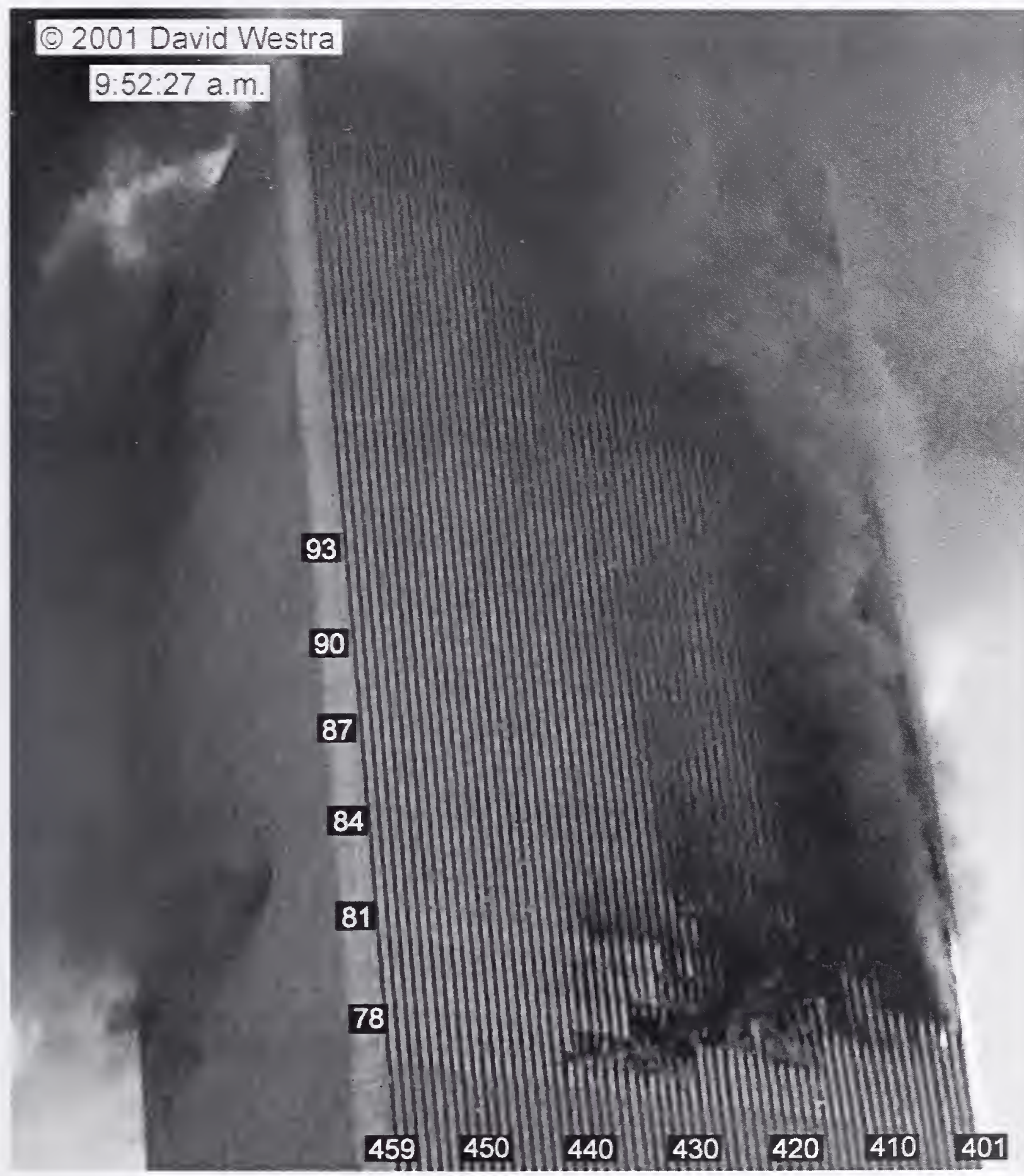

Figure 9-84. This cropped photograph shows the south and west faces of WTC 2 at 9:52:27 a.m. The intensity levels have been adjusted, and floor numbers have been added. 


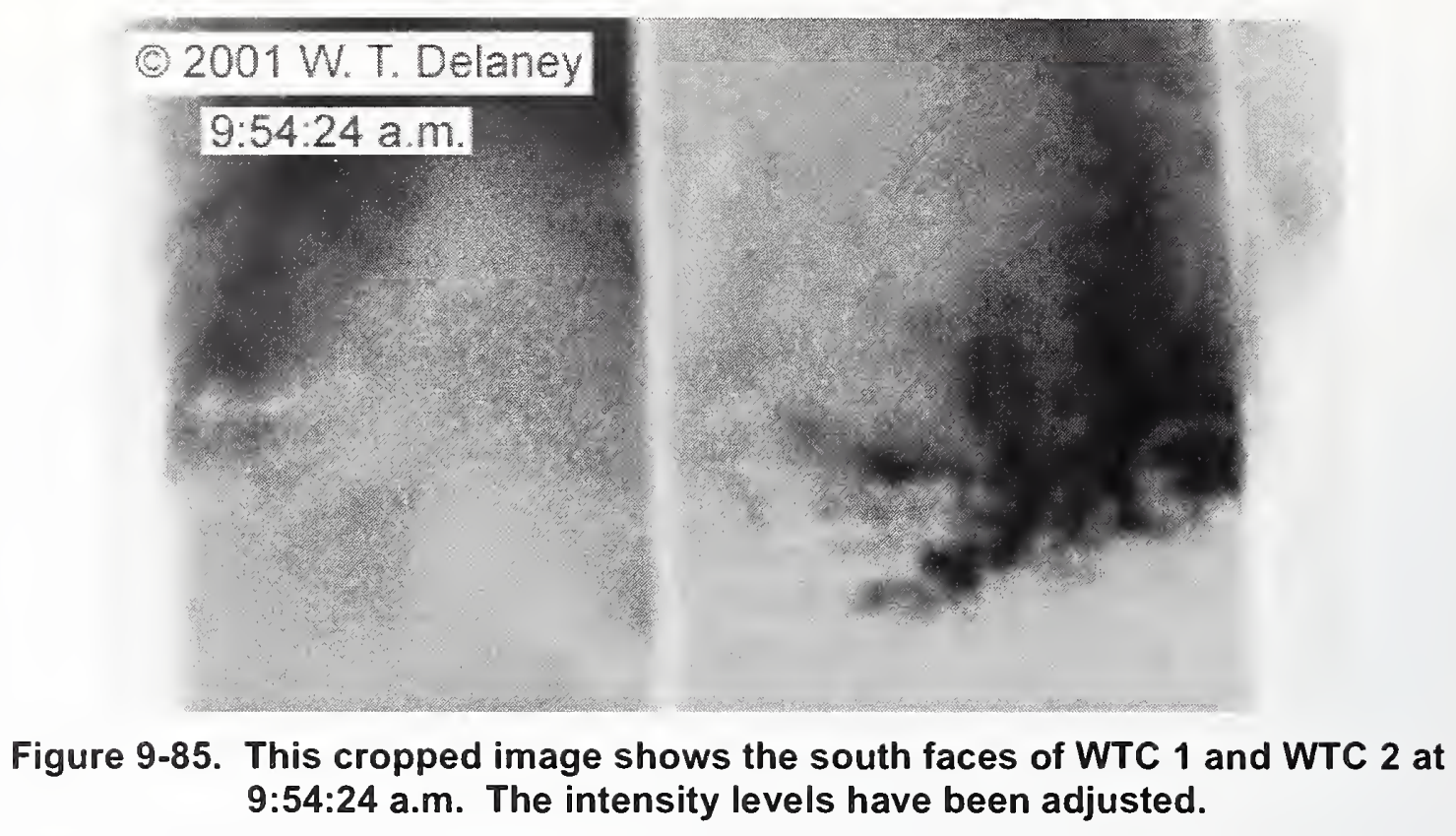

A view of the south face of WTC 2 captured from a video at 9:58:49 a.m. is shown in Figure 9-87. It was taken just $10 \mathrm{~s}$ before the start of the collapse of the tower. Flames are visible on the 80th floor from windows $80-440$ to $80-452$, and smoke is flowing from open windows $80-438$ to $80-455$. The aerial video shows that the bright region of flame near window 80-450 flared up briefly for several seconds around this time. There are no distinct flames visible at other locations on the face. In particular, the flames visible inside the aircraft impact cavity in Figure 9-86 are not seen from this angle.

When WTC 2 collapsed at 9:58:59 a.m. the only significant flames pushed out of open windows on the south face came from the fire area to the west of the aircraft impact cavity on the 80th floor. This supports the conclusion, based on visual observations, that this was the only extensive fire burning on this face at the time of collapse.

Views of the west face of WTC 2 at 9:52:27 a.m. and 9:57:49 a.m. are provided in Figure 9-84 and Figure 9-86, respectively. Smoke is coming from many of the open windows previously identified earlier as possibly having been broken by people. There are no indications of additional windows having opened, and no flames are visible. The visual evidence indicates that fires capable of breaking out window glass had not reached the west face of WTC 2 prior to the collapse.

During the period between 9:45 a.m. and the start of the global collapse of WTC 2 at 9:58:59 a.m., there were numerous indications that significant changes were taking place within the tower. These included 1) repeated pressure pulses that pushed smoke and/or dust, and in some cases flames, from multiple open windows simultaneously over multiple floors, 2) flows of molten metal (assumed to be aluminum) from the tops of windows, 3) visible changes in the location of hanging objects, 4) sudden changes in fire location and intensity, 5) inward bowing of exterior columns, and 6) a large number of pieces of debris falling from the north side of the east face just prior to the collapse. The available evidence suggests that many, if not all, of the hanging objects were possibly floor slabs that had become detached from and settled down below the spandrel to which they were originally attached. Certain of the behaviors, such as the flows of molten metal, were temporally correlated with pressure pulses, and it is considered likely that the events responsible for changes in hanging object locations also generated such pulses. 


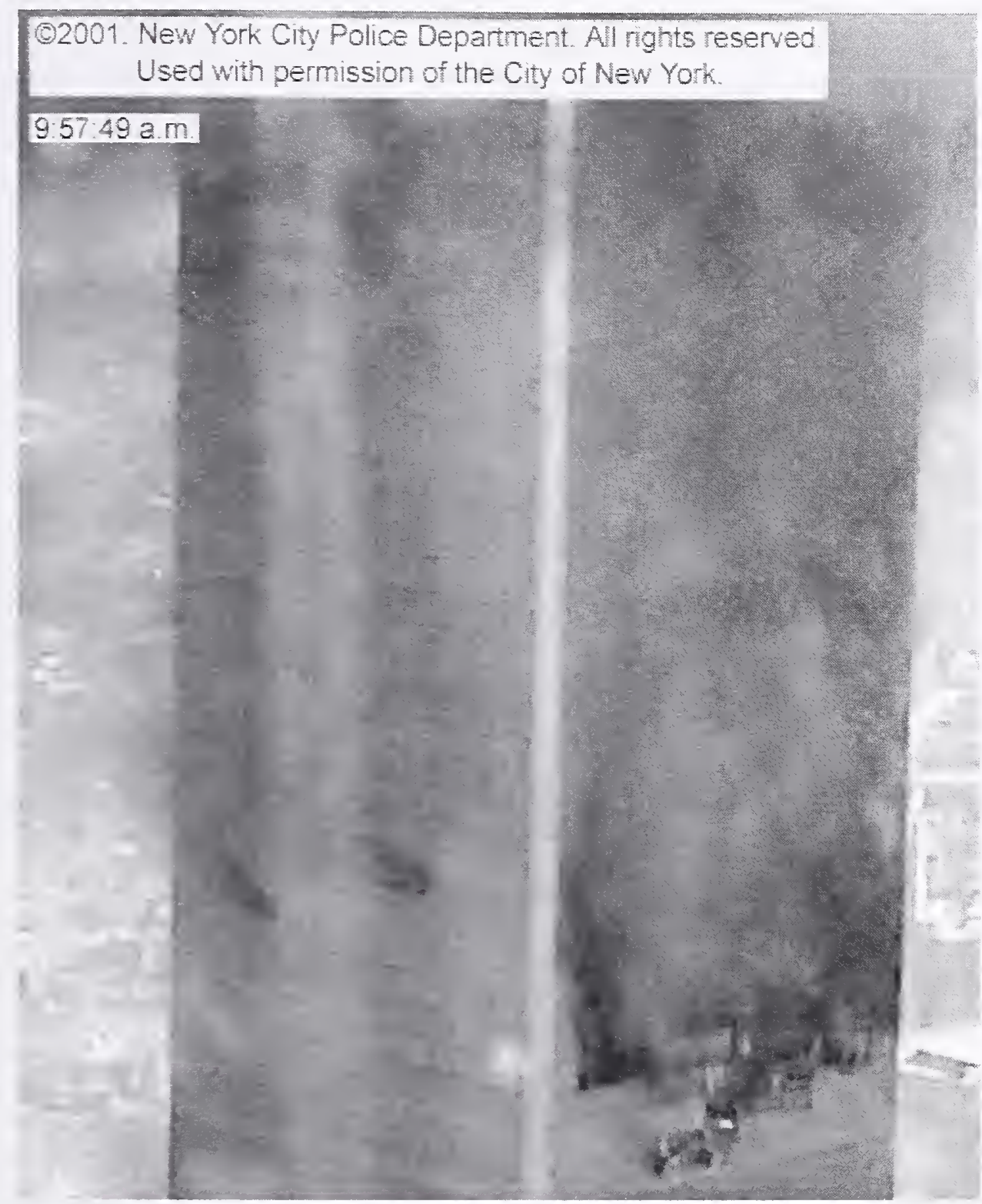

Figure 9-86. This cropped photograph showing the west and south faces of WTC 2 was shot from a helicopter at 9:57:49 a.m. The intensity levels have been adjusted.

Façade maps of integrated fire intensities for the period from 9:45 a.m. to 9:59 a.m. are shown in Figure 9-88. Comparison with the corresponding maps for the 9:29 a.m. to 9:45 a.m. period shown in Figure 9-60 is helpful for tracking the changes in fire distributions during the current period.

Many of the fires observed during earlier periods were either no longer visible or were dying down during the final interval prior to the tower collapse. Fires dying down included those burning on debris piles located in the northeast corner of the tower and near the center of the north face on the 79th floor, a fire that had earlier burned from south to north across the 82 nd floor on the east face, and numerous small fires ignited around the aircraft impact cavity on the south face. 


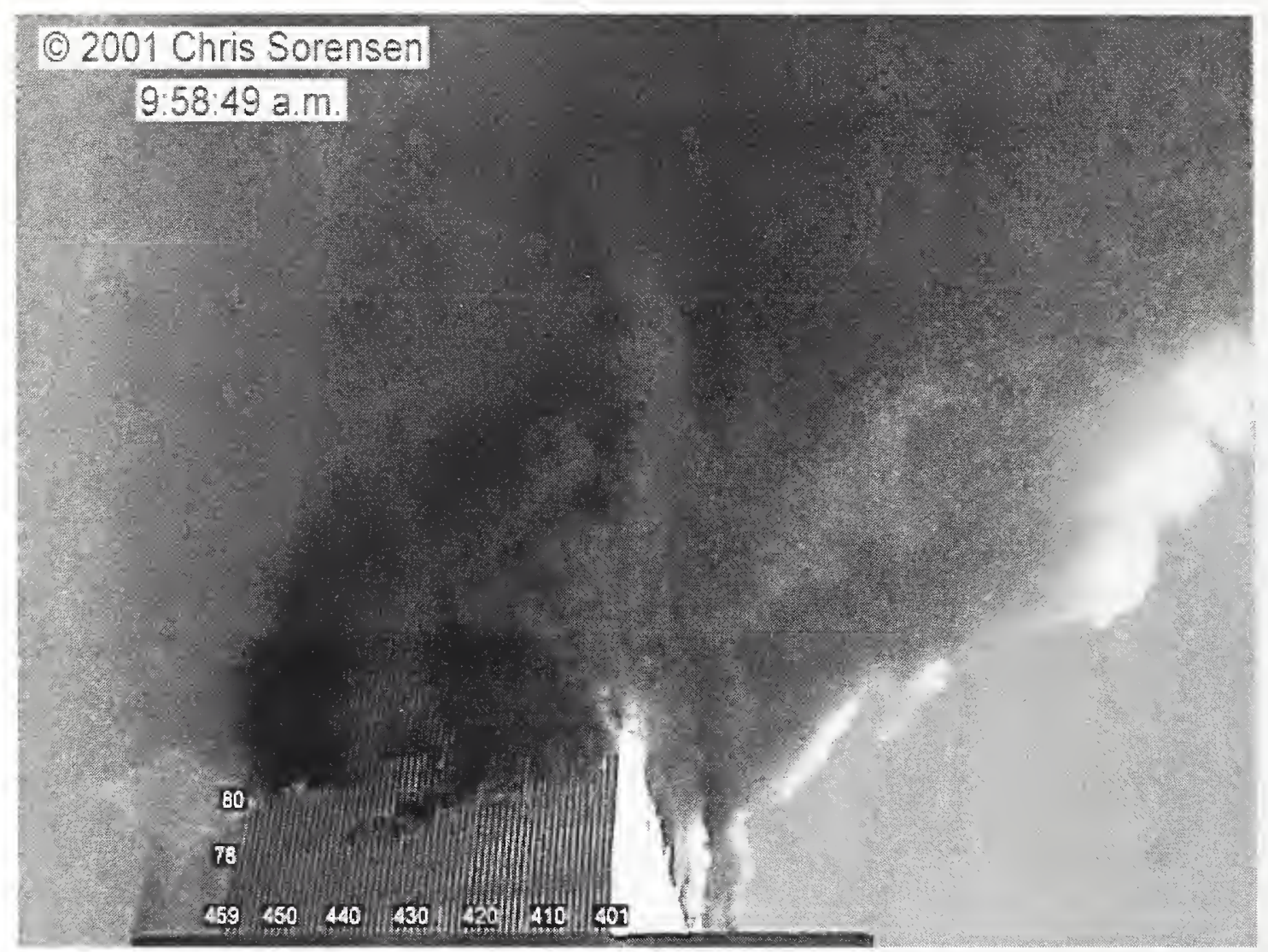

Figure 9-87. This image of the south face of WTC 2 is a frame captured from a video recorded at 9:58:49 a.m. The intensity levels have been adjusted, and column and floor numbers have been added.

At the same time, fires spread to areas of the faces that had not been involved earlier, including the north face toward the west on the $79 \mathrm{th}, 82 \mathrm{nd}$, and $83 \mathrm{rd}$ floors. There was also an indication that fire may have spread upward on the north face from the 83 rd to the 84 th floor. The fire growth and local spread on the 79 th floor of the north face appeared to be rapid and took place at a location that was apparently removed from earlier fires. This may be an indication that fire spread to this area on the faces occurred from interior locations within the tower that were not visible from outside. The new areas of fire which appeared on both the 79th and 83rd floors developed rapidly and remained localized at a few windows. These behaviors suggest that these fires had spread into rooms with walls that terminated at nearby façade columns and limited further fire spread. On the 79th floor, flames were seen in window 79-209 to window 79-212. A floor plan layout for the 79th floor is shown in Figure B-10 in Appendix B. It indicates that a room was not present behind these windows. A room is indicated in the northwest corner with walls at columns 79-208 and 79-154. The fire on the 83rd floor appeared in windows 83-221 to 83-225. Unfortunately, a floor plan layout for this floor is not available for comparison. The visual evidence indicates that none of the spreading fires on the north face reached as far as the west face prior to the tower collapse.

Some flames were observed in the cold spot on the north face during the period. Even so, no large fires grew in the area, and very little smoke flowed from the open windows. 

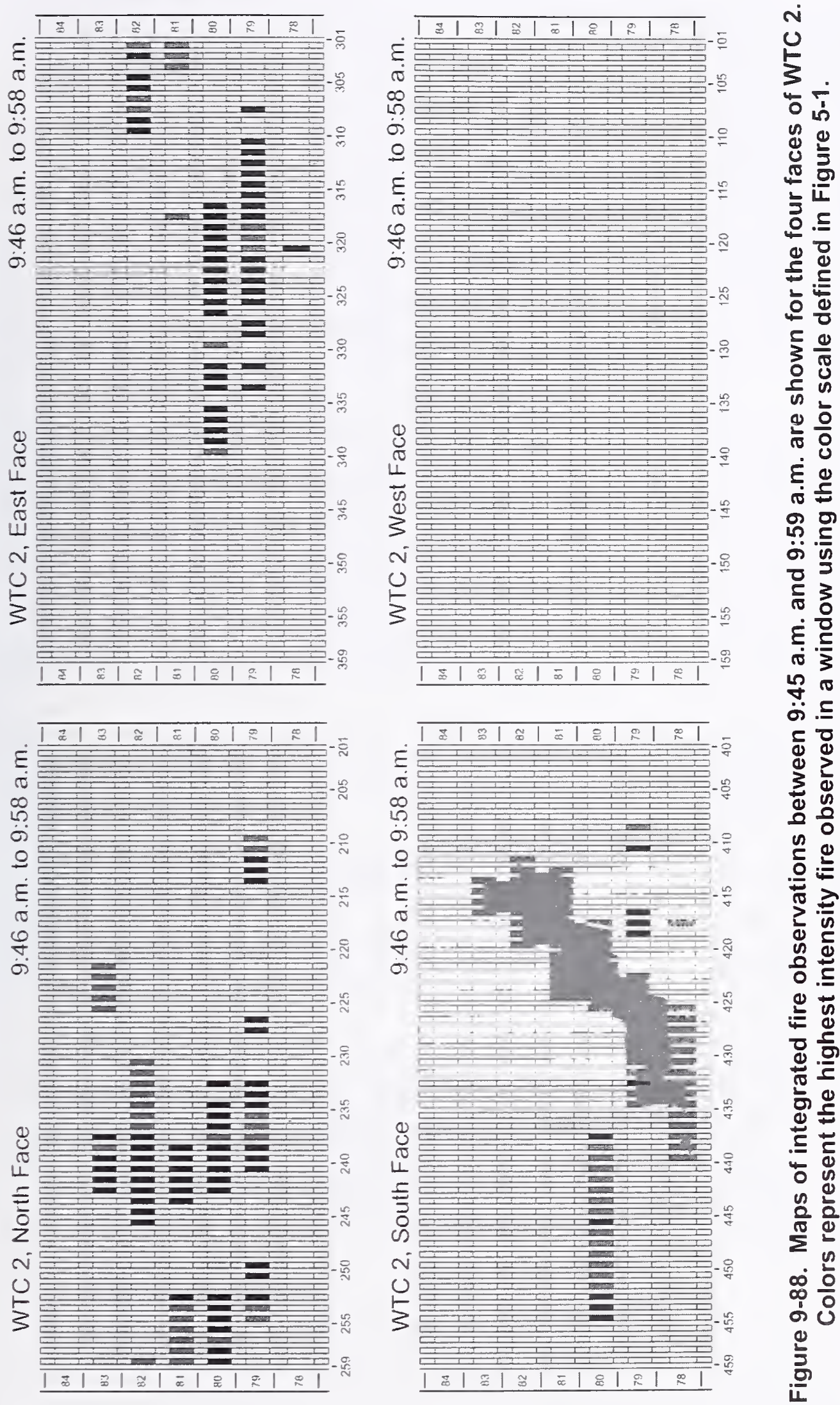
Between 9:45 a.m. and 9:59 a.m. there were at least four periods during which intense smoke flows and some flames appeared suddenly from windows near the centers of the 79th and 80th floors of the east face, only to subside roughly a minute later. Similar, but more intense, behaviors had been observed between 9:29 a.m. and 9:45 a.m. and were tentatively attributed to burning of pools of aviation fuel located somewhere within the interior of the tower. During the period, sustained fires, with behaviors more typical of building fires, were also observed on the 79th and 80th floors. On the 79th floor these flames extended from roughly windows $79-310$ to $79-320$ and on the 80th floor from windows 80-315 to 80-326. At the start of the period, the flames on the 79th floor were heavier and generated smoke that partially obscured the 80 th floor. By the end of the period, these flames had died down and had receded into the building, and little smoke was coming from the nearby windows. The fire on the 80th floor did not appear to generate external flames, and little smoke was visible coming from nearby windows. A fire that had been burning on a pile of debris in the northeast corner of the 81 st floor since the aircraft impact was still present.

On the south face, several relatively small fires that had developed earlier around the aircraft impact cavity continued to die down or disappeared from view. More substantial fires that had been present at the eastern edges of the face on the 81st and 82nd had also died down. By around 9:50 a.m. very little fire was visible on the façade. Between 9:52 a.m. and 9:54 a.m. a fire became visible and rapidly spread along the west side of the face on the 80th floor. The only earlier indication of fire development at this location was a short flame flare up observed from window 80-439 at 9:47:50 a.m. and a small flame at this location. Just prior to 9:57 a.m. the fire grew rapidly, and large flames were observed coming from windows in the area. At the time of the tower collapse, this fire extended from roughly window $80-437$ to 80-454.

On the west face, the only indication of the fires in the tower was smoke flow from open windows above the fire floors. There was no indication that the fires spreading toward the west on the north and south faces had reached the west face by 9:58:59 a.m.

\subsection{SUMMARY AND DISCUSSION OF OBSERVATIONS FOR WTC 2}

Appendices $\mathrm{G}$ to $\mathrm{J}$ contain façade maps that summarize the details of visual analyses for window condition, fires, and smoke for the four faces of WTC 2 as a function of time. The fire behaviors are briefly summarized here.

Compared to WTC 1, the amount of fire spread in WTC 2 was less extensive during the 56 min between the impact of United Airlines Flight 175 and the collapse of the tower. One approach for characterizing the fire spread in WTC 2 is to compare integrated façade maps for the entire period from 9:03 a.m. to 9:59 a.m. with the initial fire distributions shortly after the aircraft impact. Figure 9-89 shows fire maps for the four faces obtained by integrating the fire datasheets over the entire period. These maps are compared with the corresponding fire distributions around 9:04 a.m. in Figure 9-90. 

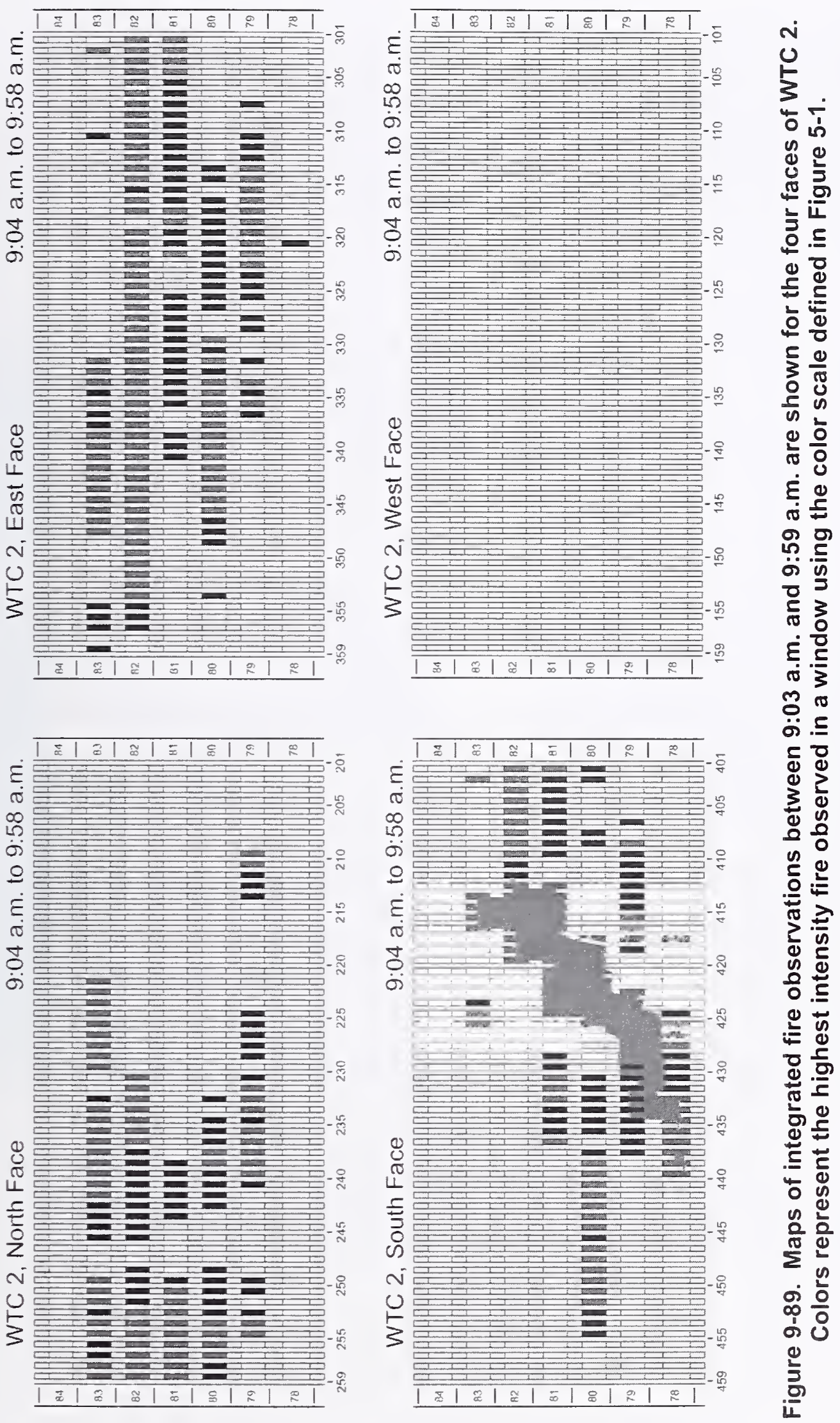

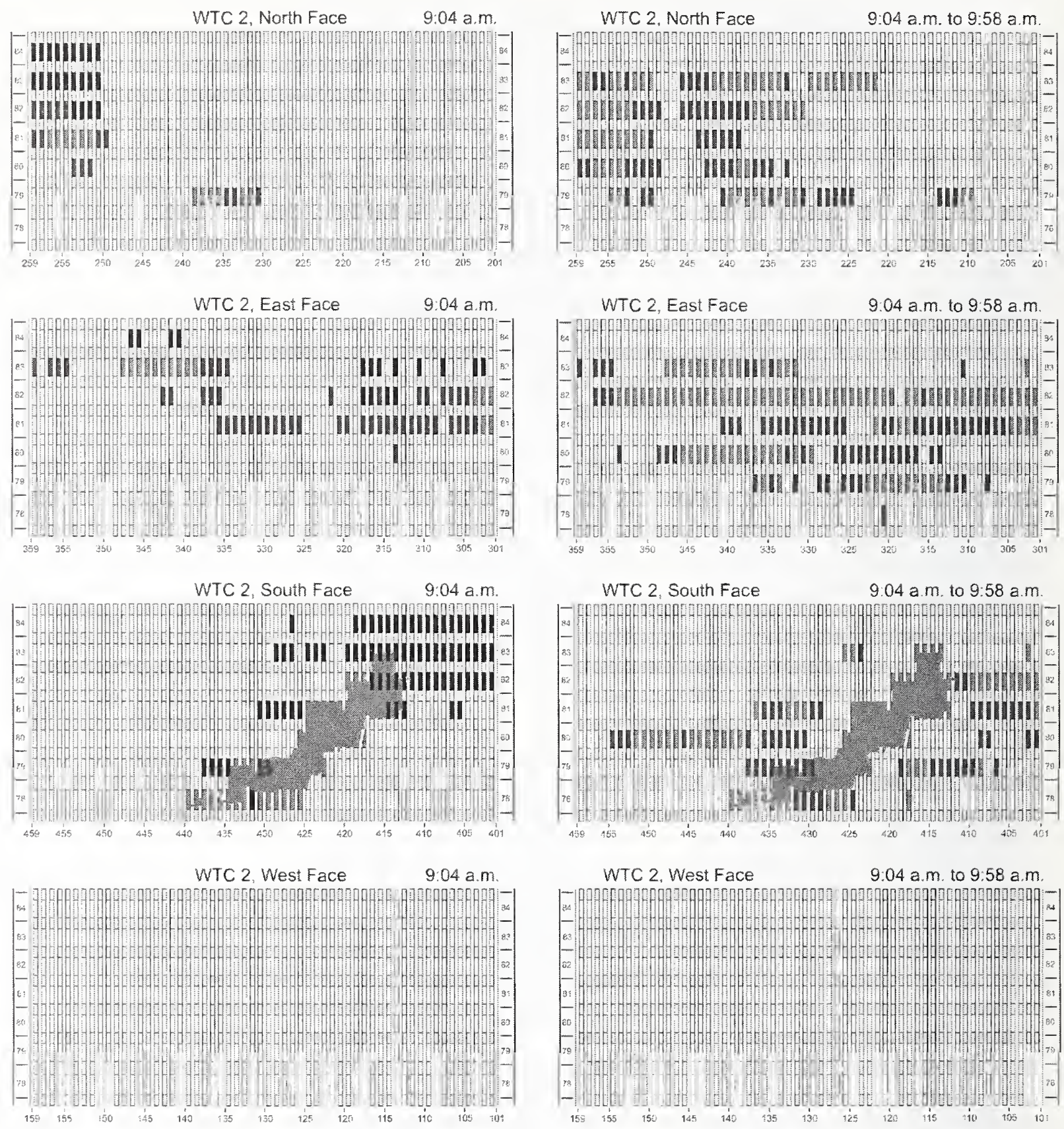

Figure 9-90. Maps of observed fire distributions on the four faces of WTC 2 shortly after the aircraft impact (near 9:04 a.m.) are compared with the corresponding integrated fire observations for the entire period between 9:03 a.m. and 9:59 a.m. The data for 9:04 a.m. are represented using the color scale in Figure 5-1. For the integrated results, the same color scale is used to represent the highest intensity fire observed in a given window during the period. 
Similar to the analyses included in Chapter 8, changes in window condition can also be used as a rough indicator of fire spread. This analysis method has been used to the complement the integrated fire data. The results are shown in Figure 9-91. It should be kept in mind that this approach only reveals fire spread that occurs to locations where windows were originally unopened. This is particularly important for WTC 2 because the number of windows opened by the aircraft impact and subsequent fireballs was much larger than for WTC 1. Even so, the analysis does provide a good estimate for fire spread into areas where window glass was still in place immediately following the aircraft impact.

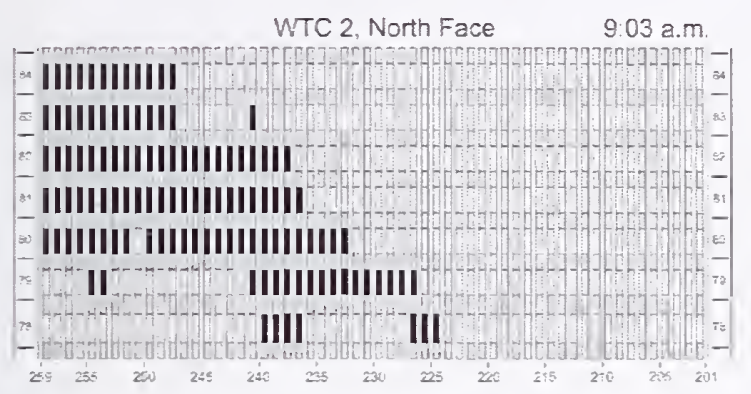

WTC 2, East Face
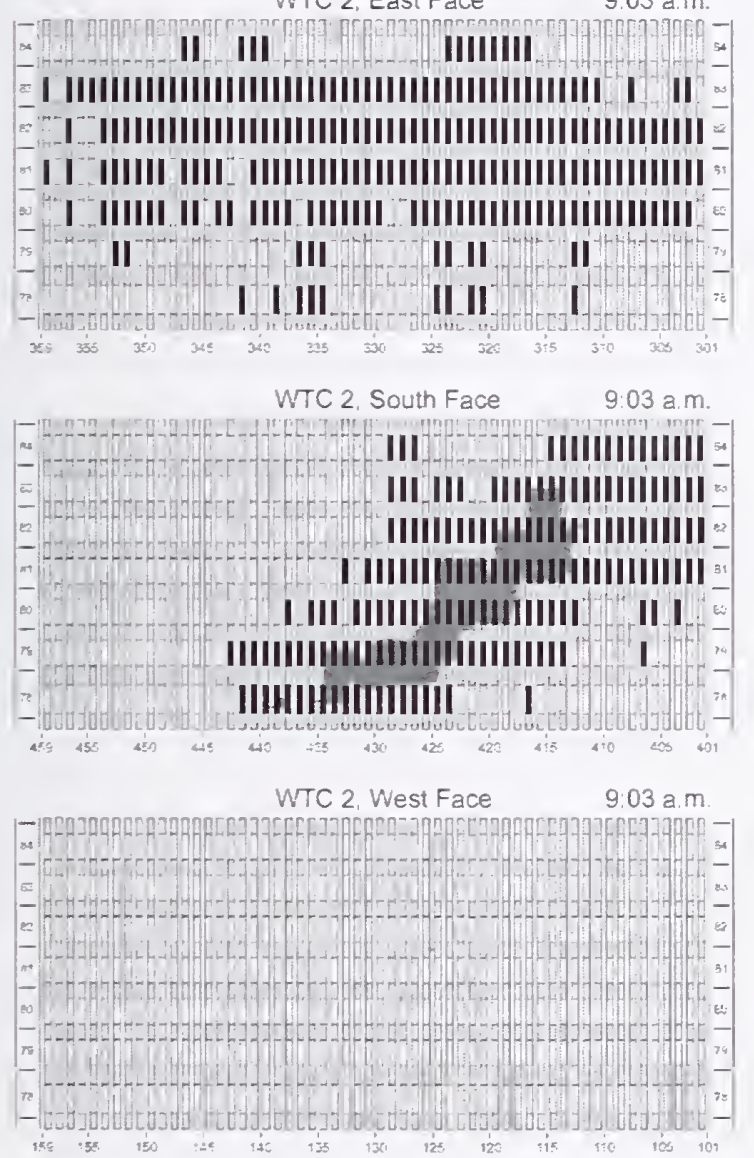

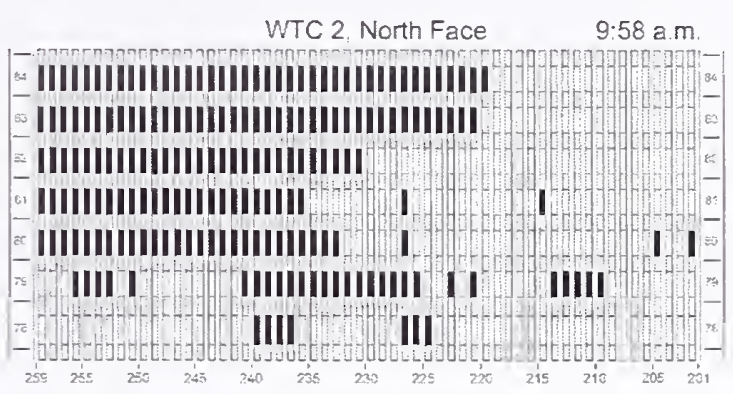

WTC 2, East Face

9.58 a.m.

: IIIIIIIIIIIIIIIIIIIIIIIIIIIIIIIIIIIIIIIIIIIIIIIIIIIIIIIIII IIIIIIIIIIIIIIIIIIIIIIIIIIIIIIIIIIIIIIIIIIIIIIIIIIIII III |IIIIIIIIIIIIIIIIIIIIIIIIIIIIIIIIIIIIIIIIIIIIIIIIIIIIIIIII

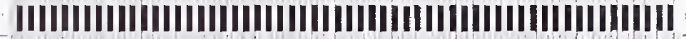
IIIIIIIIIIIIIIIIIIIIIIIIIIIIII IIIIIIIIIIIIIIIIIIIIIIII

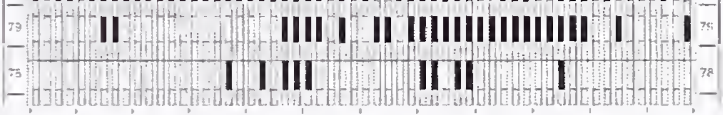
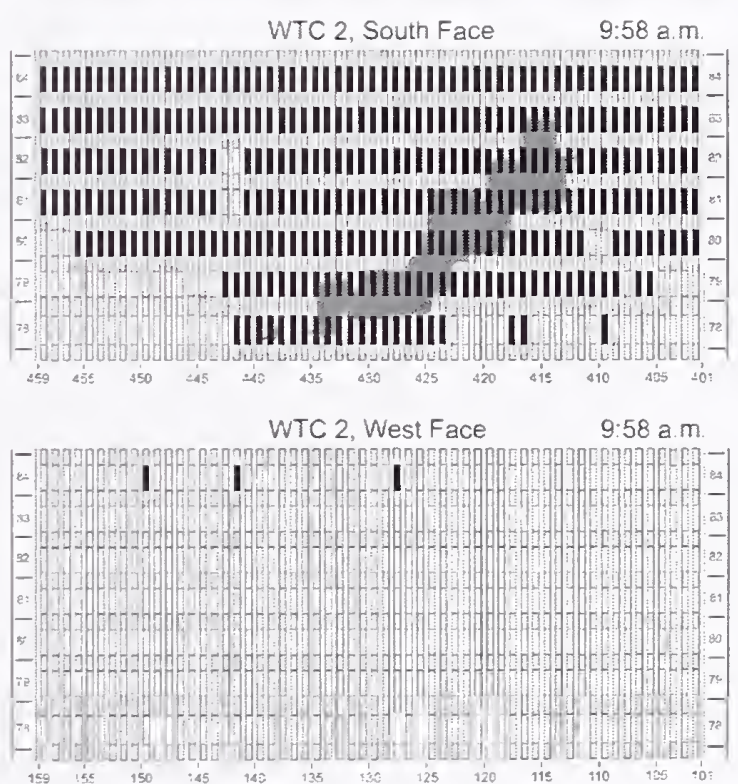

Figure 9-91. Maps for the 78th through the 84th floors compare missing window glass for the four faces of WTC 2 at two times, immediately following the aircraft impact at 9:02:59 a.m. and just prior to the collapse of WTC 1 at 9:58:59 a.m. 
On the north face significant fire spread took place on the 79th, 82nd, and 83rd floors. The effects of this fire spread are revealed by areas of fire (Figure 9-90) and opened windows (Figure 9-91) on the west sides of the areas of fire and open windows that were present shortly following the aircraft impact and fireballs. On the 79th floor this fire growth took place from the large fire burning on a pile of debris just to the east of the center of the face. Despite the presence of this sustained fire, westward fire spread on this floor was relatively slow and sporadic. The fire spread on the $82 \mathrm{nd}$ floor occurred just to the west of the cold spot (an area on the 80th, 81st, and 82nd floors between columns 238 and 250). Fire spread into this area was not observed until around 9:54 a.m. On the 83rd floor fire spread occurred in discrete segments that suggested fire was spreading across a series of rooms. The first large fire appeared just above the cold spot around 9:29 a.m. None of the fires that spread to the west on the north face appeared to reach the western edge of the tower by the time of collapse.

The summary of observations of open windows included in Table 9-1 and Table 9-2 indicates that approximately 70 windows were opened by the fires on the north face and that the majority of these were on the 79th, 82nd, and 83rd floors, with the largest number on the 83rd floor.

An important fire behavior on the north face that is not captured well by the fire maps and the window summary façade maps and tabulated results are the long-burning fires observed on the 79th, $81 \mathrm{st}$, and 82 nd floors. These fires were located on piles of debris created during the aircraft impact in the northeast corners of the 81 st and 82nd floors and near the center of the north face on the 79th and 80th floors. Intense fires were present at these locations immediately following the aircraft impact and continued to burn until the tower collapsed. Most fires observed elsewhere in the towers tended to die down faster than these fires. This suggests that large amounts of combustible material were present in the debris piles.

Another north face observation that is not captured by the window results is the existence of the cold spot. Since all of the windows in the area (along with most of the exterior curtain wall) were removed during the aircraft impact and resulting fireball, window changes were impossible. On the other hand, the location of the cold spot is readily identifiable in the integrated fire maps shown in Figure 9-89 and Figure 9-90 as the area on the 80th through 82nd floors near column 245 in which no fire was observed during the entire event. Flames were observed on both sides of this area on each of the three floors.

Recall that the cold spot was the location where the largest fireball was generated on the north face during the aircraft impact and subsequent fireballs. Despite the formation of the fireball, the area stood out as being nearly smoke and fire free shortly after the aircraft impact, even though large fires were present nearby on the 79th, 81st, and 82nd floors. Infrared images showed that temperatures in most of the cold spot were near the ambient outside temperature at 9:15 a.m.

As reflected in the integrated fire maps, flames were occasionally observed at some locations inside the cold spot, which is defined as covering windows 238 to 249 on the $80 \mathrm{th}, 81 \mathrm{st}$, and $82 \mathrm{nd}$ floor. The firefree area indicated by the maps is considerably narrower than this. The most significant of these fires was in the lower right-hand corner, where a fire spread onto a pile of debris on the 79th floor and burned gently for a long period. Small flames were observed for short times at other locations, including some near the center of the area, such as on the 82nd floor. At many of these locations the flames appeared very dark and were barely detectable. Despite the detection of some flames in the area, the cold spot remained unexpectedly smoke and fire free until the tower collapsed near 9:59 a.m. 
Table 9-1. Comparison of Window Conditions on the Four Faces of WTC 2 Near the Times of Aircraft Impact on WTC 2 and Collapse of WTC 2

\begin{tabular}{|c|c|c|c|c|c|c|}
\hline & \multicolumn{3}{|c|}{ Around 9:03 a.m. } & \multicolumn{3}{|c|}{ Around 9:58 a.m. } \\
\hline & $\begin{array}{c}\text { Open } \\
\text { Window }\end{array}$ & $\begin{array}{c}\text { Glass in } \\
\text { Place } \\
\end{array}$ & $\begin{array}{l}\text { "Can't } \\
\text { See" }\end{array}$ & $\begin{array}{c}\text { Open } \\
\text { Window }\end{array}$ & $\begin{array}{c}\text { Glass in } \\
\text { Place } \\
\end{array}$ & $\begin{array}{l}\text { "Can't } \\
\text { See" }\end{array}$ \\
\hline Floor & \multicolumn{3}{|c|}{ North Face 9:03 a.m. } & \multicolumn{3}{|c|}{ North Face 9:58 a.m. } \\
\hline 84 & 0 & 46 & 12 & 5 & 18 & 35 \\
\hline 83 & 4 & 45 & 9 & 39 & 19 & 0 \\
\hline 82 & 22 & 36 & 0 & 29 & 29 & 0 \\
\hline 81 & 23 & 35 & 0 & 25 & 32 & 1 \\
\hline 80 & 22 & 32 & 4 & 29 & 28 & 1 \\
\hline 79 & 13 & 41 & 4 & 28 & 30 & 0 \\
\hline 78 & 7 & 51 & 0 & 7 & 51 & 0 \\
\hline Floor & \multicolumn{3}{|c|}{ East Face 9:03 a.m. } & \multicolumn{3}{|c|}{ East Face 9:58 a.m. } \\
\hline 84 & 0 & 45 & 13 & 0 & 0 & 58 \\
\hline 83 & 49 & 7 & 2 & 57 & 0 & 1 \\
\hline 82 & 54 & 4 & 0 & 58 & 0 & 0 \\
\hline 81 & 52 & 6 & 0 & 56 & 0 & 2 \\
\hline 80 & 47 & 11 & 0 & 54 & 3 & 1 \\
\hline 79 & 11 & 47 & 0 & 36 & 8 & 14 \\
\hline 78 & 9 & 48 & 1 & 13 & 45 & 0 \\
\hline Floor & \multicolumn{3}{|c|}{ South Face 9:03 a.m. } & \multicolumn{3}{|c|}{ South Face $9: 58$ a.m. } \\
\hline 84 & 0 & 41 & 17 & 3 & 0 & 55 \\
\hline 83 & 0 & 33 & 25 & 23 & 0 & 35 \\
\hline 82 & 17 & 30 & 11 & 27 & 2 & 29 \\
\hline 81 & 31 & 27 & 0 & 39 & 2 & 17 \\
\hline 80 & 27 & 31 & 0 & 50 & 5 & 3 \\
\hline 79 & 31 & 27 & 0 & 37 & 21 & 0 \\
\hline 78 & 20 & 38 & 0 & 22 & 36 & 0 \\
\hline Floor & \multicolumn{3}{|c|}{ West Face 9:03 a.m. } & \multicolumn{3}{|c|}{ West Face $9: 58$ a.m. } \\
\hline 84 & 0 & 58 & 0 & 3 & 55 & 0 \\
\hline 83 & 0 & 58 & 0 & 0 & 58 & 0 \\
\hline 82 & 0 & 58 & 0 & 0 & 58 & 0 \\
\hline 81 & 0 & 58 & 0 & 0 & 58 & 0 \\
\hline 80 & 0 & 58 & 0 & 0 & 58 & 0 \\
\hline 79 & 0 & 58 & 0 & 0 & 58 & 0 \\
\hline 78 & 0 & 58 & 0 & 0 & 58 & 0 \\
\hline
\end{tabular}


Table 9-2. Summary of Open Windows Observed on Faces (78th to 84th Floors) of WTC 2 at 9:03 a.m. and 9:58 a.m.

\begin{tabular}{|c|c|c|}
\hline Face & $\begin{array}{c}\text { Number of Open Windows } \\
\text { 9:03 a.m. }\end{array}$ & $\begin{array}{c}\text { Number of Open Windows } \\
\text { 9:58 a.m. }\end{array}$ \\
\hline North & 91 & 162 \\
\hline East & 222 & 274 \\
\hline South & 126 & 201 \\
\hline West & 0 & 7 \\
\hline & & 637 \\
\hline Total & 439 & 6 \\
\hline
\end{tabular}

The existence of the cold spot implies that little fuel was available in the immediate area to support burning. Since fires could be observed many feet into the building, the area of limited fuel must have extended some unknown, but significant, distance from the outer wall. A number of possible explanations were considered for the absence of fuel along lengths corresponding to roughly one sixth of the width of the building over three floors. One possibility is that the area was vacant and that there was no fuel present prior to the aircraft impact. NIST is unaware of any information to support such a conclusion.

A second possibility is that the fireballs that exited through the area were so intense that they pushed most available fuel in the area to the sides or out through the opened windows. The argument against this mechanism is that it was not observed elsewhere in the towers. The outer steel structure in the cold spot was intact. In the northeast corner this framework acted as a net, catching and forming large piles of debris. Large amounts of small debris were expelled from the tower, but ground shots of the area immediately below did not indicate the presence of large pieces of debris such as furniture or aircraft parts.

A third possibility to explain the presence of the cold spot is that the floor slabs, particularly on the 81 st and 82nd floors, in the immediate area collapsed during the aircraft impact and fireballs, in effect, creating a cavity behind the cold spot. The presence of the large fireball supports such a possibility since the overpressures associated with the fireballs have been implicated in floor slab collapse elsewhere in the tower. Ironically, the observation of hanging objects, as well as small fires, through open windows in the cold spot suggests that, while the floor slabs in the area may well have partially collapsed and may have even continued to do so during the event, the floor slabs maintained some strength and should have been capable of supporting some fuel and hence burning. Several close-up images provide little visual evidence that indicates the possibility of extensive local floor slab collapses. While it could be argued that the debris piles observed on the 79th and 80th floors just to the east of the cold spot were formed by such localized collapses, observations summarized in Table 7.1 suggest that debris from the aircraft impact reached the north face of the tower near this location prior to the initiation of the fireball and, thus, could explain the presence of the debris pile(s).

NIST concludes that insufficient information is available to allow a likely formation mechanism for the cold spot to be postulated.

While the mechanism responsible for the formation of the cold spot is not known, it appears that its presence strongly influenced fire spread on the north face, acting, in effect, as a fire break. It is 
significant that fire spread to the west of the spot occurred on the 83 rd floor before it took place on the 80 th, 81 st, or $82 \mathrm{nd}$ floors, despite the presence of large fires on the $81 \mathrm{st}$ and $82 \mathrm{nd}$ floors to the east of the cold spot immediately following the aircraft impact. Flames were observed on the 83 rd floor above the cold spot. suggesting that this floor slab was at least partially in place and, thus, providing a path for fire spread to the west. When fire spread and growth finally occurred to the west of the cold spot on the $82 \mathrm{nd}$ floor, it appeared to come from a fire that developed near the center of the cold spot. No significant fire spread occurred to the west of the cold spot on the 81 st floor.

Hanging objects were observed elsewhere on the north face, which might possibly indicate the presence of partially collapsed floor slabs. These observations are summarized below.

The fire behaviors observed on the east face were complex and somewhat unusual. As a result, the summary of window conditions and integrated fire observations do not capture these behaviors well. Even comparisons of the integrated fire maps for the four periods are inadequate for this purpose. It is perhaps most useful to refer to the discussions for the individual time periods.

During the first minute following the aircraft impact and fireballs, large flames were present on the $81 \mathrm{st}$, $82 \mathrm{nd}$. and 83 rd floors. The fire on the 83 rd floor was burning primarily on the southern half of the face. Some flames in this area were also visible near the tops of windows on the 82 nd floor. These flames were primarily located above a hanging object that may possibly have been the floor slab from the 83rd floor. Most of the fires observed on the 81 st and 82 nd floors were burning on the northern side of the face. These fires were particularly intense near the northeast corner where large piles of debris were present, and intense burning was taking place on these floors from an opening filled with debris in the northeast corner. Only spot fires were visible on the 79 th and 80 th floors.

Within four minutes of the aircraft impact, the total area of visible flames on the east face had decreased markedly. The large fire on the south side of the 83 rd floor had died down considerably, and flames were only present just to the south of the center of the face. Meanwhile, an intense fire with flames out of the windows had grown on the south side of the 82nd floor between roughly window 82-342 and window 82-350. Much of the flame on the north side of the 82 nd floor had died down, with the exception of the fire burning near and in the northeast comer. The fires on the north side of the 81 st floor were also dying down, and the only external flames coming from open windows were near the north edge of the face and from the northwest corner. Isolated fires were present on the 80th and 81 st floors near the center of the east face.

Over the next several minutes the fire that had grown on the south side of the 82nd floor on the east face began to spread to the north and intensify. This fire seemed to be going through a "normal" cycle in which flames that appeared earlier were beginning to die down, while areas of recent fire spread burned intensely with external flaming. At the same time, fires elsewhere on the face continued to decrease in intensity. Even so, fires on the north sides of the 81 st and 82 nd floors continued to burn with flames that either filled the windows or extended from windows.

During the first ten minutes following the aircraft impact, the only flames observed on the 79th or 80th floors were small spot fires on the 80th floor near the center of the face. Windows on these floors were essentially smoke-free, except for the short smoke puffs discussed further below. 
An infrared image of the east face, taken around 9:15 a.m., confirmed the general findings summarized above. Much of the 81 st and 82 nd floors were heated by the intense fires burning on these floors, while only localized heated areas were evident on the 79 th and 80 th floors.

Beginning as early as 9:12:45 a.m., short-lived smoke puffs with durations of a few seconds were observed coming from open windows on the east face. These puffs typically occurred from multiple windows on two or more floors. The puffs often came from windows on the 79th and 80th floors. In many cases the puffs appeared to move across the face in sequence. At times, similar puffs were observed simultaneously on the north and south faces. The evidence suggests that these smoke puffs resulted from pressure pulses generated within the tower and transmitted to other locations. It should be $\mathrm{kcpt}$ in mind that these pressure pulscs were likely of sufficient magnitude to affect smoke flows over multiple faces and floors, but were much too small to affect structural components within the tower. For most of the smoke puffs, there was no visual indication of the location of the event that generated the pressure pulse. In a few cases, such as when molten metal poured from the tower, circumstantial evidence indicated that the puffs could be associated with specific events.

Even though the smoke puffs had short durations, they were frequently seen. Table 9-3 lists the times for the initiation of observed smoke puffs based on a review of a long-distance, continuous video of the east face of WTC 2. It should be kept in mind that the amount of smoke released varied from puff to puff, so there is some uncertainty associated with whether a weak smoke flow represents a smoke puff or not. Since the puffs tended to move across the face over periods lasting several seconds, there are also uncertainties associated with the start times for the puffs. As indicated by the results in Table 9-3, the smoke puffs first appeared around 9:13 a.m. and were observed numerous times until WTC 2 collapsed at 9:58:59 a.m.

Table 9-3. Start Times for Smoke Puffs Observed on the East Face of WTC 2

\begin{tabular}{|c|c|c|c|c|}
\hline 9:12:48 a.m. & 9:14:31 a.m. & 9:14:57 a.m. & 9:15:20 a.m. & 9:16:06 a.m. \\
\hline $9: 17: 16$ a.m. & 9:18:07 a.m. & 9:18:35 a.m. & 9:19:05 a.m. & 9:19:20 a.m. \\
\hline 9:19:47 a.m. & 9:20:58 a.m. & 9:21:12 a.m. & 9:21:22 a.m. & 9:21:37 a.m. \\
\hline 9:21:46 a.m. & 9:22:34 a.m. & 9:23:01 a.m. & 9:23:14 a.m. & 9:23:57 a.m. \\
\hline 9:24:10 a.m. & 9:24:31 a.m. & 9:25:04 a.m. & 9:25:18 a.m. & 9:25:43 a.m. \\
\hline 9:26:11 a.m. & 9:26:25 a.m. & 9:27:16 a.m. & 9:27:33 a.m. & 9:28:05 a.m. \\
\hline 9:28:09 a.m. & 9:28:41 a.m. & 9:28:55 a.m. & 9:29:05 a.m. & 9:29:24 a.m. \\
\hline 9:30:15 a.m. & 9:30:46 a.m. & 9:31:27 a.m. & 9:31:39 a.m. & 9:31:55 a.m. \\
\hline 9:32:26 a.m. & 9:33:20 a.m. & 9:33:39 a.m. & 9:34:04 a.m. & 9:37:05 a.m. \\
\hline 9:37:42 a.m. & 9:38:02 a.m. & 9:38:13 a.m. & 9:38:29 a.m. & 9:39:37 a.m. \\
\hline 9:40:47 a.m. & 9:42:58 a.m. & 9:48:36 a.m. & 9:49:16 a.m. & 9:49:23 a.m. \\
\hline 9:50:00 a.m. & 9:50:37 a.m. & 9:52:13 a.m. & 9:52:30 a.m. & 9:53:10 a.m. \\
\hline 9:53:46 a.m. & 9:54:16 a.m. & 9:54:37 a.m. & 9:57:02 a.m. & 9:57:19 a.m. \\
\hline 9:57:28 a.m. & & & & \\
\hline
\end{tabular}


In addition to the smoke puffs seen on the east face of WTC 2, there were also periods lasting considerably longer, typically about a minute, during which heavy smoke would suddenly start to flow from open windows over large areas of the face that had been essentially free of smoke. The smoke flows tended to be particularly heavy near the centers of the 79th and 80th floors. Figure 9-44 shows the east face of WTC 2 during one of these periods. As evident in this photograph, flames were also occasionally observed coming from the windows. At the end of a period, the smoke flow would subside as quickly as it started. These periods of heavy smoke flow were easily distinguished from the smoke puffs (pressure pulses) described above.

Seven periods of increased smoke flow were identified starting around 9:36 a.m. Table 9-4 lists the approximate start and end times for the pulses along with their durations. Even though the smoke flow density varied for the seven periods, the lengths of the periods were remarkably consistent.

\section{Table 9-4. Start and End Times for Periods of Heavy Smoke Flow (and External Flaming)} from Numerous Windows on 79th and 80th Floors of the East Face of WTC 2

\begin{tabular}{|c|c|c|}
\hline Start Time & End Time & Duration \\
\hline $9: 35: 42$ a.m. & $9: 36: 49$ a.m. & $1 \mathrm{~min} 7 \mathrm{~s}$ \\
\hline $9: 39: 41$ a.m. & $9: 40: 41$ a.m. & $1 \mathrm{~min} 0 \mathrm{~s}$ \\
\hline $9: 41: 13$ a.m. & $9: 42: 24$ a.m. & $1 \mathrm{~min} 1 \mathrm{~s}$ \\
\hline $9: 44: 58$ a.m. & $9: 46: 08$ a.m. & $1 \mathrm{~min} 10 \mathrm{~s}$ \\
\hline $9: 46: 53$ a.m. & $9: 47: 53$ a.m. \\
\hline $9: 50: 57$ a.m. & $9: 51: 50$ a.m. & $53 \mathrm{~s}$ \\
\hline $9: 55: 29$ a.m. & $9: 56: 43$ a.m. & $1 \mathrm{~min} 14 \mathrm{~s}$ \\
\hline
\end{tabular}

The short-term release of large amounts of smoke. along with external flaming over large areas of a building façade, is not a typical building fire behavior. Solid-fueled fires in buildings more typically go through the stages of growth, sustained burning, and decay observed elsewhere in both towers. As postulated earlier, the burning of isolated unburned pools of aviation fuel deposited inside the building during the aircraft impact is viewed as the most likely explanation for the short periods of heavy smoke release. As discussed in Chapter 7, the impact location of the aircraft indicates that much of the aviation fuel from the port tank should have been deposited on the 79th floor. The fact that the smoke releases occurred simultaneously on both the 79th and 80th floors may be an indication that there were one or more internal connections, such as open elevator shafts, ventilation shafts, or locally collapsed floor slabs, that allowed the flame gases to vent to open windows on both floors.

Prior to the start of the short-term heavy smoke releases, only relatively small spot fires were observed on the east face of the 80th floor, and the 79th floor appeared to be free of fire. Very little smoke came from windows on these floors. The infrared images recorded around 9:15 a.m. indicated that there was a small heated area on the 79th floor that may have been due to a small, unseen fire. Starting shortly after 9:28 a.m., a substantial fire began to grow on the 79th floor between windows 79-315 and 79-320. Prior to this time, the glass had been in place in these windows. This fire spread to cover windows $79-310$ to 79-320 and was still burning when the tower collapsed.

As can be seen in Figure 9-43 and the blow-ups shown in Figure 9-44, which was taken during the first period of heavy smoke release from the 79th and 80th floors at 9:36:14 a.m., heavy smoke and flame were pushed from numerous open windows on these floors during these releases. It is noteworthy that the relative amounts of smoke and fire from the area on the 79th floor where an active fire had been observed 
earlier, i.e., windows 79-310 to 79-320, is relatively light compared to windows further to the south on the same floor. These figures provide a good indication of how heavy the smoke flow and flames were during this particular smoke release. By the time Figure 9-46 was taken at 9:37:32 a.m., the only heavy smoke and flames visible on the 79th floor were again coming from windows between 79-315 and 79-320. The contrast between the two conditions is stark and vividly demonstrates how unusual the periods of heavy smoke release were.

Given the possibility that a large amount of aviation fuel was spread across the 79th floor during the aircraft impact, perhaps the most noteworthy observation on the east face is the absence of large fires on the 79th and 80th floors for roughly $25 \mathrm{~min}$ following the distribution of the fuel. The fire behaviors during the short periods of heavy smoke release are consistent with rapid burning of aviation fuel at interior locations within the tower. The identification of seven such occurrences suggests that, if the aviation fuel was responsible, it had collected at several different locations that separately ignited and burned.

During the period when the heavy smoke releases were occurring on the 79th and 80th floors, fires continued to burn on the 81 st and 82 nd floors. The fire near and in the northeast corner of the 81 st floor continued to die down slowly. At various times it would appear to flare up at different locations. An unusually bright, relatively small flame, lasting from roughly 9:30 a.m. to 9:40 a.m., was observed in the northeast corner. It was attributed to metal combustion.

The fire on the $82 \mathrm{nd}$ floor that initially appeared near the southern edge of the east face shortly after the aircraft impact continued to spread north. Around 9:37 a.m. the fire had appeared as far north as window 82-320. At 9:47:10 a.m. fire was visible in windows $82-305$ to $82-310$, and by $9: 53$ a.m. flames were present on this floor near the northern edge.

Starting around 9:50 a.m., a substantial fire was observed on the 80th floor. By 9:55 a.m. flames were visible inside windows $80-316$ to $80-326$. When the tower collapsed, a small amount of flames was forced from the tower near window 80-326.

The fires on the east face of WTC 2 were relatively isolated when the tower collapsed, being primarily localized on the 79th through 81 st floors toward the north side of the face.

The fire behavior observed on the south face of WTC 2 seemed to be determined to a large extent by the locations of small fires ignited in and around the aircraft impact cavity by the fireball following the impact. The locations of some of these initial fires are indicated by the fire distribution map for the face around 9:04 a.m. included in Figure 9-90.

During the initial period following impact, small, slowly developing fires were seen on the 78th through 81 st floors on either side of the impact cavity. The only location where a fire was observed to grow rapidly was in the area on the 81 st floor between the aircraft impact cavity and the eastern edge of the face. Around 9:16 a.m. a similar fire to that burning on the 81 st floor became visible on the 82 nd floor between the aircraft impact cavity and east face.

By 9:30 a.m. the larger fires that had grown on the east side of the 81 st and 82 nd floors had moved through their maximum intensity phase and were dying down. Two small fires ignited on either side of the aircraft impact cavity on the 79th floor grew and spread slowly in both directions away from the 
cavity. Similarly, a small fire that was initially on the 78th floor to the east of the aircraft cavity spread slowly to the east.

Around 9:38 a.m. a fire burning in the area between the aircraft impact area and the eastern edge of the 80th floor was observed. By 9:44 a.m. this fire had already died down. The fire on the 79th floor to the east of the aircraft impact cavity had continued to spread very slowly toward the east. The fire that had earlier grown and spread west from the aircraft cavity on the 81 st floor appeared to be dying down. There was evidence that a small fire to the west of the aircraft impact cavity on the 80th floor was slowly spreading westward. During the next eight minutes (up to 9:52 a.m.), the appearance of the face did not change significantly, and the amount of visible fire seemed to decrease.

Around 9:52 a.m. a fire deep inside the tower was observed on the 80th floor to the west of the aircraft impact cavity. This fire rapidly developed over the next few minutes following a release of smoke from the area just before 9:53 a.m., and by 9:54:24 a.m. flames were visible from the ground over a line of open windows from roughly window $80-440$ to window 80-450. Just before 9:57 a.m. flames began to come from a number of windows on the west side of the 80 th floor. By the time the tower collapsed at 9:58:59 a.m., fire was visible from roughly window 80-440 to window 80-455. Flames were pushed from the area by the pressure wave generated by the collapse. The fire spread on this area of the 80 th floor was the most rapid and extensive observed in WTC 2. It took place after a long period of relative inactivity on the south face.

The effects of the fire behaviors discussed above are reflected in the integrated fire intensities for each face, shown in Figure 9-89 and Figure 9-90.

The façade maps of window conditions for the south face shown in Figure 9-91 also provide a good indication of the how the fires tended to move away from the aircraft impact cavity between the time of aircraft impact and tower collapse. In particular, the result of the rapid fire spread on the 80th floor stands out. As evident from Table 9-2, the number of open windows on the face increased by roughly $45 \%$ during the period between the aircraft impact and collapse.

As discussed. fire spread toward the west was observed on both the north and south faces, but fires did not appear to reach the western edge of either face. This is consistent with the fact that fire was not observed on the west face of WTC 2 prior to its collapse. Smoke observed coming from the face may possibly be attributed to windows being broken open by people.

Table 9-2 indicates that the windows broken open in WTC 2 during the time following the aircraft impact increased in total by roughly $45 \%$ over that present immediately following impact. This can be compared with the corresponding increase in WTC 1, which exceeded a factor of 4.2. This large difference reflects several factors. The initial number of open windows following the aircraft impact was nearly twice as high for WTC 2, due primarily to the large number of windows broken open on the east face by the impact and subsequent fireballs. The amount of time available for WTC 2 was less than for WTC 1.56 min versus nearly $1 \mathrm{hr} 42 \mathrm{~min}$. Even given the shorter period of time, the rates of development of fires during the period shortly following impact and subsequent fire spread were much slower for WTC 2 than those observed for WTC 1. As a result of the large difference in the amount of additional ventilation area generated by the fires, it is likely that changes in the number of open window due to fire spread were much less important for the fire behavior of WTC 2 as compared to WTC 1. 
Numerous visual observations suggest that important changes, which might have had structural relevance, were taking place in WTC 2 in the period following the aircraft impact until collapse. These observations include hanging objects, some of which, based on appearance, may have been locally dislodged floor slabs that had settled down to locations below the spandrel, at scveral locations on the north and east faces, changes in the positions of the hanging objects during the period, the occurrence of numerous pressure pulses identified by smoke and/or dust puffs generated over multiple windows and floors, the appearance of molten metal pouring from the tops of open windows, and bowing of the outer steel framework. These observations are summarized below. Additional discussion and analysis concerning these observations are prescnted in other Investigation reports. (NIST NCSTAR 1-6; NIST NCSTAR 1-3C)

Observations concerning the hanging objects seen through the windows are summarized in Table 9-5. The entries are grouped by location. The entry under floor indicates the floor on which the observation was madc. Changes in appearance at a given location are noted in the comments.

Hanging objects were obscrved on the east face on the 82nd floor and on the north face of the 79th through 82 nd floors. The object on the east face appeared to be the floor slab from the 83rd floor that had become detached from the spandrel over a long length of the face and settled downward. The object was visible shortly after the aircraft impact and fireballs, and it is likely that it was first created during this period. Assuming that observations on the north and south ends represent a single object, there were at least three times when the object settled further downward. The exact times of these localized changes are not known. The approximate time ranges are indicated in Table 9-5.

Hanging objects were visible at several locations on the north face. In the area of the cold spot, objects were seen on the 80 th and 81 st floors. On both floors the appearance of the objects changed during the event. On the 80 th floor there was one major change, while on the 81 st floor there were at least two. Time ranges for when these changes took place are included in Table 9-5. At the eastern edge of the north face on the 82nd floor, a portion of what appeared to be the 83rd floor slab was visible sinking away from the spandrel at the base of the 83 rd floor. This object appeared to be separated into two pieces, possibly along the boundary where two prefabricated sections of the floor slab had been joined. Other hanging objects were not viewed as clearly. As a result, the visual evidence for identifying these objects as possibly being dislodged floor slabs is weaker.

As discussed above, the sources for the pressure pulses that created the wide-spread smoke and/or dust puffs observed on multiple faces of WTC 2 are unknown. There arc numerous possibilities. However, it is possible that some fraction of these pulses could have been associated with structural changes in the tower, even though definitive visual evidence is not available to support such a hypothesis. There. is evidence that some of the puffs were correlated with releases of molten aluminum, which could possibly be associated with local floor slabs movement (see below).

The sudden release of molten material (possibly aluminum) that began pouring from window $80-255$ on the north side of the 80th floor at 9:51:51 a.m. provides evidence for the extensive heating that had taken place from the fire that had been burning in the area for nearly $50 \mathrm{~min}$. The melting point range for the relevant aluminum alloys varies from $475^{\circ} \mathrm{C}$ to $635^{\circ} \mathrm{C}$, and a great deal of heat would have been required to melt the large volume of liquid metal observed pouring from the tower. The sudden appearance of the flow at the top of the window was likely the result of the formation of a pathway from 
the 81 st floor, where the aluminum possibly had pooled on top of the floor slab as it melted. This, in turn, suggests that the 81 st floor slab possibly sank down or pulled away from the spandrel at this time.

Table 9-5. Summary of Observations of Hanging Objects on the North and East Faces of WTC 2

\begin{tabular}{|c|c|c|c|c|}
\hline Face & $\begin{array}{c}\text { Time } \\
\text { Observed } \\
\end{array}$ & Floor & $\begin{array}{l}\text { Window } \\
\text { Range }\end{array}$ & Comments \\
\hline East & $\begin{array}{l}\text { 9:03:42 a.m. } \\
\text { 9:18:38 a.m. }\end{array}$ & 82 & $82-321$ to $82-340$ & $\begin{array}{l}\text { Appears to be draped across tops of } \\
\text { windows, only edge visible, flames visible } \\
\text { above object }\end{array}$ \\
\hline East & $\begin{array}{l}\text { 9:25:57 a.m. } \\
\text { 9:26:20 a.m. } \\
\text { 9:27:35 a.m. } \\
\text { 9:34:40 a.m. }\end{array}$ & 82 & $82-329$ to $82-342$ & $\begin{array}{l}\text { Object seen earlier appears to have settled } \\
\text { down to point where the top and bottom } \\
\text { surfaces can be seen, lowest point near } \\
\text { window } 82-336 \text { is below half height of } \\
\text { window, fire above object }\end{array}$ \\
\hline East & $\begin{array}{l}\text { 9:38:22 a.m. } \\
\text { 9:47:10 a.m. } \\
\text { 9:55:04 a.m. }\end{array}$ & 82 & $82-332$ to $82-342$ & $\begin{array}{l}\text { Slopes from left to right from near top of } \\
\text { window to base of window, object seen } \\
\text { earlier appears to have settled down further } \\
\text { and a long portion (at least as far as } \\
82-330 \text { ) is hidden below bottom of window }\end{array}$ \\
\hline East & $\begin{array}{l}\text { 9:38:22 a.m. } \\
\text { 9:47:10 a.m. }\end{array}$ & 82 & $82-311$ to $82-317$ & $\begin{array}{l}\text { Slopes gently from right to left near tops of } \\
\text { windows }\end{array}$ \\
\hline East & 9:55:04 a.m. & 82 & $82-310$ to $82-317$ & $\begin{array}{l}\text { Slopes rapidly from right to left from near } \\
\text { top of window to near bottom, may be } \\
\text { object above, which has settled down } \\
\text { further }\end{array}$ \\
\hline North & 9:46:32 a.m. & 79 & $79-229$ to $79-233$ & $\begin{array}{l}\text { Slopes from left to right starting near top of } \\
\text { window and settling to near center of } \\
\text { window, has different appearance than } \\
\text { elsewhere }\end{array}$ \\
\hline North & 9:58:37 a.m. & 79 & $79-252$ to $79-254$ & $\begin{array}{l}\text { Appears to be draped across windows at } \\
\text { roughly } 3 / 4 \text { of the height of the window }\end{array}$ \\
\hline North & $\begin{array}{l}\text { 9:10:01 a.m. } \\
\text { 9:14:03 a.m. } \\
\text { 9:19:19 a.m. } \\
\text { 9:27:04 a.m. }\end{array}$ & 80 & $80-243$ to $80-255$ & $\begin{array}{l}\text { Roughly horizontal at roughly } 2 / 3 \text { of } \\
\text { windows height, flame visible above object }\end{array}$ \\
\hline North & $\begin{array}{l}\text { 9:10:01 a.m. } \\
\text { 9:14:03 a.m. } \\
\text { 9:19:19 a.m. } \\
\text { 9:27:04 a.m. }\end{array}$ & 80 & $80-241$ to $80-247$ & $\begin{array}{l}\text { Slopes downward from near top of window } \\
\text { on left to roughly } 2 / 3 \text { of window height on } \\
\text { the right }\end{array}$ \\
\hline North & $\begin{array}{l}\text { 9:42:31 a.m. } \\
\text { 9:46:32 a.m. } \\
\text { 9:50:55 a.m. } \\
\text { 9:52:51 a.m. } \\
\text { 9:58:37 a.m. }\end{array}$ & 80 & $80-245$ to $80-248$ & $\begin{array}{l}\text { Slopes from near top of window on left to } \\
\text { base of window on right, this object has } \\
\text { replaced the two objects seen earlier }\end{array}$ \\
\hline North & 9:14:03 a.m. & 80 & $80-252$ to $80-254$ & $\begin{array}{l}\text { Near top of window, fire is visible burning } \\
\text { above object }\end{array}$ \\
\hline North & 9:14:03 a.m. & 80 & $80-252$ & $\begin{array}{l}\text { Slopes downward from left to right near } \\
\text { mid height of window, area hidden in later } \\
\text { images }\end{array}$ \\
\hline North & 9:14:03 a.m. & 80 & $80-252$ to $80-254$ & Located in lower $1 / 3$ of windows \\
\hline
\end{tabular}




\begin{tabular}{|c|c|l|l|l|}
\hline Face & $\begin{array}{c}\text { Time } \\
\text { Observed }\end{array}$ & Floor & \multicolumn{1}{|c|}{$\begin{array}{c}\text { Window } \\
\text { Range }\end{array}$} & \multicolumn{1}{c|}{ Comments } \\
\hline North & $\begin{array}{l}9: 14: 03 \text { a.m. } \\
9: 29: 42 \text { a.m. }\end{array}$ & 81 & $81-240$ to $81-243$ & $\begin{array}{l}\text { Top of two closely spaced objects, slopes } \\
\text { slowly downward from left to right starting } \\
\text { near top of window }\end{array}$ \\
\hline North & $\begin{array}{l}9: 14: 03 \text { a.m. } \\
9: 29: 42 \text { a.m. }\end{array}$ & 81 & $81-238$ to $81-242$ & $\begin{array}{l}\text { Bottom of two closely spaced objects, } \\
\text { slopes downward from left to right starting } \\
\text { near top of window and ending near } 2 / 3 \\
\text { window height }\end{array}$ \\
\hline North & $\begin{array}{l}9: 42: 31 \text { a.m. } \\
9: 46: 32 \text { a.m. }\end{array}$ & 81 & $\begin{array}{l}81-240 \text { to } 81-245, \\
81-248 \text { to } 81-250\end{array}$ & $\begin{array}{l}\text { Right side slopes from near top of window } \\
\text { on left to base of window on right, left side } \\
\text { near top of windows, fire above object, this } \\
\text { object replaced two others seen at } 9: 29: 42 \\
\text { a.m., not visible at 9:52:51 a.m. }\end{array}$ \\
\hline North & $9: 58: 37$ a.m. & 81 & $81-247$ to $81-250$ & $\begin{array}{l}\text { Slopes downward from near top of window } \\
\text { on left to roughly } 1 / 2 \text { of the window height } \\
\text { on right, this object has replaced single } \\
\text { object present at 9:46:32 a.m. }\end{array}$ \\
\hline North & $\begin{array}{l}9: 53: 51 \text { a.m. } \\
9: 58: 37 \text { a.m. }\end{array}$ & 82 & $82-250$ to $81-258$ & $\begin{array}{l}\text { In right three windows see the edge of a } \\
\text { sheet below the spandrel, on left appears to } \\
\text { be a sheet that slopes away from the } \\
\text { spandrel at the edge of building, sheet is } \\
\text { separated into two sections }\end{array}$ \\
\hline
\end{tabular}

During the $7 \mathrm{~min}$ between when the flow of molten metal was first observed and the tower collapsed, the amount of material flowing from the 80th floor increased and decreased repeatedly. At one point the flow shifted from window 80-255 to window 80-256. The change in the source window for the liquid suggests that the lowest local point with pooled aluminum somehow moved to the east. These observations suggest that the 81 st floor slab in the immediate vicinity was possibly shifting almost continuously during this time, and, in the process, spilling more and more of the pooled liquid.

A similar release of liquid occurred from window 78-238 on the 78th floor around 9:27 a.m. It is possible that this material came from the pile of debris immediately above on the 79th floor. Since this flow was observed for only a few seconds, it is not appropriate to speculate further concerning its source.

Images of the east face of WTC 2 reveal that the exterior columns were distinctly bowed inward as early as 9:21 a.m. This bowing appeared to be greatest near the center of the face and to disappear near the edges. Analyses discussed elsewhere have estimated the degree of inward bowing as a function of time. (NIST NCSTAR 1-3B).

The general picture that emerges from the observations is that WTC 2 possibly underwent a prolonged series of subtle structural changes that began shortly after the aircraft impact and that these changes continued until the tower collapsed. These changes occurred as the structure adjusted to the initial damage inflicted by the aircraft and associated fireballs and the additional deformation caused by the fires that subsequently developed. While there is similar direct evidence for structural changes in WTC 1 as for WTC 2, e.g., bowing columns, events, such as smoke puffs, that might be possible telltales for internal changes taking place within the tower, such events were considerably less frequent in WTC 1 than observed for WTC 2. 
It has been pointed out numerous times that the fires in WTC 1 and WTC 2 had different charactcristics. These differences extended to human behaviors, which provide additional indications about these differences. In WTC 1 people broke out numerous windows above the immediate floors that were burning and were often observed in open windows. While some windows were broken open in WTC 2, the locations were limited and people were infrequently observed. Numerous people were observed falling from the upper floors on the four faces of WTC 1, while the three people observed falling from WTC 2 came from a single window on the east side of the 79th floor. These differences suggest that conditions on the upper floors in WTC 2 did not degenerate as quickly, nor become as dirc, as on the upper floors of WTC 1.

The difference in conditions on the upper floors of the towers was likely due to the zonal structures of the ventilation system. In WTC 1 the stories above the aircraft impact floors were serviced by a ventilation system connected to the mechanical equipment room located on the 108 th and 109 th floors. As a result, there were ventilation pathways passing throughout the area that could transport smoke and noxious gases directly from the fire floors. In WTC 2 the aircraft impacted floors that were serviced by the mechanical equipment room on the 75 th and 76th floors. As a result, floors above the $93 \mathrm{rd}$ floor were connected to a separate system, and the ventilation pathways were not available to directly transport smoke and gas from the floors with active fires. Visual evidence does indicate that smoke reached the upper levels of WTC 2. This was most likely transported through compromised elevator shafts that passed through the fire floors and extended to the upper floors of the tower.

In WTC 1 a large number of streamers were observed falling from the tops of windows. These were attributed to smoldering polyurethane that was originally located above the window head casing. The polyurethane was frequently exposed and burned when the head casing dropped down. As the polyurethane burned, it eventually fell, flamed out, and began to smolder, forming a streamer as it dropped. In contrast, very few streamers were observed for WTC 2. The relative absence of streamers is like due to the relatively limited fire spread that occurred in WTC 2 and because large areas of the aluminum curtain wall that contained the polyurethane insulation were removed by the aircraft impact and resulting fireballs.

\subsection{REFERENCES}

McAllister. T., ed. 2002. World Trade Center Building Performance Study: Data Collection, Preliminary Observations, and Recommendations. FEMA 403. Federal Emergency Management Agency. Washington, DC, May.

The Aluminum Association. 2003. Aluminum Standards and Data - 2003. The Aluminum Association, Inc., Washington, DC. 
This page intentionally left blank. 


\section{Appendix A \\ VENTILATION LOUVER LOCATIONS}

This appendix contains drawings that show locations of intake and exhaust louvers relative to the exterior façade for the mechanical equipment rooms on the 108th and 109th floors of World Trade Center

(WTC) 1 and the 75th and 76th floors of WTC 2 (see Figure A-1 through Figure A-8). The drawings for WTC 1 can be applied to WTC 2 by rotating counterclockwise 90 degrees, and those for WTC 2 can applied to WTC 1 by rotating 90 degrees clockwise. The locations of louvers are indicated by the solid green color.

The mechanical equipment room on the 108th and 109th floors of WTC 1 provided services for the 92nd to 110 th floors, and the mechanical equipment room on the 75 th and 76 th floors provided services for the 77 th to 91 st floors. The air intakes and exhausts were located on louvered walls recessed $4 \mathrm{ft}$ from the outer façade of the tower. Sections of the louvered wall lined up with the exterior columns, but the widths of the sections were wider than the spacing between the exterior column covers. For the mechanical equipment room on the 75th and 76th floors, the base of the louvered wall extended 19 in below the aluminum spandrel cover for the base of the 75th floor, while for the upper mechanical floor, the base of the louvered wall was above the spandrel cover for the 108th floor. 


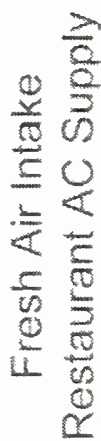

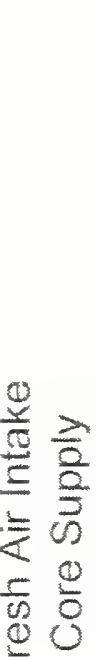

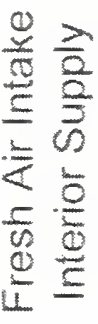

는

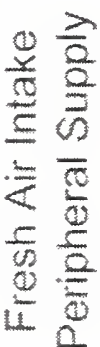

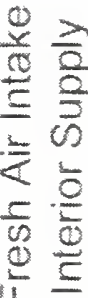

$\amalg$ 드

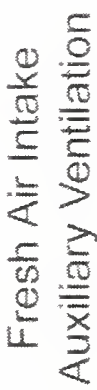

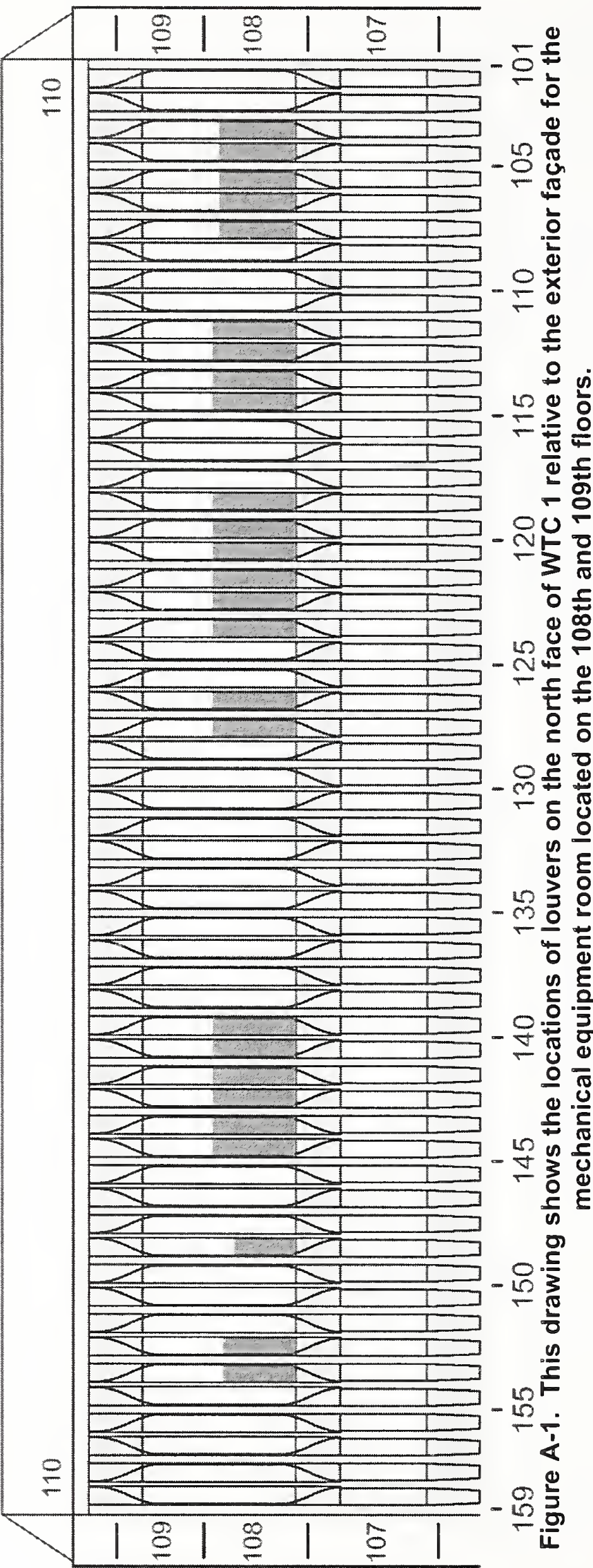




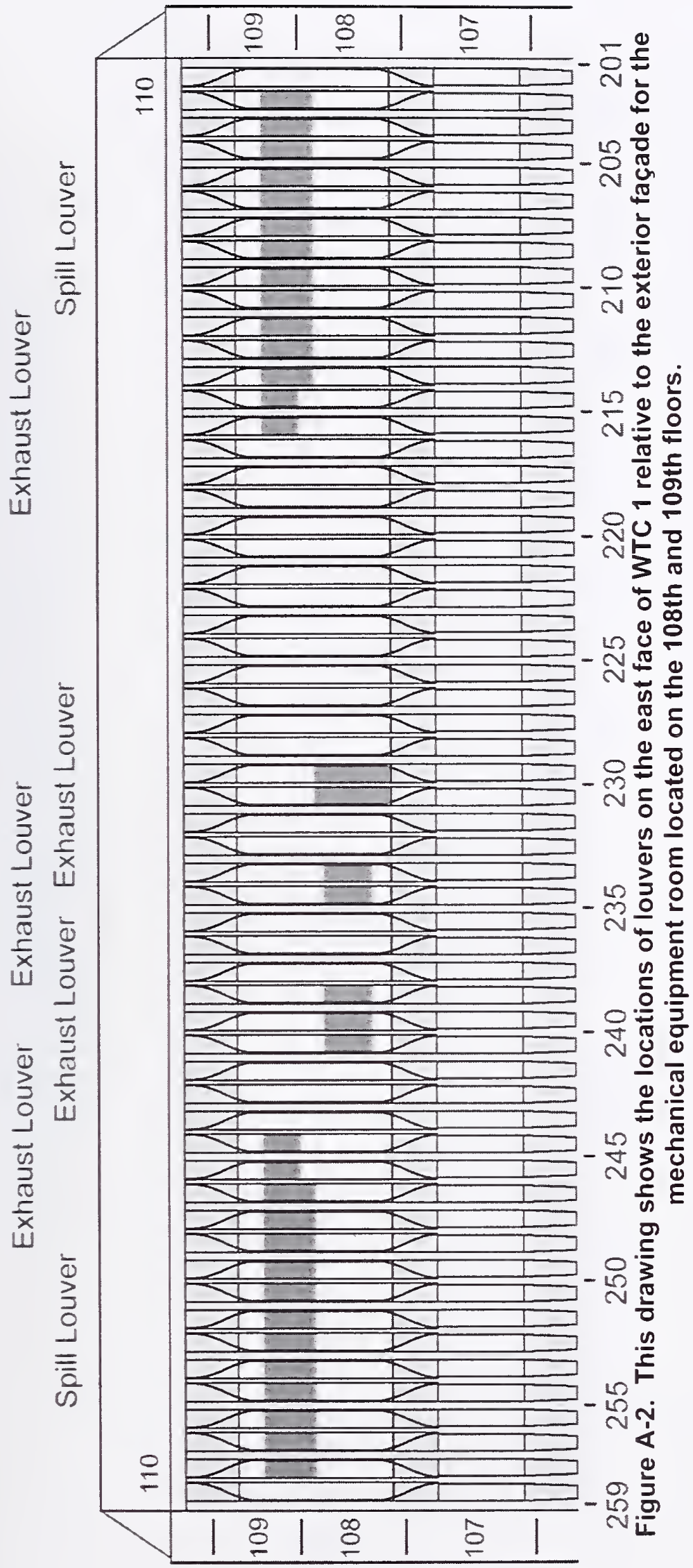




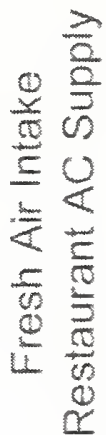

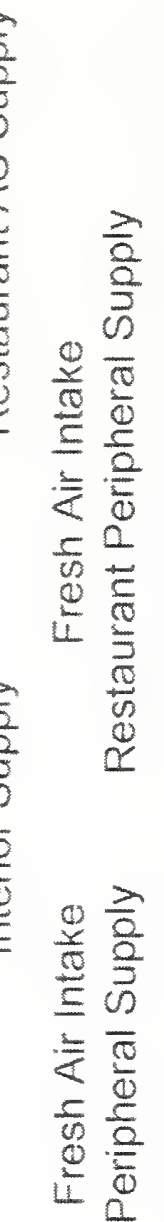

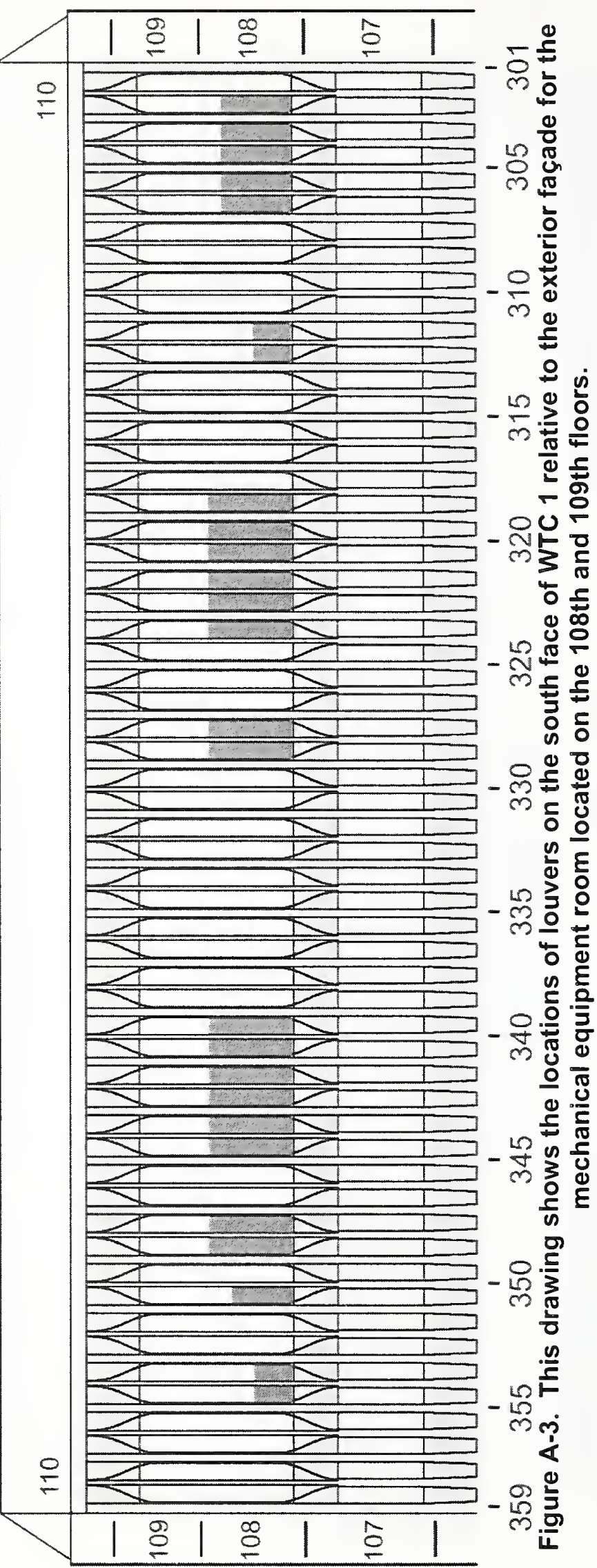




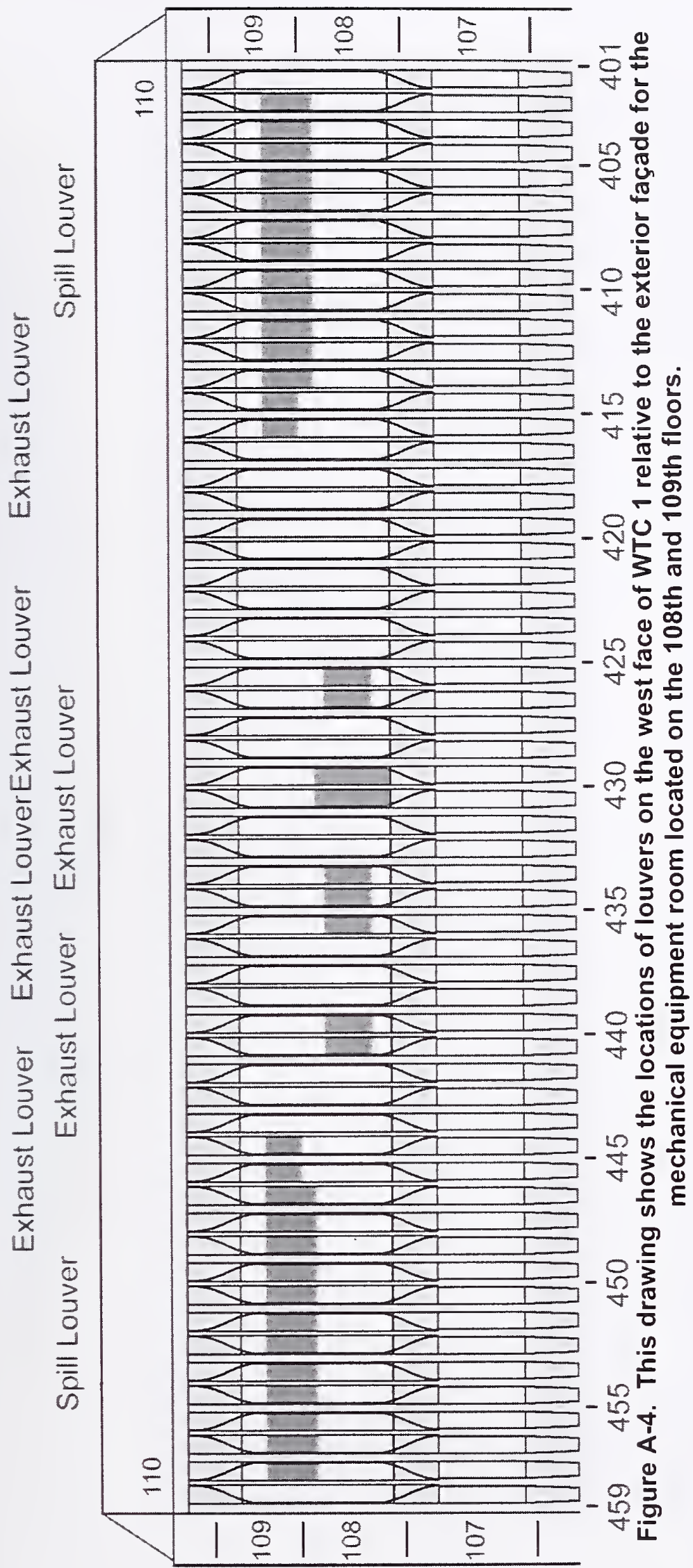




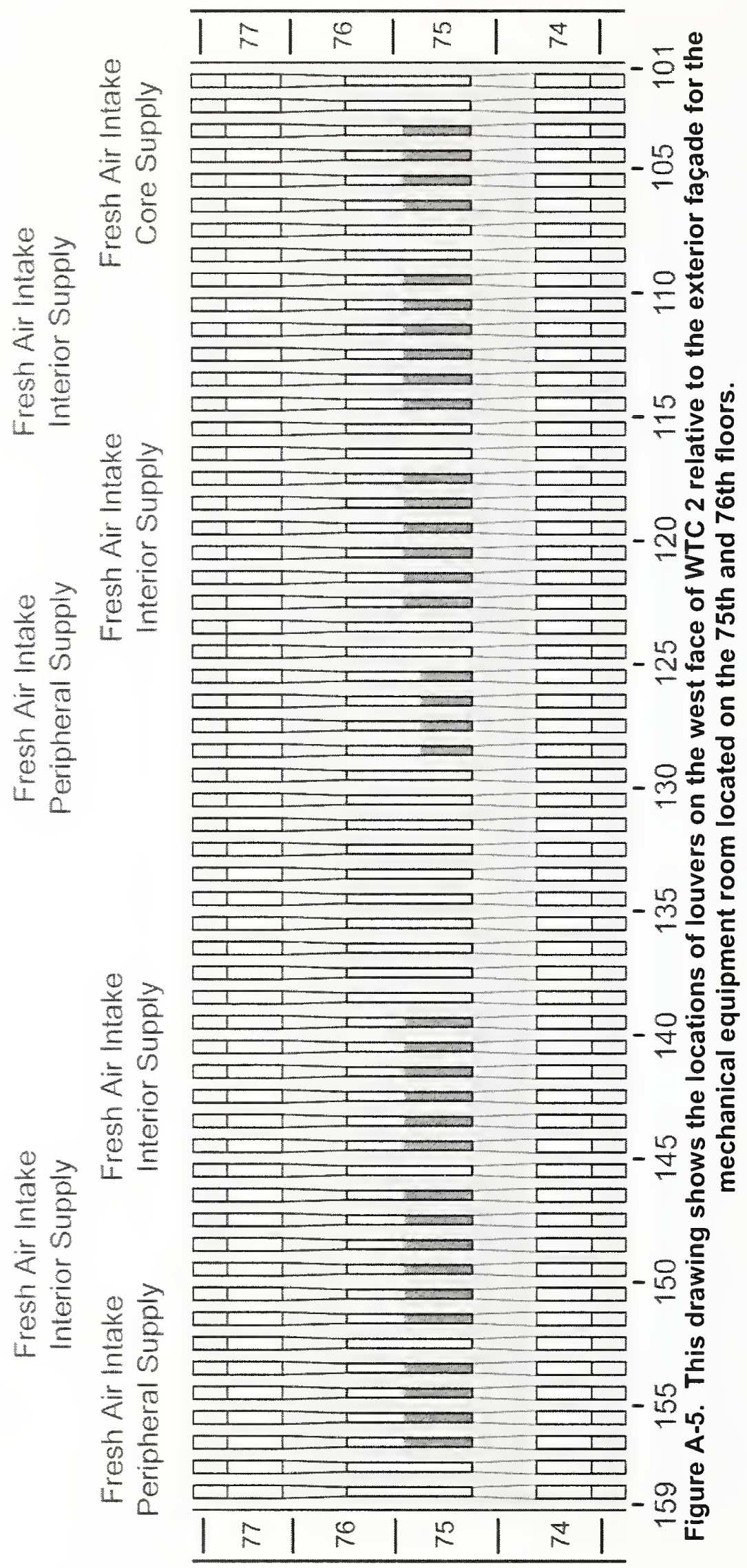




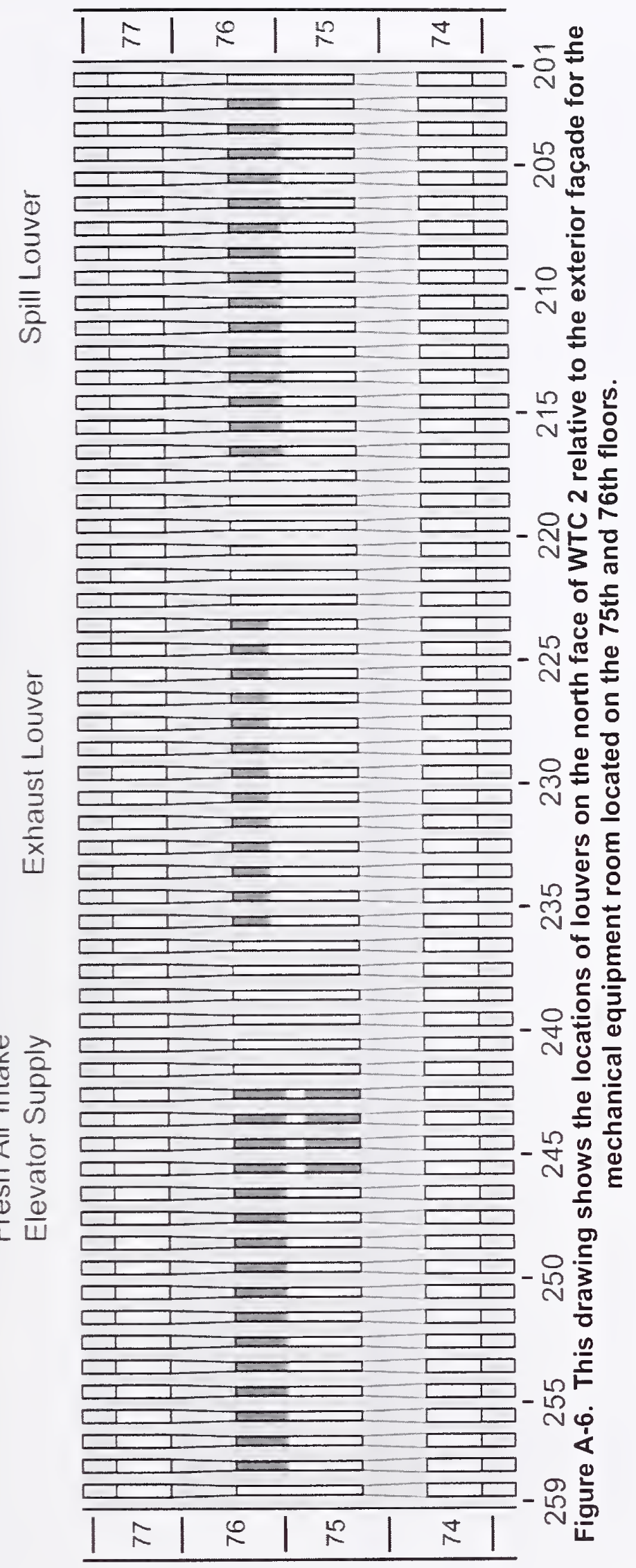




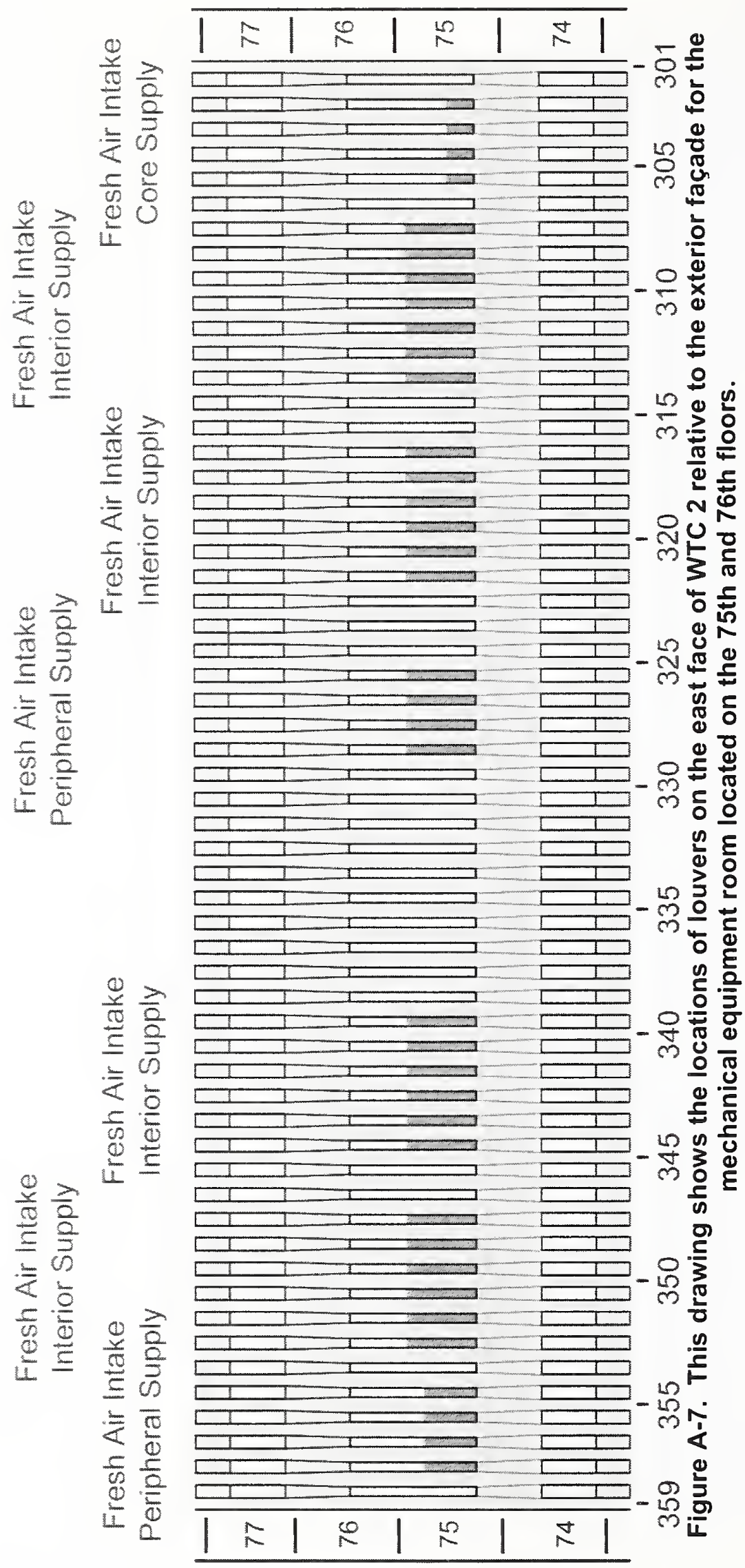




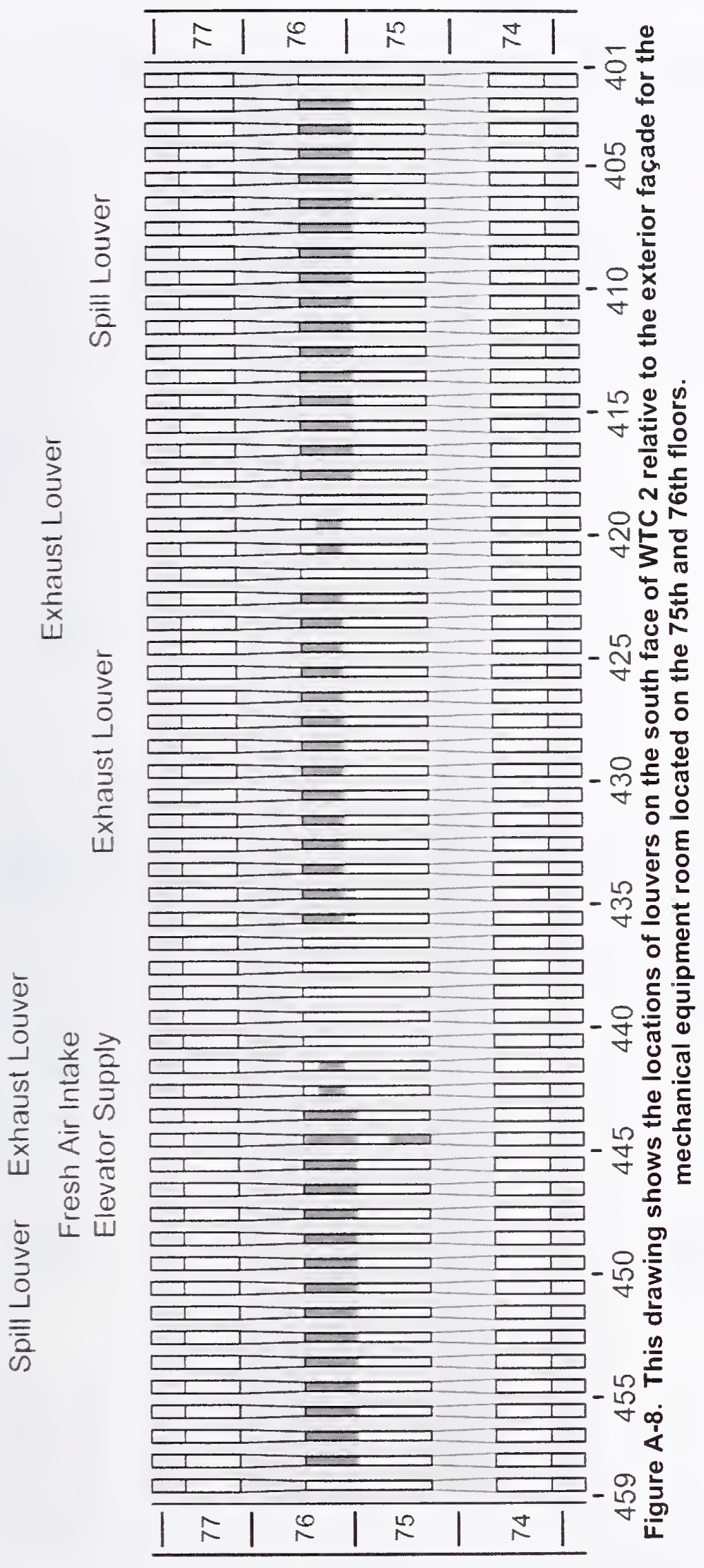


This page intentionally left blank. 


\section{Appendix B \\ FLOOR PLAN LAYOUTS FOR SELECTED WTC 1 AND WTC2 FLOORS}

This appendix reproduces floor plan layouts (Figure B-1 through Figure B-16) generated from original sources provided to National Institute of Standards and Technology (NIST) as part of the Investigation of the World Trade Center Disaster. The layouts were produced as part of the task "Fire Dynamics Modeling" (NIST NCSTAR 1-5F), one of the components that made up of the Investigation Project 5-Reconstruction of Fires in the WTC Towers (NCSTAR 1-5). They are included here for easy reference.

The following key summarizes the various colors and symbols used to represent different objects on the layouts.

$340 \quad$ Exterior columns and spandrels

Gypsum wall in core

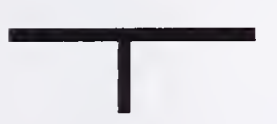

Glass wall

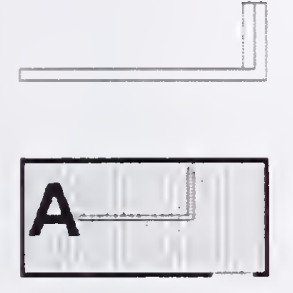

Stairwell

6 Cargo elevator for upper sector

85

Local elevator for floor

Enclosed Vertical Shaft

\section{Core column \\ 1001}
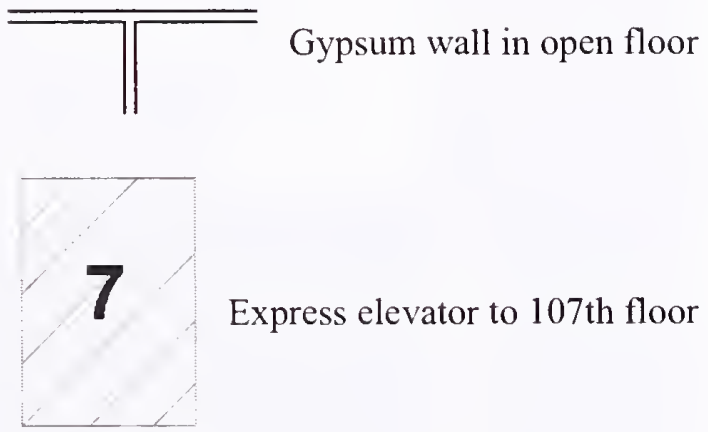

Express elevator to 107 th floor

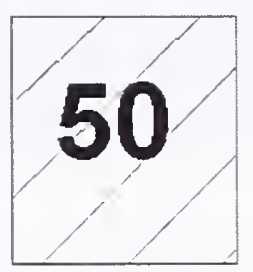

Freight elevator

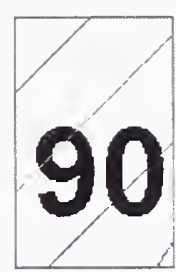

Local elevator for higher floor

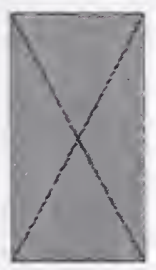




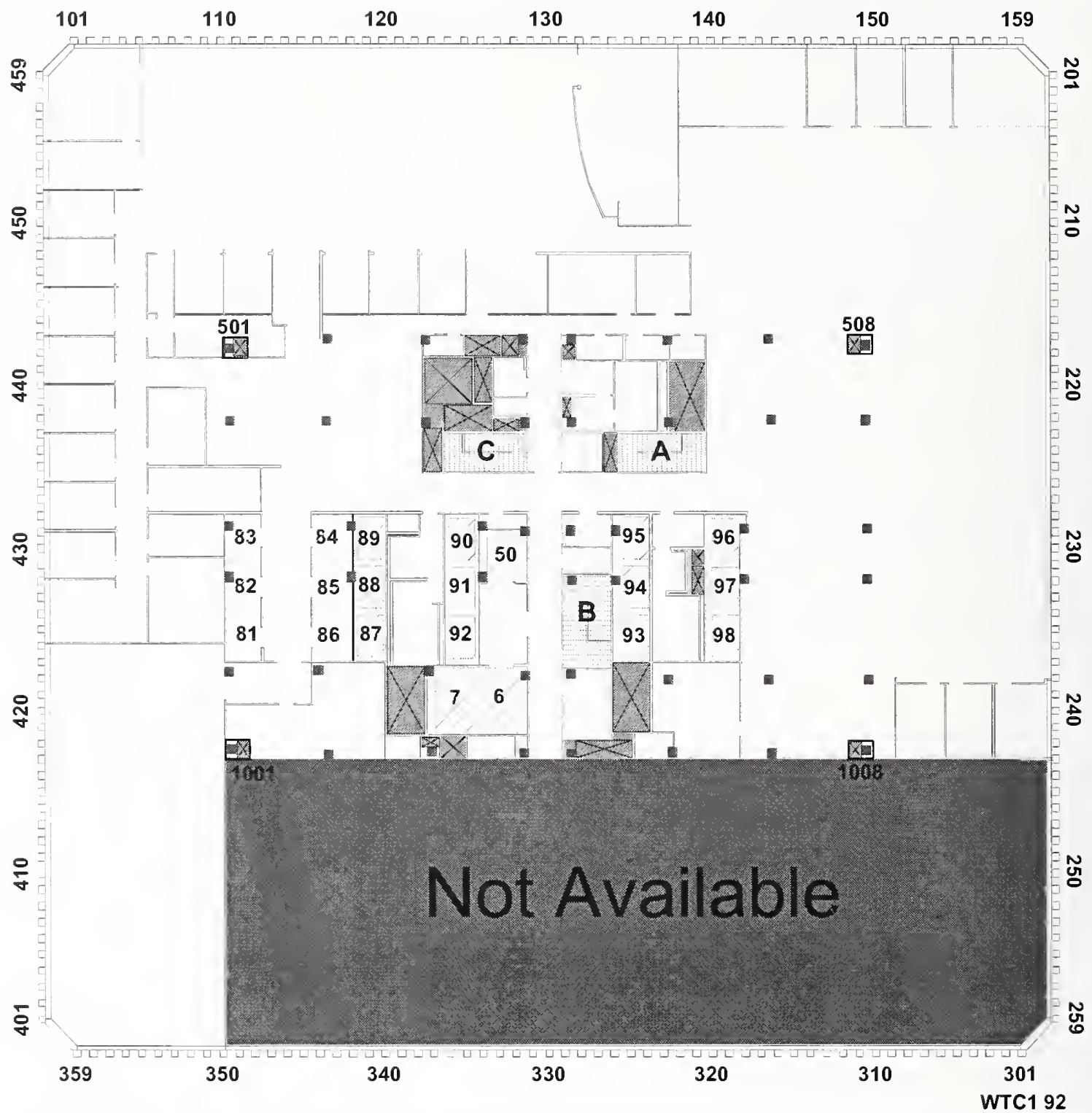

Figure B-1. This drawing shows the floor plan layout for the 92 nd floor of WTC 1. 


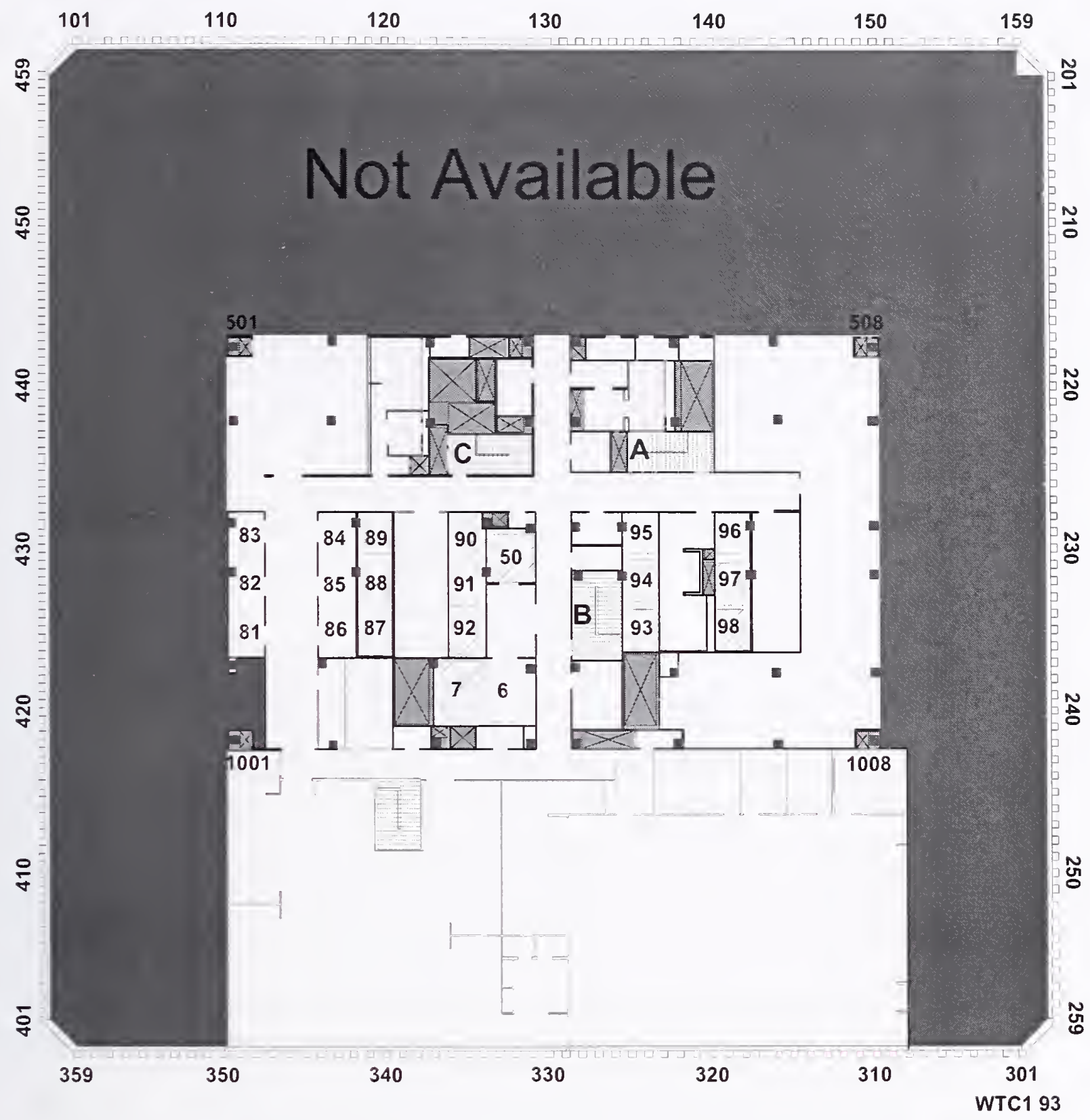

Figure B-2. This drawing shows the floor plan layout for the 93rd floor of WTC 1. 


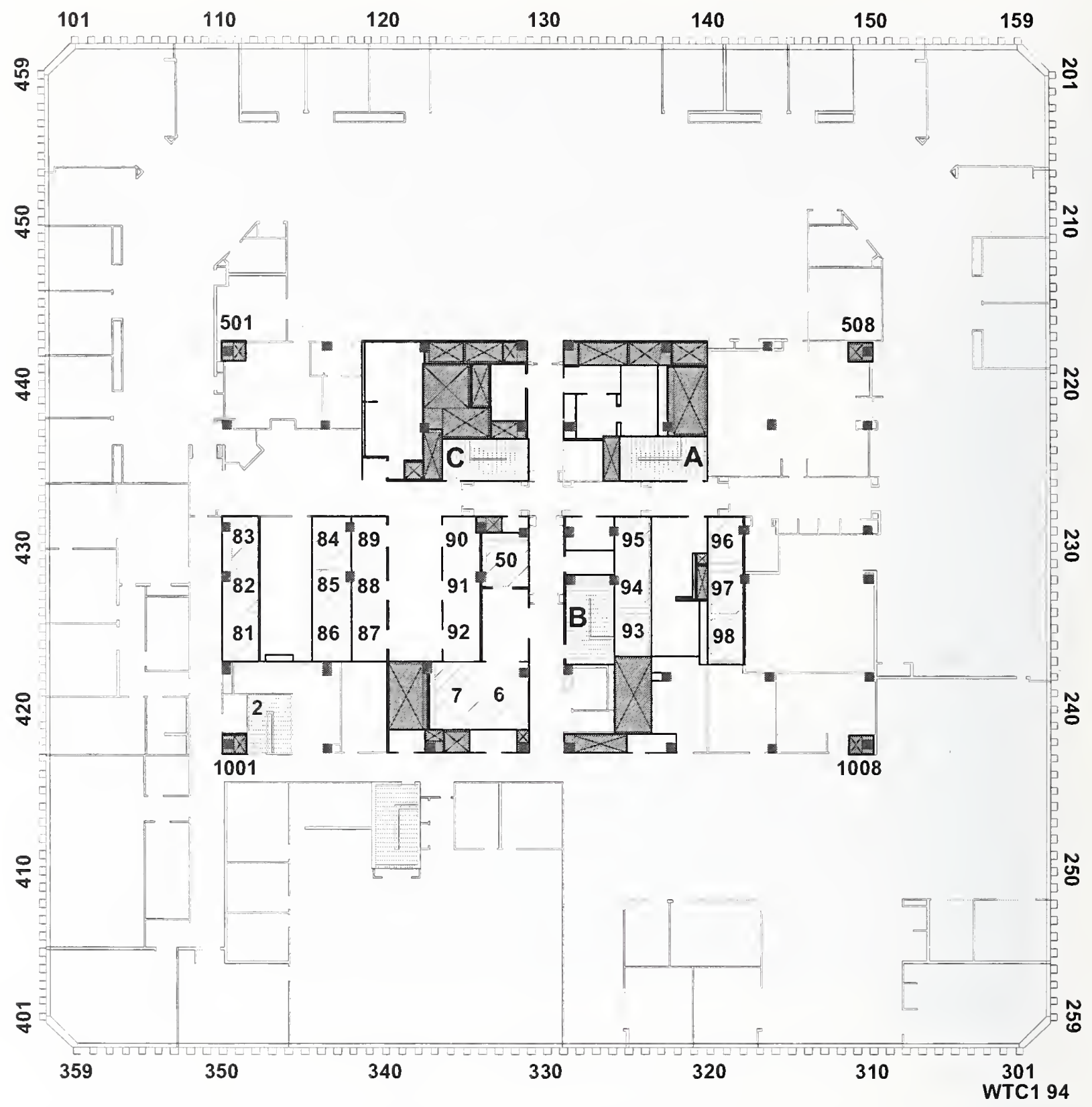

Figure B-3. This drawing shows the floor plan layout for the 94th floor of WTC 1. 


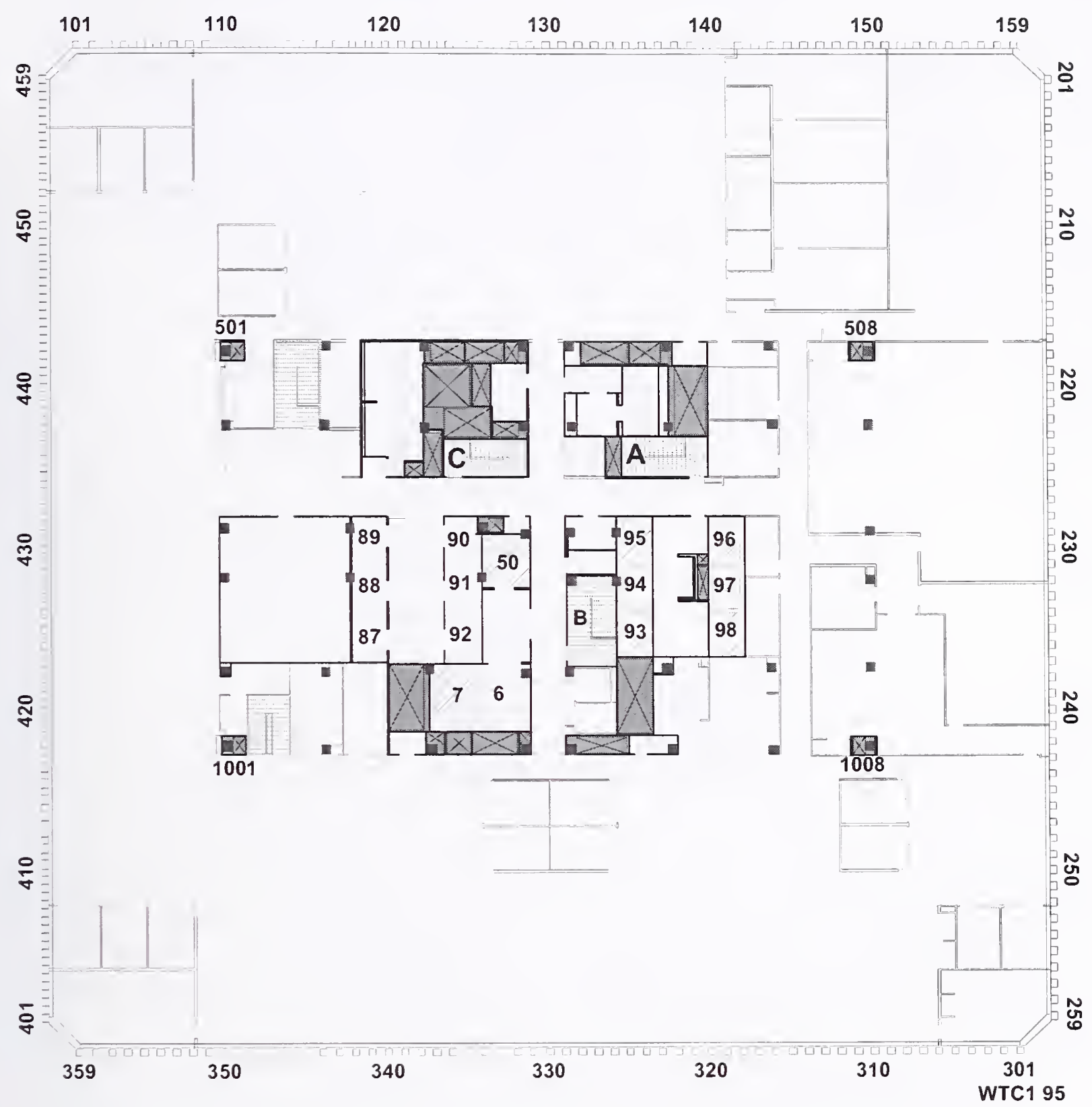

Figure B-4. This drawing shows the floor plan layout for the 95th floor of WTC 1. 


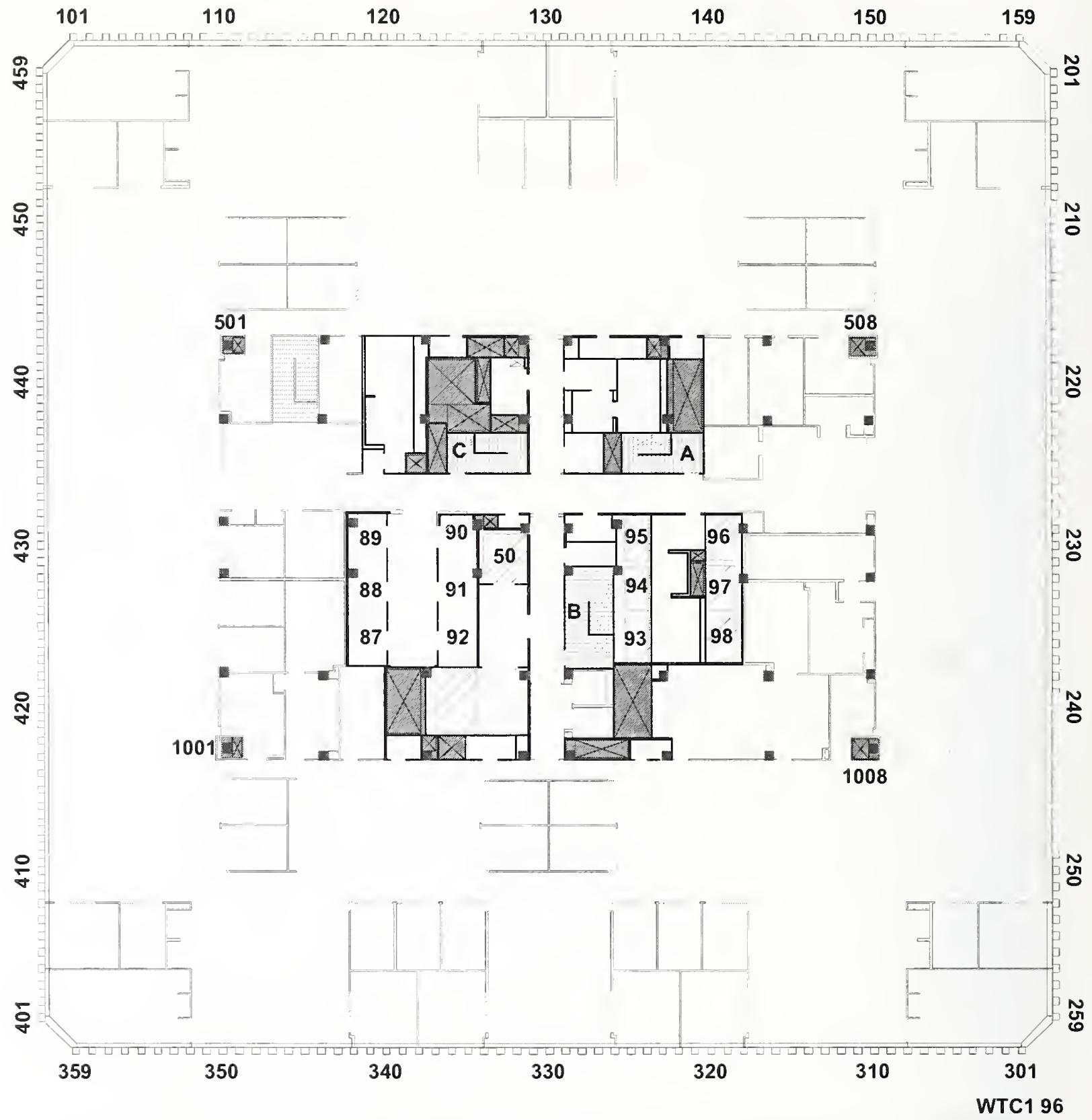

Figure B-5. This drawing shows the floor plan layout for the 96th floor of WTC 1 . 


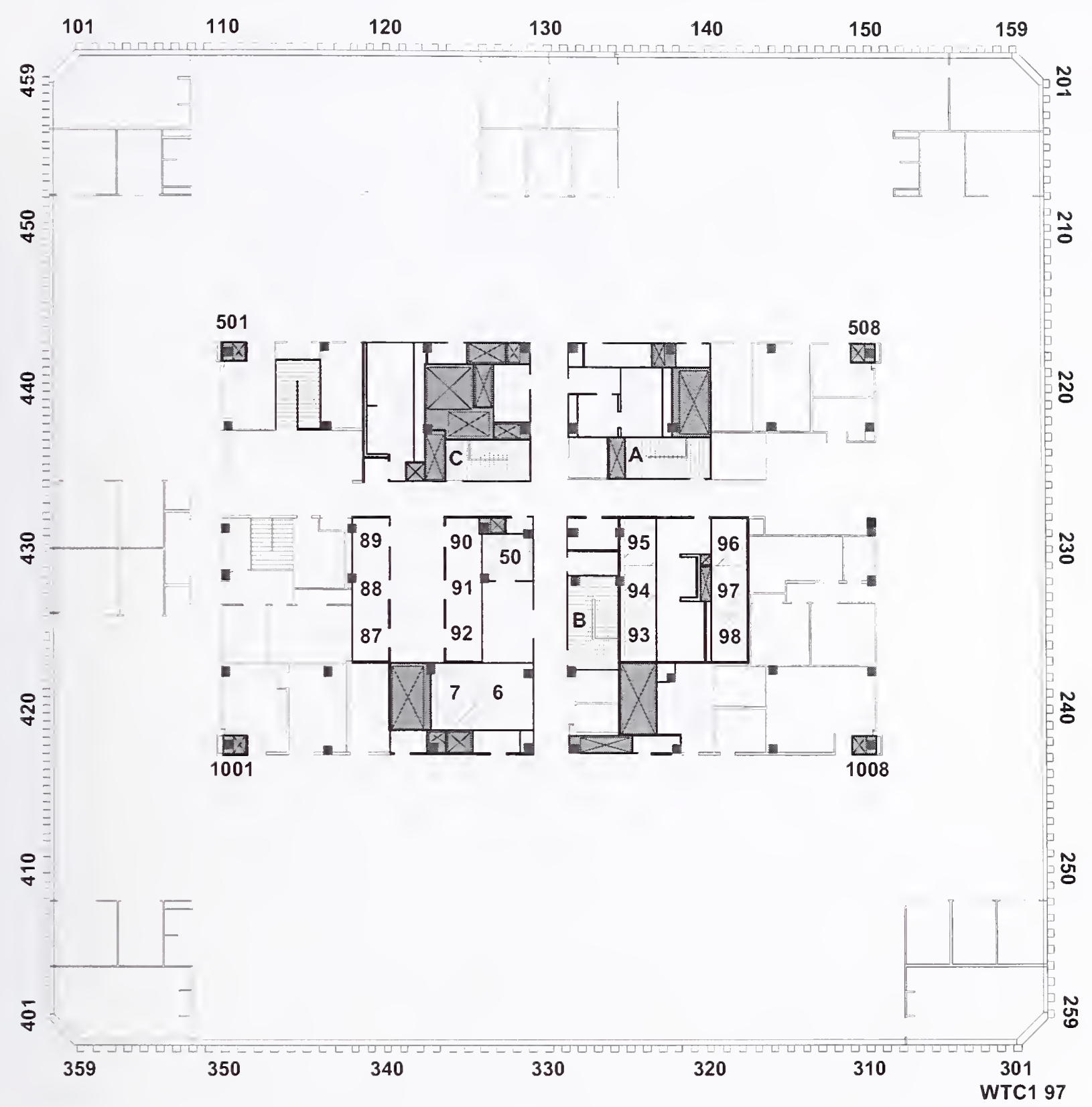

Figure B-6. This drawing shows the floor plan layout for the 97th floor of WTC 1. 


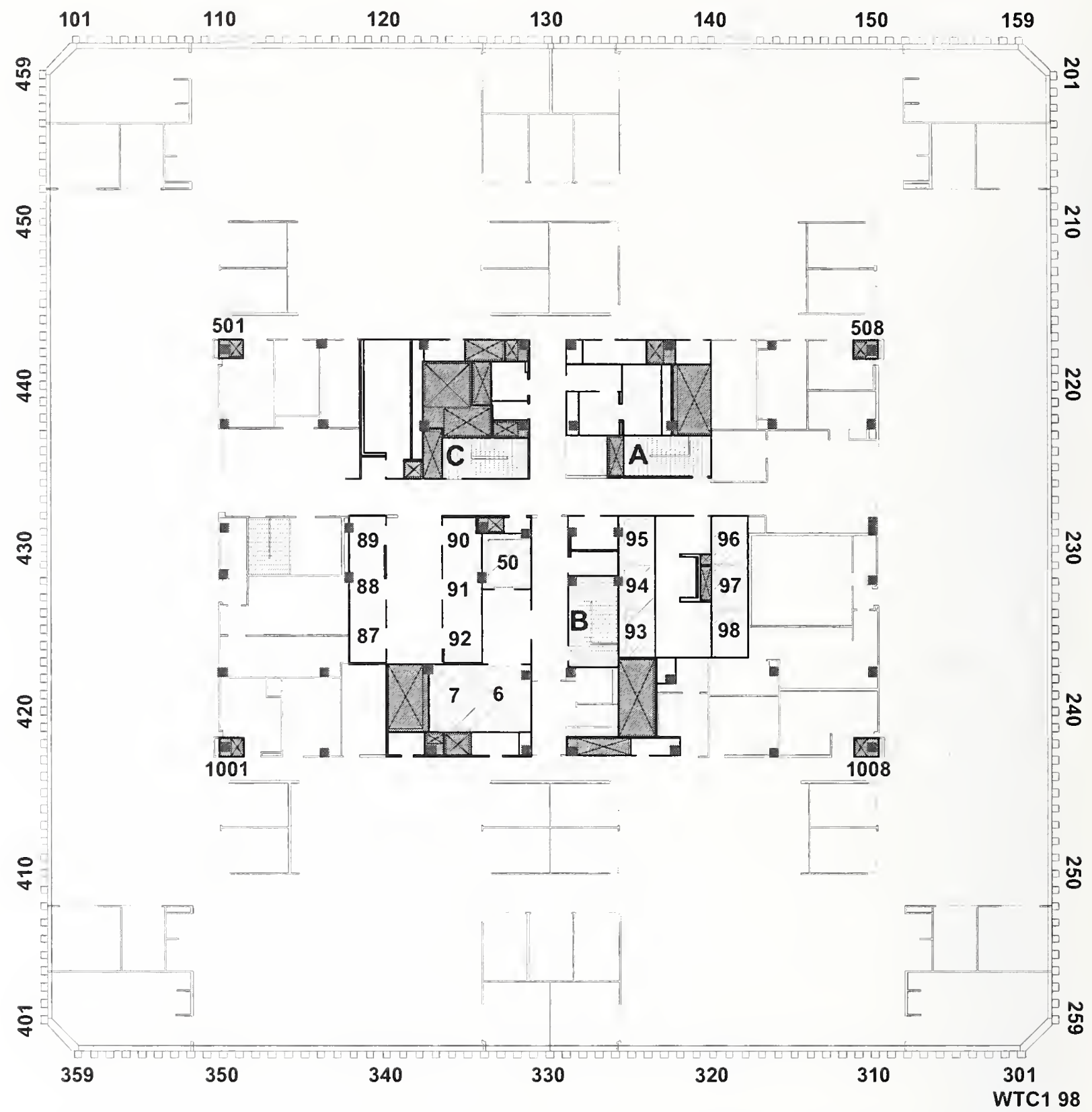

Figure B-7. This drawing shows the floor plan layout for the 98th floor of WTC 1. 


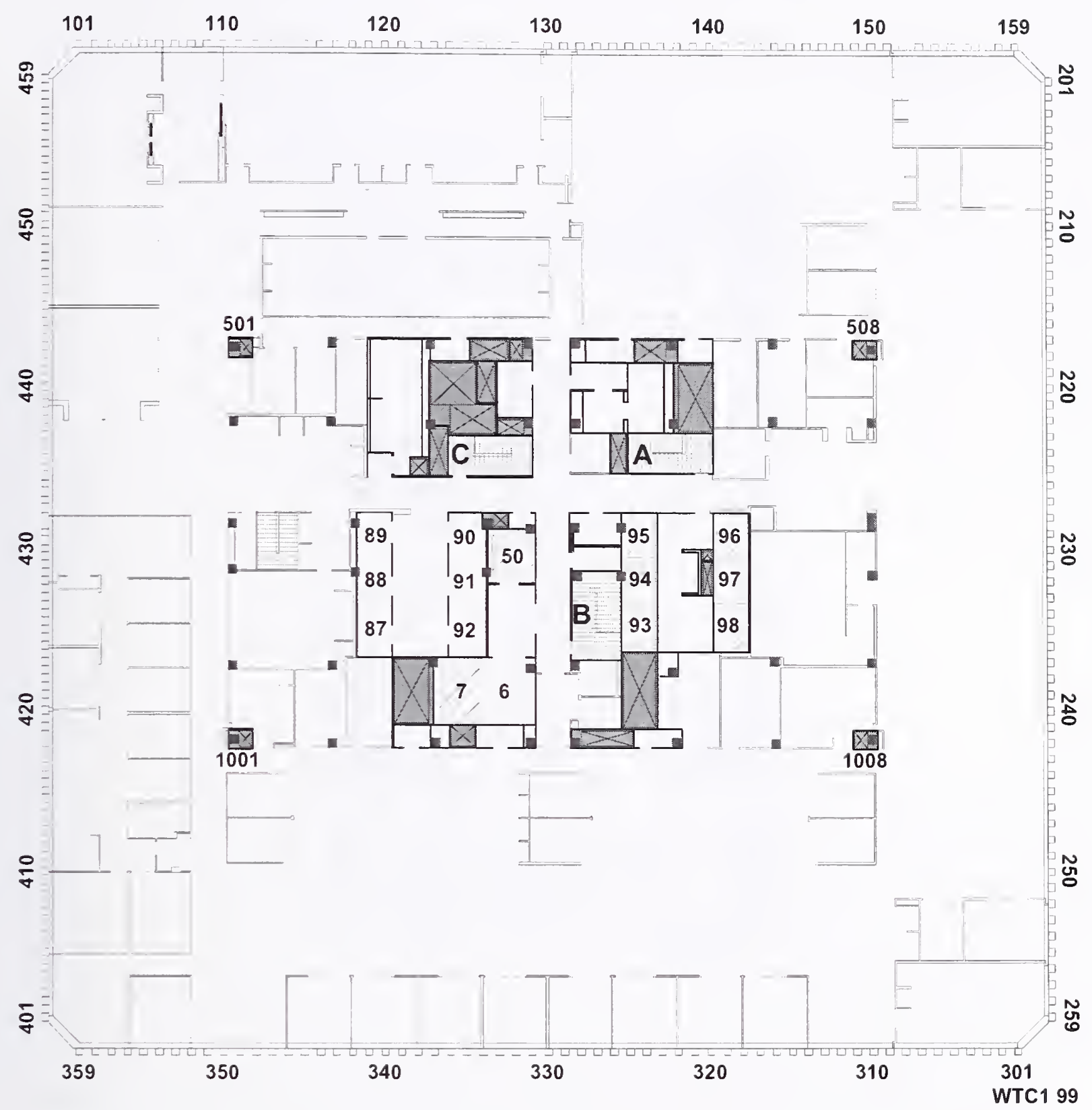

Figure B-8. This drawing shows the floor plan layout for the 99th floor of WTC 1. 


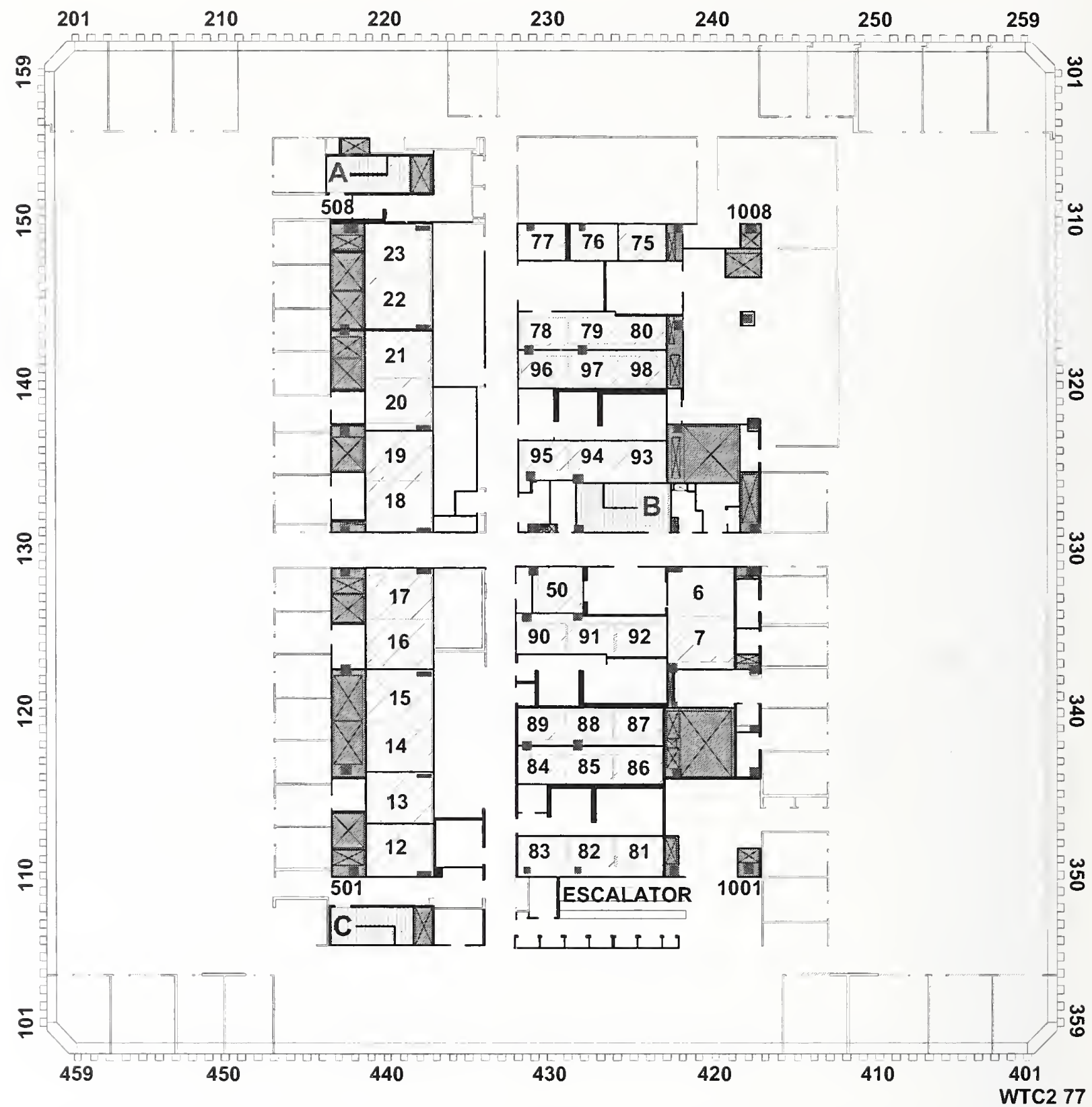

Figure B-9. This drawing shows the floor plan layout for the 77th floor of WTC 2. 


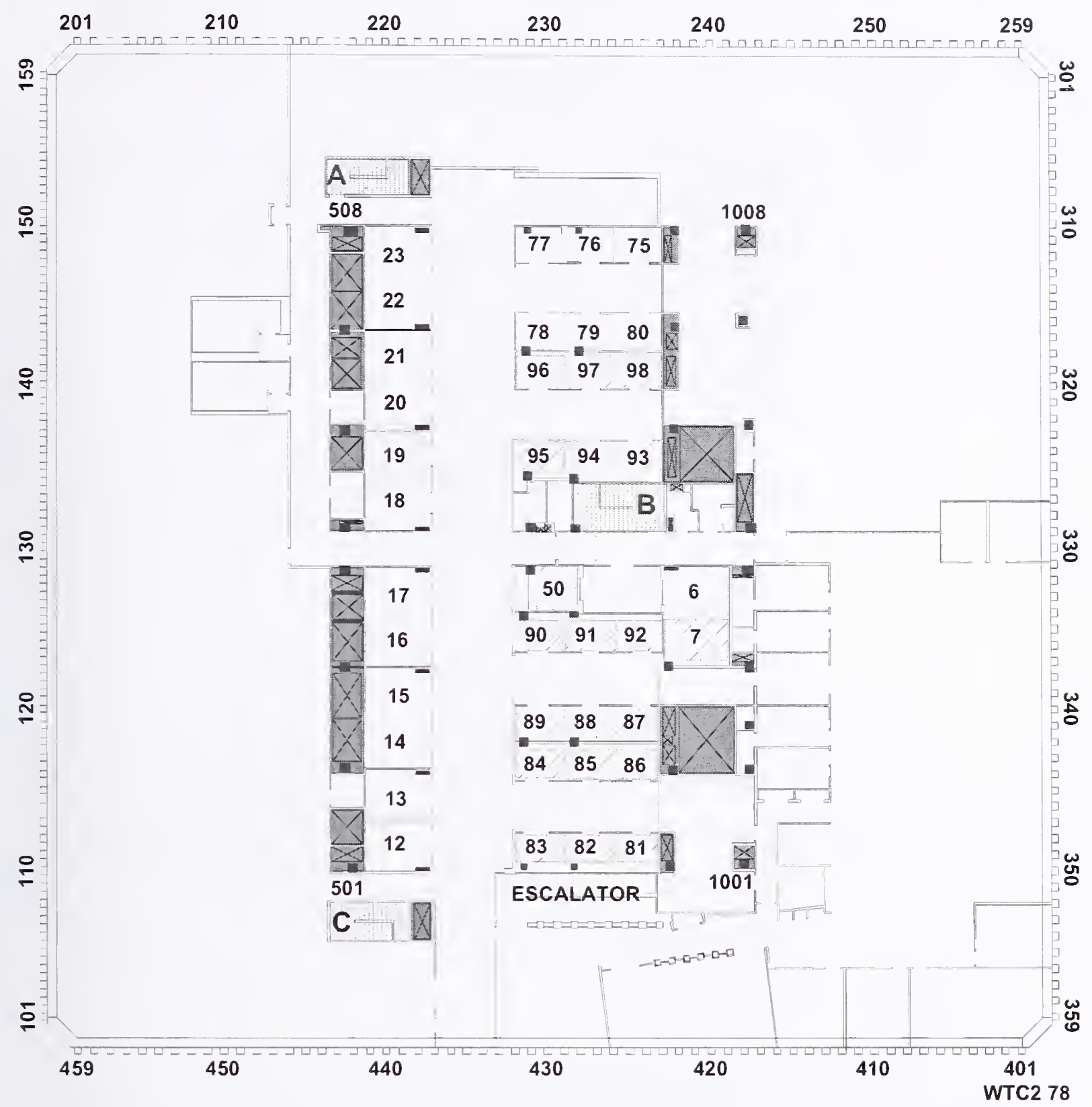

Figure B-10. This drawing shows the floor plan layout for the 78th floor of WTC 2. 


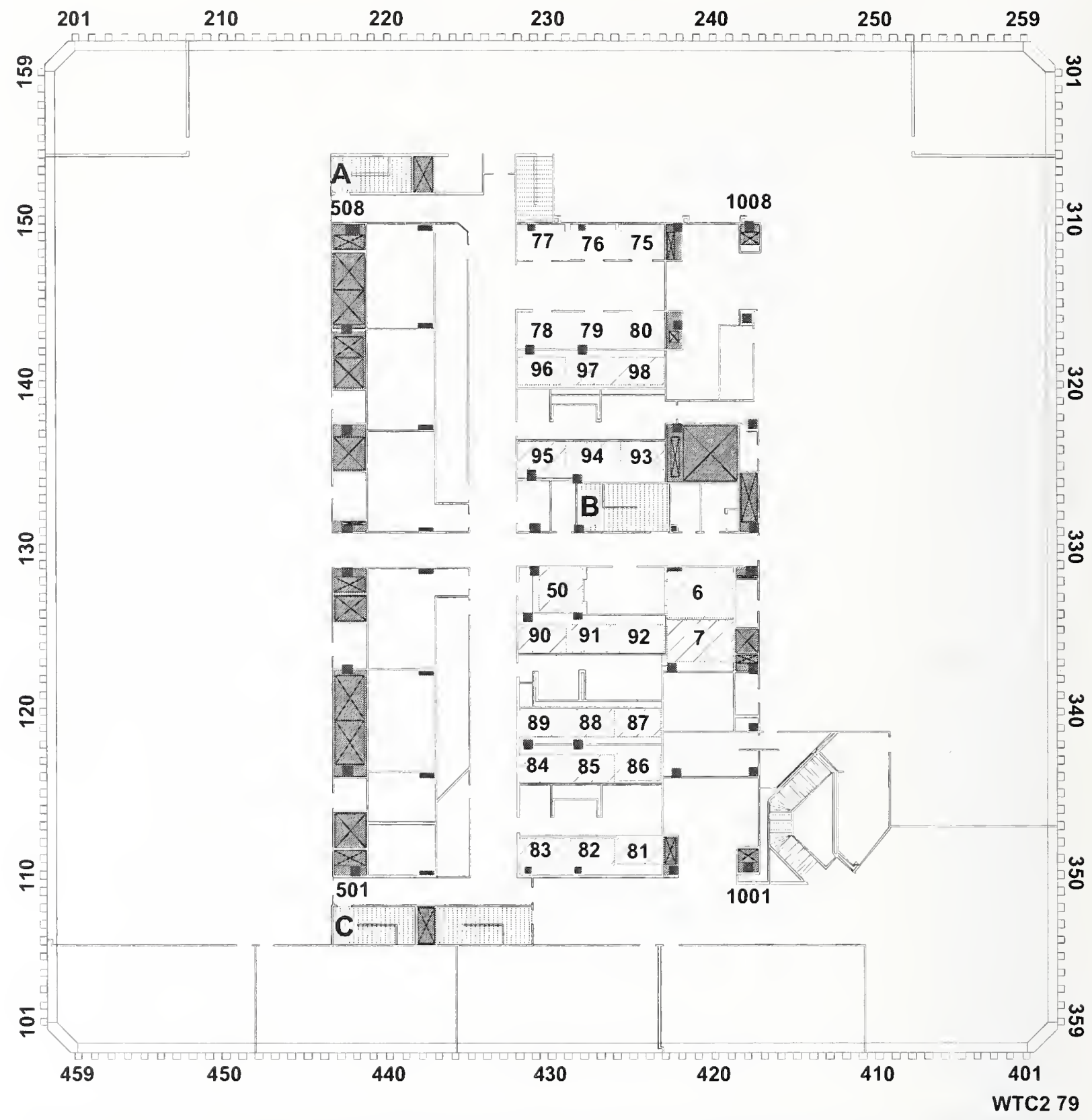

Figure B-11. This drawing shows the floor plan layout for the 79th floor of WTC 2. 


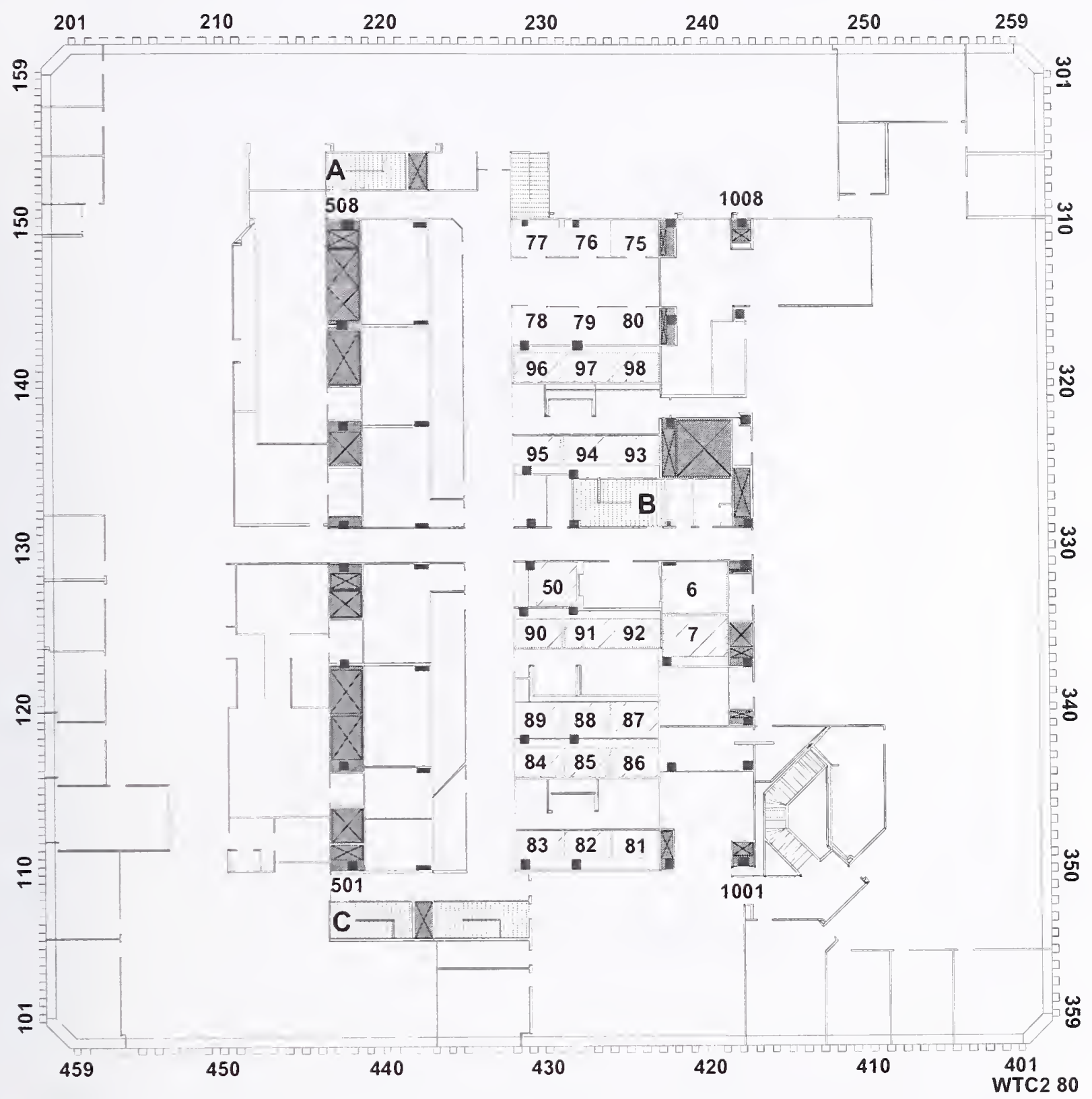

Figure B-12. This drawing shows the floor plan layout for the 80th floor of WTC 2. 


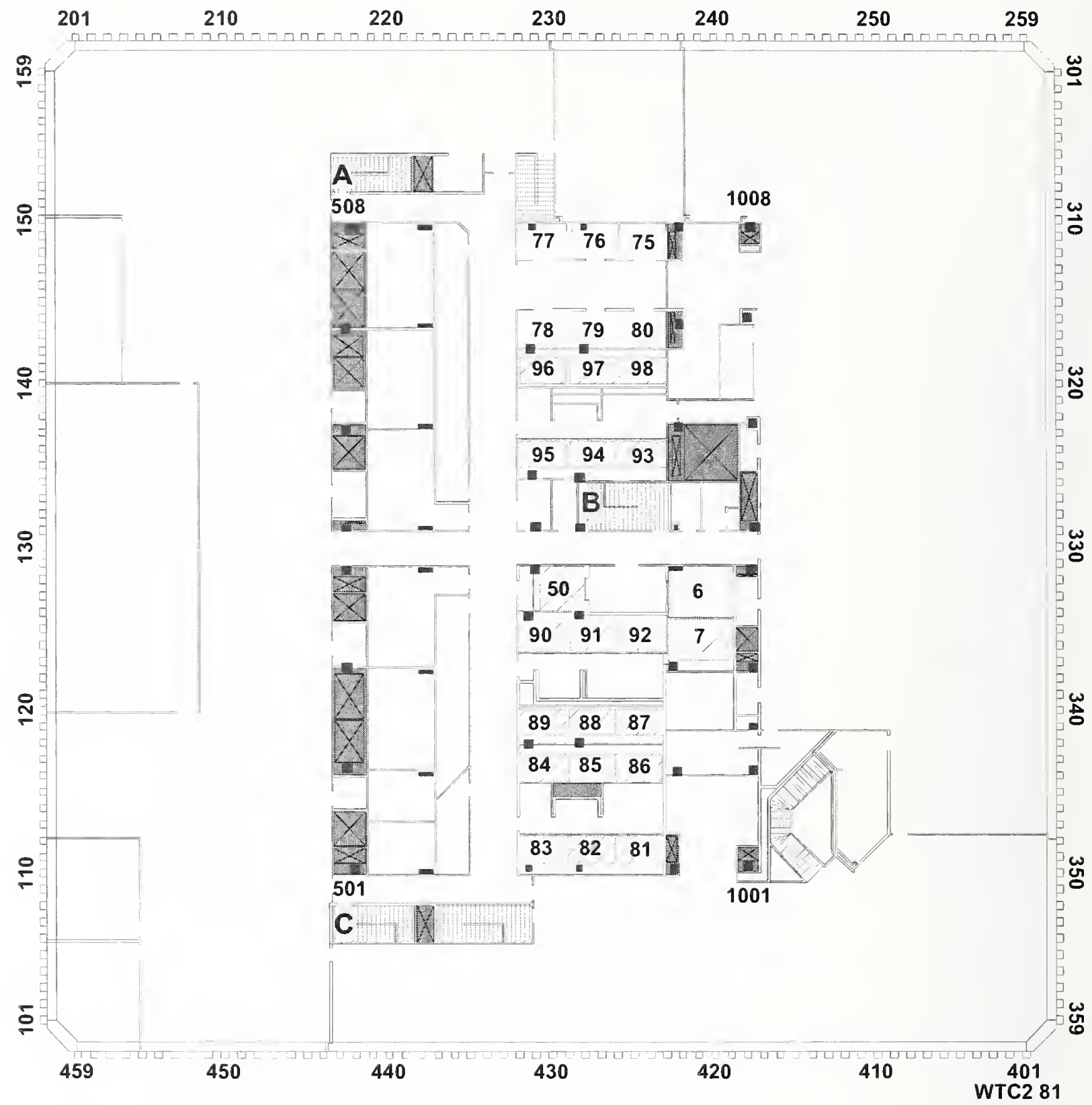

Figure B-13. This drawing shows the floor plan layout for the 81st floor of WTC 2 . 


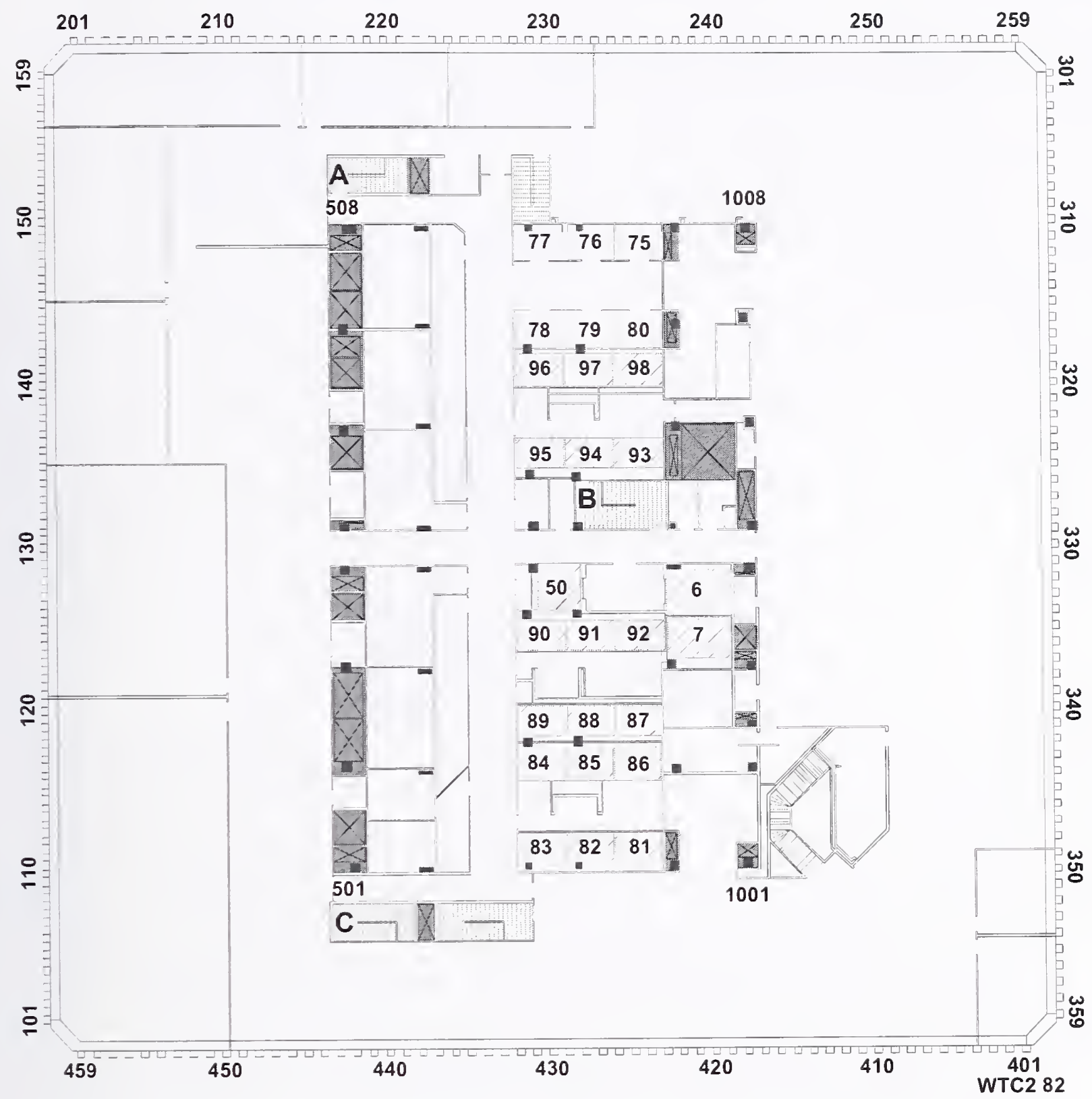

Figure B-14. This drawing shows the floor plan layout for the 82nd floor of WTC 2. 


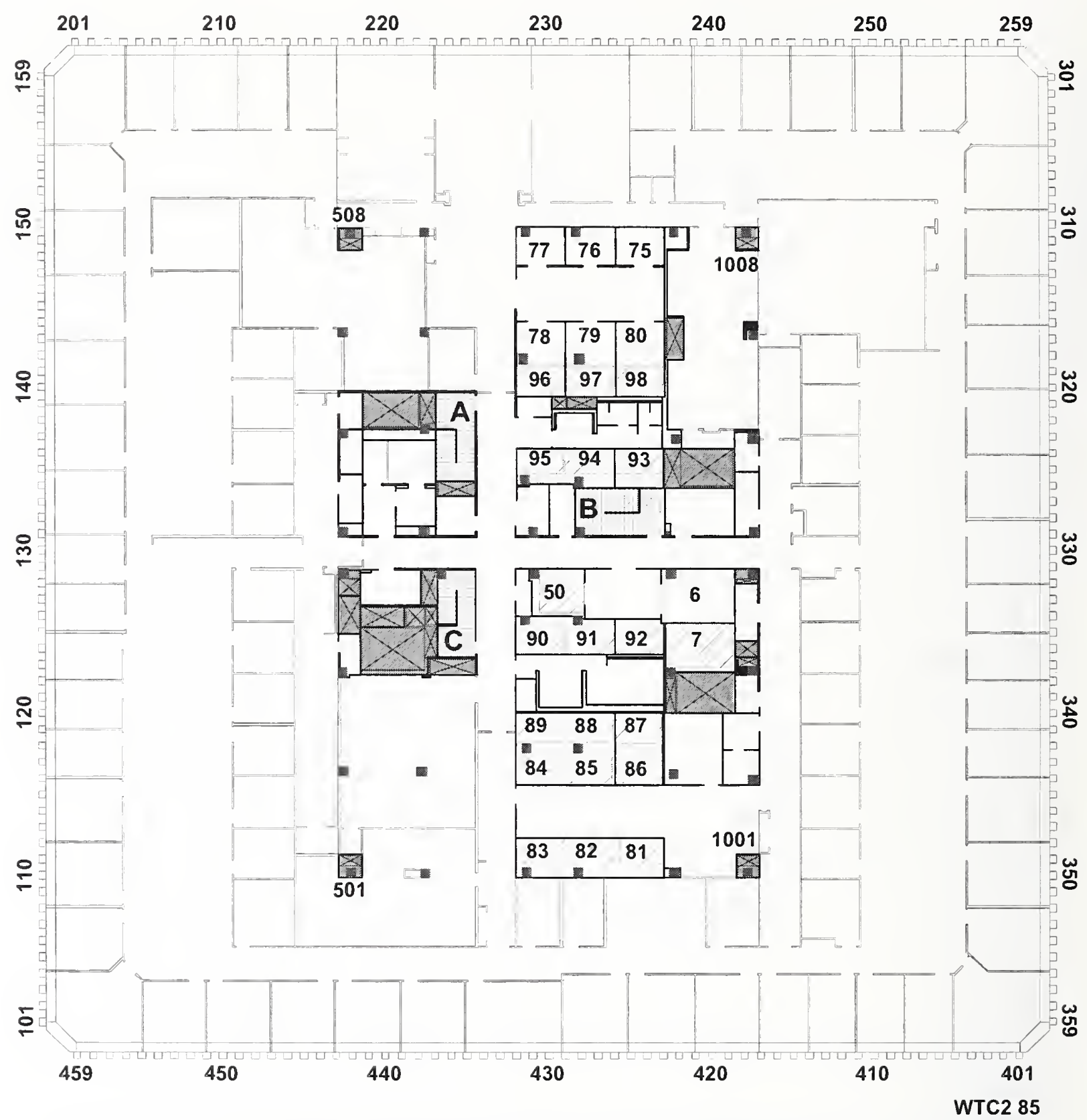

Figure B-15. This drawing shows the floor plan layout for the 85th floor of WTC 2. 


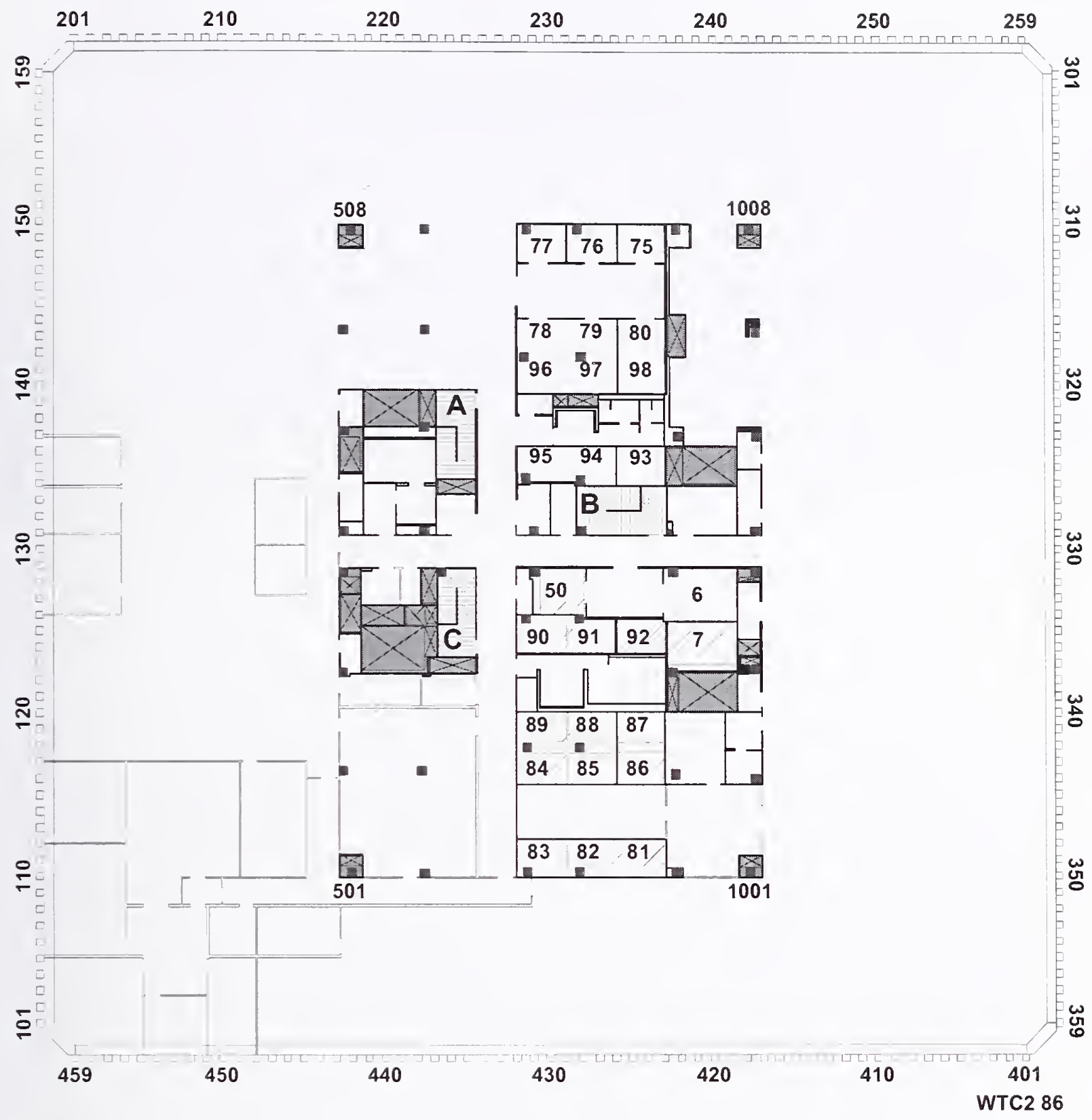

Figure B-16. This drawing shows the floor plan layout for the 86th floor of WTC 2. 
This page intentionally left blank. 


\section{Appendix C \\ WTC 1 NORTH FACE COMBINED FIRE AND WINDOW CONDITION AND WINDOW SMOKE CONDITION}

This appendix provides visual representations of the data contained in data sheets describing observations of fire. smoke, and window condition on a window-by-window basis as a function of time for the north face of WTC 1. The data for window condition and fire observations are combined into single representations, with the data for fire taking precedence. Observations are represented using the key provided in Figure 5-1 as follows: - - window glass in place, - window open, - spot fire, - fire visible inside, - external flaming, and - not visible. The data for smoke are shown in separate figures using the key from Figure 5-1 as follows: — - no smoke visible, - light smoke, - heavy smoke, and - not visible. For a given time, the window condition and fire data are shown first. 
WTC 1, North Face 8:47 a.m.

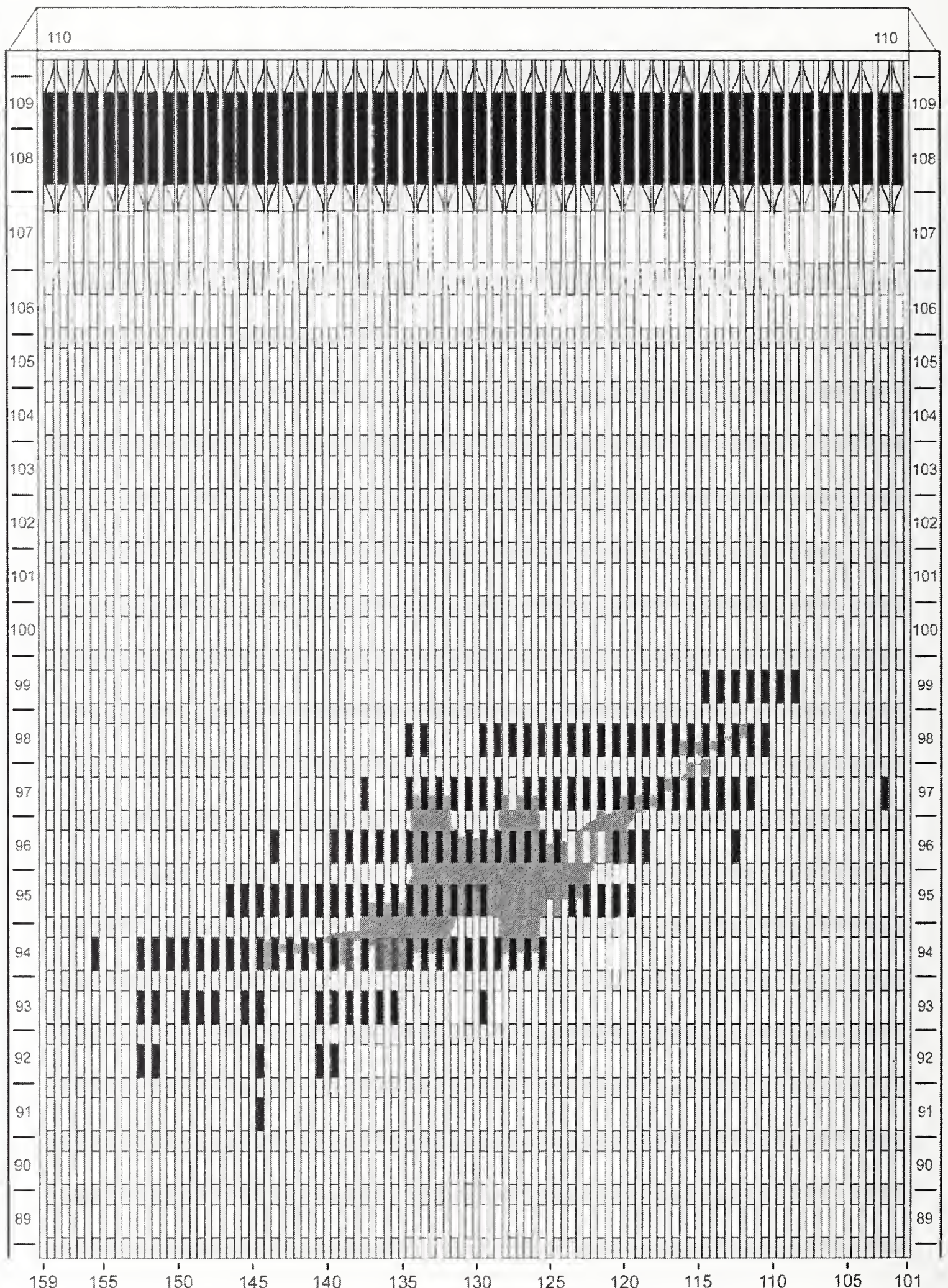

Figure C-1. Diagram of the north face of WTC 1 for floors 89 to 110 at 8:47 a.m. showing the condition of windows and locations of fires. 
WTC 1, North Face $\quad 8: 47$ a.m.

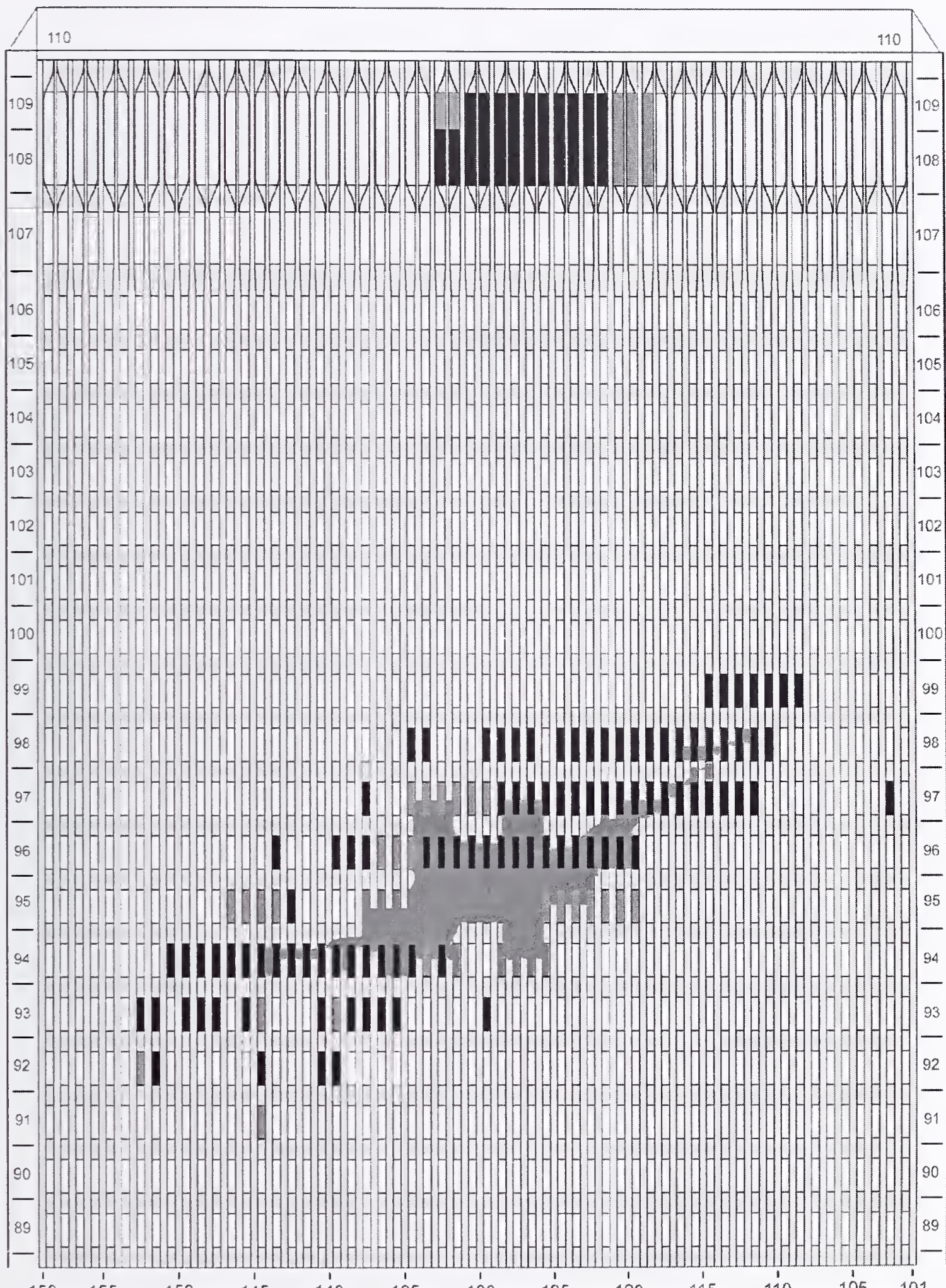

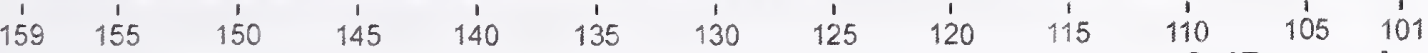

Figure C-2. Diagram of the north face of WTC 1 for floors 89 to 110 at $8: 47$ a.m. showing windows where smoke was observed and those that were hidden from view. 
WTC 1, North Face $\quad 8: 48$ a.m.

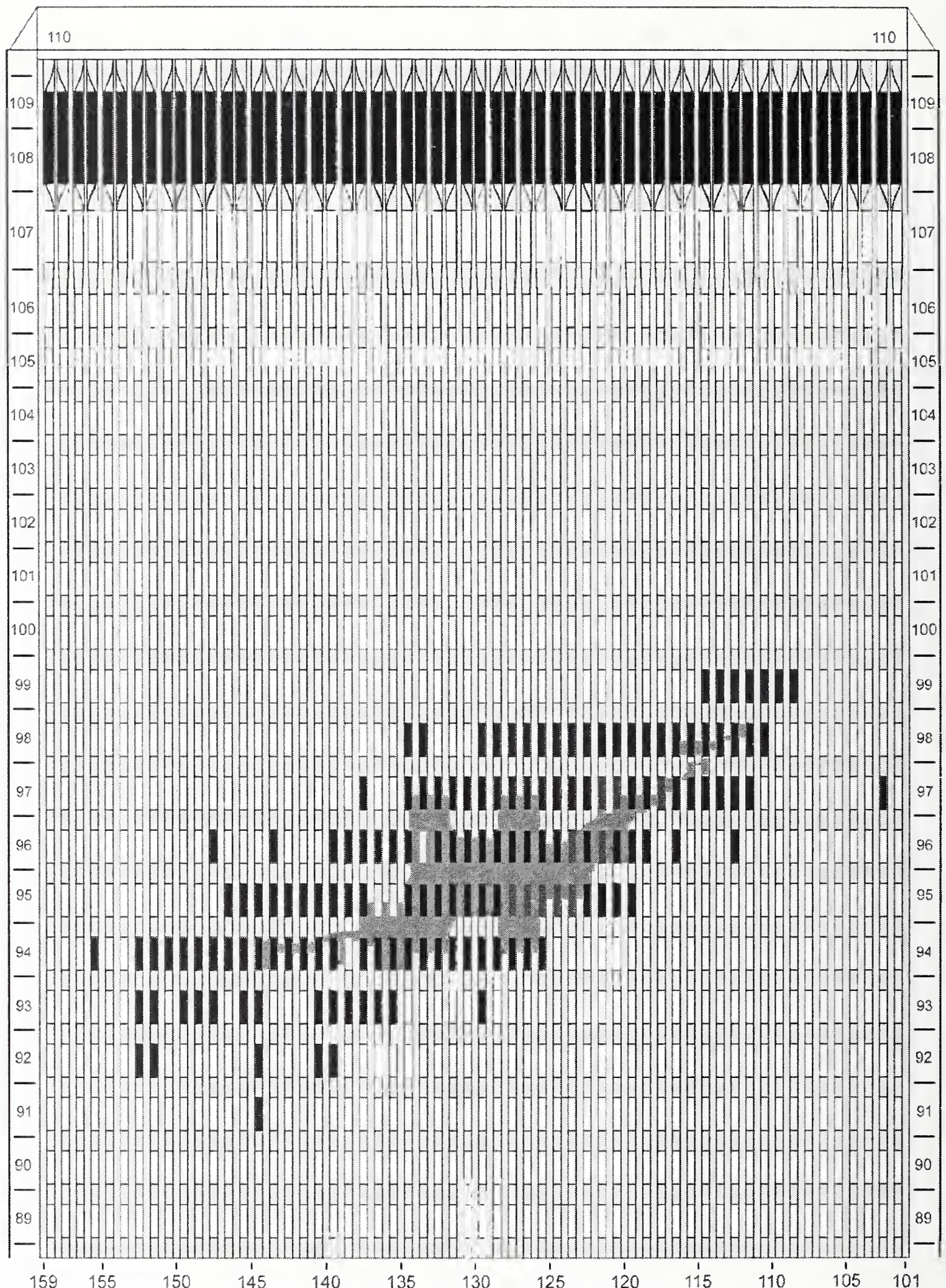

Figure C-3. Diagram of the north face of WTC 1 for floors 89 to 110 at 8:48 a.m. showing the condition of windows and locations of fires. 
WTC 1, North Face 8:48 a.m.

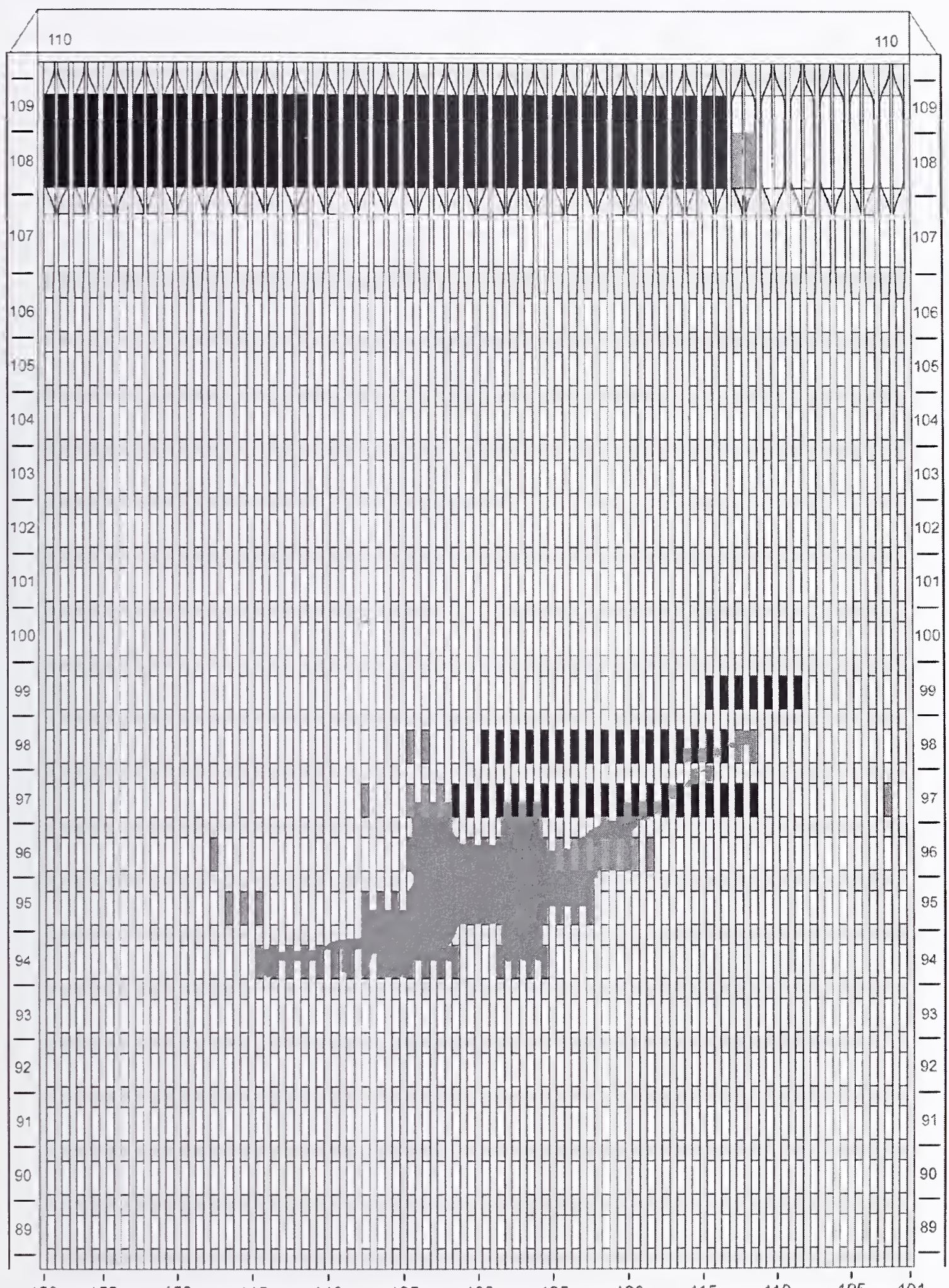

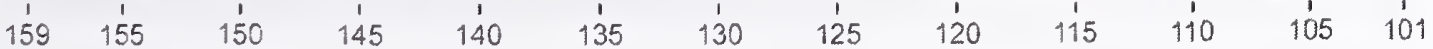

Figure C-4. Diagram of the north face of WTC 1 for floors 89 to 110 at $8: 48$ a.m. showing windows where smoke was observed and those that were hidden from view. 


$$
\text { WTC 1, North Face } \quad \text { 8:50 a.m. }
$$

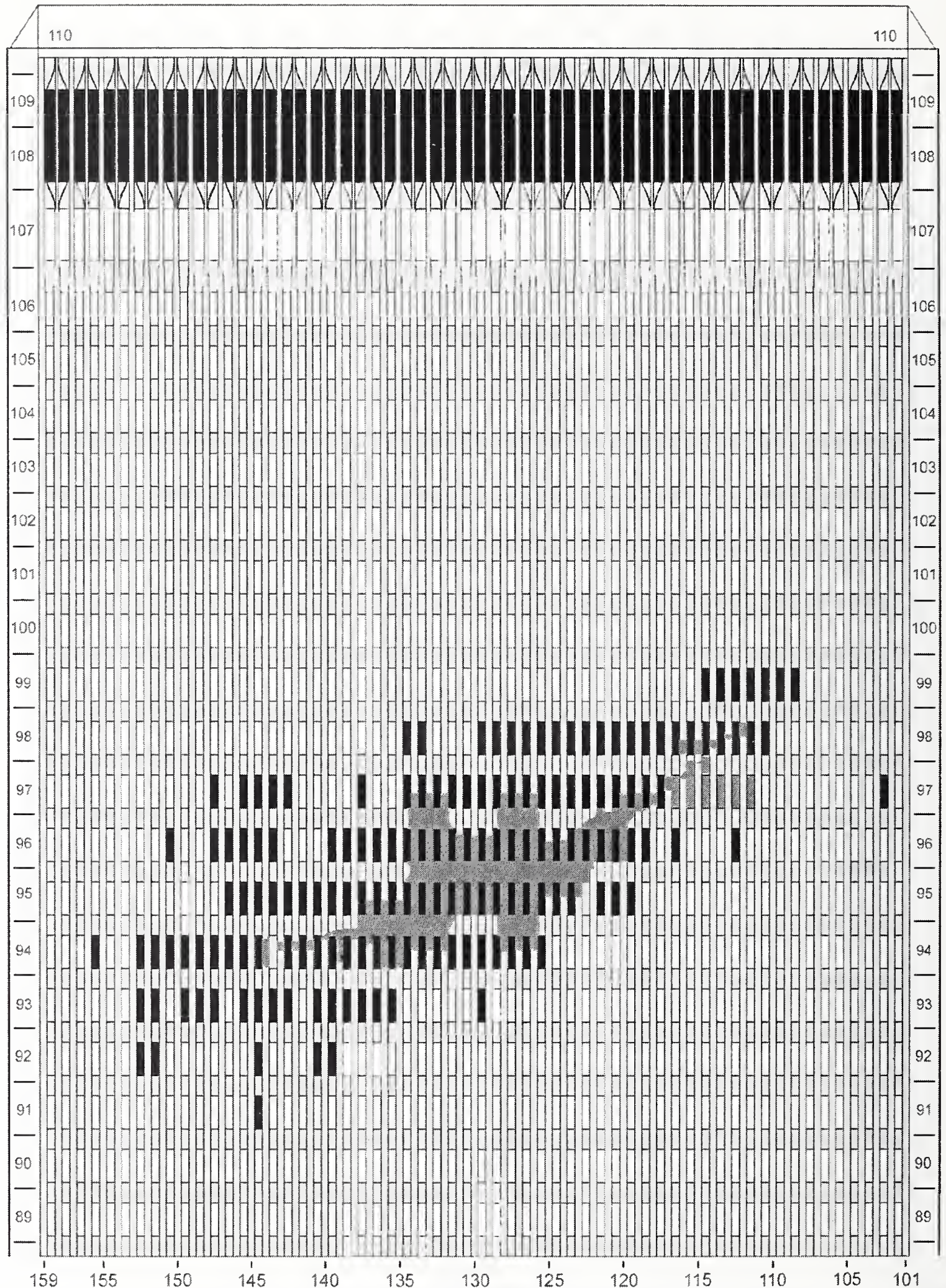

Figure C-5. Diagram of the north face of WTC 1 for floors 89 to 110 at 8:50 a.m. showing the condition of windows and locations of fires. 
WTC 1, North Face $\quad 8: 50$ a.m.

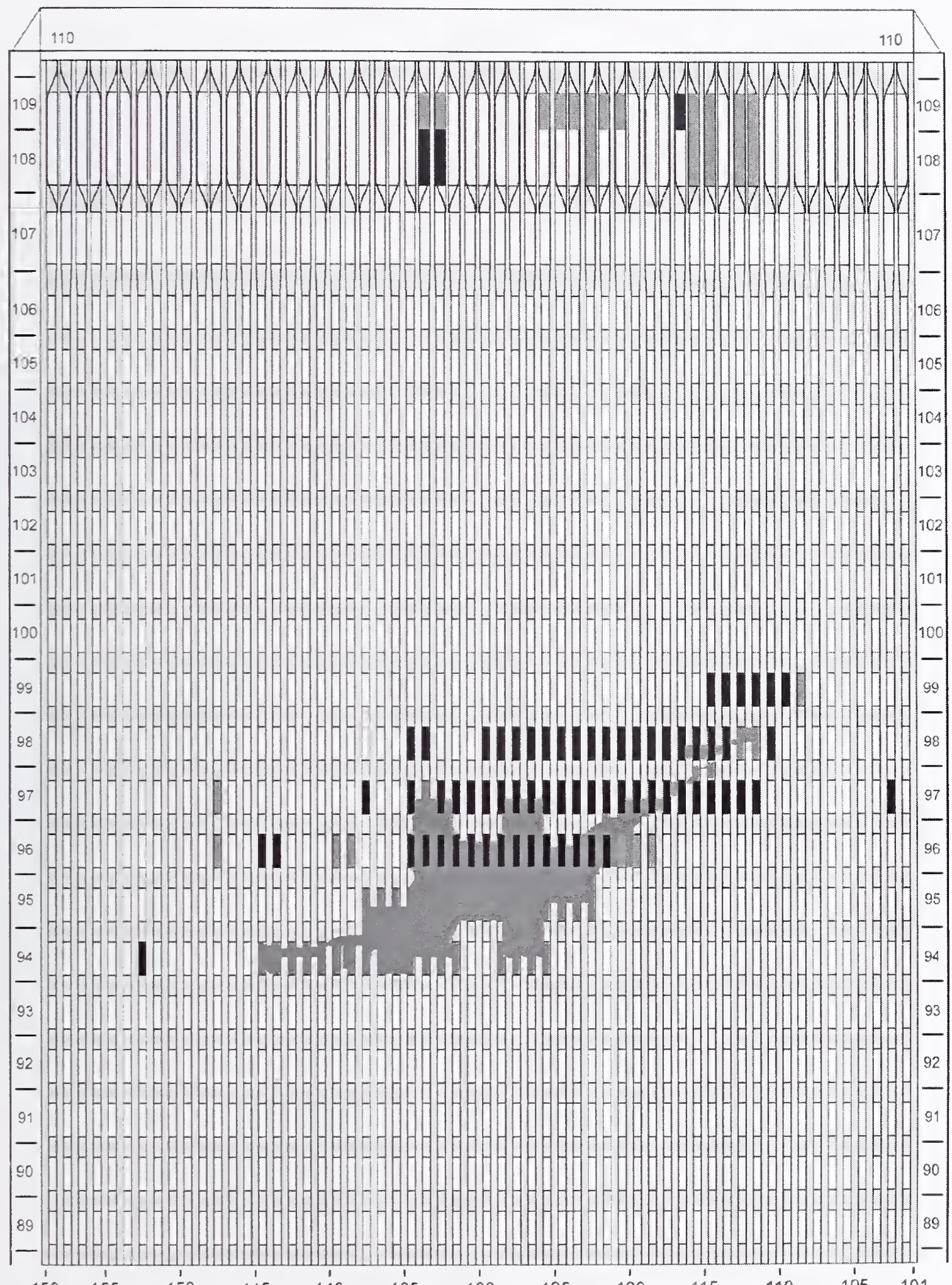

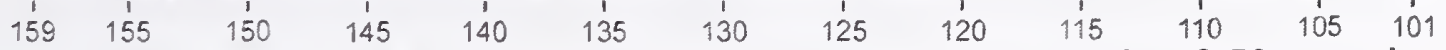

Figure C-6. Diagram of the north face of WTC 1 for floors 89 to 110 at 8:50 a.m. showing windows where smoke was observed and those that were hidden from view. 
WTC 1, North Face $\quad$ 8:52 a.m.

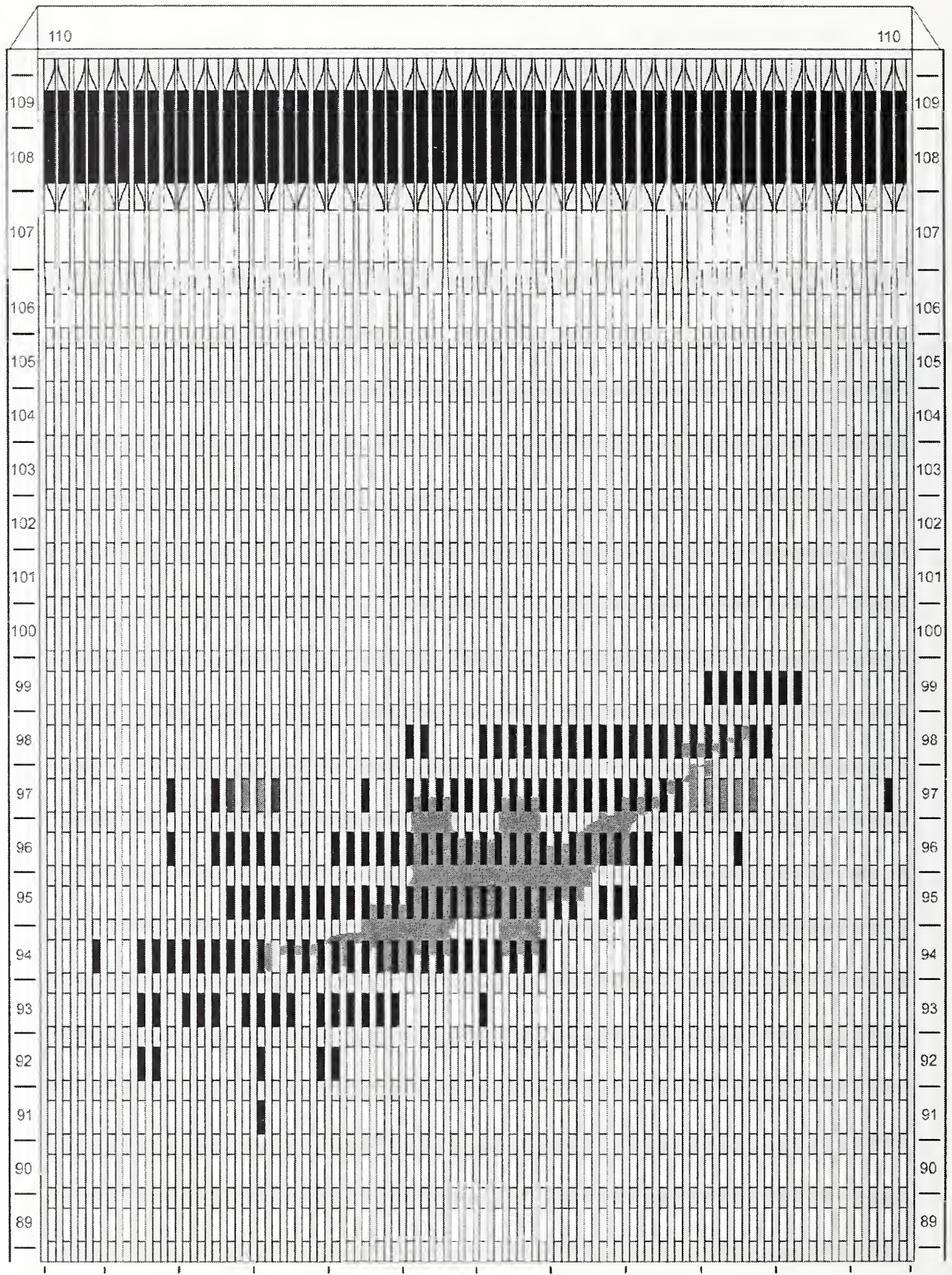

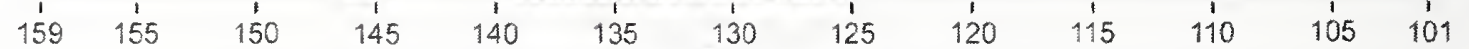

Figure C-7. Diagram of the north face of WTC 1 for floors 89 to 110 at 8:52 a.m. showing the condition of windows and locations of fires. 
WTC 1, North Face $\quad$ 8:52 a.m.

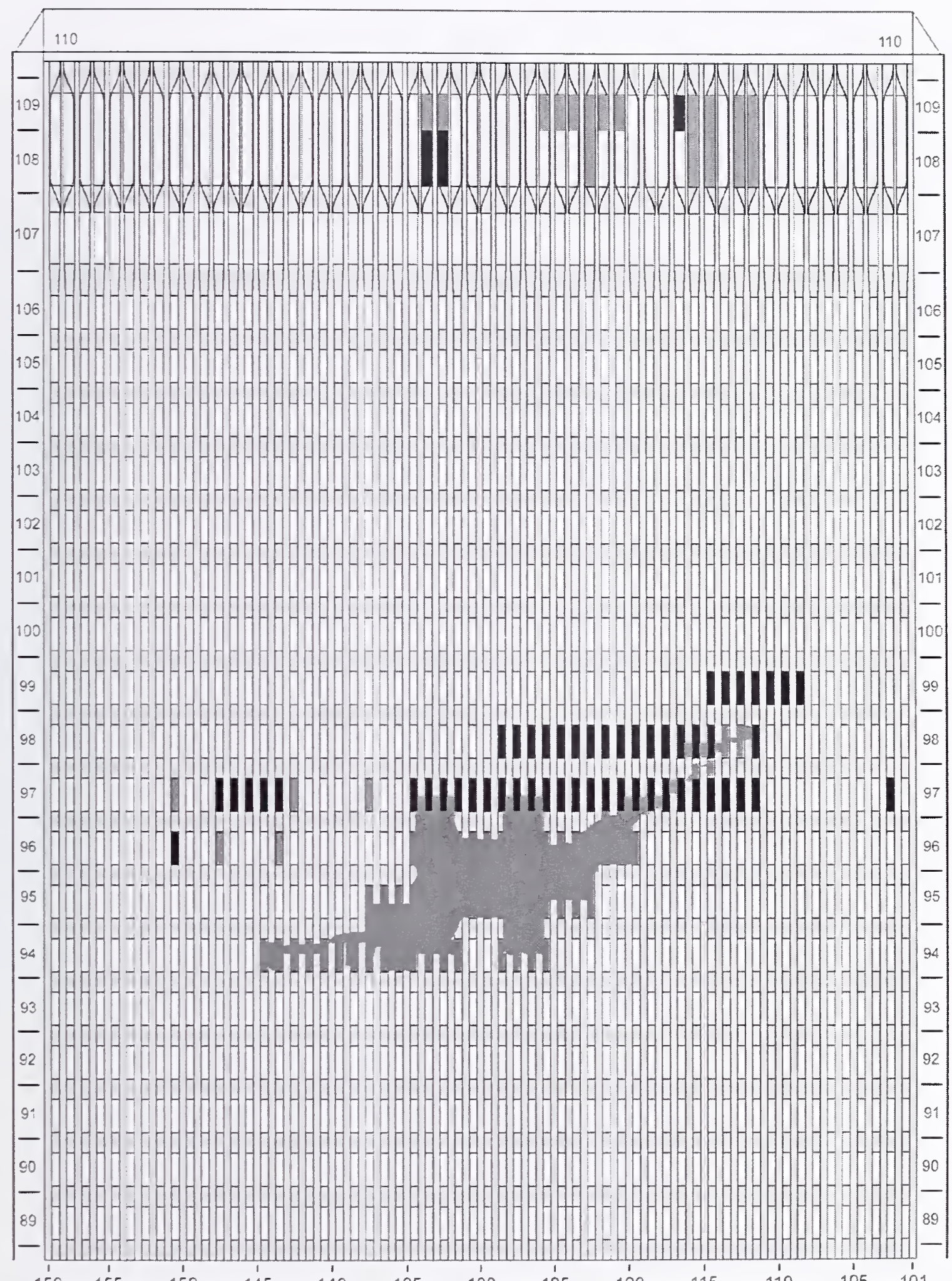

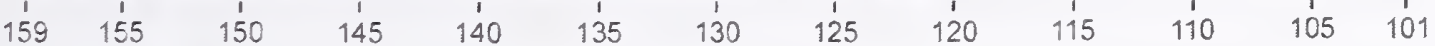

Figure C-8. Diagram of the north face of WTC 1 for floors 89 to 110 at $8: 52$ a.m. showing windows where smoke was observed and those that were hidden from view. 
WTC 1, North Face $\quad$ 8:54 a.m.

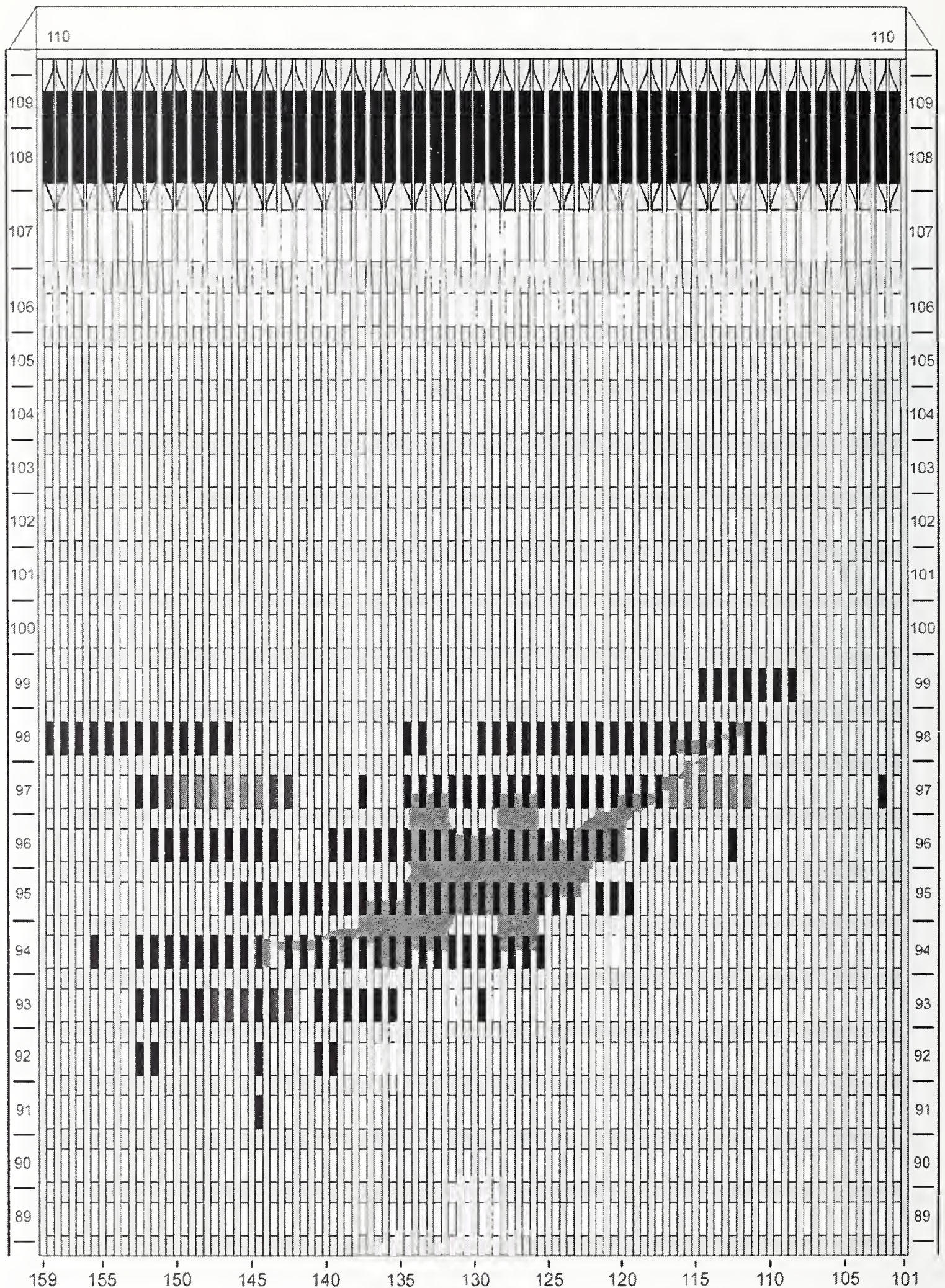

Figure C-9. Diagram of the north face of WTC 1 for floors 89 to 110 at 8:54 a.m. showing the condition of windows and locations of fires. 


$$
\text { WTC 1, North Face } \quad 8: 54 \text { a.m. }
$$

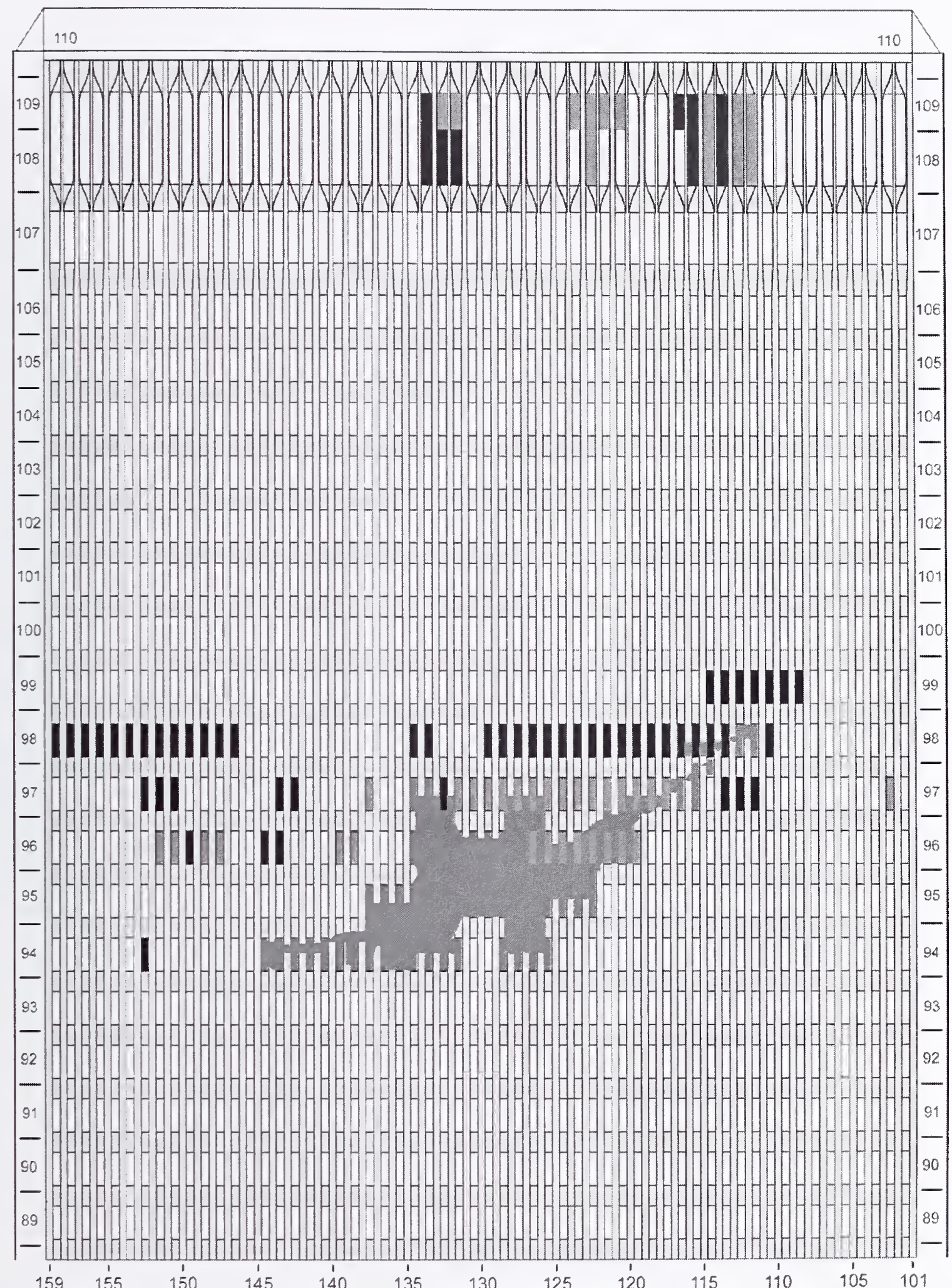

Figure $\mathrm{C}-10$. Diagram of the north face of WTC 1 for floors 89 to 110 at $8: 54$ a.m. showing windows where smoke was observed and those that were hidden from view. 

WTC 1, North Face
8:56 a.m.

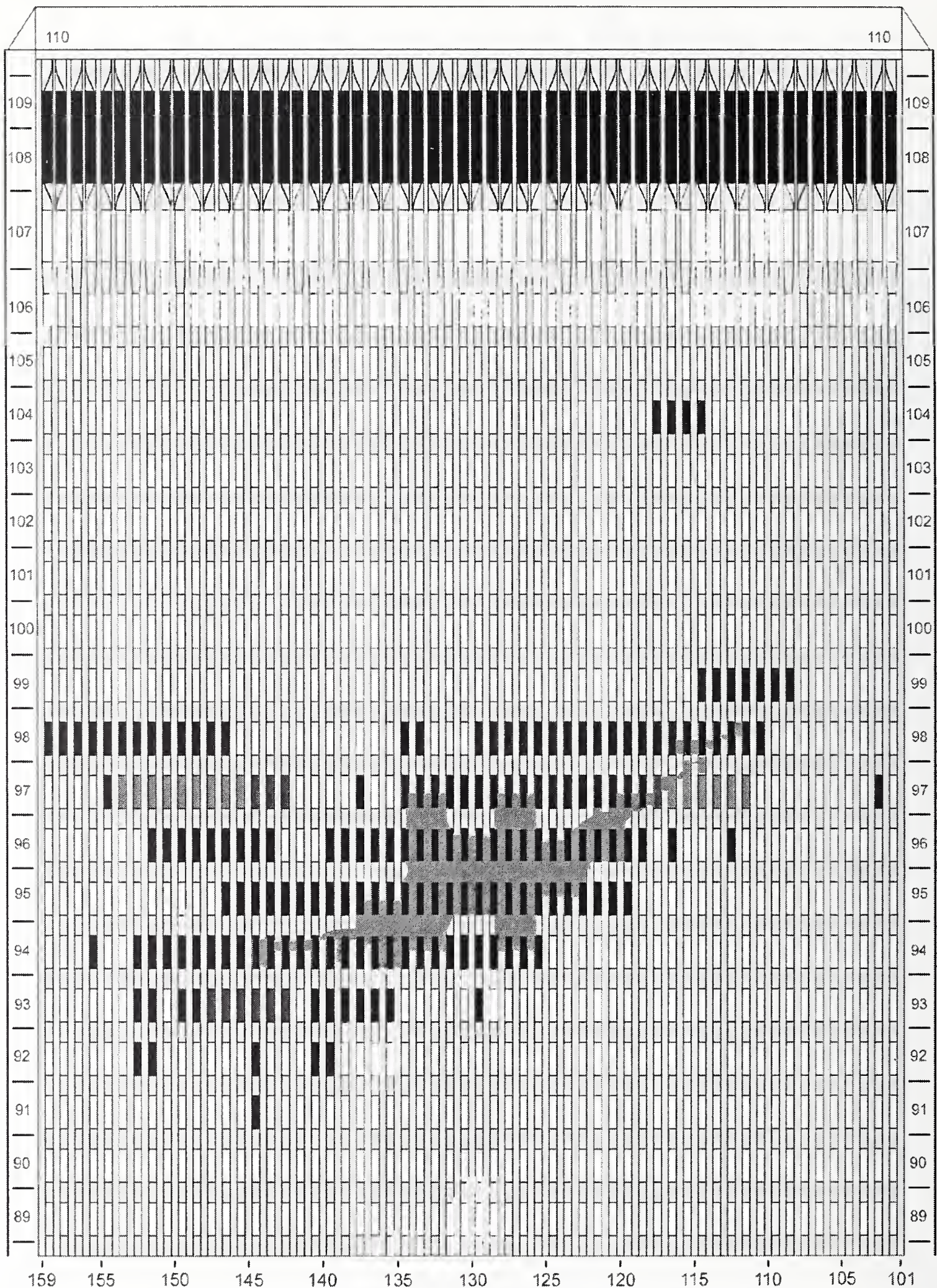

Figure C-11. Diagram of the north face of WTC 1 for floors 89 to 110 at 8:56 a.m. showing the condition of windows and locations of lines. 


\section{WTC 1, North Face $\quad$ 8:56 a.m.}

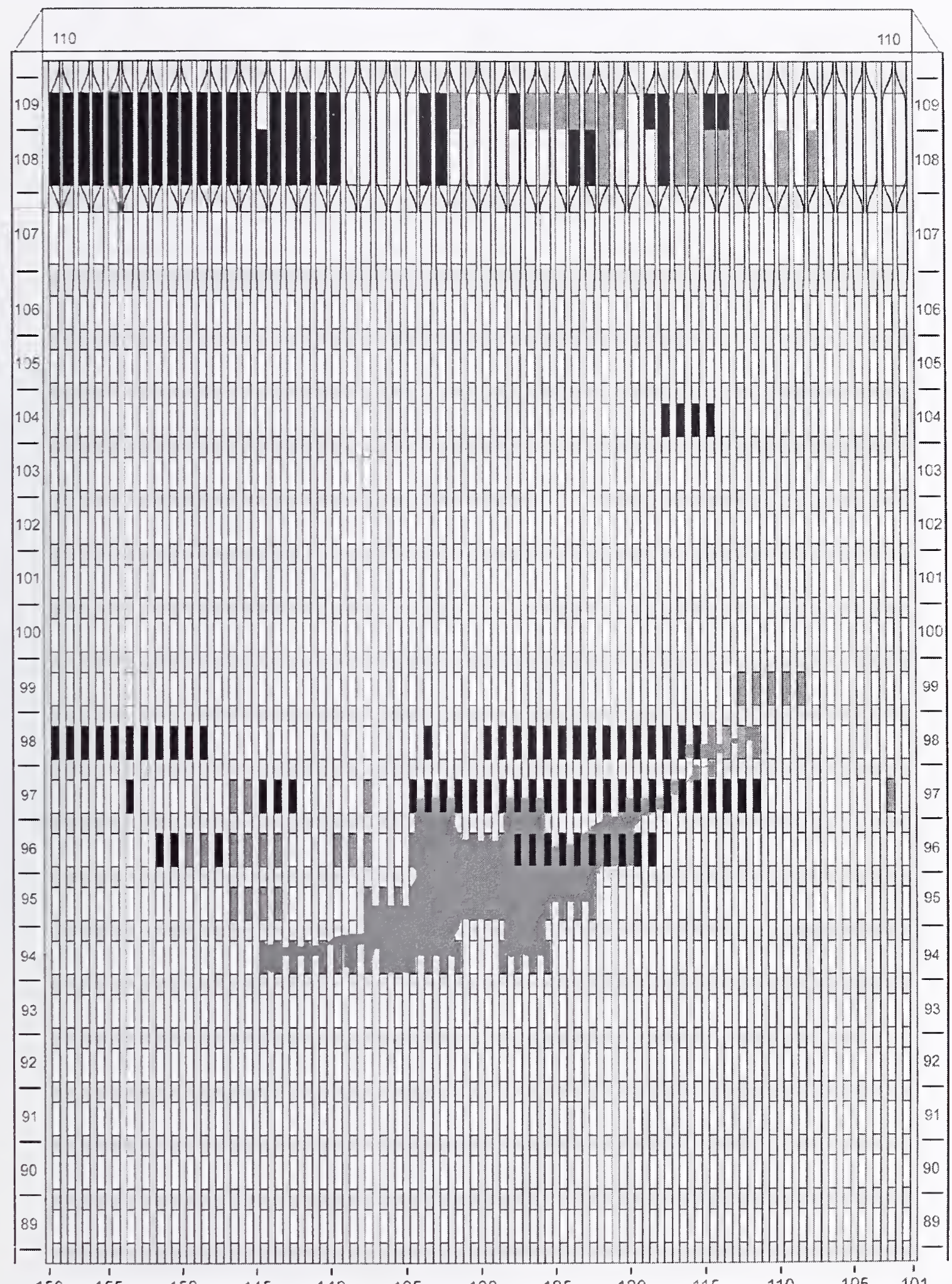

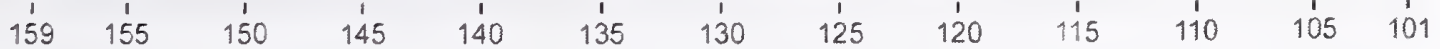

Figure C-12. Diagram of the north face of WTC 1 for floors 89 to 110 at $8: 56$ a.m. showing windows where smoke was observed and those that were hidden from view. 
WTC 1, North Face $\quad$ 8:58 a.m.

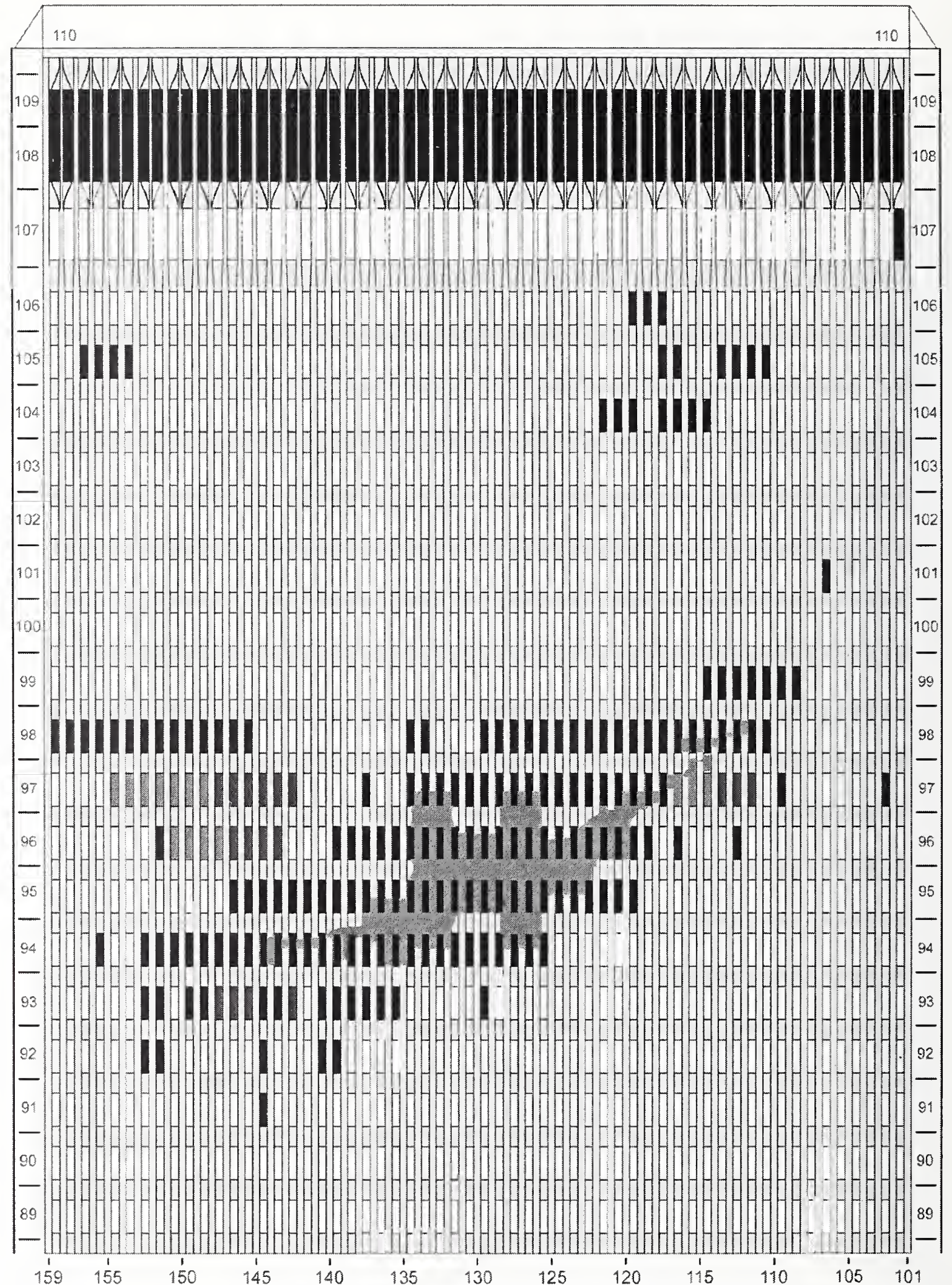

Figure C-13. Diagram of the north face of WTC 1 for floors 89 to 110 at $8: 58$ a.m. showing the condition of windows and locations of fires. 


$$
\text { WTC 1, North Face } \quad \text { 8:58 a.m. }
$$

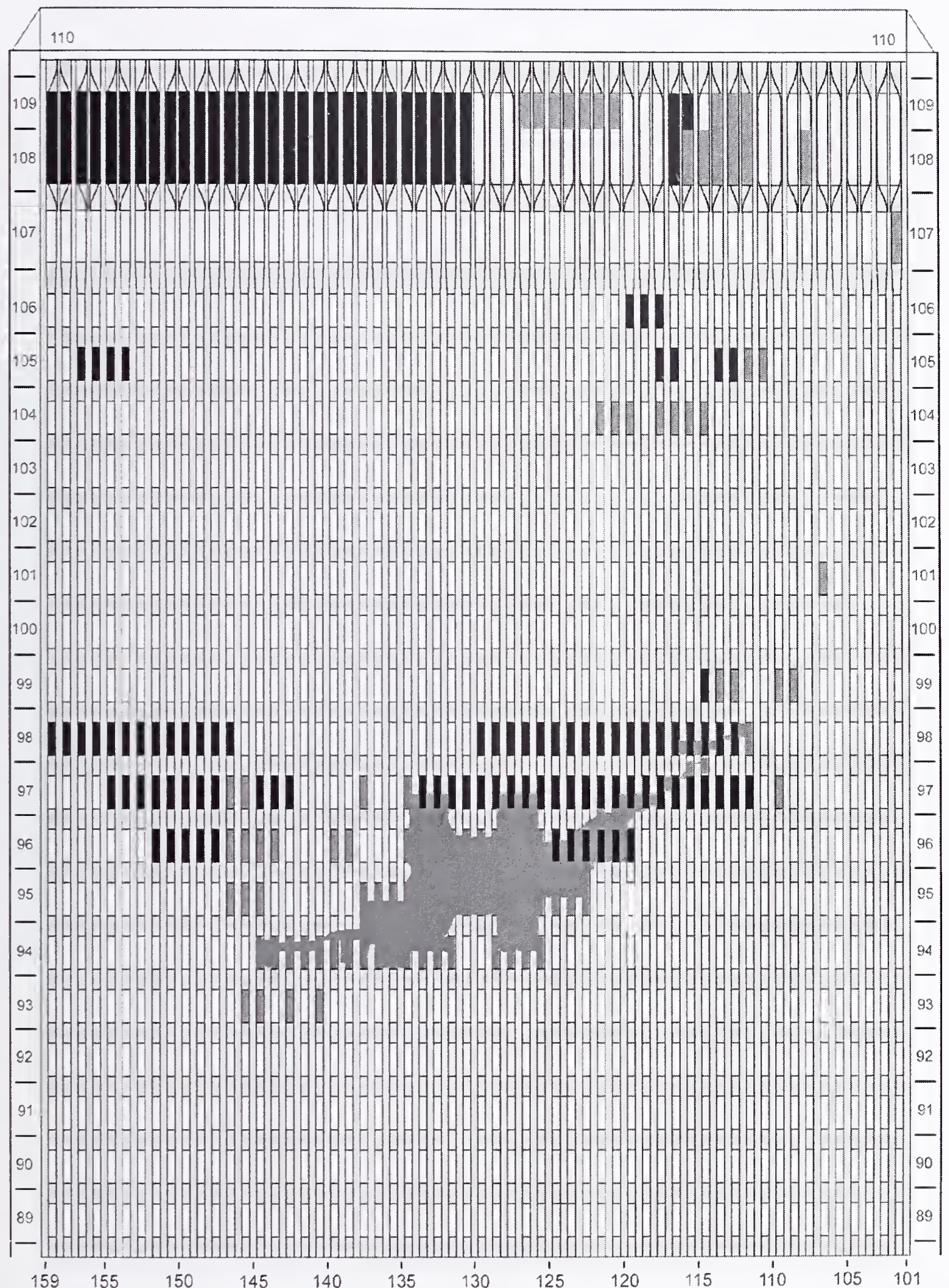

Figure C-14. Diagram of the north face of WTC 1 for floors 89 to 110 at $8: 58$ a.m. showing windows where smoke was observed and those that were hidden from view. 
WTC 1, North Face 9:00 a.m.

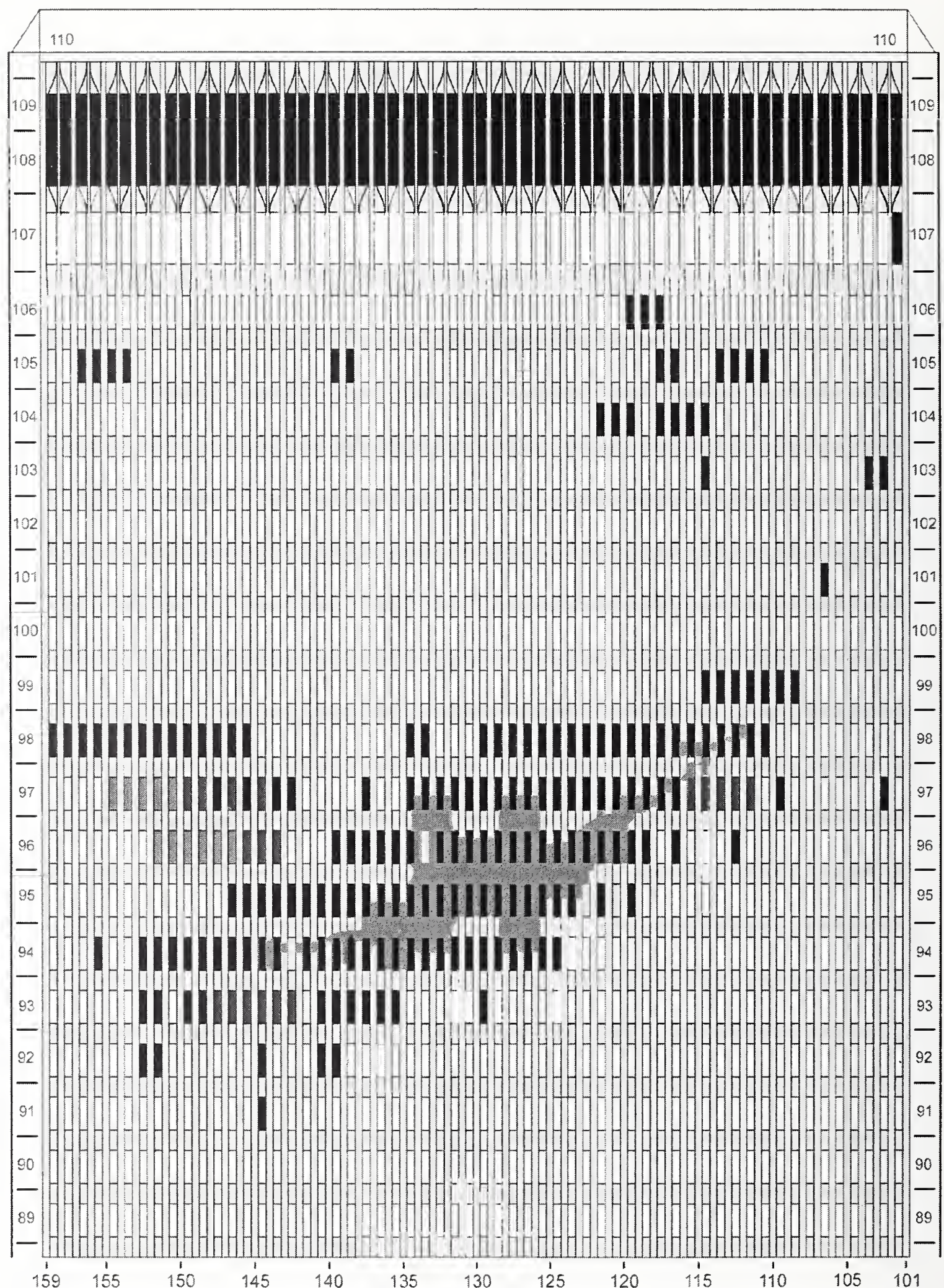

Figure C-15. Diagram of the north face of WTC 1 for floors 89 to 110 at 9:00 a.m. showing the condition of windows and locations of fires. 


\section{WTC 1, North Face $\quad$ 9:00 a.m.}

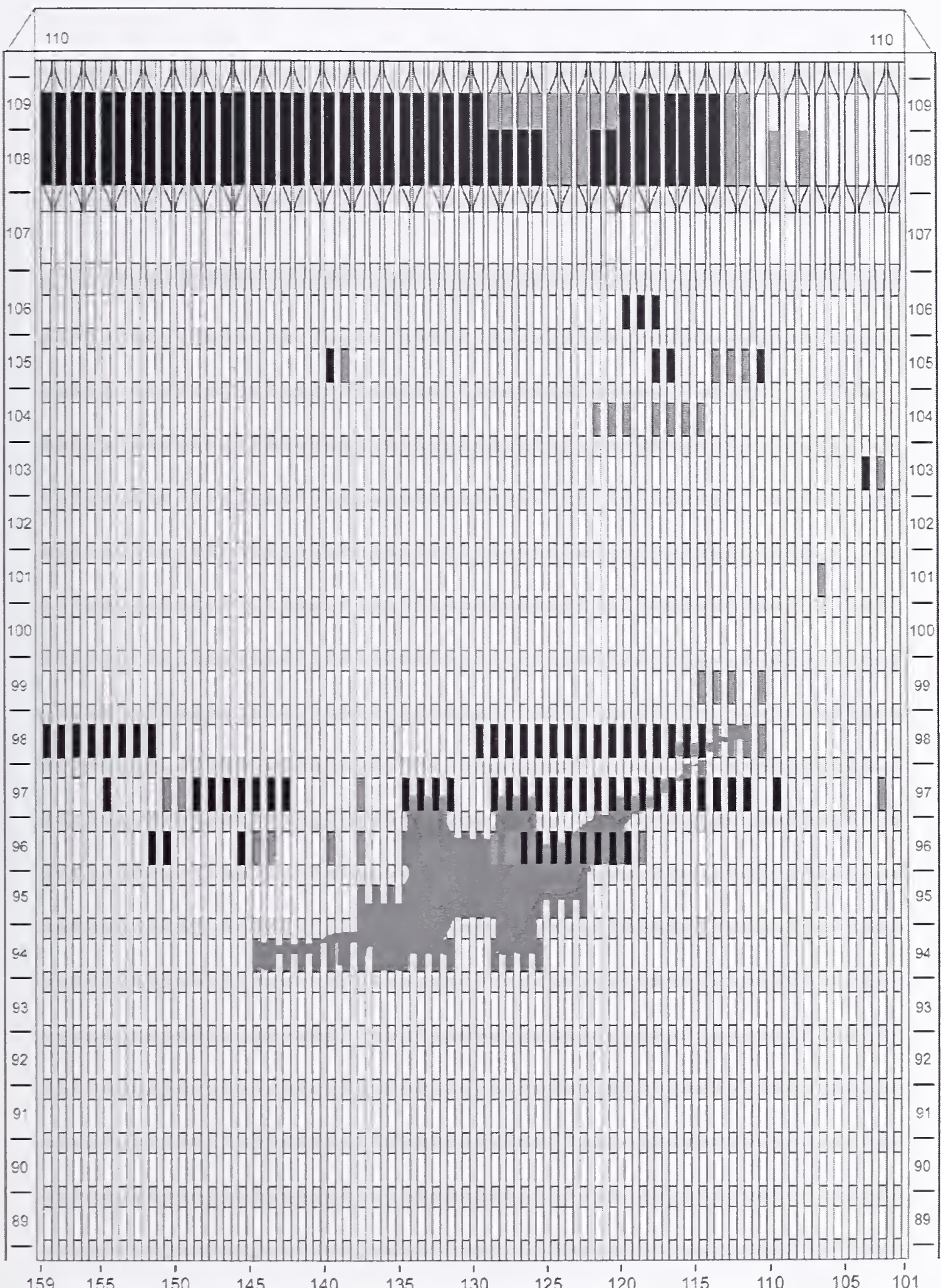

Figure C-16. Diagram of the north face of WTC 1 for floors 89 to 110 at 9:00 a.m. showing windows where smoke was observed and those that were hidden from view. 


$$
\text { WTC 1, North Face } \quad 9: 02 \text { a.m. }
$$

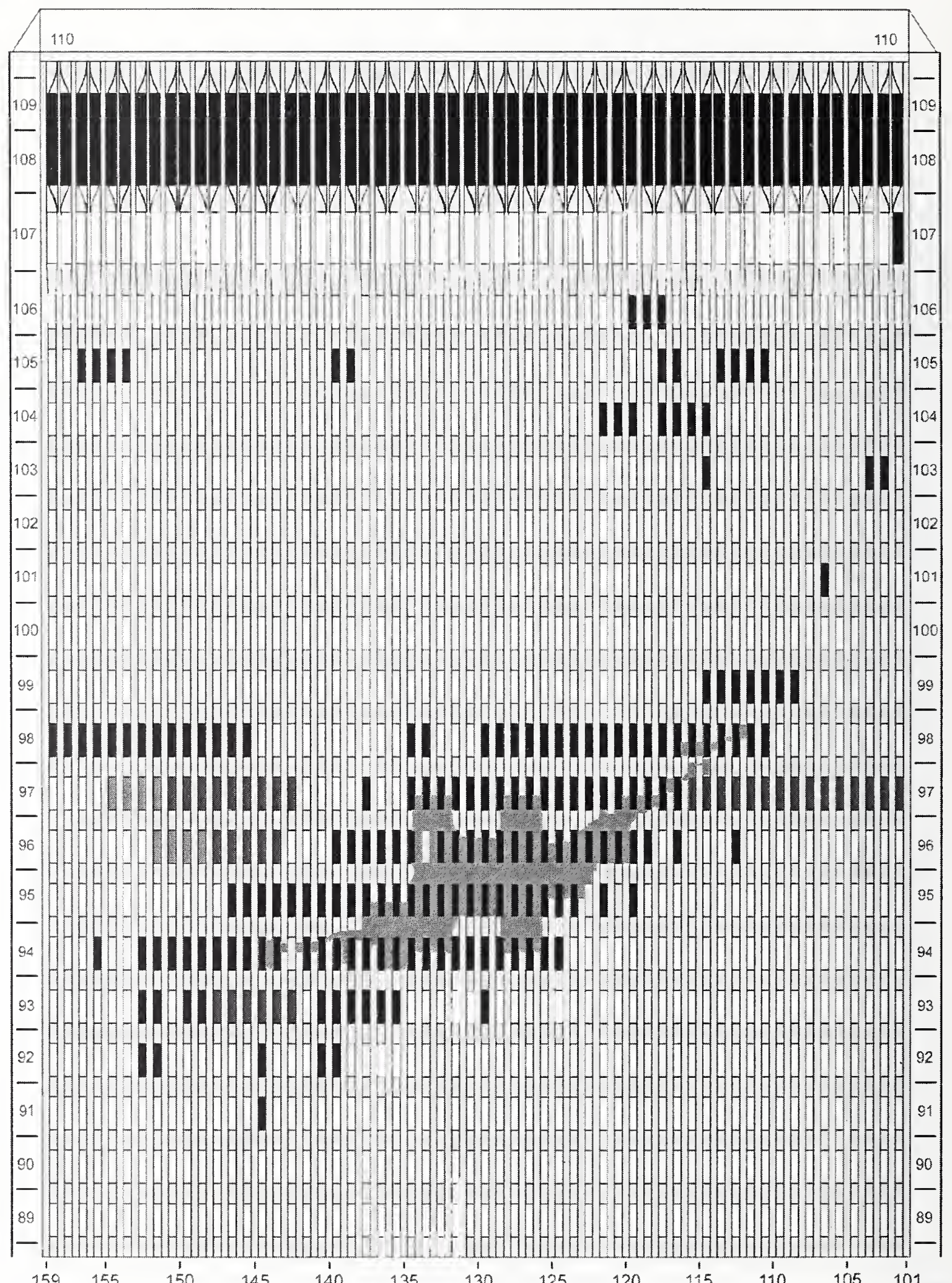

Figure C-17. Diagram of the north face of WTC 1 for floors 89 to 110 at 9:02 a.m. showing the condition of windows and locations of fires. 
WTC 1, North Face 9:02 a.m.

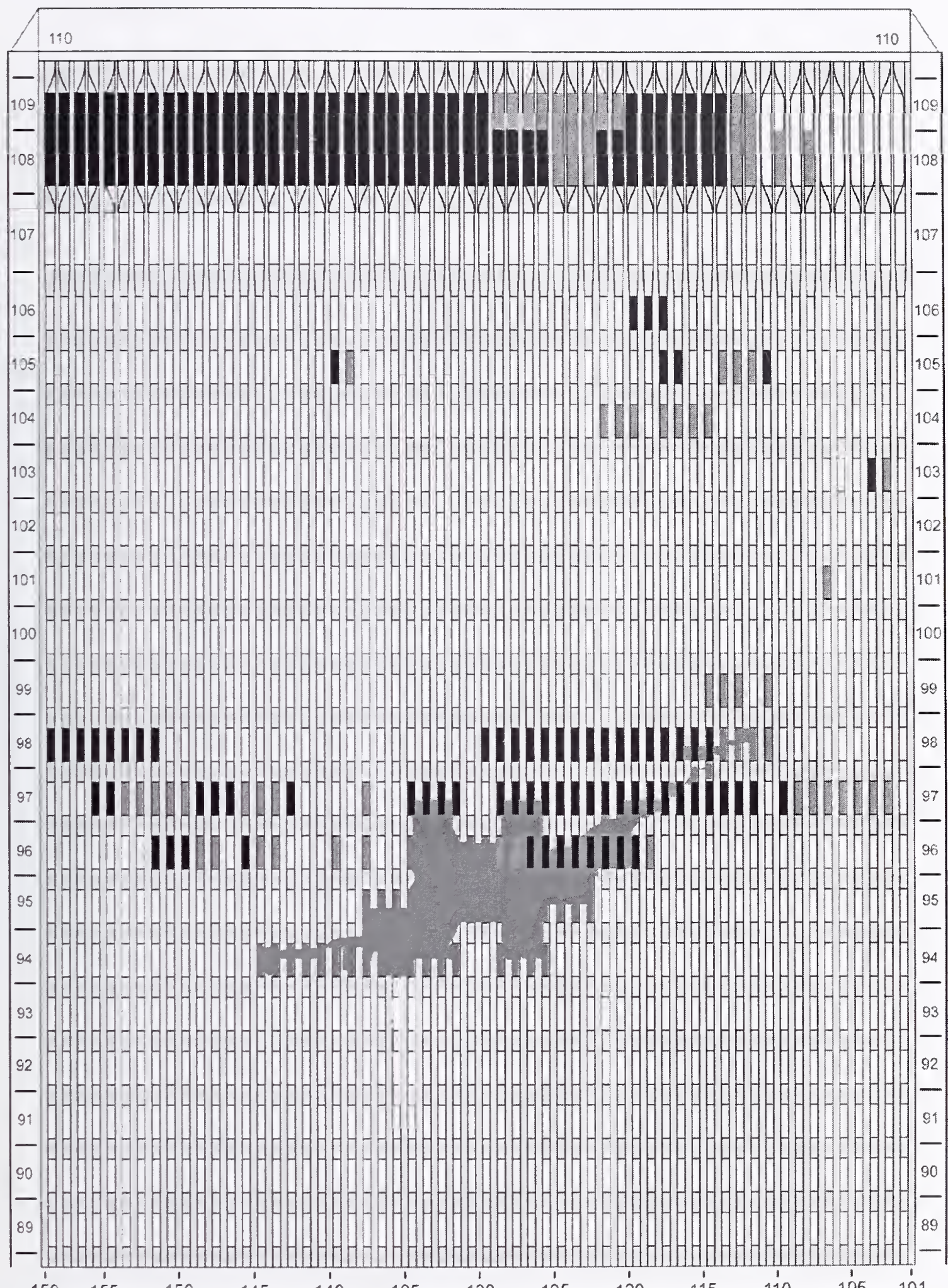

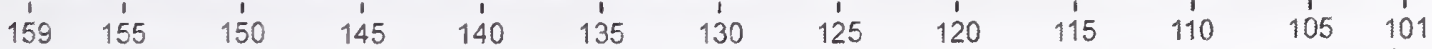

Figure C-18. Diagram of the north face of WTC 1 for floors 89 to 110 at 9:02 a.m. showing windows where smoke was observed and those that were hidden from view. 
WTC 1, North Face 9:04 a.m.

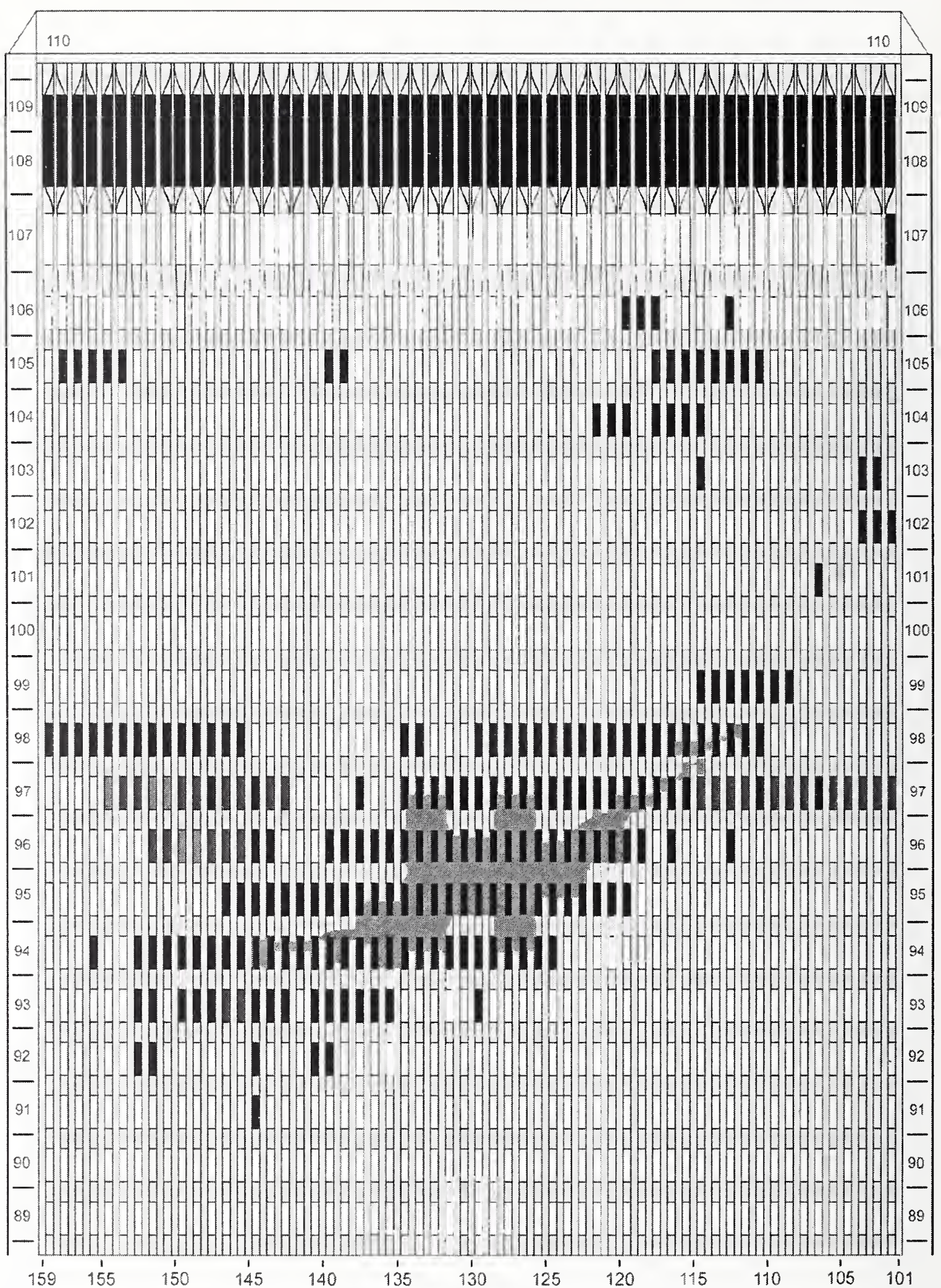

Figure C-19. Diagram of the north face of WTC 1 for floors 89 to 110 at 9:04 a.m. showing the condition of windows and locations of fires. 


\section{WTC 1, North Face 9:04 a.m.}

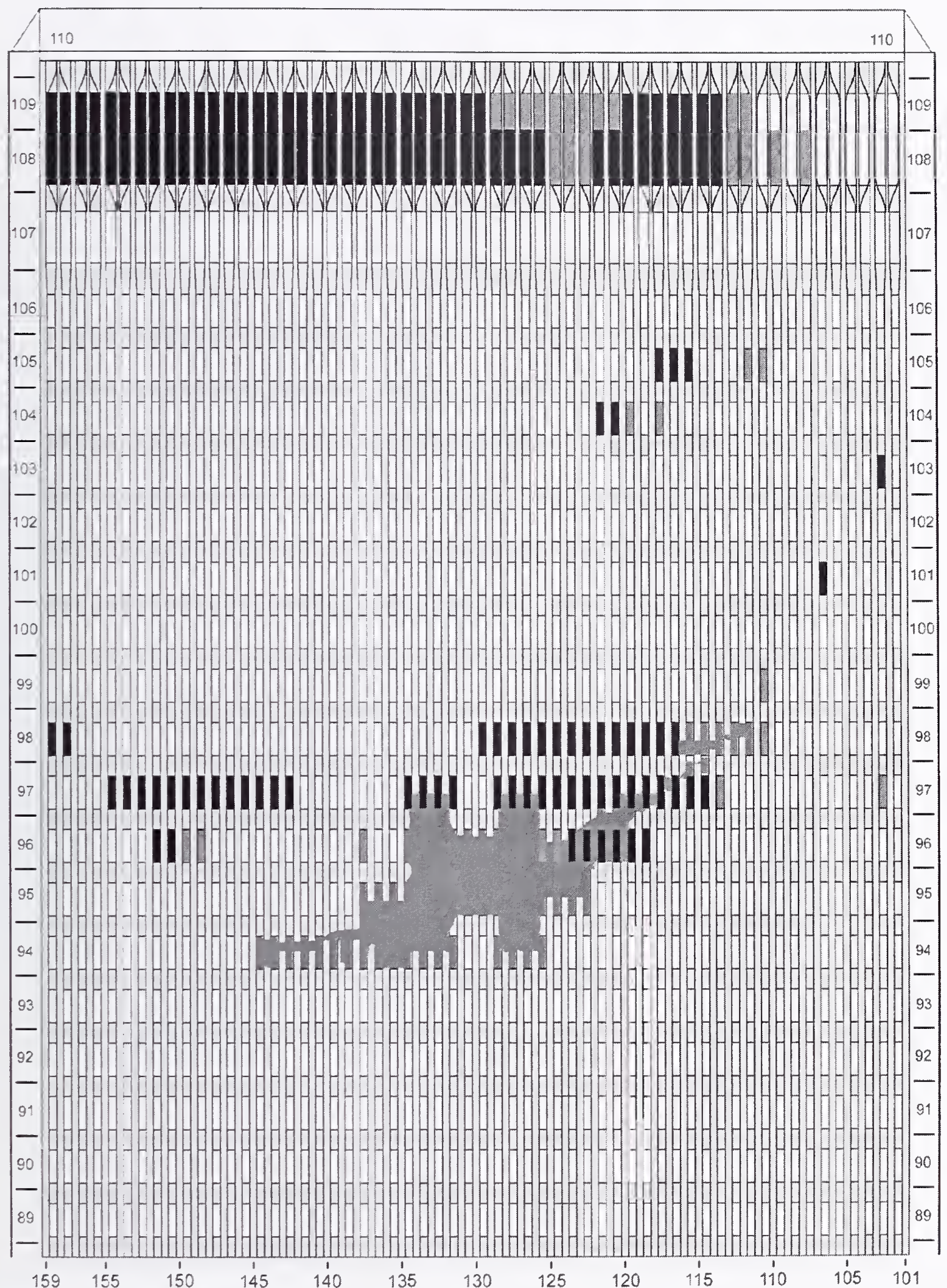

Figure C-20. Diagram of the north face of WTC 1 for floors 89 to 110 at 9:04 a.m. showing windows where smoke was observed and those that were hidden from view. 
WTC 1, North Face $\quad$ 9:10 a.m.

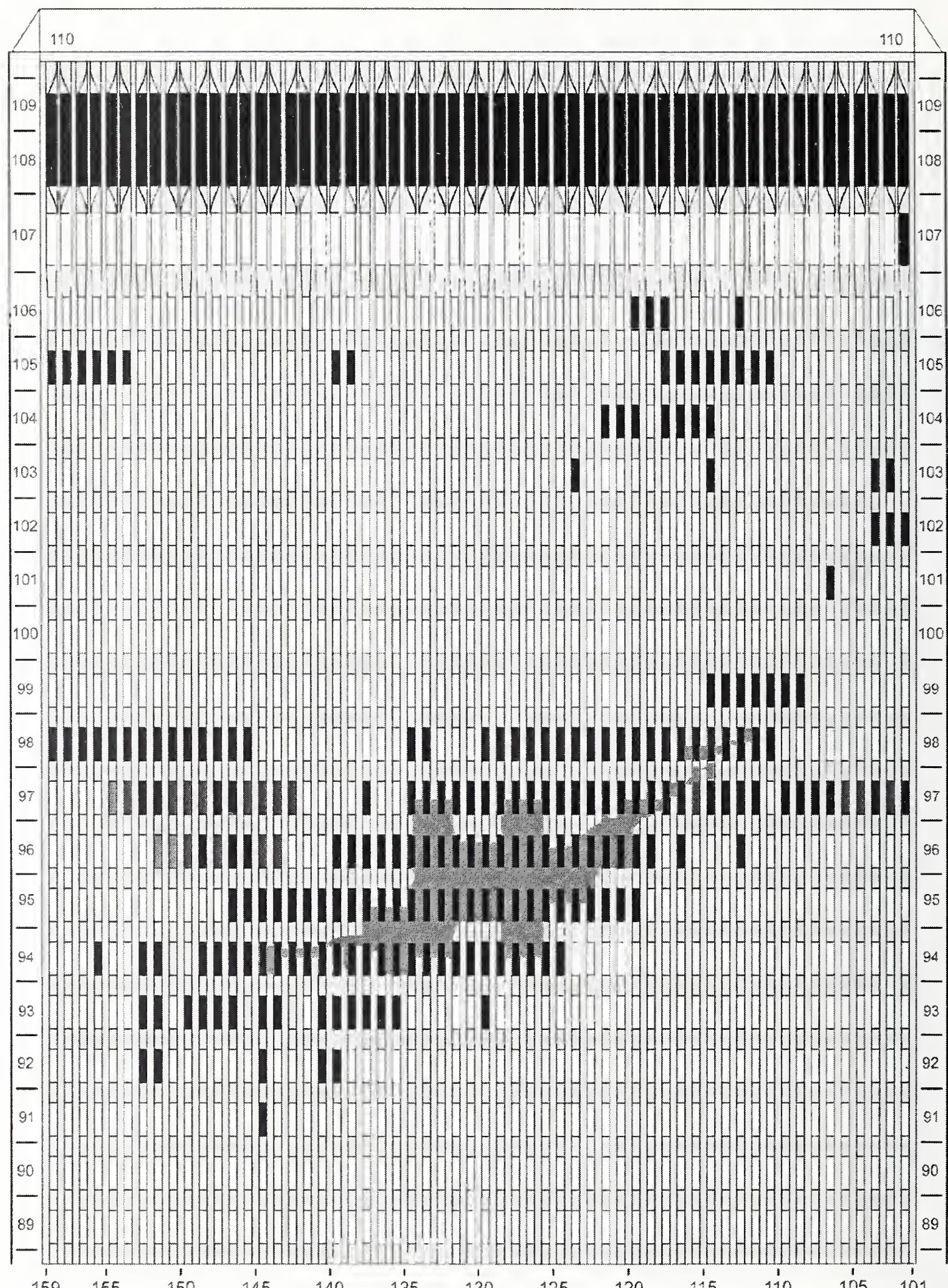

Figure C-21. Diagram of the north face of WTC 1 for floors 89 to 110 at 9:10 a.m. showing the condition of windows and locations of fires. 
WTC 1, North Face 9:10 a.m.

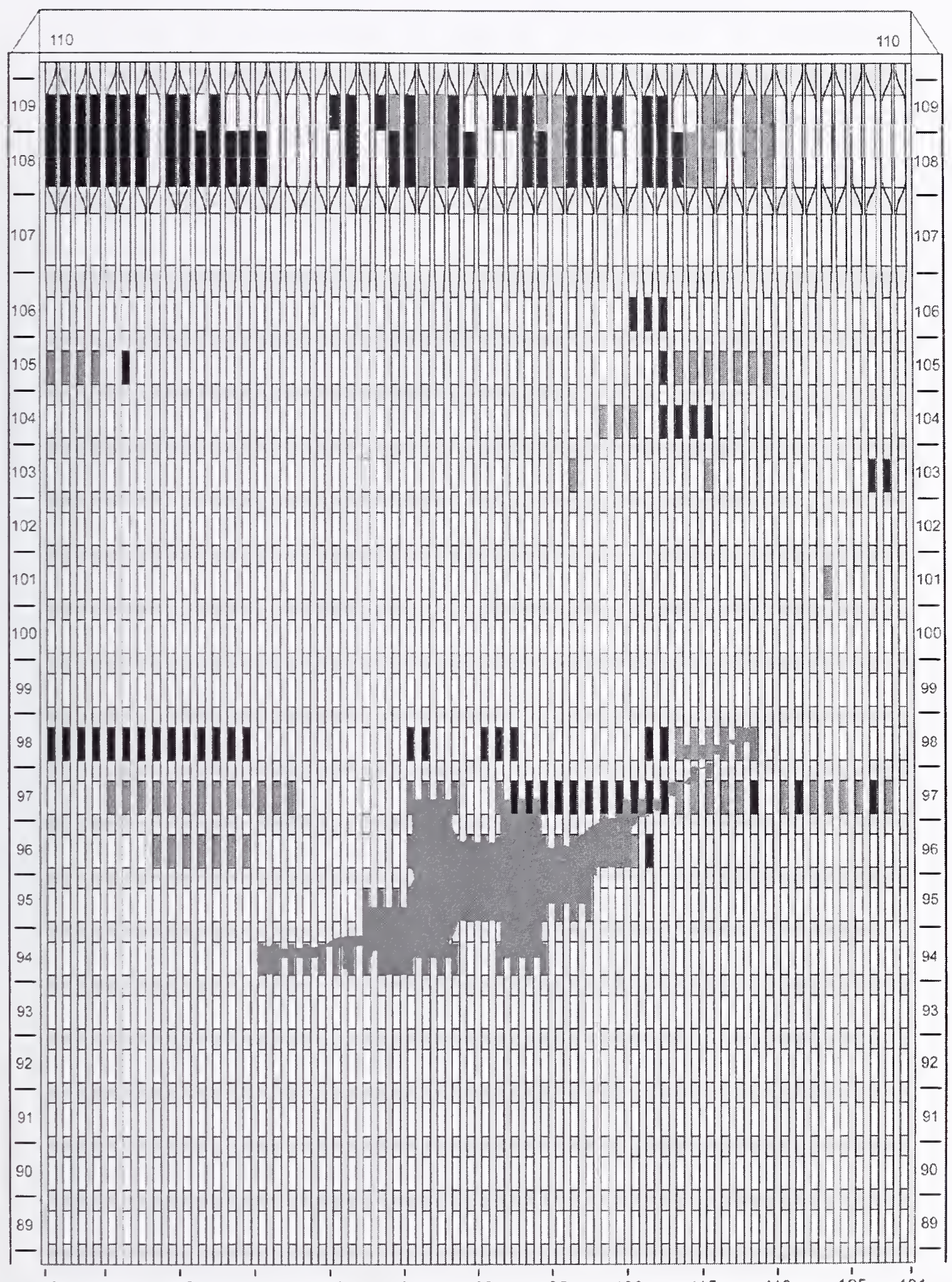

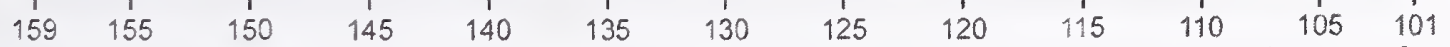

Figure C-22. Diagram of the north face of WTC 1 for floors 89 to 110 at 9:10 a.m. showing windows where smoke was observed and those that were hidden from view. 
WTC 1, North Face 9:14 a.m.

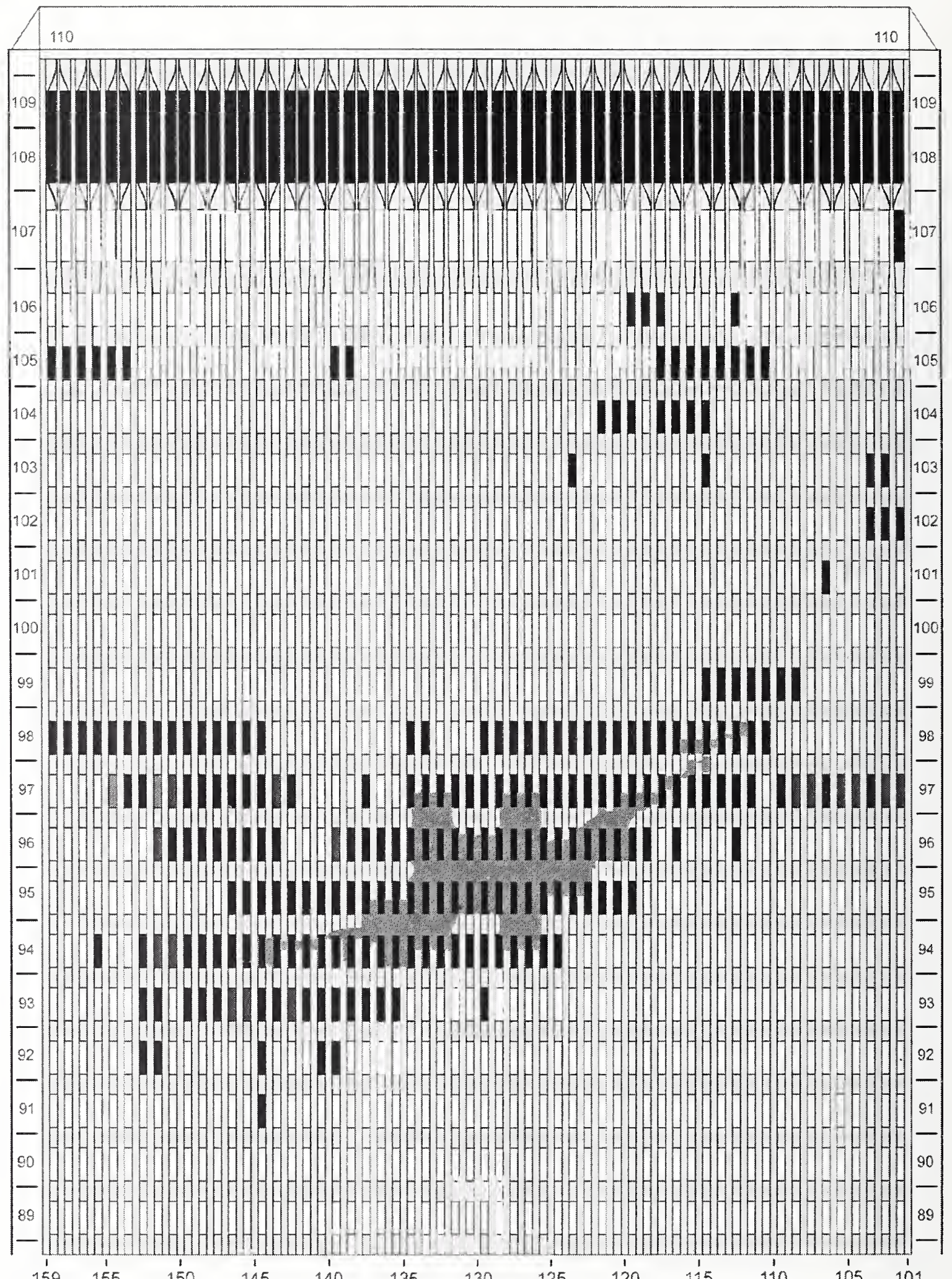

Figure C-23. Diagram of the north face of WTC 1 for floors 89 to 110 at 9:14 a.m. showing the condition of windows and locations of fires. 
WTC 1, North Face $\quad$ 9:14 a.m.

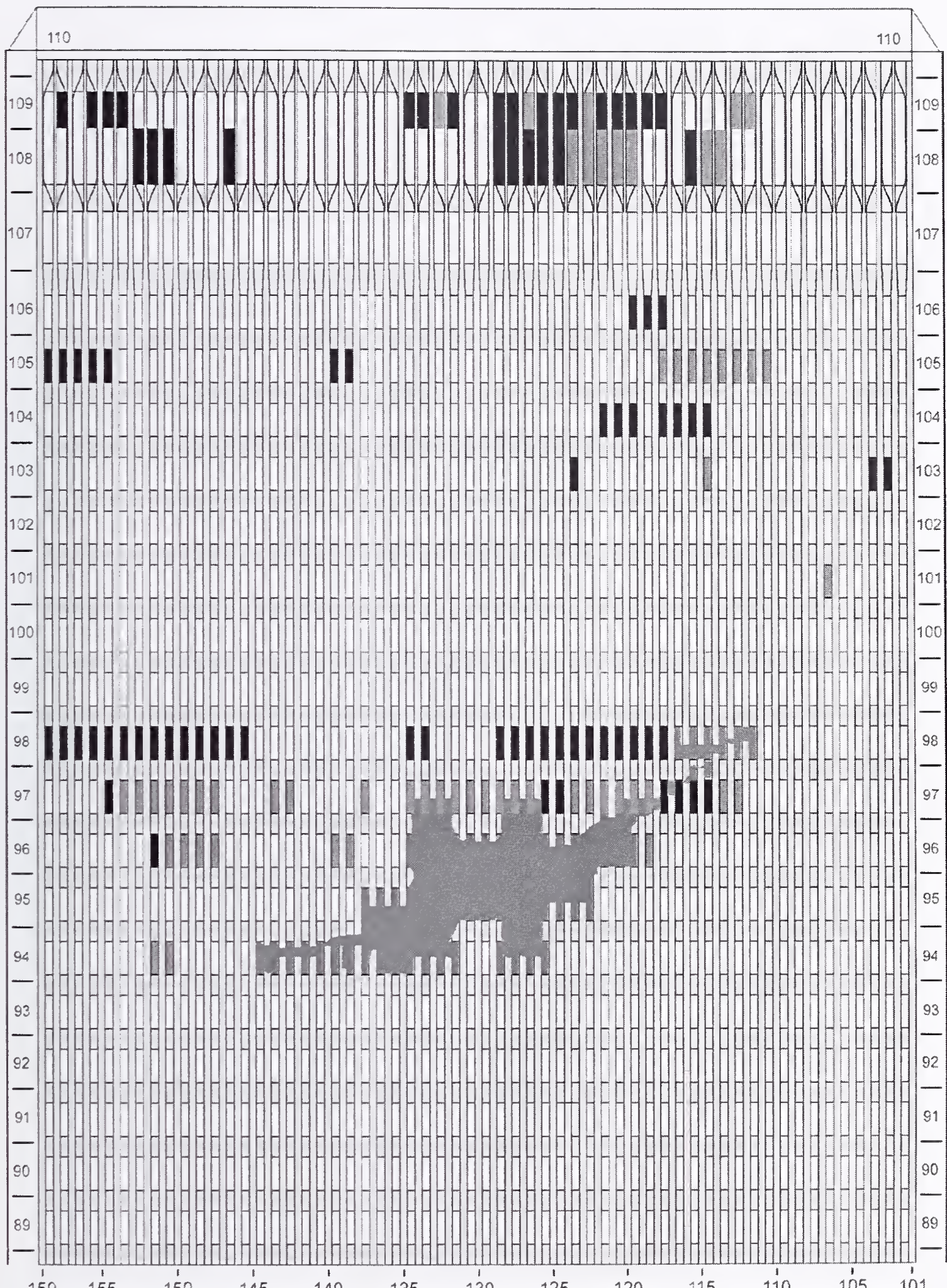

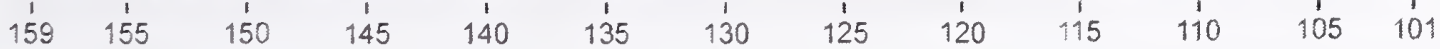

Figure C-24. Diagram of the north face of WTC 1 for floors 89 to 110 at 9:14 a.m. showing windows where smoke was observed and those that were hidden from view. 
WTC 1, North Face 9:18 a.m.

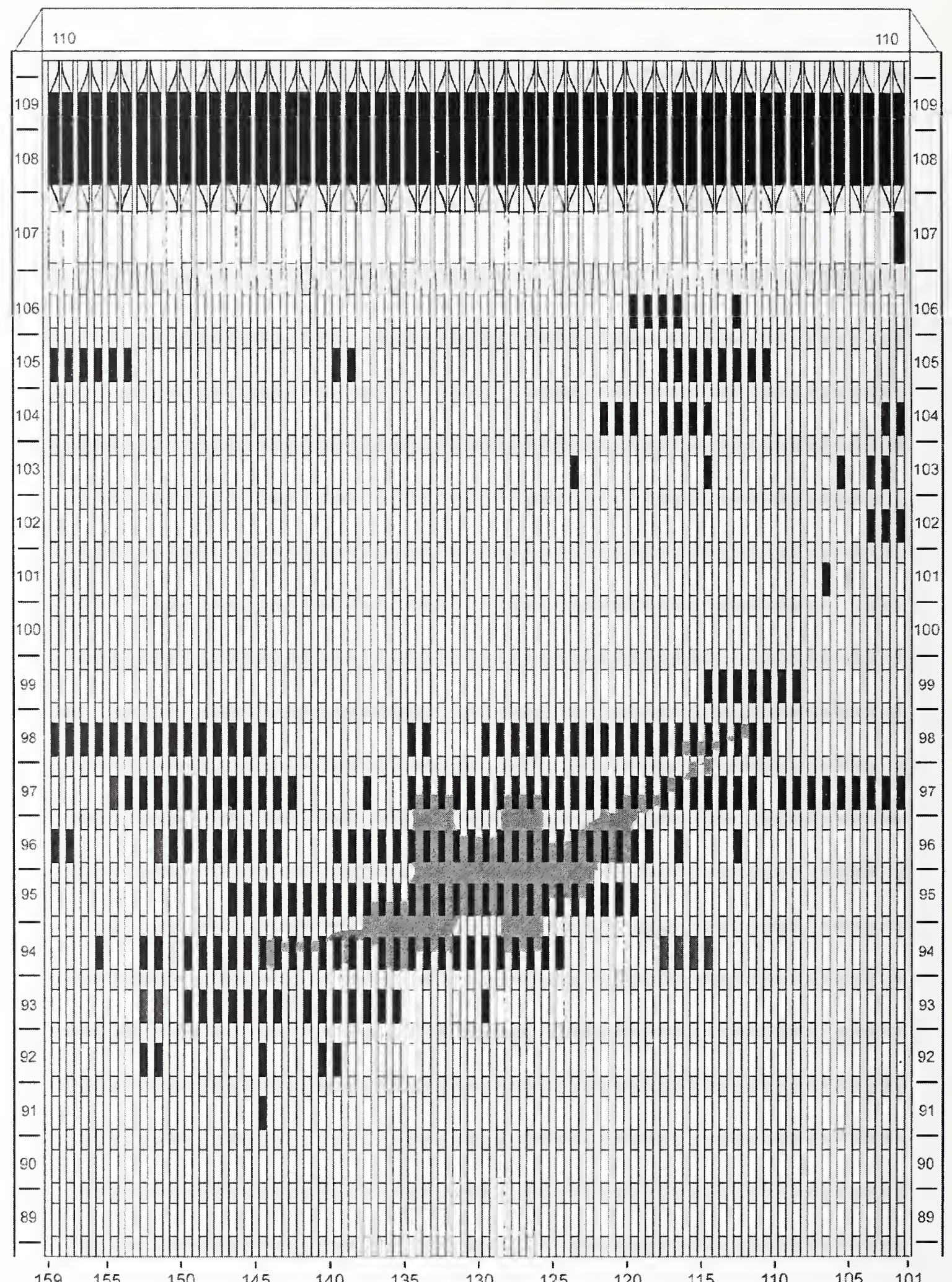

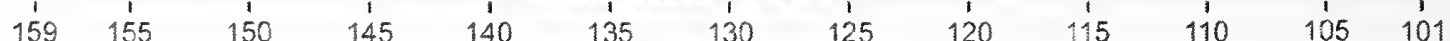

Figure C-25. Diagram of the north face of WTC 1 for floors 89 to 110 at 9:18 a.m. showing the condition of windows and locations of fires. 


$$
\text { WTC 1, North Face } \quad 9: 18 \text { a.m. }
$$

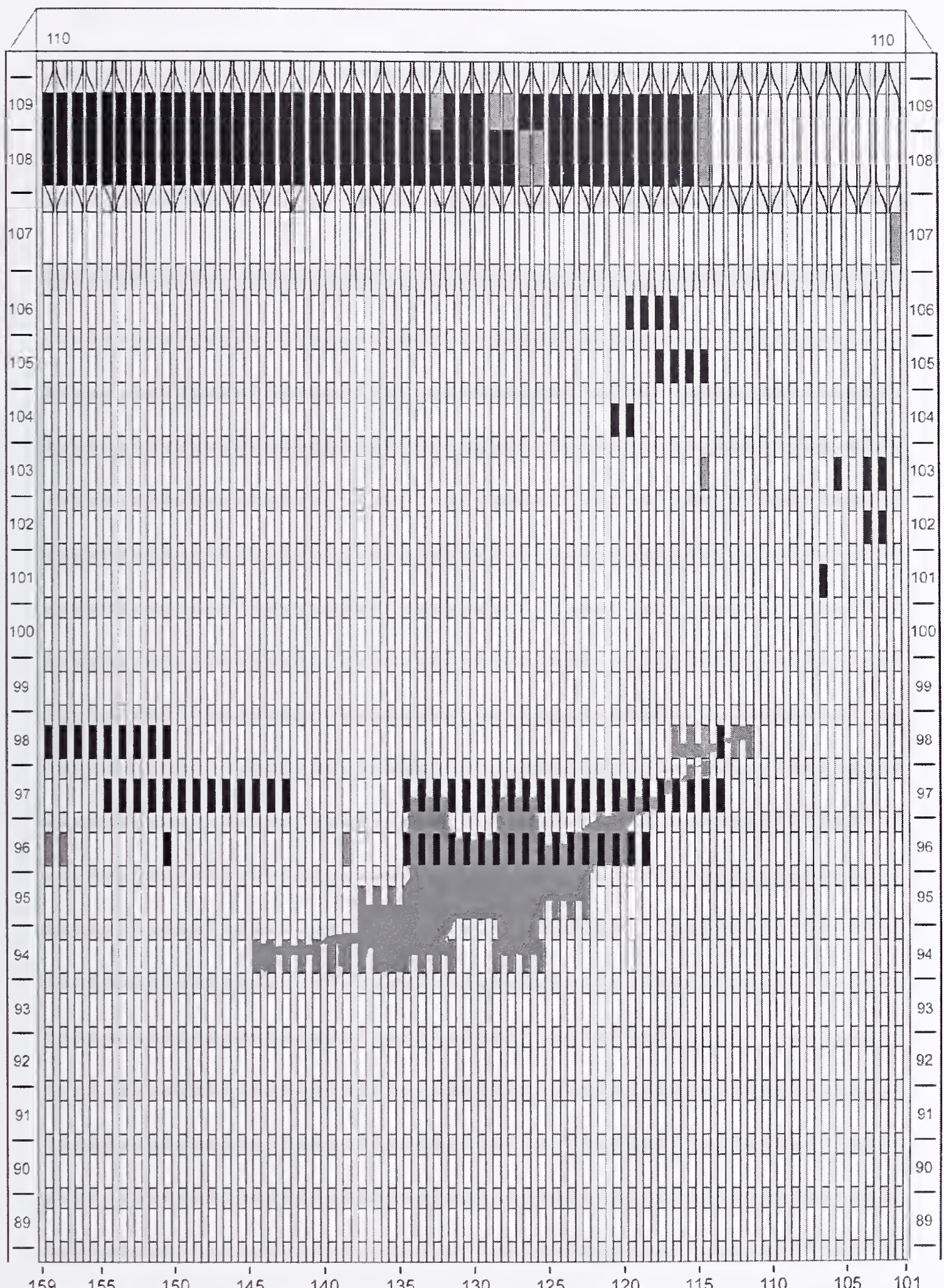

Figure C-26. Diagram of the north face of WTC 1 for floors 89 to 110 at $9: 18$ a.m. showing windows where smoke was observed and those that were hidden from view. 
WTC 1, North Face 9:20 a.m.

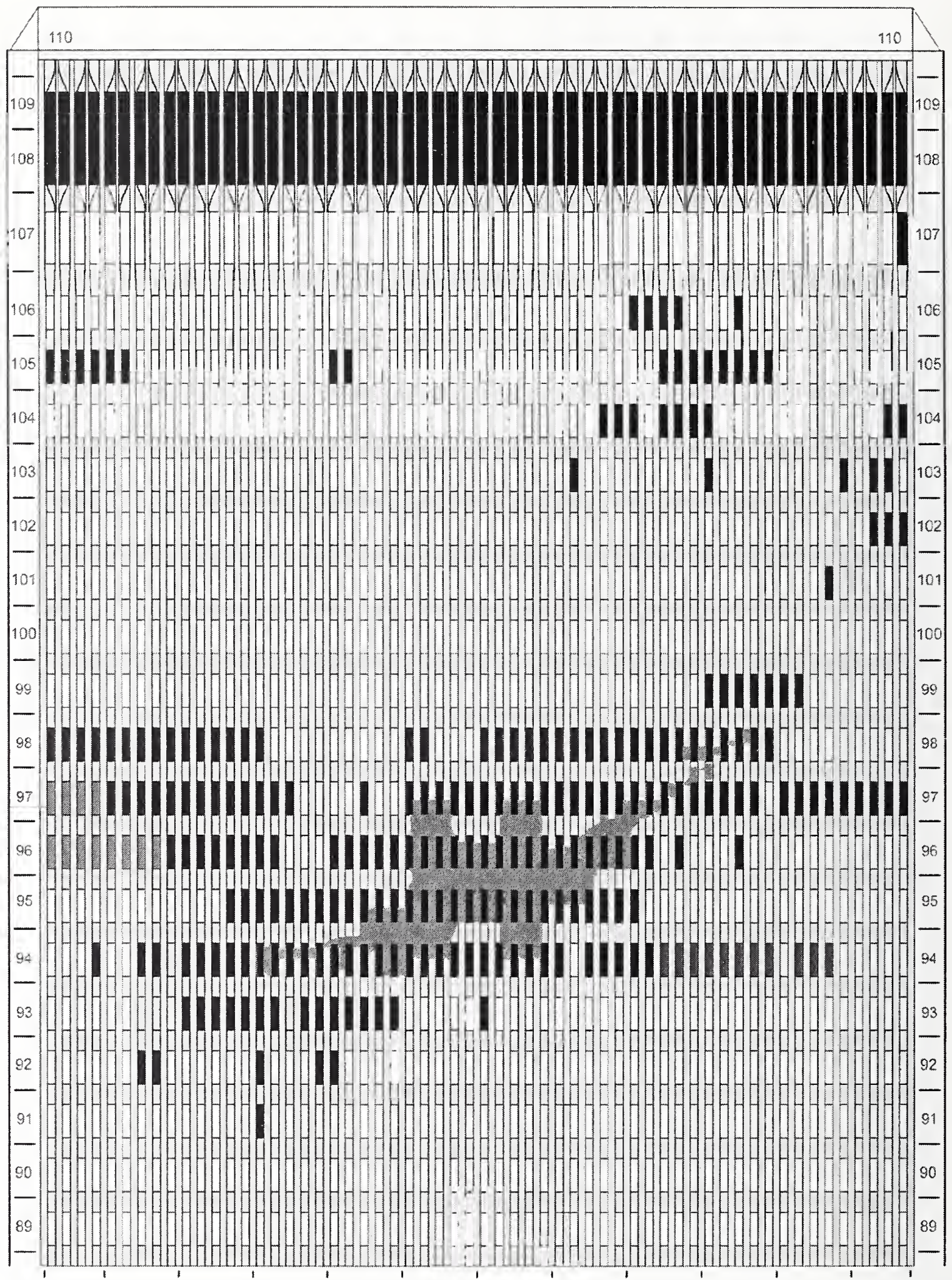

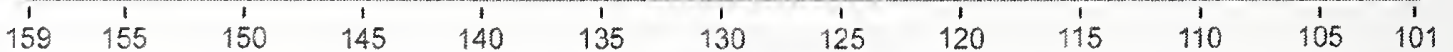

Figure C-27. Diagram of the north face of WTC 1 for floors 89 to 110 at 9:20 a.m. showing the condition of windows and locations of fires. 

WTC 1, North Face
9:20 a.m.

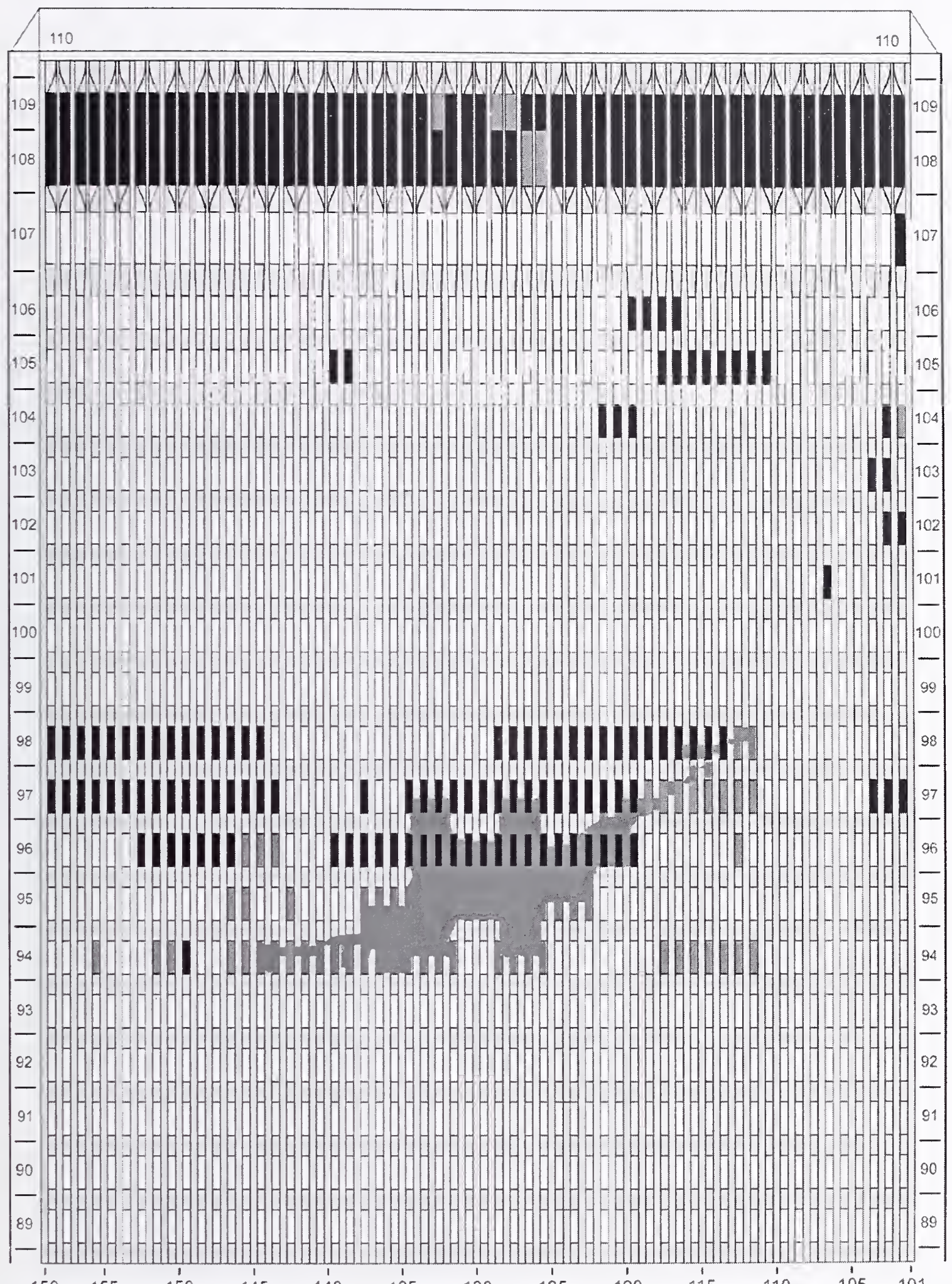

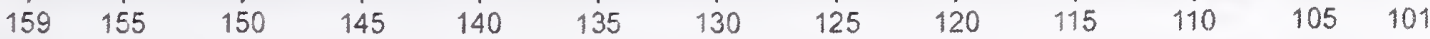

Figure C-28. Diagram of the north face of WTC 1 for floors 89 to 110 at 9:20 a.m. showing windows where smoke was observed and those that were hidden from view. 
WTC 1, North Face 9:26 a.m.

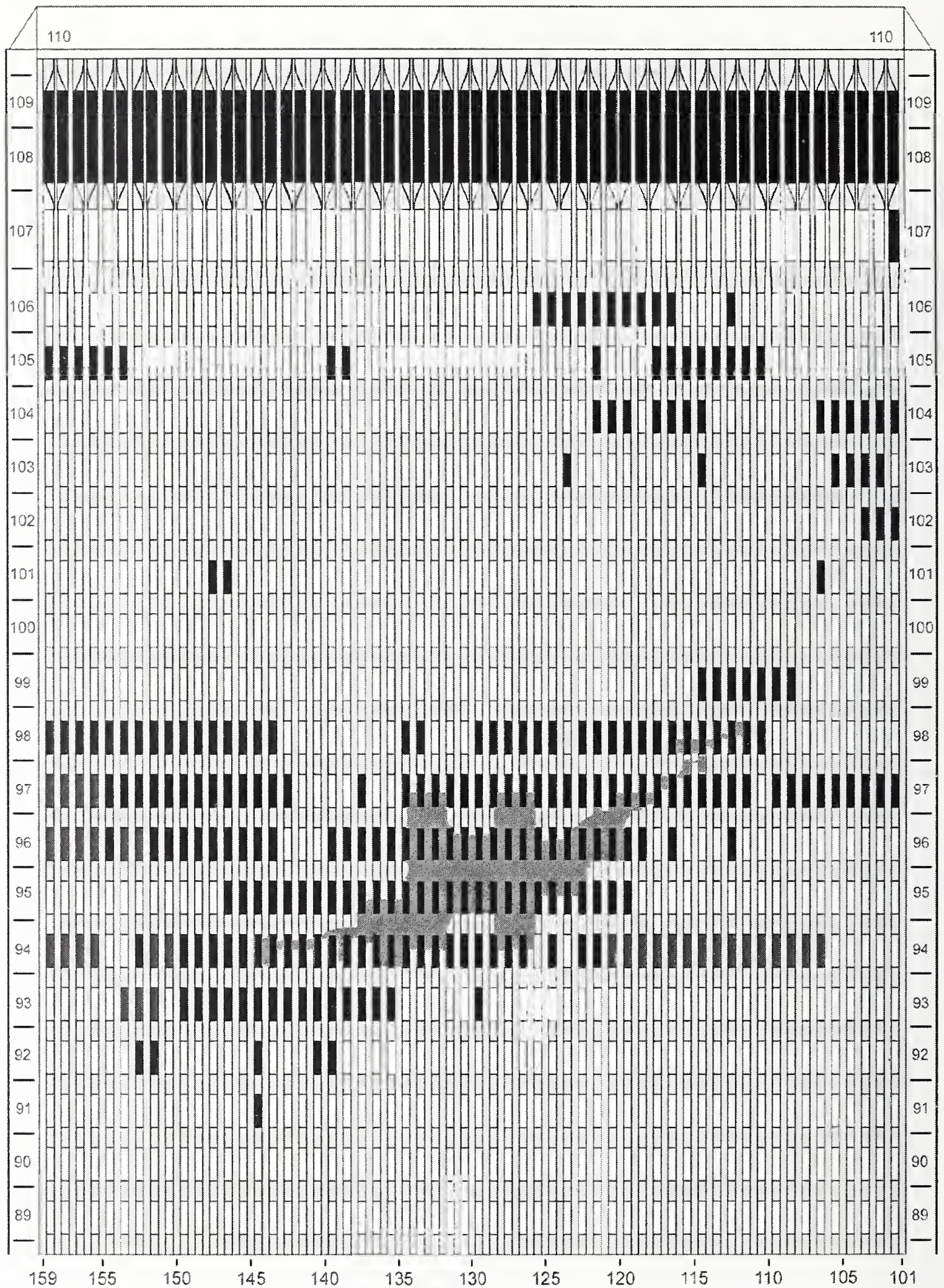

Figure C-29. Diagram of the north face of WTC 1 for floors 89 to 110 at 9:26 a.m. showing the condition of windows and locations of fires. 
WTC 1, North Face $\quad 9: 26$ a.m.

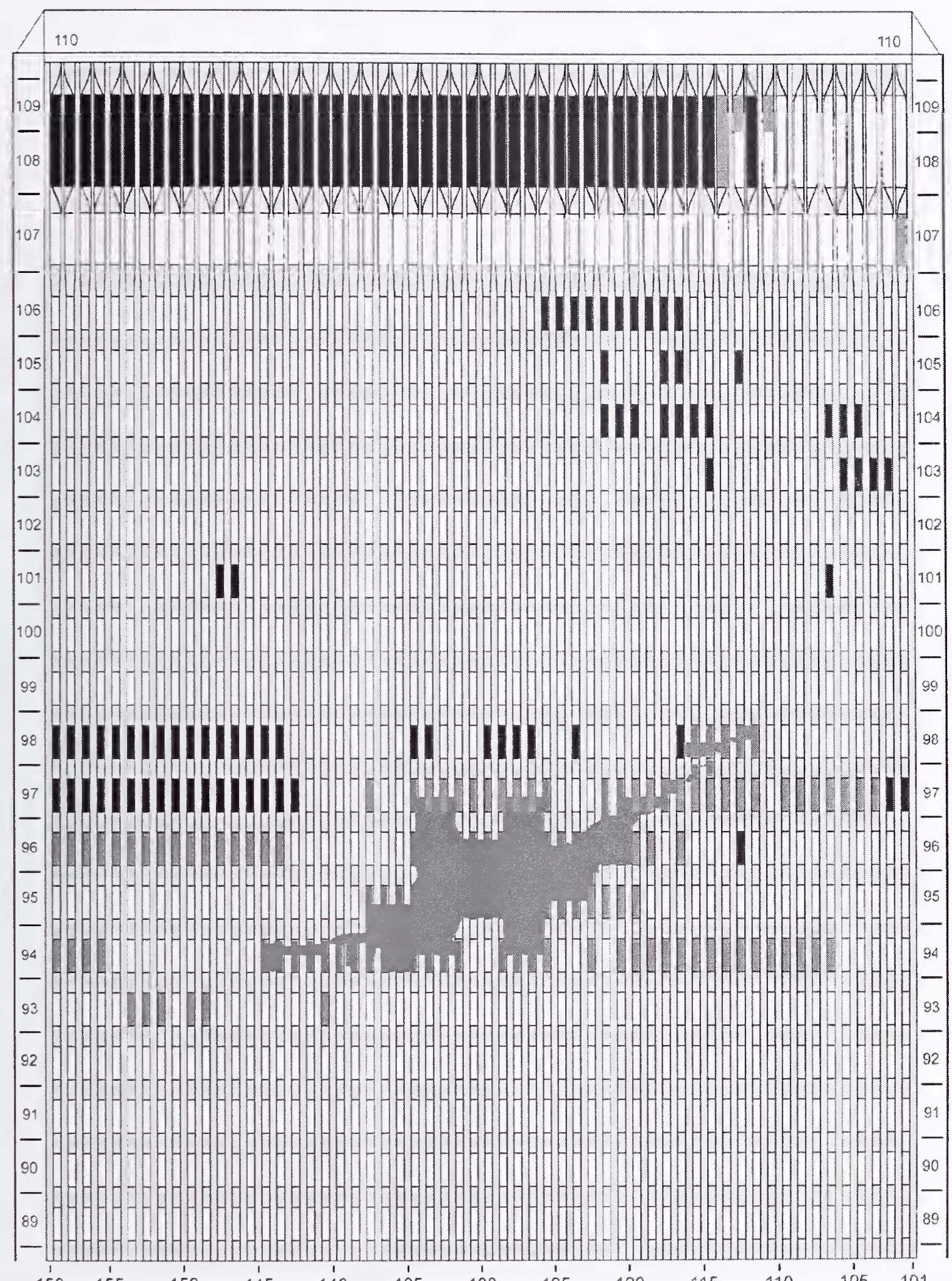

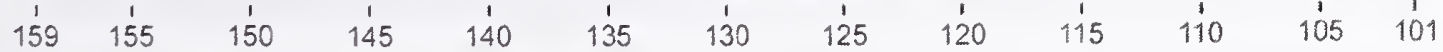

Figure C-30. Diagram of the north face of WTC 1 for floors 89 to 110 at 9:26 a.m. showing windows where smoke was observed and those that were hidden from view. 

WTC 1, North Face
9:32 a.m.

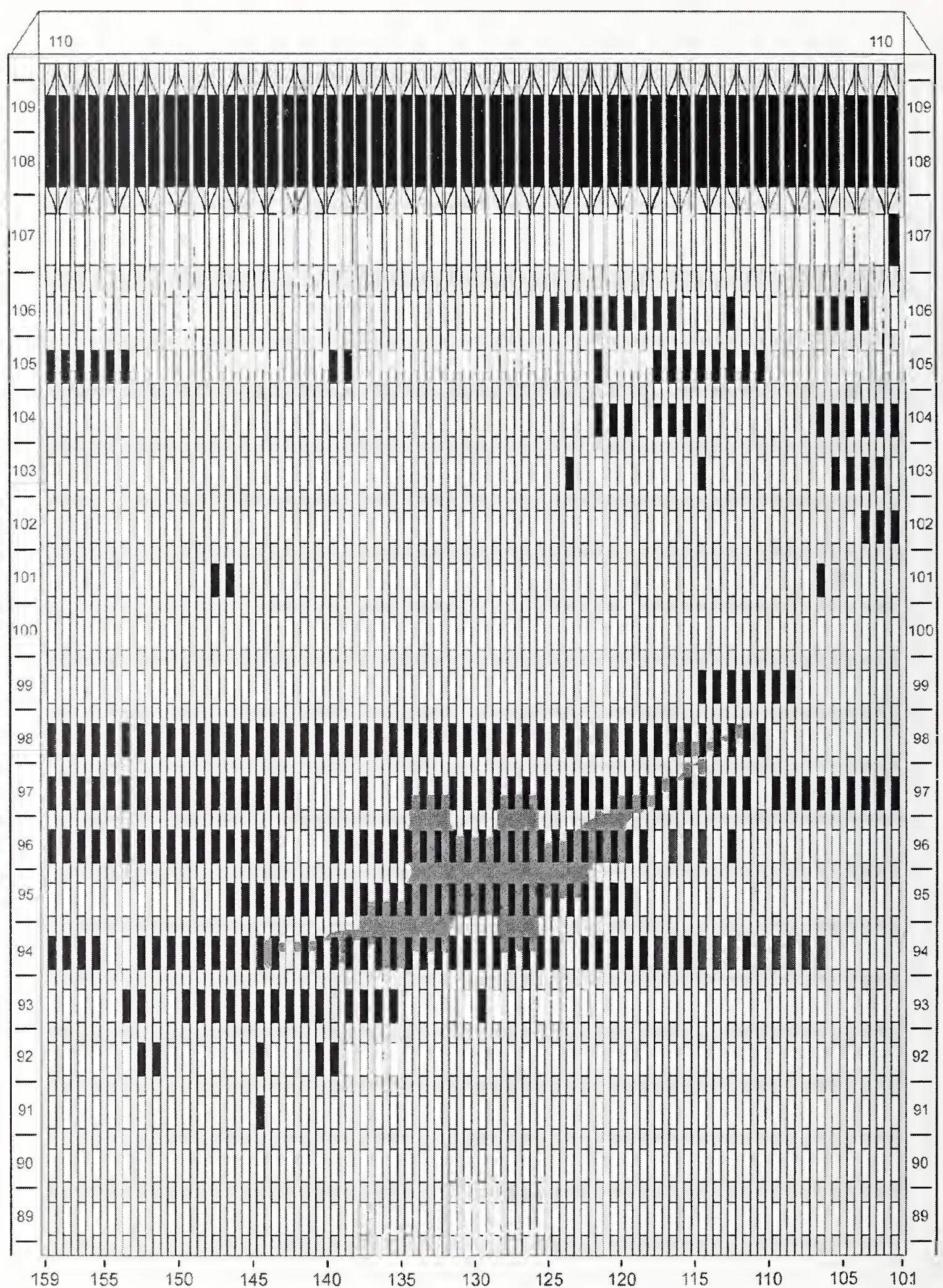

Figure C-31. Diagram of the north face of WTC 1 for floors 89 to 110 at 9:32 a.m. showing the condition of windows and locations of fires. 

WTC 1, North Face
9:32 a.m.

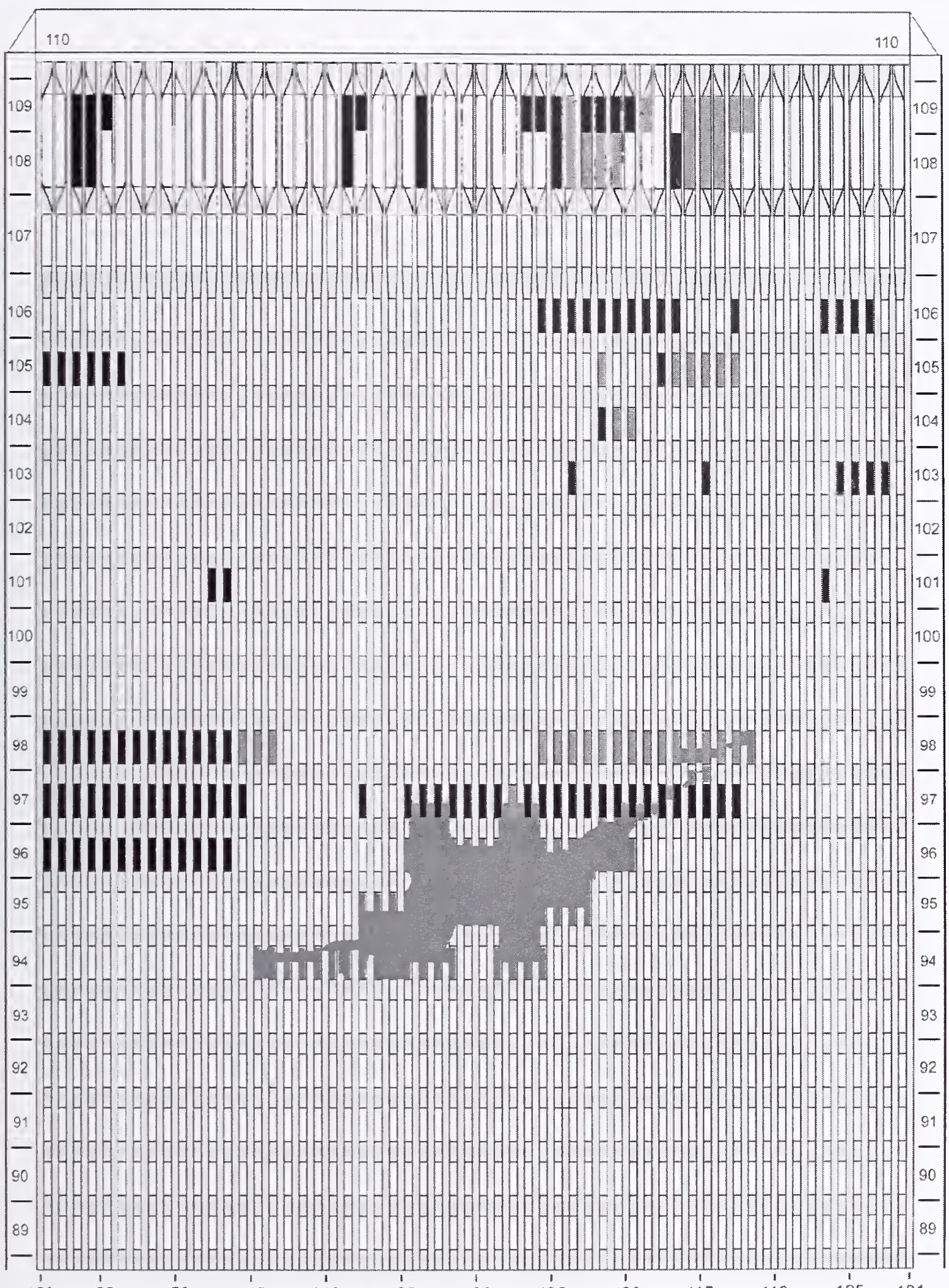

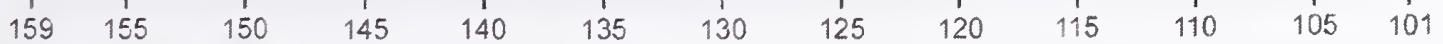

Figure C-32. Diagram of the north face of WTC 1 for floors 89 to 110 at 9:32 a.m. showing windows where smoke was observed and those that were hidden from view. 


\section{WTC 1, North Face 9:38 a.m.}

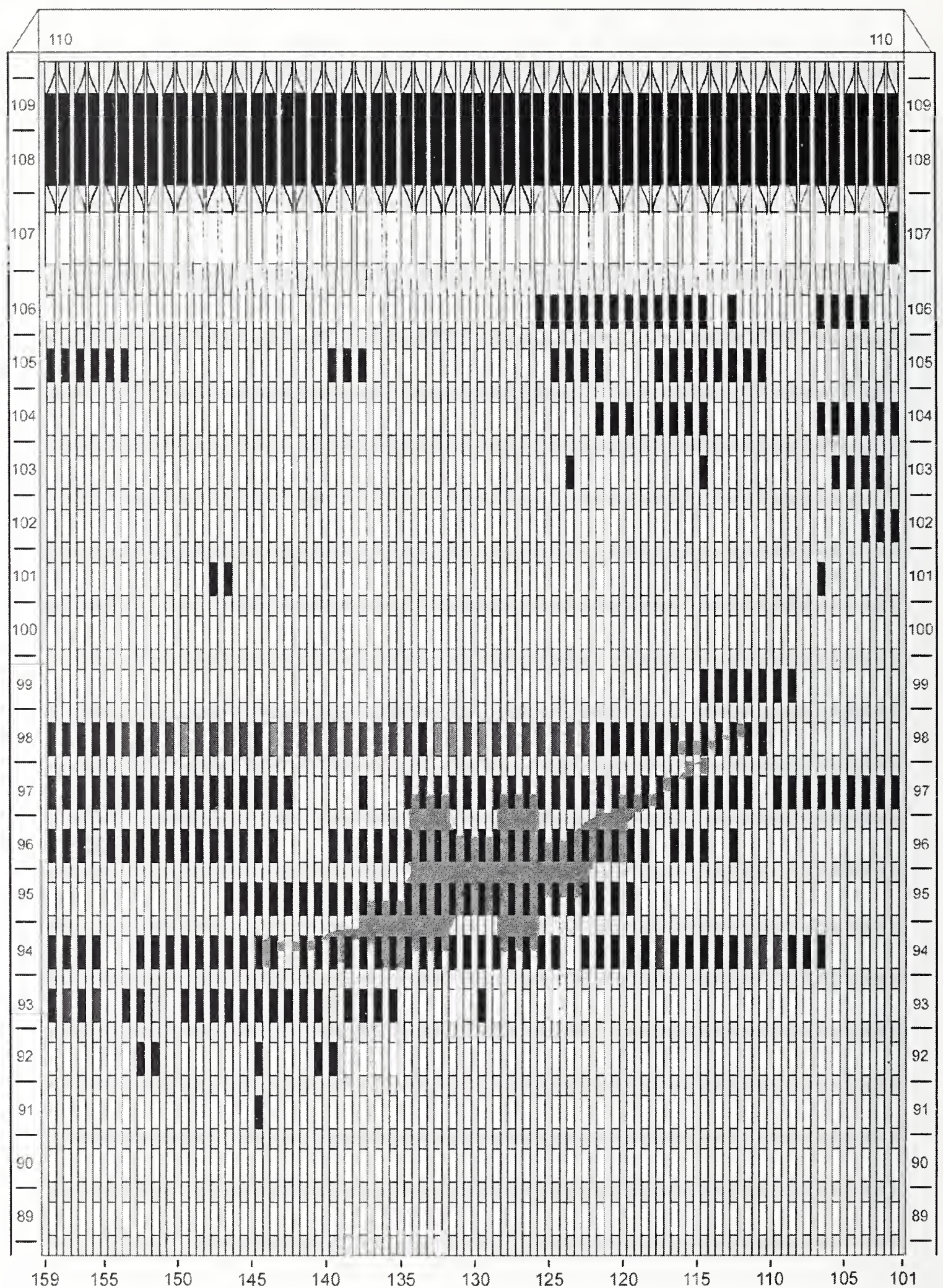

Figure C-33. Diagram of the north face of WTC 1 for floors 89 to 110 at 9:38 a.m. showing the condition of windows and locations of fires. 


\section{WTC 1, North Face 9:38 a.m.}

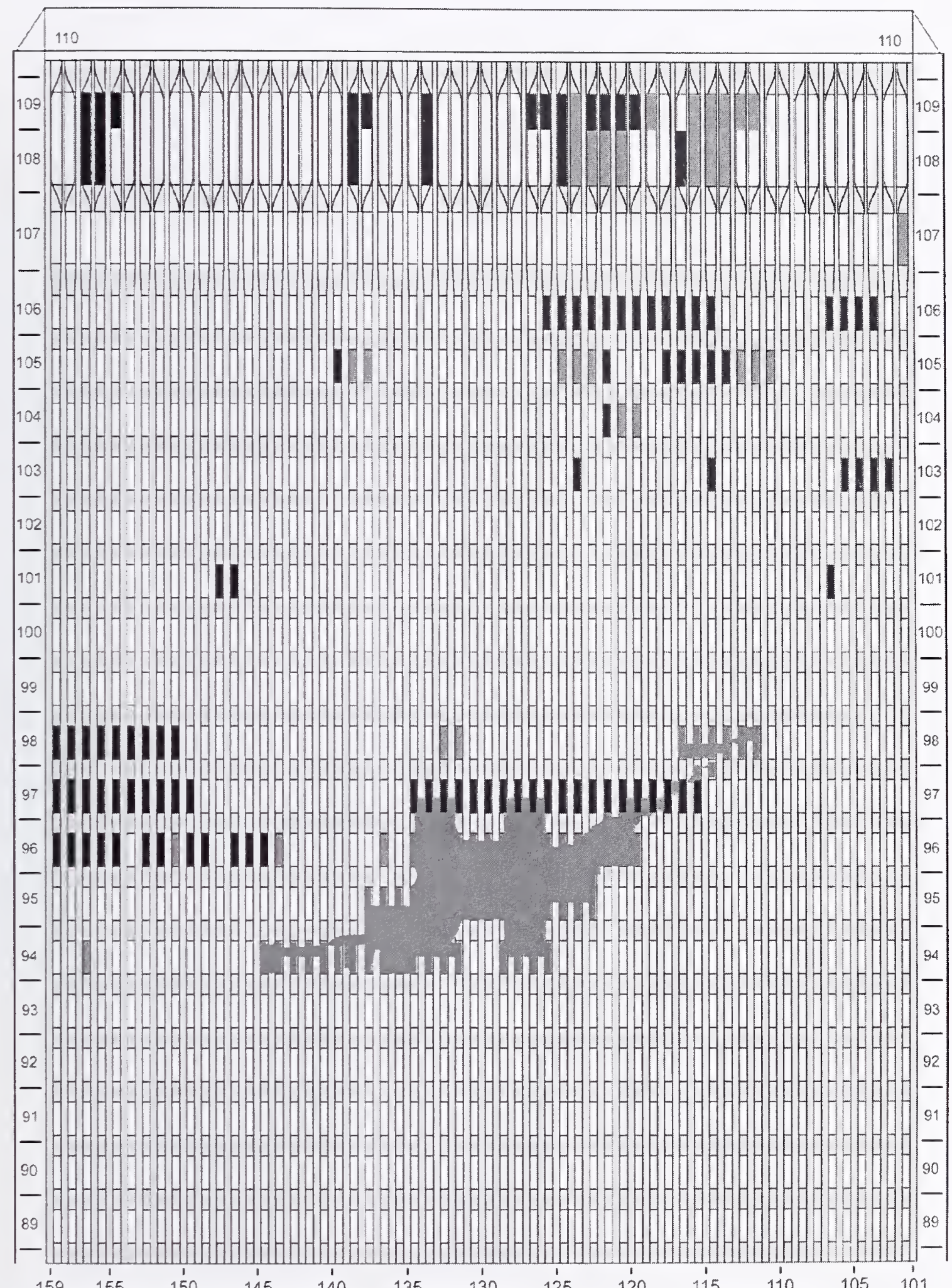

Figure C-34. Diagram of the north face of WTC 1 for floors 89 to 110 at 9:38 a.m. showing windows where smoke was observed and those that were hidden from view. 

WTC 1, North Face
9:42 a.m.

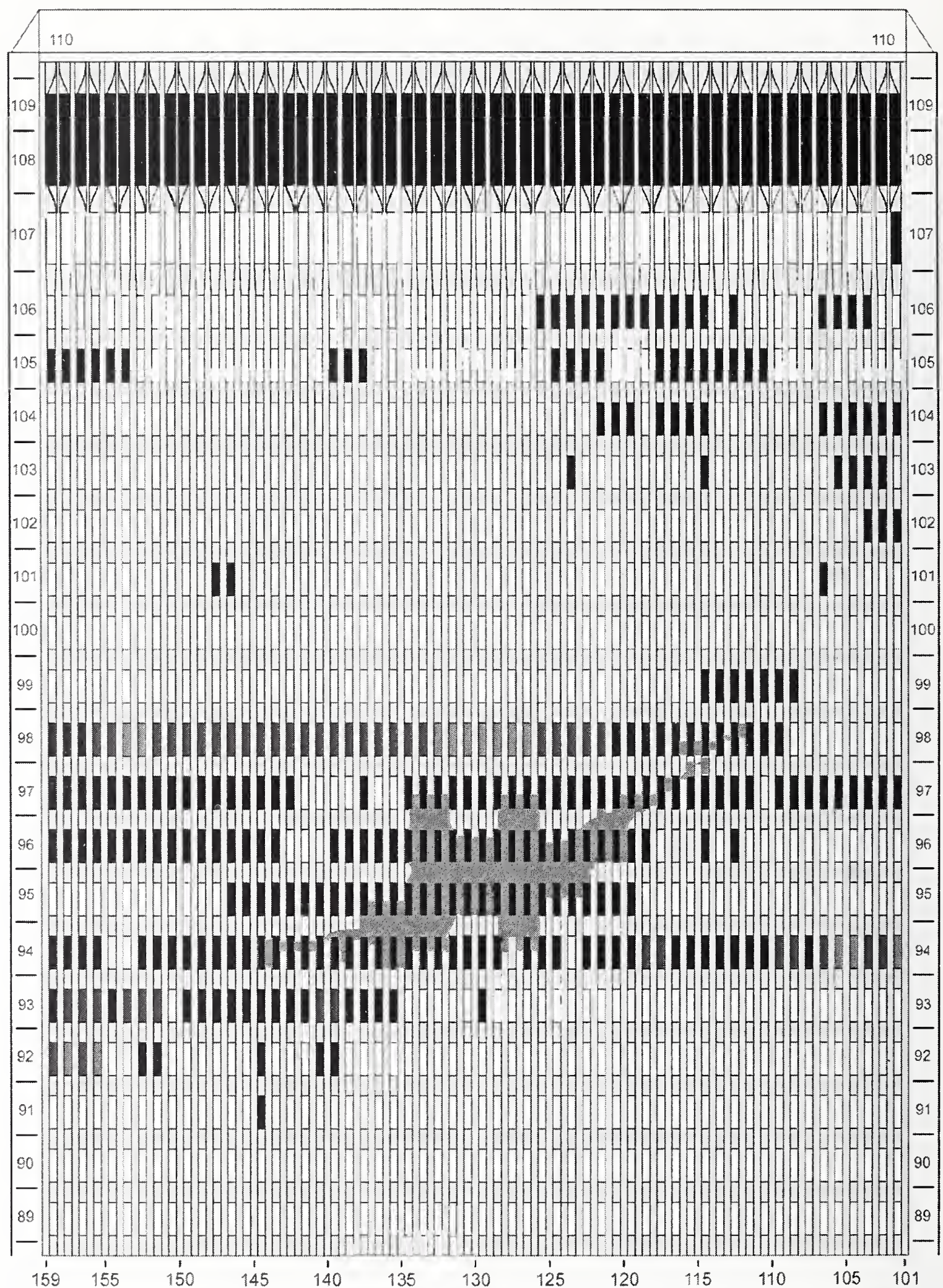

Figure C-35. Diagram of the north face of WTC 1 for floors 89 to 110 at 9:42 a.m. showing the condition of windows and locations of fires. 

WTC 1, North Face
9:42 a.m.

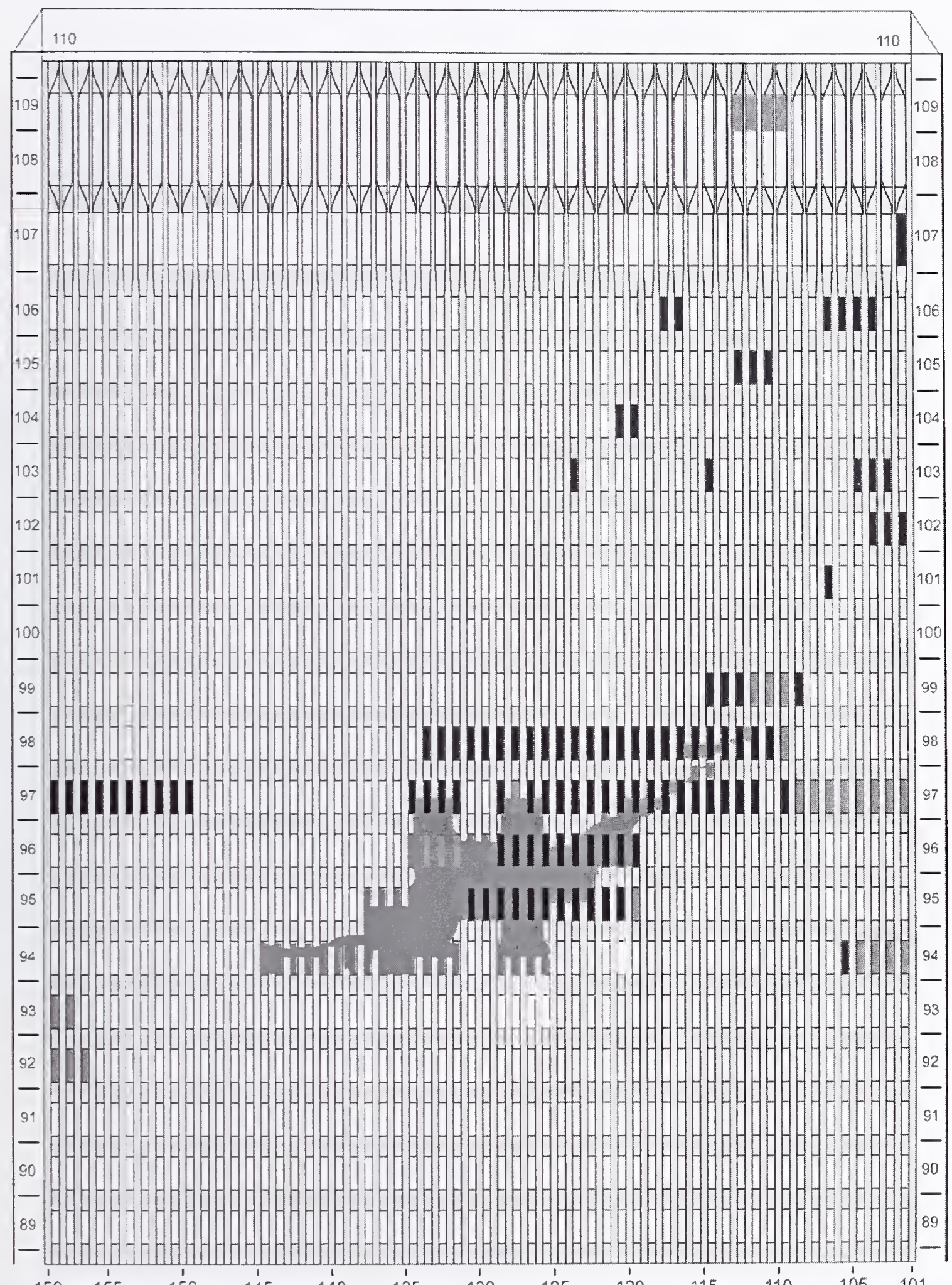

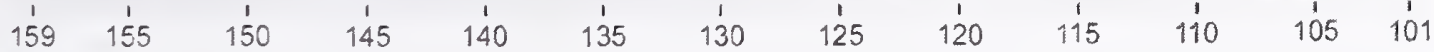

Figure C-36. Diagram of the north face of WTC 1 for floors 89 to 110 at 9:42 a.m. showing windows where smoke was observed and those that were hidden from view. 


\section{WTC 1, North Face 9:46 a.m.}

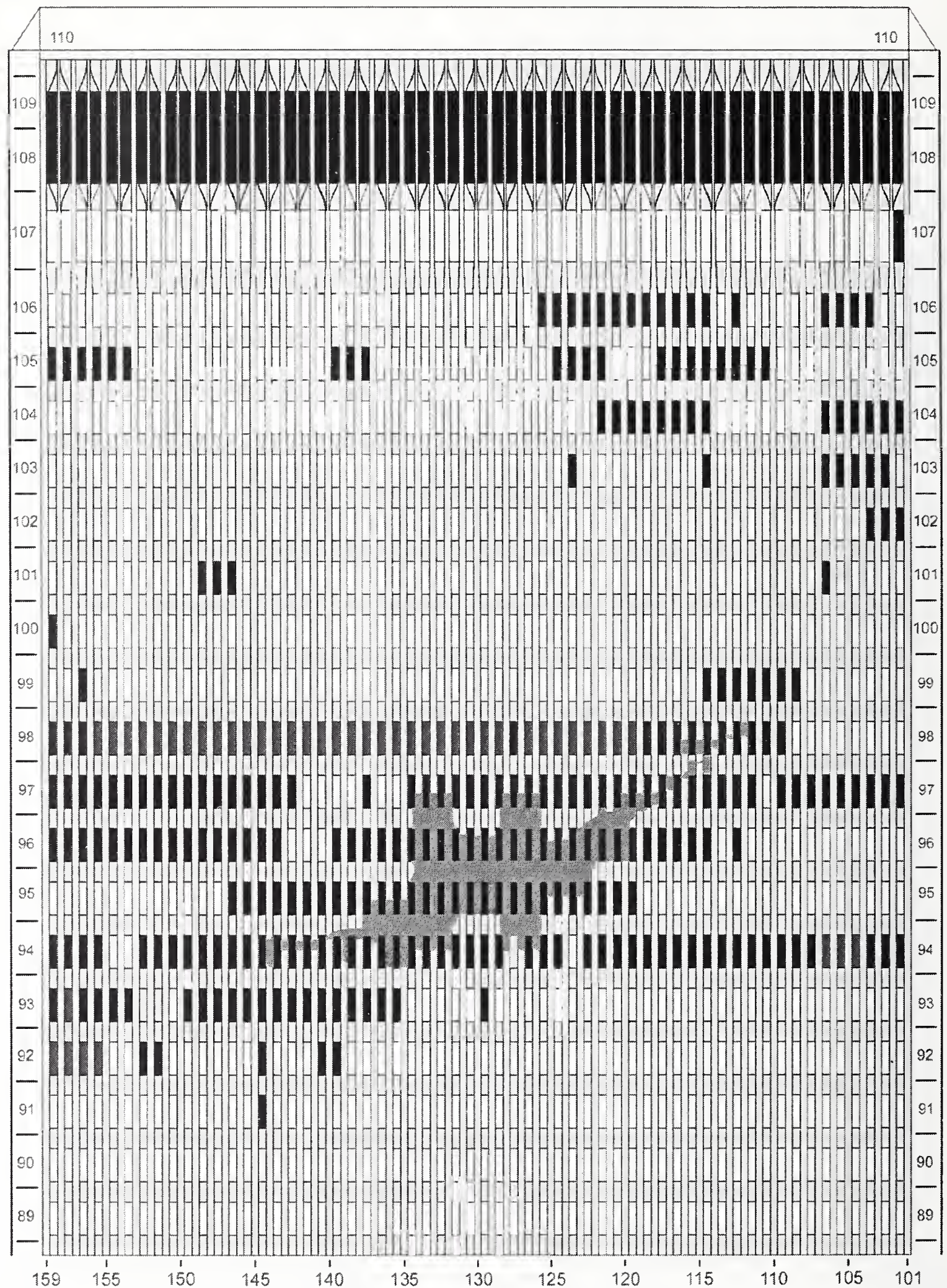

Figure C-37. Diagram of the north face of WTC 1 for floors 89 to 110 at 9:46 a.m. showing the condition of windows and locations of fires. 
WTC 1, North Face 9:46 a.m.

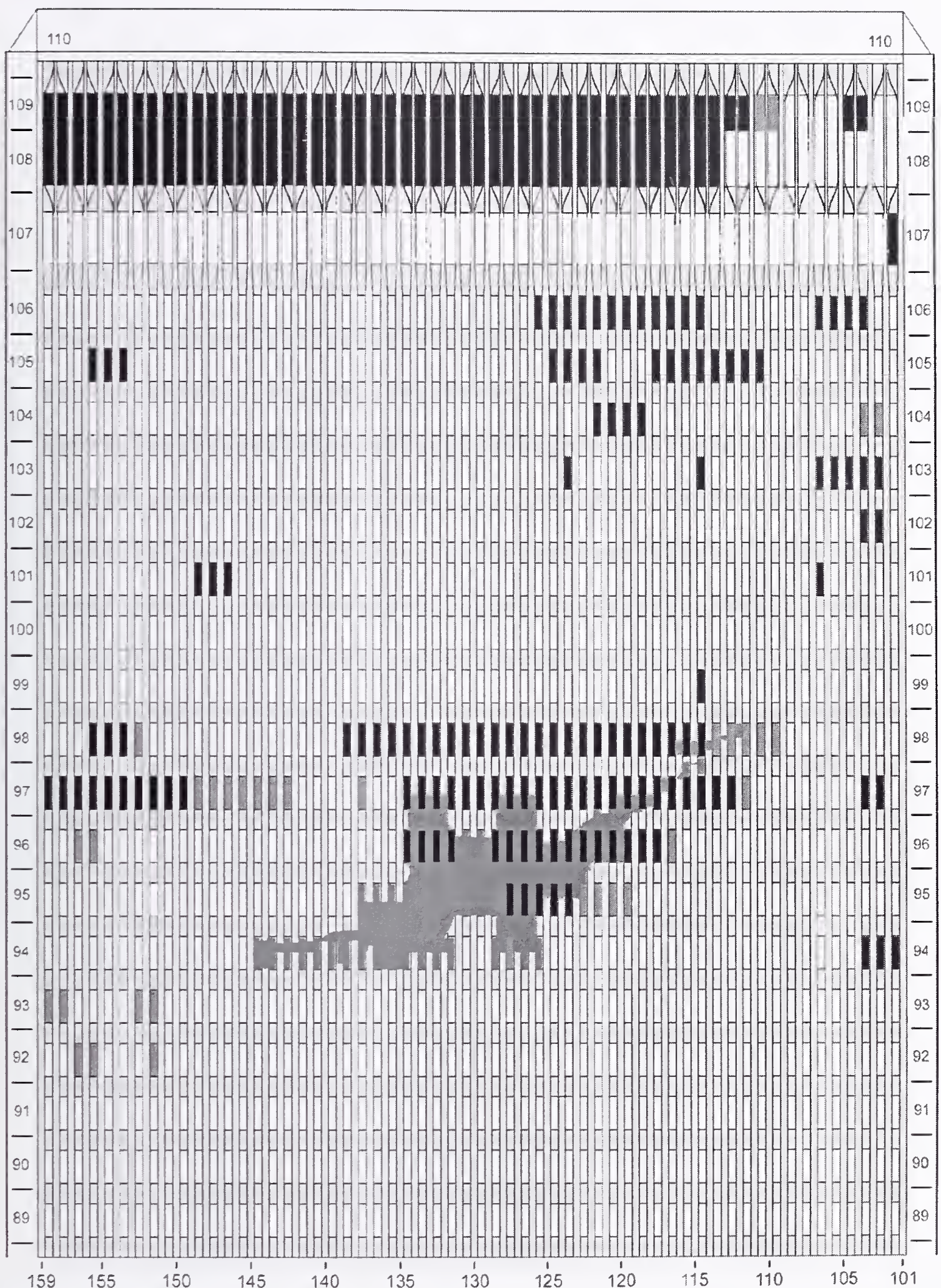

Figure C-38. Diagram of the north face of WTC 1 for floors 89 to 110 at 9:46 a.m. showing windows where smoke was observed and those that were hidden from view. 


$$
\text { WTC 1, North Face 9:52 a.m. }
$$

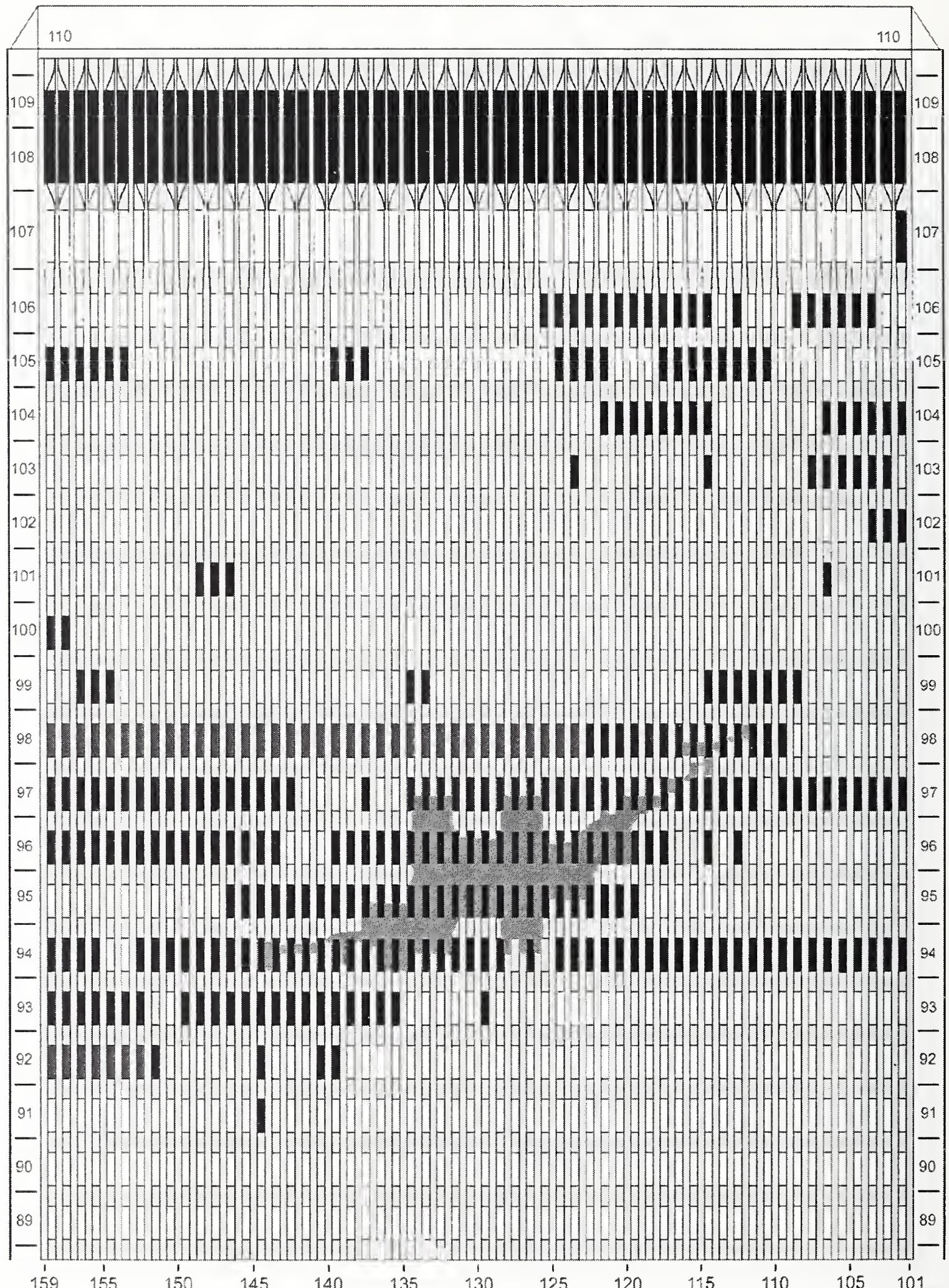

Figure C-39. Diagram of the north face of WTC 1 for floors 89 to 110 at 9:52 a.m. showing the condition of windows and locations of fires. 
WTC 1, North Face 9:52 a.m.

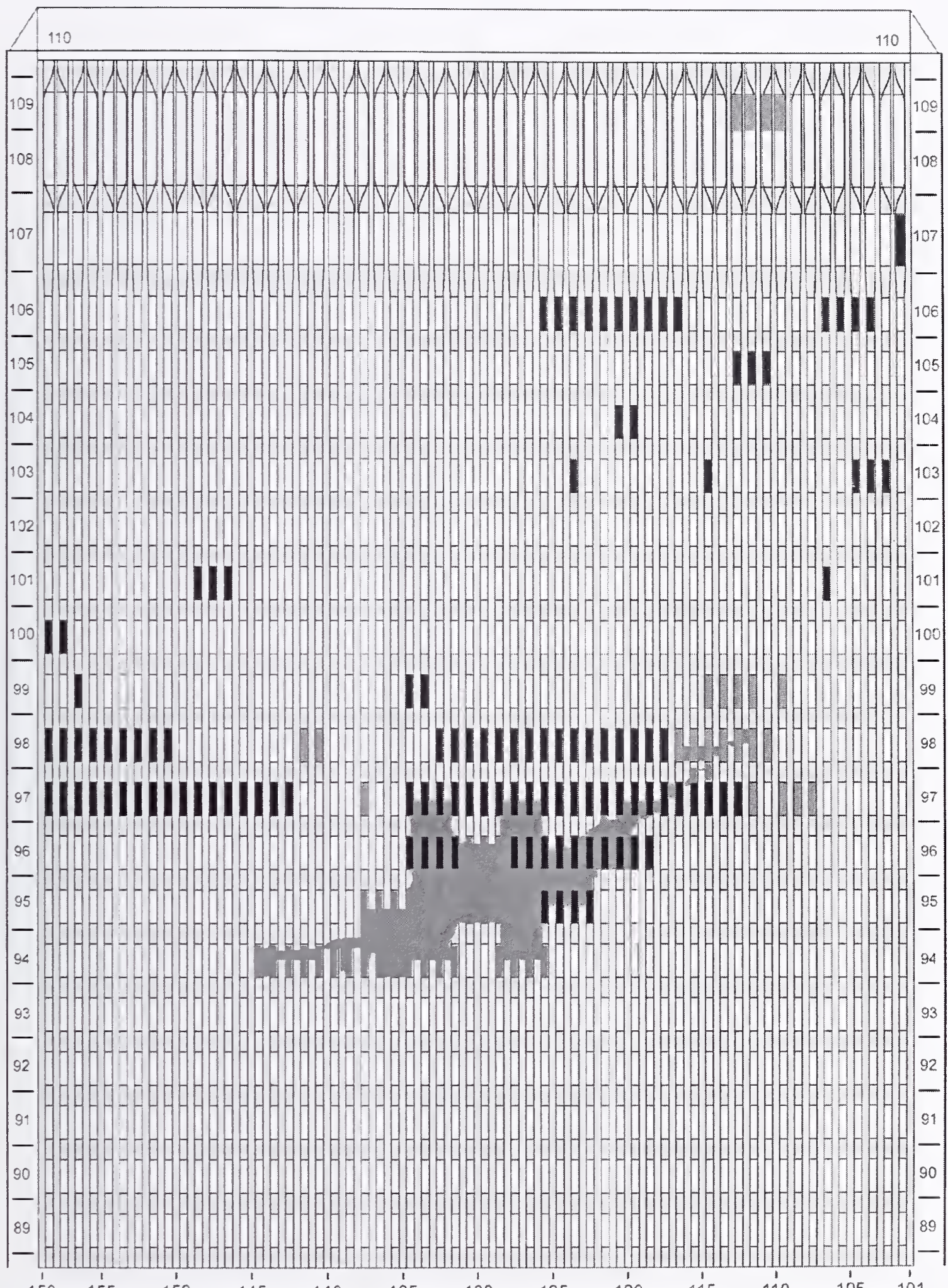

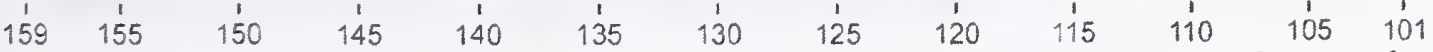

Figure C-40. Diagram of the north face of WTC 1 for floors 89 to 110 at 9:52 a.m. showing windows where smoke was observed and those that were hidden from view. 
WTC 1, North Face $\quad 9: 54$ a.m.

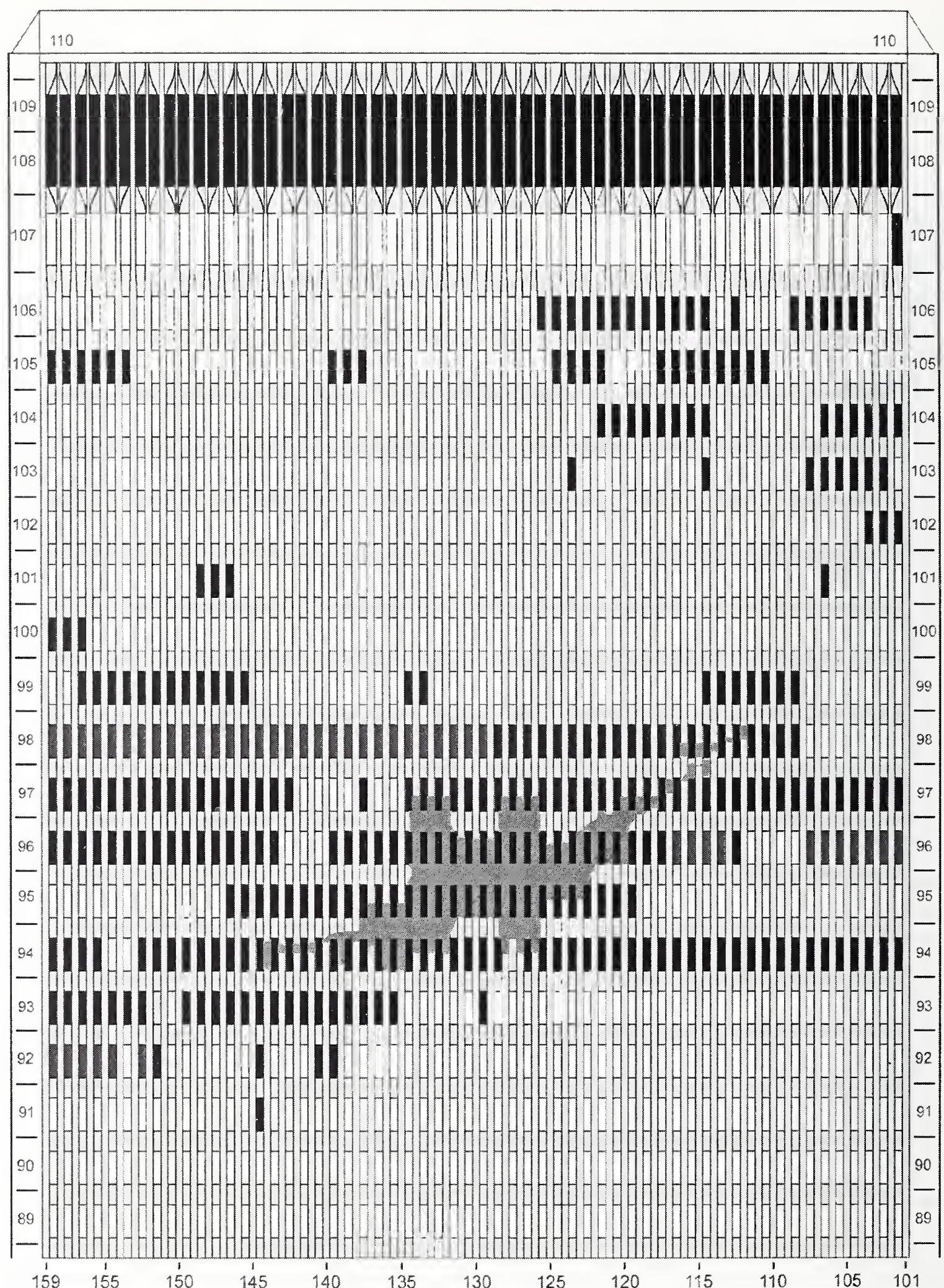

Figure C-41. Diagram of the north face of WTC 1 for floors 89 to 110 at 9:54 a.m. showing the condition of windows and locations of fires. 


$$
\text { WTC 1, North Face 9:54 a.m. }
$$

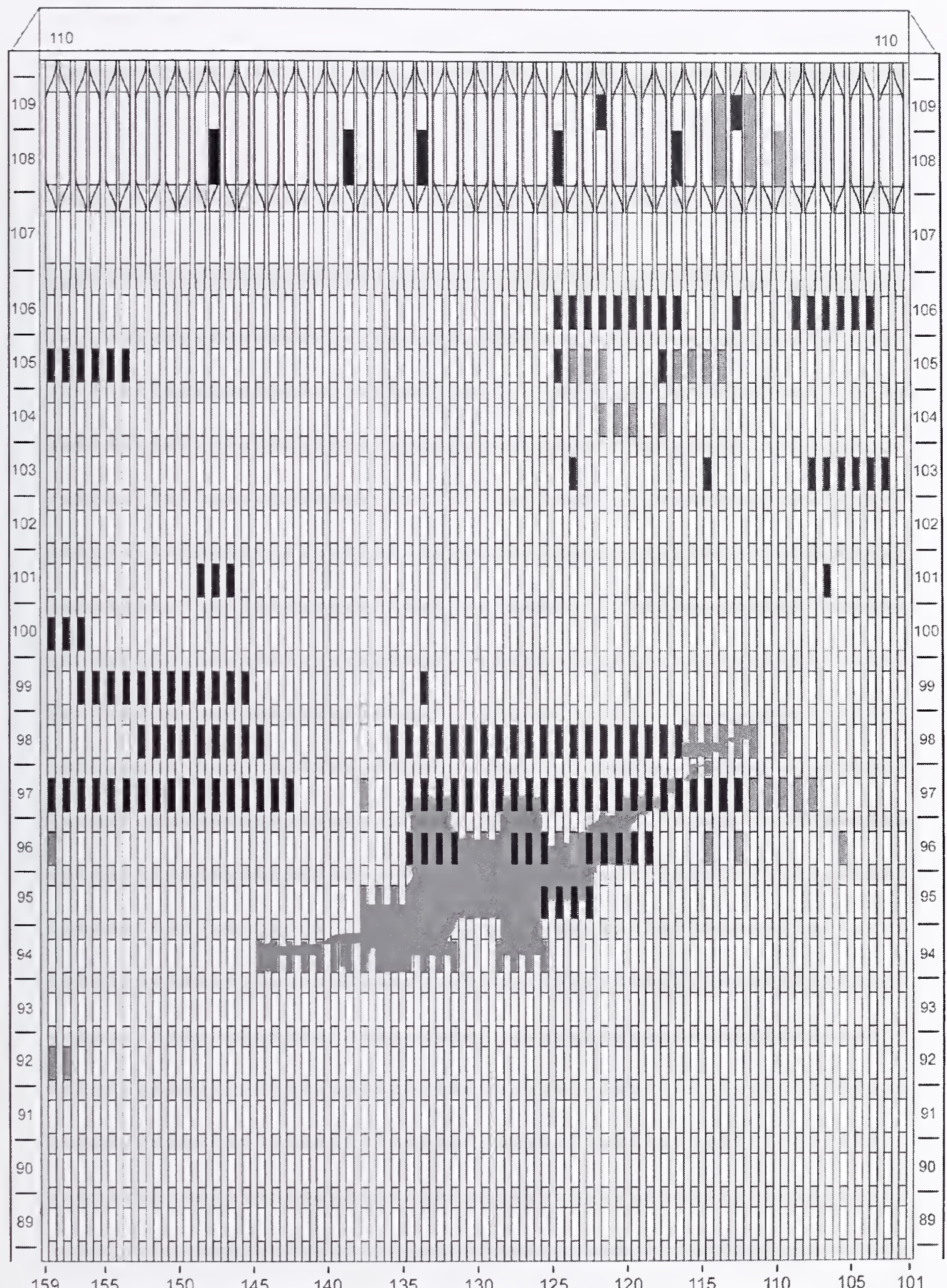

Figure C-42. Diagram of the north face of WTC 1 for floors 89 to 110 at 9:54 a.m. showing windows where smoke was observed and those that were hidden from view. 


$$
\text { WTC 1, North Face } \quad 9: 58 \text { a.m. }
$$

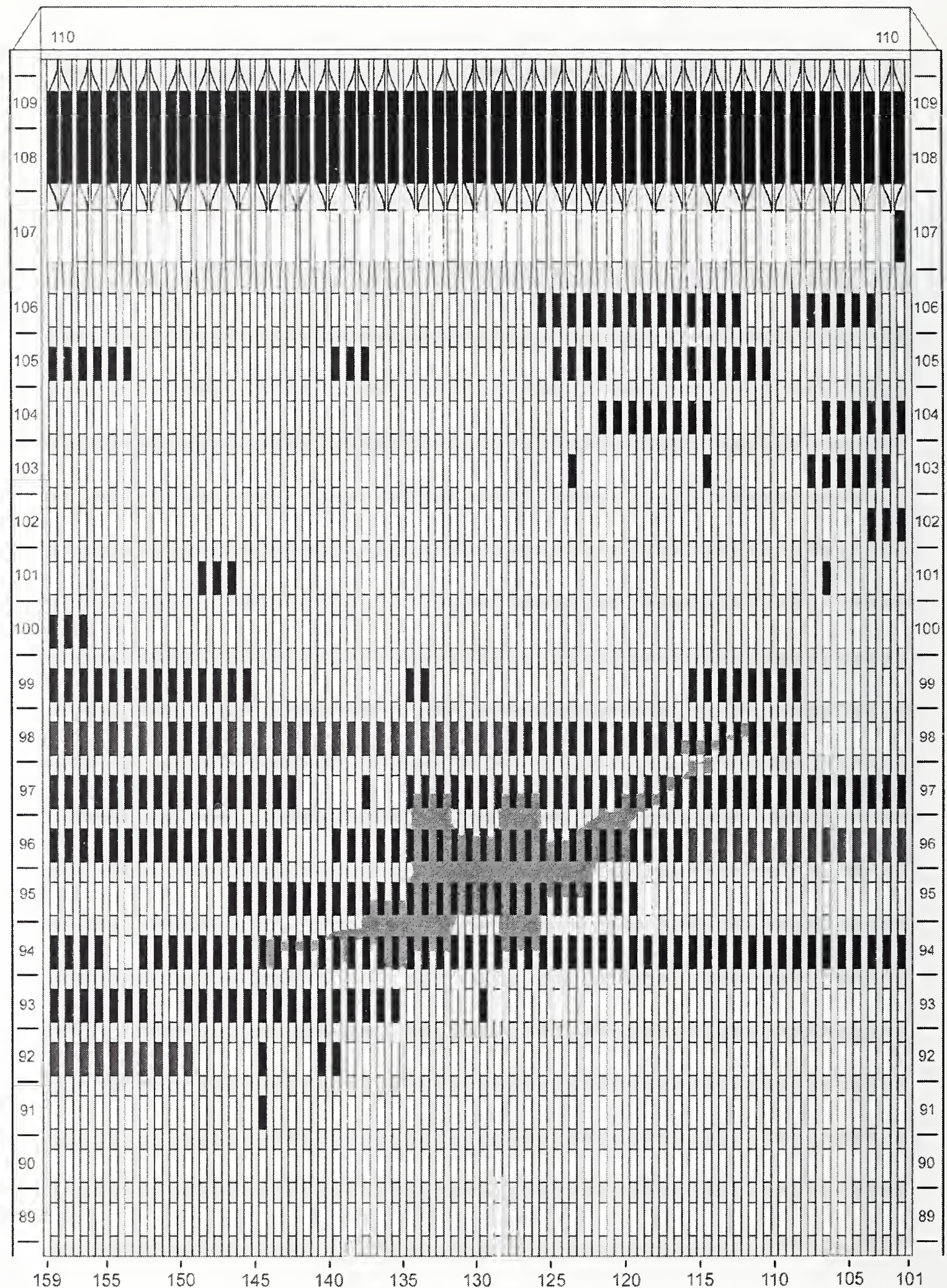

Figure C-43. Diagram of the north face of WTC 1 for floors 89 to 110 at 9:58 a.m. showing the condition of windows and locations of fires. 


$$
\text { WTC 1, North Face 9:58 a.m. }
$$

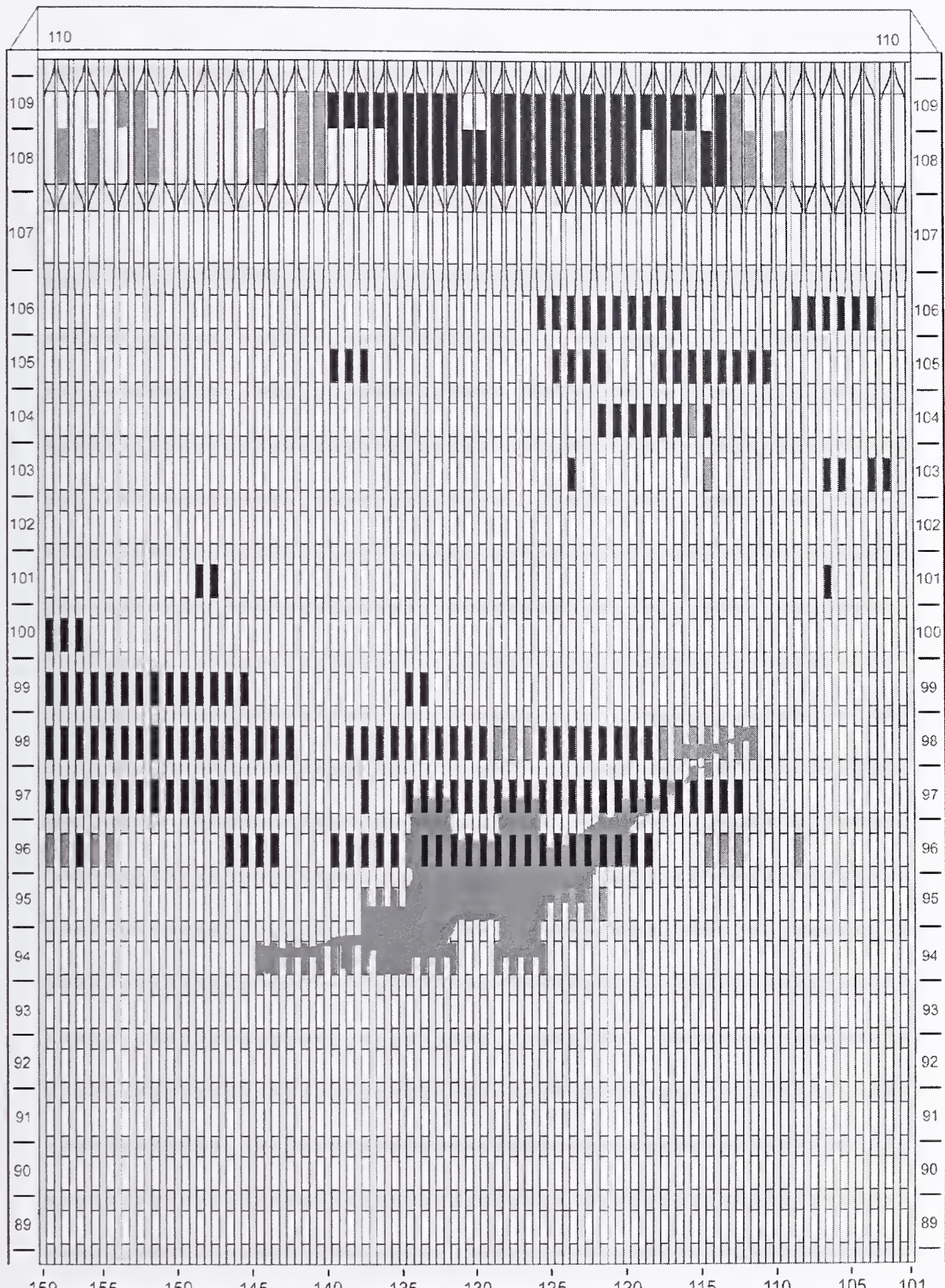

Figure C-44. Diagram of the north face of WTC 1 for floors 89 to 110 at 9:58 a.m. showing windows where smoke was observed and those that were hidden from view. 
WTC 1, North Face 10:04 a.m.

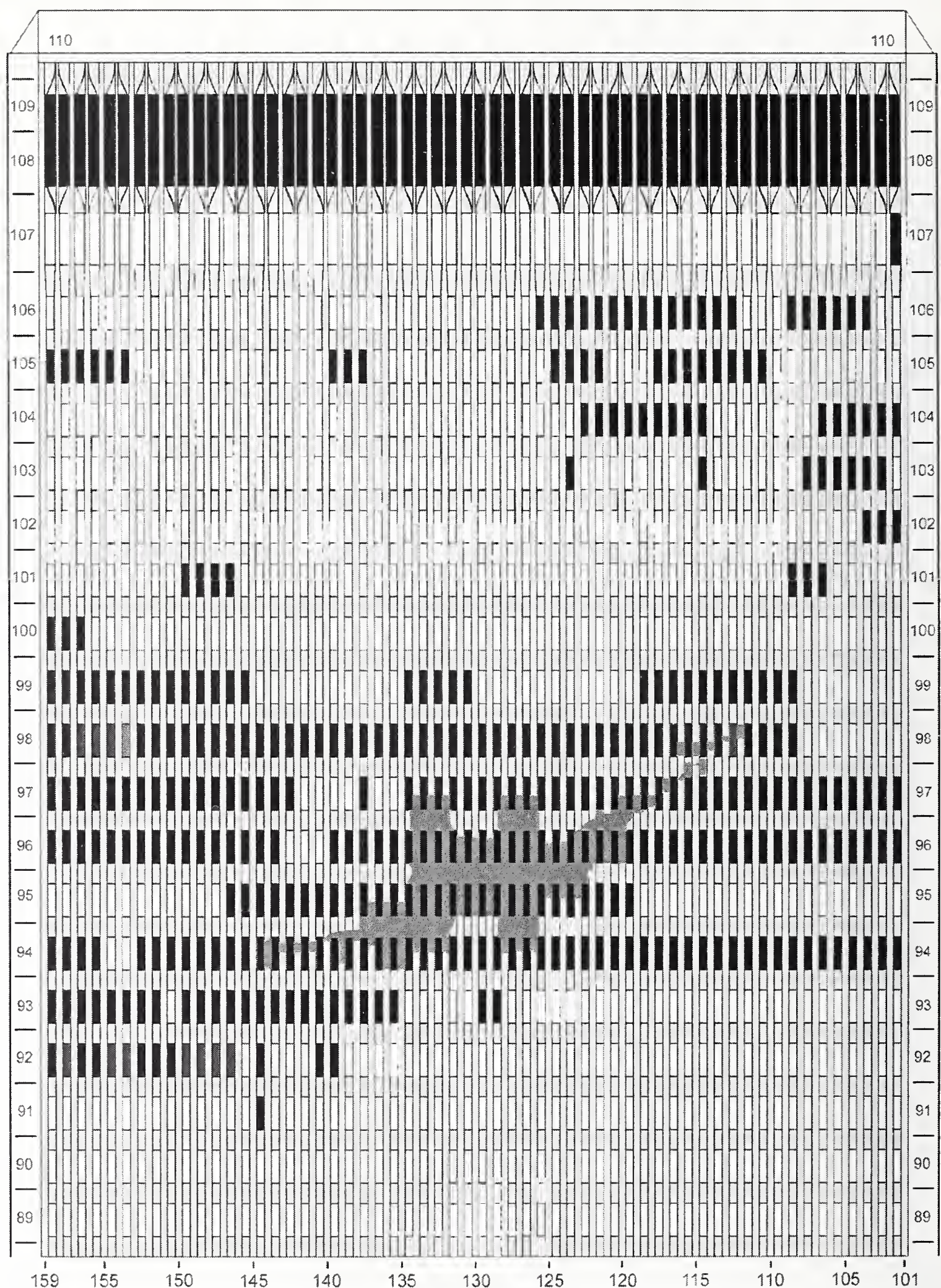

Figure C-45. Diagram of the north face of WTC 1 for floors 89 to 110 at 10:04 a.m. showing the condition of windows and locations of fires. 


\section{WTC 1, North Face $\quad$ 10:04 a.m.}

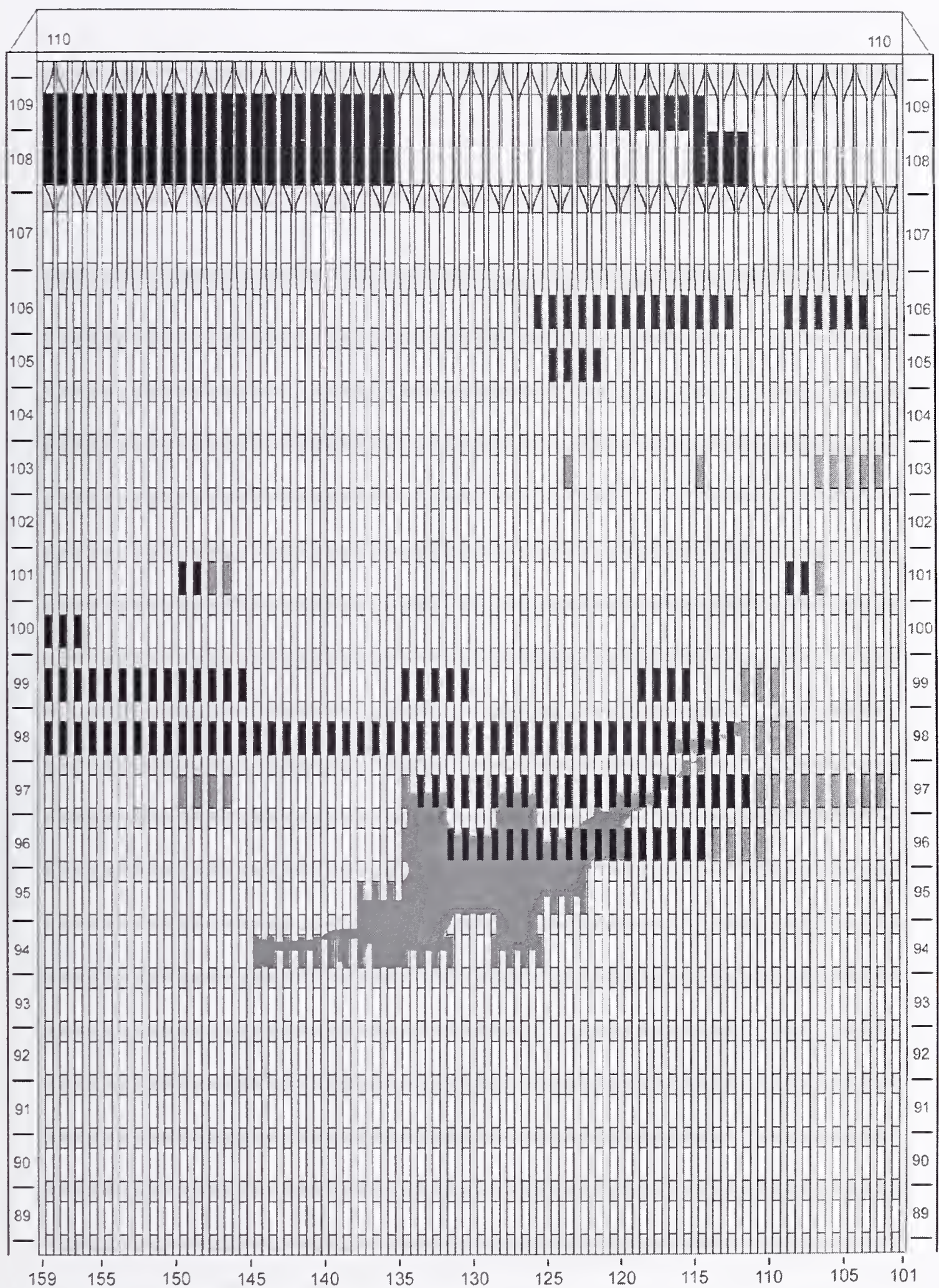

Figure C-46. Diagram of the north face of WTC 1 for floors 89 to 110 at 10:04 a.m. showing windows where smoke was observed and those that were hidden from view. 


$$
\text { WTC 1, North Face 10:10 a.m. }
$$

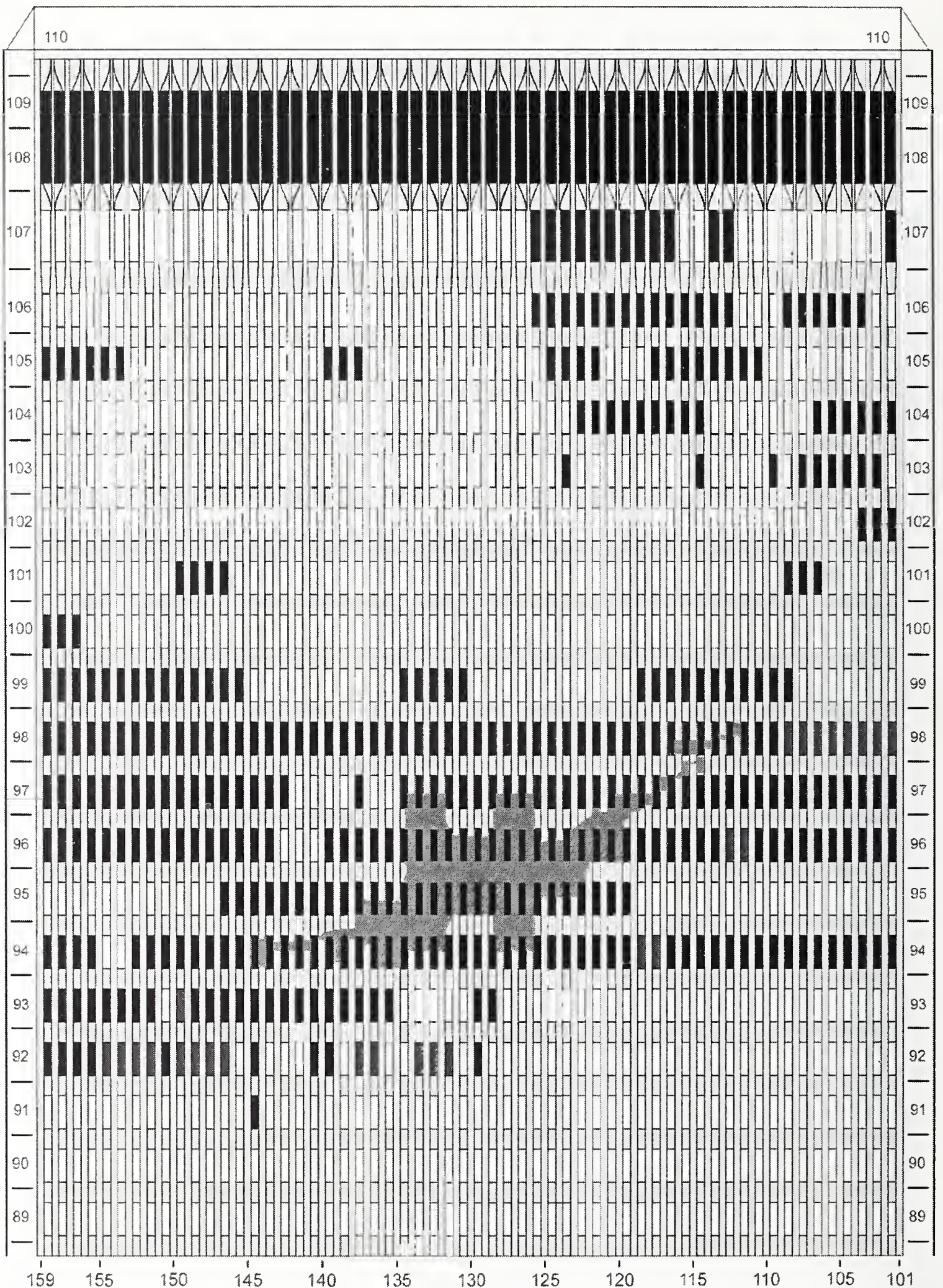

Figure C-47. Diagram of the north face of WTC 1 for floors 89 to 110 at 10:10 a.m. showing the condition of windows and locations of fires. 


\section{WTC 1, North Face $\quad$ 10:10 a.m.}

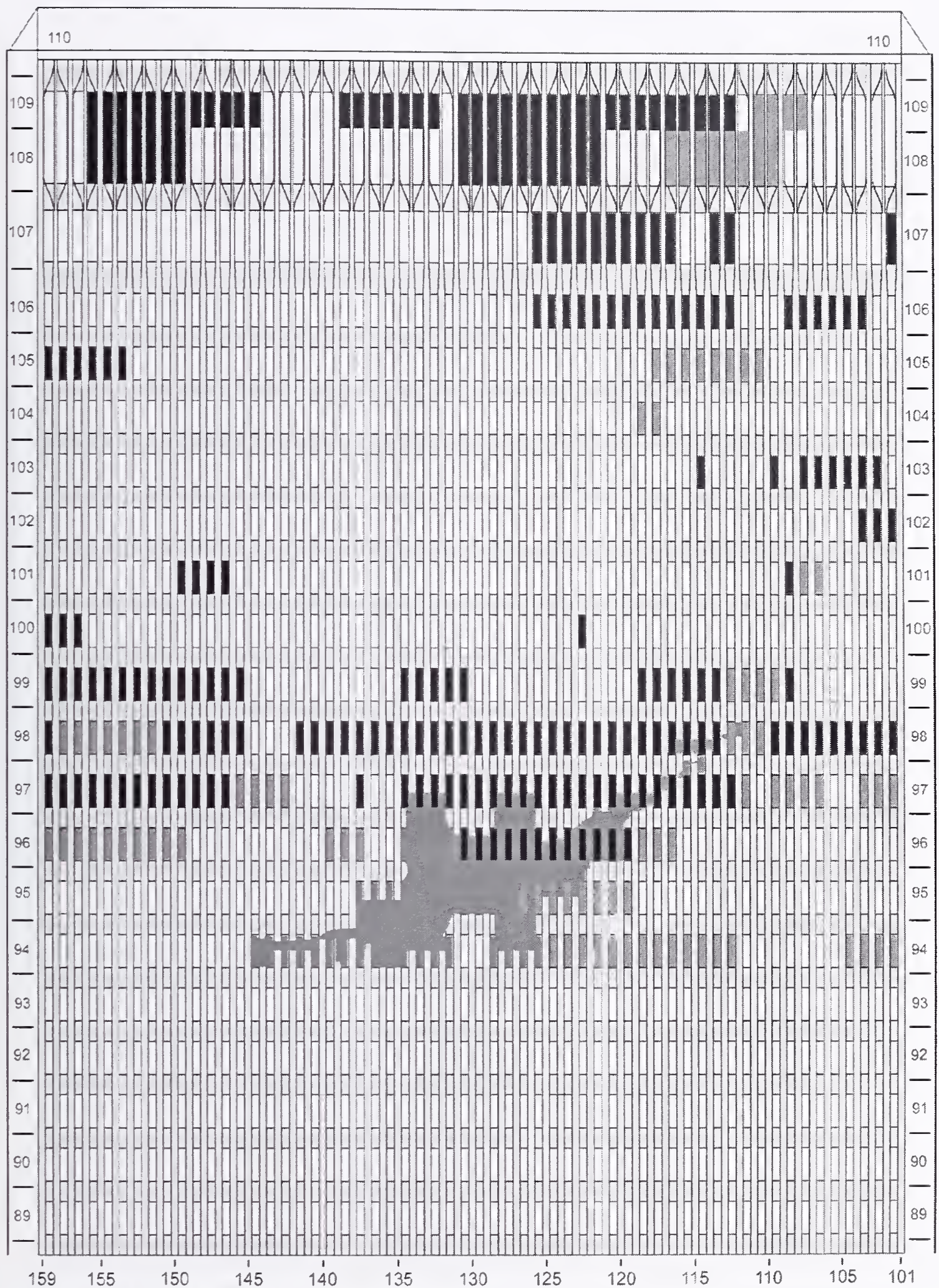

Figure C-48. Diagram of the north face of WTC 1 for floors 89 to 110 at 10:10 a.m. showing windows where smoke was observed and those that were hidden from view. 


$$
\text { WTC 1, North Face } \quad \text { 10:14 a.m. }
$$

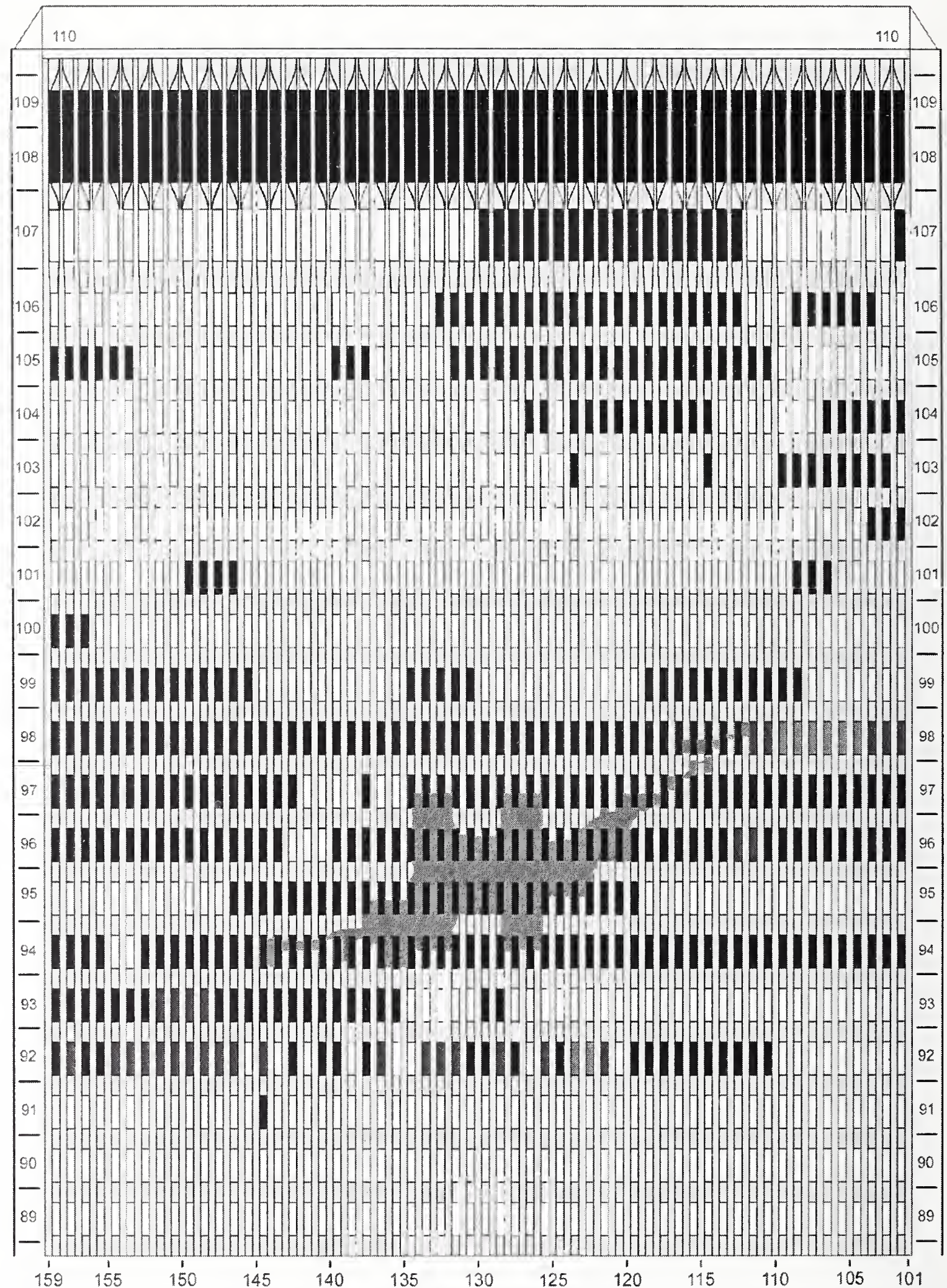

Figure C-49. Diagram of the north face of WTC 1 for floors 89 to 110 at 10:14 a.m. showing the condition of windows and locations of fires. 


$$
\text { WTC 1, North Face 10:14 a.m. }
$$

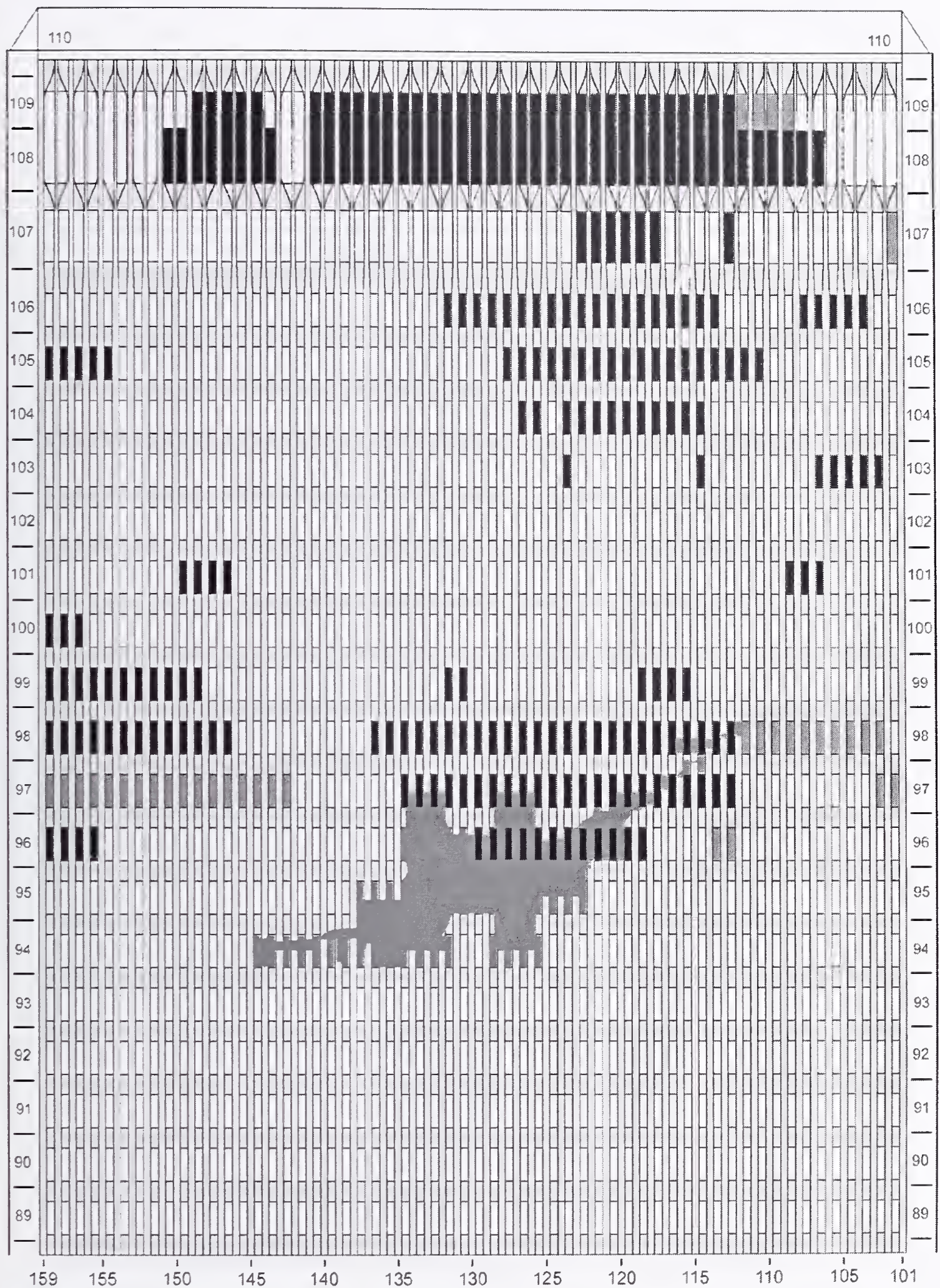

Figure C-50. Diagram of the north face of WTC 1 for floors 89 to 110 at 10:14 a.m. showing windows where smoke was observed and those that were hidden from view. 
WTC 1, North Face 10:18 a.m.

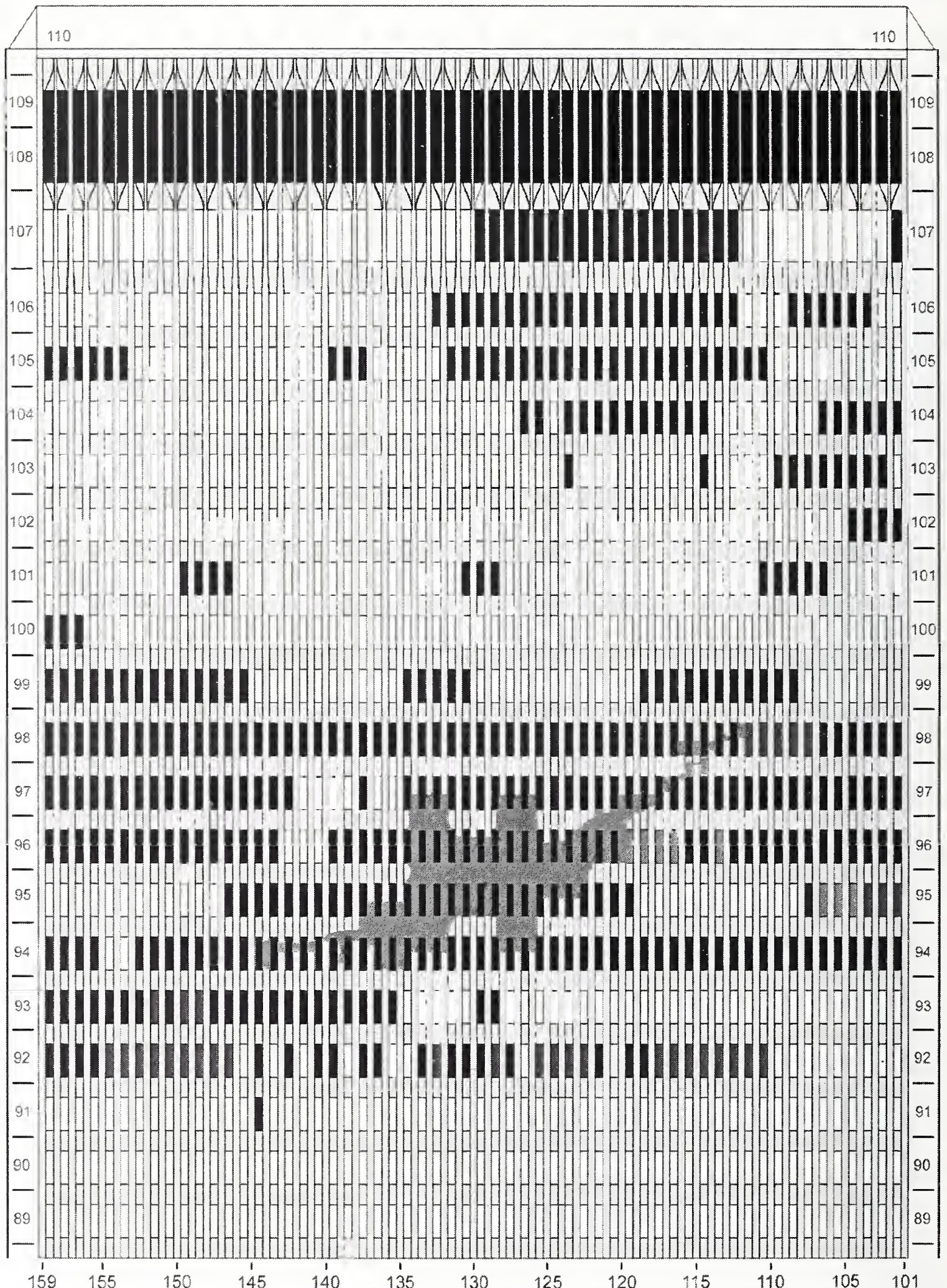

Figure C-51. Diagram of the north face of WTC 1 for floors 89 to 110 at 10:18 a.m. showing the condition of windows and locations of fires. 


$$
\text { WTC 1, North Face } \quad 10: 18 \text { a.m. }
$$

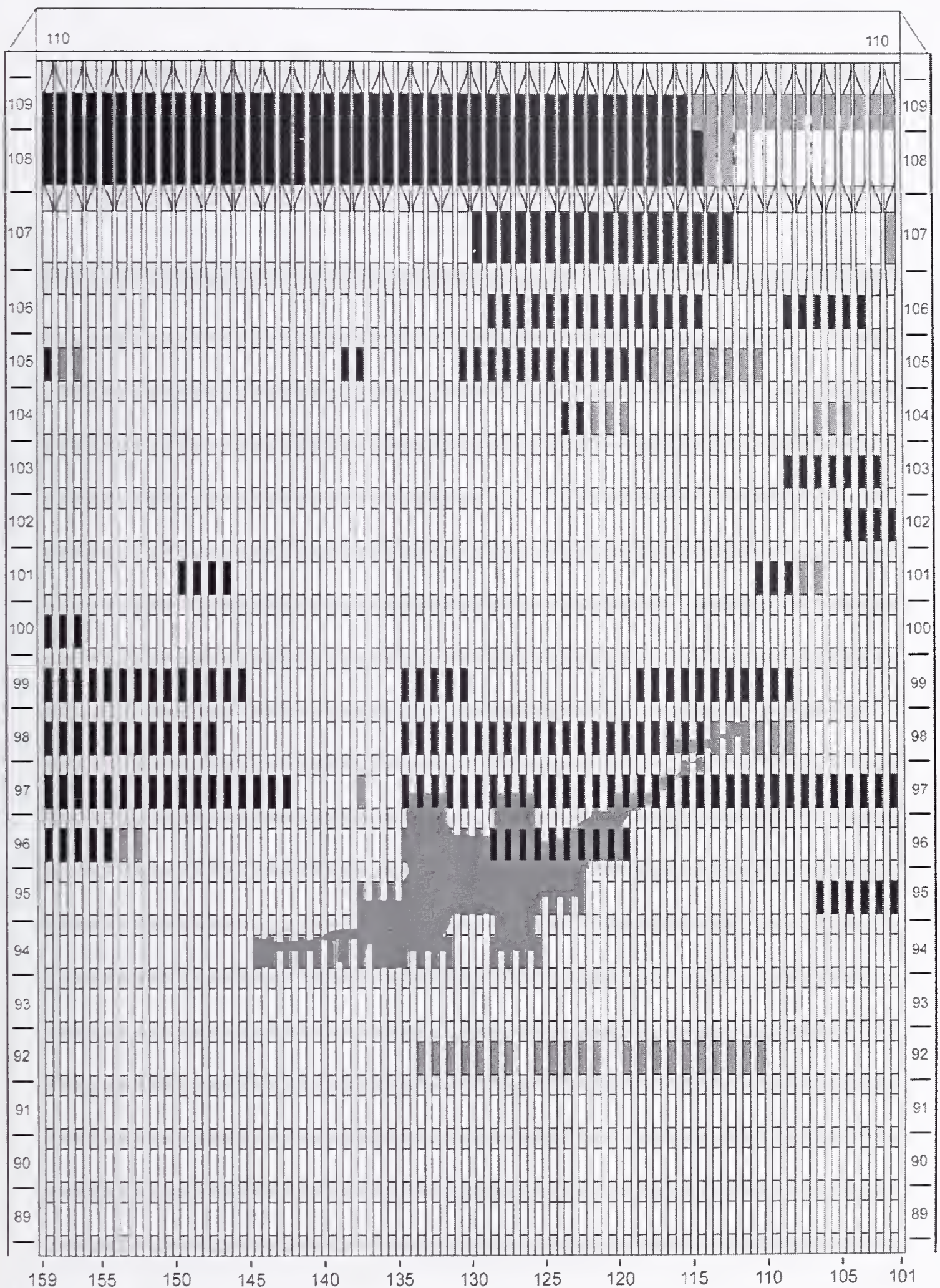

Figure C-52. Diagram of the north face of WTC 1 for floors 89 to 110 at $10: 18$ a.m. showing windows where smoke was observed and those that were hidden from view. 


$$
\text { WTC 1, North Face 10:20 a.m. }
$$

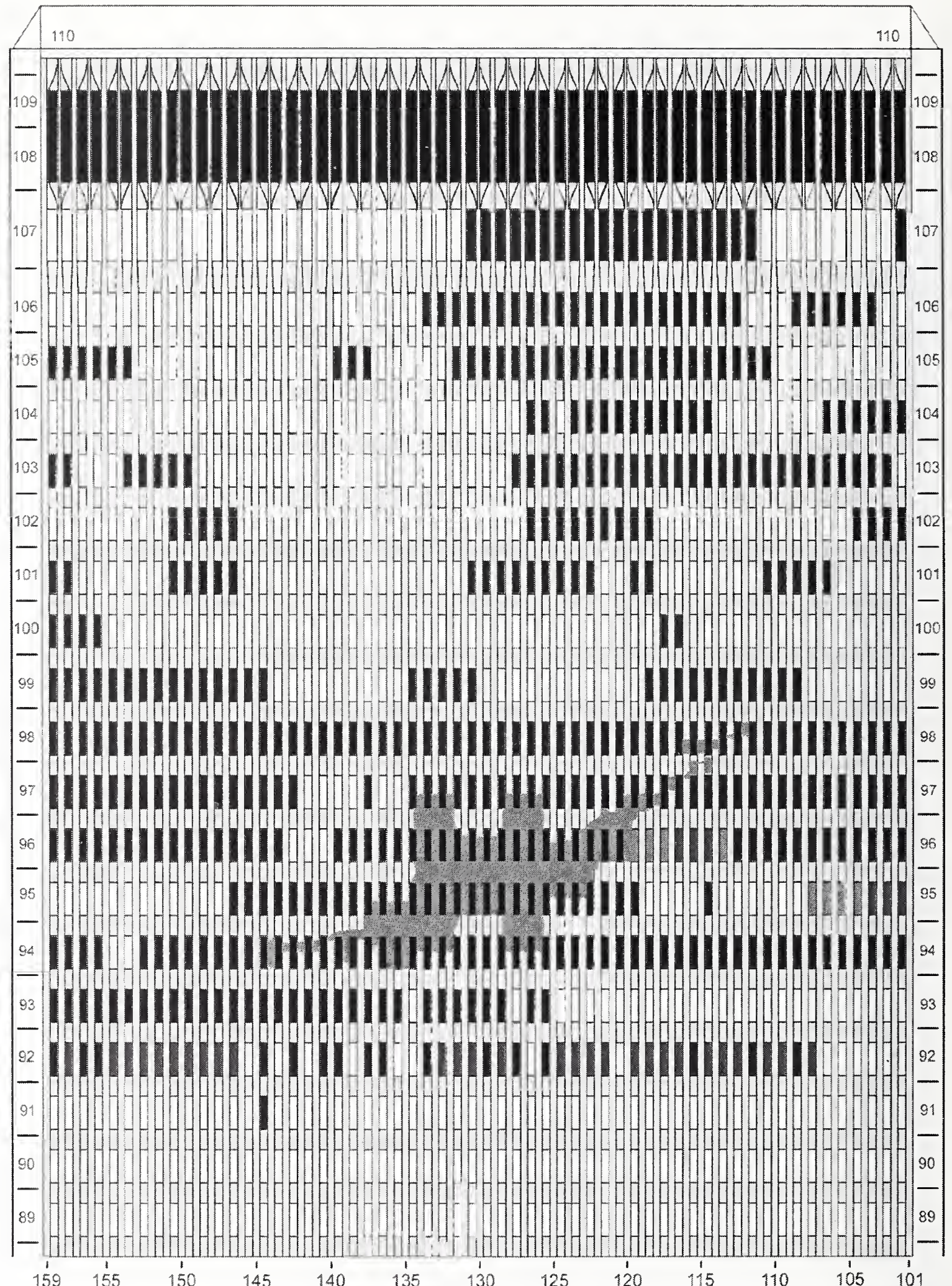

Figure C-53. Diagram of the north face of WTC 1 for floors 89 to 110 at 10:20 a.m. showing the condition of windows and locations of fires. 
WTC 1, North Face $\quad$ 10:20 a.m.

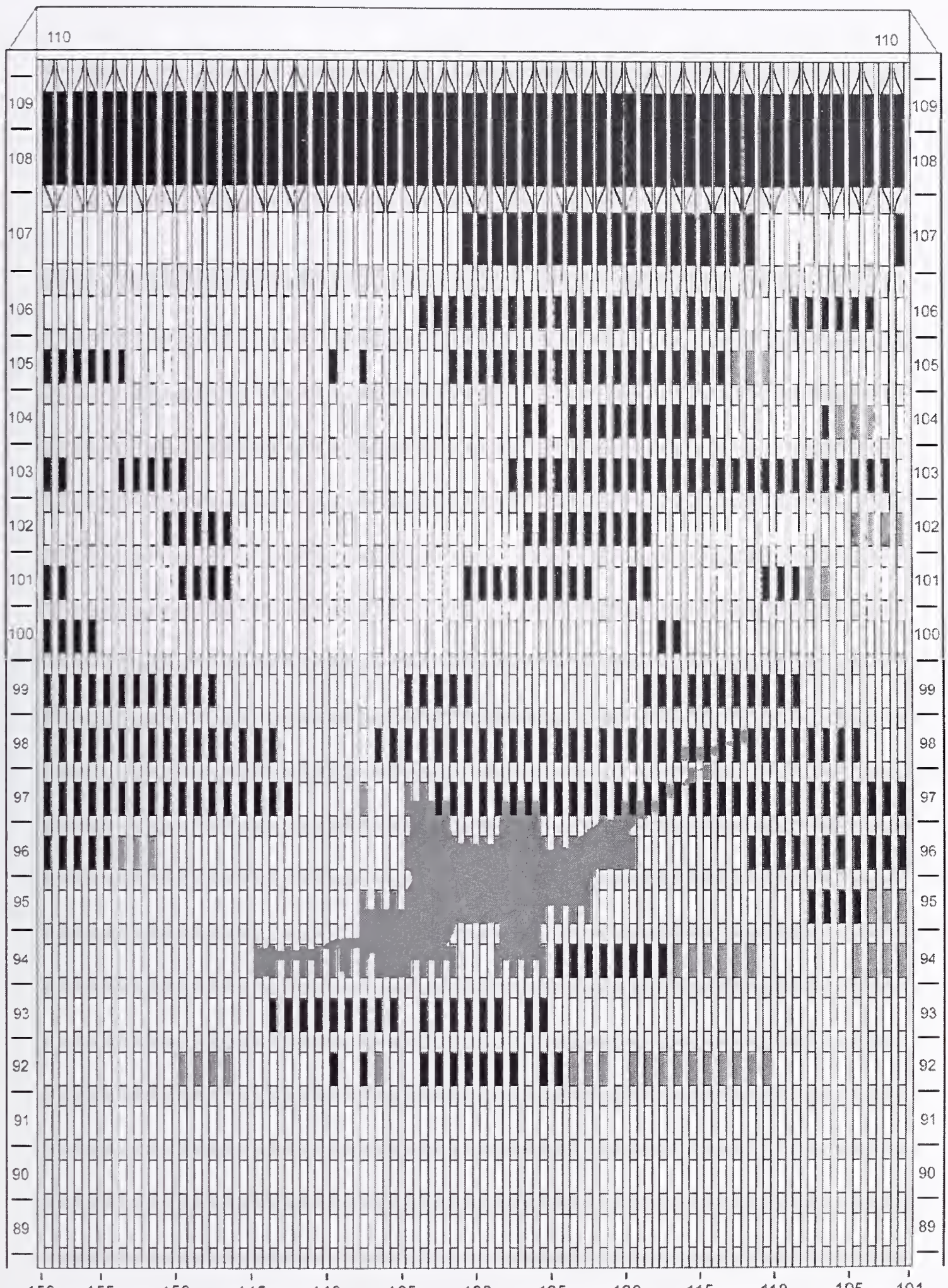

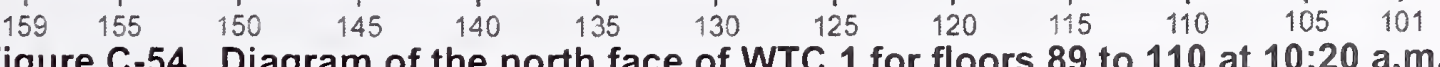
showing windows where smoke was observed and those that were hidden from view. 


$$
\text { WTC 1, North Face 10:24 a.m. }
$$

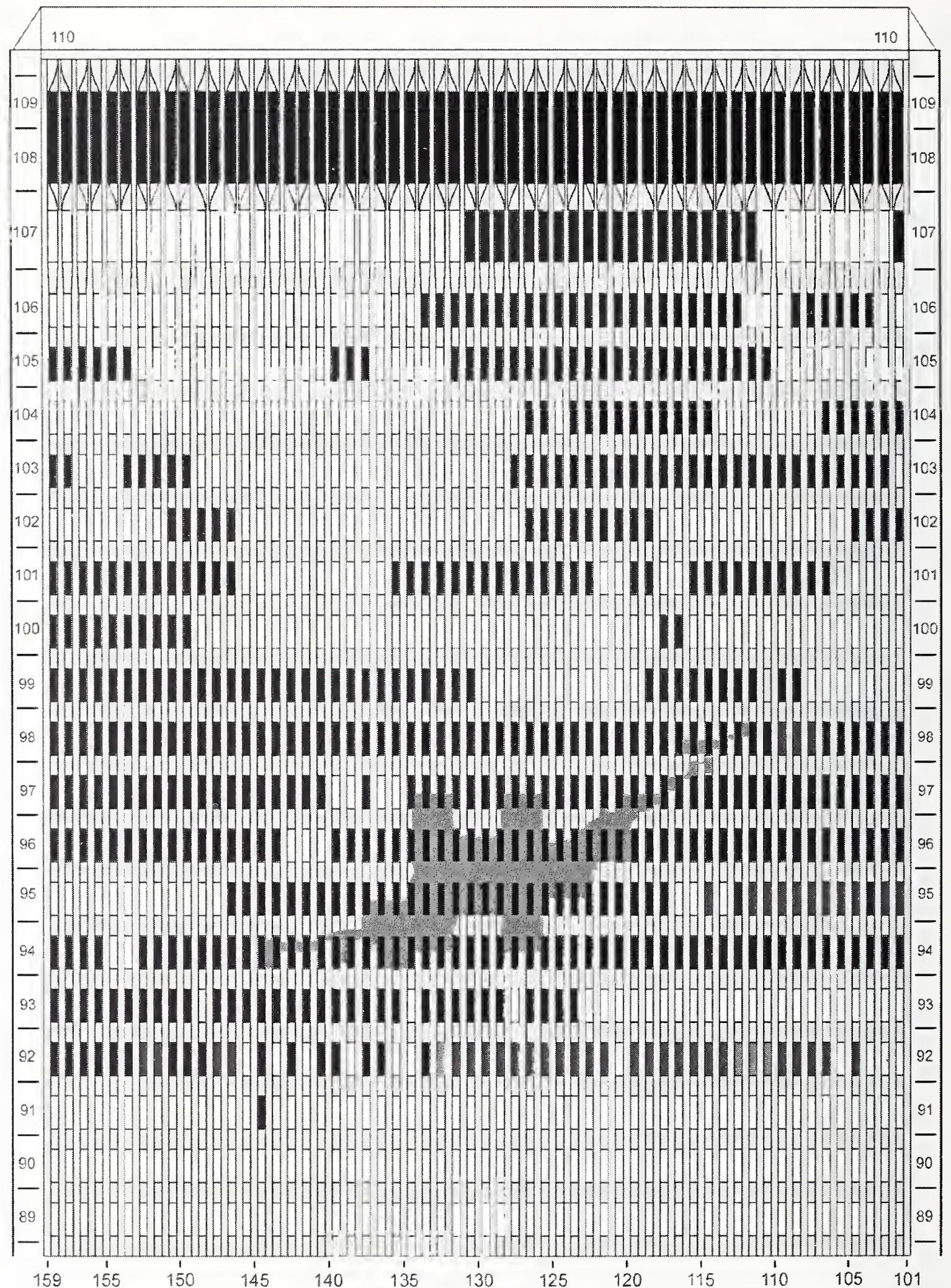

Figure C-55. Diagram of the north face of WTC 1 for floors 89 to 110 at 10:24 a.m. showing the condition of windows and locations of fires. 


$$
\text { WTC 1, North Face } \quad \text { 10:24 a.m. }
$$

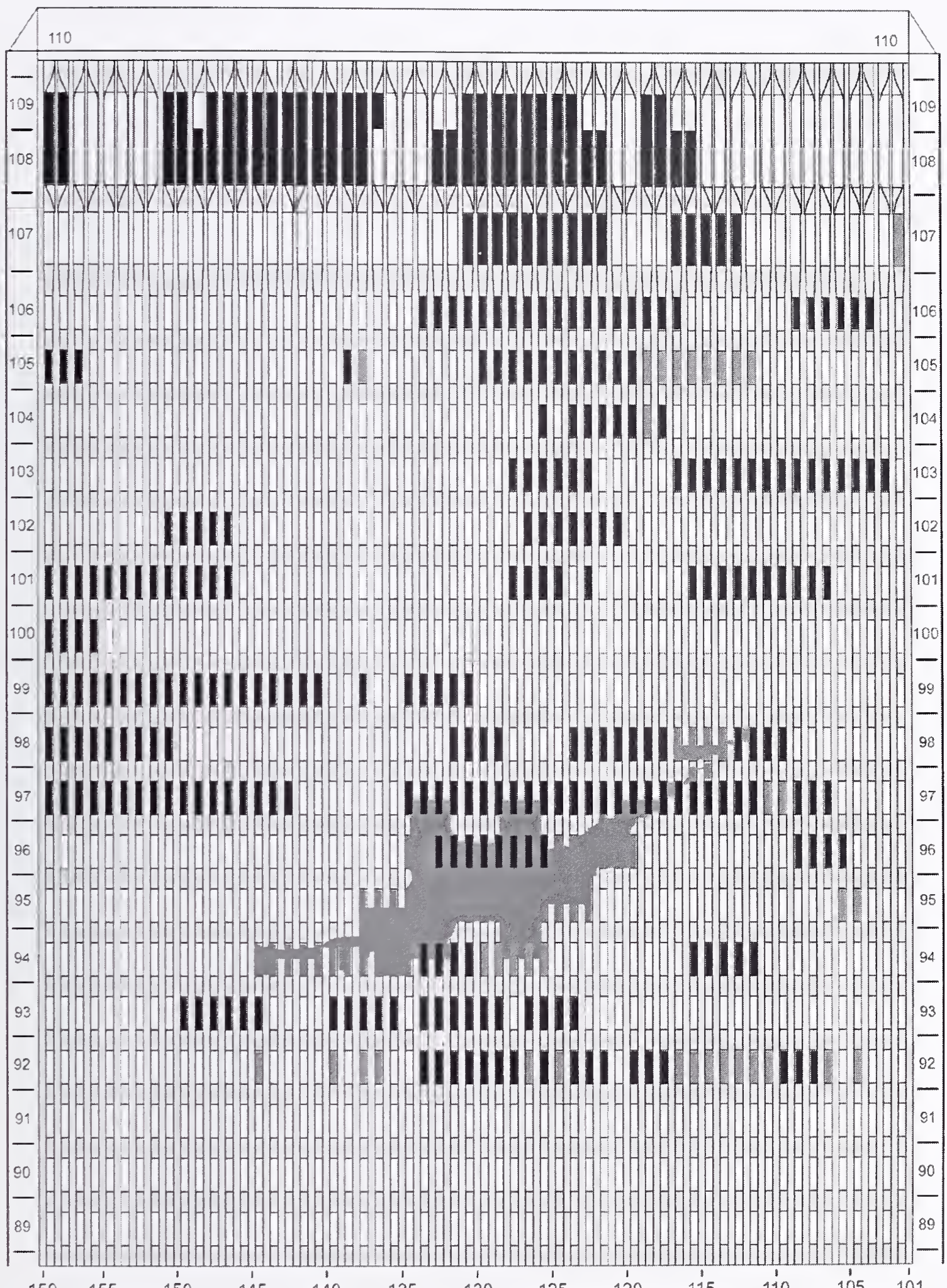

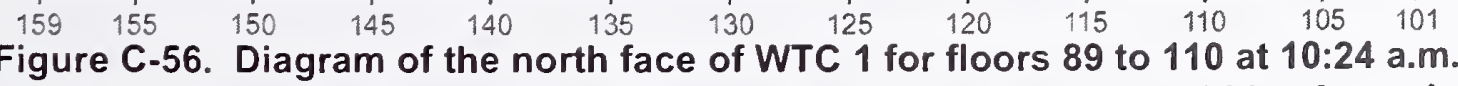
showing windows where smoke was observed and those that were hidden from view. 

WTC 1, North Face
10:28 a.m.

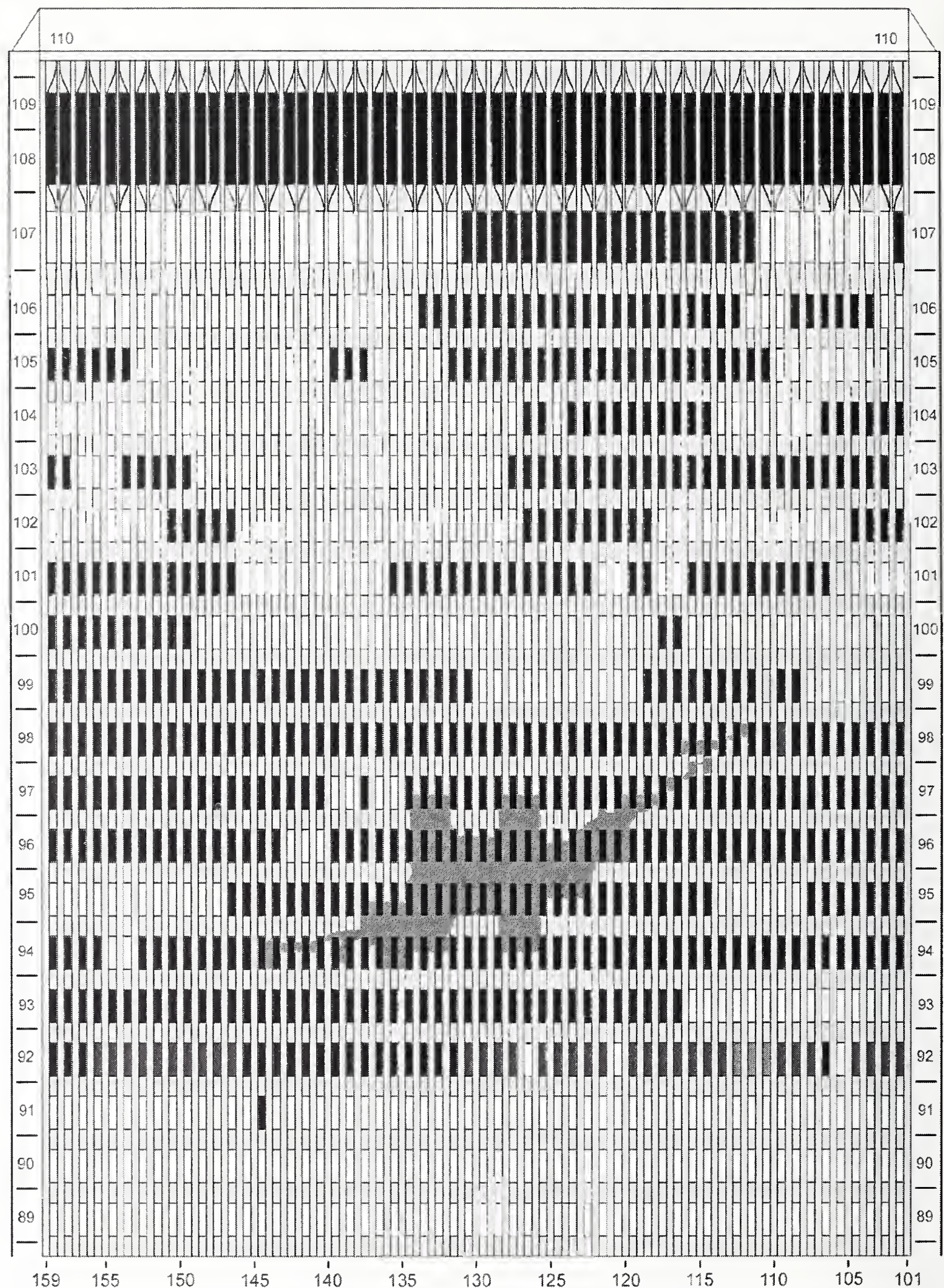

Figure C-57. Diagram of the north face of WTC 1 for floors 89 to 110 at 10:28 a.m. showing the condition of windows and locations of fires. 


$$
\text { WTC 1, North Face 10:28 a.m. }
$$

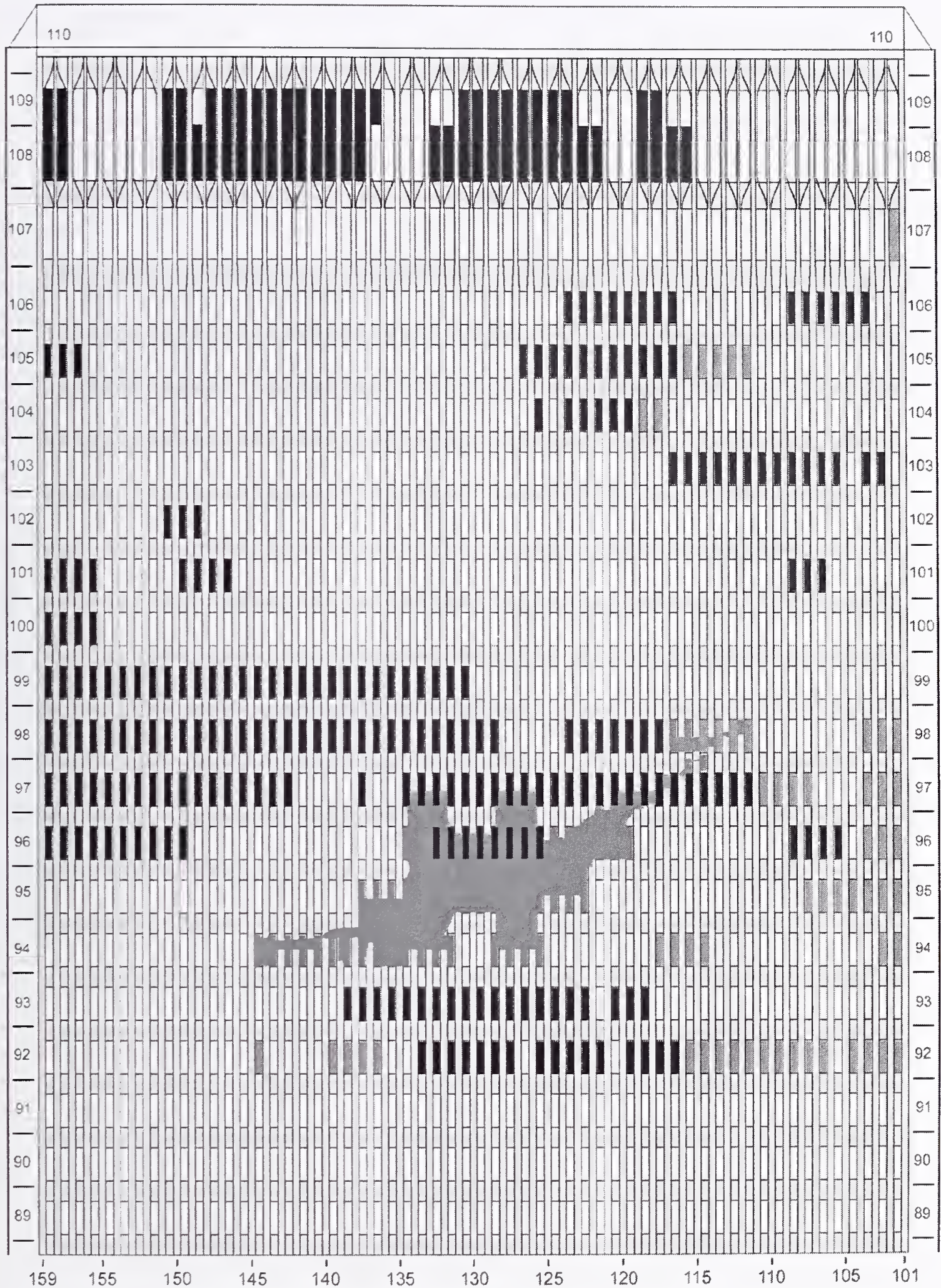

Figure C-58. Diagram of the north face of WTC 1 for floors 89 to 110 at 10:28 a.m. showing windows where smoke was observed and those that were hidden from view. 
This page intentionally left blank. 


\section{Appendix C \\ WTC 1 NORTH FACE COMBINED FIRE AND WINDOW CONDITION AND WINDOW SMOKE CONDITION}

This appendix provides visual representations of the data contained in data sheets describing observations of fire, smoke, and window condition on a window-by-window basis as a function of time for the north face of WTC 1. The data for window condition and fire observations are combined into single representations, with the data for fire taking precedence. Observations are represented using the key provided in Figure 5-1 as follows: - window glass in place, - window open, - spot fire, \- fire visible inside, - external flaming, and - not visible. The data for smoke are shown in separate figures using the key from Figure 5-1 as follows: _ no smoke visible, - light smoke, - heavy smoke, and I- not visible. For a given time, the window condition and fire data are shown first. 
WTC 1, North Face $\quad$ 8:47 a.m.

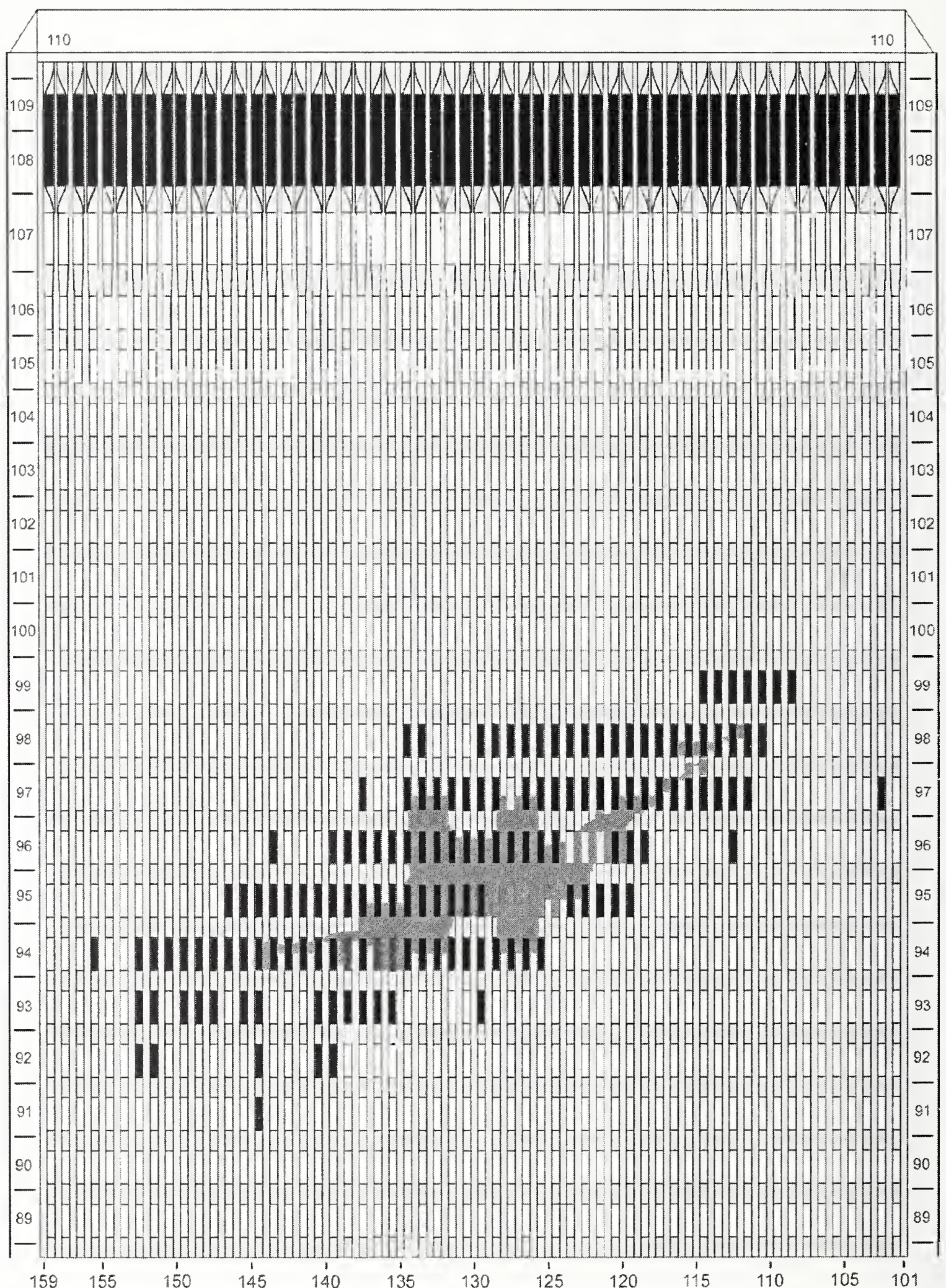

Figure C-1. Diagram of the north face of WTC 1 for floors 89 to 110 at $8: 47$ a.m. showing the condition of windows and locations of fires. 


\section{WTC 1, North Face $\quad$ 8:47 a.m.}

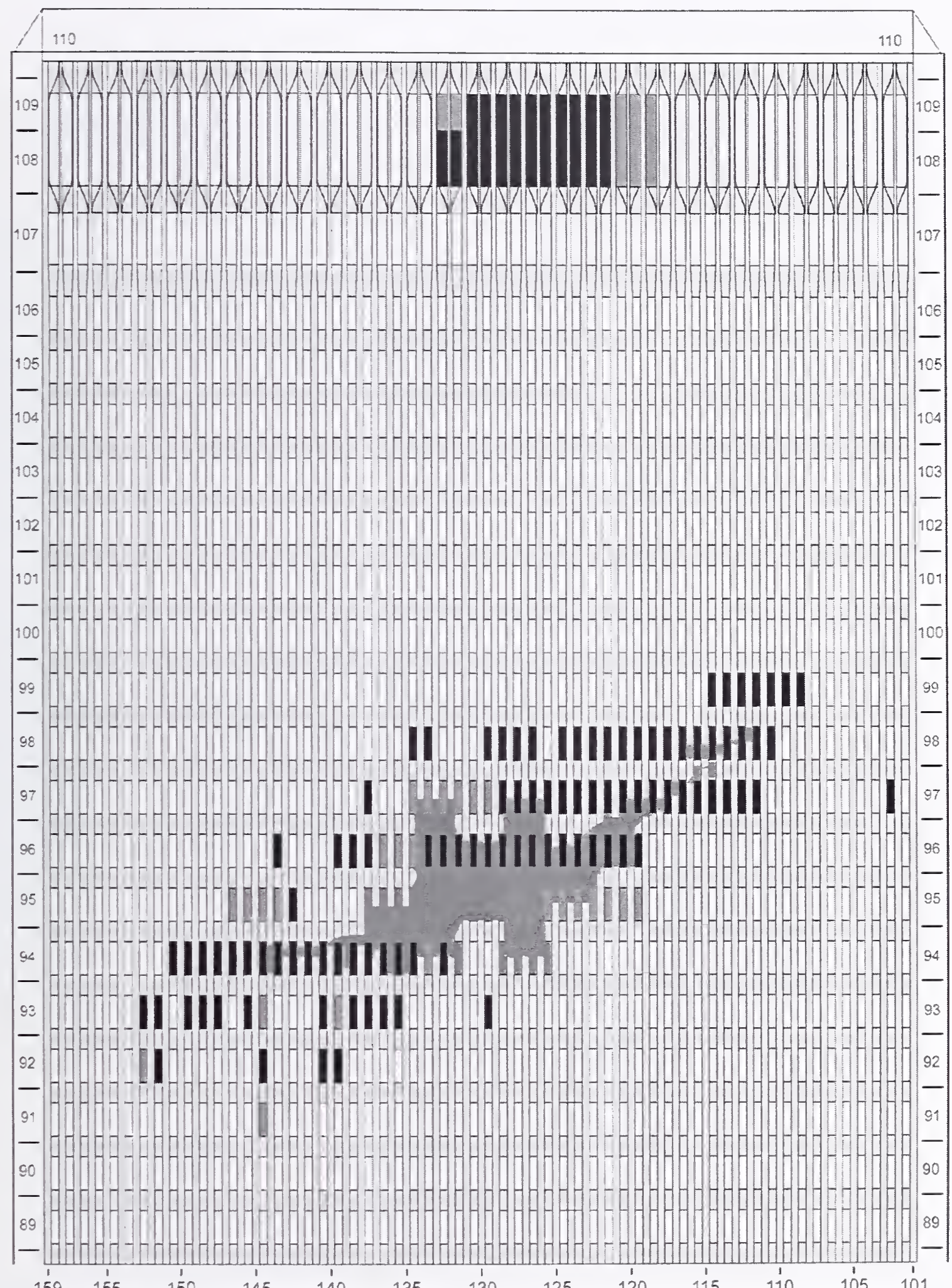

Figure C-2. Diagram of the north face of WTC 1 for floors 89 to 110 at $8: 47$ a.m. showing windows where smoke was observed and those that were hidden from view. 
WTC 1, North Face $\quad$ 8:48 a.m.

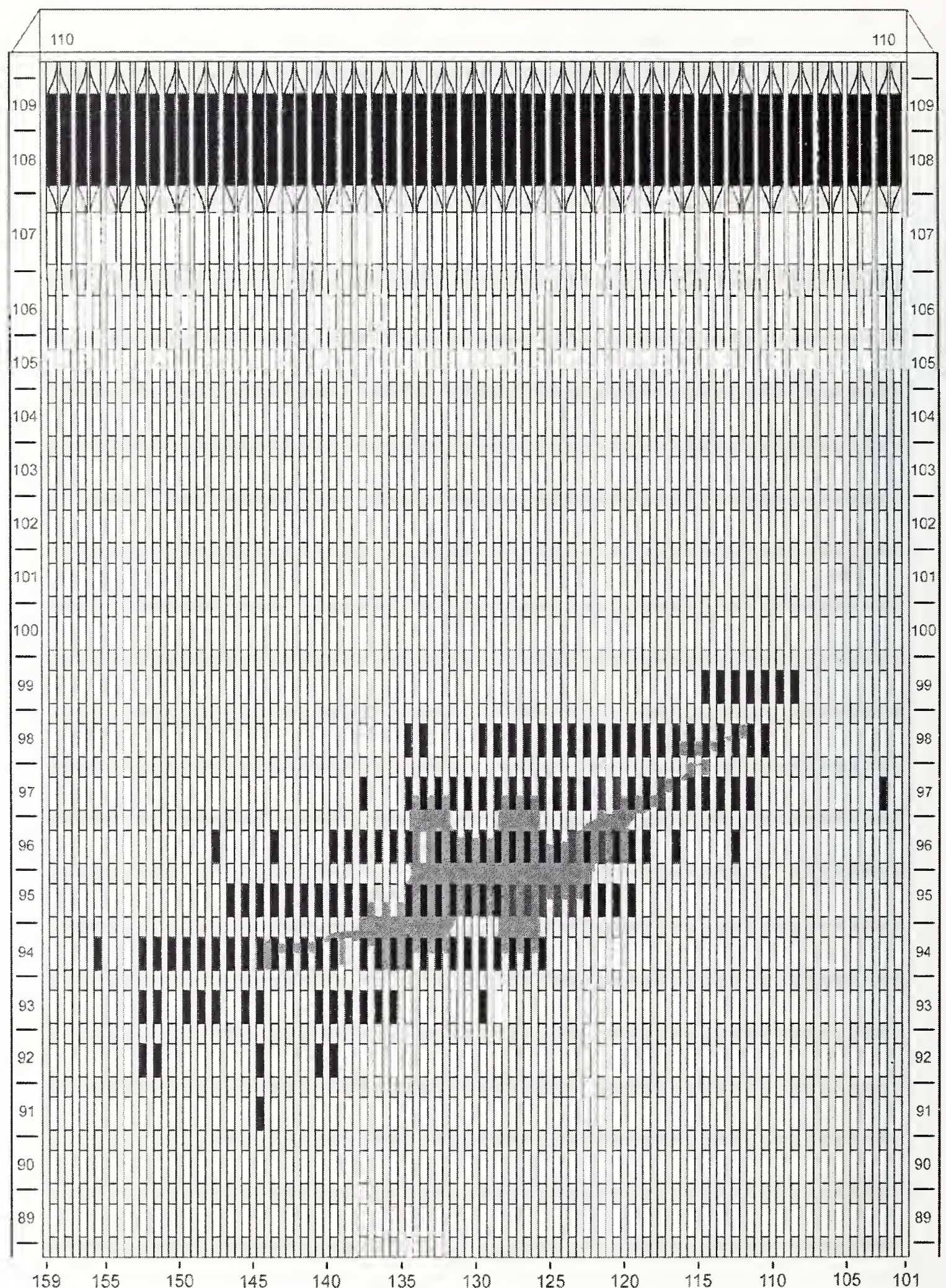

Figure C-3. Diagram of the north face of WTC 1 for floors 89 to 110 at 8:48 a.m. showing the condition of windows and locations of fires. 
WTC 1, North Face $\quad 8: 48$ a.m.

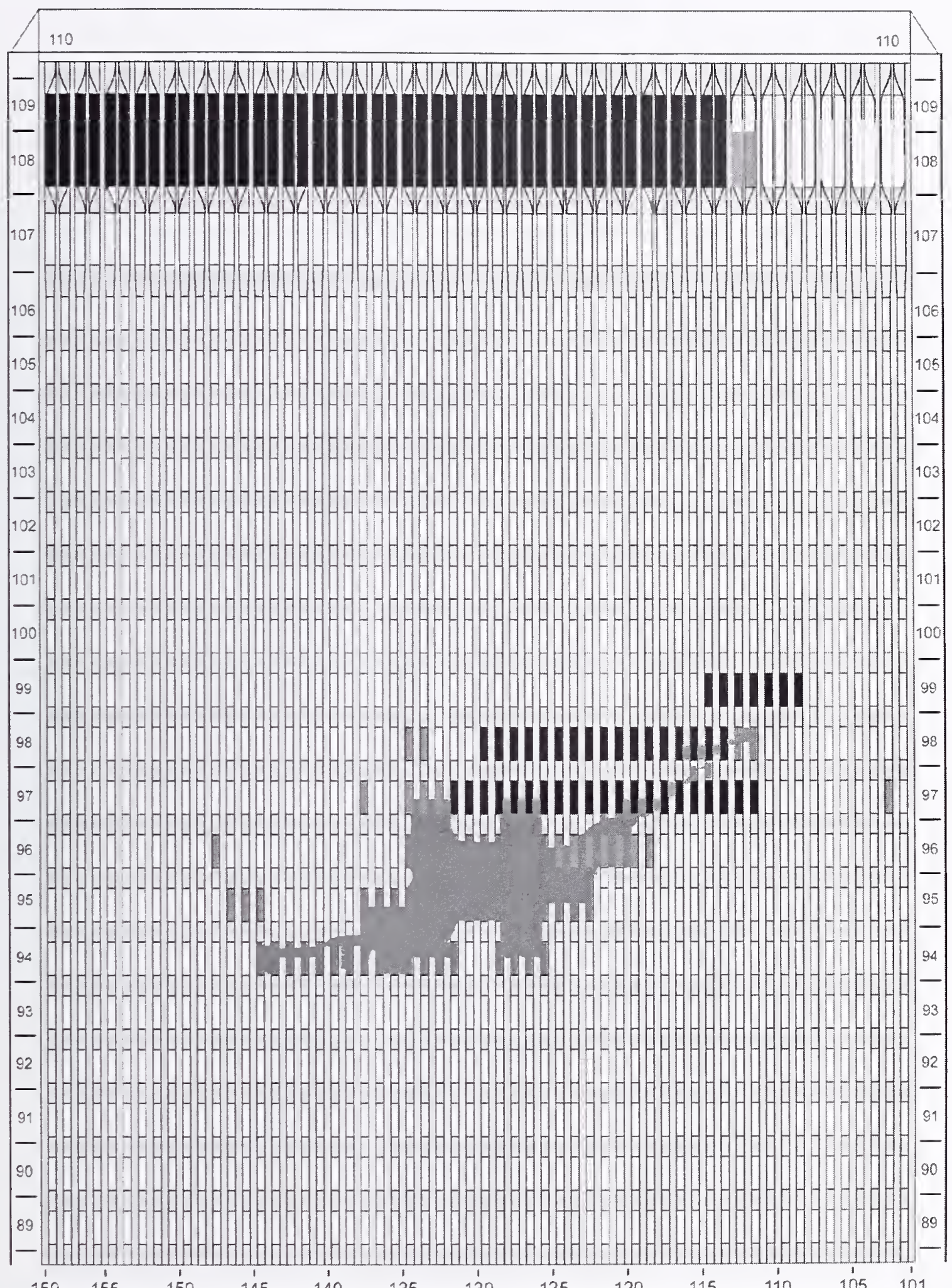

Figure C-4. Diagram of the north face of WTC 1 for floors 89 to 110 at $8: 48$ a.m. showing windows where smoke was observed and those that were hidden from view. 


\section{WTC 1, North Face $\quad$ 8:50 a.m.}

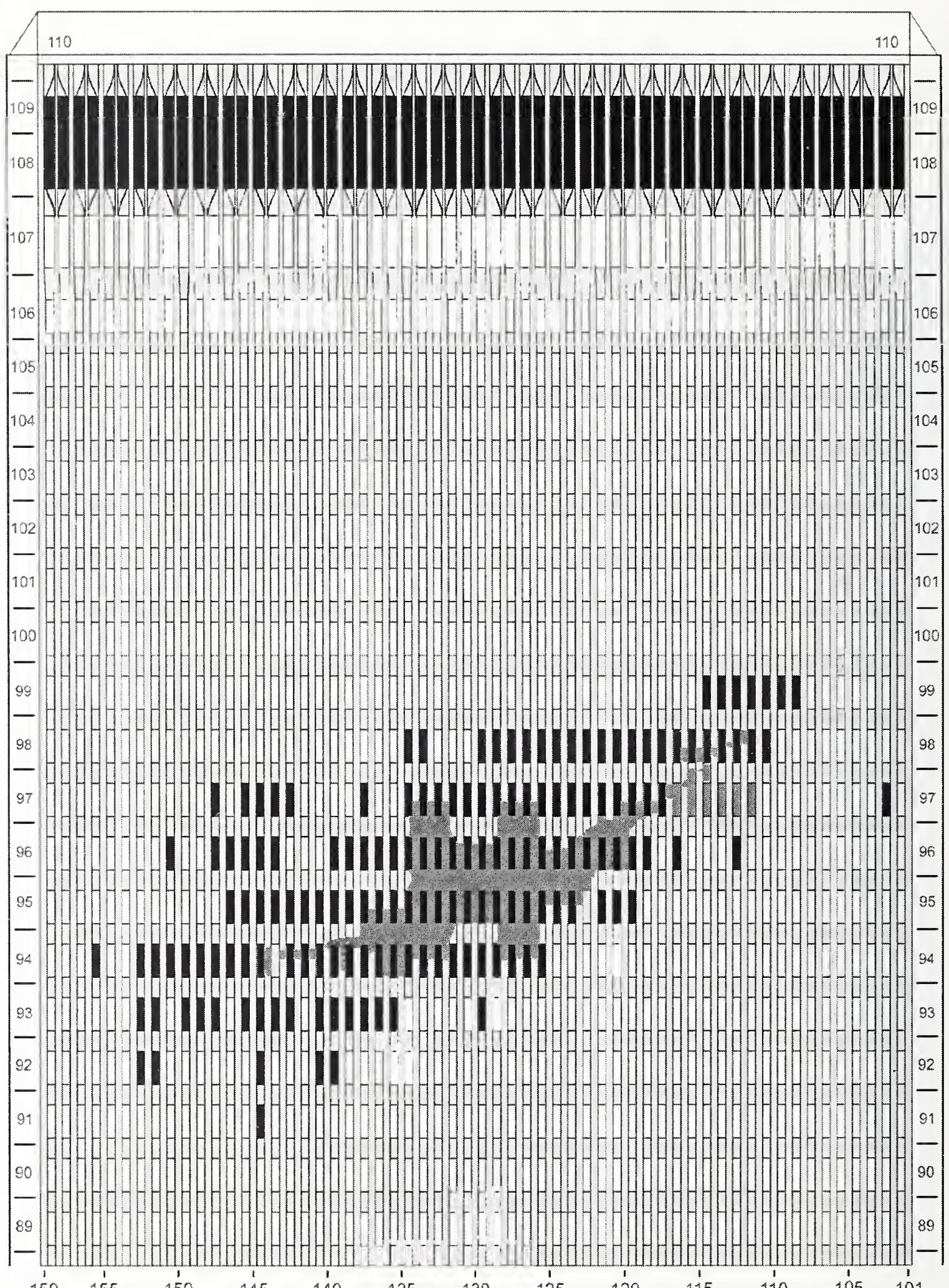

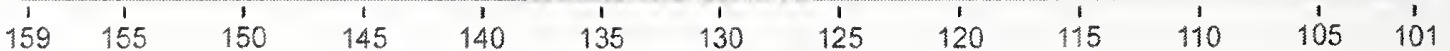

Figure C-5. Diagram of the north face of WTC 1 for floors 89 to 110 at 8:50 a.m. showing the condition of windows and locations of fires. 


\section{WTC 1, North Face $\quad$ 8:50 a.m.}

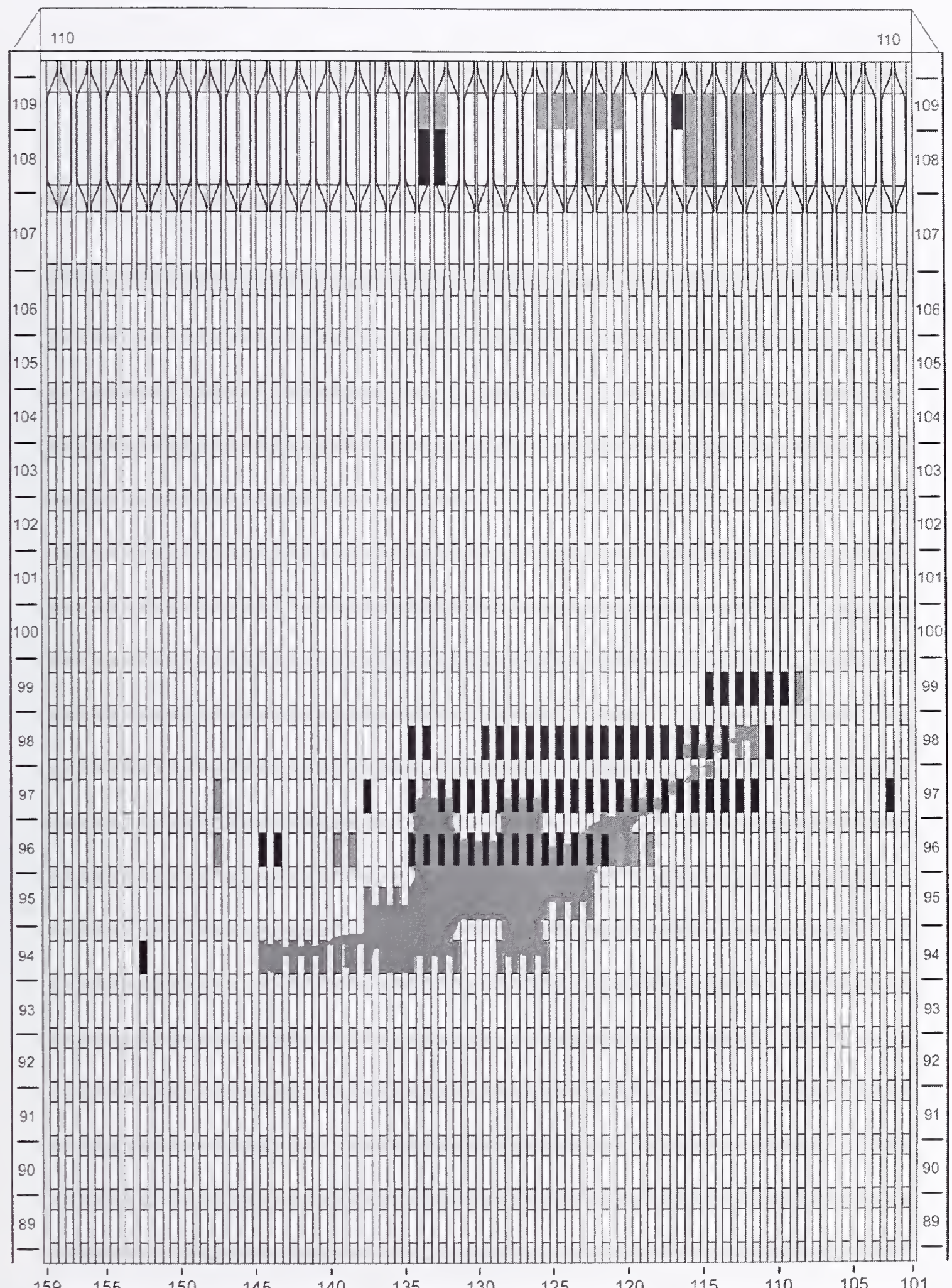

Figure C-6. Diagram of the north face of WTC 1 for floors 89 to 110 at $8: 50$ a.m. showing windows where smoke was observed and those that were hidden from view. 


\section{WTC 1, North Face $\quad$ 8:52 a.m.}

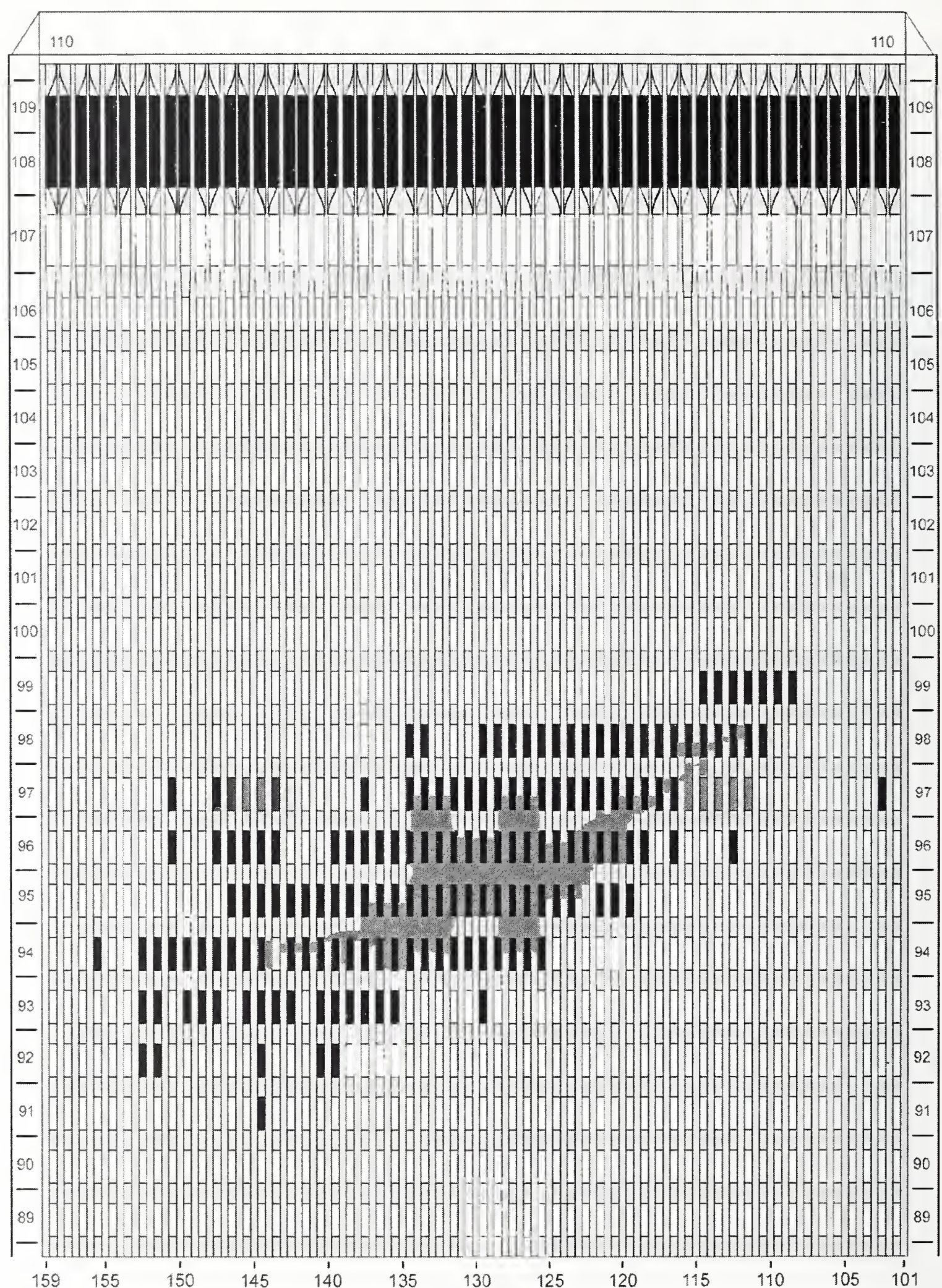

Figure C-7. Diagram of the north face of WTC 1 for floors 89 to 110 at 8:52 a.m. showing the condition of windows and locations of fires. 
WTC 1, North Face $\quad$ 8:52 a.m.

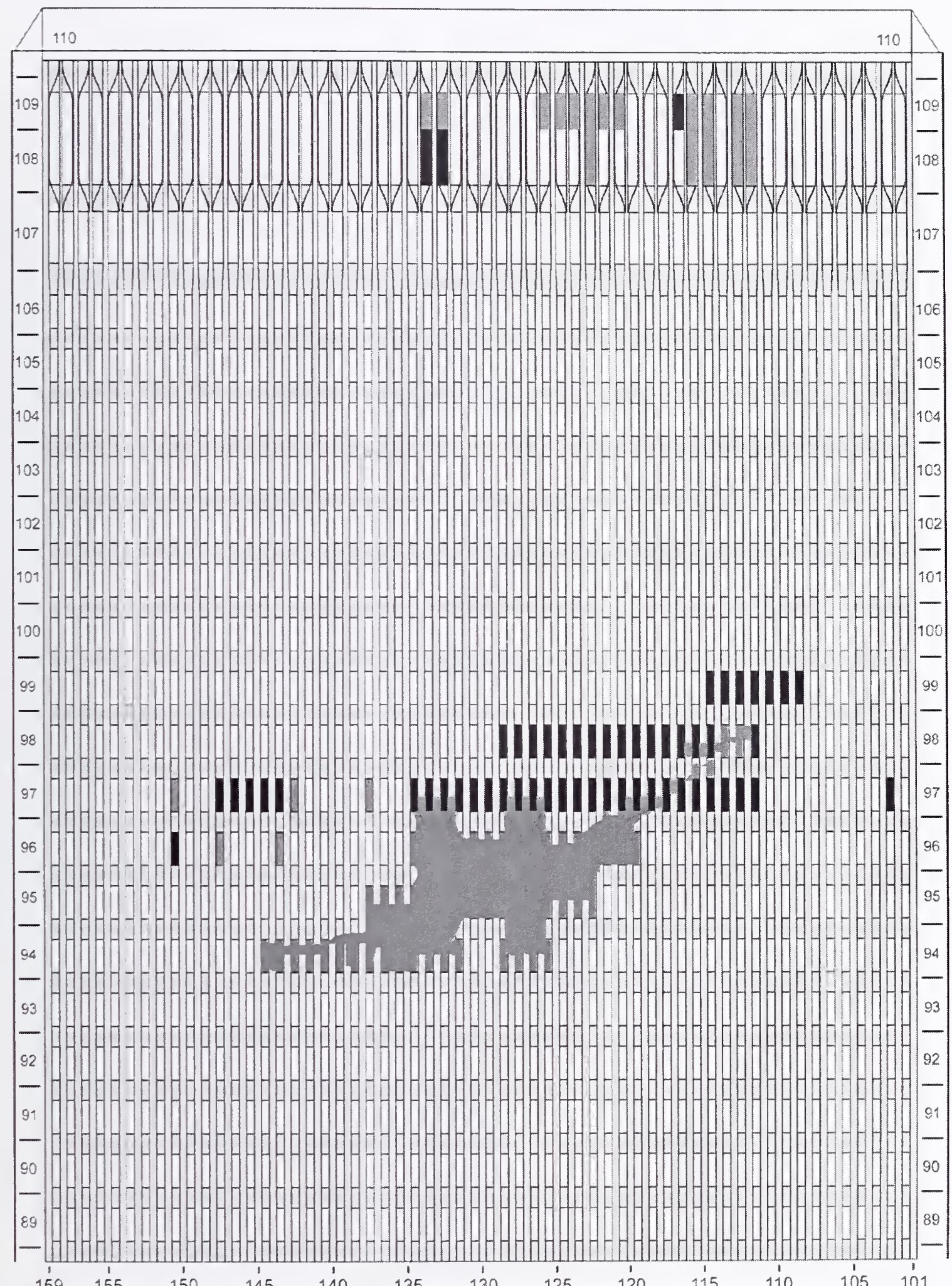

Figure C-8. Diagram of the north face of WTC 1 for floors 89 to 110 at 8:52 a.m. showing windows where smoke was observed and those that were hidden from view. 


\section{WTC 1, North Face 8:54 a.m.}

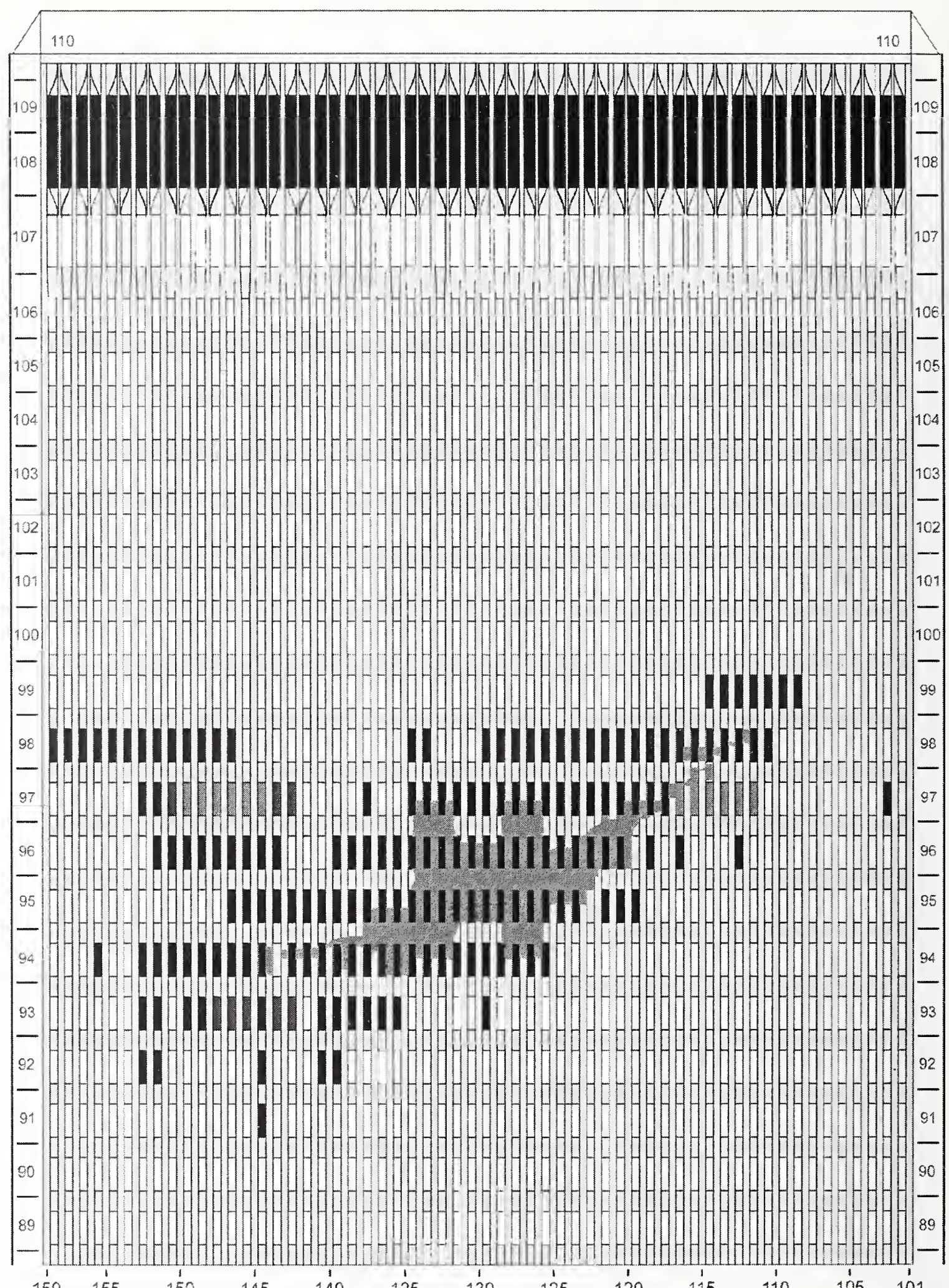

Figure C-9. Diagram of the north face of WTC 1 for floors 89 to 110 at 8:54 a.m. showing the condition of windows and locations of fires. 


\section{WTC 1, North Face $\quad$ 8:54 a.m.}

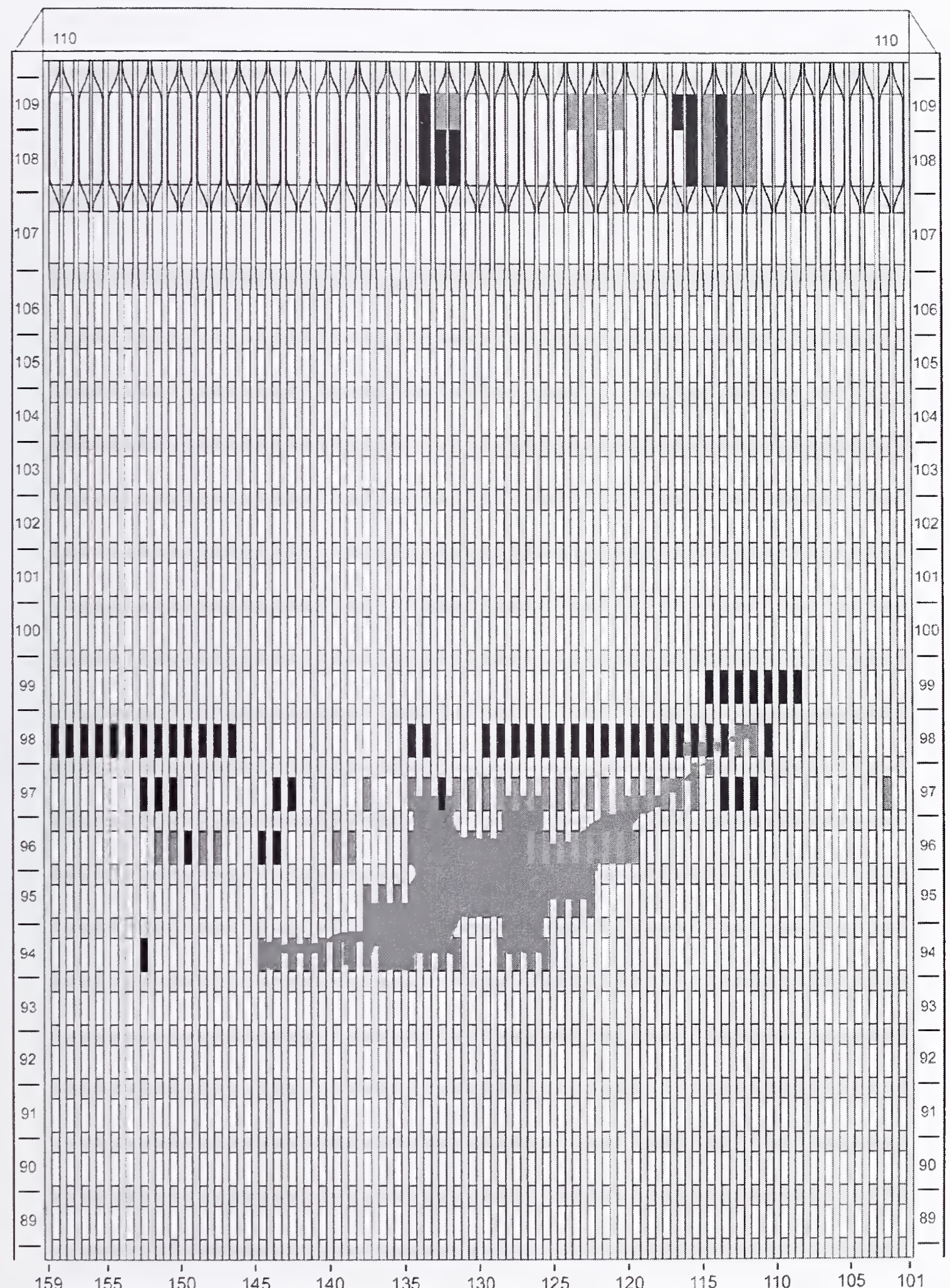

Figure C-10. Diagram of the north face of WTC 1 for floors 89 to 110 at $8: 54$ a.m. showing windows where smoke was observed and those that were hidden from view. 


\section{WTC 1, North Face $\quad 8: 56$ a.m.}

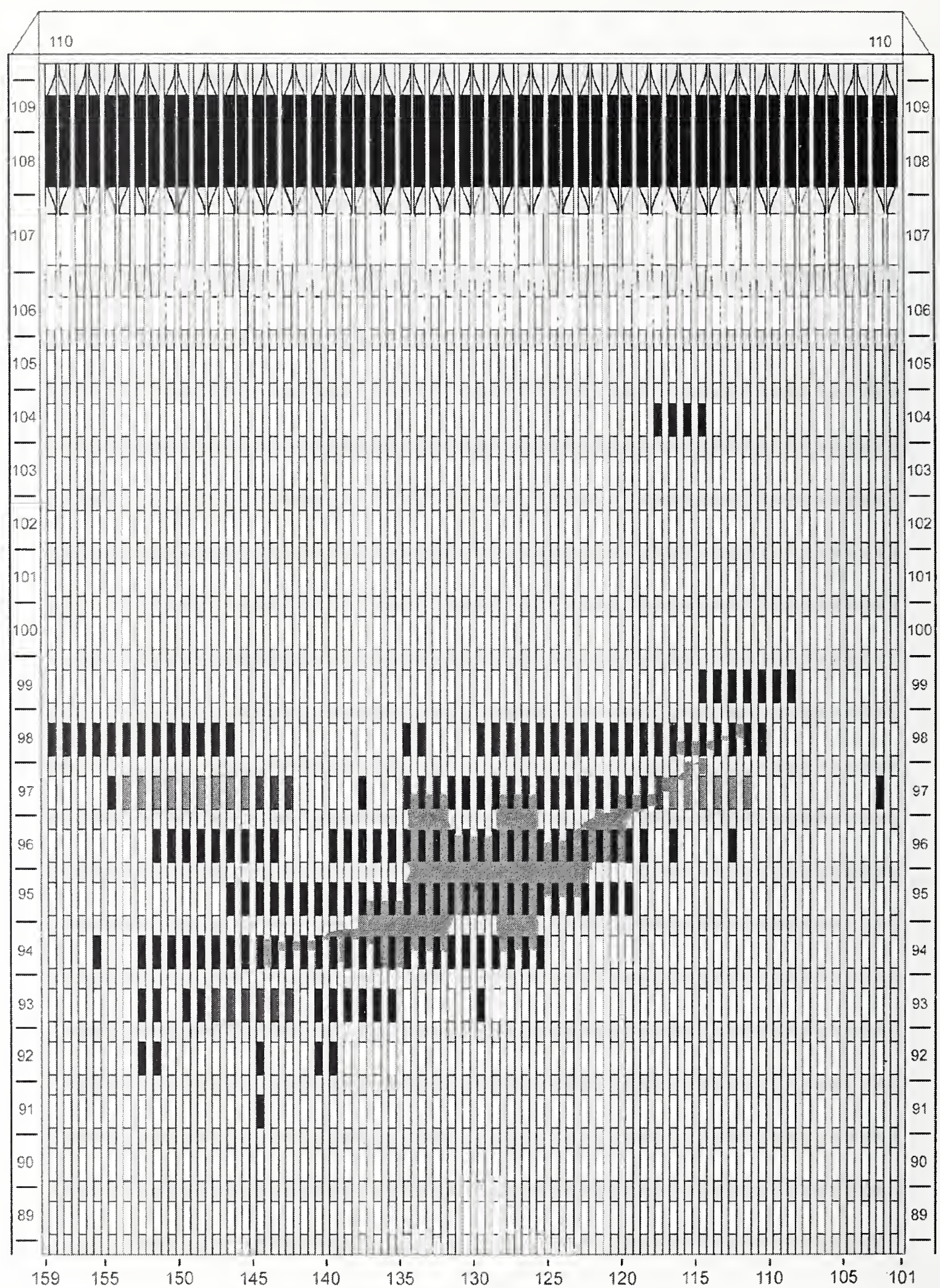

Figure C-11. Diagram of the north face of WTC 1 for floors 89 to 110 at $8: 56$ a.m. showing the condition of windows and locations of lines. 


\section{WTC 1, North Face $\quad$ 8:56 a.m.}

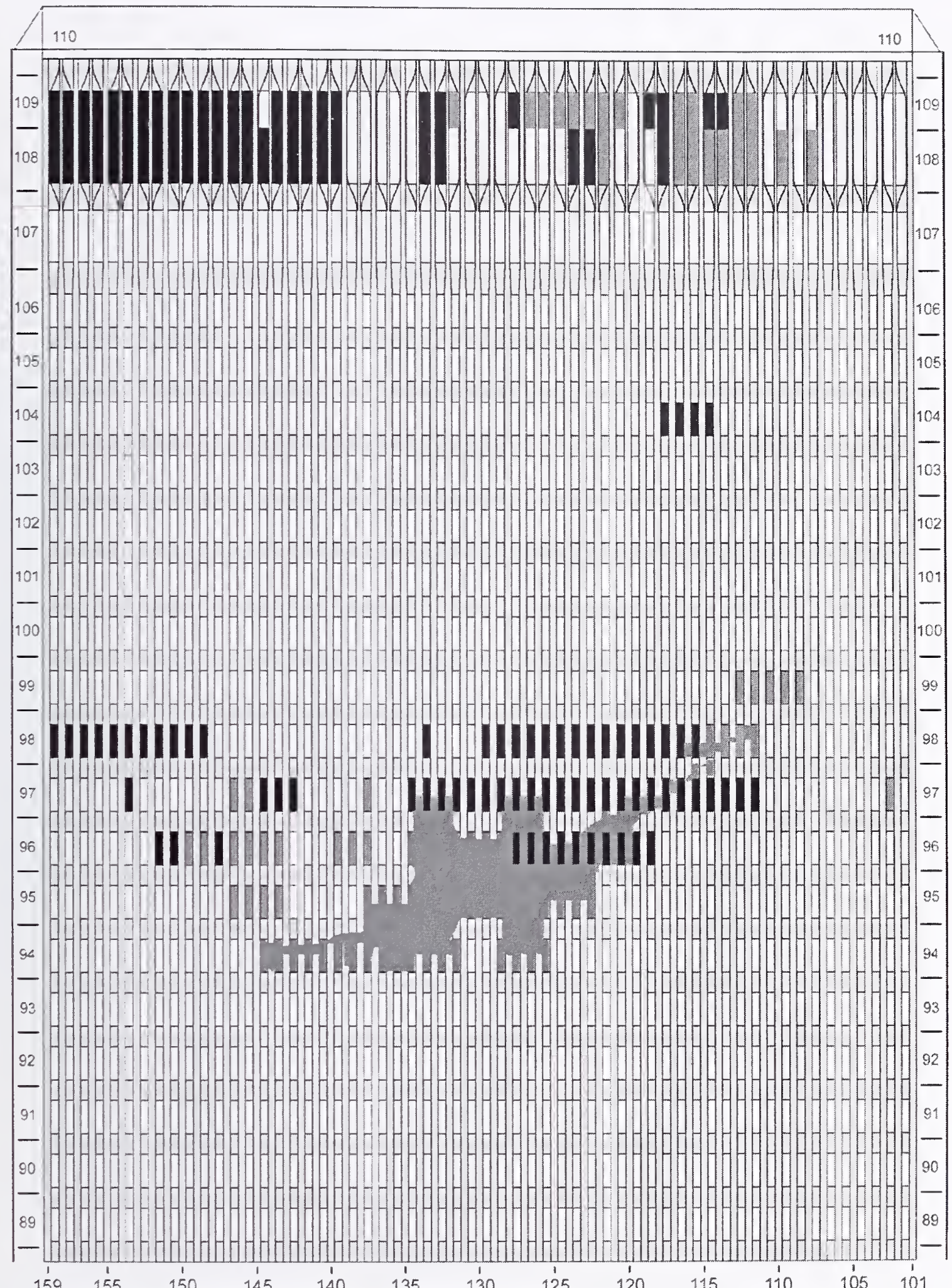

Figure C-12. Diagram of the north face of WTC 1 for floors 89 to 110 at $8: 56$ a.m. showing windows where smoke was observed and those that were hidden from view. 

WTC 1, North Face
8:58 a.m.

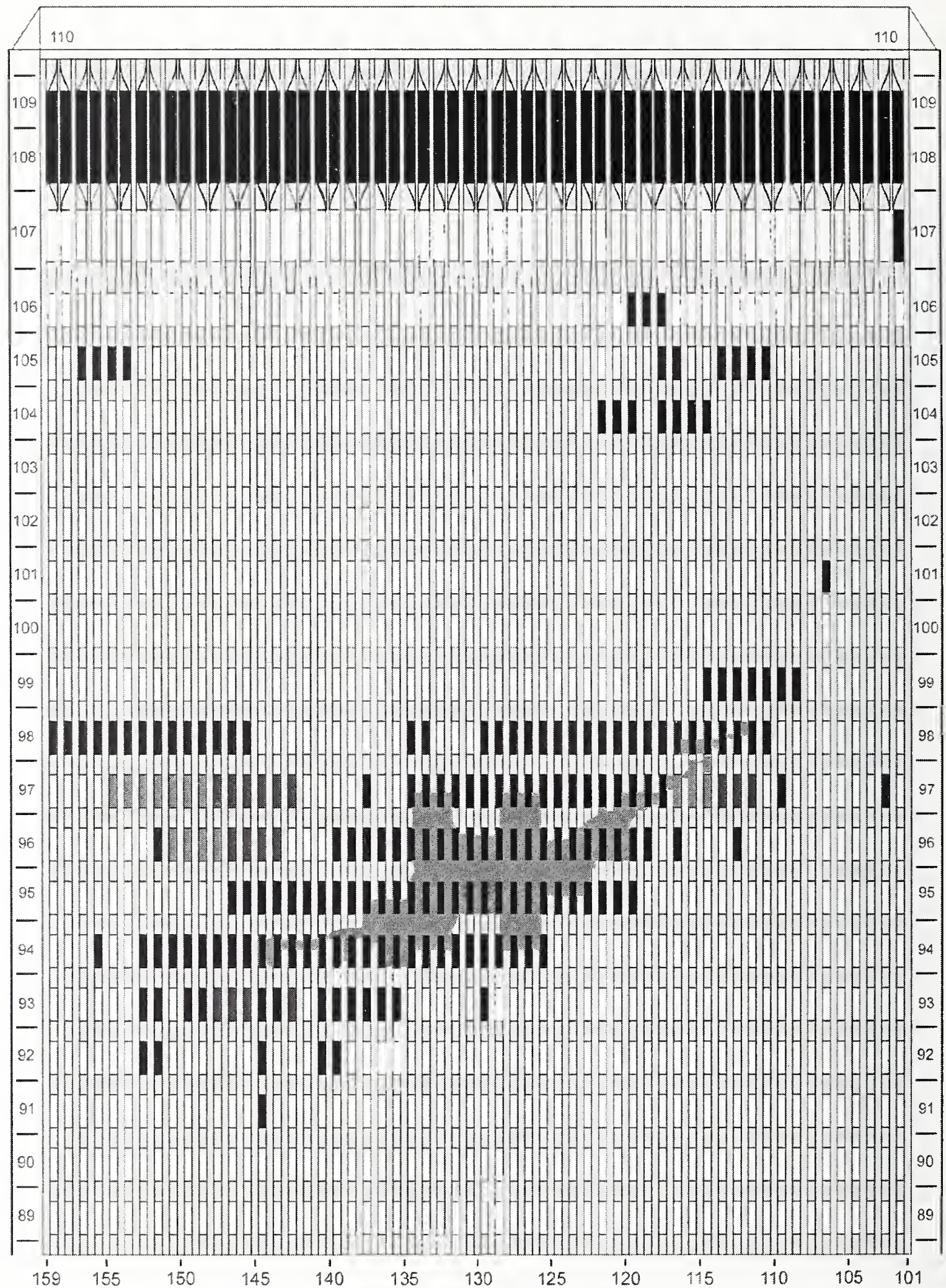

Figure C-13. Diagram of the north face of WTC 1 for floors 89 to 110 at 8:58 a.m. showing the condition of windows and locations of fires. 


$$
\text { WTC 1, North Face } \quad 8: 58 \text { a.m. }
$$

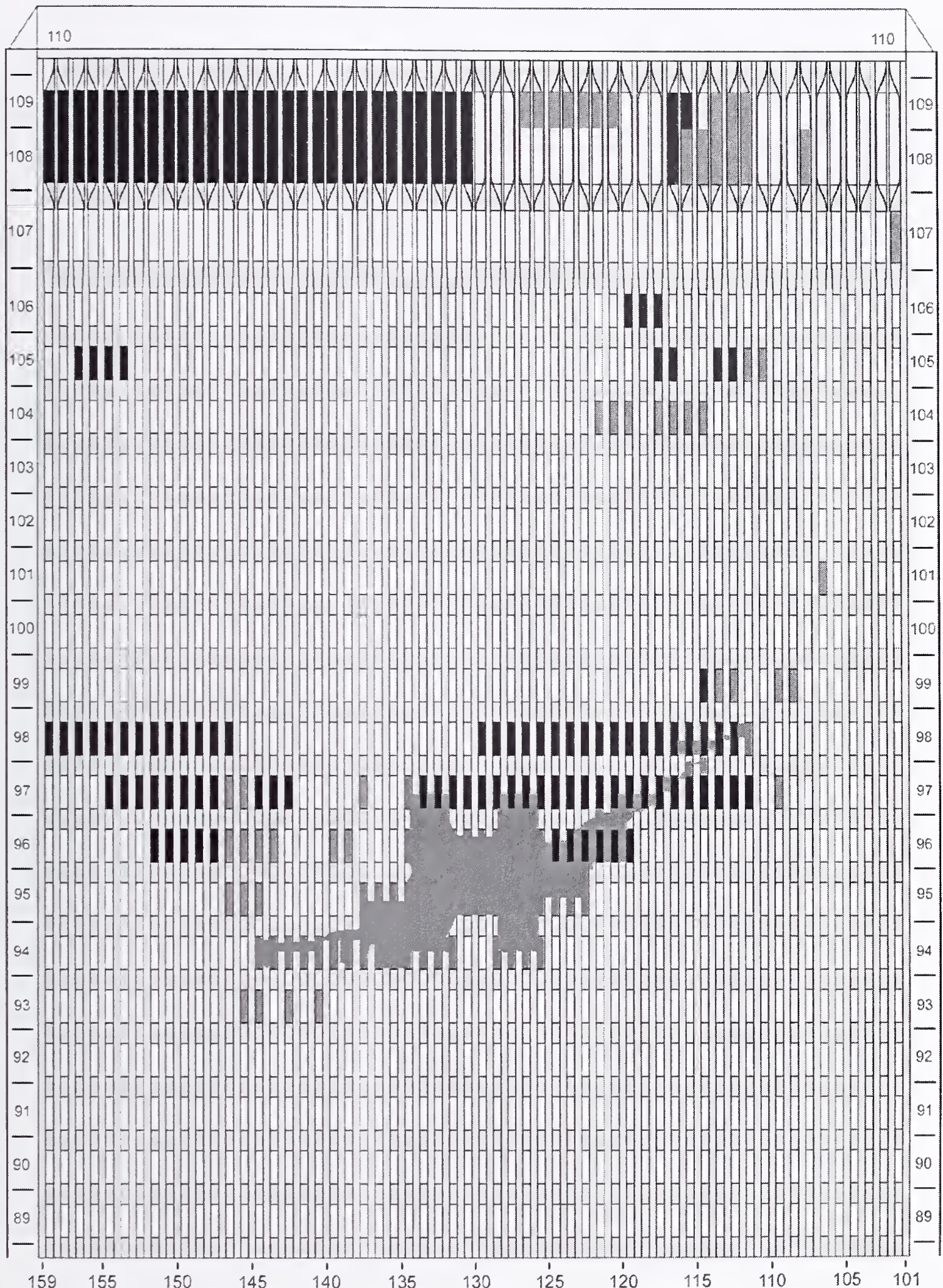

Figure C-14. Diagram of the north face of WTC 1 for floors 89 to 110 at $8: 58$ a.m. showing windows where smoke was observed and those that were hidden from view. 
WTC 1, North Face 9:00 a.m.

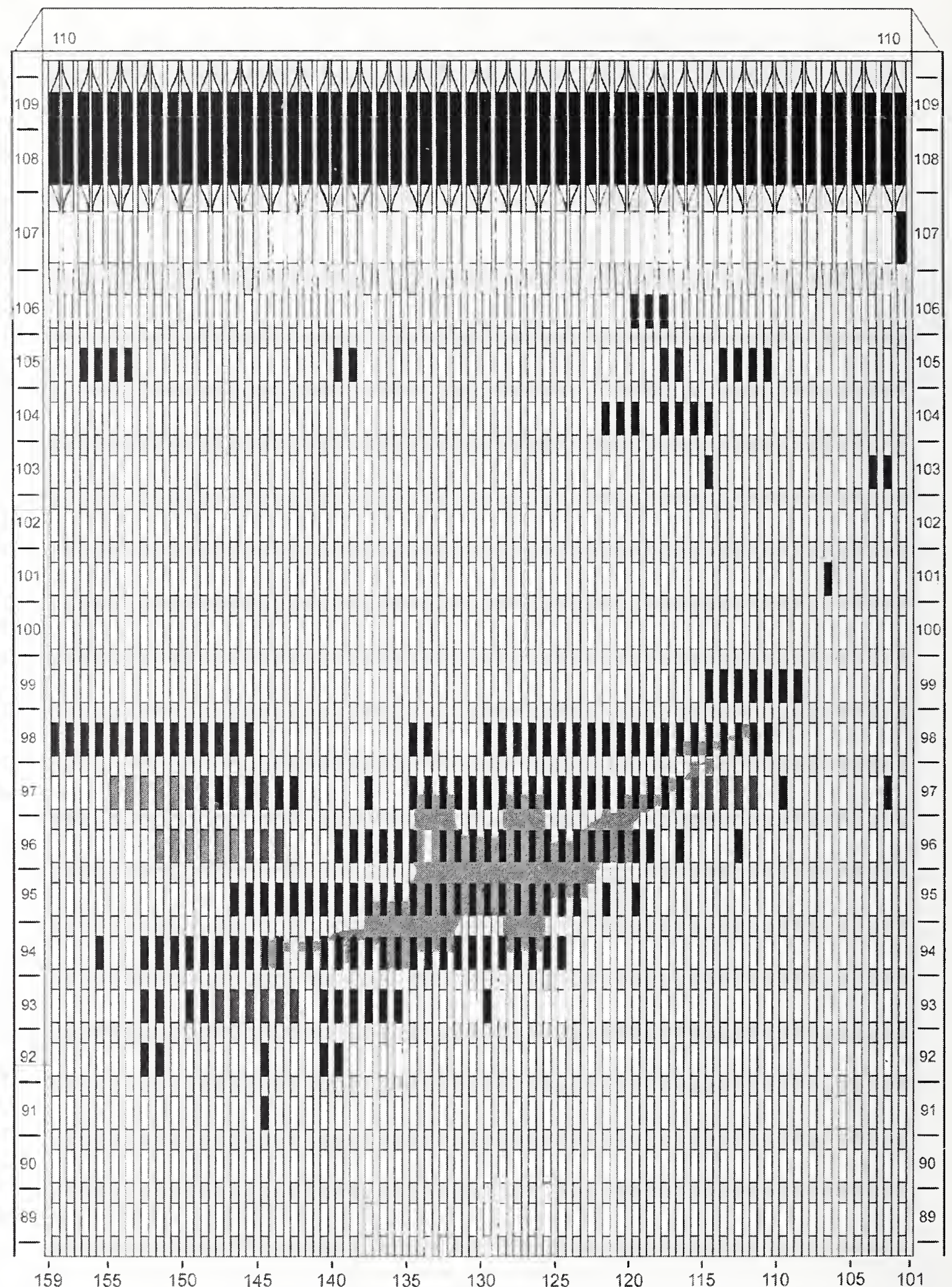

Figure C-15. Diagram of the north face of WTC 1 for floors 89 to 110 at 9:00 a.m. showing the condition of windows and locations of fires. 


\section{WTC 1, North Face 9:00 a.m.}

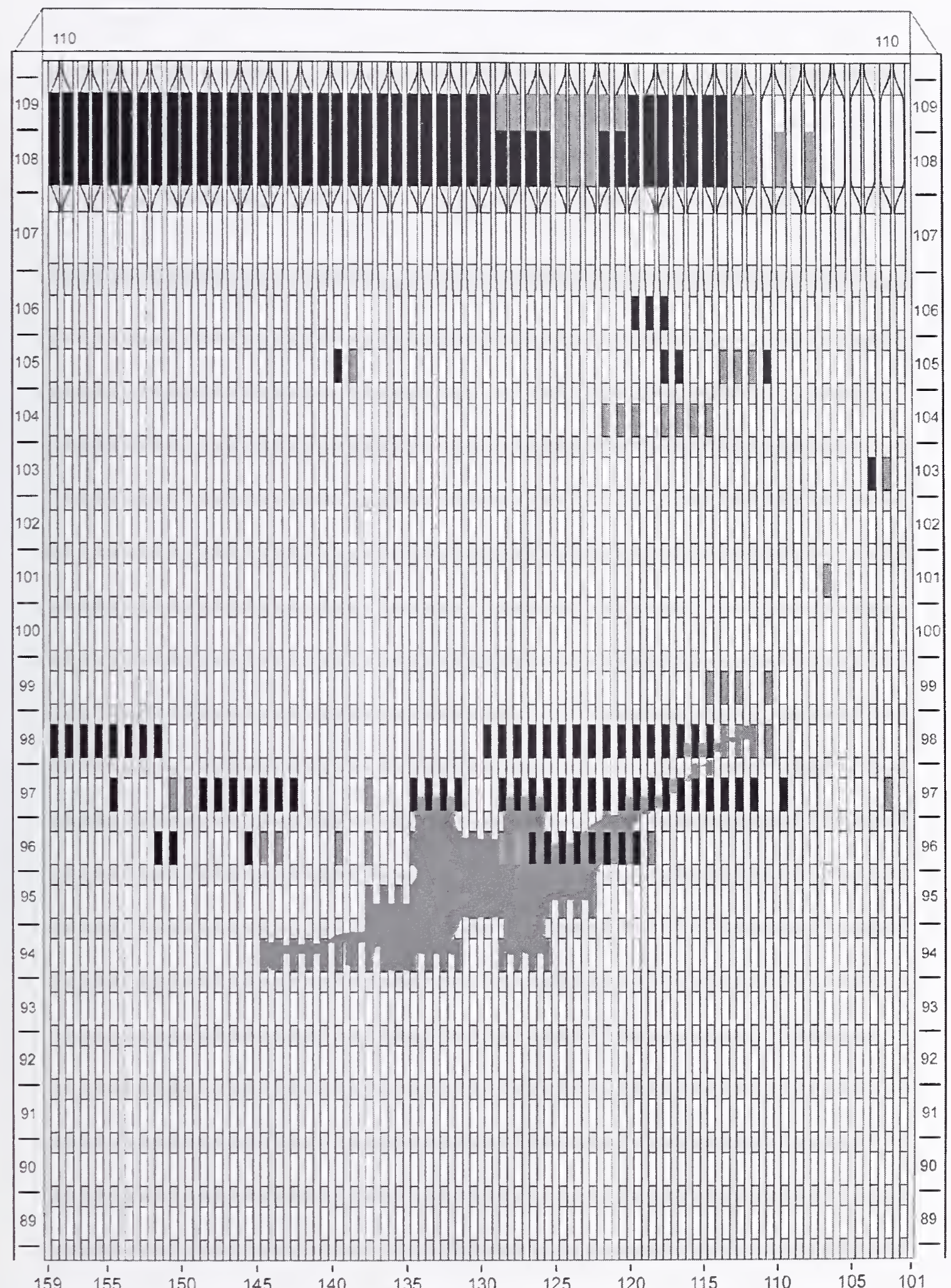

Figure C-16. Diagram of the north face of WTC 1 for floors 89 to 110 at 9:00 a.m. showing windows where smoke was observed and those that were hidden from view. 
WTC 1, North Face 9:02 a.m.

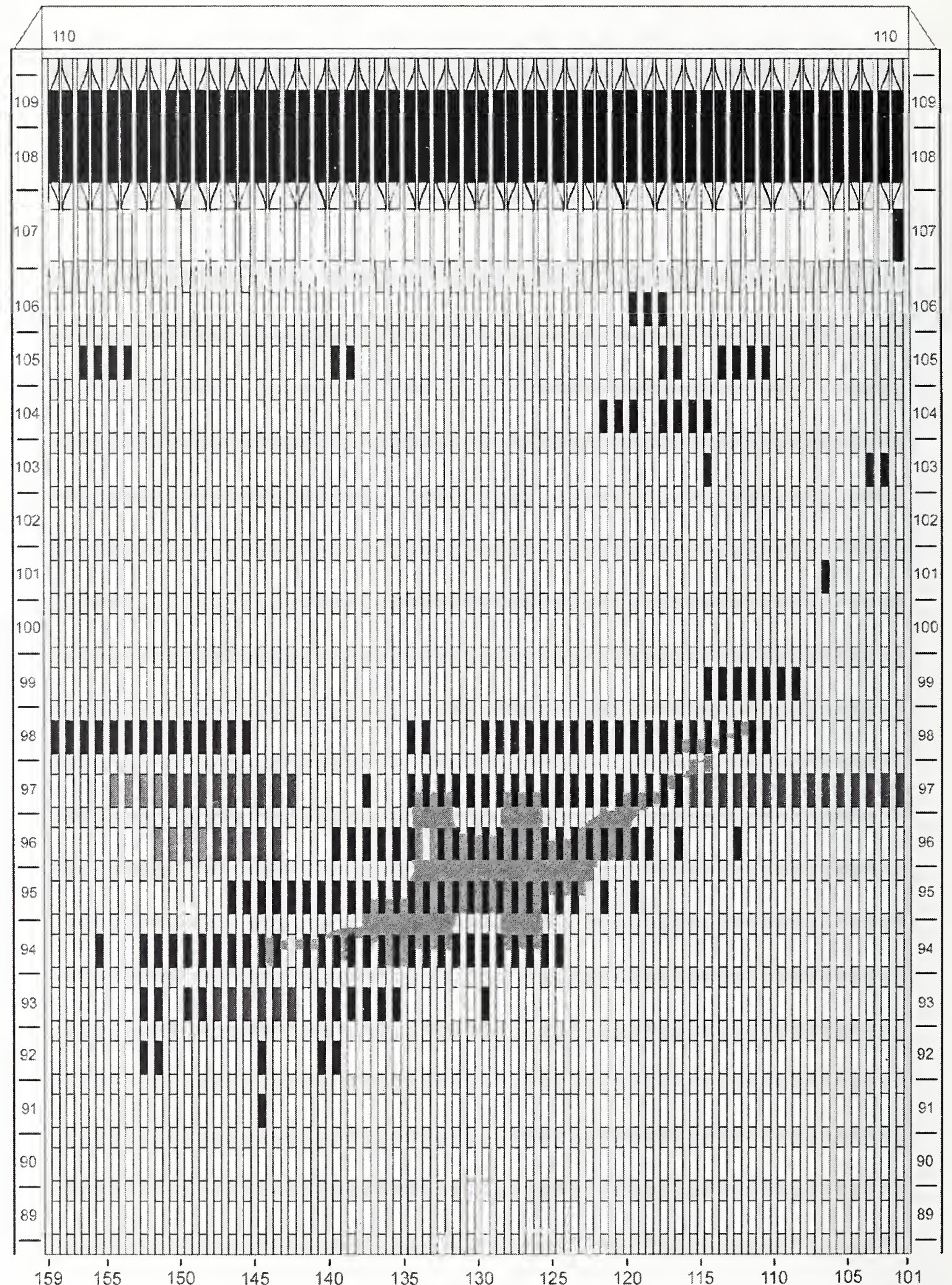

Figure C-17. Diagram of the north face of WTC 1 for floors 89 to 110 at 9:02 a.m. showing the condition of windows and locations of fires. 


\section{WTC 1, North Face 9:02 a.m.}

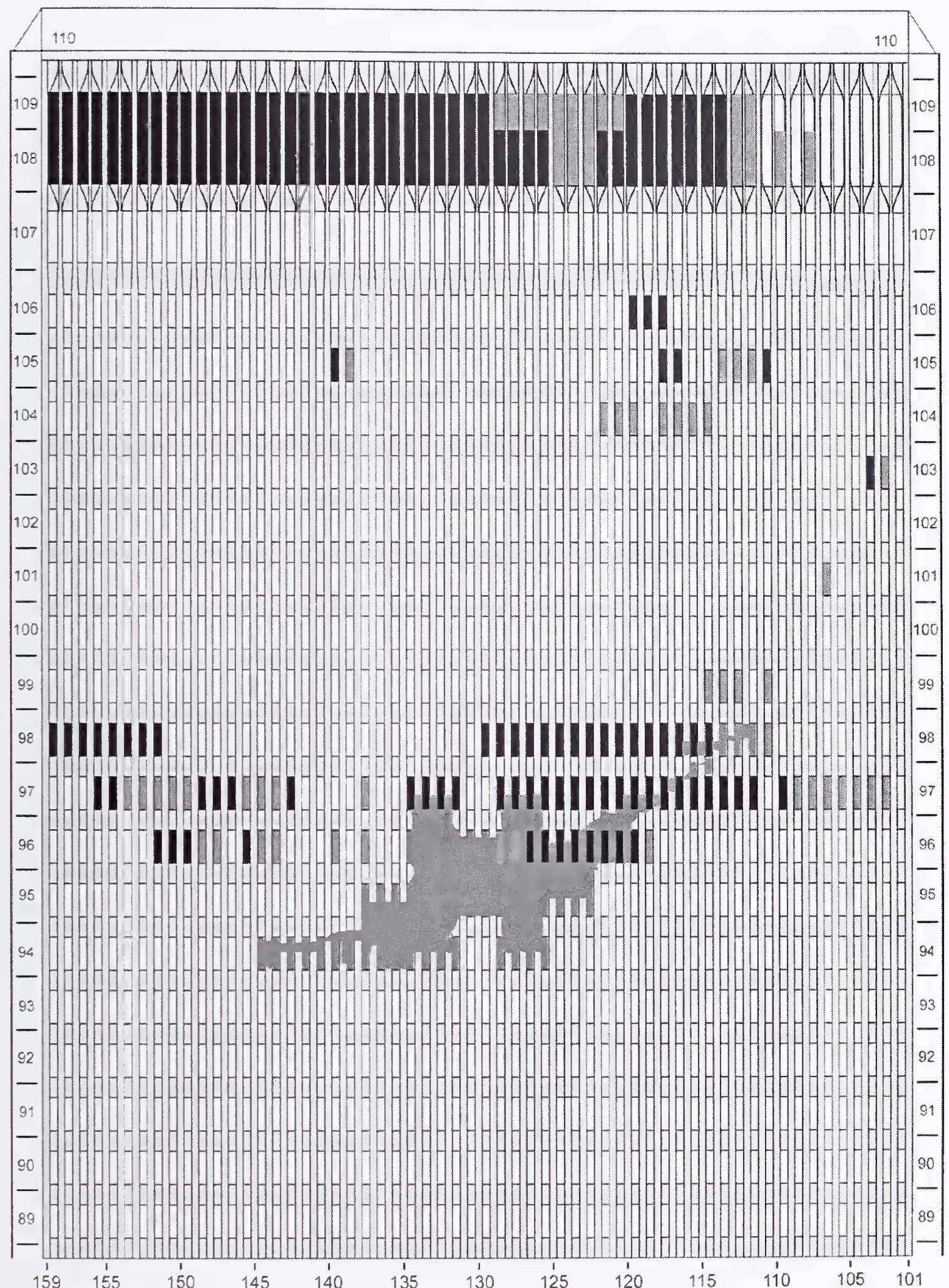

Figure C-18. Diagram of the north face of WTC 1 for floors 89 to 110 at 9:02 a.m. showing windows where smoke was observed and those that were hidden from view. 
WTC 1, North Face 9:04 a.m.

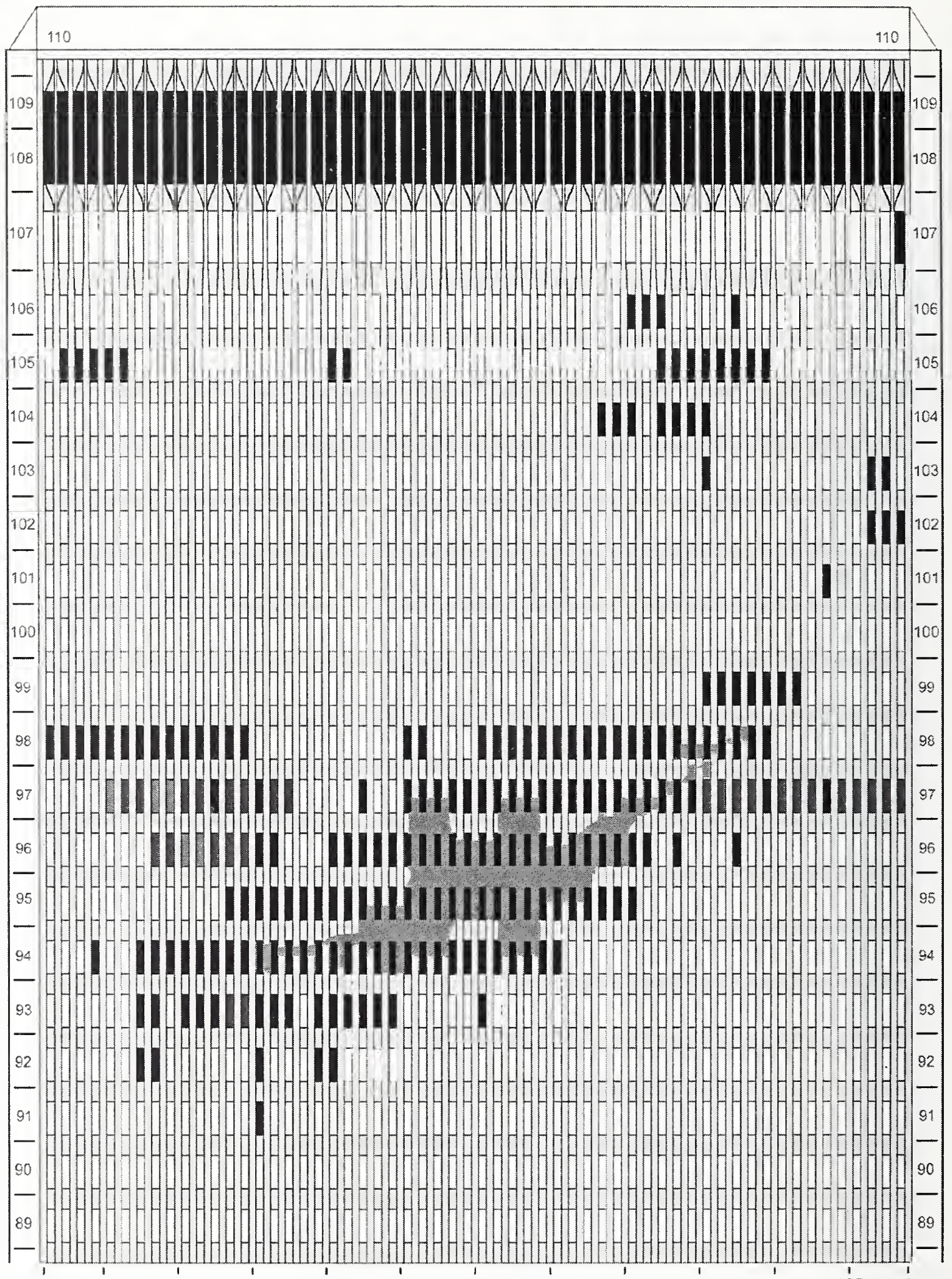

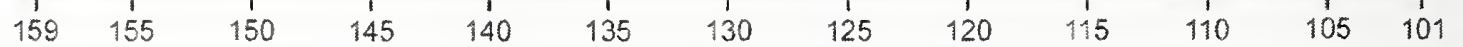

Figure C-19. Diagram of the north face of WTC 1 for floors 89 to 110 at 9:04 a.m. showing the condition of windows and locations of fires. 


\section{WTC 1, North Face 9:04 a.m.}

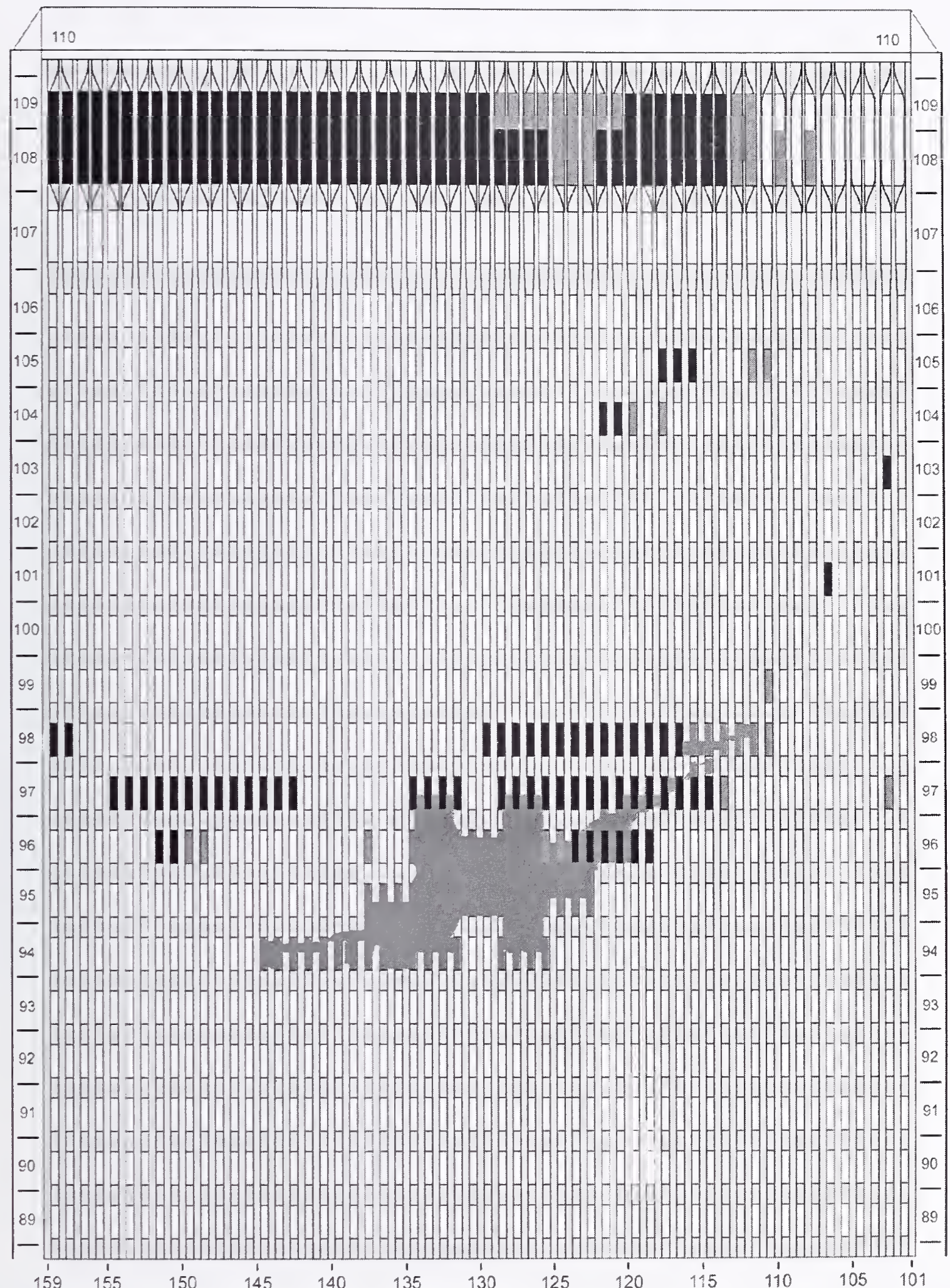

Figure C-20. Diagram of the north face of WTC 1 for floors 89 to 110 at 9:04 a.m. showing windows where smoke was observed and those that were hidden from view. 

WTC 1, North Face
9:10 a.m.

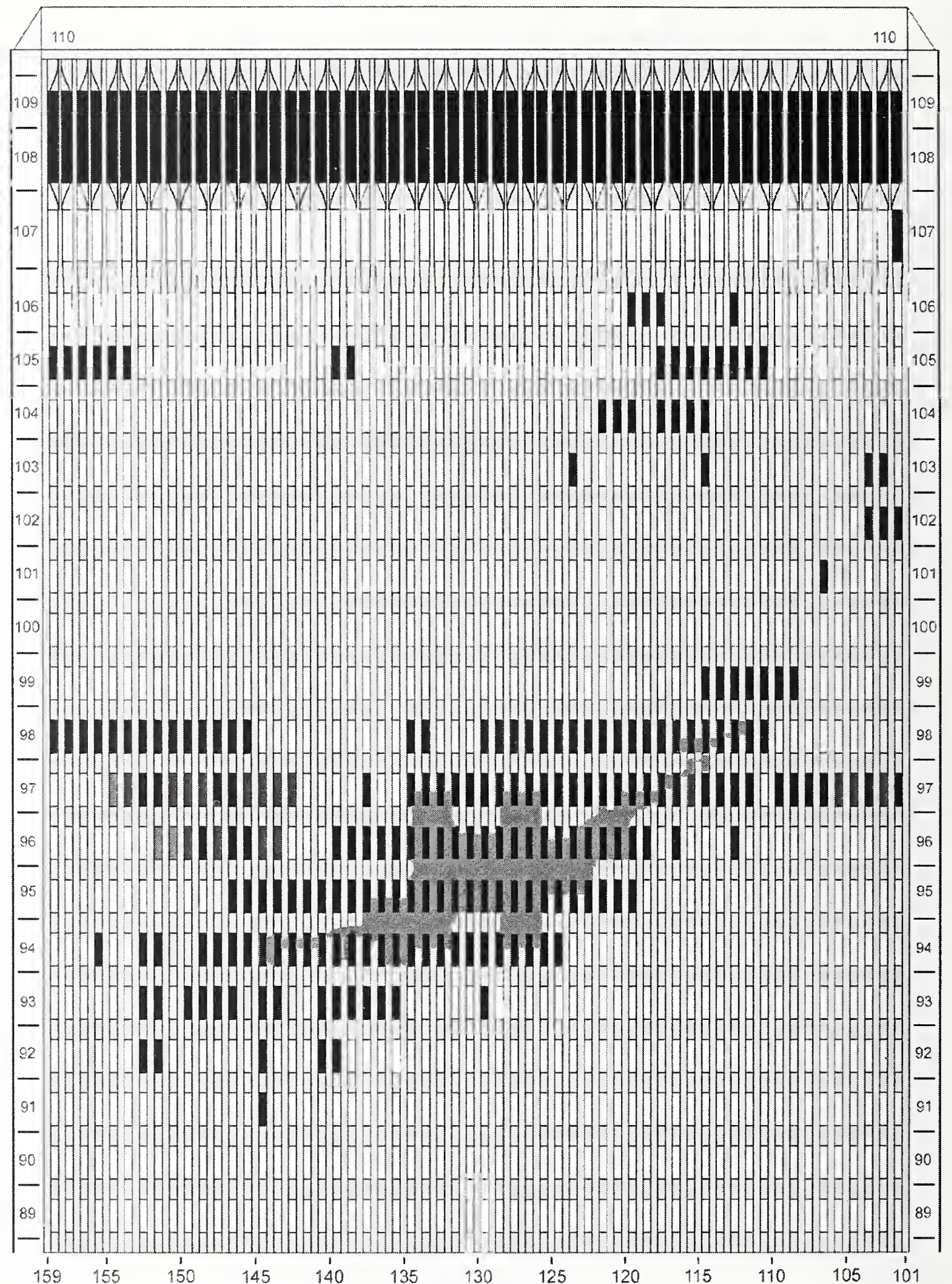

Figure C-21. Diagram of the north face of WTC 1 for floors 89 to 110 at 9:10 a.m. showing the condition of windows and locations of fires. 


\section{WTC 1, North Face 9:10 a.m.}

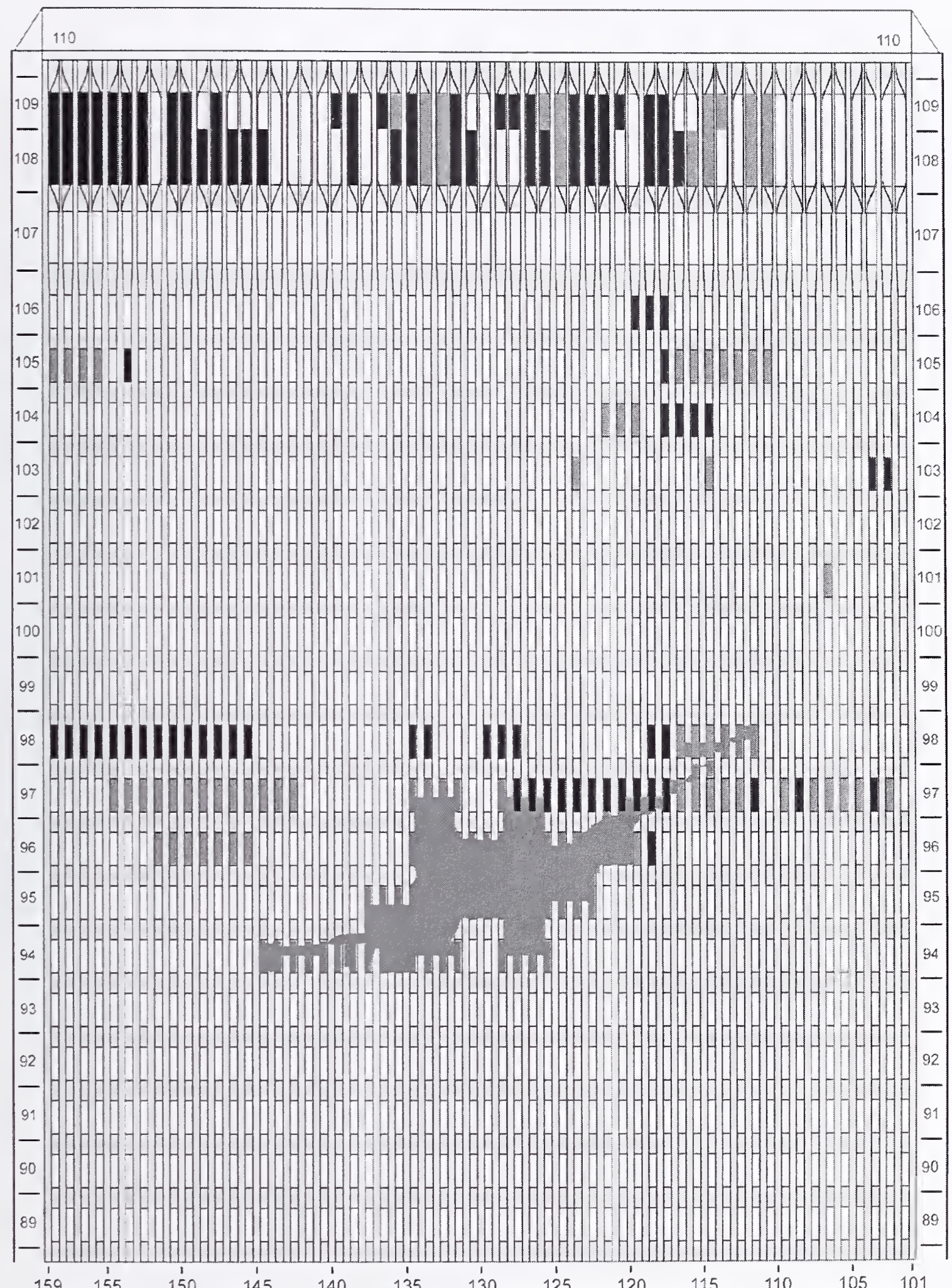

Figure C-22. Diagram of the north face of WTC 1 for floors 89 to 110 at $9: 10$ a.m. showing windows where smoke was observed and those that were hidden from view. 


$$
\text { WTC 1, North Face } \quad \text { 9:14 a.m. }
$$

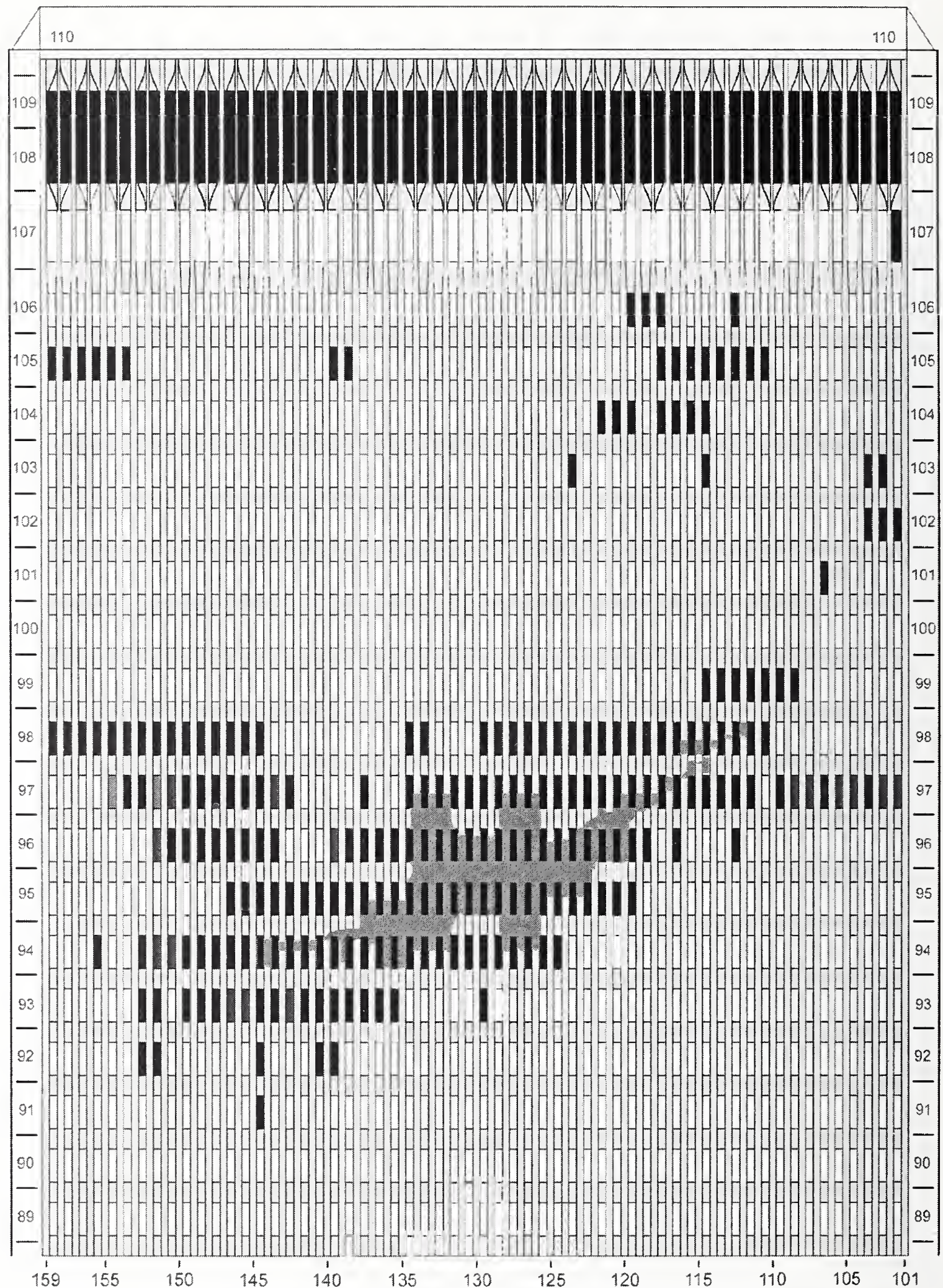

Figure C-23. Diagram of the north face of WTC 1 for floors 89 to 110 at 9:14 a.m. showing the condition of windows and locations of fires. 


\section{WTC 1, North Face $\quad$ 9:14 a.m.}

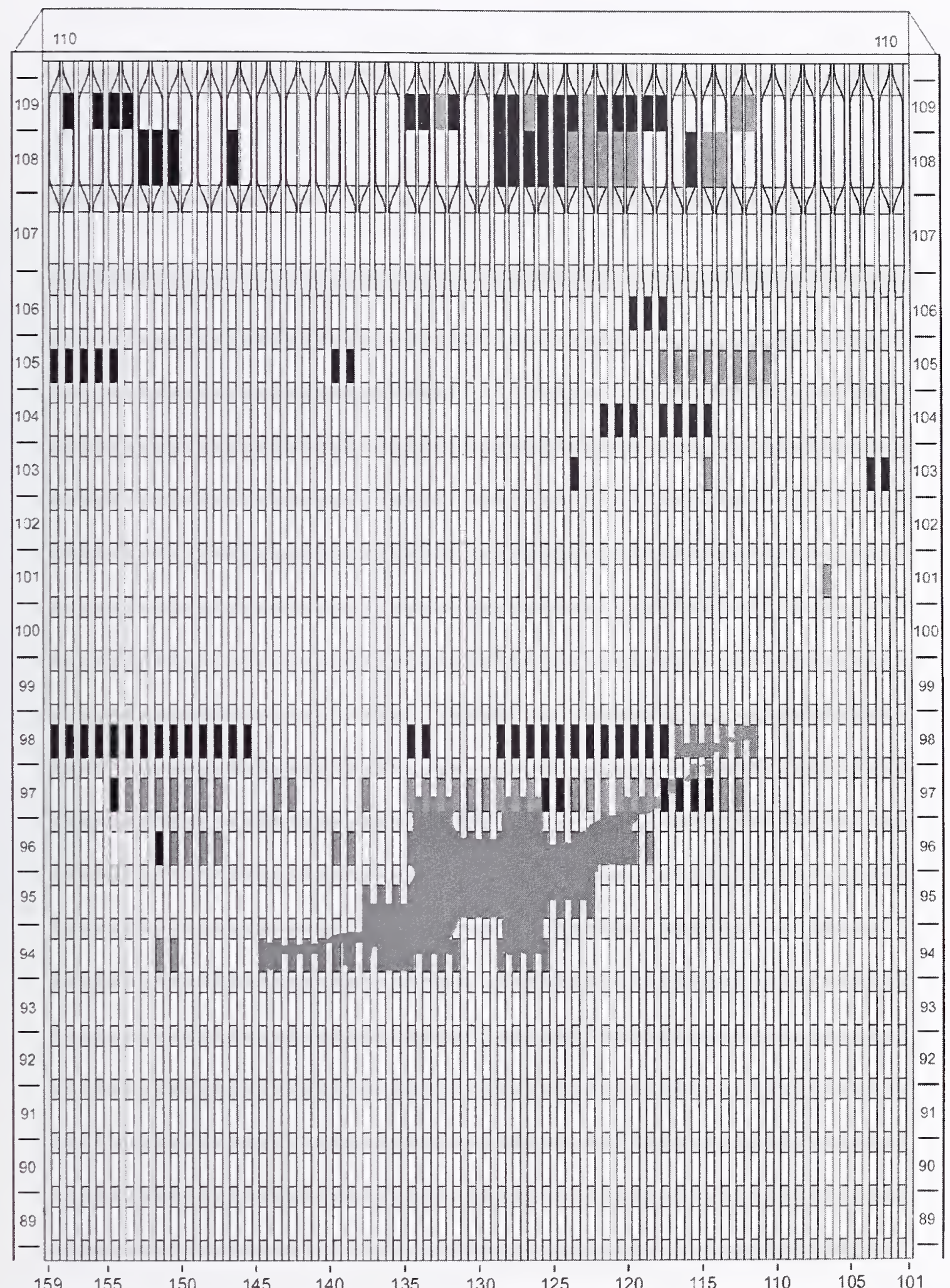

Figure C-24. Diagram of the north face of WTC 1 for floors 89 to 110 at 9:14 a.m. showing windows where smoke was observed and those that were hidden from view. 
WTC 1, North Face 9:18 a.m.

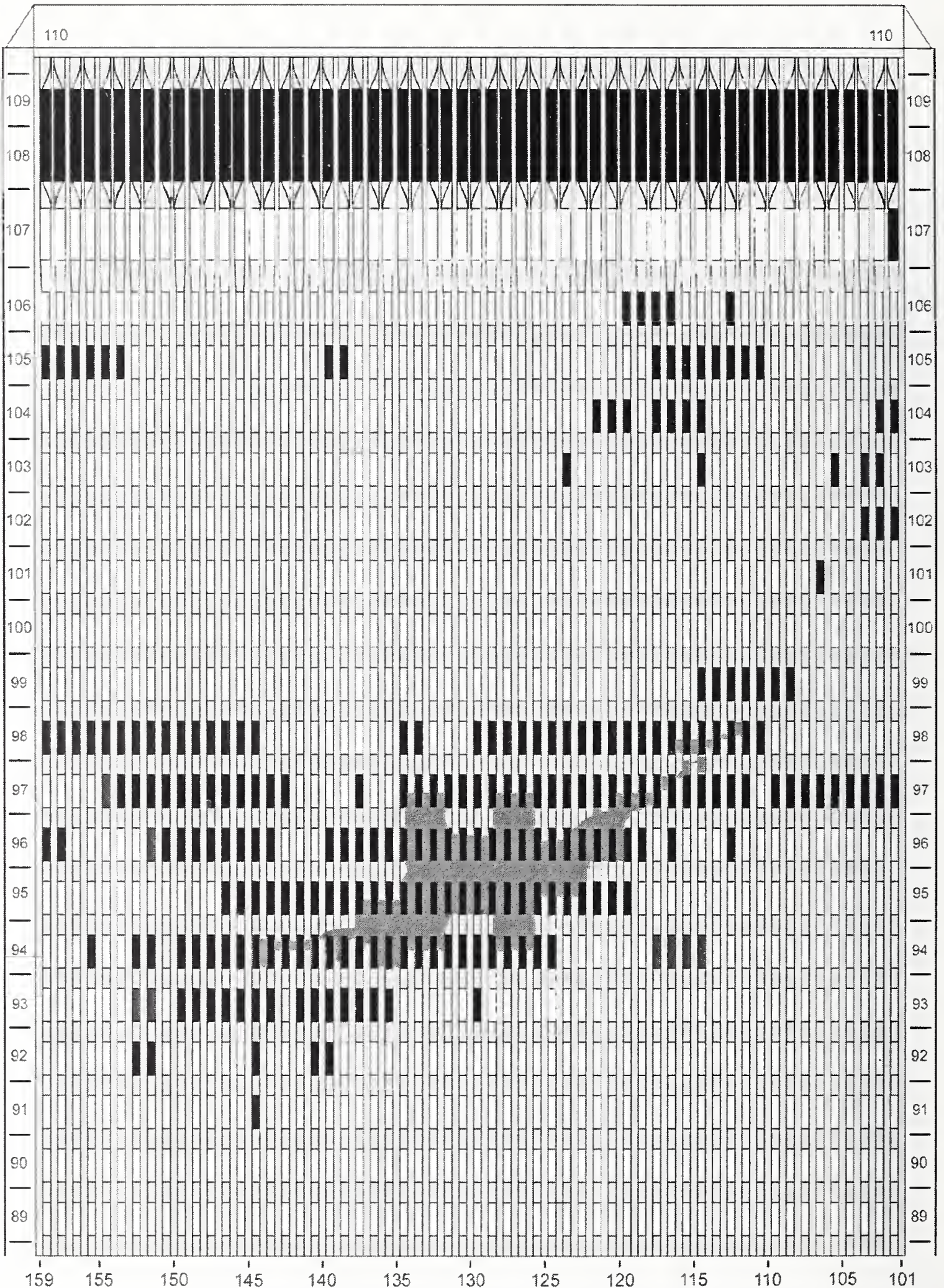

Figure C-25. Diagram of the north face of WTC 1 for floors 89 to 110 at 9:18 a.m. showing the condition of windows and locations of fires. 


\section{WTC 1, North Face 9:18 a.m.}

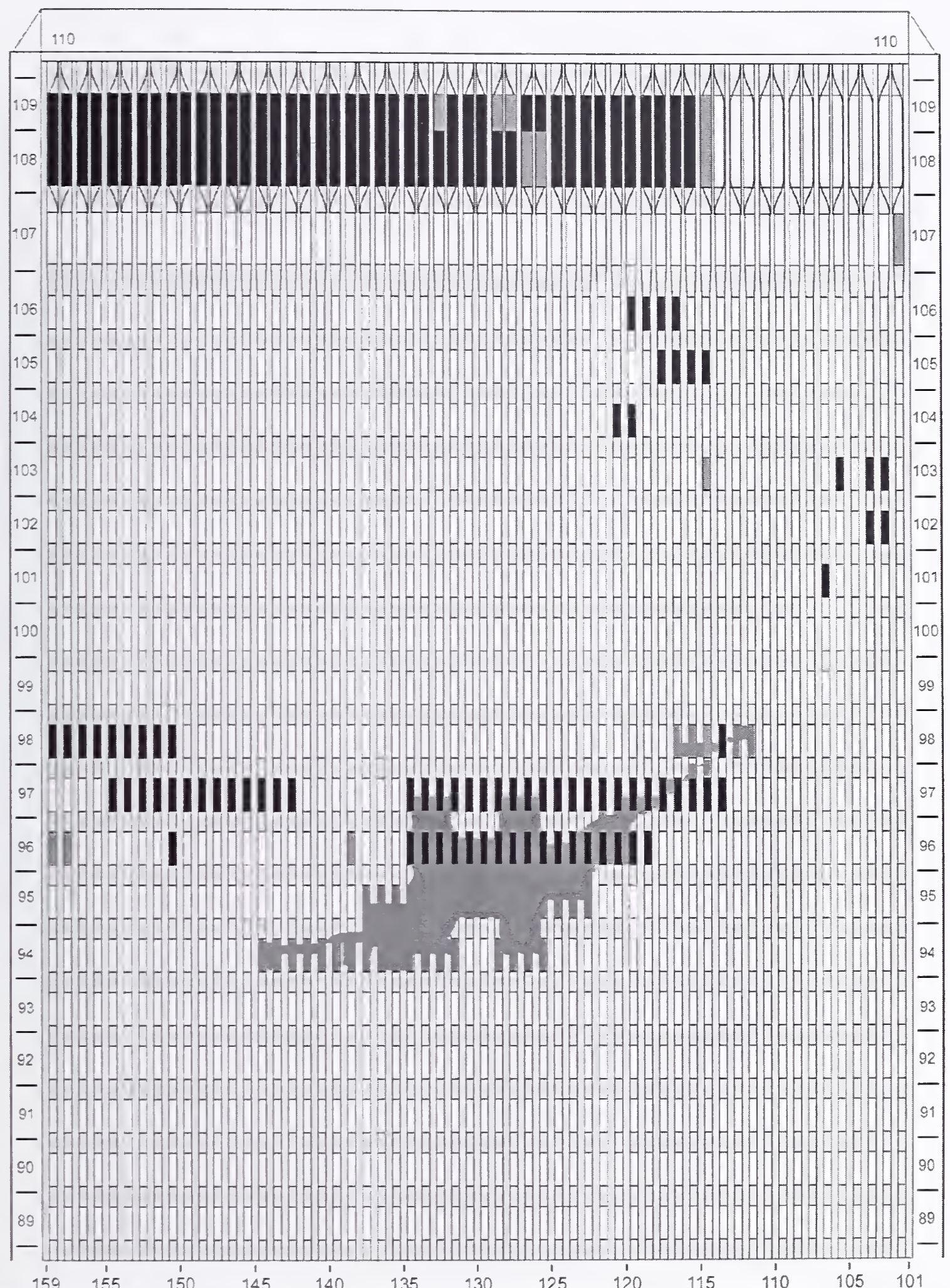

Figure C-26. Diagram of the north face of WTC 1 for floors 89 to 110 at 9:18 a.m. showing windows where smoke was observed and those that were hidden from view. 
WTC 1, North Face 9:20 a.m.

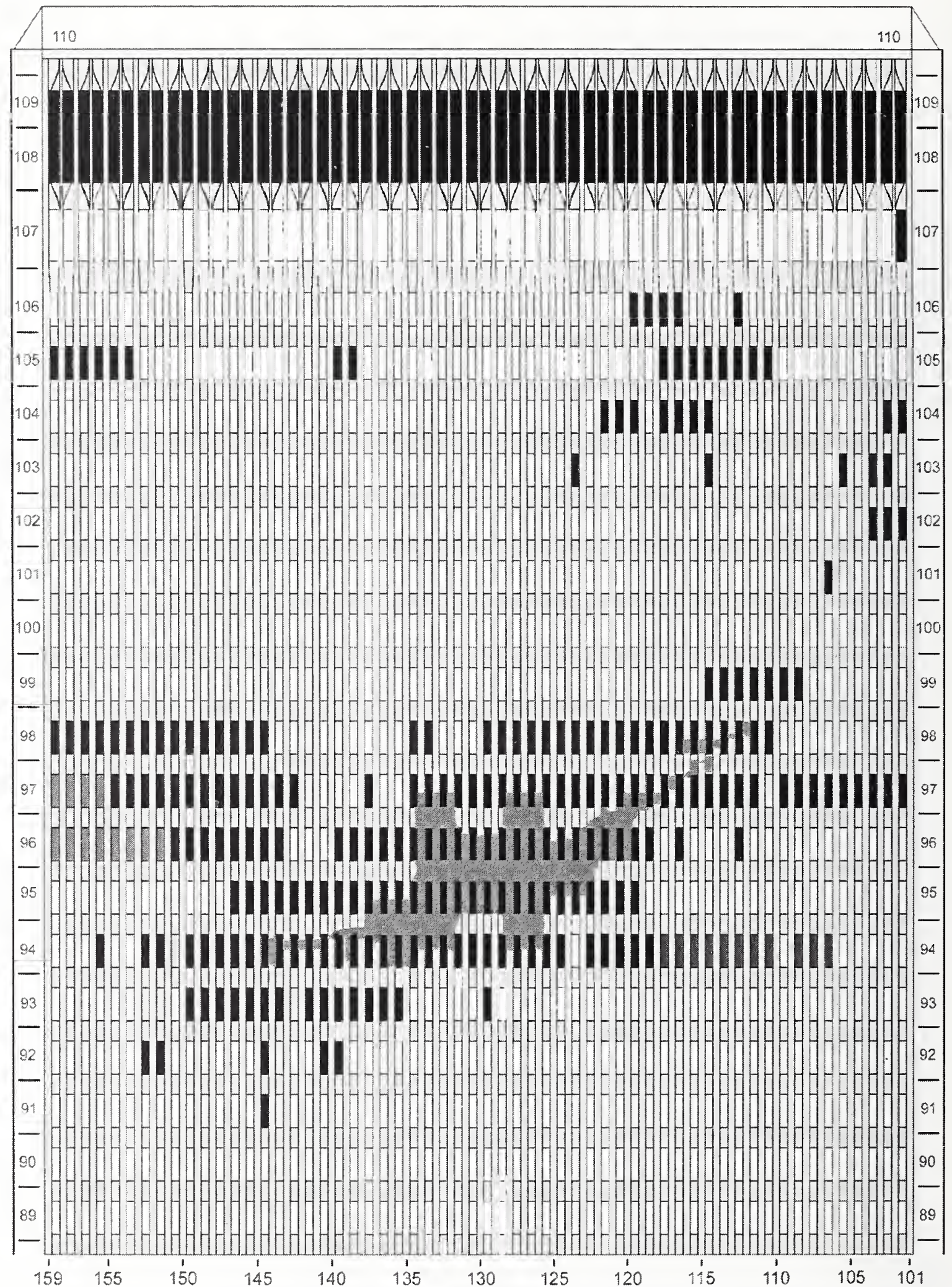

Figure C-27. Diagram of the north face of WTC 1 for floors 89 to 110 at 9:20 a.m. showing the condition of windows and locations of fires. 


$$
\text { WTC 1, North Face } \quad \text { 9:20 a.m. }
$$

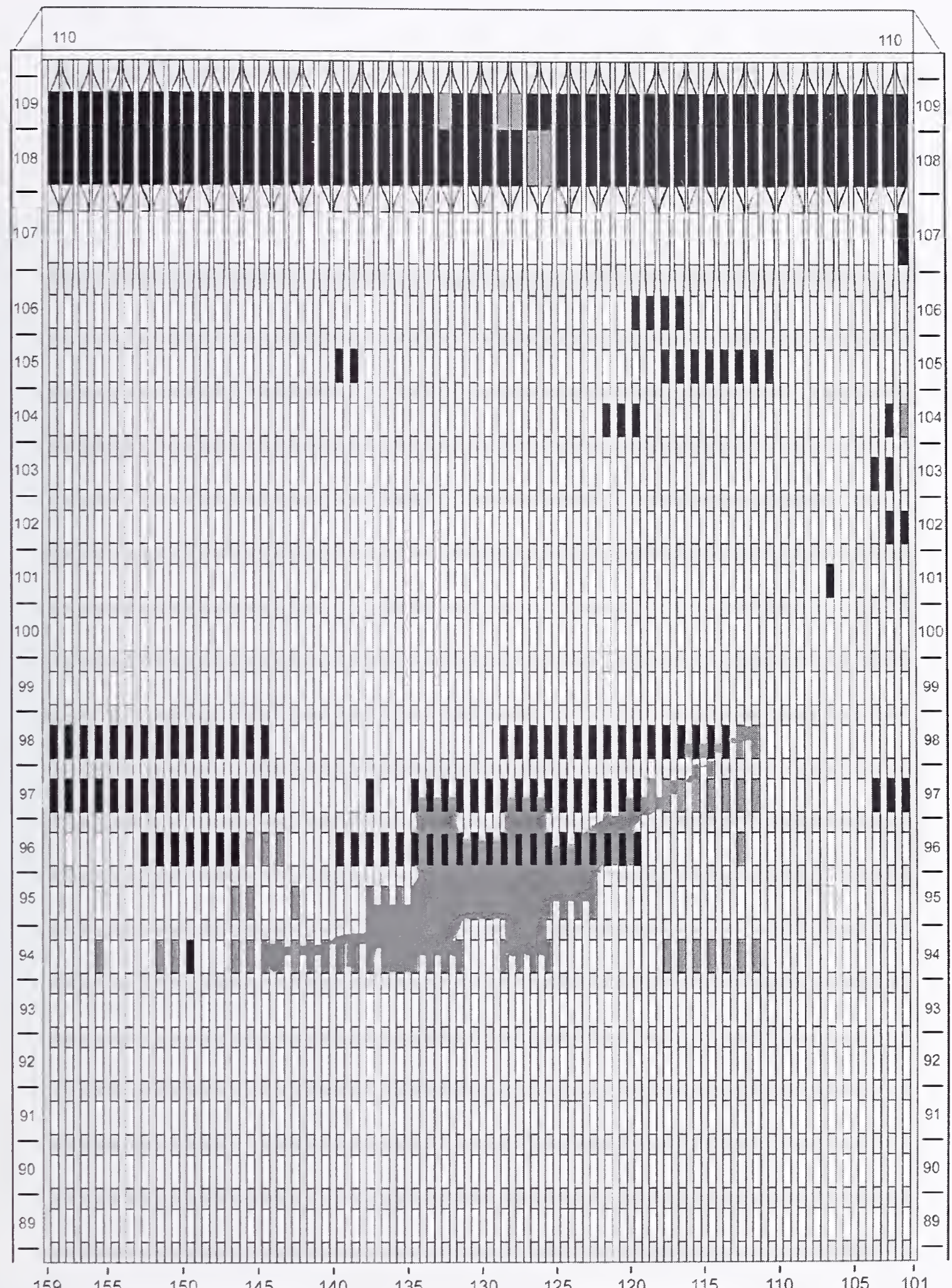

Figure C-28. Diagram of the north face of WTC 1 for floors 89 to 110 at 9:20 a.m. showing windows where smoke was observed and those that were hidden from view. 


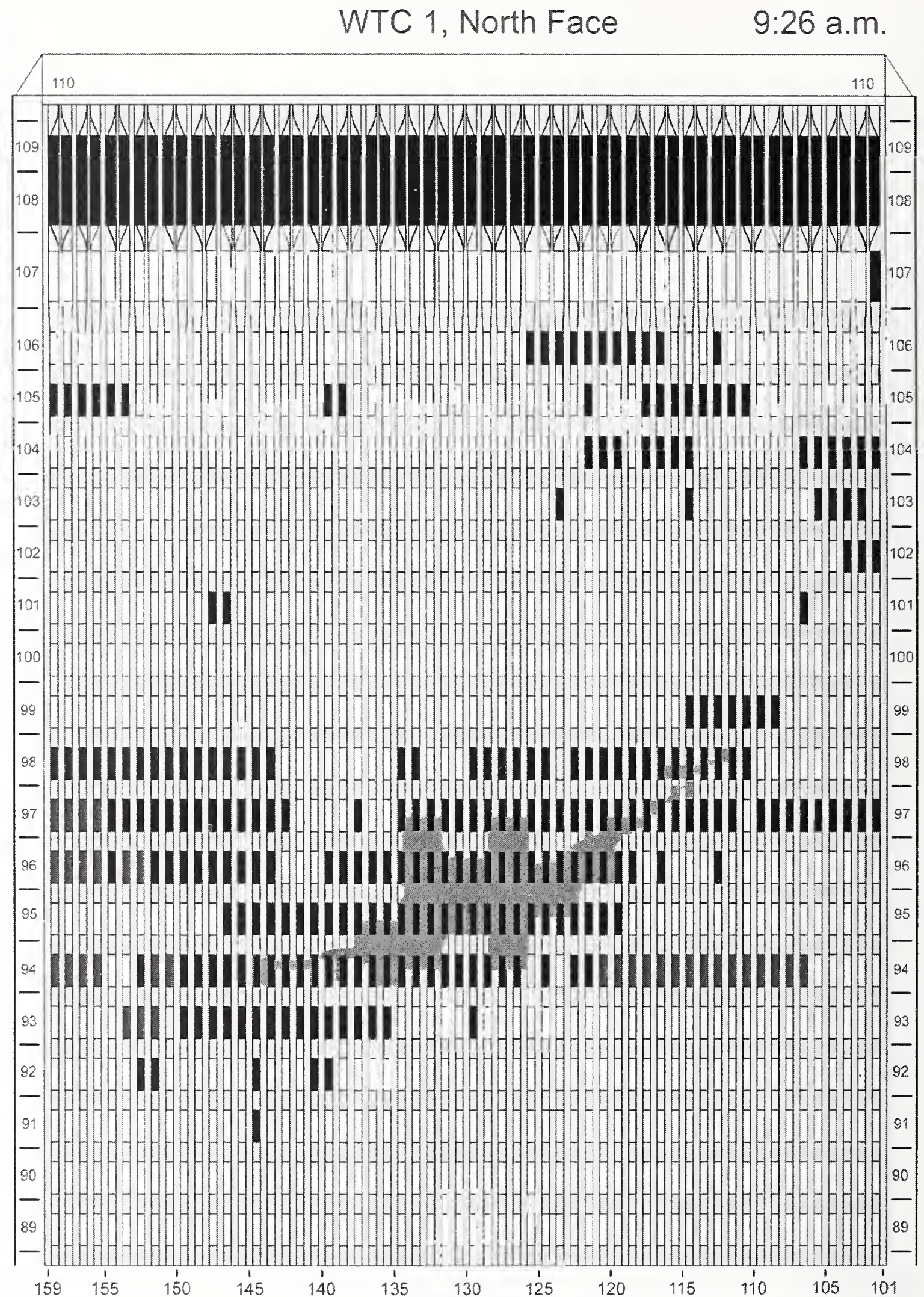

Figure C-29. Diagram of the north face of WTC 1 for floors 89 to 110 at 9:26 a.m. showing the condition of windows and locations of fires. 


$$
\text { WTC 1, North Face 9:26 a.m. }
$$

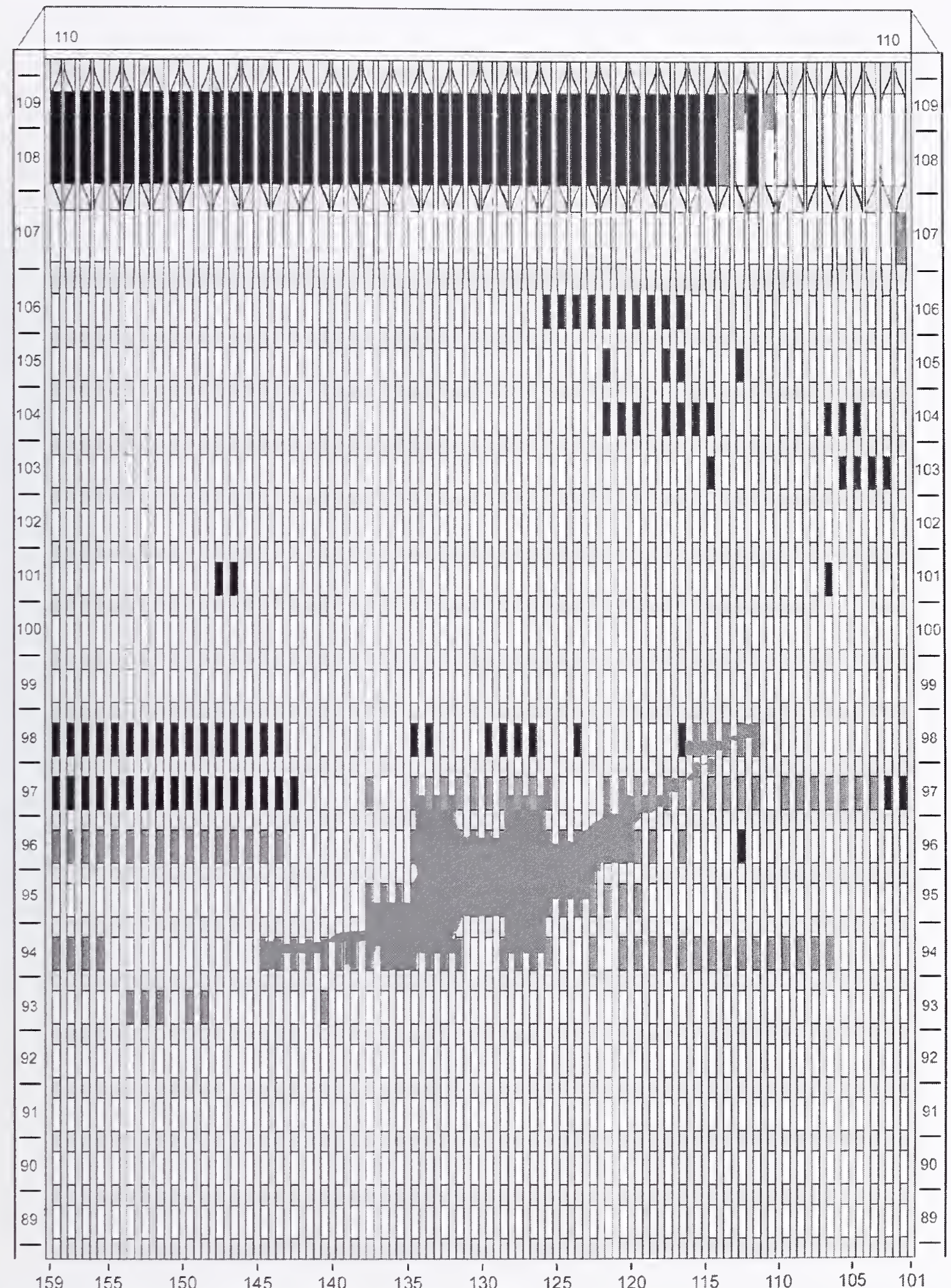

Figure C-30. Diagram of the north face of WTC 1 for floors 89 to 110 at 9:26 a.m. showing windows where smoke was observed and those that were hidden from view. 


$$
\text { WTC 1, North Face } \quad 9: 32 \text { a.m. }
$$

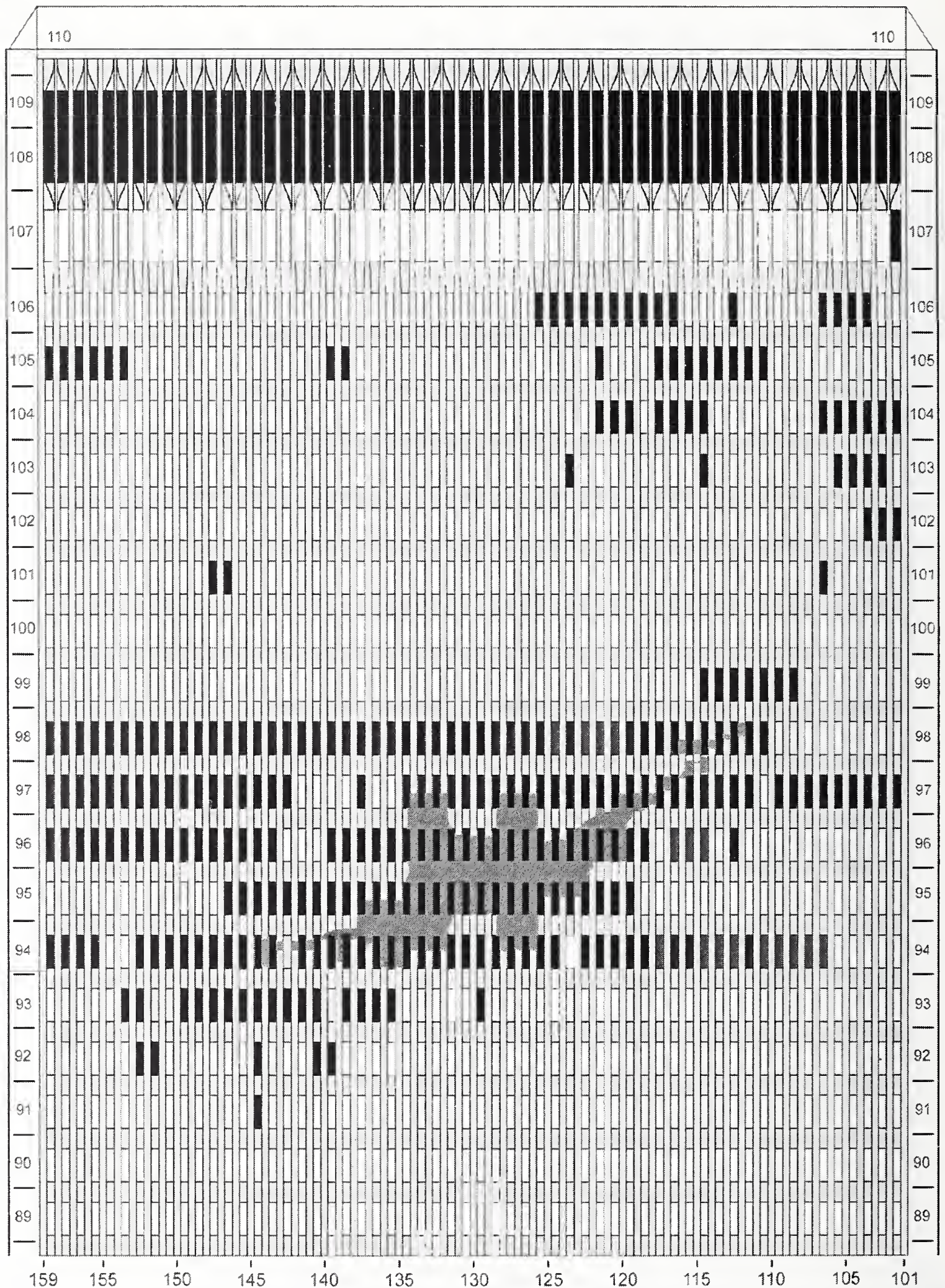

Figure C-31. Diagram of the north face of WTC 1 for floors 89 to 110 at 9:32 a.m. showing the condition of windows and locations of fires. 
WTC 1, North Face 9:32 a.m.

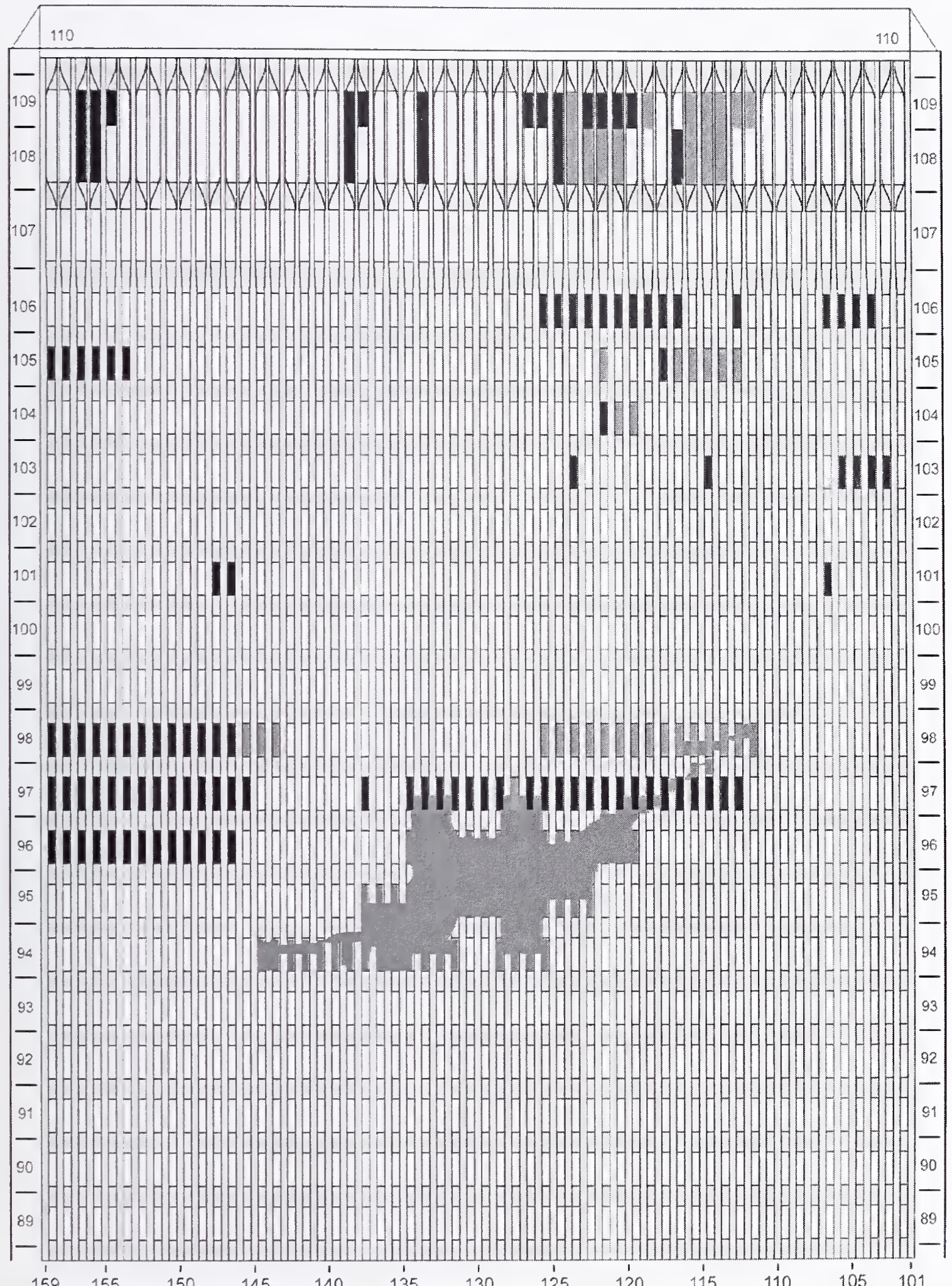

Figure C-32. Diagram of the north face of WTC 1 for floors 89 to 110 at 9:32 a.m. showing windows where smoke was observed and those that were hidden from view. 
WTC 1, North Face $\quad 9: 38$ a.m.

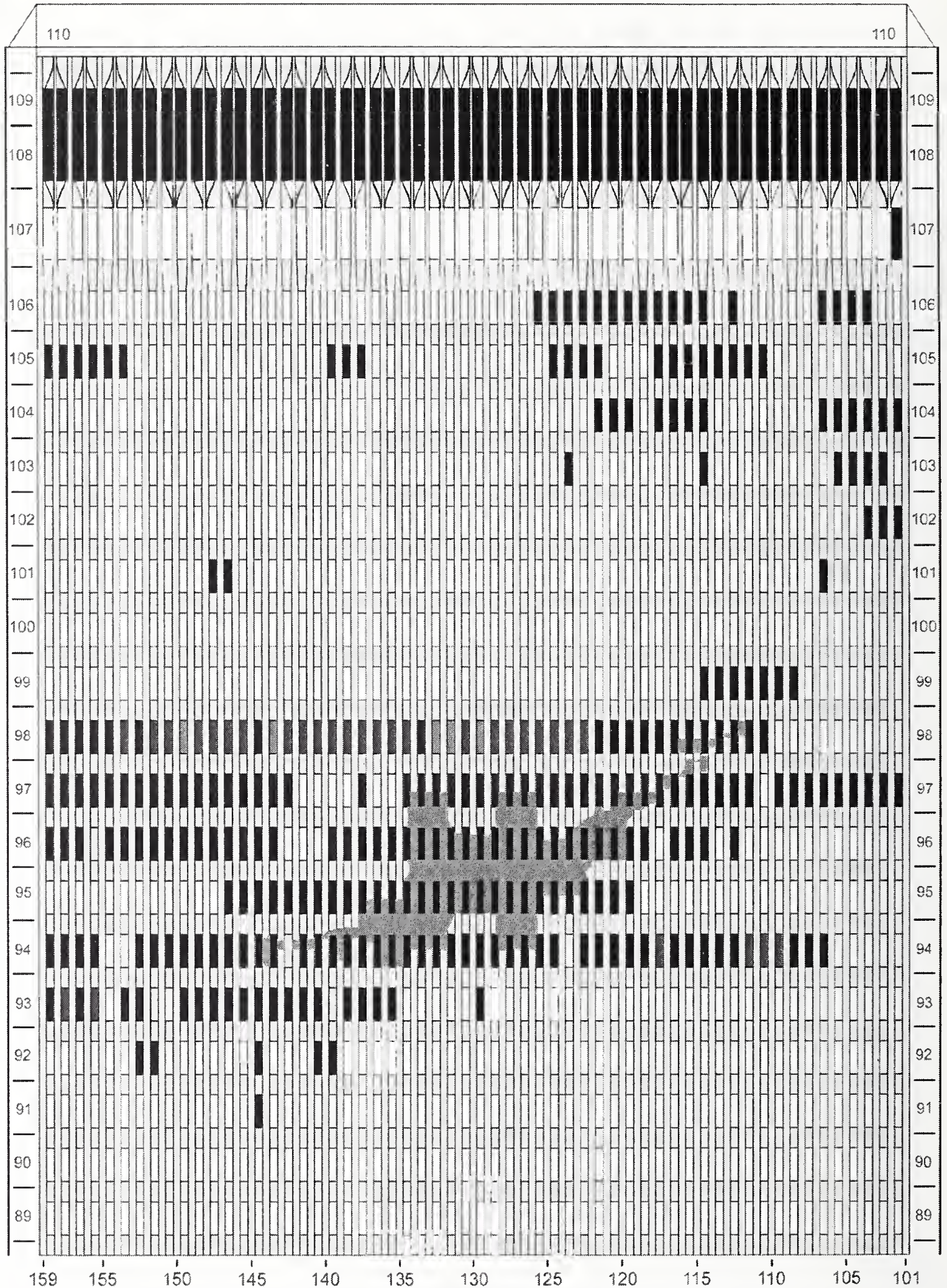

Figure C-33. Diagram of the north face of WTC 1 for floors 89 to 110 at 9:38 a.m. showing the condition of windows and locations of fires. 


\section{WTC 1, North Face 9:38 a.m.}

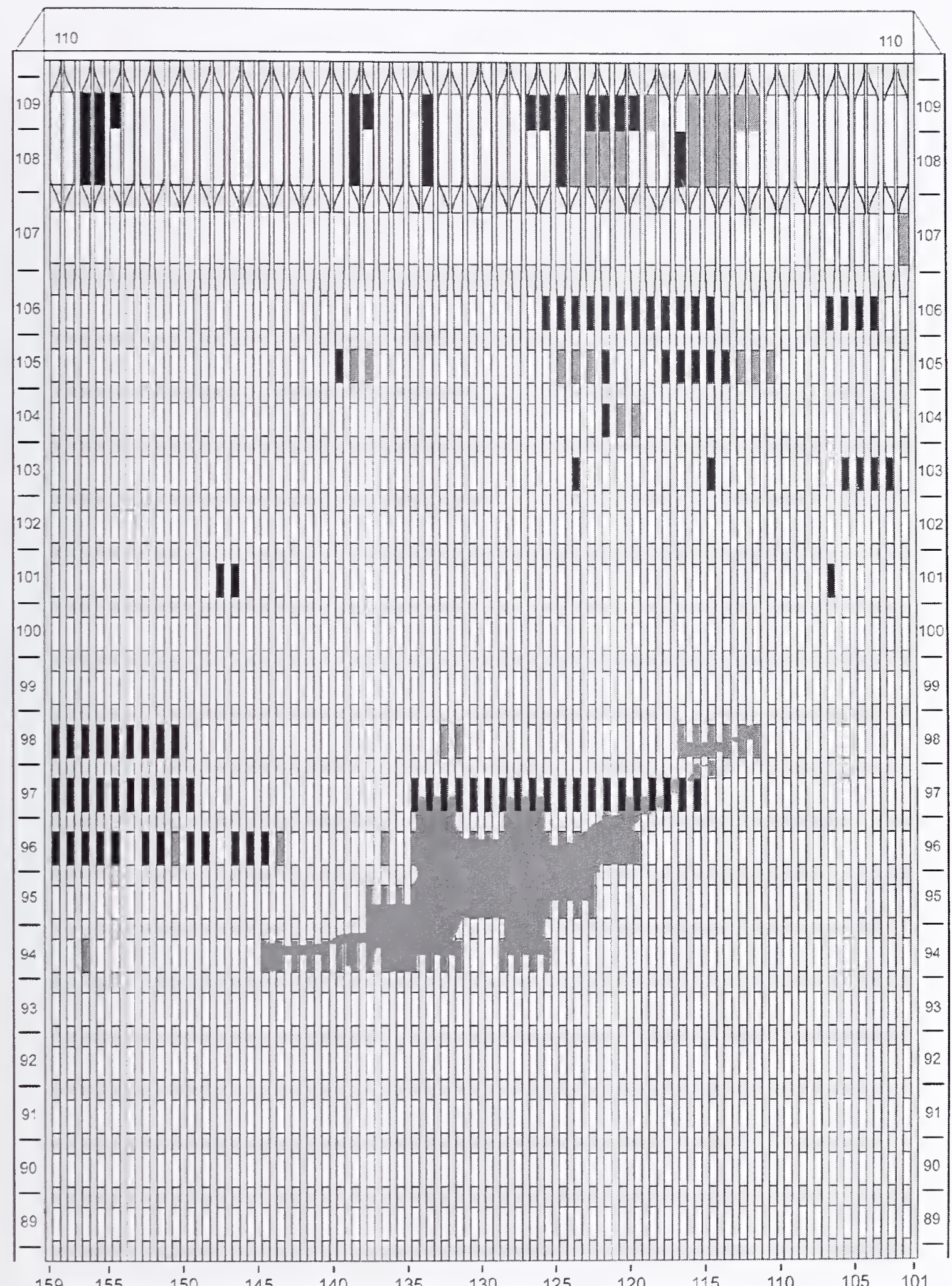

Figure C-34. Diagram of the north face of WTC 1 for floors 89 to 110 at $9: 38$ a.m. showing windows where smoke was observed and those that were hidden from view. 
WTC 1, North Face 9:42 a.m.

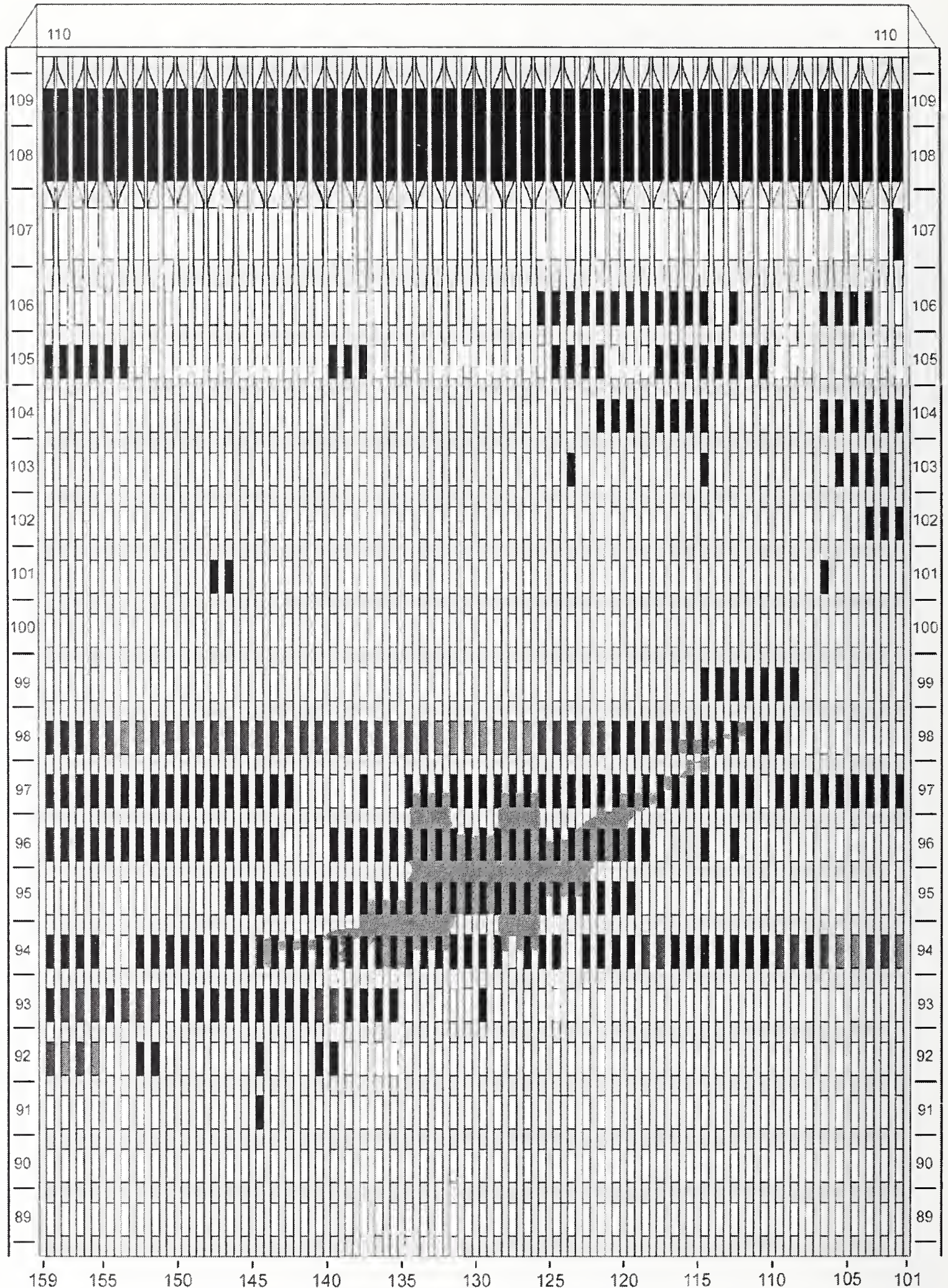

Figure C-35. Diagram of the north face of WTC 1 for floors 89 to 110 at 9:42 a.m. showing the condition of windows and locations of fires. 
WTC 1, North Face 9:42 a.m.

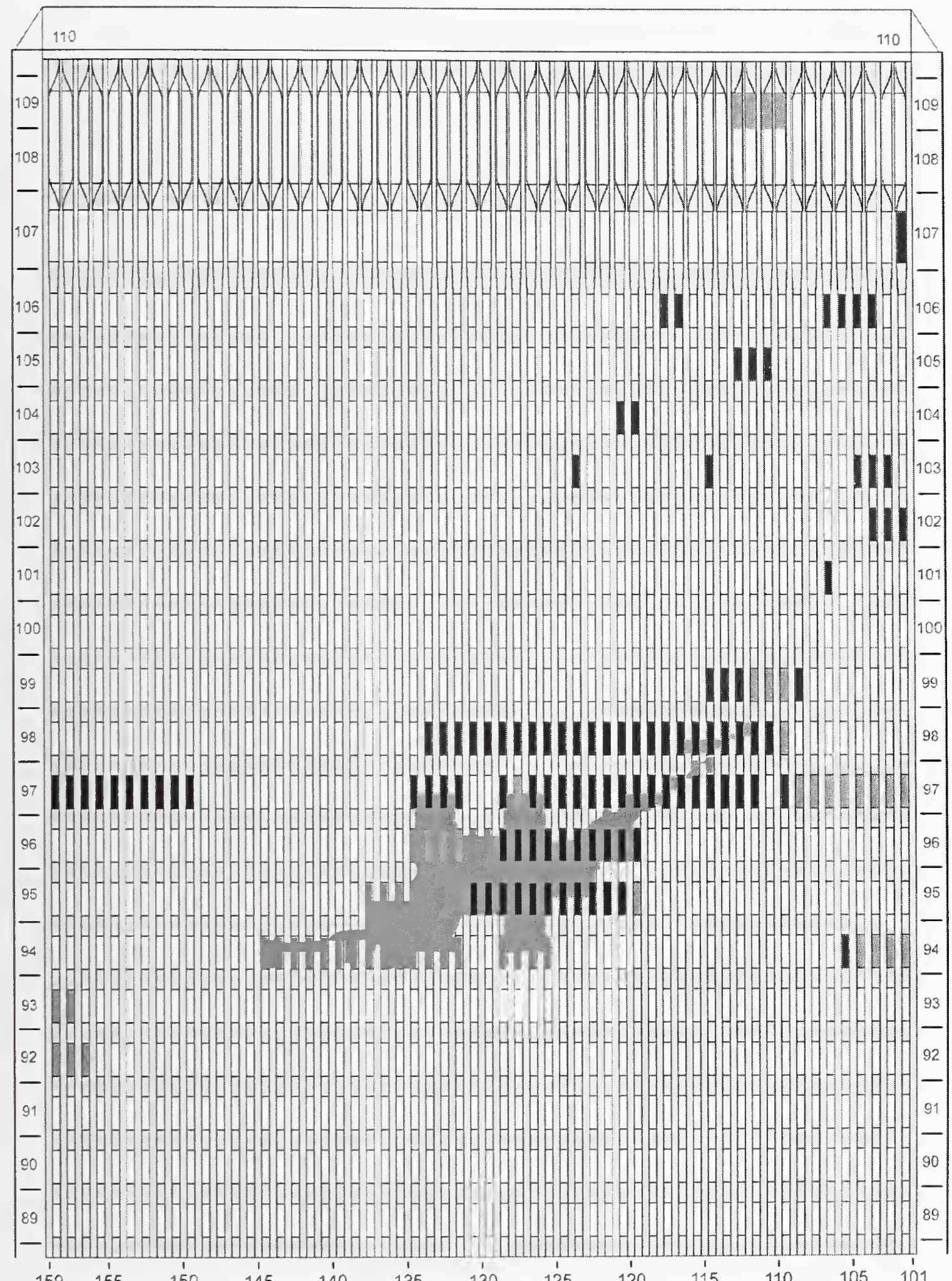

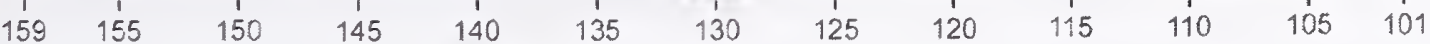

Figure C-36. Diagram of the north face of WTC 1 for floors 89 to 110 at 9:42 a.m. showing windows where smoke was observed and those that were hidden from view. 
WTC 1, North Face

9:46 a.m.

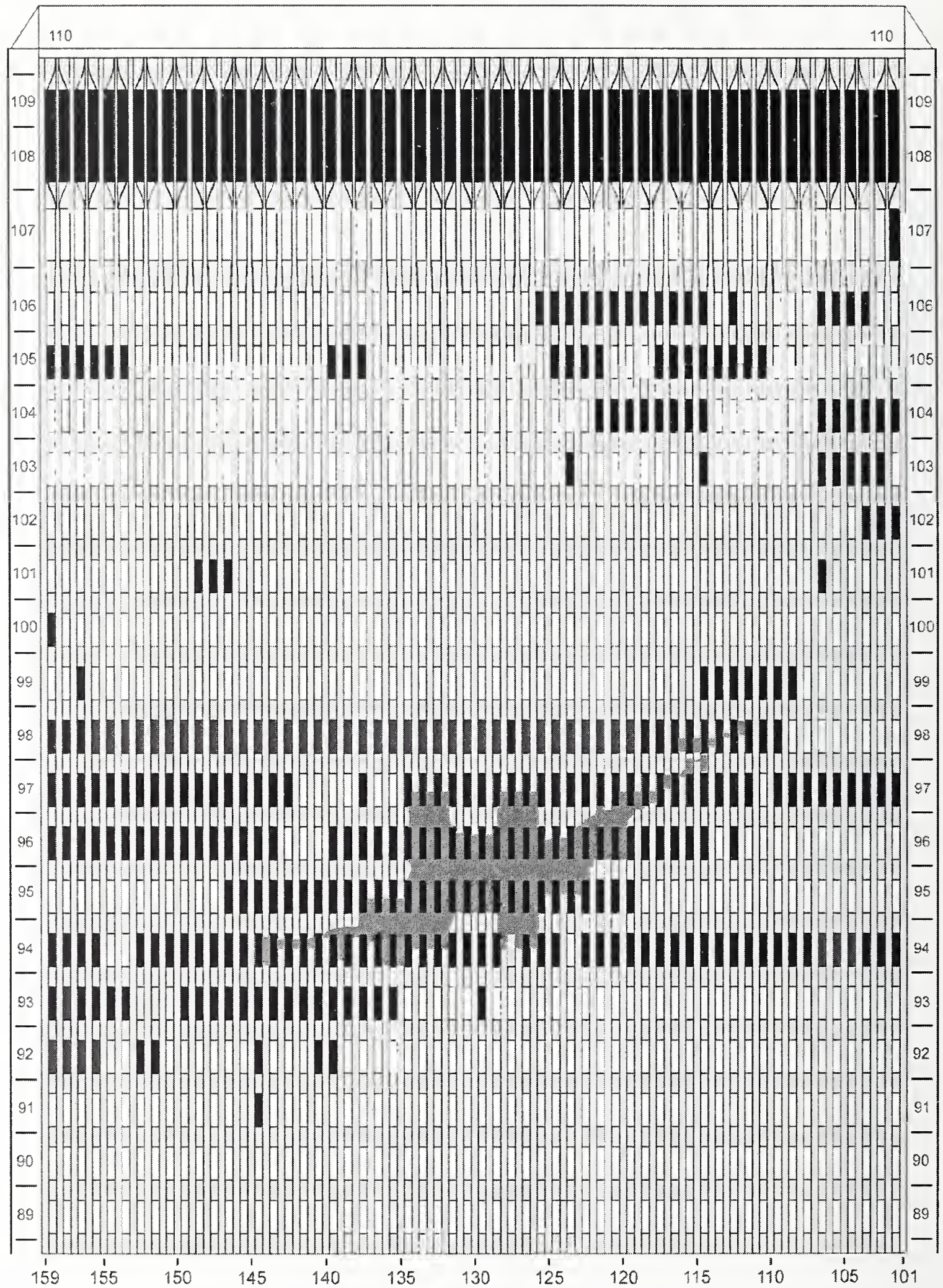

Figure C-37. Diagram of the north face of WTC 1 for floors 89 to 110 at 9:46 a.m. showing the condition of windows and locations of fires. 


$$
\text { WTC 1, North Face 9:46 a.m. }
$$

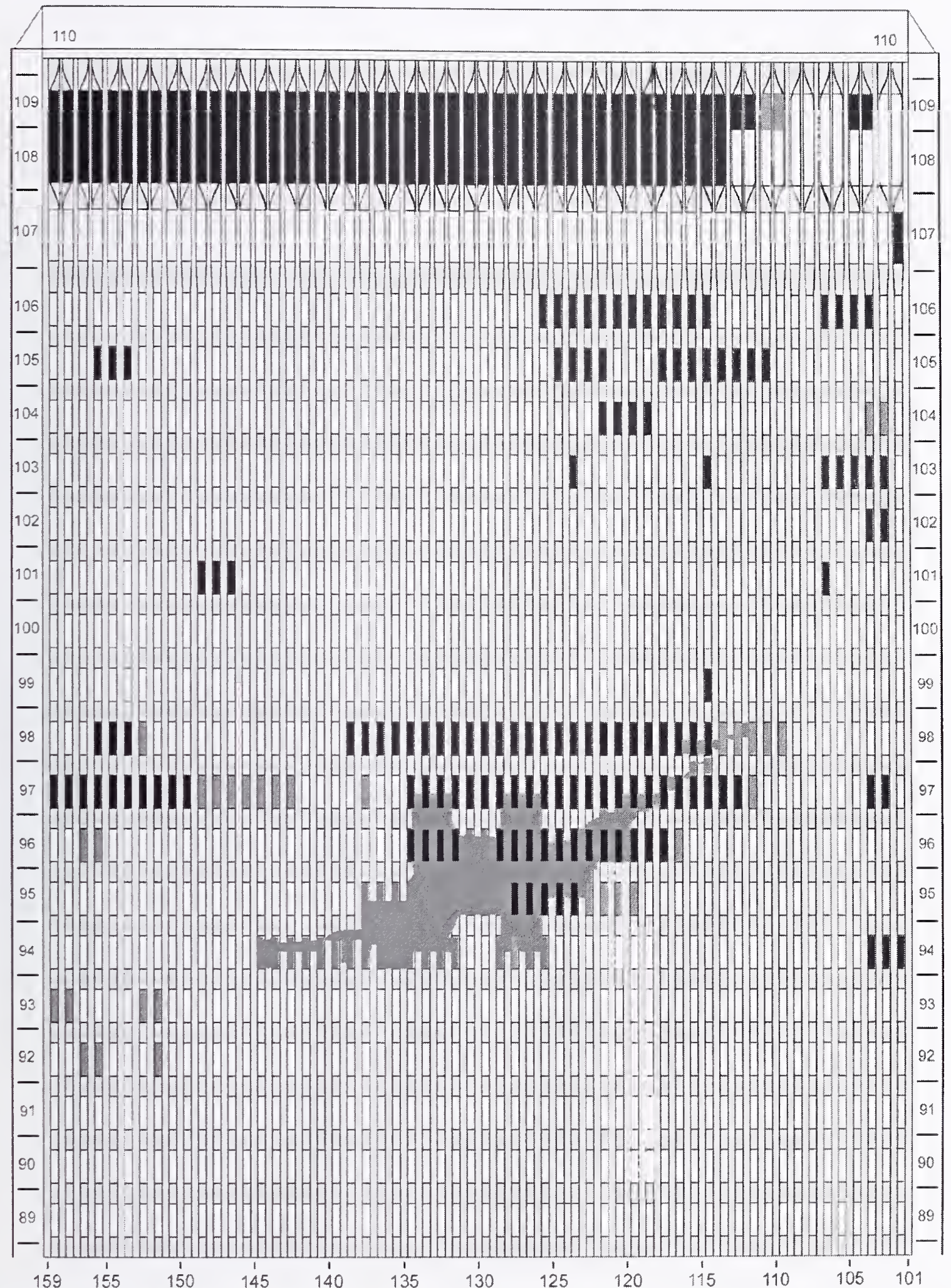

Figure C-38. Diagram of the north face of WTC 1 for floors 89 to 110 at 9:46 a.m. showing windows where smoke was observed and those that were hidden from view. 


$$
\text { WTC 1, North Face 9:52 a.m. }
$$

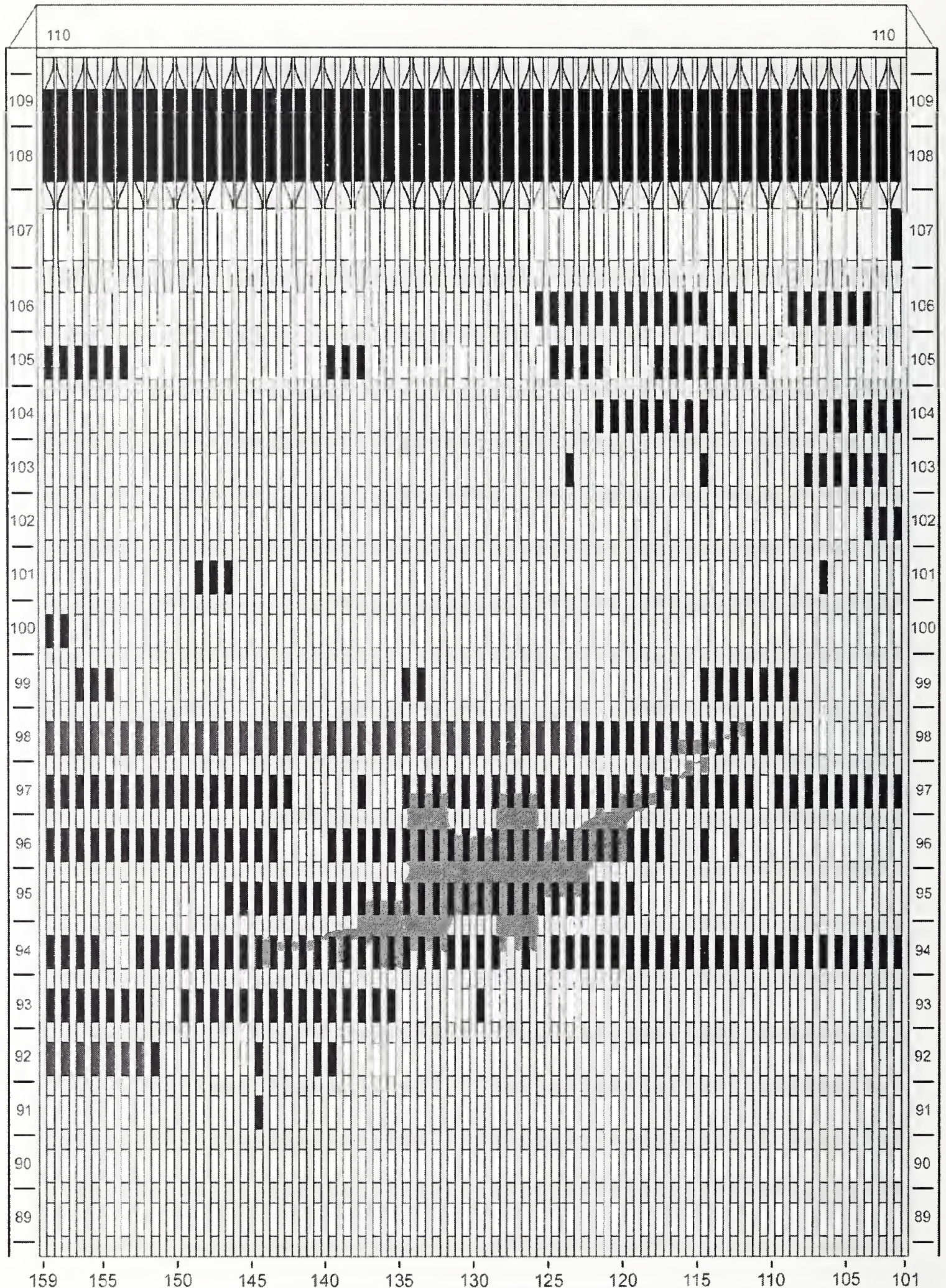

Figure C-39. Diagram of the north face of WTC 1 for floors 89 to 110 at 9:52 a.m. showing the condition of windows and locations of fires. 


$$
\text { WTC 1, North Face 9:52 a.m. }
$$

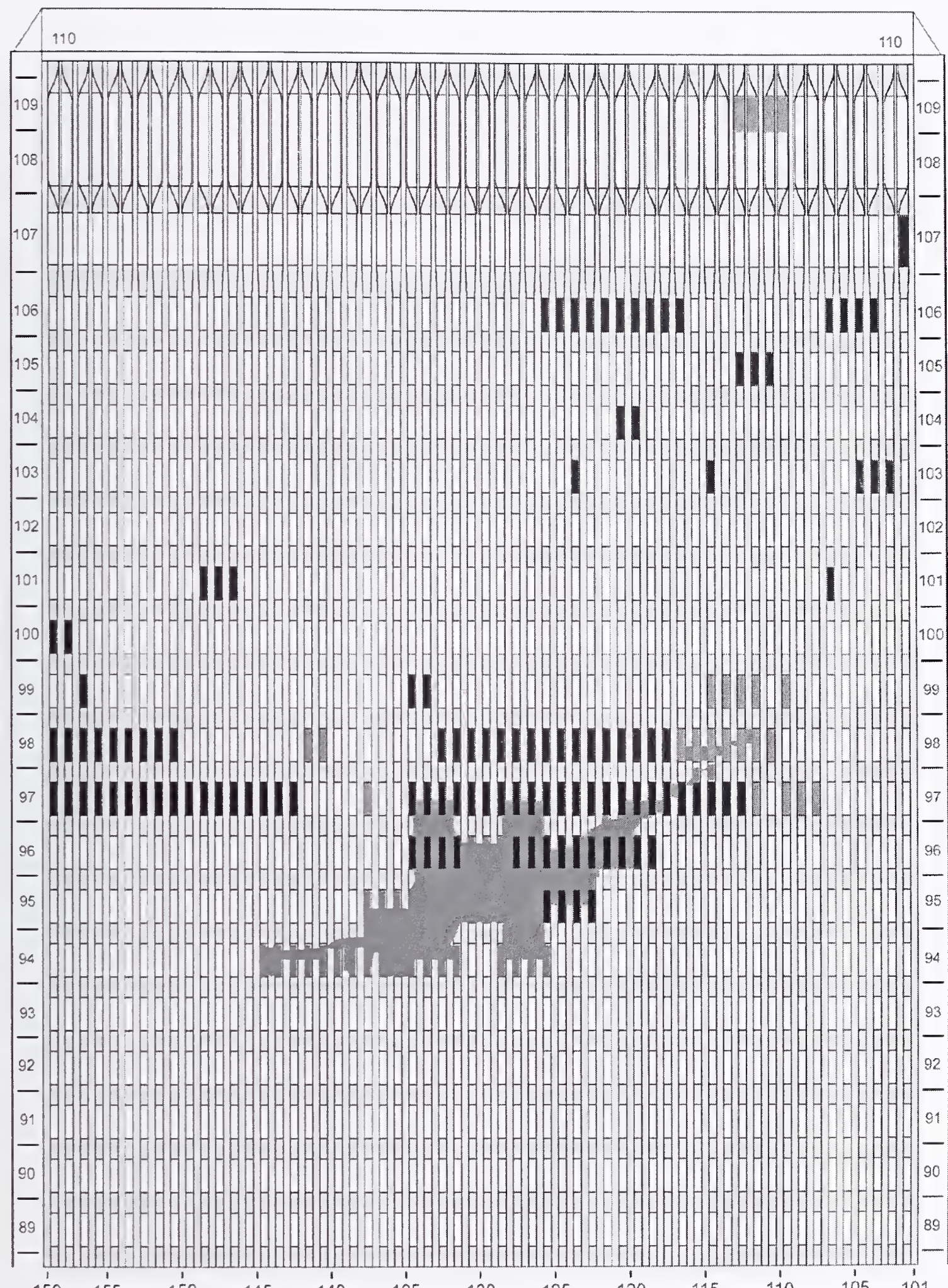

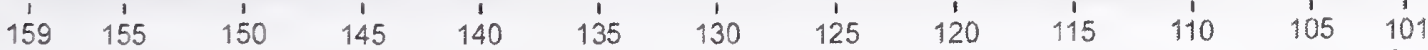

Figure C-40. Diagram of the north face of WTC 1 for floors 89 to 110 at 9:52 a.m. showing windows where smoke was observed and those that were hidden from view. 
WTC 1, North Face 9:54 a.m.

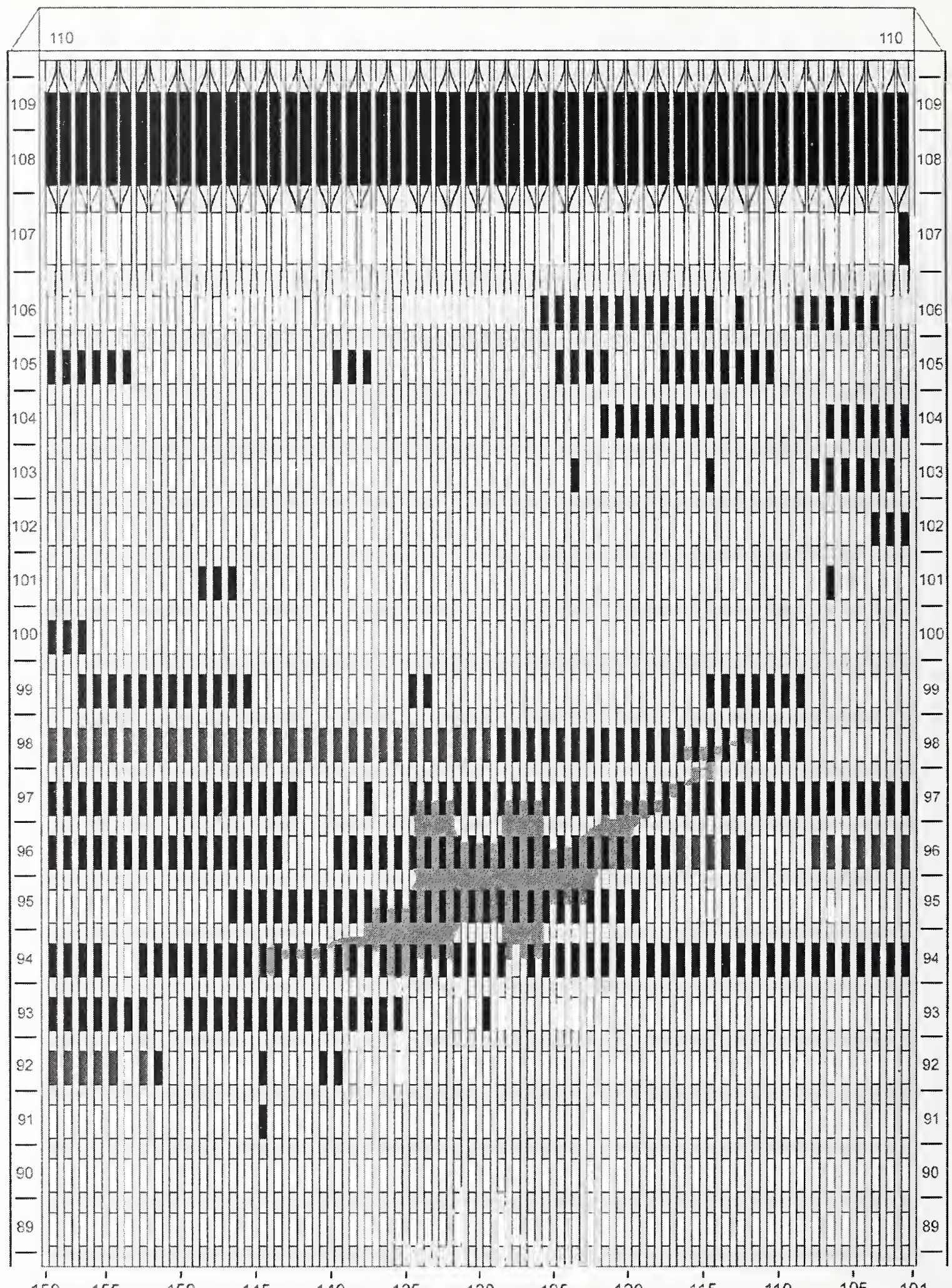

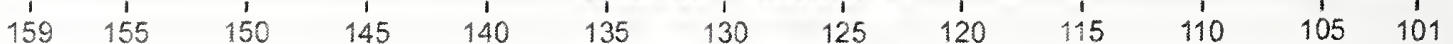

Figure C-41. Diagram of the north face of WTC 1 for floors 89 to 110 at 9:54 a.m. showing the condition of windows and locations of fires. 


$$
\text { WTC 1, North Face 9:54 a.m. }
$$

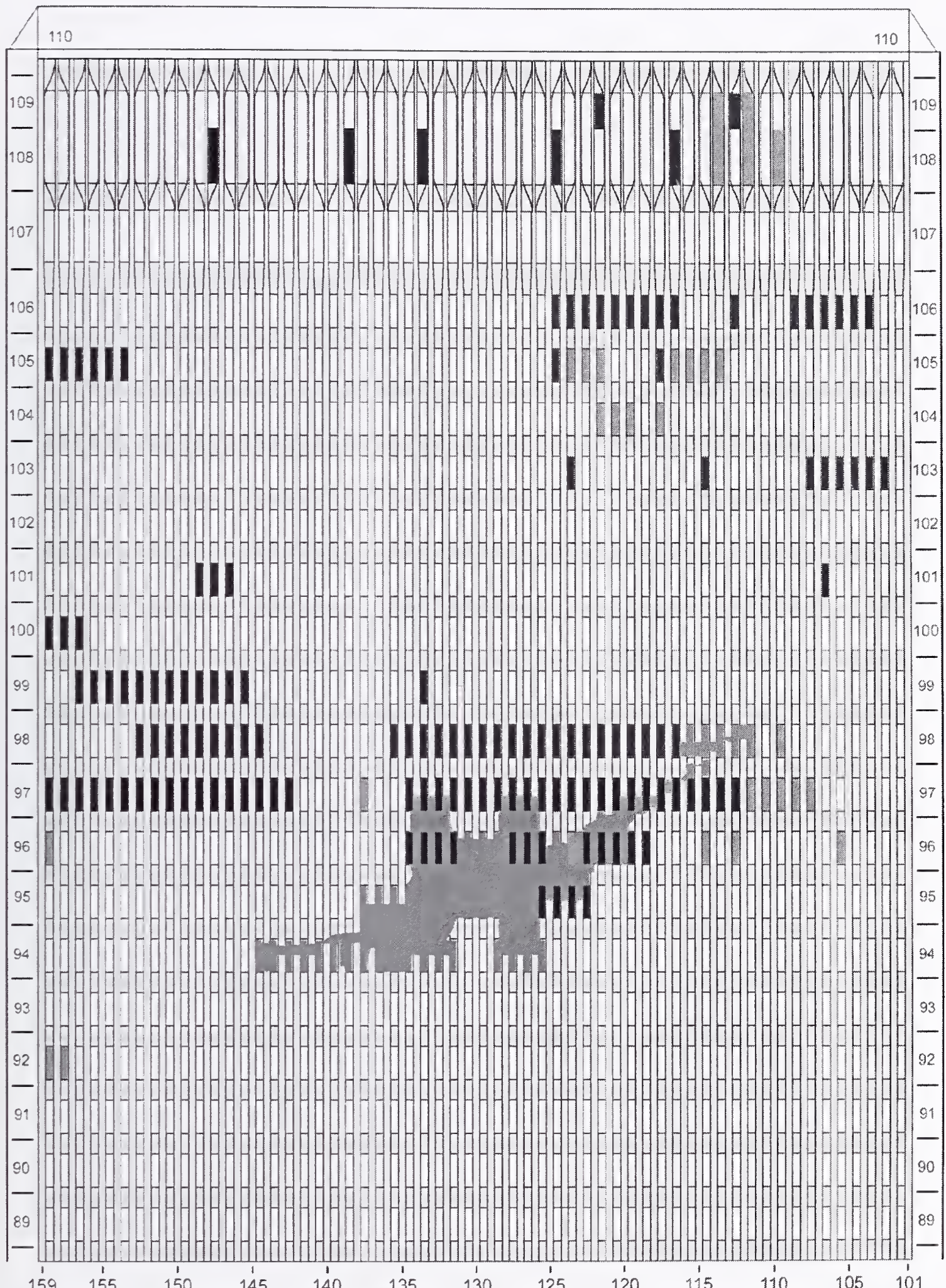

Figure C-42. Diagram of the north face of WTC 1 for floors 89 to 110 at $9: 54 \mathrm{a} . \mathrm{m}$. showing windows where smoke was observed and those that were hidden from view. 


\section{WTC 1, North Face $\quad$ 9:58 a.m.}

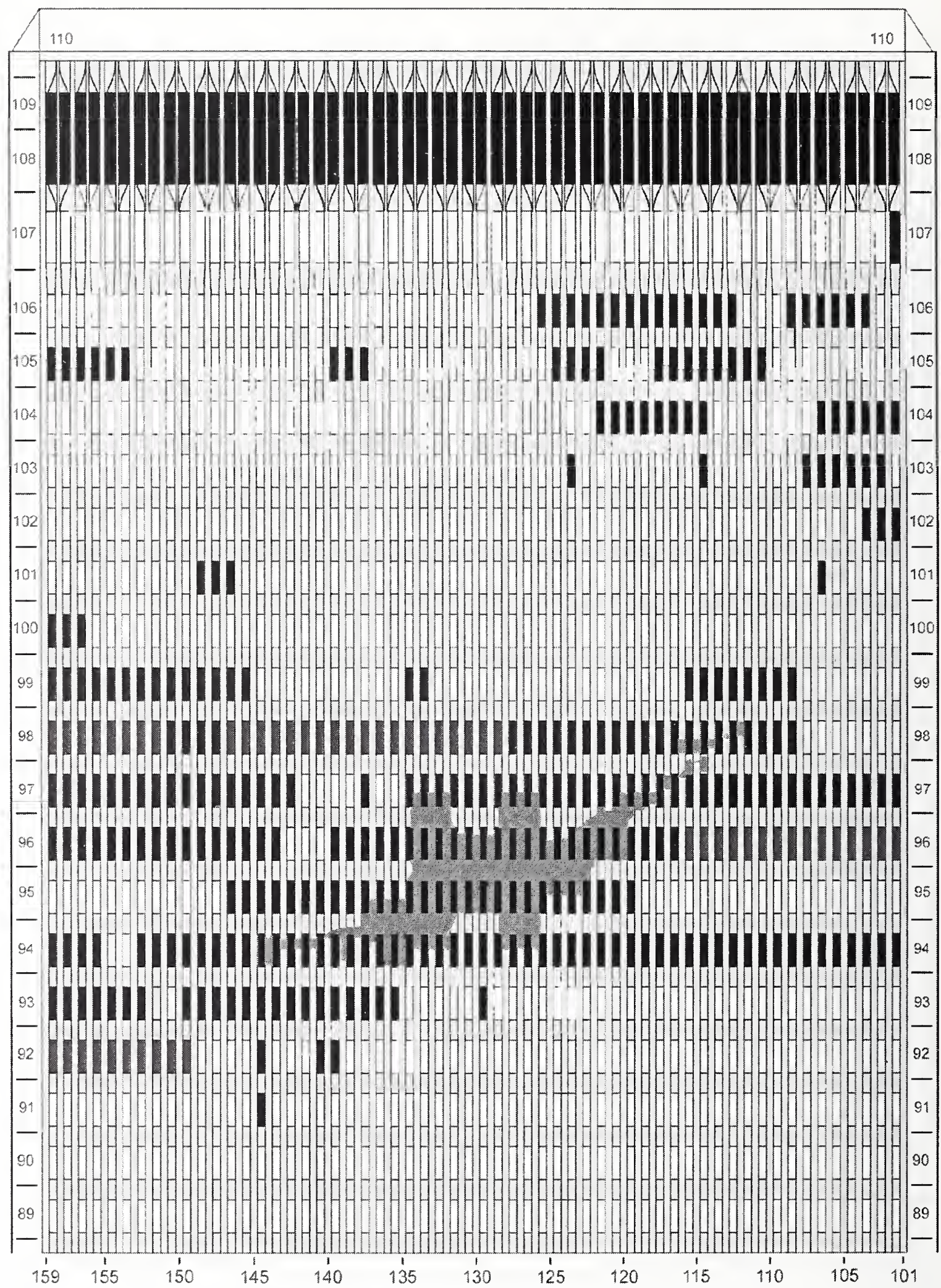

Figure C-43. Diagram of the north face of WTC 1 for floors 89 to 110 at 9:58 a.m. showing the condition of windows and locations of fires. 


$$
\text { WTC 1, North Face 9:58 a.m. }
$$

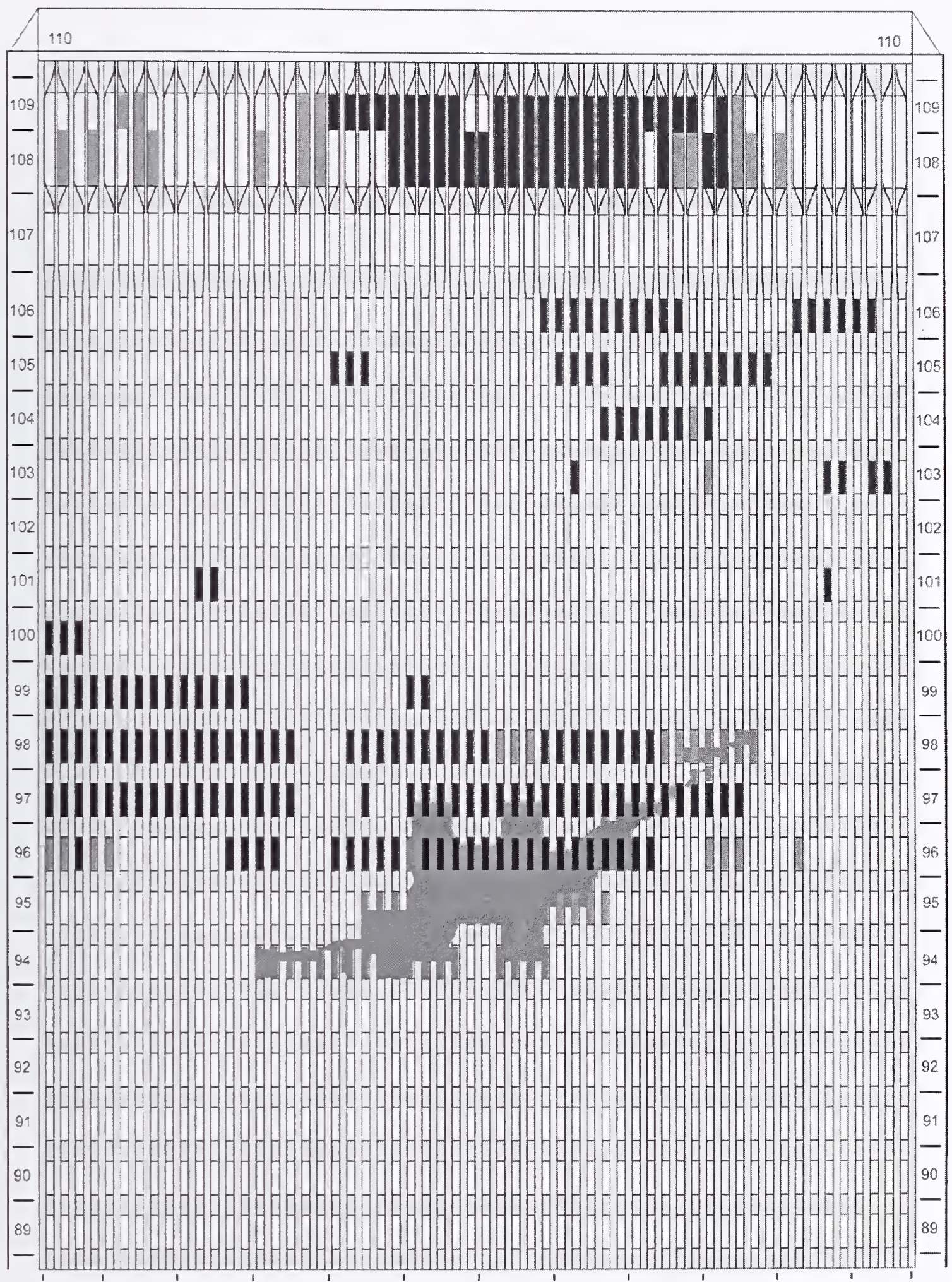

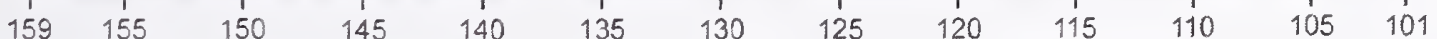

Figure C-44. Diagram of the north face of WTC 1 for floors 89 to 110 at 9:58 a.m. showing windows where smoke was observed and those that were hidden from view. 


\section{WTC 1, North Face 10:04 a.m.}

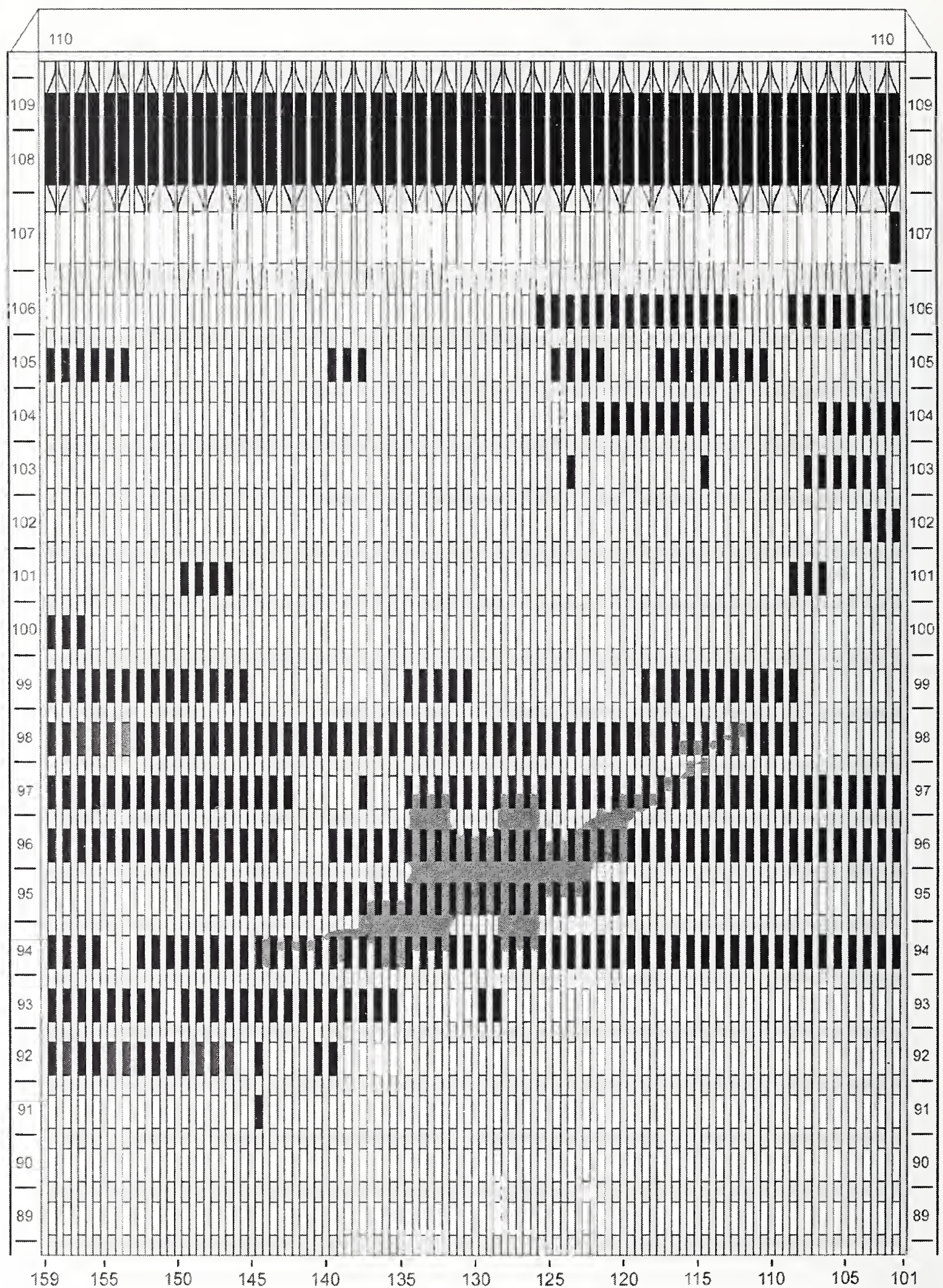

Figure C-45. Diagram of the north face of WTC 1 for floors 89 to 110 at 10:04 a.m. showing the condition of windows and locations of fires. 


$$
\text { WTC 1, North Face 10:04 a.m. }
$$

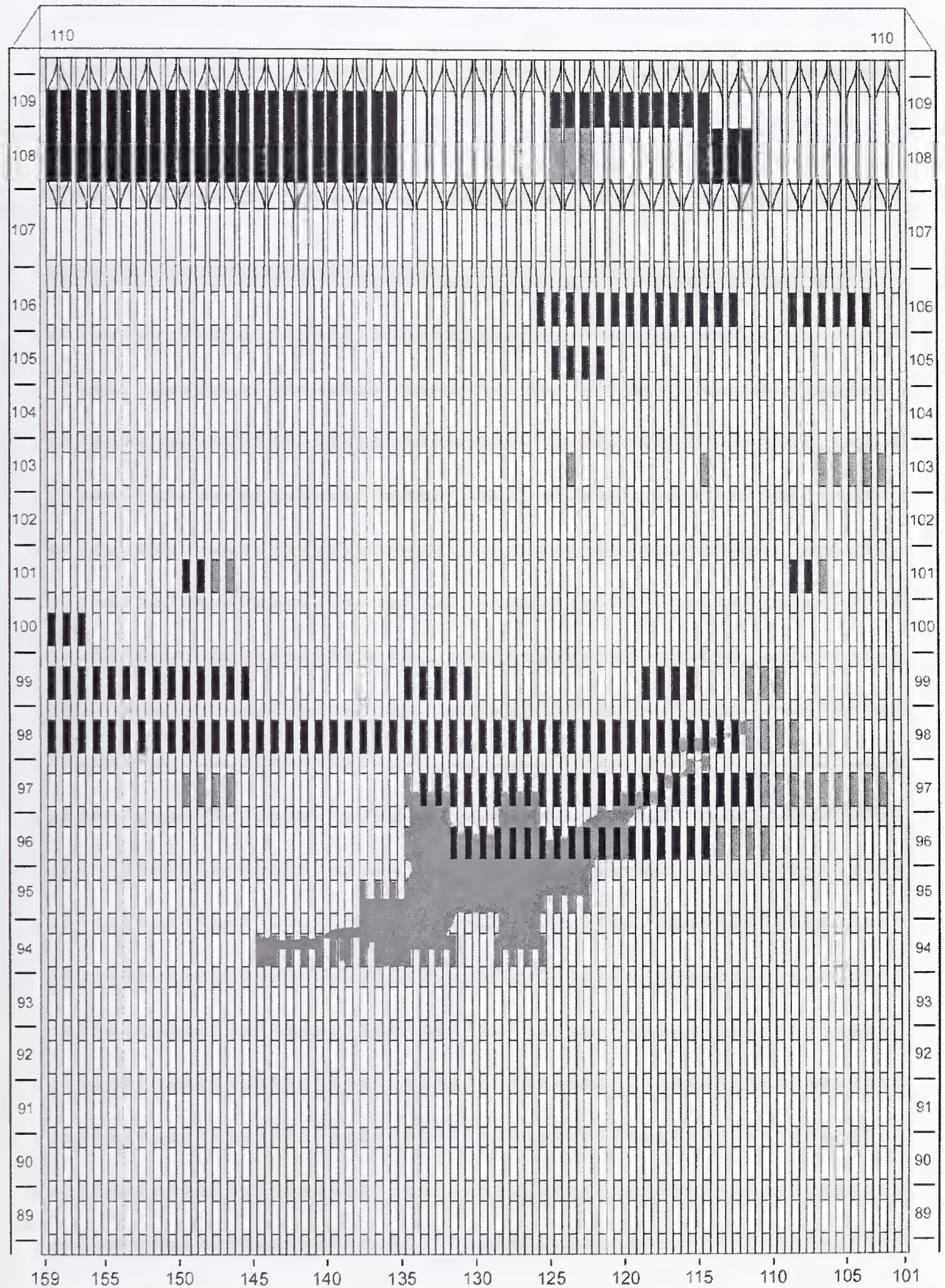

Figure C-46. Diagram of the north face of WTC 1 for floors 89 to 110 at 10:04 a.m. showing windows where smoke was observed and those that were hidden from view. 


\section{WTC 1, North Face $\quad$ 10:10 a.m.}

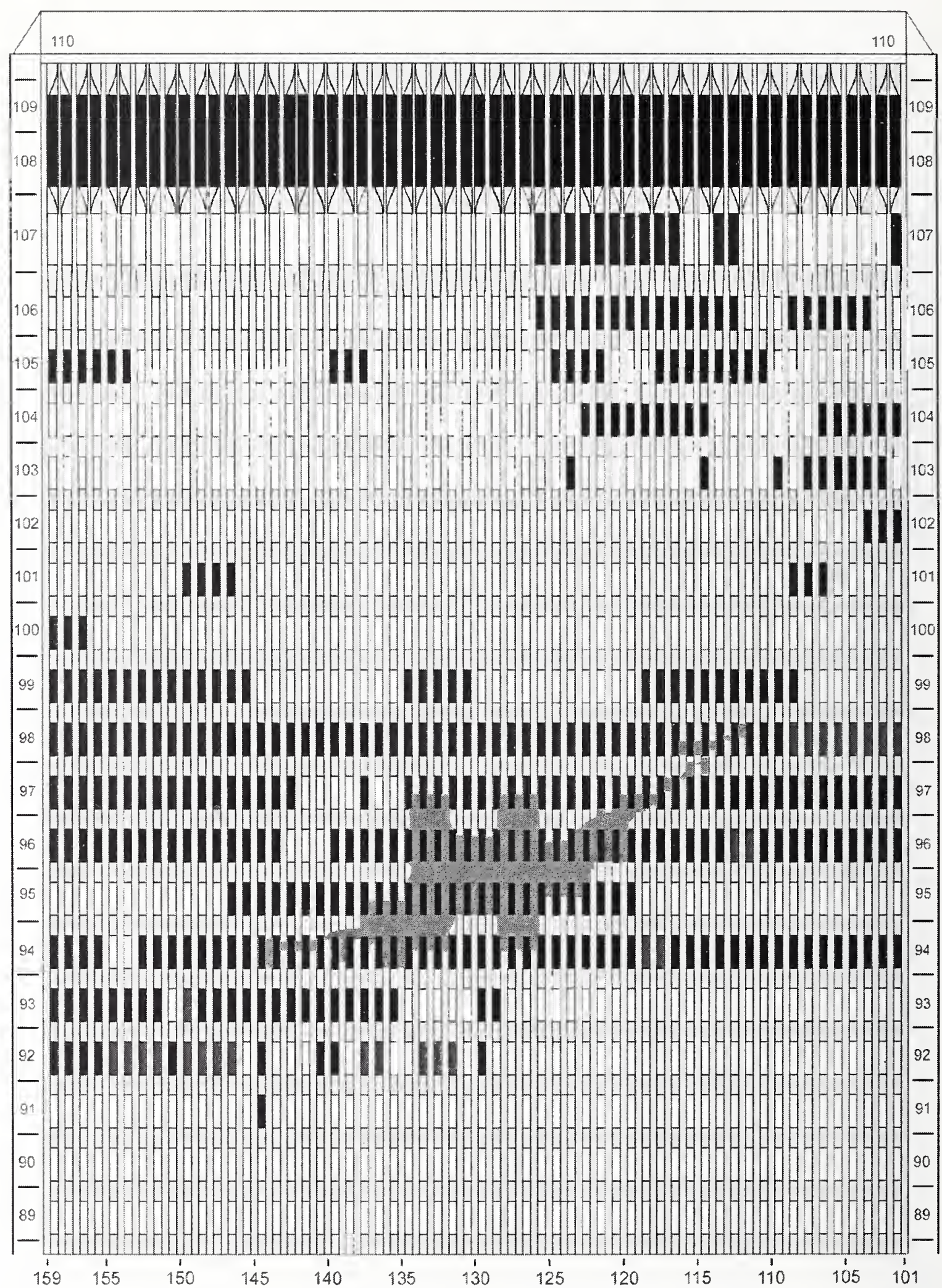

Figure C-47. Diagram of the north face of WTC 1 for floors 89 to 110 at 10:10 a.m. showing the condition of windows and locations of fires. 
WTC 1, North Face 10:10 a.m.

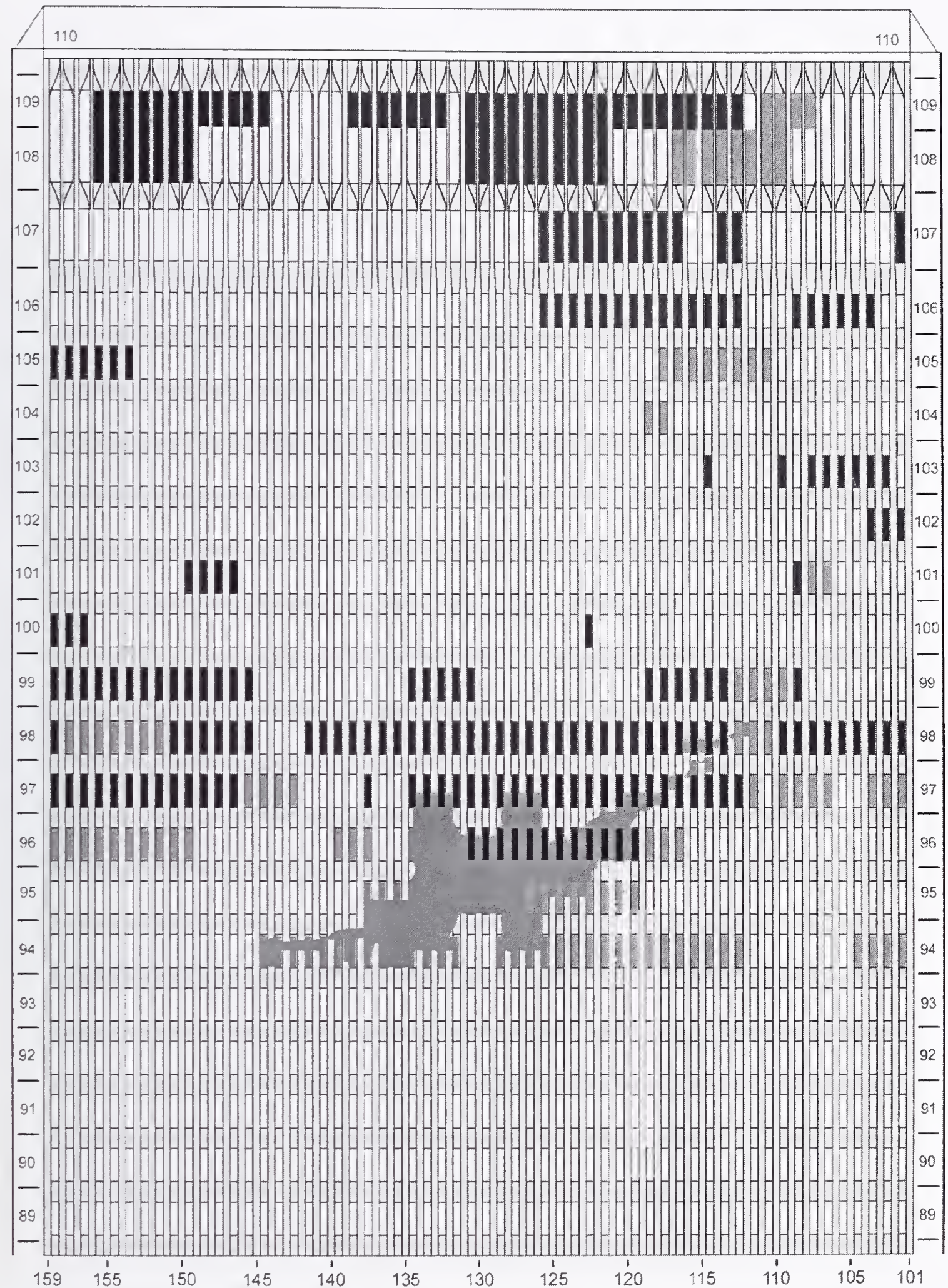

Figure C-48. Diagram of the north face of WTC 1 for floors 89 to 110 at 10:10 a.m. showing windows where smoke was observed and those that were hidden from view. 
WTC 1, North Face $\quad$ 10:14 a.m.

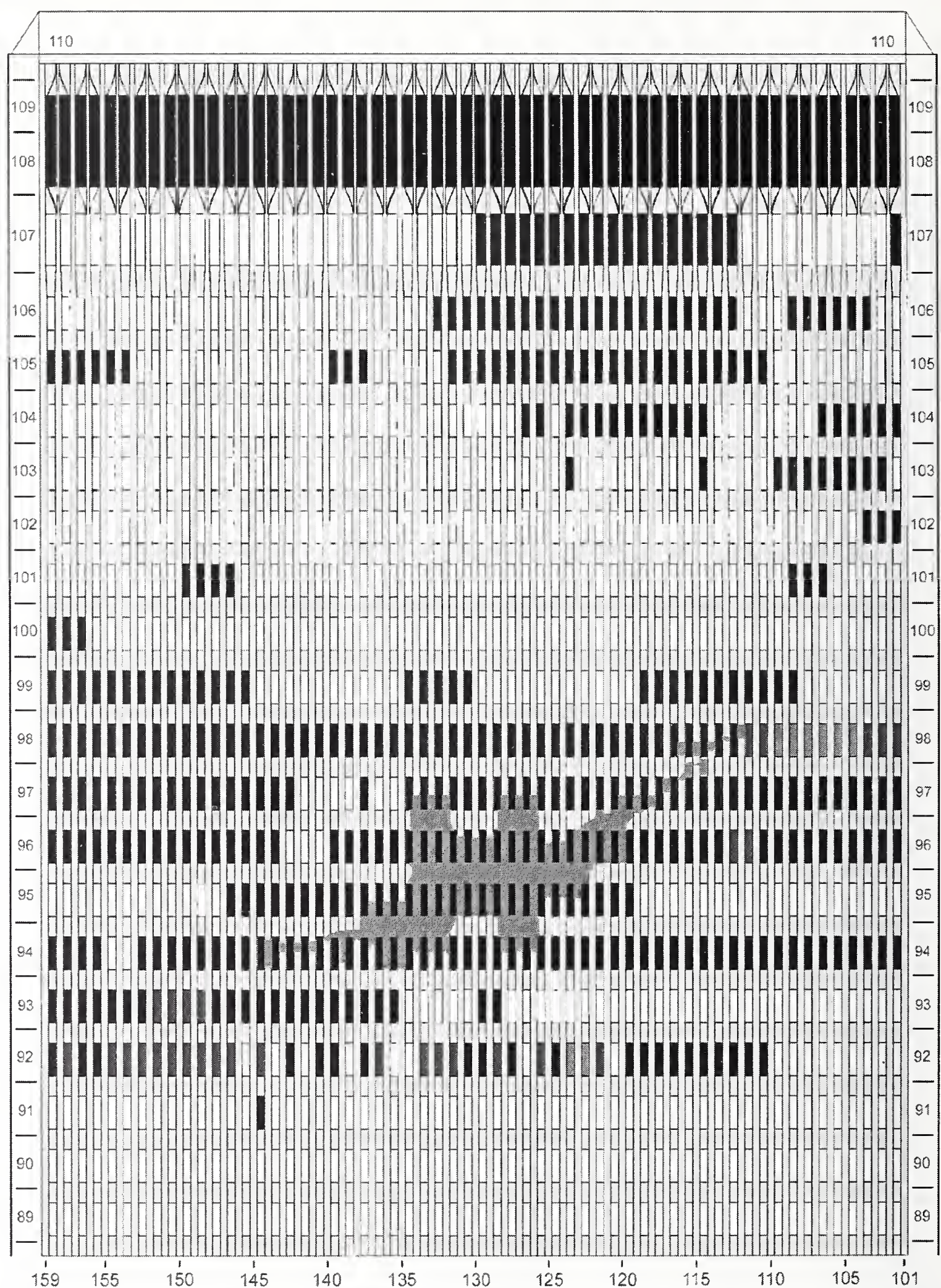

Figure C-49. Diagram of the north face of WTC 1 for floors 89 to 110 at 10:14 a.m. showing the condition of windows and locations of fires. 
WTC 1, North Face $\quad$ 10:14 a.m.

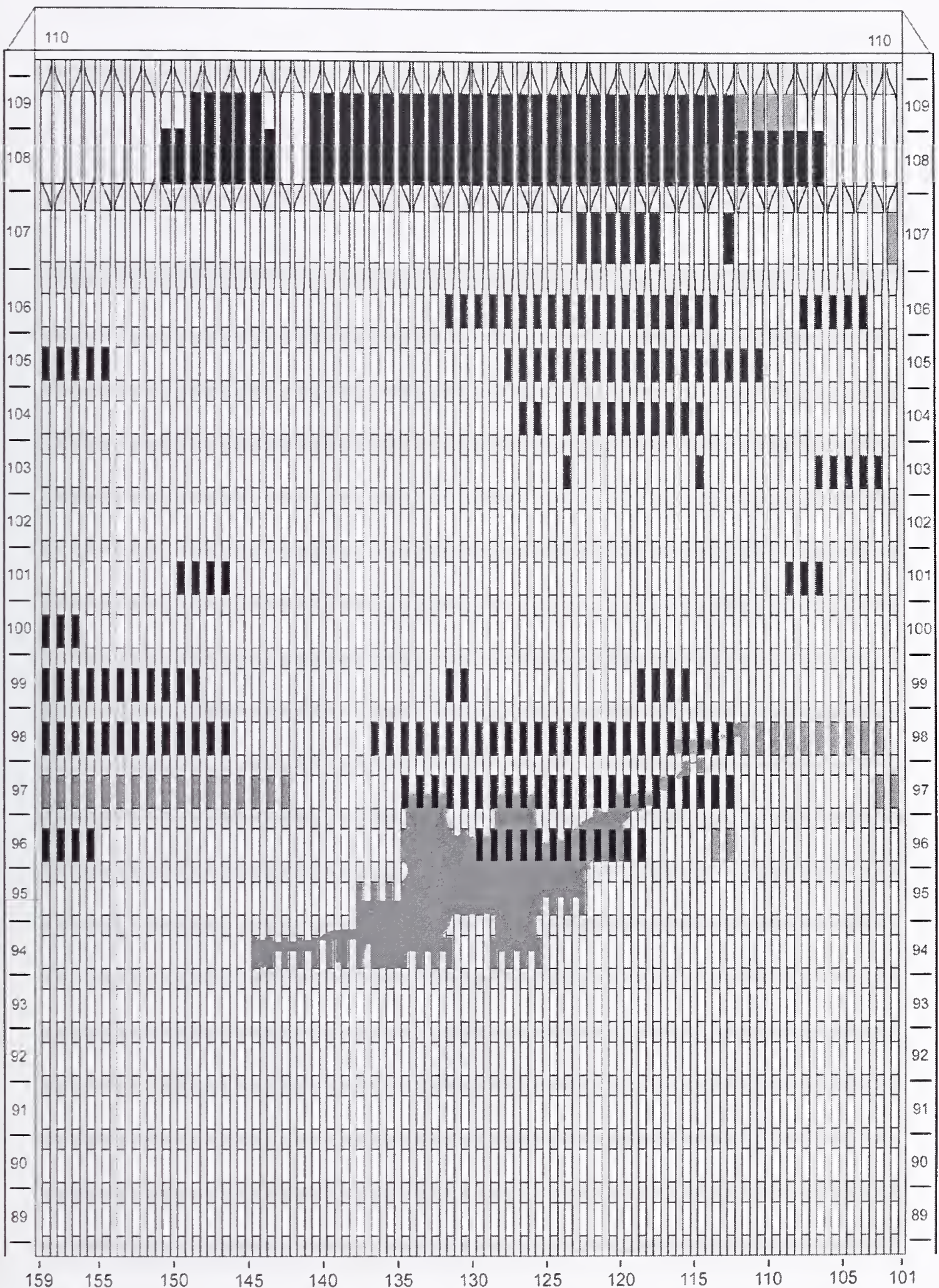

Figure C-50. Diagram of the north face of WTC 1 for floors 89 to 110 at $10: 14$ a.m. showing windows where smoke was observed and those that were hidden from view. 
WTC 1, North Face 10:18 a.m.

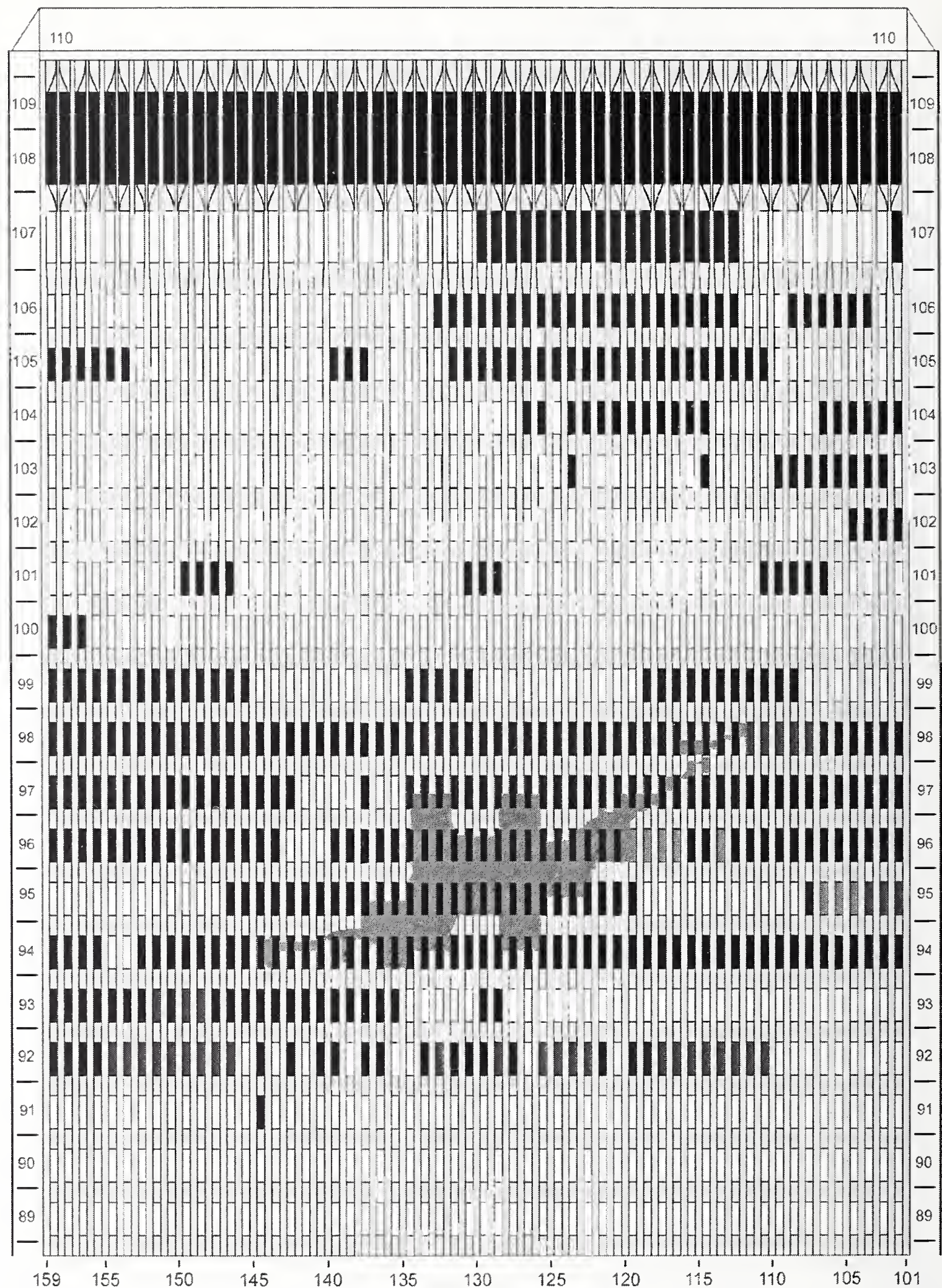

Figure C-51. Diagram of the north face of WTC 1 for floors 89 to 110 at 10:18 a.m. showing the condition of windows and locations of fires. 


$$
\text { WTC 1, North Face 10:18 a.m. }
$$

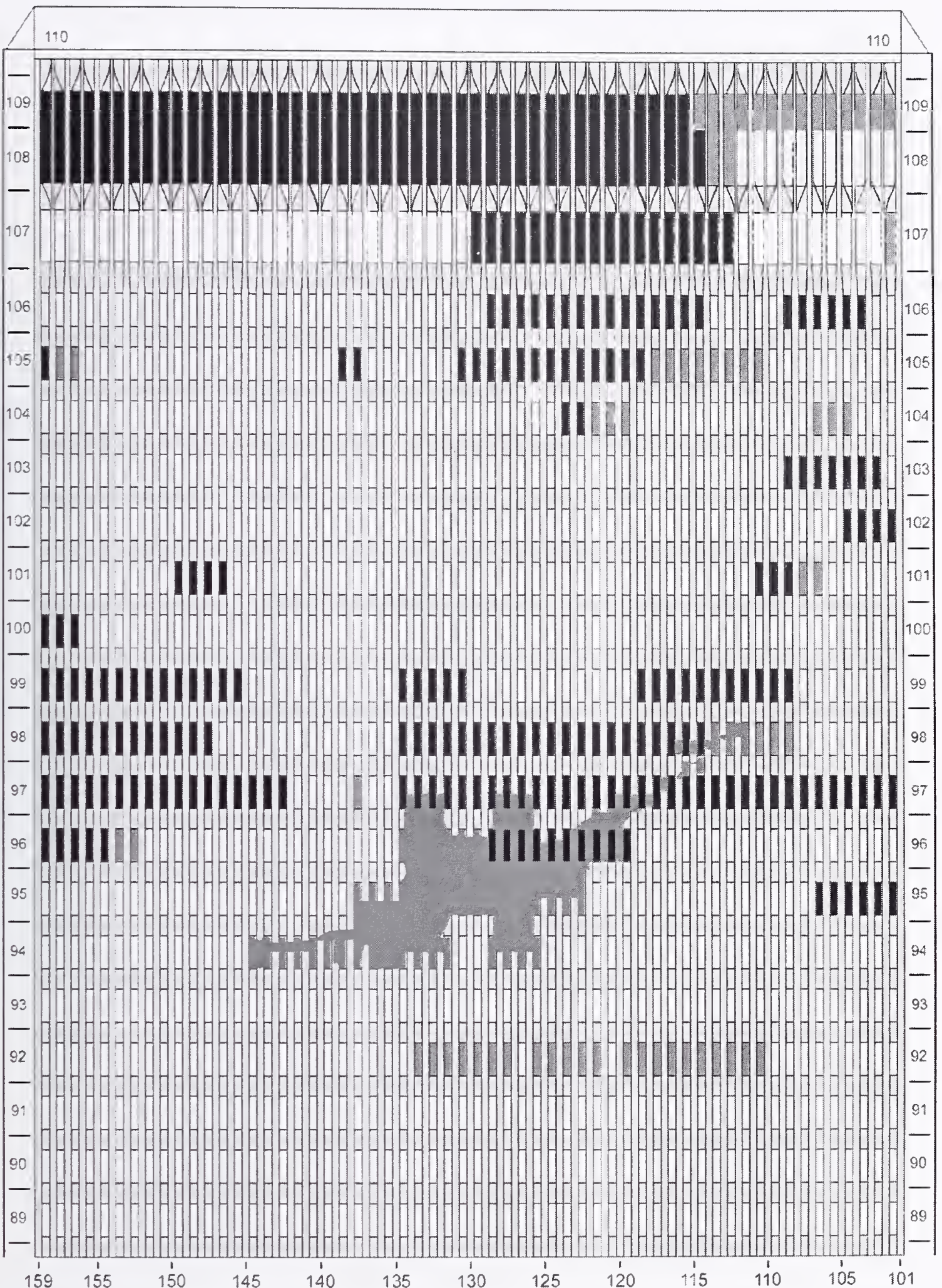

Figure C-52. Diagram of the north face of WTC 1 for floors 89 to 110 at 10:18 a.m. showing windows where smoke was observed and those that were hidden from view. 


\section{WTC 1, North Face 10:20 a.m.}

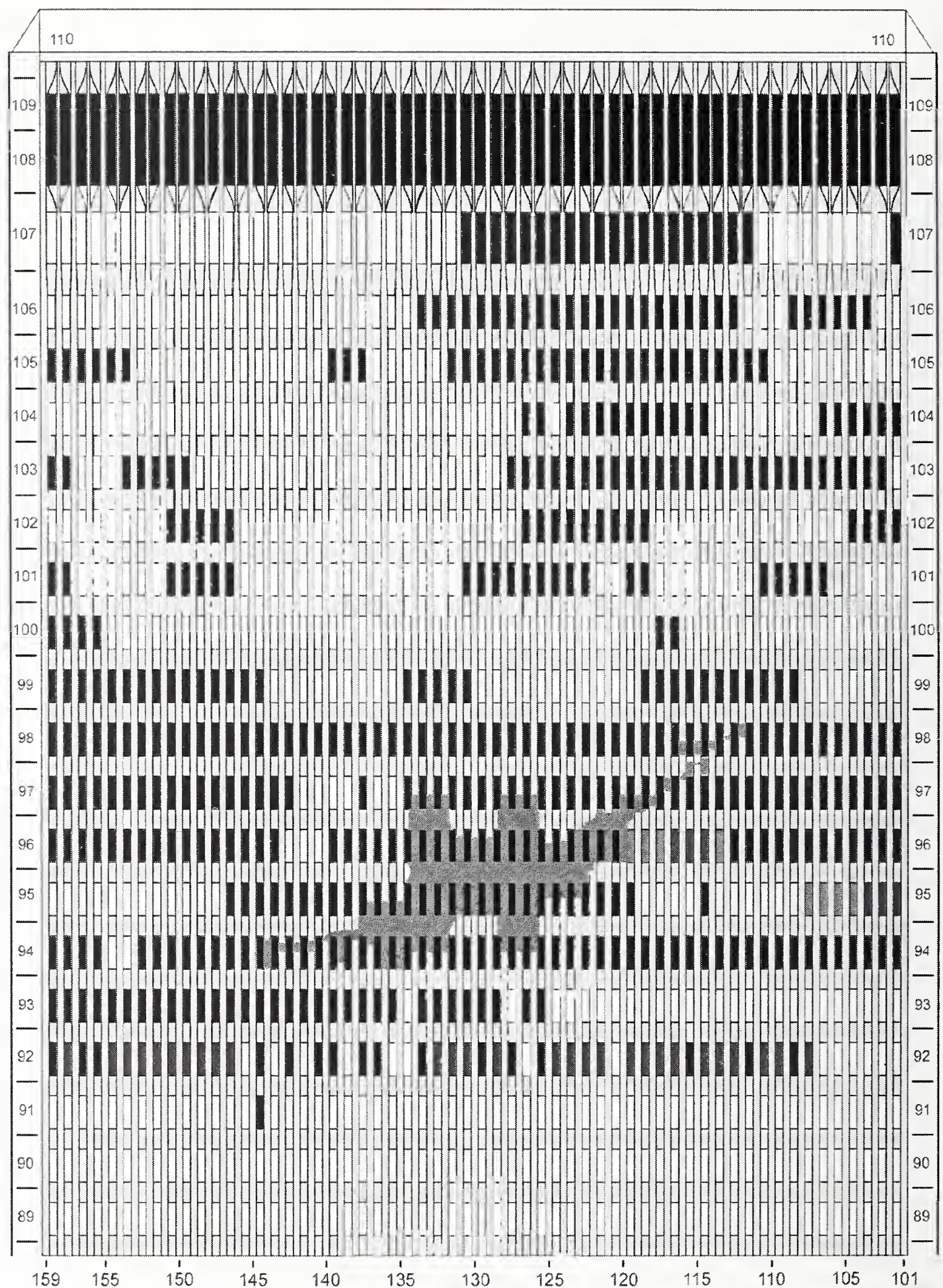

Figure C-53. Diagram of the north face of WTC 1 for floors 89 to 110 at 10:20 a.m. showing the condition of windows and locations of fires. 


$$
\text { WTC 1, North Face 10:20 a.m. }
$$

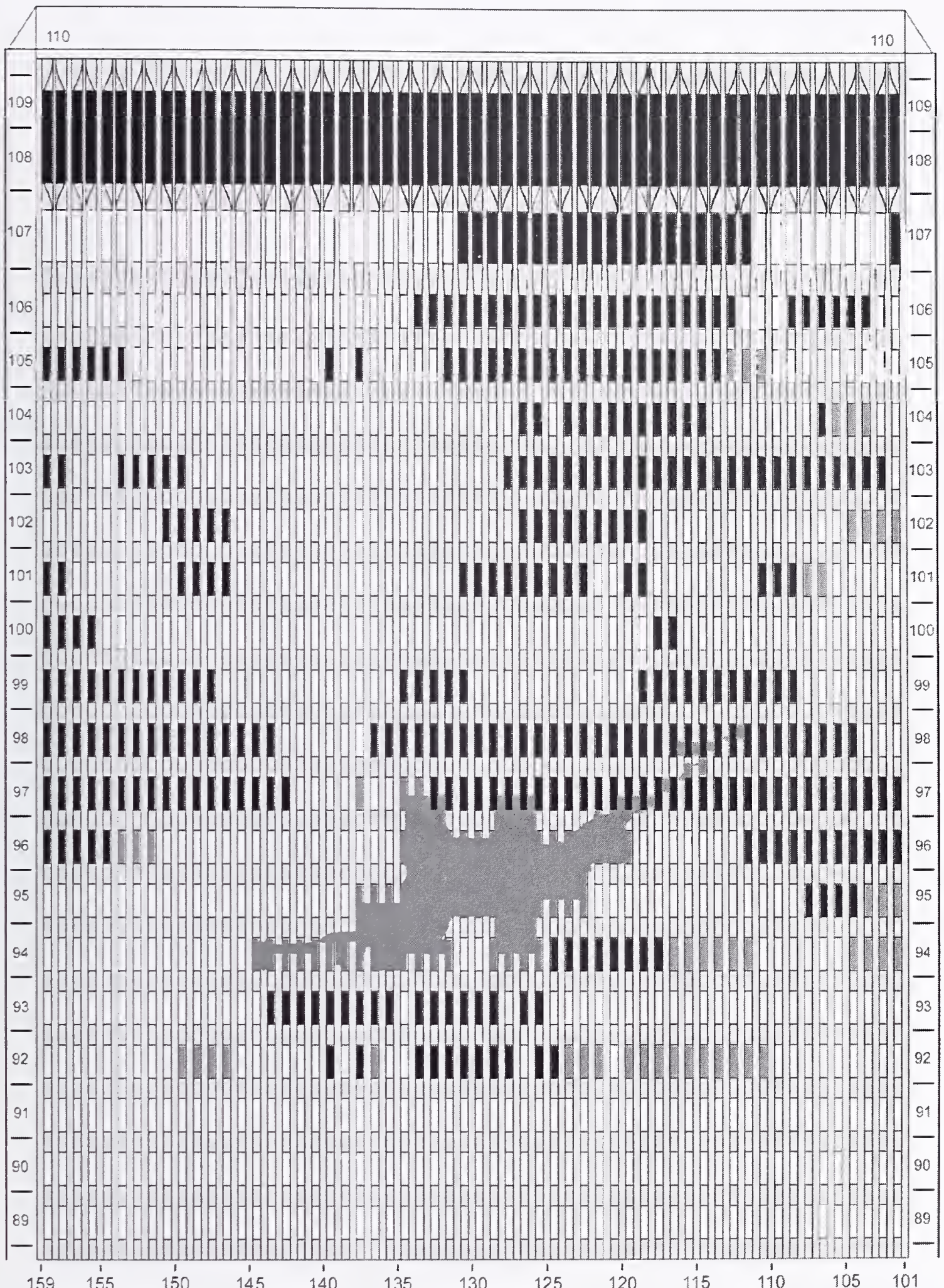

Figure C-54. Diagram of the north face of WTC 1 for floors 89 to 110 at 10:20 a.m. showing windows where smoke was observed and those that were hidden from view. 
WTC 1, North Face 10:24 a.m.

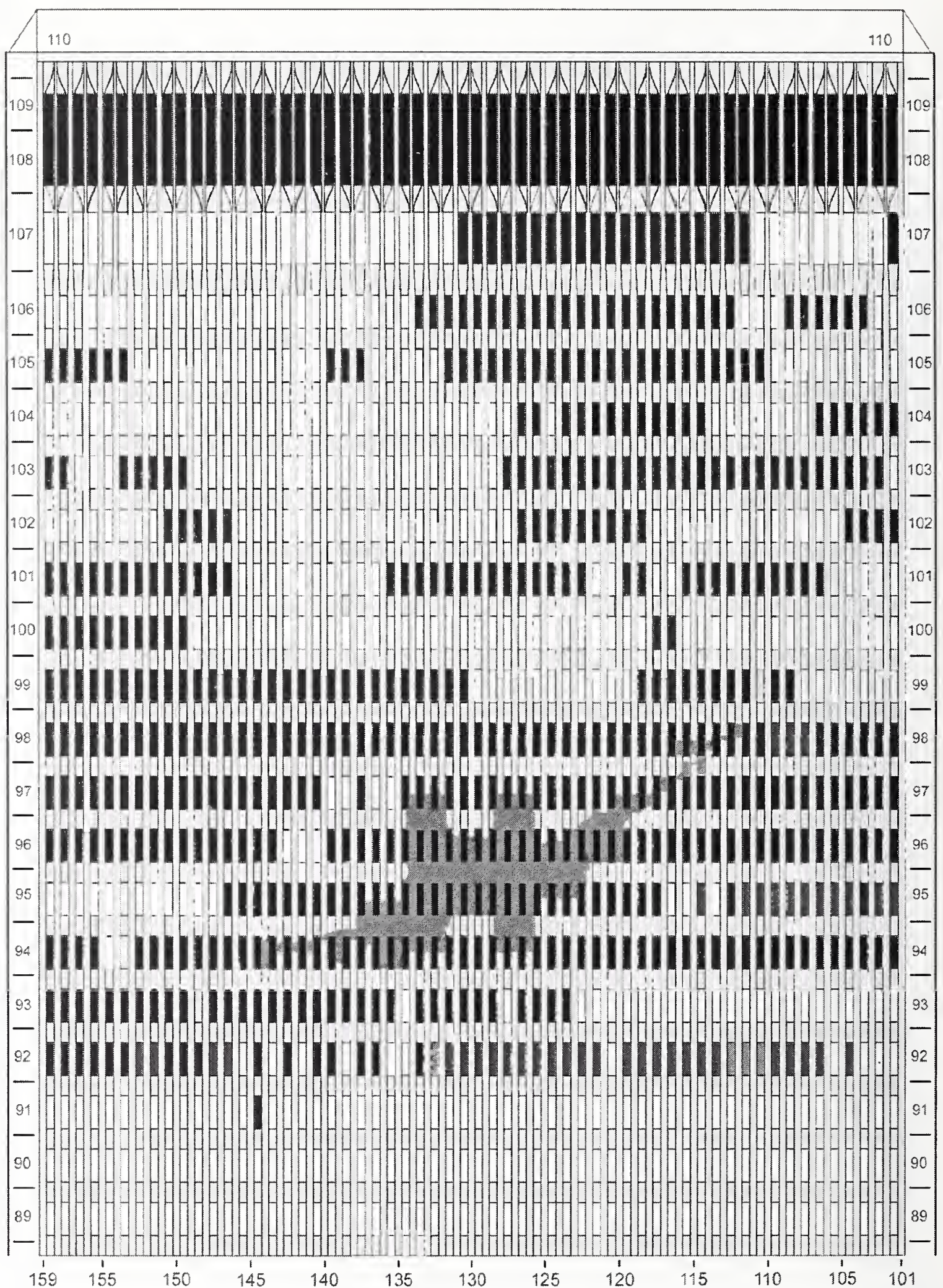

Figure C-55. Diagram of the north face of WTC 1 for floors 89 to 110 at 10:24 a.m. showing the condition of windows and locations of fires. 


$$
\text { WTC 1, North Face 10:24 a.m. }
$$

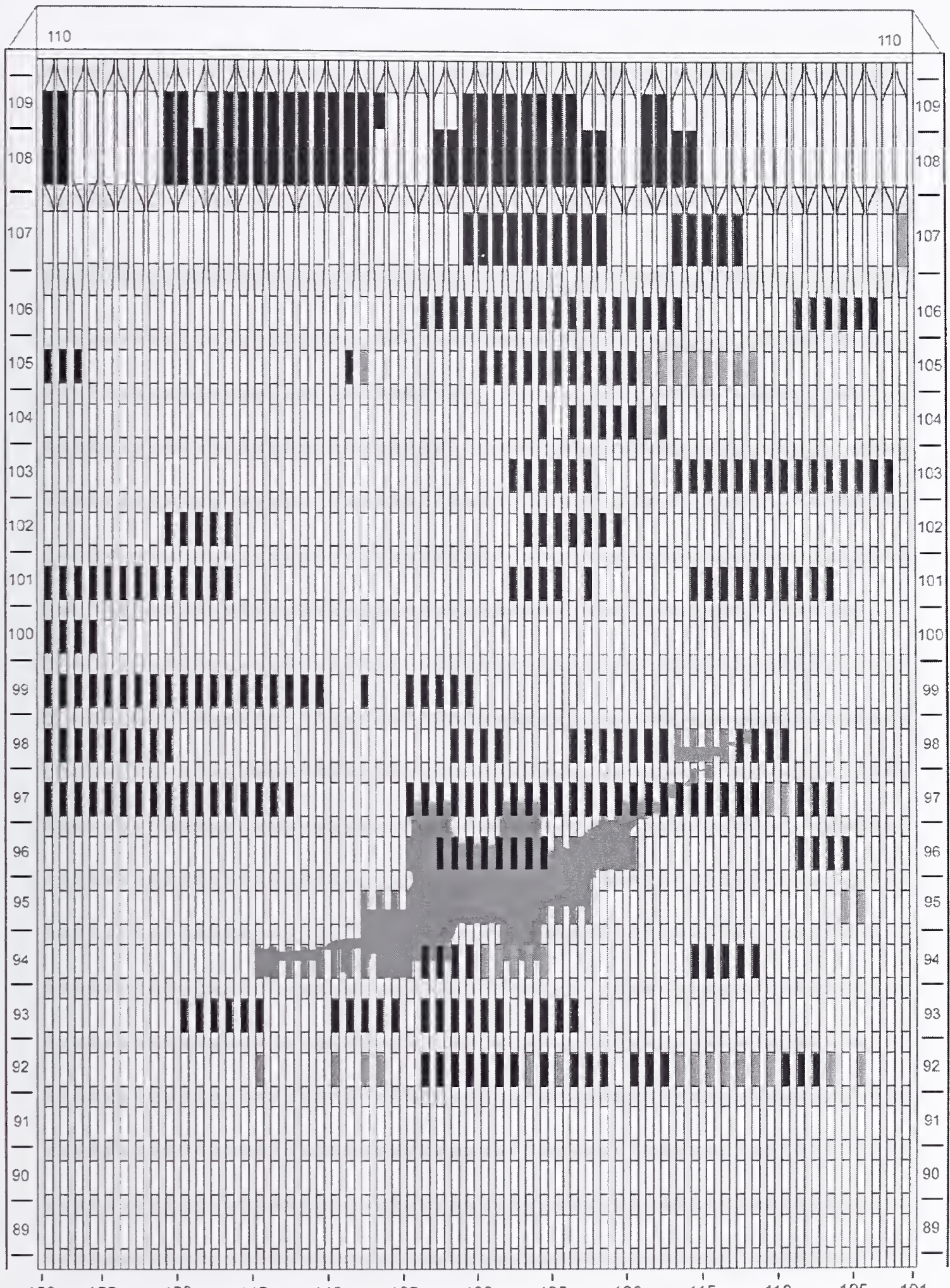

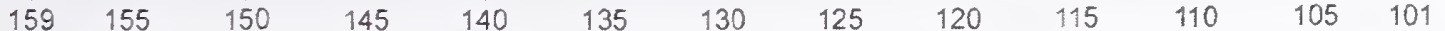

Figure C-56. Diagram of the north face of WTC 1 for floors 89 to 110 at 10:24 a.m. showing windows where smoke was observed and those that were hidden from view. 


$$
\text { WTC 1, North Face } \quad 10: 28 \text { a.m. }
$$

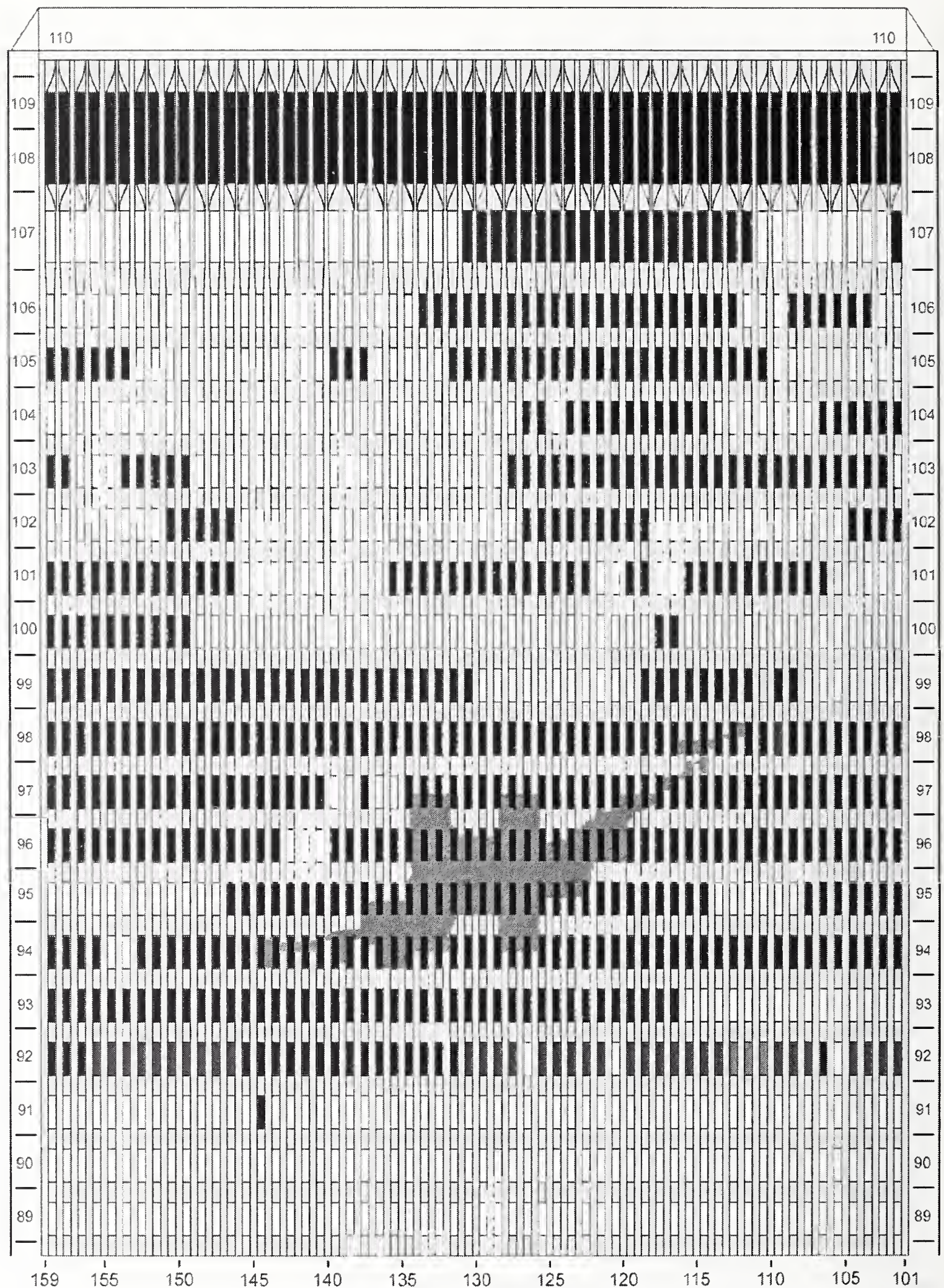

Figure C-57. Diagram of the north face of WTC 1 for floors 89 to 110 at 10:28 a.m. showing the condition of windows and locations of fires. 


$$
\text { WTC 1, North Face 10:28 a.m. }
$$

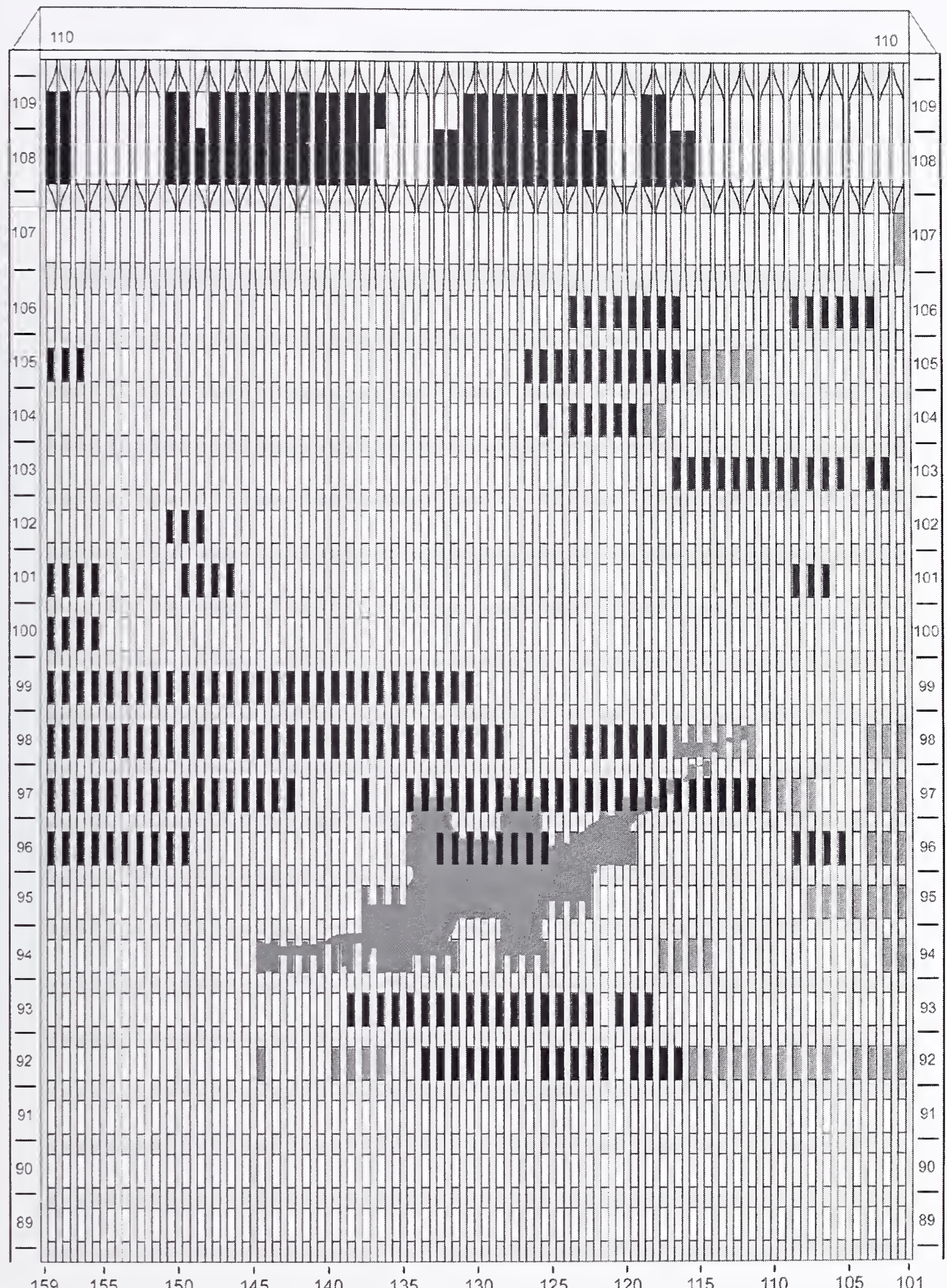

Figure C-58. Diagram of the north face of WTC 1 for floors 89 to 110 at 10:28 a.m. showing windows where smoke was observed and those that were hidden from view. 
This page intentionally left blank. 
